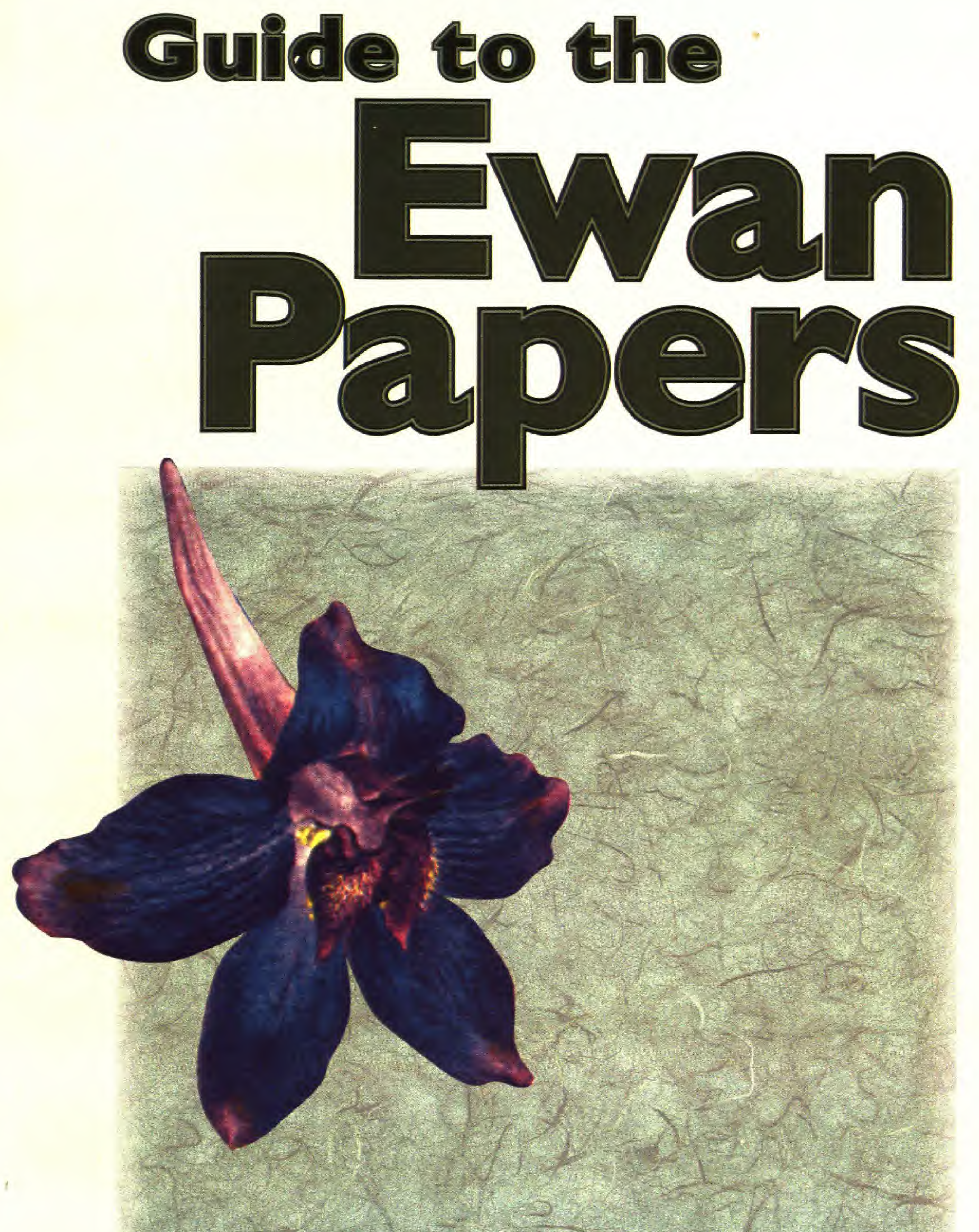

Douglas Holland Martha Riley Mary Stiffler

* Missouri Botanical Garden 


$$
\text { . }
$$




\section{GUIDE to the Ewan Papers}


The cover illustration, based on a water color by Keith West, is Delphinium treleasei Bush ex K. C. Davis, an Orzark endemic that reflects the Ewans' long association with the Missouri Botanical Garden and Joe's early interest in Delphinium. 


\section{Guide to the Ewan Papers}

Compiled By:

Douglas Holland

Martha Riley

Mary Stiffler

MISSOURI BOTANICAL GARDEN PRESS

St. Louis, Missouri 1997

\section{敞。}


Compilation of this Guide was made possible by a grant from the National Historical Publications and Records Commission to the Missouri Botanical Garden.

ISBN 0-915279-45-2

COMPILERS' ADDRESS

Missouri Botanical Garden

P. O. Box 299

St. Louis, Missouri 63166-0299, U.S.A.

Copyright C1997 by Missouri Botanical Garden.

All rights reserved.

Printed in U.S.A. 
No one can be more fully aware than we are of the numerous imperfections in our records. Such a work must of necessity contain errors both of commission and of omission, and few who consult it can form an adequate opinion of the labour involved in collecting information which, when set down, may appear of the most meagre character.

James Britten and G. S. Boulger, A Biographical Index of British and Irish Botanists, 1893 



\section{Table of Contents}

Preface $\quad \ldots \ldots \ldots \ldots \ldots \ldots \ldots \ldots \ldots \ldots \ldots \ldots \ldots \ldots \ldots$ ix

Introduction $\ldots \ldots \ldots \ldots \ldots \ldots \ldots \ldots \ldots \ldots \ldots \ldots \ldots \ldots \ldots$

Scope $\ldots \ldots \ldots \ldots \ldots \ldots \ldots \ldots \ldots \ldots \ldots \ldots \ldots \ldots \ldots \ldots \ldots$

Series Listing $\ldots \ldots \ldots \ldots \ldots \ldots \ldots \ldots \ldots \ldots \ldots \ldots \ldots \ldots \ldots \ldots \ldots$

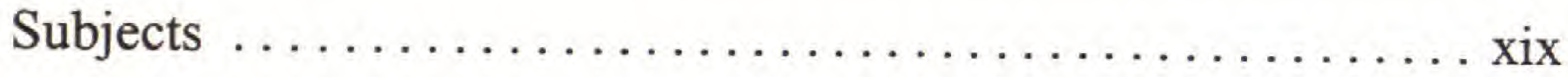

Chronology $\ldots \ldots \ldots \ldots \ldots \ldots \ldots \ldots \ldots \ldots \ldots \ldots \ldots \ldots \ldots \ldots \ldots \ldots$

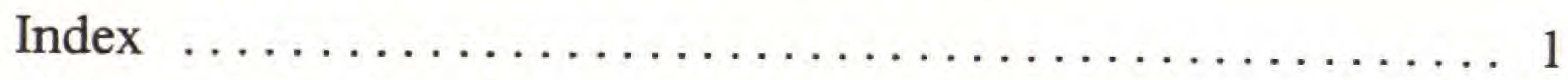





\section{Preface}

Joseph Ewan is the author of more than 400 books, essays, and reviews on the history of natural history, plant taxonomy, and biogeography. Since their marriage in 1934, Nesta Ewan, biologist and librarian, has been his research assistant, co-author, editor, and critic. "We've been like the motto of Sigma Xi, the scientific research society: 'Companions in zealous research."' For more than sixty years, Joseph and Nesta Ewan sought books and tidbits of fact about the people who have assembled our knowledge of natural history. The result is an immense archive of personal papers consisting of research files and correspondence and a library of 5000 rare and unusual books on the history and biography of natural history. The Missouri Botanical Garden purchased the entire collection in 1986. Since then, the Ewans have added 600 titles to complement the original collection.

In 1994-1995, the National Historical Publications and Records Commission (NHPRC) funded an eight-month project to arrange and describe the personal papers of Joseph Ewan. This alphabetical index to 10,000 names in the biographical research files and correspondence is a result of this project.

The project combined the efforts of many people who identified, arranged, and unfolded documents, removed harmful fasteners and stored the papers in acidfree containers, described documents in a database, and edited and produced the guide. Joseph and Nesta Ewan identified correspondents. Douglas Holland labored at arrangement, description, and preservation, and aided with editing and proofing. Mary Stiffler assisted with arrangement, preservation, description, editing, and proofing. Mary also formatted the index, after setting up and maintaining the database and importing data into WordPerfect. I directed day-today work, had primary editorial responsibility, and worked throughout on arrangement, description, and preservation. Eight volunteers worked hard to complete the project: Lucy Baker, Dorothy Baumann, Sven Eliasson, James Gossum, Jane Kahn, Barbara Loeb, Priscilla Rodriguez, and Mary Lou Waldman. Joseph Ewan made suggestions on format and content of the guide and wrote the chronology. Constance Wolf was project director. Dan Stokes of the NHPRC gave advice and encouragement throughout the project.

Martha Riley

Archivist 



\section{Introduction}

\section{Scope of the Guide}

This guide is an alphabetical name index to the research files and correspondence of Joseph and Nesta Ewan housed at the Missouri Botanical Garden. It is an introduction to the papers that will allow scholars to locate research materials on 10,000 individuals or institutions who were the Ewans' correspondents or their subjects of study.

Scholars wanting more description of the correspondence or a search by subject may request these from the library or do their own search of the database used to create the guide. A list of subjects is on page xvii of this guide. The Ewan database is available for searches in the archives and will be loaded on the archives section of the Missouri Botanical Garden on-line catalog in 1997. Through the Internet, this catalog will be available worldwide.

\section{Use of this Guide}

Overall this guide is an index of names noting the series in which documents can be found. Each entry is a description of a person or institution and the documents pertaining to them. Not all elements in the samples below appear in every entry.
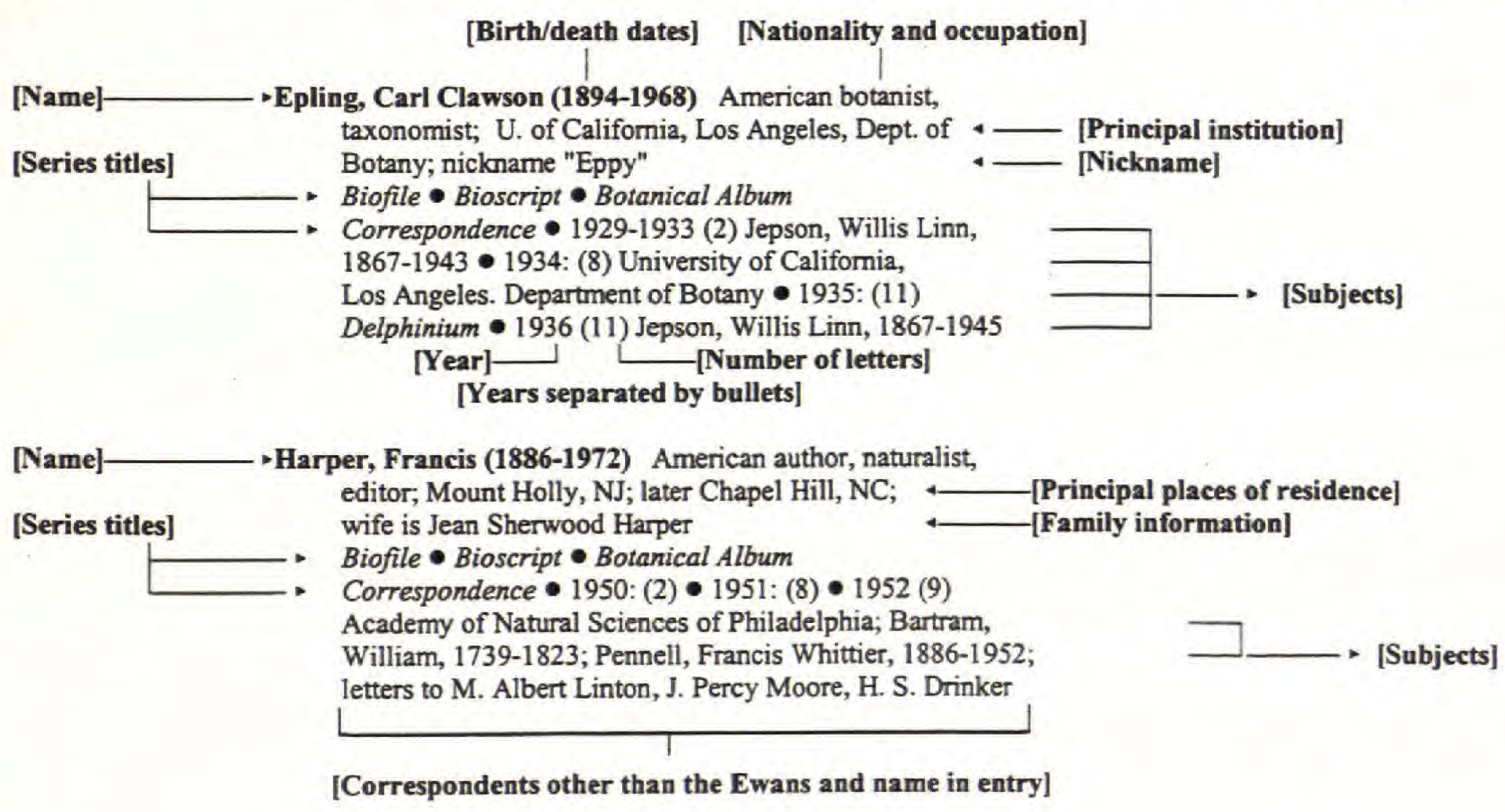
Information on names was taken from documents or folders in the Ewan papers and the following reference sources: American Men of Science, 11th ed. (1965-1967); American Men and Women of Science: Biology (1977); Taxonomic Literature, 2nd ed. (1976-); Index Herbariorum, Part II, Collectors (1954-1988); Authors of Plant Names (1992); Rocky Mountain Naturalists, 2nd ed. (1984); and Biographical Dictionary of Botanists Represented in the Hunt Institute Portrait Collection (1972). The intent was to differentiate surnames and institutions, not to give full biographical or historical information.

Description of the documents consists of a series title for biofile, bioscript, or botanical album. A more complex description is given for correspondence including year, number of letters, subject (optional), and other correspondents (optional). Since the year is the most important division of correspondence, a bullet $(\bullet)$ is used between dates for emphasis and clarity. When one encounters a ( 0 ) for number of letters, it represents documents other than letters or letters filed under another name, which is stated. Subjects indexed were limited to the list on pages xix-xxii of major projects undertaken by the Ewans and people or institutions known to them who shaped the history of natural history in the 20th century.

\section{Reference Service}

Researchers wishing to visit the Ewan collection should make appointments in advance to discuss holdings related to the research problem, accommodations, and hours. The collection will provide photocopies on a limited basis to researchers unable to visit $\mathrm{St}$. Louis. Fax and photographs are also available. Address inquiries to: Librarian, Missouri Botanical Garden Library, P.O. Box 299, St. Louis, MO 63166-0299. Phone: 314-577-5156; Fax: 314-577-9590; E-mail: molibref@mobot.org.

\section{Recommended}

Researchers are advised to consult Ewaniana: The Writings of Joe and Nesta Ewan, compiled by Keith Crotz, Chillicothe, IL: The American Botanist, Booksellers, 1989. This bibliography of 358 titles, 1928-1988, is indexed by subject and available from the 


\section{Permission and Copyright}

The Missouri Botanical Garden requires that researchers obtain written permission to publish quotations from its archival collections. Except in rare cases, permission to quote will be granted.

In many cases, the Garden may not control copyright of a document. The archivist will inform researchers of copyright information when it is known, but researchers assume legal responsibility for observing copyright, property, and libel laws. 



\section{Guide to the Ewan Papers}

\section{Scope}

The research files of the Ewans as described here were begun on their arrival in Colorado in 1937 to prepare essays on the botanical explorers of the state. Illustrated articles were written on C. C. Parry, Edwin James, J. C. Frémont, H. N. Patterson, T. C. Porter, and M. E. Jones, and published in the publication of the Colorado Mountain Club, Trail and Timberline. The six illustrated essays were published in Rocky Mountain Naturalists (1950) with a roster of natural history collectors, artists, and explorers based on museum records, correspondence, and publications. Aware of the reference value of such digests, companion "Naturalists of the Old South" was planned after moving to New Orleans in 1947. Though this book was left in the fact-gathering-stage, biographical facts collected were incorporated in the publications on Frederick Pursh and William Bartram that followed. The research materials grew with the tenure of a Guggenheim Fellowship based at London 1954-1955. Meanwhile, items were collected for a "Botanical Album," begun at Berkeley in 1933. All these research materials were organized into three series: Biofile, Bioscript, and the already extensive Botanical Album, after moving to the Missouri Botanical Garden in 1986. A full-length biography of Benjamin Smith Barton, a "keystone physician-naturalist of the Jefferson period," has been completed for publication. "Andes and Amazon," a biobibliography of travel narratives of naturalists in South America is in progress. These two projects are separate units not yet incorporated in the research files described here.

The correspondence dating back to 1921 with natural scientists around the world sought biographical facts, identifications, and exchange of ideas, reprints, and specimens relating to the life sciences. Nesta Ewan's letters are encountered throughout after their marriage in 1934, confirming her role as research assistant, critic, editor, librarian, and co-author. This international 20th century correspondence offers insight into the lives of scientists, their travels, publication travails, and shared happiness at a time of transition in the natural world when the environment passed from nearly pristine to the devastation of today. Some of the scientific correspondents include Tim Berra, Edith and Frederic Clements, T. D. A. and Wilmatte Cockerell, Lincoln Constance, Carl Epling, F. R. Fosberg, Samuel Wood Geiser, John Thomas Howell, W. L. Jepson, George H. M. Lawrence, David D. Keck, C. V. Morton, P. A. Munz, Robert M. Peck, Peter Raven, E. D. Rudolph, A. C. Smith, Sir George Taylor, and George Van Schaack.

Pursuant to Joseph Ewan's career in teaching the history of biology at different institutions with their curricula ranging from premedicine, to forestry or fisheries biology, the correspondence varied widely. The history of natural history, world-wide 
exploration, the lives of artists-naturalists, biographies especially of minor figures, gave a focus to their book collecting. The critical influence of book dealers who appear in the correspondence is noteworthy. From experience in writing and publishing the Ewans were often able to assist fellow biohistorians. Acknowledgement of their assistance may be recognized in the writings of John Cawte Beaglehole, Edmund and Dorothy Berkeley, Ray Desmond, Francis Harper, Ida Langman, Susan Delano McKelvey, E. Charles Nelson, Frans Stafleu, and Frans Verdoorn.

Additional information and commentary on persons listed in the Guide may be pursued in the Ewan book collection. (Dust wrappers that may carry facts or hyperbole not in the book were conscientiously preserved.) For example, a quest for information on Loye Holmes Miller, "Padre," may be found in his autobiography and an oral history transcript, in the collection of over 600 biographies, as well as Correspondence, Biofile, Bioscript, or Botanical Album, described hereafter. 


\section{Series Listing}

\section{Series 1 Research files}

Series 1 is made up of three series of biographical files on naturalists, botanists, geologists, scientific illustrators, entomologists, ornithologists, conchologists, or influential figures in the history of natural history. These files were assembled material for potential publications. Mary Lynn Ricketts initially sorted and identified the persons in the files.

\section{Series 1/1 Bioscript 24 linear feet}

Bioscript contains mostly undated manuscript material on 4026 persons and is arranged alphabetically by person. The research notes by the Ewans and Francis Harper (originals at University of Kansas, Lawrence) and copies of books and letters serve as a finding guide to material in book and manuscript collections they visited in North America and Europe. In addition, the bioscript contains usually short newspaper obituaries, rough notes for Joseph Ewan's correspondence related to a person's life and activities, notes on interviews, drafts of publications by Joseph Ewan and others, and published photographs. The file is a rich resource, but notes and copies of letters and other manuscripts may be difficult to read. Copies are often not able to be reproduced, because they are Contoura copies or because of copyright. The institution who holds the original is noted so the researcher can contact it for usable copies and permissions.

\section{Series 1/2 Biofile 11 linear feet}

Biofile is a file of reprints of published biographies of 1232 naturalists arranged alphabetically by person and then chronologically by date of publication. Often the articles are obituaries or full biographies, but the file contains other articles giving clues to the identities of living or obscure people who do not have a published biography.

\section{Series 1/3 Botanical Album 5 linear feet}

Album consists of fragile ephemera and original manuscript material on 1634 individuals and institutions and is arranged alphabetically. Types of material include original letters not connected with the Ewans, Christmas greetings of Ewan correspondents, portraits, herbarium labels, publication flyers, and programs of events. The album is used for identification of handwriting as well as information on the life of the person. It is arranged alphabetically by name 


\section{Introduction}

with a few folders of group portraits at the beginning. Housed in the album are letters of selected South African correspondents of Adele Lewis Grant.

\section{Series 2 Personal Papers}

Personal papers consist of correspondence and other papers to be added at a later time.

Series 2/1 Correspondence, 1921-1993 48 linear feet

Series $2 / 1$ consists of correspondence, 1921-1993, arranged first chronologically and then alphabetically within. Outgoing correspondence begins with the year 1940. The file contains some outgoing family correspondence. The Ewan chronology, page xiii-xxiv, will add another dimension, place of residence, to the sequence of the letters. 


\section{Subjects}

\section{Note on topic or content}

For institutions, societies, or organizations, the interest is development, policies, conflicts, "rise" and "fall" of the organization.

For persons, the interest is appraisal from others (not direct correspondence), traits of character, reported in letters of friends or contacts, "success"- items that would be of interest to a biographer. Incidental poignant commentary.

For plant genera or titles of books, the interest centers on the progress of a revision, book, or extended study, and on persons who were associated with the research or publication.

\section{Academy of Natural Sciences of Philadelphia}

\section{American Fern Society}

\section{Arnold Arboretum}

Banister, John, 1650-1692 (as to publication(s) in progress)

Banks, Joseph, Sir, 1743-1800 (as to publication in progress)

Barton, Benjamin Smith, 1766-1815 (as to publication(s) in progress)

Bartram, William, 1739-1823 (as to publication(s) in progress)

Beaglehole, John Cawte (biographer of James Cook, etc.)

Biology-Instruction and study.

See also Science-Instruction and Study

Book collectors (methods, traits, "nuggets" of biographical interest)

British Museum (Natural History). Dept. of Botany 
Introduction

Chronica Botanica.

See also Verdoorn, Frans Antonie, 1906-1984

Cockerell, Theodore Dru Alison, 1866-1948 (of Colorado)

\section{Delphinium}

Eastwood, Alice, 1859-1953 (San Francisco)

Epling, Carl Clawson, 1894-1968 (UCLA)

Fairchild Tropical Garden (Coconut Grove, FL)

Geiser, Samuel Wood, 1890- (Southern Methodist University, author)

Grant, Adele Lewis, 1881-1967

Harper, Francis, 1886-1972 (AL, NJ, PA, etc.)

Harper, Roland McMillan, 1878-1966 (AL, GA, NJ, PA, etc.)

Henry E. Huntington Botanical Garden.

See Henry E. Huntington Library and Art Gallery

Henry E. Huntington Library and Art Gallery

Jepson, Willis Linn, 1867-1946 (University of California, Berkeley)

Jones, Marcus Eugene, 1852-1934

Langman, Ida Kaplan, 1904

Lawrence, George Hill Mathewson, 1910-1978 (Cornell; Hunt Botanical Library,
Pittsburgh)

Lyon, John, 1764-1814 (Anglo-American "plant hunter"-as a subject of study) 
Maxon, William Ralph, 1877-1948 (Smithsonian, curator of United States National Museum)

Merrill, Elmer Drew, 1876-1956 (Berkeley, Harvard, "American Linneaus")

Missouri Botanical Garden (St. Louis)

Morrison, Benjamin Yoe, 1891-1966

Munz, Philip Alexander, 1892-1974 (Pomona College)

National Science Foundation

Nuttall, Thomas, 1786-1859 (Philadelphia, vs. Frederick Pursh)

Pacific Tropical Botanical Garden (Kauai)

Peattie, Donald Culross, 1898-1964

Pennell, Francis Whittier, 1886-1952

Pursh, Frederick, 1774-1820 (Philadelphia)

Rocky Mountain Naturalists (1950; ed. 2, 1981)

"Rocky Mountain Vegetation" (ined.)

Royal Botanic Gardens, Kew

Science-Instruction and study.

See also Biology-Instruction and Study

Setchell, William Albert, 1864-1943 (University of California, Berkeley)

Species (Species question: criteria, limits, evidence)

Stafleu, Frans Antonie, 1921- (Utrecht) 
Introduction

Taylor, George, Sir, 1904-1993 (British Museum (Natural History) and Kew)

Teaching methods: (criteria, priorities, tests, etc.)

See Science-Instruction and study; Biology-Instruction and study

Tulane University. Department of Botany

University of California, Berkeley. Department of Botany

("star performers," programs, relations with Stanford, UCLA, etc.)

University of California, Los Angeles. Department of Botany (move from Vermont Ave. campus to Westwood, growth, from undergraduate to graduate, after our years at UCLA, "star performers")

University of Colorado, Boulder. Department of Biology (development of summer session field programs, emphasis on ecology, University of Colorado Museum and Colorado Museum Natural History, Denver, contacts)

Van Schaack, George Booth, 1903-1983 (Missouri Botanical Garden, Morton Arboretum, and Eugene, $\mathrm{OR}$ )

Verdoorn, Frans Antonie, 1906-1984 (Waltham, MA, and Chronica Botanica)

Vismia 


\section{Guide to the Ewan Papers}

\section{Chronology}

A Ewan Chronology especially of Travel and Residence

1908 November 8, Nesta Ewan was born near Langemeade, Saskatchewan.

1909 October 24, Joseph Ewan was born in Philadelphia, Pennsylvania. (Hereafter, events refer to Joseph Ewan except where noted.)

1911 Following the death of his mother, Emma Magill Ewan, Joseph Ewan lived with paternal aunt, Sara Seranne and her husband, John Lewis Andorfer, at Finley, Cumberland Co., New Jersey.

1912 Moved to San Gabriel, California. After three months residence removed to Los Angeles to 1631 Shatto Street until August, 1933.

1927 Graduated from Belmont High School. Influenced by Nettie and Charles E. Merrill (biology), Flora Abbott Herring (Latin), and Marjorie Van Deusen, Librarian. President, Belsci Science Club; contributed to Belmont Sentinel.

1928 Freshman year, University of California at Los Angeles, Vermont campus. Botany major. Extracurricular employment, UCLA Library. Joe Ewan met Nesta Dunn in botany class.

1929 University moved to Westwood. Botany major, zoology and geography minors. Extracurricular employment, Los Angeles Public Library.

1933 Moved to Berkeley. Botany major, University of California. Research assistant to Prof. W. L. Jepson, twenty hours a week, 1933-1937.

1934 Graduated, A. B. Joe Ewan married Nesta Dunn August 20.

1937 Moved to Boulder. Appointed Instructor, Biology, University of Colorado, Boulder. Evening classes, University of Colorado, Denver. Class continued as "Colorado Seminar in Botany." 
1944 Appointed Botanist, Cinchona Division, Foreign Economic Administration, Bogotá, Colombia. Field operations chiefly in Dept. Nariño, out of Pasto, April 1944 - April 1945.

1945 Moved to Arlington, Virginia. Appointed Assistant Curator, Division of Plants, Smithsonian Institution, Washington, D. C.

1946 Appointed Associate Botanist, Bureau Plant Industry, U. S. D. A., Beltsville, MD.

1947 Tulane University, New Orleans, Louisiana, appointed Assistant Professor.

1954 Awarded Guggenheim Fellowship, 1954-1955. Based at British Museum (Natural History), London.

1967 Appointed Visiting Instructor, Botany, Summer Session, University of Hawaii, Honolulu.

1972 Tulane University. Awarded Ida Richardson Professorship of Biology.

1974 Reappointed Summer Session, Visiting Professor of Botany, University of

1978 Appointed Summer Session Visiting Professor of Botany, University of Oregon, Eugene. 1981 Reappointed University of Oregon, Eugene, Summer session Visiting
Professor of Botany.

1982 Appointed Ohio State University Visiting Distinguished Professor, Spring
term.

1984 Appointed Regents' Fellow 1984-1985, Smithsonian Institution, Washington,
D. C.

1986 Moved to St. Louis. Research Associate, Missouri Botanical Garden. 


\section{$\boldsymbol{A}$}

Aaron, Isador Morris (1904-) Paleobotanist Correspondence $\bullet$ 1950: (5)

Abadie, E. H. Bioscript

Abbe, Elfriede Martha (1919- ) Artist, sculptor Bioscript

Abbey, Edward Correspondence • 1973: (2)

Abbot, Charles C. Bioscript

Abbot, John (1751-1840) American lepidopterist, ornithological artist, entomologist Biofile $\bullet$ Bioscript $\bullet$ Botanical Album

Abbott, Clinton G. Natural History Museum, San Diego, California

Correspondence - 1929-1933: (1) • 1936: (4) Species • 1941: (1) - 1943: (3)

Abbott, Isabella Aiona (1919-) Hopkins Marine Station, Pacific Grove, CA Correspondence $\bullet$ 1969: (1)

Abbott, M. Jessamine U. of California, Dept. of Biology, Library Correspondence $\bullet$ 1938: (2)

Abbott, R. Tucker American Malacologists, Inc., Greensville, DE, President

Botanical Album

Correspondence $\bullet$ 1974: (3) • 1975: (2) • 1977: (2) • 1979: (4) • 1987: (2)

Abelson, P. H. Science, Editor Correspondence - 1973: (0) Letter filed under George Booth Van Schaack

Aberbach, Alan D. Simon Fraser U., Burnaby, British Columbia, Dept. of History Correspondence - 1988: (2)

Aberg, Ewert 7th International Botanical Congress, Secretary Correspondence $\bullet$ 1950: (1)

Abert, James William (1820-1897) American topographical engineer, U. S. Army

Bioscript

Abraham, Charles Bioscript

Abraham, Herbert J. U. S. State Dept., Group Relations Branch

Correspondence 1946: (3) Verdoorn, Frans Antonie, 1906-1984

Abraham, Terry Washington State U., Pullman, Library Correspondence $\bullet$ 1977: (3)

Abrahams Magazine Service Maurice White, Manager Correspondence 1956: (2)

Abrams, Leroy (1874-1956) American botanist; Stanford U., Dudley Herbarium Biofile $\bullet$ Bioscript $\bullet$ Botanical Album Correspondence $\bullet$ 1929-1933: (4) $\bullet$ 1935: (2) $\bullet$ 1938: (3) Letter to T. D. A. Cockerell • 1939: (3) • 1949: (1)

Abrams, Rochonne Science writer, St. Louis, MO Correspondence $\bullet$ 1990: (1)

Abramson, Ben Ben Abramson Bookseller Correspondence $\bullet$ 1954: (4)

Academy of Natural Sciences of Philadelphia
Botanical Album (Filed under Philadelphia)

Correspondence • 1964: (1) • 1970: (2) • 1976: (1) • 1980: (1) • 1983: (1) • 1988: (1)

Accokeek Foundation

Botanical Album

Achert, Edward E.

Bioscript

Achord, Vivian C. New Orleans, LA Correspondence $\bullet$ 1981: (3) Species • 1983: (1)

Ackermann, John M. U. of the South Pacific Correspondence 1974: (1)

Ackley, Helen M. Los Angeles, CA; husband is Leroy Ackley

Correspondence 1942 : (1)

Ackley, May M.

Botanical Album $\bullet$ Bioscript

Correspondence • 1929-1933: (2) • 1938: (2)

Acosta, José de, Father (1539-1600) Spanish writer and Jesuit Missionary in Peru Bioscript

Acosta-Solis, Micael Botanical Album Correspondence • 1948: (2)

Acrelius, Israel (1714-1800) Swedish-American Divine Bioscript

Acuña Galé, Julián Baldomero (1900-) Estación Experimental Agronómica, Santiago de las Vegas, Cuba, Director

Correspondence • 1939: (1)

Adair, James M. Bioscript

Adams, C. B. Bioscript

Adams, C. Dennis (1920- ) London, England Correspondence • 1989: (4)

Adams, Charles Correspondence $\bullet$ 1990: (1)

Adams, Charles Christopher (1873-1955) American ecologist, zoologist

Biofile $\bullet$ Bioscript

Correspondence • 1929-1933: (1)

Adams, Dennis

Botanical Album

Adams, Franklin

Correspondence • 1973: (1)

Adams, Janine Missouri Botanical Garden Correspondence • 1991: (2)

Adams, J. E. Correspondence $\bullet$ 1935: (3)

Adams, James Capen Bioscript

Adams, Joseph Edison (1904-1981) U, of North Carolina, Chapel Hill Correspondence • 1943: (1)

Adams, Lee Artist of tropical fruits Correspondence $\bullet$ 1952: (2)

Adams, Mark Boyer U. of Pennsylvania, Philadelphia, Dept. of History and Sociology of Science Correspondence $\bullet$ 1971: (1) • 1974: (3) 


\section{Guide to the Ewan Papers}

Adauns, Presten DePaw U., Greencastle, IN, Dept. of Botany

Correspondence • 1962: (2)

Adams, Robert (1846-1906) American lawyer Bloscript

Adams, Robert McCormick (1926-) American anchropologist Bioflle

Adams, Roger U. of Illinois, Dept. of Chemistry Correspondence $\bullet$ 1939: (3)

Adams, Thomas R. Librarian; Brown U., Providence, RI, The Jotan Carter Brown Library

Correspondence • 1962: (3) • 1963: (5) Book collectors

Adams, William Muskingum College, New

Concord, $\mathrm{OH}$

Correspondence • 1965: (2) • 1967: (2)

Adams, William Howard National Gallery of Art; member, International Steering Committee for exhibition Correspondence • 1976: (0) Filed under Peter Raven • 1976: (0) Filed under National Gallery of Art

Adansoa, Michel (1727-1806) French naturalist Bioflle

Adelhelm, C. E.

Correspondence $\bullet 1944$ : (1)

Adher, A. \& Co.

Correspondence • 1951: (1)

Adilakstumi, Lahohin

Botanical Album

Adler, Kraig Cornell U., Zoology

Correspondence • 1990: (5) • 1991: (2)

Adlum, John

Bloscript

Adrouny, Sonia A. Arnold Arboretum; student of J. Ewan at Tulane

Correspondence • 1969: (2) • 1969: (0) Filed under Gordon DeWolf • 1976: (1)

Aellen, Pawl (1896-1973) Swiss botanist Biofite

Affolter, Nancy

Correspondence $\bullet 1980$ : (1)

Affolter, Vie

Correspondence • 1978: (2)

Agar, Percival K. Dominica, B. W. I. Correspondence • 1951: (1) Species • 1955: (1)

Agarilh, Cari Adolph (1785-1859) Biofile • Bioscript

Agardh, Jacob Georg (1813-1901) Bioscript

Agassia, Alecander Emmanuel Rudolpe (1835-1910) American marine biologist and oceanographer Blofile • Bioscript

Agassiz, Jean Louis Rodolphe (1807-1873) Swiss naturalist; Biofile $\bullet$ Botanical Album of Comparative Zoology

Aricultural

Davis; Vivian B. Whiteh, U.S.D.A. U. of California, Correspondence 1980 : (2) Historical Assistant

Ahern, George Patrick Botanical Album Ahles, Hiarry E. (1924-1981) U. of North Carolina,
Chapel Hill, Dept. of Botany, Curator
Ahles, Harry E. (continued Bioscript

Correspondence • 1961: (1) • 1962: (1)

AIBS (American Institute of Biological Sciences)

See American Institute of Biological Sciences

Aiken, Charles Edward Howard (1850-1936) American naturalist, taxidermist

Bioscript

Aiken, William E.

Bioscript

Aikin, William Edward Augustin (1807-1888)

Botanical Album

Ainslie, George

Bioscript

Aiton, George Briggs (1856- )

Bioscript

Aiton, William (1731-1793)

Bioscript

Akeley, Mary Jobe

Bioscript

Akeley, Osa Johnson

Botanical Album

Akerman, Alfred American forester Biofile

Alabama Conservancy

Correspondence • 1974: (1)

Alabama State Archives Dept. of Archives and History, Montgomery, AL

Correspondence • 1952: (2)

Alain, Hermano

Botanical Album

Albany Hotel

Correspondence • 1991: (1)

Albert, A. S.

Correspondence • 1936: (2) Delphinium

Albertini, Johannes Baptista von (1769-1831) Bioscript

Albin, Eleazer

Bioscript

Albritton, Errett C. American Institute of Biological Sciences; Editor of the Handbook of Biological Data, Washington, D.C.

Correspondence • 1952: (1) • 1953: (2)

Albury, W. Randall U. of New South Wales, Kensington, N. S. W., Australia

Correspondence • 1974: (5) Letters to/from L. E. Fredman

Alden, Bradley Ripley (1811-1870) Bioscript

Alderson, Mary L. Bioscript

Alderson, Rufus Davis (1858-1932) American botanist Biofile $\bullet$ Bioscript

Aldridge, Robert D. St. Louis U., Dept. of Biology Correspondence 1990 : (1)

Alexander, Annie Montague (1867-1950) American naturalist

Biofile • Bioscript

Alexander, Edward Gordon (1901-1973) American zoologist; U. of Colorado, Boulder, Dept. of Biology Bioscript $\bullet$ Botanical Album

Correspondence $\bullet 1940:$ 
Alexander, Edward Gordon (continued)

1943: (9) - 1943: (0) Filed under William G. Houk $\bullet$ 1945: (4) $\bullet$ 1946: (6) • 1947: (4) Vismia • 1948: (5)

Tulane U. Dept. of Botany - 1949: (8) Tulane U. Dept. of Botany $\bullet$ 1950: (4) • 1951: (1) • 1952: (2) • 1953: (5)

"Rocky Mountain Vegetation" $\bullet$ 1957: (1) 1963 : (1) 1966: (2) • 1970: (2) • 1971: (2) • 1973: (1)

Alexander, Edward Johnston (1901-1985) New York Botanical Garden, NY, Associate Curator Bioscript • Botanical Album Correspondence • 1959: (1)

Alexander, Edward P. Museum Studies, U. of Deleware, Newark Correspondence - 1982: (0) Filed under Richard D. Altick

Alexander, Gordon See Alexander, Edward Gordon

Alexander, Harry, Mrs. Correspondence - 1941: (1)

Alexander, James (d. 1778) Bioscript

Alexander, Jo Oregon State U. Press, Managing Editor Correspondence - 1991: (8) Botanical Exploration of the Trans-Mississippi West $\bullet$ 1992: (0) Letter from Frank A. Lang filed under Eric Groves

Alexander, Taylor R. U. of Miami Correspondence $\bullet$ 1947: (1)

Alexander, William M. Bioscript

Alexander, William P. Bioscript

Alexandrov, V. Ya. Komarov Botanical Institute, Leningrad, USSR, Laboratory of Cytophysiology Botanical Album Correspondence • 1972: (2)

Alford, Ruth B. Eastern Michigan U., Ypsilanti, Michigan, Curator of the Herbarium Correspondence • 1972: (1) • 1974: (1)

Alfred A. Knopf, Inc. Harold Strauss, New York, NY Correspondence $\bullet$ 1969: (3)

Alibert, Jean Louis, Baron (1768-1837) Bioscript

Allan, Grace Alesworth, Suffolk, England Correspondence $\bullet$ 1981: (1)

Allan, Mea British botanical author; Alesworth, Suffolk, England Botanical Album

Correspondence $\bullet 1963:(9) \bullet 1964:$ (1) $\bullet$ 1966: (1) $\bullet$ 1968: (1) • 1969: (4) • 1970: (1) • 1974: (1) • 1976: (3) • 1977: (4) • 1979: (6) Banks, Joseph, Sir, 1743-1800 • 1980: (2)

Allard, Harry Ardell Bioscript • Botanical Album

Allee, Ralph Inter-American Institute of Agricultural Sciences Correspondence • 1947: (2)

Allen, A. T. Bioscript

Allen, Arthur A. American ornithologist Biofile • Botanical Album Correspondence $\bullet$ 1958: (4) $\bullet$ 1961: (0)
Allen, Bruce H. (1952- ) American bryologist; Missouri Botanical Garden Correspondence • 1987: (1) • 1988: (1) • 1990: (1) • 1991: (1)

Allen, Charles Andrew (1841- ) Bioscript

Allen, Charles M. U. of Southwestern Louisiana, Lafayette, LA, Dept. Biology

Correspondence - 1976: (0) Letter to Roland Treubig, filed under Louisiana Forestry Commission - 1982: (2)

Allen, David Elliston (1932- ) English amateur botanist; London

Correspondence $\bullet$ 1964: (2) $\bullet$ 1965: (1) 1966: (1) 1968 :

(2) $\bullet$ 1969: (7) • 1976: (3) - 1977: (3) - 1978: (3) • 1979:

(3) $\bullet$ 1983: (2) 1 1984: (2) 1 1988: (2) $\bullet$ 1990: (4) $\bullet 1991$ :

(2) 1992: (1)

Allen, David G. Ithaca, NY; son of Elsa Allen Correspondence • 1969: (1) • 1970: (2) • 1971: (1)

Allen, Donald R. Chelsea Physic Garden, Chelsea, England Correspondence • 1956: (2)

Allen, Dorothy Botanical Album (Filed under Paul Allen)

Allen, Elsa Guerdrum Cornell U., Ithaca, New York State College of Agriculture, Laboratory of Ornithology Botanical Album Correspondence - 1952: (8) • 1953: (2) - 1954: (7) 1955: (2) • 1956: (9) • 1957: (5) • 1958: (11) • 1959: (13) - 1960: (1) • 1961: (1) • 1962: (7) • $1963:(1) \bullet 1964:$

(4)

Allen, Ethel Kullman Botanical Album (Filed under Oscar Nelson Allen)

Allen, F. F. Bioscript

Allen, George William H. Allen Co., Booksellers, Philadelphia, PA

Correspondence $\bullet$ 1959: (2) $\bullet$ 1964: (1) • 1968: (2) • 1975: (2) Book collectors $\bullet$ 1980: (4) Book collectors $\bullet$ 1981: (1) • 1982: (2) • 1983: (1) • 1984: (2) • 1985: (1) • 1986: (5) • 1989: (7) • 1991: (1) • 1992: (4) • 1993: (3) Book collectors

Allen, George Nelson (-1877) Naturalist; Oberlin, Prof. of Natural History Bioscript

Allen, Harrison Bioscript

Allen, Jessie M. Correspondence $\bullet$ 1935: (2)

Allen, Joel Asaph (1838-1921) American zoologist Biofile $\bullet$ Bioscript

Allen, John Fisk (1807-1876) Bioscript

Allen, O. D. Bioscript

Allen, Oscar Nelson Botanical Album

Allen, Paul Hamilton (1911-1963) Plant explorer, taxonomist Bioscript • Botanical Album

Allen, Ruth Florence (1879-1963) American plant pathologist Biofile 
Alese, Walter S. American Fern Society

Cormepondence • 1967: (6) American Fern Society • 1964: (1) American Fern Society • 1950: (3)

Allen, Wuan H. American boobseller

See abo Willes H. Alles Co.

Bingtie

Cimepondence • 1951: (2) Book collectors • 1957: (2) • 1960: (1) • 1964: (1) • 1973: (1) • 1977: (2) • 1981: (1) • 1982: (1) • 194: (1)

Aller, Alin Ray (1906-) Plast ecologist, taxonomist;

Northent Natarene College

Comesondence • 1951: (3) Delphinium

Allertoe, Robert

Alasorit:

Allescher, Andras (1828-1903)

Bigtie $\bullet$ Bloscript

Allerate, Senela Regina Servico Publico Federal, Brail, Litrarias

Corremonience $\bullet$ 1985: (2)

ABsed, Carh Ledovice (1728-1804) Italian physician, taxonomint

Bigith

Allove, Asdrew

Biancript

Crrepondonce * 1965: (1)

Allisea, Frascis

Blascrist

Alisoe, Willam B.

Blisscripe • Botanical Album

Comcrpoedence • 1952: (7) • 1958: (1) Species

Alled, J, B., Mrs. New Orleans, LA

Comesondence • 1960: (3)

Allred, Kelly W. (1949) New Mexico State U.

Blatcript

Combeondence $\bullet$ 1991: (2)

Alimeda, Frank (1946-) California Academy of Sciences,
Ditretior of Research

Comepondence • 1985: (1)

Almeida, J. F. R. D. (1845-1916) Indian botanist

Blyfle $\bullet$ Bloscript

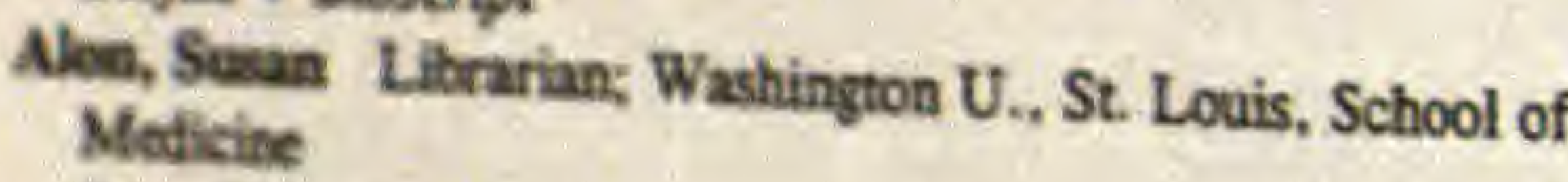

Slascript

Comreondence • 1991: (4) • 1992: (1) • 1993: (3) Aperin, Richard J. Community College of Philadelphia,
Peiladelphia, PA

Comespondence $\bullet$ 1972: (2)

Alpert, Harry National Science Foundation, Program Director for Socio-Physical Sciences

Comerpondence • 1956: (1) • 1957:

Apert, Riba New York, NY.

$$
\text { Carrespondence - 1988: (0) Fi }
$$

Alstoen, Arther Hagle taxonomist; British Garfit (1902-1958) English Bigfile * Botanical Album (Natural History) Carrerasentence $\bullet$ 1945:

1953: (1) • 1956: (6) • (6) • 1948: (1) • 1951: (1) •

Abton, J. M.

Correspondence $\bullet 1978$ : (2)
Alstroemer, Johan (Jonas) (1685-1761) Swedish botanist, industrialist

Bioscript

Altevogt, Raymond Fred (1940-) Plant geneticist; Missouri Botanical Garden

Correspondence $\bullet$ 1966: (1)

Altick, Richard D. Ohio State U., Dept. of English

Correspondence $\bullet$ 1982: (4) • 1987: (1)

Altschul, Siri Sylvia Patrick von Reis (1931- ) Botanical Album

Alvarez Conde, José See Conde, José Alvarez

Alverson, Andrew Halstead (1845-1916) American botanist, minerologist, zoologist

Biofile $\bullet$ Bioscript $\bullet$ Botanical Album

Amadon, Dean American Museum of Natural History, NY Correspondence $\bullet$ 1944: (2) • 1973: (2)

American Academy of Arts and Sciences Correspondence $\bullet$ 1973: (7) $\bullet$ 1974: (3) • 1975: (5) • 1976: (1)

American Antiquarian Society Worcester, MA Correspondence • 1960: (1) • 1975: (2) • 1976: (3) • 1978: (2) $\bullet$ 1979: (0) Flyers announcing lectures and deadline for fellowships $\bullet$ 1981: (1) $\bullet$ 1982: (3) 1 1983: (3) Barton, Benjamin Smith 1766-1815 • 1984: (2) • 1985: (1) - 1986: (1) • 1988: (1) • 1991: (1) • 1992: (1)

American Association for the Advancement of Science William Bevin, Exec. Officer, AAAS, Washington, D.C. Correspondence $\bullet$ 1971: (2)

American Association of Petroleum Geologists Tulsa, OK

Correspondence • 1949: (1)

American Association of University Women Correspondence • 1950: (0) Recommendation letter filed under Ida Langman

American Begonia Society

Correspondence $\bullet 1965$ : (1) $\bullet$ 1966: (1)

American Broadcasting Co. New York, NY Correspondence • 1969: (1)

American Documentation Institute Washington, D.C. Correspondence • 1949: (2) U. of Colorado, Boulder, Dept. of Biology

American Fern Society Correspondence $\bullet$ 1969: (1)

American Forest Institute Correspondence $\bullet$ 1972: (2)

American Geographical Society Lynn S. Mullins Correspondence $\bullet$ 1965: (2)

American Historical Review Correspondence 1975: (1) American Institute of Biological Sciences

Correspondence • 1962: (1) • 1968: (3) • 1976: (4)

American Irish Fistorical Society New York, NY Correspondence 1977 : (1)

American Joumal of Botany Correspondence 1987: (1)

American Library Association Correspondence - 1974: (1)

American Men and Women of Science Correspondence $\bullet$ 1985: (1) 
American Men of Science

Correspondence $\bullet$ 1965: (2)

American Midland Naturalist U. of Notre Dame, Notre Dame, IN

Correspondence • 1949: (1) • 1964: (3) • 1989: (2)

American Motors Conservation Awards Program Southfiels, MI

Correspondence $\bullet$ 1977: (1)

American Museum of Natural History Correspondence $\bullet$ 1974: (5)

American National Biography American Council of Learned Societies; Oxford U. Press, Cary, NC Correspondence $\bullet$ 1992: (2)

American Ornithologists Union I. Lehr Brisbin, Chairman, Membership Committee

Correspondence $\bullet$ 1988: (2)

American Philosophical Society Philadelphia, PA Correspondence $\bullet$ 1945: (1) 1950: (1) • 1959: (0) Invitation to dedication of Library Hall, American Philosophical Society 1 1972: (3) • 1974: (1) - 1976: (1) 1978: (4) • 1979: (1) • 1980: (1) • 1981: (1) • 1983: (6) • 1984: (5) • 1985: (1) • 1989: (4) • 1990: (1) 1991: (1)

American Philosophical Society Library, Friends of Correspondence • 1993: (1)

American Plant Life Society Correspondence $\bullet$ 1984: (2)

American Rhododendron Society Correspondence • 1979: (5) • 1980: (3)

American Scientist Correspondence • 1975: (2) • 1980: (2)

American Security Bank Correspondence • 1985: (1)

American Society of Plant Taxonomists Correspondence • 1974: (1) • 1975: (4) • 1990: (1)

Americanist-Bookseller Norman Kane Correspondence • 1974: (3)

Americans for Democratic Action Leon Shull Correspondence • 1976: (1)

Amerson, Peggy A. Correspondence • 1970: (0) Filed under Calaway Dodson

Ames, Mary E. Pulsifer (1845-1902) Botanist, plant collector

Bioscript

Ames, Oakes (1874-1950) American taxonomist, orchidologist Biofile $\bullet$ Bioscript $\bullet$ Botanical Album

Amherst College Correspondence • 1969: (2)

Anderson, Alexander (1748-1811) Bioscript

Anderson, Berta Correspondence • 1976: (4) Rocky Mountain Naturalists

Anderson, Charles Lewis (1827-1910) Taxonomist Bioscript

Anderson, Edgar Shannon (1897-1969) American plant geneticist; Missouri Botanical Garden, St. Louis, MO Biofile $\bullet$ Bioscript $\bullet$ Botanical Album Correspondence • 1945: (1) - 1948: (2) Missouri Botanical Garden • 1949: (3) • 1952: (2) Pennell, Francis Whittier, 1886-1952 • 1953: (4) • 1954: (2) National Science Foundation; See also Barbour, Evelyn, Assistant to
Anderson, Edgar Shannon (continued) Edgar Anderson • 1959: (1) • 1961: (0) Letter filed under Mrs. James W. Reily • 1966: (1)

Anderson, Elsie Jean Correspondence • 1921-1928: (3)

Anderson, Ethel A. Arnold Arboretum, Secretary to Dr. I. M. Johnston Correspondence • 1929-1933: (1)

Anderson, Frederick William (1866-1891) American botanist Bioscript

Anderson, Gilberta T. Husband is William H. Anderson, Jr. Botanical Album Correspondence • 1969: (5)

Anderson, Gregory Joseph (1944) Botanical Society of America; U. of Connecticut, Storrs, Ecology and Evolutionary Biology Correspondence • 1989: (0) Filed under Steven Darwin

Anderson, Harvey Wife is Alice Anderson Bioscript $\bullet$ Botanical Album Correspondence • 1948: (1) • 1974: (1)

Anderson, J. Denton Graduate Men's Club, Norman, OK Correspondence $\bullet$ 1942: (2)

Anderson, Jacob Peter (1874-1953) American horticulturist, phytogeographer, taxonomist; Iowa State College, Ames, Dept. of Botany Bioscript Correspondence • 1929-1933: (3) • 1944: (2) Delphinium - 1949: (2) • 1951: (1)

Anderson, John Bioscript

Anderson, Lewis Edward (1912- ) Taxonomist; Duke U., Durham, NC Correspondence • 1952: (2)

Anderson, Loran Crittendon (1936- ) Taxonomist; Florida State U., Tallahassee, Herbarium Correspondence • 1976: (3) Barton, Benjamin Smith, 1766-1815 • 1977: (3) • 1978: (2) • 1986: (1)

Anderson, Maureen Tulane U., Library Correspondence $\bullet$ 1980: (1)

Anderson, , Mrs. Correspondence $\bullet$ 1943:

Anderson, Paul G. Washington U., School of Medicine, St. Louis, MO, Archives and Rare Books, Associate Director Correspondence • 1989: (2)

Anderson, Paul K. U. of Calgary, Professor of Zoology Correspondence • 1976: (2)

Anderson, R. M. Canada Dept. of Resources Correspondence • 1941: (3)

Anderson, Robert (1818-1856) Bioscript

Anderson, Robert D. Dayton, $\mathrm{OH}$ Correspondence $\bullet$ 1960: (1) Book collectors $\bullet$ 1961: (6) $\bullet$ 1964: (2)

Anderson, Susan Heller Pittsburg, PA Correspondence • 1969: (1)

Anderson, Walt Environmental Studies, Prescott College, Prescott, AZ

Correspondence • 1992: (2) 
Anderson, Watter laglis

Bosanical Album

Andernce, Wullam (1766-1840) Chelsea Physic Garden Blascrilt

Anderson, Willam Arthur (1900-1949) Taxonomist; U of lowa

Correspondence • 1946: (2) American Fern Society

Andersoo, Willasm H., Jr. Denver Botanic Gardens, Denver, $C 0$

Cornapondence $\bullet 1974:(4) \bullet 1977$ : (6) Cockerell, Theodore Drs Alison, 1866-1948

Anderson, Wullian Russell (1942-) U. of Michigan, Hertarium, Amin Arbor, MI

Correspondence $\bullet 1971$ : (3) $\bullet 1977:$ (2) $\bullet 1978:$ (1)

Andersen-Henry, laxe

Biascript

Andersoen, Nils Johan (1821-1880) Swedish botanist, plant explortr Bloscripe

Andes, Sarah Dublin Parls \& Recreation, Dublin, $\mathrm{OH}$,

Parks Horticulnirist

Comespondence • 1989: (2)

Andorfer, Johama

Correspondence • 1948: (1)

Andre, Edoward Frangois (1840-1911) Landscape architect, plant collector, horticulturist

Bigfile • Biloscript

Andre, Joha (1751-1780)

Blascript

Andresen, John William (1925.) Taxonomist, dendrologiat: Michigan State U., East Lansing, Dept. of Forestry, Asst. Prof, of Forestry

Correspondence • 1962: (1) • 1963 : (2) "Rocky Mountain Vegeation"

Andrew, Warren Indiana U., Indianapolis, Professor Comropondence * 1978: (2)

Andrews, Charles William (1866-1924) Paleontologist; Memorial U. of Newfoundland, St. John's

Corremondence • 1957: (1)

Andrews, David Alpine, NJ

Correpondence • 1989: (2) • 1990; (2) • 1992: (3) • 1993: (2)

Andrem, Darwin Masson (1869-1938) Nurseryman; Boulder, $\mathrm{CO}$

Blascript * Botanical Album

Andrews, Benry Nathanie, Jr. (1910-) American paleobotanist; Laconia, NH

Correspondence $\bullet 1977$ : (1)

Andrews, Mary W. Boralder, CO, husband is Darwin Andrews

Correspondence • 1949: ( 9 )

Andrews, Ralph U.S. Dept. of the Interior, Fish \& Wildlife Serv., Laurel, MD, Chief, Sect. of Wetland Ecology

Correspondence $\bullet 1971$ : (1) Andrews, Roy Chapman American paleontologist
Botanical Album Andrews, Timothy Langdon (1819-1908) California
medical botanist

Biescripr
Angell, Lydia

Correspondence $\bullet$ 1950: (1)

Angely, João Alberto (1917- ) Brazilian botanist Biofile

Angier, Belle Summer Bioscript

Angus and Robertson, Ltd. Technical book seller Correspondence $\bullet$ 1951: (0) Correspondence filed under Frans Verdoorn

Angwin, Florence

Correspondence $\bullet$ 1938: (3) - 1939: (10) $\bullet$ 1940: (3)

Letter from Robert L. Stearns • 1942: (1) • 1943: (1) • 1944: (2) • 1945: (3) • 1947: (1) Jepson, Willis Linn, 1867-1946 • 1949: (3) • 1951: (1) • 1952: (3)

Ankenef, Margaret E. Colorado Education Assoc. Correspondence • 1941: (1)

Annals of Science Correspondence $\bullet$ 1982: (1)

Anspach, G. Bioscript

Anstruther, John Bioscript

Anthony, Alfred Webster (1865-1939) Field naturalist, mining engineer

Biofile $\bullet$ Bioscript $\bullet$ Botanical Album

Anthony, Charles A. Son of A. W. Anthony Correspondence $\bullet 1963:(5) \bullet 1964:$ (2)

Anthony, Harold Elmer (1890-1970) American zoologist; American Museum of Natural History; son of A. W. Anthony

Biofile $\bullet$ Bioscript $\bullet$ Botanical Album

Correspondence • 1951: (3) 1963 : (1) $\bullet$ 1964: (1)

Anthony, John G. Bioscript

Anthony, John Gould (1804-1877) American conchologist Biofile

Antill, Edward (1701-1770)

Bioscript

Antin, Doris Tulane U., Howard-Tilton Memorial Library, Archivist

Correspondence • 1980: (1) $\bullet$ 1981: (2)

Antiquarian Booksellers Association of America New York, NY

Correspondence • 1971: (2)

Antisell, Thomas (1817-1893) Irish-born American physician and chemist Bioscript

Apfelbaum, Joan Academy of Natural Sciences, Philadelphia, PA, Dept. of Botany, Research Assistant Correspondence - 1969: (2) Nuttall, Thomas, 1786-1859 1974: (3) • 1975: (6) Barton, Benjamin Smith, 1766-1815 - 1976: (6) • 1977: (3) • 1978: (2) • 1979: (1)

Apgar, Austin Craig Bioscript • Botanical Album

Appalachian Cultural Center Charles Alan Watkins, Director

Correspondence • 1985: (1)

Appalachian Trail

Botanical Album 
Appelbaum, Stuart Tulane Gift Report, Project Coordinator

Correspondence • 1979: (1)

Applegate, Elmer Ivan (1867-1949) Plant collector, taxonomist; Stanford U., Dudley Herbarium, Bioscript

Correspondence $\bullet$ 1935: (2) • 1937: (4) Epling, Carl Clawson, 1894-1968; Species

Applegate, Susan Ewan family member Correspondence $\bullet$ 1929-1933: (2) • 1951: (1) • 1952: (2) - 1958: (2)

Applegate, T. S. Albany, NY Botanical Album

Arader, W. Graham, III American antiquarian bookdealer Biofile $\bullet$ Bioscript Correspondence $\bullet$ 1984: (4)

Arata, Andrew A. World Health Organization, Geneva, Switzerland Correspondence • 1965: (1) • 1966: (3) • 1967: (1) • 1973: (1) • 1987: (1) • 1993: (3)

Arbeláez, Enrique Pérez (1896-1972) Congreso Botanico Suramericano Botanical Album

Correspondence $\bullet$ 1951: (2)

Arber, Agnes Robertson (1879-1960) Botanist, morphologist Biofile $\bullet$ Botanical Album

Arceneaux, George International Research Service, Pass Christian, MS, Director Correspondence • 1956: (2) • 1959: (4) • 1967: (2)

Archer, John (1777-1830) Bioscript

Archer, William Andrew (1894-1973) Mexican-born American economic botanist, plant geographer, archivist; U. S. Dept. of Agriculture, Herbarium, Plant Industry Station, Beltsville, MD; nickname "Andy"

Biofile $\bullet$ Bioscript $\bullet$ Botanical Album

Correspondence - 1947: (1) - 1948: (7) Letter to Ewan from Harold N. Moldenke 1949: (2) Maxon, William Ralph, 1877-1948 • 1951: (1) • 1952: (3) • 1953: (4) "Rocky Mountain Vegetation" - 1954: (2) Delphinium • 1956: (1) 1961: (1) 1965: (1) • 1966: (1) 1968: (2) 1969: (2) • 1971: (5) - 1972: (5)

Archibald, Jennie Maude Husband is J. C. Blake Bioscript

Archives Internationals D'Histoire Des Sciences Correspondence 1982 : (1)

Arctowski, Henry Biofile

Ardène, Jean-Paul de Rome d' Biofile

Arènes, M. J. Museum National D'Histoire Naturelle Correspondence $\bullet$ 1946: (1)

Arete Publishing Company, Inc. Lawrence K. Lustig, Editor-In-Chief Correspondence 1978: (3)

Argus, George William (1929-) Taxonomist; National Museum of Natural Sciences, Canada Correspondence $\bullet$ 1983: (2)
Arion Press

Correspondence $\bullet$ 1986: (2)

Aristeguieta, Leandro (1923- ) Botanist; Instituto Botánico, República de Venezuela, Caracas

Correspondence • 1959: (3)

Arizona State University Correspondence $\bullet$ 1985: (3)

Arkansas, Conway Conway Chamber of Commerce Correspondence • 1975: (1) Nuttall, Thomas, 1786-1859

Arkway, Richard B., Inc. Bookseller Correspondence $\bullet$ 1985: (2)

Armentano, Thomas U. of Tennessee, Knoxville, graduate assistant Correspondence $\bullet$ 1964: (1)

Armitage, Thomas Tulane U., Superintendent, Buildings and Grounds Correspondence $\bullet$ 1983: (1)

Armstrong, Alan W. First Pennsylvania Bank, Philadelphia, Senior Vice-President; Haversford, PA

Bioscript • Botanical Album

Correspondence $\bullet$ 1984: (28) Letter to Whitfield Bell $\bullet$ 1985: (13) - 1986: (8) Letters to and from Edmund Berkeley $\bullet$ 1987: (14) $\bullet$ 1988: (6) $\bullet$ 1989: (5) Barton, Benjamin Smith, 1766-1815 • 1990: (3) • 1992: (2) • 1993: (1)

Armstrong, Margaret Bioscript

Armstrong, Martha B.

Correspondence • 1972: (0) Correspondence filed under College of William and Mary

Army Medical Library Correspondence • 1951: (1) Barton, Benjamin Smith, 1766-1815

Arnaud, Paul H. California Academy of Sciences, Curator and Chairman, Dept. of Entomology

Correspondence • 1974: (1)

Arnett, Florence

Correspondence • 1943: (1)

Arnett, Ross H. Oxycopis Pond Research Station, Kinderhook, New York Correspondence $\bullet$ 1981: (4)

Arnold Arboretum Bioscript • Botanical Album Correspondence $\bullet$ 1982: (7) $\bullet$ 1983: (4) $\bullet$ 1984: (4) $\bullet$ 1985: (4)

Arnold, Chester Arnold (1901- ) Paleobotanist; U. of Michigan, Ann Arbor, Botanical Museum Correspondence • 1950: (2) • 1968: (12) • 1969: (1)

Arnold, John G. Loyola U., New Orleans, LA Correspondence • 1949: (1)

Arnold, Lillian Eleanore (1895- ) American taxonomist; U. of Florida, Gainesville, Herbarium Correspondence • 1949: (2)

Arnold, Robert C. Office of Economic Warfare Correspondence • 1943: (1)

Arnold, Thurman Bioscript

Arnott, George Arnold Walker (1799-1868) Scottish botanist Bioscript 
Arnott, Howard Joseph (1928-) Plant anatomist and morphologist; Northwestern U., Evanston, II, Assistant Professor

Correspondence $\bullet$ 1961: (2)

Arnow, Lois U. of Utah, Salt Lake City, Dept. of Botany Correspondence $\bullet 1968$ : (2)

Arny, Sam Amn Arbor, Michigan

Correspondence $\bullet$ 1949: (1)

Arps, Louisa Ward

Correspondence $\bullet$ 1965: (0) Letter filed under Anthony W. Shipps

Arsène, Brother Gerfroy (1867-1938) Botanist

See also Brouard, Arsène Gustave Joseph Bioscript

Artedi, Peter (1705-1735) Ichthyologist Biofile

Arthur, Ann Tulane U.

Correspondence $\bullet$ 1975: (2)

Arthur, John S. New Orleans, LA

Correspondence $\bullet 1963:(1) \bullet 1964$ : (0) Filed with Waldemar H. Fries

Arthur, Joseph Charles (1850-1942) American pioneer, plant parhologist

Bioscript

Arthur, Stanley Clisby

Correspondence $\bullet$ 1948: (1)

Artigas, Jorge N. Universidad de Concepción, Instituto Central de Biología, Concepción, Chile

Correspondence $\bullet 1968:(2)$

Artigue, Gloria Tulane U., Secretary to the Art Dept. Correspondence $\bullet 1968$ : (2)

Artjushenko, Zinaida T. (1916-) Botanical Institute, Leningrad, Russia

Botanical Album

Correspondence $\bullet 1971$ : (1) $\bullet 1972$ : (1)

Arundel, Walter Brown

Bioscript

Arwidsson, Th. Amold Arboretum Correspondence $\bullet 1929-1933:$ (2)

Arzeni, Carlo (Charles) Basel (1925-) Eastern Ilinois U., Charleston, Associate Prof. of Botany; Morris Harvey College, Charleston, WV; formerly at Tulane Correspondence • 1961: (3) $\bullet 1966$ : (3)

Ashbey, Anna New Orleans, LA Correspondence $\bullet 1957$ : (1) $\bullet 1958$ : (1)

Ashby, C. P.

Correspondence $\bullet$ 1951: (2) American Fern Society

Ashby, William Clark (1922-) Plant ecologist and physiologist, Ecological Society of America, Dept. of Botany Correspondence $1970:(0)$ Correspondence filed under
Willis Alexander Eggler

Ashe, Thomas (-1835)

Bioscript

Ashe, William Willard (1872-1932)

plant collector Biofile • Bioscript

Asheville Citizen Times Asheville, NC Correspondence • 1962: (1) Lyon, John, 1764-1814
Ashmead, William Harris (1855-1908) American entomologist

Bioscript

Ashmole, Elias (1617-1692) Astrologer, naturalist Bioscript

Ashmun, Edward Houghton (1853-1904) American malacologist, Congregational minister

Bioscript

Ashton, Peter Shaw (1934) English dendrologist, texonomist

Bioscript

Ashton, Ruth Elisabeth Nelson (1896-) American bryologist

Biofile • Bioscript $\bullet$ Botanical Album

Assie, G. L. Ambassador of France, New York. NY, The Cultural Counselor

Correspondence $\bullet$ 1952: (1)

Association for Tropical Biology, Ine. Cifford Evans Correspondence $\bullet$ 1976: (1)

Association of Beneficial Plant Research Carmel Valley. CA

Correspondence $\bullet 1980$ : (1)

Atherton, Edward H.

Correspondence • 1921-1928: (4) • 1929-1933: (5)

Atherton, May

Correspondence • 1952: (2)

Atkins, F. L. (1862-1930)

Bioscript

Atkinson, George Francis (18541918) American mycologist

Botanical Album

Atkinson, Lenette May Rogers (1899-) Plant cytologist and morphologist; Amherst, MA

Correspondence $\bullet 1963:$ (1) $\bullet 1969:$ (2)

Allas New York, NY; Eleanor Davidson Worley, Publisher-Editor

Correspondence • 1961: (1)

Atlee, Edwin A.

Bioscript

Atmus, R. E.

Correspondence • 1966: (2)

Atsatt, Sarah Rogers (188s-) American zoologist; Los Angeles, CA

Correspondence • 1944: (1) • 1949: (1)

Attenborough, David Frederick (1926-) British naturalist, film-maker, author

Biofile

Atwater, Betty Ransom Ransom Seed Laboratories Botanical Album (Filed under Eugene Arwater) Correspondence - 1936: (1) Species

Atwater, Eugene

Botanical Album

Atwood, Howland Huntingwon Library, San Marino, CA Correspondence * 1953: (2) • 1954: (1) Aublet, Jean Baptiste Christophe Fuske (1720-1778)
French botanist

Biofile - Bioscript

Audibert (of Tarascon)

Bioscript 
Audubon House and Gardens Molly Wylly, Research Historian

Correspondence $\bullet$ 1986: (3)

Audubon, John James (1785-1851) Ornithologist, artist Biofile $\bullet$ Bioscript $\bullet$ Botanical Album

Audubon, John Woodhouse (1812-1862) Bioscript

Audubon Park and Zoological Park, New Orleans Correspondence $\bullet$ 1968: (1) $\bullet$ 1981: (3) $\bullet$ 1982: (2)

Audubon, Victor Gifford (1809-1860) Painter; elder son of J. J. Audubon

Bioscript

Aufrere, George Bioscript

Augusta College Augusta, GA Correspondence $\bullet$ 1992: (2)

Auld, Jean M. Correspondence $\bullet 1963$ : (3)

Aulin, Fredrik Rutger (1841-1923) Swedish taxonomist, plant collector Biofile $\bullet$ Bioscript

Aulls, Ina T. Public Library, Denver, CO Correspondence • 1948: (4) - 1949: (5) Rocky Mountain Naturalists • 1950: (1) • 1951: (2) Rocky Mountain Naturalists

Ault, Quentin L. Tulane U., Public Relations Dept. Correspondence • 1949: (1)

Aungst, John W., Jr. Correspondence $\bullet$ 1985: (1)

Austin, Elizabeth S. U. of Florida, Gainesville; husband is Oliver L. Austin

Correspondence $\bullet$ 1965: (4) $\bullet$ 1971: (1)

Austin, Rachel Merritt (1832-1919) American botanist Biofile $\bullet$ Bioscript $\bullet$ Botanical Album

Australia National Library Botanical Album

Australia Week Correspondence $\bullet$ 1990: (1)

Auty, Phyllis Simon Fraser U., History Dept; Cook Conference, "Captain James Cook \& His Times" Correspondence • 1978: (3)

Avant, William H. Tulane U. student Correspondence - 1949: (2)

Avery, George Sherman (1903-) Plant physiologist; Brooklyn Botanic Garden, Director Bioscript $\bullet$ Botanical Album

Correspondence • 1968: (4) • 1969: (3) • 1970: (2) • 1973: (2)

Avinoff, Audrey Nikolaivich (1884-1949) RussianAmerican lepidopterist and painter Biofile - Bioscript

Axelrod, Daniel Isaac (1910- ) Bioscript $\bullet$ Botanical Album Correspondence $\bullet$ 1952: (1) $\bullet$ 1967: (2)

Axelrod, Nancy U. of California, Berkeley, Librarian Correspondence • 1986: (1)

Aycock, James F. Correspondence $\bullet$ 1950: (2)

Ayers, Tina J. (1957- ) Correspondence • 1990: (1) • 1992: (1)
Ayres, Horace Beemer (1856-) American timber inspector and geologist Bioscript

Ayres, William Orville (1817-1891) Bioscript

Azara, Félix de (1746-1821) Spanish geographer, naturalist Biofile

\section{$\boldsymbol{B}$}

Baatz, __, Dr. Correspondence • 1989: (1)

Babcock, Dean (1888-1968) American artist, naturalist Bioscript

Babcock, Ernest Brown (1877-1954) American plant geneticist, taxonomist; U. of California, Berkeley, Division of Genetics

Bioscript • Botanical Album

Correspondence $\bullet$ 1934: (1) • 1935: (1) • 1951: (2)

1952: (2) • 1953: (6) • 1954: (4)

Babcock, Henry Homes (1832-1881) American botanist, plant collector Bioscript • Botanical Album

Babcock, James M. Detroit Public Library Correspondence • 1964: (2)

Babcock, Orville Gorman Bioscript $\bullet$ Botanical Album

Babington, Churchill (1821-1889) English lichenologist, ornithologist Bioscript

Bach, Johannn Sebastian (1685-1750) Bioscript

Bach, L. M. N. (Matt) Tulane U., Dept. of Physiology; Correspondence • 1970: (1) Letter from Fred Sowell, related to Fred Rugel

Bach, Susan Bioscript

Bache, Alexander Dallis (1806-1867) American physicist and chemist Biofile

Bachman, John (1790-1874) American clerygman, ornithologist, and botanist at Charleston Biofile $\bullet$ Bioscript $\bullet$ Botanical Album

Bacialupi, Rimo Carlo Felice (1901-) American botanist; U. of California, Berkeley, Jepson Herbarium, Dept. of Botany; nickname "Batch" or "Bach" Bioscript $\bullet$ Botanical Album Correspondence • 1937: (4) • 1948: (4) • 1950: (2) 1951: (4) Jepson, Willis Linn, 1867-1946 • 1952: (4) • 1953: (1) • 1954: (3) Jepson, Willis Linn, 1867-1946 - 1956: (1) • 1960: (2) - 1962: (5) $11963:(5) \bullet 1964:$ (3) $\bullet$ 1965: (3) $\bullet$ 1966: (11) $\bullet 1977:$ (2) $\bullet$ 1985: (1)

Back, George, Sir (1796-1878) Bioscript

Backeberg, Curt (1894-1966) German author on succulent plants Botanical Album

Bacon, Francis (1561-1626) Biofile $\bullet$ Bioscript

Bade, C. H. Bioscript 


\section{Guide to the Ewan Papers}

Baden-Powel, Georse Sayth (1847-1898) Author, politician

Bloscript • Botunical Album

Baedelier, Karl (1801-1859) German publisher Bioftil

Barhail, Charles Geova (1906-1984) Swiss taxonomist, plant morphologist; Conservatoire et Jardin Botaniques, Geneva, Switzerland

Bioflie • Bloscript • Botenical Album

Corrapondence • 1948: (3) $\bullet$ 1950: (2) $\bullet$ 1951: (1) • 1953: (1)

Baeza, Don Victor Manvel (1859-1944) Chilean botanist Bloftle • Blascript

Bagert, Betty WYES-TV

Correspondence • 1976: (1) Baruam, William, 1739-1823; Harper, Francis, 1886-1972

Bacley, Wullam C. Society for the Advancement of Edscanoe

Comerpondence $\bullet$ 1942: (3)

Baglietto, F.

Bloscripe

Bailly, Aifred Mariball (1894-1978) American

ornithologist; Denver Museum of Natural History, Denver. co

Bicucript

Comespondence • 1943: (1) • 1944: (5) • 1946: (8) •

1947: (8) • 1949: (8) Rocky Mountain Naturalists • 1951

(2) $\bullet 1971:(4)$

Bailey, Charies

Bloscripe • Botanical Album

Baile, D. K. Physicist, book collector; Fort Collins, CO

Botunical Abum

Boilly, Dthed Zoe (1889-1983) American botanist and borticulturist

Biofile

Balley, Ference Merriam (1863-1948) American omithologist

Bloftie • Bloscript

Bailey, Harold Edwands (1906-) American mycologist; Wayne U., Detroir

Bloscripr • Botanical Album

Correspondence • 1939: (6) • 1950: (1) • 1959: (0) Letters filed under Virginia Bailey • 1979: (I) • 1985: (1) • 1986: (1)

Balley, J. Atzstin Calgary, Alberta

Correspondence $\bullet 1970$ : (1)

Balley, Jacob Whitman (1811-1857) American botanist, chemist, geologist

Bigfile • Bioscript • Botanical Album

Baliey, Jane Mother of Jacob Whitman Bailey Bloscript

Bailey, Laura Rocktoridge Alum Springs Biological Laboratory, Goshen, VA Correspondence • 1966: (2) • 1967: (1)

Bailey, Liberty Hyde (1858-1954) Americ Biofile • Blotcriot * Botuica American horticulturist Correspondence

(3) Species $\bullet$ 1947: 1937: (1) • 1938: (4) Species $\bullet$ 1939: - 1951: (3) $1942:$ (2) 1 1945: (2) $\bullet$ 1948: (2) $\bullet$ 1949: (2) Bioscript
Bailey, Vernon Orlando (1864-1942) American naturalist; wife is Florence Merriam Bailey

Biofile $\bullet$ Bioscript $\bullet$ Botanical Album

Bailey, Virginia Edith Long, (1908- ) Husband is Harold Bailey; W. L. Jepson's botanical artist

Correspondence • 1937: (4) Jepson, Willis Linn, 1867-1946

- 1940: (2) • 1951: (2) Species • 1954: (3) "Rocky

Mountain Vegetation" $\bullet$ 1955: (6) Species $\bullet$ 1956: (4) • 1959: (2) • 1960: (2)

Bailey, William Whitman (1843-1914) American botanist, taxonomist

Bioscript

Bailkin, David New Orleans, LA Correspondence • 1961: (1)

Baillie, James L. Royal Ontario Museum of Zoology and Palaeontology, Toronto

Correspondence $\bullet$ 1955: (2)

Baillon, Henri Ernest (1827-1895) French natural historian, physician Biofile $\bullet$ Bioscript

Bain, John Bioscript

Bainbridge, 0. Cullom and Gertner, Publisher Correspondence $\bullet$ 1936:

Baird, Spencer Fullerton (1823-1887) American naturalist Biofile $\bullet$ Bioscript $\bullet$ Botanical Album

Baisoletto, B. Bioscript

Baker, Charles Fuller (1872-1927) American entomologist, botanist, plant explorer

Biofile • Bioscript $\bullet$ Botanical Album

Baker, Edmund Gilbert (1864-1949) Bioscript

Baker, Herbert George (1920-) English plant ecologist; U. of California, Berkeley

Bioscript • Botanical Album

Correspondence $\bullet$ 1967: (2) Letters to and from Adolph E. Waller; letter from A. E. Dimond $\bullet$ 1968: (7) $\bullet$ 1969: (4) $\bullet$ 1974: (3) • 1977: (1) • 1983: (1)

Baker, Horace Burrington (1889-1971) American zoologist, conchologist; U. of Pennsylvania, PA Bioscript

Correspondence • 1957: (2) • 1958: (1)

Baker, Hugh Potter (1878-1950) American educator, forester

Bioscript

Baker, John Gilbert (1834-1920)

Bioscript

Baker, Kenneth Frank (1908-) American plant pathologist; U. of California, Berkeley

Correspondence • 1967: (3) • 1968: (2) • 1970: (2)

Baker, Marjorie Dillman Vanderbilt U., Dept. of Biology; student of R. B. Channell Correspondence 1961 : (2)

Baker, Milo Samuel (1868-1961) American botanist Bioscript $\bullet$ Botanical Album

Correspondence - 1935: (4) Delphinium • 1938: (4) Species • 1939: (4) • 1940: (5) Delphinium • 1943: (2) • 1944: (2) • 1948: (1) 1949: (7) Species $\bullet$ 1951: (3) Delphinium $\bullet$ 1952: (4) $\bullet$ 1953: (2) $\bullet$ 1960: (1) 
Baker, Richard Eric Defoe (1908-1954) Imperial College of Tropical Agriculture, Trinidad, B. W. I.

Correspondence - 1949: (2)

Baker, Richard C. American bookbinder; St. Louis, MO Botanical Album

Correspondence $\bullet$ 1993: (1)

Baker, Sylva Academy of Natural Sciences, Philadelphia, Head Librarian

Correspondence $\bullet$ 1982: (3) $\bullet$ 1983: (1)

Baker, Walter U. of California, Los Angeles Botanical Album

Correspondence • 1939: (2) - 1943: (1)

Baker, William Hudson (1911-1985) American taxonomist; U. of Idaho, Moscow

Botanical Album

Correspondence • 1948: (1) • 1949: (2) Delphinium • 1950: (1)

Bakewell, Lucy (1786-1874) Mill Grove, PA; husband is J. J. Audubon

Bioscript

Bakke, Arthur Lawrence (1886- ) Correspondence $\bullet$ 1953: (1)

Balbis, Giovanni B. Horticulturist Bioscript

Baldinger, Ernst Gottfried (1738-1804) German physician, medical writer Biofile

Baldwin, Benjamin Baldwin-Machado Correspondence $\bullet$ 1953: (2)

Baldwin, John Thomas (1910-) American cytogeneticist; College of William and Mary, Williamsburg, VA

Bioscript $\bullet$ Botanical Album Correspondence $\bullet$ 1939: (3) $\bullet$ 1940: (1) $\bullet$ 1947: (1) $\bullet$ 1948: (1) • 1949: (3) Species • 1962: (1) • 1963: (1) • 1964: (1) 1970: (1)

Baldwin, Samuel Prentiss (1868-1938) American ornithologist, lawyer Bioscript

Baldwin, William (1779-1819) Physician, botanist Biofile • Bioscript

Baley, Sallie W. Correspondence $1921-1928$ : (1)

Balfour, A. M. Daughter of Gertrude Balfour; husband is F. R. S.

Correspondence $\bullet$ 1955: (3)

Balfour, Andrew Bioscript

Balfour, Gertrude Correspondence $\bullet$ 1955: (4)

Balfour, I. B. Bioscript

Balfour, John Hutton (1808-1884) Scottish physician, botanist Biofile • Bioscript

Balfour-Gourlay, William (c. 1879-1966) Bioscript

Ball, Anne E. (1908-1872) Irish algologist Biofile

Ball, Carleton Roy (1873-1950) American taxonomist, plant collector; U. S. Dept. of Agriculture

Bioscript $\bullet$ Botanical Album

Correspondence • 1929-1933: (3) • 1939: (2) • 1940: (2)
Ball, Carleton Roy (1873-1950) (continued)

- 1942: (4) • 1947: (1) • 1948: (3) • 1949: (2) • 1950: (7)

- 1951: (6) "Rocky Mountain Vegetation" Species

Ball, Elias

Biofile

Ball, H. W. British Museum (Natural History), London, England

Correspondence - 1958: (2)

Ball, John (1794-1884) Geologist, Rocky Mountains Bioscript

Ball, John (1818-1889) Irish naturalist, plant collector and explorer Bioscript

Ball, Lizzie Second wife of Isaac Martindale Bioscript

Ball, M. F. Correspondence $\bullet$ 1935: (1)

Ballard, Ernesta D. (1920- ) American horticulturist; Pennsylvania Horticultural Society

Botanical Album

Correspondence • 1974: (3) • 1975: (9) • 1976: (10) Includes correspondence with Julie Morris $\bullet$ 1977: (3) • 1978: (2)

Ballard, J. O.

Correspondence $\bullet$ 1957: (2)

Ballard, Olive M. Husband is of Roy Page Correspondence - 1944: (4)

Ballou, Ruth Correspondence • 1929-1933: (1) Delphinium

Ballow, Jack Montchanin, DE Correspondence • 1970: (1)

Balls, Edward Botanical Album

Balmis, Francisco Xavier (fl. 1794) Spanish physician, botanist Biofile

Balogh, Pamela Correspondence • 1976: (1)

Bamforth, Stuart Newcomb College, Zoology Correspondence • 1958: (1) • 1964: (3) • 1966: (1) • 1972: (1) - 1976: (1) Copy of correspondence between Edwin Gould and Stuart Bamforth • 1986: (1) 1991 : (4)

Bampus, George C. (fl. 1901) Hyde Park Botanical Album

Bancroft, Edward (1744-1821) Chemist, naturalist; British Guiana Bioscript

Bancroft, Edward Nathaniel (1772-1842) Botanist; Kingston, Jamaica Biofile

Bancroft, Eleanor U. of California, Bancroft Library Correspondence • 1943: (7) • 1946: (1)

Bancroft Library U. of California Correspondence $\bullet$ 1938: (1) • 1960: (1) • 1966: (1) • 1984: (2)

Bandelier, Adolph Francis Alphonse (1840-1914) Swiss archeologist, ethnologist Bioscript

Bane, Ellin C. New Orleans, LA; husband is Ray Bane Correspondence - 1957: (2)

Bang-Hofman, Niels (1776-1855) Bioscript 


\section{Guide to the Ewan Papers}

Bang, Miguel (1853-1936)

Bioscript $\bullet$ Botanical Album

Bange, Chr. Université Claude Bernard, France Correspondence $\bullet$ 1987: (2)

Banister, John (1650-1692) English naturalist; Virginia Biofile • Bioscript

Banker, Howard James (1866-1940) American botanist Biofile

Banker, Isaac Alexander Bioscript

Banks, Donald Jack (1930-) American taxonomist, agrostologist Correspondence • 1965: (4) • 1966: (1)

Banks, Harlan Parker (1913-) American paleobotanist; Cornell U.; Botanical Society of America Correspondence • 1966: (1) • 1967: (2)

Banks, Joseph (1743-1820) British botanist, plant explorer, patron

Biofile $\bullet$ Bioscript $\bullet$ Botanical Album

Bankston, Aurelia E.

Correspondence • 1951: (1) American Fern Society

Barabino, Joseph Biofile $\bullet$ Bioscript

Barber, Charles Melvin (1876-)

Bioscript • Botanical Album

Barber, J. H. Horticulturist; Santa Monica, CA Bioscript $\bullet$ Botanical Album

Barber, John Threlfall (1937- ) English-born American plant physiologist; Tulane U.

Correspondence $\bullet 1969 \bullet 1974$ : (2)

Barbey, William (1842-1914) Swiss botanist, taxonomist, plant collector Bioscript

Barbier, J. B. Bioscript

Barbour, Clyde U. of Utah, Salt Lake, Dept. of Zoology Correspondence • 1967: (2)

Barbour, Evelyn Missouri Botanical Garden; assistant to Edgar Anderson

Correspondence $\bullet$ 1954: (1)

Barbour, Roger William (1919)

Correspondence $\bullet 1991$ : (1) Barbour, Thomas (1884) American zoologist
Biofile

Barbre, Clarence American horticulturist; Clarence Barbre, Azaleas and Roses, Webster Groves, MO Correspondence $\bullet$ 1961: (2) Nutrall, Tho

(2) Nuttall, Thomas, 1786-1859 Correspondencer (1932-) Plant explorer, taxomist

Barclay, George Biofile $\bullet$ Bioscript

Barclay, Harriet George (1910) American plant ecologist; Bioscript Dept. of Life Sciences, Professor of Botany

Correspondence • 1961: (2) • 1962: (2)

Barclay, Robert Bioscript

Bard, wiliam Bioscript
Barde, Fred (1869-1916) American journalist and wildlife photographer

Biofile

Barfield, Jon U. of Alabama, Biology

Correspondence $\bullet$ 1991: (1)

Barfoot, Joseph Lindsey (1816-1882) English-born curatot of Deseret (Salt Lake City) Museum Bioscript

Barkelew, Frederick E. Bioscript

Barker, Byron W. Correspondence • 1929-1933: (1)

Barker, John Correspondence $\bullet$ 1979: (1)

Barkley, Fred Alexander (1908-) Taxonomist; Montana State U., Missoula

Botanical Album

Correspondence • 1938: (2) • 1939: (2) Species • 1940: (2) $\bullet$ 1942: (3) $\bullet$ 1945: (1) $\bullet 1950$ : (2)

Barkley, Grace (1878-1930) American botanist Biofile

Barkley, Theodore Mitchell (1934) American texonomist; Kansas State U., Manhattan, Division of Biology Botanical Album

Correspondence • 1974: (4) • 1976: (3) • 1979: (3) • 1987: (1) • 1988: (3) Van Schaack, George Booth, 1903 1983

Barlow, Chester (1874-1902) Bioscript

Barlow, Henry Correspondence • 1993: (1)

Barlow, Max G. Correspondence $\bullet 1964:$ (2)

Barman, Roderick J. Hispanic and Italian Studies, U. of British Columbia Correspondence $\bullet$ 1984: (3)

Barnard, Julia A. American Antiquarian Socicty Correspondence • 1978: (0) Filed under American Antiquarian Society

Barmard, T. Bioscript

Barneby, Rupert Charles (1911-) English-born taxonomist; New York Botanical Garden Biofile • Bioscript Correspondence • 1948: (5) • 1950: (2) • 1975: (2) Species Barnes, Albert Coombs (1872-1951) Bioscript

Barnes Arboretum Correspondence $\bullet 1972$ : (1)

Barnes, Charles Reid (1858-1910) American bryologist Bioscript

Barnes, Claude Teancum (1884) Mammalogist, Ornithologist, banker; First National Bank, Salt Lake City,

Bioscript

Correspondence $\bullet$ 1949: (1)

Bames, Harry Elmer Bioscript

Barnes, Kate Amateur botanist; hasband is Charles Barnes 
Barnes \& Noble Bookstores, Inc. New York, NY Correspondence • 1980: (1)

Barnes, Richard Magoon Cooper Ornithological Club Correspondence • 1921-1928: • 1929-1933: (1)

Barnes, William (1860-1930) American surgeon, entomologist Bioscript

Barnett, Adrian Correspondence - 1992: (1)

Barnett, C. E. Northrup, King and Co., Seed Merchants Correspondence $\bullet$ 1937: (1)

Barnett, Carol Books, Portland, OR Correspondence • 1992: (2)

Barnett, James F. Meriden Gravure Company, publisher Correspondence $\bullet$ 1965: (0) Correspondence filed under G. W. Corner

Barnhart, Carl H. Correspondence • 1940: (3) • 1941: (3) U. of Colorado, Boulder. Dept. of Biology

Barnhart, John Hendley (1871-1949) American botanical bibliographer; New York Botanical Garden Bioscript $\bullet$ Botanical Album Correspondence • 1936: (10) • 1941: (1) • 1942: (1)

Barnhill, Ethel $\mathbf{M}$. Correspondence $\bullet$ 1947: (1) $\bullet$ 1954: (2)

Barnsley, Godfrey (1805-1873) Bioscript

Barnum, Phineas Taylor (-1891) American showman and collector of natural history

Biofile $\bullet$ Bioscript

Baron, Alexander (of Charleston) Bioscript

Baron, Richard (1847-1907) English missionary, geologist, botanist

Biofile

Barr, Alwyn Texas Tech U., Lubbock, Dept. of History Correspondence • 1983: (2)

Barr, Guinn Correspondence • 1938: (1) Delphinium

Barratt, John P. Bioscript

Barratt, Joseph (1796-1882) English-born American botanist Bioscript

Barreth, D. Bioscript

Barrett, Ellen C. Los Angeles Public Library, Genealogy and Local History Div.

Correspondence $\bullet$ 1959: (2)

Barrett, P. G. Bioscript

Barrett, Robert Le Moyne American geographer Biofile

Barriga, H. Garcia Bioscript $\bullet$ Botanical Album

Barrington, Daines (1727-1800) Bioscript

Barrington, Davis S. U. of Vermont, Burlington, Associate Professor of Botany, Pringle Herbarium Correspondence • 1986: (1) • 1988: (1)
Barroeta, G.

Bioscript

Barron, W. Bioscript

Barrott, Bioscript (of South Carolina)

Barrow, John Bioscript

Barrows, John S. Correspondence • 1943: (0) Two letters filed under Robert E. More re. Horace Greeley

Barry, B. C. Bioscript

Barry, Edward Free Press Correspondence $\bullet 1975$ :

Barsagita, , Dr.

Bioscript

Bart, Ellen U. of Pennsylvania, PA Correspondence $\bullet$ 1992: (3)

Bartholomew, Bruce Monroe (1946-) U. of California, Berkeley, Dept. of Botany, Curator Correspondence • 1976: (2)

Bartholomew, Elam (1852-1934) American mycologist, politician Bioscript

Bartholomew, Elizabeth Ann West Virginia U., Morgantown, Southern Appalachian Botanical Club, Secretary Bioscript • Botanical Album Correspondence $\bullet$ 1958: (1) • 1968: (1)

Bartine, John G. Judge; Circuit Court of South Dakota Correspondence • 1942: (2)

Bartle, Ida B. Bioscript • Botanical Album

Bartlett, A. P., Mrs. Bioscript

Bartlett, Albert William (1875-1943) Bioscript

Bartlett, Harley Harris (1886-1960) American plant explorer, plant geneticist; U. of Michigan, Ann Arbor Biofile $\bullet$ Bioscript $\bullet$ Botanical Album Correspondence • 1947: (1) - 1952: (5) • 1953: (1) • 1957: (4) • 1958: (1) - 1961: (0) See letter from Nordin-Petterson to Bartlett

Bartley, Floyd (1888- ) Bioscript Correspondence $\bullet$ 1950: (3) $\bullet$ 1951: (5)

Bartling, F. G. Bioscript

Barton, A. L. Bioscript

Barton, Barbara J. Correspondence $\bullet$ (2)

Barton, Benjamin Smith (1766-1815) American physician, naturalist

Biofile • Bioscript

Barton, David Rittenhouse Bioscript

Barton, Edward ( -1821) Bioscript 


\section{Guide to the Ewan Papers}

Barton, Edward Hall (1797-1859) Bioscript

Barton, Mary Husband is Benjamin Smith Barton Botanical Album

Barton, Matthias

Bioscript

Barton, Richard (1706-1751)

Bioscript

Barton, Thomas (1730-1780) Irish American minister and botanist

Biofile $\bullet$ Bioscript

Barton, Thomas W. (d. 1818)

Bioscript

Barton, William ( -1817)

Bioscript

Barton, William Paul Crillon (1786-1856) American physician and botanist

Biofile $\bullet$ Bioscript $\bullet$ Botanical Album

Bartram Association

See John Bartran Association

Bartram, Edwin Bunting (1878-1964) American bryologist, plant collector

Bioscript • Botanical Album

Bartram George, Jr. Bioscript

Bartram, John Bioscript • Botanical Album

Bartram, John B., M.D. Philadelphia, PA

Correspondence $\bullet 1977:$ (2)

Bartram, John, Jr.

Botanical Album

Bartram Trail Conference Correspondence • 1991: (1)

Bartram Trail Society

Correspondence • 1975: (1)

Bartram, William

Bioscript • Botanical Album

Bartsch, F.

Bioscript

Bary, H. A. de Bioscript

Basalla, George U. of Texas, Dept. of History, Austin Correspondence 1 1967: (2)

Basile, Ralph R. Former student

Correspondence 1 1967: (1)

Basilisk Press \& Bookshop Hampstead, London, England, Charlene Garry, Director (USA) Correspondence • 1976: (1) • 1979: (1) • 1981: (1) •
1983: (2) 1983: (2)

Baskett, James Newton (1849-1925) American mamulist author Bioscript

Baskin, Jerry Mack (1940-) American plant coologist: of Kentucky, Lexington, T. H. Morgan School of Bio. Sciences

Correspondence • 1979: (2) • 1985: (2)

Baskin, Samuel Pleasants Bioscript

Bass, Thomas Bioscript
Bass, William W. Carson-Newman College, Jefferson City, TN

Correspondence $\bullet$ 1953: (2)

Bassett, Douglas National Museum of Wales, Director Correspondence $\bullet$ 1986: (1)

Bassett, Frank N. Los Angeles bookseller, natural sciences Correspondence • 1954: (1) Book collectors $\bullet$ 1960: (2) • 1963 : (3) Book collectors • 1965: (1)

Bassett, Victor H. Bioscript

Bastard, T. J. Bioscript

Baster, Job (1711-1775) Bioscript

Batcheler, Freda Thomas Cook and Son Correspondence $\bullet 1964$ : (1)

Bateman, John (fl. 1665-1700) English botanist Biofile

Bateman, Robert American botanical artist Bioscript • Botanical Album

Bates, David M. Cornell U., L. H. Bailey Hortorium Correspondence • 1965: (2) • 1966: (1)

Bates, Henry Walter (1825-1892) English entomologist Bioscript • Botanical Album

Bates, John Mallory (1846-1930) American Episcopal clergyman, botanist, ornithologist Bioscript

Bateson, William (1861-1926) English horticulturist, plant geneticist

Biofile • Bioscript • Botanical Albwm

Batsch, August Jobann Georg Carl (1761-1802) German botanist at Jena

Bioscript

Batson, Wade T. U. of South Carolina, Columbia, Dept. of Biology, Acting Head

Botanical Album

Correspondence $\bullet$ 1959: (2)

Batty, Joseph H. (1847-1906) Taxidermist; New York, NY Bioscript

Baudin, Nicolas Thomas (1750-1803) French navigator, plant collector, phytogeographer Biofile

Bauer, Ferdinand (1760-1826) Austrian botanical artist Biofile (Filed under Franz Bauer)

Bauer, Francis Bioscript

Bauer, Franz (1758-1840) Austrian botanical artist Biofile

Baver, Harry Lloyd (1892-) Santa Monica Junior College Bioscript

Correspondence • 1929-1933: (1)

Bauhin, Jean Gaspard (1606-1685) Swiss botanist. physician

Bioscript

Baumann, J. P. Bioscript

Baur, $\mathbf{F}$. Bioscript

Baxevanis, John J. E. Stroudsburg U., E. Stroodsbure. PA, Geography Dept.

Correspondence $\bullet 1989:$ : 
Baxter, W. H.

Bioscript

Bay, Jens Christian (1871-1962) Danish-American bibliographer; John Crerar Library, Chicago, IL Biofile Correspondence 1929-1933: (1) • 1949: (1)

Bayer, Frederick M. U. of Miami, Institute of Marine Science Correspondence • 1965: (1) • 1973 (2)

Bayer, Sophia Otto Bioscript

Bayless, William A. Rockefeller U. Press Correspondence • 1967: (2)

Beach, Kay H. U. of California, Berkeley Correspondence • 1949: (2)

Beach, Spencer Ambrose (1860-1922) American horticulturist Biofile

Beadle, C. D. Bioscript

Beaglehole, Elsie Wife of John Cawte Beaglehole Correspondence • 1974: (4) 1976 (1)

Beaglehole, John Cawte (1901-1971) Victoria U., Wellington, New Zealand Biofile - Botanical Album Correspondence $\bullet$ 1956: (2) $\bullet$ 1957: (6) $\bullet$ 1959: (5) Banks, Joseph, Sir, 1743-1800 - 1960: (12) Banks, Joseph, Sir, 1743-1800 • 1960: (0) Banks, Joseph, Sir, 1743-1800; Filed under Lysaght $\bullet$ 1961: (2) Banks, Joseph, Sir, 1743-1800 • 1962: (13) • $1963:$ (8) National Science Foundation; Banks, Joseph, Sir, 1743-1800 • 1964: (1) • 1965: (1) • 1966: (1) • 1967: (1) • 1968: (2) • 1974: (1) Correspondence with Elsie Beaglehole

Beaglehole, T. H. Victoria U., Australia, Dept. of History Correspondence • 1983: (1)

Beal, J. M. U. of Chicago, Dept. of Botany Correspondence • 1942: (1) • 1943: (1)

Beal, Mary Botanical Album

Beal, William James (1833-1924) American botanist, horticulturist, teacher

Biofile $\bullet$ Bioscript $\bullet$ Botanical Album

Beale, Georgia Robison Washington, D.C. Botanical Album

Correspondence • 1987: (5) Harper, Francis, 1886-1972 - 1988: (1) • 1989: (3) • 1992: (1)

Beaman, John Homer (1929-) American taxonomist; Michigan State U., East Lansing, Dept. of Botany Correspondence • 1964: (3) • 1966: (1) • 1973: (1) • 1980: (3) • 1981: (1)

Bean, T. L. Laggan, Alberta, Canada Correspondence • 1949: (1)

Beard, John Stanley (1916-) Australian plant ecologist Bioscript $\bullet$ Botanical Album Correspondence • 1945: (4) - 1946: (18) • 1947: (0) Correspondence filed under J. R. Edmudson $\bullet$ 1948: (5) • 1949: (6) • 1950: (4) • 1951: (3) • 1952: (1) • 1954: (1) • 1955: (1) • 1956: (1) • 1957: (1) • 1958: (1) • 1964: (2) 1965: (1) • 1968: (2) • 1970: (3) • 1971: (3) $\bullet$ 1973: (5) $\bullet$ 1974: (7) • 1978: (0) • 1981: (3) • 1983: (3) • 1984: (6) • 1992: (1) • 1993: (4)
Beard, Letitia Loyola U., Dept. of Biological Sciences Correspondence $\bullet$ 1978: (1)

Beard, Pamela Husband is J. S. Beard Correspondence • 1951: (1) • 1976: (1) • 1978: (1)

Beardslee, Henry Curtis (1865-1948) Bioscript

Beardsley, A. F. (fl. 1852-1861) Bioscript

Beardsley, Arthur Eugene (1853- ) American zoologist Bioscript

Beath, Orville Andrew (1884 ) American research chemist; U. of Wyoming, Laramie Agricultural Research Chemistry Bioscript • Botanical Album Correspondence • 1939: (2) • 1954: (2) Delphinium • 1955: (6) Species

Beatley, Janice Carson (1919-1987) American plant ecologist

Bioscript

Beattie, Rolla Kent (1875-1960) American plant pathologist, taxonomist

Biofile $\bullet$ Bioscript $\bullet$ Botanical Album

Beatty, M. E. Correspondence $\bullet$ 1941: (2)

Beaudry, Jean Roumald (1917- ) Taxonomist; Institut Botanique, Université de Montréal, Montréal, Canada Botanical Album Correspondence $\bullet$ 1957: (7) • 1958: (1) • 1967: (1)

Beaumont, J. F. Bioscript

Beaumont, John E. New Orleans; student of George van Schaack

Correspondence • 1978: (1) • 1979: (1)

Beauvois, Palisot de (1752-1820) French botanist Biofile • Bioscript

Beaver, Paul C. Tulane U., New Orleans, LA Correspondence • 1952: (1) • 1977: (1) • 1983: (3) • 1984: (4)

Bebb, Michael Schuck (1833-1895) American taxonomist, plant collector Bioscript $\bullet$ Botanical Album

Bebb, William Bioscript $\bullet$ Botanical Album

Beccari, E. Bioscript

Becherer, Alfred (1897-1977) Swiss phytogeographer, taxonomist, plant collector

Biofile

Bechstein, J. M. Bioscript

Beck, Lewis Caleb (1798-1853) American geologist and botanist Biofile • Bioscript

Beck, Rollo Howard (1870-) Bioscript

Beck, Theodoric Romeyn (1791-1855) American physician, botanist Bioscript

Becker, R. B. U. of Florida, Gainesville, Agricultural Experiment Stations, Dept. of Dairy Science Correspondence $1963:$ (2) $\bullet$ 1968: (1) • 1969: (4) 


\section{Guide to the Ewan Papers}

Beckett, John Edgar (1878-1934) Bioscript

Beckett, T. W. Naylor (1839-1906) Bryologist Bloscript

Becklhars, Charles Wickliffe (1856-1888) American ornithologist, lawyer Btoseript

Beckley, John James (1757?-1807) American lawyer Biofile

Beckwith, Edwin Griffin (1838-1881) American soldier Bloscript

Beckwith, Florence (1843-1929) Bloscript

Becael, Wm. K. Correspondence $\bullet 1965:$ (1)

Beddall, Barbara Kennett Square, PA Correspondence • 1979: (4) • 1984: (2) • 1985: (2) • 1988: (2) Book collectors

Bedell, Forence J. Correspondence $\bullet$ 1948: (2)

Bedford, Duke of J. Barton, Comptroller to Duke Botanical Album Correspondence $\bullet 1965:(4)$

Beebe, C. William (1877-1962) American zoologist Biofile * Bioscript

Beecher, Blla Bloscript

Beechey, Frederick William (1796-1856) Bloscript

Beclswy der, Bloscript

Beers, Alma Holland U. of North Carolina, Chapel Hill Correspondence • 1949: (2)

Beesley, Correspondence $\bullet$ 1970: (0) Correspondence filed under Longue Vue Gardens

Beetle, Alan Ackermann (1913- ) American taxonomist, agrostologist

Bioscript • Botanical Album

Correspondence - 1937: (3) - 1938: (14) Species Delphinion • 1939: (2) • 1941: (4) • 1943: (1) • 1944 (2) $\bullet 1992:$ : (1)

Behem, [or Behin or Beheim], Martin Bioscript

Behle, William Harroun (1909-) American ornithologist; U. of Utah, Salt Lake City, Dept. of Biology, Dept. Head
Bioscript

Correspondence • 1949: (2) • 1976: (3)

Behinke, John A. The Ronald Press Co., New York, NY Correspondence • 1957: (1) "Rocky Mountain Vegetation" 1958: (3) - 1959: (6) "Rocky Mountain Vegetation" • 1960: (2) "Rocky Moumain Vegetation" - 1962: (2) • 1814, "Rocky Moun, John, 1650-1692; Lyon, John, 1764 1814, "Rocky Mountain Vegetation:" National Science Foundation • 1964: (2) • 1972: (4)

Behr, Hans Herman (1818-1904) (4) taxonomist in California Bioscript

Behr, Otto (1901-1957)

Correspondence $\bullet$ 1938: (2) • 1939: (1)
Beidleman, Richard Gooch (1923-) Colorado College, Colorado Springs, CO

Botanical Album

Correspondence - 1953: (8) Chronica Botanica; Rocky Mountain Naturalists • 1954: (3) "Rocky Mountain Vegetation" • 1956: (2) • 1958: (2) • 1959: (8) $\bullet$ 1960: (1) Nuttall, Thomas, 1786-1859 • 1962: (2) • 1968: (3) • 1970: (3) Rocky Mountain Naturalists - 1973: (1) - 1974:

(1) • 1977: (2) • 1978: (2) • 1979: (1) • 1980: (1) • 1983:

$(1) \bullet 1984:(1) \bullet 1985:(1) \bullet 1987:(1) \bullet$ 1988: (1) $\bullet$ 1989:

(3) $\bullet$ 1990: (1) $\bullet$ 1992: (5) $\bullet$ 1993: (2)

Beiswanger, William Thomas Jefferson Memorial Foundation

Correspondence $\bullet$ 1986: (1)

Belanger, Charles Bioscript

Belanger, Terry Columbia U., School of Library Science Correspondence $\bullet$ 1986: (1)

Belden, Louise Correspondence $\bullet$ 1973: (0) correspondence filed under Frans Stafleu

Belder, Robert de Bioscript

Belding, Lyman (1829-1917) Bioscript

Belknap, Jeremy American botanist Biofile

Bell, Charles Ritchie (1921-) American taxonomist, cytotaxonomist; U. of North Carolina, Chapel Hill Correspondence • 1966: (2) Bartram, William, 1739-1823 - 1967: (2) • 1974: (3)

Bell, ___ Dr. Ornithological Conference XX Correspondence • 1988: (0) Filed under Ornithological Conference Bell, (Ernest) Arthur (1926-) Bioscript

Bell, Ernest Layton (1874-1964) American entomologist; American Museum of Natural History, Dept. of Insects Correspondence $\bullet$ 1949: (4)

Bell, Gail Brigham Young U. Press, Provo, UT, Senior Editor

Correspondence $\bullet$ 1977: (0) Filed under Robert William Kiger

Bell, J. G. Stanford U. Press, Editor Correspondence • 1967: (0) Filed under George Booth Van Schaack

Bell, Janet American librarian; U. of Hawaii, Pacific Collection Botanical Album

Correspondence • 1967: (5) • 1968: (6) • 1969: (4) Banister, John, 1650-1692; Beaglehole, John Cawte • 1970: (4) $\bullet$ 1971: (8) • 1974: (7) • 1975: (3) • 1976: (2) $\bullet 1977$ : (2) $1978:(2) \bullet 1980:$ (1)

Bell, John Graham (1812-1889) Bioscript

Bell, Malcolm Savannah, Georgia Correspondence $\bullet$ 1972: (1)

Bell, Phoebe L. B. Husband Correspondence $\bullet$ 1959; (2)

Bell, R. G.

Correspondence 1948: (1) 
Bell, Richard C.

Correspondence $\bullet$ 1964: (6) • 1965: (4) • 1966: (4) • 1967: (1)

Bell, Rita

Correspondence • 1989: (1)

Bell, S. L.

Bioscript

Bell, Thomas

Bioscript

Bell, Whitfield Jenks American Philosophical Society Botanical Album

Correspondence $\bullet$ 1957: (2) $\bullet 1963:$ (1) $\bullet$ 1966: (3) $\bullet$ 1967: (2) • 1968: (2) 1970 : (3) Barton, Benjamin Smith, 1766-1815 • 1971: (6) • 1972: (7) • 1973: (6) • 1974: (10) - 1975: (4) - 1976: (3) Barton, Benjamin Smith, 1766-1815 • 1977: (1) • 1978: (1) • 1979: (10) Barton, Benjamin Smith, 1766-1815 • 1980: (15) • 1981: (1) 1982: (1) - 1984: (0) Filed under Alan W. Armstrong • 1986: (5) Missouri Botanical Garden - 1988: (7) Barton, Benjamin Smith, 1766-1815 • 1989: (5) • 1990: (4) • 1991: (6) • 1992: (4)

Bell, William Abraham (1841-1921) English-born American railroad officer, plant collector

Bioscript $\bullet$ Botanical Album

Bellamy, David

Botanical Album

Bellardt, C. A. L.

Bioscript

Belling, John (1866-1933)

Bioscript

Bellis, Genevieve H. Bogotá, Colombia

Correspondence $\bullet$ 1948: (2)

Belshaw, Charles

Botanical Album

Correspondence • 1936: (2) • 1937: (1)

Belt, Elmer Bioscript

Belt, Thomas (1832-1878) English geologist Bioscript

Belvedere Scientific Fund Correspondence • 1968: (2)

Bemis, Dorothy Botanical Album

Bender, George A. Park Davis \& Co., Detroit, MI Correspondence $\bullet$ 1959: (1)

Bendire, Charles Emil (1836-1897) German-born American ornithologist and U. S. Army officer

Bioscript

Benedict, Ralph Curtis (1883-1965) American biologist, taxonomist; American Fern Society

Botanical Album

Correspondence • 1929-1933: (2) • 1935: (2) • 1941: (3) • 1947: (8) • 1950: (3) • 1952: (4) • 1953: (2) • 1954: (1) • 1955: (4) • 1958: (1)

Benezet, Samuel Bioscript

Benjamin, Curtis G. McGraw-Hill publishers Correspondence $\bullet$ 1941: (1)

Benke, Hermann Conrad (1869-1946) German-born American botanist, photographer Bioscript
Benner, Walter M. Philadelphia, PA

Bioscript $\bullet$ Botanical Album

Correspondence $\bullet$ 1949: (2)

Bennett, A. W.

Bioscript

Bennett, B. A. (nee Bulmer) Manvnau Central, New Zealand

Correspondence $\bullet$ 1988: (1)

Bennett, Bradley Charlton Institute of Economic Botany, Carrboro, NC Correspondence $\bullet$ 1991: (1)

Bennett, Cora Bioscript

Bennett, H. R. Hornblower \& Weeks Correspondence 1963 : (3) Delphinium

Bennett, Harry J. Louisiana State U., Baton Rouge Correspondence $\bullet$ 1975: (0) Filed under Edward Sturtevant Hathaway $\bullet$ 1979: (1)

Bennett, Joan Wennstrom (1942- ) American plant geneticist, mycologist Bioscript Correspondence $\bullet$ 1971: (1) $\bullet$ 1983: (1)

Bennett, John Joseph (1801-1876) English botanist Bioscript

Bennett, M. K. Bioscript

Bennett \& Marshall Antiquarian booksellers Correspondence $\bullet$ 1957: (2)

Bennett, Warren C. Bennett \& Marshall Antiquarian Booksellers Correspondence $\bullet$ 1954: (1)

Bennett, William Bioscript

Bennett's Photo New Orleans, LA Correspondence • 1949: (1) • 1950: (1)

Benson, Lyman David (1909- ) American taxonomist; U. of Arizona, Tucson

Bioscript $\bullet$ Botanical Album

Correspondence • 1929-1933: (3) Delphinium - 1938: (1) - 1939: (8) Delphinium - 1941: (14) - 1942: (5) - 1943: (4) - 1944: (3) - 1945: (8) Jones, Marcus Eugene, 1852-1934 • 1946: (1) - 1948: (5) • 1949: (8) • 1950: (4) - 1951: (7) Setchell, William Albert, 1864-1943 - 1952: (10) - 1953: (3) - 1954: (1) • 1957: (5) • 1959: (1) • 1962: (1) • 1967: (1) • 1984: (1)

Benson, Maxine Kansas State Historical Society Correspondence $\bullet$ 1983: (2)

Benson, Seth B. Museum of Vertebrate Zoology, Berkeley, CA Correspondence • 1936: (1) - 1949: (2)

Bent, Arthur Cleveland (1865-1954) Ornithologist Biofile

Bentham, George (1800-1884) English botanist Biofile • Bioscript

Bentham, Lady Bioscript

Bentinck, C. Bioscript

Benton, Fred Correspondence $\bullet$ 1976: (1) 
Bem, Bruce F. Universidad de Guadalajara Correspondence $\bullet 1990$ : (1)

Bercaw, Louise O. U. S. Dept. of Agriculture Library, Chief

Correspondence $\bullet 1951:(1)$

Berckmans, Prosper Julius Alphonso (1830-1910) Belgian-American horticulurist Biofile $\bullet$ Bioscript

Berendt, $\mathbf{H}$. Bioscript

Berg, N. K. Plant collector, agriculturist; Broomfield, CO Bioscript

Berg, Roir Yngvar (1925-) Taxodomist; Universitetets Botaniske Museum, Oslo, Norway Correspondence • 1958: (1)

Berg, Thomas M. Pennsylvania Dept. of Environmental Resources Correspandence $\bullet 1984$ : (1)

Berg, Wieland Deutsche Akademie der Naturforscher Leopoldina, Halle Correspondence • 1979: (5)

Bergendal, David (1855-1908) Swedish botanist Biofile - Bioscript

Berger, Charles A. Fordham U., New York, NY; Correspondence • 1950: (1) • 1951: (4) • 1952: (2) • 1955: (1) • 1959: (1)

Bergseng, Margaret S. Sacramento, CA Correspondence • 1958: (2)

Beringer, Johann Bartholomew Adam (1667-1740) German geologist

Biofile

Berkeley, Derothy A. Richmond, VA; husband is Edmund Berkeley

Correspondence • 1958: (3) • 1959: (2) • 1960: (2) Banister, John, 1650-1692 - 1963 : (12) Banister, John, 1650-1692; Lyon, John, 1764-1814 - 1969: (0) Filed under Jerry Willmer Stannard $\bullet 1970$ : (7) Barton, Benjamin Smith, 1766-1815 • 1975: (3) Bartram, William, 1739-1823 - 1976: (10) Banister, John, 1650-1692; Barton, Benjamin Smith, 1766-1815; Rocky Mountain Naturalists: Bartram, William, 1739-1823 • 1979: (6) • 1980: (7) • 1990: (1)

Berkeley, Edmund (1912-) Biohistorian; Lynchburg, VA; wife is Dorothy A. Berkeley

Bloscript • Botanical Album

Correspondence • 1960: (2) • 1961: (6) • 1962: (7) • 1963

1964: Copy of correspondence to John Warren Cooke $\bullet$ 1964: (16) • 1965: (2) • 1966: (9) • 1967: (12) • 1968: (20) $\bullet$ 1969: (8) $\bullet$ 1970: (9) Barton, Benjamin Smith, (4) - 1973: (5) $\bullet 1974$ : (3) Stannard $\bullet 1971$ : (2) $\bullet 1972$ : (4) $1973:$ (5) $\bullet 1974$ : (3) Bartram, William, 1739-1823 1977: (2) - 1978: (3) Bartram, William, 1739-1823 • 1980: (5) - 1981: (3) • 1982: (6) • 1983: (9) $\bullet$ 1984: (10) 1985: (10) $\bullet 1986:$ (4) Letters to and from Alan Armstrong • 1987: (2) • 1988: (4) Barton, Benjamin Smith, 1766-1815 • 1991: (3) • 1992: (9) • 1993: (17)

Berkeley, Francis L. Jr. U. of Virginia, Charlottesville, Associate Librarian

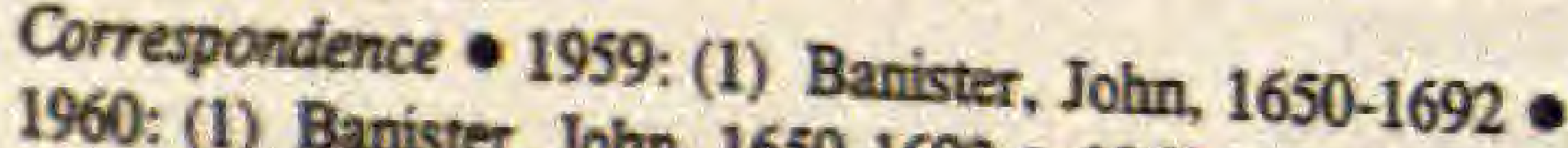
1960: (1) Banister, John, 1650-1692 1963 : (3) Banister,
John, 1650-1692
Berkeley, M. J.

Bioscript

Berkenhout, John (1730-1791) Bioscript

Berkett, George D. B. Physician; Metairie, LA Botanical Album

Correspondence $\bullet$ 1978: (2) • 1985: (2) • 1986: (1) $\bullet$ 1988: (1) • 1993: (1)

Berlandier, Jean Louis (1805-1881) Belgian explorer in N. America and Mexico

Bioscript $\bullet$ Botanical Album

Bernard, George R. U. of Notre Dame, IN; American Midland Naturalist

Correspondence • 1958: (1)

Bernard, Jean-Paul (1921-) Canadian cytotaxonomist; Universite Laval, Quebec, Canada Correspondence $\bullet$ 1985: (2)

Bernard Quaritch, Ltd. Antiquarian Booksellers, London, England, Natural History Department Correspondence • 1977: (3) Book collectors

Bernard, Richard U. of California, Berkeley, Reference Librarian Correspondence $\bullet$ 1957: (2)

Bernhardi, Johann Jakob (1774-1859) German botanist Biofile $\bullet$ Bioscript

Bernhardt, Peter American botanist Biofile $\bullet$ Bioscript $\bullet$ Botanical Album Correspondence $\bullet$ 1987: (1) • 1988: (2) • 1992: (4)

Bernice P. Bishop Museum Library Correspondence $\bullet 1974$ : (1)

Bernstein, Leonard Botanical Album

Berra, Paul Correspondence 1954 : (1)

Berra, Tim Martin (1943-) American ichthyologist, zoogeographer, anthropologist; Ohio State U.

Biofile $\bullet$ Bioscript $\bullet$ Botanical Album Correspondence • 1969: (10) - 1970: (28) • 1971: (17) • 1972: (15) Barton, Benjamin Smith, 1766-1815 - 1973: $(10) \bullet 1974:(18) \bullet 1975:(8) \bullet 1976:(18) \bullet 1977:(7) \bullet$ 1978: (8) - 1979: (5) Book collectors 1 1980: (13) - 1981: $(11) \bullet 1982(8) \bullet 1983:(9) \bullet 1984:(7) \bullet 1985:(9) \bullet$ 1986: (15) • 1987: (13) • 1988: (8) Bartram, William, 1739-1823 • 1989: (10) • 1990: (6) • 1991: (9) • 1992: (11) - 1993: (10)

Berringer, Kerry Brooklyn Botanic Garden Correspondence 1989 : (1)

Berry, Edward Willard (1900-) Geologist, paleontologist Bioscript - Botanical Album

Berry, Elmer G. (1907-) American malacologist Bioscript

Berry, Emma L. New Orleans, LA Correspondence $\bullet$ 1962: (2)

Berry, Moses E.

Correspondence • 1964: (4) • 1965: (1)

Berry, Paul Edward (1952-) Missouri Botanical Garden, St. Louis, MO, Assoc. Curator

Correspondence - 1989: (2) Corres. with E. Charles Nelson
Berry, S. Stillman Correspondence $\bullet$ 1964: (2) 
Bertea, Albert U. of California, Los Angeles

Correspondence $\bullet$ 1935: (1)

Berthoud, Edward L. (1828-1908) Swiss-born American engineer, naturalist

Bioscript

Bertoloni, Antonio (1775-1869) Physician, botanist Biofile $\bullet$ Bioscript

Bertoloni, G.

Bioscript

Beruin-Duvallon Bioscript

Besant, John W. Glasnevin Botanic Gardens, Glasnevin, Dublin

Correspondence • 1939: (2) Species • 1942: (2)

Bessey, Charles Edwin (1845-1915) American botanist Biofile $\bullet$ Bioscript $\bullet$ Botanical Album

Bessey, Ernst Athearn (1877-1957) American mycologist, taxonomist; son of C. E. Bessey

Bioscript

Bestor, Norman

Bioscript

Bethel, Ellsworth (1863-1925) American mycologist, plant pathologist

Bioscript $\bullet$ Botanical Album

Bethune, Alexander Contura Photocopying Ltd., London Correspondence $\bullet$ 1954: (7) $\bullet$ 1955: (1)

Bett, Hugh Maggs Bros. Ltd. Rare Books Correspondence • 1990: (0) Filed under Maggs Bros. Ltd.

Betton, Samuel Bioscript

Betts, Mary Hall

Correspondence • 1964: (1)

Betts, Norman deWitt (1880-1917) American ornithologist Bioscript

Beutinck, Margaret Cavendish Bioscript

Beverley, Robert (c. 1673-1722)

Bioscript

Beyrich, Carl Bioscript

Bibliographical Society of America, Papers of Ruth Mortimer, Editor

Correspondence $\bullet$ 1983: (2)

Bick, George Hermann (1923- ) American entomologist; Lafayette, LA; Saint Mary's College, Notre Dame, IN, Biology Dept.

Correspondence - 1958: (1) 1971: (2)

Bicknell, Eugene Pintard (1859-1925) American banker, botanist, ornithologist Biofile - Bioscript

Bicknell, F. J., Mrs. California Audubon Society Correspondence • 1929-1933: (1)

Biddle, Clement Bioscript

Biddle, Nicholas (1786-1844) Bioscript

Biddlecome, Hannah Jane Bryologist; Springfield and Columbus, $\mathrm{OH}$

Bioscript

Bidwell, Annie E. Kennedy Husband is John Bidwell Bioscript

Bidwell, John

Bioscript
Bidwill, John Carne (1815-1853) Geographer, botanist; England and Australia Biofile

Bieberstein, L. B. Friderico Marshall von Bioscript

Bieman, Jim U. of Southwestern Louisiana, Lafayette Correspondence • 1983: (1)

Bierhorst, David William (1924-1966) American plant anatomist and morphologist

Biofile • Bioscript

Correspondence $\bullet$ 1960: (2)

Bierstadt, Albert (1830-1902) German-born American artist Bioscript

Biesterfeldt, Adrienne Missouri Botanical Garden, Garden Guides, Program Chairman

Correspondence • 1988: (4)

Bieweiler, Louis C. Botanical Album

Bigelow, Jacob (1787-1879) American physician, botanist at Harvard

Biofile • Bioscript

Bigelow, John Milton (1804-1878) American surgeon, botanist on the Whipple Expedition

Biofile $\bullet$ Bioscript

Bi-Jun Yao

See Yao, Bi-Jun

Bilebof, Correspondence $\bullet$ 1972: (1)

Bill Moyer's Journal New York, NY Correspondence $\bullet$ 1979: (1)

Billot, C.

Bioscript

Binford, Janet Friends of the Berry Botanic Garden, Portland, OR; husband is Thomas P. Binford Correspondence • 1980: (3)

Binford, Laurence C. Correspondence - 1990: (2)

Bingham, Caroline P. Lord Bioscript

Bingham, Lloyd A. U. of Michigan, Ann Arbor, Clements Library, Director Correspondence $\bullet$ 1959: (2) • 1973: (8)

Bingham, R. F. Bioscript

Binkley, Frances U. of Colorado Correspondence • 1943: (1)

Binney, W. G. Bioscript

Binnings, Lee P. Unit Design Interiors, Inc., Covington, LA Correspondence - 1981: (4) Book collectors

Binns, Blodwen Lloyd U. of Strathclyde, Glasgow, Dept. of Biology Correspondence $\bullet$ 1970: (2) • 1971: (3) • 1972: (6) $\bullet$ 1975: (6) • 1976: (2) • 1977: (2)

Bioletti, Frederick Theodore (1865-1939) Bioscript • Botanical Album

Biological Abstracts Correspondence $\bullet$ 1942: (0) Letter filed under Robert William Pennak • 1970: (2)

Biological Society of Nevada Correspondence • 1967: (1) 


\section{Guide to the Ewan Papers}

Biological Society of Washington Washington, D.C. Correspondence $\bullet 1977:(1)$

BioScience Book Review Editor Correspondence $\bullet$ 1968: (2)

BioSciences Info Service Philadelphia, PA Correspondence • 1971: (1)

Biossat, Suzanne Former student Correspondence • 1942: (1) Bircher, Warda J. Botanical Album

Bird, Charles Durham (1932-) Canadian phytogeographer, plant ecologist; U. of Calgary, Alberta, Canada, Dept. of Biology

Correspondence • 1969: (2) Rocky Mountain Naturalists

Bird, Isabella Lucy (1831-1904) English traveller Biofile $\bullet$ Bioscript

Birger, Selim Gotthard (1879-1931) Swedish taxonomist Bioscript $\bullet$ Botanical Album

Birkbeck, Morris Bioscript

Biroli, G. Bioscript

Birtel, Frank T. Tulane Univ., New Orleans, LA Correspondence • 1977: (1) • 1980: (1)

Bischoff, Ferdinand Taxidermist Bioscript

Bischoff, G. Botanical Album

Bishop, Alexander Bioscript

Bishop Museum See Bernice P. Bishop Museum

Bishop, Francis Marion, Capt. (1843-1933) Bioscript

Bishop, Mabel Lowell Hood College Correspondence $\bullet$ 1939: (1)

Bishop, Sherman C. Bioscript Correspondence $\bullet$ 1953: (1)

Bixby, John W. Bioscript $\bullet$ Botanical Album

Bixby, William $\mathbf{K}$. Bioscript

Black, George (fl. 1850-1855) Bioscript

Black, Joseph Bioscript

Blackburn, Benjamin Coleman (1908-) Correspondence $\bullet$ 1948: (2)

Blackburn, Julia Correspondence • 1986: (5) • 1987: (1)

Blackburn, Robert English research student Correspondence 1984 : (2)

Blackburne, Anna (1726-1793) English botanist. museum keeper Biofile

Blackburne, Ashton (c. 1730-1787) English ornithologist
Biofile Blackburne, John Bioscript Blackie, George Stodart (1834-1881)
Bioscript
Blackwell,

Correspondence $\bullet 1974$ : (1)

Blackwell, William Hoyle, Jr. (1939- ) Taxonomist; Miami U., Oxford, OH; Missouri Botanical Garden

Botanical Album

Correspondence • 1967; (3)

Blackwell's Bookseller; Oxford, England

Botanical Album

Correspondence • 1945: (1) • 1946: (1) • 1947: (3) Book collectors • 1986: (2)

Blagden, Charles Bioscript

Blainville, M. H. D. de (1777-1850) Bioscript

Blair, James P. Botanical Album

Blair, William D. Botanical Album

Blaisdell, Frank Ellsworth (1862-1946) Bioscript

Blake, Anson Society of California Pioneers Bioscript

Correspondence - 1942: (1) Recommendation for Fred M. Packard • 1955: (2) Jepson, Willis Linn, 1867-1946 • 1958: (2) • 1959: (1)

Blake, Doris Holmes (1892-) American coleopterist; Smithsonian Institution Bioscript - Botanical Album

Blake, Heubert T. Sutton and Sons Correspondence • 1929-1933: (1)

Blake, J. S. Correspondence $\bullet$ 1952: (1)

Blake, James Bioscript

Blake, James (1815-) English-American chemist, physiologist Biofile

Blake, Sidney Fay (1892-1959) American botanist Biofile • Bioscript • Botanical Album Correspondence $\bullet 1929-1933:$ (7) $\bullet$ 1936: (3) • 1937: (4) - 1938: (1) • 1940: (2) • 1941: (2) • 1944: (4) • 1947: (1) - 1948: (3) • 1949: (3) Species • 1950: (7) • 1951: (3) • 1956: (1) • 1957: (2) • 1958: (1)

Blake, William Phipps (1826-1910) American geologist, mining engineer Bioscript

Blakeslee, Albert Francis (1874-1954) Plant geneticist Bioscript • Botanical Album

Blakiston, Thomas Wright (1832-1891) English explorer and ormithologist Bioscript

Blanchard, Dean Bioscript

Blanchard, Ferdinand Bioscript * Botanical Albun

Blanchard, Orland J. Earlham College Correspondence $\bullet$ 1976: (1)

Blanchard, Van Anderson Forida Federation of Garden Clubs, Pensacola, FL; husband is Charles H. Blanchard Correspondence • 1977: (1) 
Bland, Theodoricus Bioscript

Blankinship, Joseph William (1862-1938) American plant pathologist

Bioscript $\bullet$ Botanical Album

Correspondence $\bullet$ 1991: (3) Letter from William J. Bonner, nephew of Blankinship

Blanton, F. S. Bioscript $\bullet$ Botanical Album

Blanton, Otto Bioscript $\bullet$ Botanical Album

Blaschka, Leopold Bioscript

Blaschka, Rudolph (1857- ) German glass modeler Biofile

Blaschke, Eduard Leontjevitch Bioscript

Blasdale, Walter Charles (1871-) Bioscript

Blasdell, Robert Ferris (1929-) Louisiana State U., New Orleans, Division of Sciences Correspondence 1960: (1)

Bleeker, Pieter (1819-1878) Dutch naturalist Biofile

Bleiweiss, Correspondence $\bullet$ 1992: (1)

Bligh, William (1754-1817) English sea captain Biofile

Bliss, Leslie E. Henry E. Huntington Library librarian Correspondence $\bullet$ 1948: (2)

Blochman, Ida Mary Twichell (1854-1931) American taxonomist, ethnobotanist Bioscript

Block, Marcus Eliefer Bioscript

Blockstein, David Edward Correspondence • 1990: (2)

Blodgett, Charles Correspondence $\bullet$ 1937: (2)

Blodgett, John Loomis (1809-1853) Bioscript $\bullet$ Biofile

Blom, Frans Botanical Album

Blom, Gertrude Botanical Album (Filed under Frans Blom)

Blome, Richard (f. 1672) Bioscript

Blommart, John Bioscript

Blomquist, Hugo Leander (1888-1964) Swedish-born American taxonomist; Duke University Correspondence - 1943: (2) - 1950: (2) - 1951: (9) Pursh, Frederick, 1774-1820 • 1952: (6) • 1954: (2) • 1955: (2) Letter co-signed by R. B. Channell $\bullet$ 1956: (1) Species

Bloodgood, Joseph Bioscript

Bloomer, Hiram G. (1819-1874) American botanist Biofile $\bullet$ Bioscript

Blount, Ainsworth Emory (1832-1911) Agronomist Bioscript
Blower, Richard Petropolis, Brazil; nickname "Dick" Botanical Album Correspondence $\bullet$ 1985: (6) • 1986: (1)

Bloxam, Andrew (1801-1878) English naturalist Bioscript

Blum, Ann S. Correspondence - 1986: (1)

Blum, Susan B. U. of Pennsylvania, Philadelphia Correspondence $\bullet$ 1975: (1) Langman, Ida Kaplan

Blumberg, Baruch S. Fox Chase Cancer Center, Philadelphia, PA

Correspondence $\bullet$ 1987: (1) $\bullet$ 1988: (1) Bartram, William, 1739-1823

Blume, Carl Ludwig (1796-1862) German-born Dutch botanist

Bioscript $\bullet$ Botanical Album

Blumenbach, J. F. Bioscript

Blumer, Jacob Corwin (1872-1948) Swiss-born American botanist Biofile $\bullet$ Bioscript

Blunt, Henry Bioscript

Blunt, Wilfred (1901- ) British botanical artist, botanical historian Bioscript $\bullet$ Botanical Album Correspondence $\bullet 1964:(1) \bullet 1979:$ (2)

Bly, Charles, Mrs. Bioscript

Blythe, Audrey D. Correspondence $\bullet$ 1952: (2)

Blytt, A. Bioscript

Blytt, M. N. Bioscript

Boas, Roger World Press, KQED, San Francisco, CA Correspondence $\bullet$ 1970: (1)

Boate, Gerard Bioscript

Bobart, Jacob Bioscript

Bock, Jane Haskett (1936-) American botanist; U. of Colorado, Boulder; husband is Carl E. Bock Correspondence $\bullet$ 1964: (1) $\bullet$ 1965: (3) $\bullet$ 1980: (2) Letter to Ewan from Peter Bretting

Bodin, J. E. Bioscript

Bodin, Susan Correspondence $\bullet$ 1987: (1)

Bodine, Edward Colorado Academy of Sciences Correspondence - 1941: (1)

Bodleian Library U. of Oxford, England, Librarian Correspondence • 1955: (2) • 1970: (1)

Bodmer, Carl (1809-1893) Swiss painter Biofile $\bullet$ Bioscript

Boerhaave, Herman (1668-1738) Dutch physician and botanist Biofile $\bullet$ Bioscript $\bullet$ Botanical Album

Boerlage, J. G. Bioscript 


\section{Guide to the Ewan Papers}

Boetjer, Barry

Correspondence $\bullet 1974:(1)$

Boetjer, Margaret Sister of Joseph Ewan

Correspondence $\bullet$ 1941: (2) • 1961: (1) • 1972: (1) • 1974: (2) - 1979: (0) Correspondence filed under Grace Clark $\bullet$ 1981: (2)

Boewe, Charles Transylvania U., Lexington, KY Correspondence • 1959: (3) • 1979: (6) • 1980: (8) • 1981: (4) • 1982: (2) • 1983: (7) $\bullet$ 1984: (7) letters to Ron Suckey $\bullet$ 1985: (1) $\bullet$ 1987: (1) $\bullet 1988:$ (7) Barton, Benjamin Smith, 1766-1815 • 1989: (11) • 1990: (3) • 1992:(3) - 1993: (6)

Bofill, Jose Bioscript

Bogert, Charles M. American Museum of Natural History Correspondence $\bullet 1938:$ (2) $\bullet$ 1968: (2)

Boggs, Lindy, M. C. U. S. House of Representatives; wife of Hale Boggs

Correspondence $\bullet$ 1979: (2) $\bullet$ 1981: (2)

Bohlin, Anders Birger (1898-) Paleobotanist Correspondence • 1972: (1) • 1973: (1)

Bohn, Scheltema \& Holkema Handelsreg, Netherlands Correspondence • 1980: (2) • 1981: (1)

Bohun, Edmund (1672-1734) Bioscript

Boice, Carrie A. Bioscript

Boisduval, Jean Alphonse (1801-1879) Bioscript

Boise, Jean R. (1952-) Correspondence • 1987: (2)

Boissier, Pierre-Edmond (1810-1885) Swiss taxonomist Biofile $\bullet$ Bioscript

Boisswain, Charles Hercules (1893-1946) Dutch bacteriologist Bioscript

Boivin, Bernard (1916-1985) Canadian botanist Biofile • Bioscript $\bullet$ Botanical Album Correspondence $\bullet$ 1951: $\bullet$ 1953: (2) • 1962: (1) • 1972: (5) - 1976: (3) - 1978: (1) Rocky Mountain Naturalists; letters between Boivin and R. W. Kiger • 1980: (5) Letter from Robert W. Kiger $\bullet$ 1981: (1) Species

Bojer, W. Bioscript

Boke, Norman Hill (1913-) American plant anatomist and morphologist Bioscript • Botanical Album

Bolander, Henry Nicholas (1831-1897) German-borm American botanist, educator, plant collector Bioscript $\bullet$ Botanical Album

Bolen, Eric G. Texas Tech U., Associate Dean \& Paul Whitfied Horn Professor Correspondence • 1988: (2) Bartram, William, 1739-1823

Boles, C. Read Correspondence $\bullet$ 1992: (1)

Boll, Jacob I. (1828-1880) Swiss-bom American naturalist
Biofile

Bollwinkel, Carl Walter (1932-) American taxonomist, plant ecologist; U. of Illinois, Carbondale Correspondence $\bullet 1966$ : (1)
Bollwinkle, J. New Orleans, LA Correspondence • 1958: (2)

Bollwinkle, John, Mrs. Correspondence • 1957: (2)

Bolten, J. F. Bioscript

Bolton, _ Dr. Correspondence • 1934: (1) Letter from W. L. Jepson

Bolton, Herbert Eugene (1870-1953) American historian Biofile

Bolton, James (fl. 1750's-1799) Canadian naturalist Biofile

Boltzius, (Rev) Biofile

Bolus, Harry (1834-1911) British-born South African businessman, botanical collector, taronomist Bioscript

Bolus, Harriet Margaret Louisa (1877-) South African botanist, taxonomist; Capetown, South Africa; niece of Harry Bolus

Bioscript $\bullet$ Botanical Album

Bomhard, Miriam Lucile (1898-1952) American palm taxonomist, ecologist

Biofile $\bullet$ Bioscript $\bullet$ Botanical Album

Bompard, $\mathbf{H}$.

Bioscript

Bonaparte, Charles Lucien (1803-1857) French ornithologist

Biofile • Bioscript • Botanical Album

Bonar, Lee M. (1891-1977) American mycologist, taxonomist

Bioscript

Correspondence • 1929-1933: (1) • 1974: (3) Rocky Mountain Naturalists

Bonato, G. A.

Bioscript

Bonavia, Edward

Bioscript

Bond, Frank (1856-1940) Ornithologist Bioscript

Bond, James (-1989) Academy of Nanural Sciences, Philadelphia, PA

Bioscript

Correspondence • 1972: (1) Species • 1973: (1)

Bondy, Earle

Bioscript • Botanical Album

Bone, Fannie Zerviah Lovell Husbend is Hugh M. Bone Correspondence • 1960: (3)

Bone, Gavin Botanical Album

Bongard, H. G. Bioscript

Bonini, Bob Pittsburgh, PA Correspondence • 1971: (2)

Bonisteel, Wm. J. Editor, Torreya Correspondence • 1941: (3)

Bonjean, J. Bioscript 
Bonner, Charles Edmond Bradlaugh (1915-1976) British bryologist, taxonomist; Conservatoire et Jardin Botanique, Genève, Switzerland Bioscript • Botanical Album

Correspondence $\bullet$ 1967: (1)

Bonner, James C. Georgia College, Milledgeville Correspondence • 1967: (4)

Bonner, John Tyler Bioscript $\bullet$ Botanical Album

Bonner, W. N. (Collections 1955-1961) English botanist Biofile

Bonner, William J.

Correspondence $\bullet$ 1991: (0) Letter from great-nephew of Joseph William Blankinship filed under Blankinship

Bonney, T. G. Bioscript

Bonnier, G. Bioscript

Bonpland, Aimé Jacques Alexander (1773-1858) Taxonomist, plant collector Biofile $\bullet$ Bioscript

Bookshop, The Bookseller; Chapel Hill, NC Correspondence • 1987: (1)

Boone, Weldon Wesley (1906-) American botanist Biofile

Boorstin, Daniel Joseph (1914 ) Library of Congress, Librarian Bioscript $\bullet$ Botanical Album

Boos, Francis Bioscript

Boos, Joseph Bioscript

Booth, J. G. Bioscript

Booth, James J. ( -1917) Bioscript

Booth, Lawrence Correspondence • 1929-1933: (17) Delphinium; Munz, Philip Alexander, 1892-1974 • 1934: (1)

Booth, Thomas J. (1829-?) Bioscript $\bullet$ Biofile

Booth, W. E. Montana State College Correspondence $\bullet$ 1947: (1)

Boott, Francis (1792-1863) American taxonomist, physician Biofile - Bioscript

Boott, William (1805-1887) Botanical Album

Borell, Adrey Edwin (1901-) American zoologist Bioscript Correspondence • 1949: (2)

Borg, James M. W. Correspondence • 1978: (1)

Borgmann, Carl W. Sigma XI, Colorado Chapter Correspondence • 1941: (1)

Borland, Hal (Harold Glen) (1900- ) American author, naturalist Correspondence • 1975: (1)

Bornstein, F. P. Correspondence $1963:(1)$
Borrer, William (1781-1862) English lichenologist, plant collector Bioscript $\bullet$ Botanical Album

Bory de St. Vincent, Jean Baptiste Geneviève Marcellin (1778-1846) French botanist, zoologist

Bioscript

Bosanquet, Louis Percival (1865-1930) Bioscript

Bosc, Louis August Guillaume (1759-1828) French naturalist, consul to Administrator Biofile • Bioscript

Bosch, Robert Bioscript

Bosniak, Dr. U. of Utah Correspondence • 1970: (1)

Bossert, Theodore W. Curator of Portraits, Hunt Institute for Botanical Documentation Correspondence $1963:$ (2) $\bullet$ 1972: (1) • 1973: (3)

Bosson, Phyllis ISIS, Seattle, WA; editorial assistant Correspondence $\bullet$ 1960: (1)

Bossu, Nicholas Bioscript

Bostain, Mary W. National Science Foundation Correspondence $\bullet$ 1965: (1)

Boston Athenaeum, Library of the Correspondence • 1983: (1)

Boston, Phyllis J. Tulane, New Orleans, Howard-Tilton Memorial Library Correspondence • 1968: (1)

Boston Public Library Correspondence • 1951: (1) Pursh, Frederick, 1774-1820 • 1957: (1)

Boston Society of Natural History Botanical Album

Botanical Congress, VII International Correspondence • 1950: (1)

Botanical Review Correspondence • 1941: (1)

Botanical Society of America Botanical Album Correspondence • 1989: (2)

Botánico Suramericano (Bogotá, Colombia) Botanical Album

Botanico Jardin Rio de Janeiro Correspondence - 1941: (1)

Botanisk Centralbibliotek Kobenhavn K., Danmark Correspondence • 1962: (1)

Botta, Carlo Guiseppe Guglielmo (1776-1837) Italian-born historian, naturalist Bioscript

Botta, Paolo Emilio (1802-1870) Bioscript

Botteri, Mateo (1808-1877) Bioscript

Boucard, Adolphe (1839-1905) Bioscript

Boucher, Pierre Bioscript

Bouderlin, Katherine Bioscript 


\section{Guide to the Ewan Papers}

Bouger,

Bioscript

Bougere, Lydia N.

Correspondence $\bullet$ 1948: (3)

Bougere, Walter C. Attorney; New Orleans, LA Correspondence $\bullet$ 1956: (1)

Boughey, Arthur Stanley (1913-) English taxonomist and plant ecologist; U. College of Rhodesia and Nyasaland, Salisbury, S. Rhodesia, Professor of Botany

Correspondence 1960 : (2)

Boulder, CO (1860)

Botanical Album

Boulderado Hotel Boulder, CO Correspondence 1 1967: (1)

Boulger, George Edward Simonds (1853-1922) British botanist

Bioscript

Boultbee, John Bioscript

Bouquet, Henry Bioscript

Boureau, Edouard (1913-) French paleobotanist; International Organization for Palaeobotany, Seine, France Correspondence $\bullet$ 1958: (1)

Bourgeau, Eugène (1813-1877) French botanical collector, taxonomist Biofile • Bioscript

Bourgeois, Frank E., Mrs. Potted Plant Club, New Orleans Correspondence $\bullet$ 1950: (3)

Bourgeois, Sidney S., Jr., Mrs. Metairie, LA Correspondence • 1961: (1)

Bourque, Juliette Public Archives of Canada, Librarian Correspondence • 1951: (4)

Bouslog, Charles S. Correspondence 1974 : (1)

Bowden, Wray Merrill (1914) Botanist; Dept. of Agriculture, Canada, Div. of Botany and Plant Pathology Correspondence • 1949: (7) Species • 1950: (2)

Bowdich, Thomas Edward (1791-1824) Bioscript

Bowditch, Fred C. Entomological collector Bioscript

Bowen, W. Wedgwood Dartmouth College Museum, Director \& Curator of Biology Correspondence • 1959: (5) 1963 : (4)

Bower, Frederick Orpen (1855-1948) English botanist, plant morphologist, paleontologist Biofile

Bowerbank, James Seott (1797-1877) British industrialist and naturalist at London Botanical Album

Bowerman, Mary Leolin (1908-) Canadian-born California taxonomist, plant ecologist Botanical Album

Correspondence • 1937: (1) 1944: (1) Bowers, Janice E. Tueson, AZ; U. S. Geological Survey,
Botanist

Correspondence • 1989: (9) • 1991: (2)
Bowers, John Z. Josiah Macy, Jr. Foundation, New York, NY, President

Correspondence $\bullet$ 1965: (4) • 1966: (5) Letter to John Duffy $\bullet$ 1971: (5)

Bowes-Lyon, David, Sir (1902-1961) Horticulturist Biofile

Bowles, Edward Augustus (1865-1954) English botanist Biofile • Bioscript

Bowman, Charles Miami, FL Correspondence $\bullet$ 1949: (2)

Bowman, Inci A. U. of Texas, Galveston, Moody Medical Library

Correspondence $\bullet 1977$ : (2)

Bowman, Isaiah Geographer Bioscript

Bowman, Thomas Clark (1941-) Plant anatomist and morphologist; Arizona State U., Tempe, Depe. of Botany Correspondence • 1968: (3)

Boyce, Bob Ohio State newspaper, On Campus Correspondence $\bullet$ 1982: (2)

Boyce Thompson Desert Botanic Garden Botanical Album

Boyd, Emma Sophia (ก. 1934) Bioscript

Boyd, Samuel T. Bioscript

Boykin, Samuel Bioscript

Boyle, Charles Elisha (1821-1870) American physician and naturalist Biofile

Boyle, Robert (1627-1691) English philosopher and scientist Biofile $\bullet$ Bioscript

Boyle, W. S. U. of California, Berkeley Correspondence • 1942: (1)

Bracelin, Nina Floy (1890-1973) American plant collector. botanical artist; U. of California, Berkeley. Depe. of Botany agent for Ines Mexia, husband is Harry Philip Bracelin; nickname "Bracie"

Bioscript • Botanical Album Correspondence • 1937: (2) • 1938: (3) • 1939: (4) • 1940: (2) Setchell, William Albert, 1864-1943; Jepson, Willis Linn, 1867-1946 • 1941: (3) • 1942: (4) • 1944: (2) - 1957: (1) • 1958: (2) Species • 1960: (4) Book collectors - 1961: (13) Book collectors; U. of California, Berkeley. Dept, of Botany • 1962: (11) • 1963: (7) Lawrence, George Hill Mathewson, 1910-1978 • 1964: (8) - 1966: (4) • 1967: (3) • 1968: (1) • 1969: (1)

Bracey, B. O. (f. 1984) Bioscript

Brackenridge, Henry Marie (1786-1871) Bioscript

Brackenridge, William Duniop (1810-1893) Scottish-born American borticulturist Biofile • Bioscript • Botanical Albw

Brackett, A. G. Bioscript 
Bradburn, Anne Tulane U., Dept. of Biology Bioscript $\bullet$ Botanical Album

Correspondence $\bullet$ 1973: (1) • 1974: (3) • 1975: (7) • 1978: (2) • 1979: (1) • 1981: (1) - 1982: (7) • 1983: (3) • 1984: (15) - 1986: (9) Tulane U. Dept. of Botany 1987 : (8) $\bullet$ 1988: (6) • 1989: (4) • 1990: (1) $\bullet$ 1991: (1) $\bullet 1993$ : (1)

Bradburn, Donald Pathologist; Touro Hospital, New Orleans

Bioscript

Correspondence • 1976: (1) $\bullet$ 1992: (4)

Bradbury, John (1762-1821) of York, Maine Bioscript

Bradbury, John (1768-1823) Scottish naturalist Biofile • Bioscript

Bradbury, W. E.

Bioscript

Brade, Alexander Curt (1881-1971) German-born architect, botanist, plant collector, Brazil; Instituto Brasileiro de Bibliografiae Documentaçao, Rio de Janeiro Correspondence $\bullet$ 1959: (1)

Bradford, Thomas G. Bioscript

Bradley, Camilla Times Picayune, New Orleans Bioscript Correspondence $\bullet$ 1946: (1)

Bradley, Cornelius Beach (1844?-) U. of California, Berkeley, English Dept. Bioscript

Bradley, George American Embassy, Ecuador-Peru Boundary Demarcation Commission Correspondence • 1944: (1)

Bradley, Richard (1688?-1732) English botanist and horticulturist Biofile $\bullet$ Bioscript

Bragg, Laura May (1881-) American taxonomist, plant collector; Charleston Museum, Charleston, SC, Director Bioscript

Bragg, Luther C. Taxidermist Bioscript

Brainerd, Ezra (1844-1924) American educator, botanist, taxonomist Biofile $\bullet$ Botanical Album

Branch, Ruth New Orleans Botanical Album Correspondence • 1985: (1)

Brandegee, Edward Newton (1865-1942) Amateur botanist Bioscript

Brandegee, Emily S. Berlin, CT Correspondence • 1949: (3)

Brandegee, Mary Katherine (Layne)(Curran) (1844-1920) American taxonomist Biofile (Filed under Townshend Stith Brandegee) - Bioscript

Brandegee, Townshend Stith (1843-1925) American civil engineer and botanist Biofile $\bullet$ Bioscript $\bullet$ Botanical Album

Brandenburg, Fredrick Harmon (1854-1922) Climatologist Bioscript

Brander, E. Bioscript
Brandes, Elmer Walker (1891-) American plant pathologist; Bureau of Plant Industry, Rubber Investigation Correspondence $\bullet$ 1943: (2)

Brandon, Ralph Board of Economic Warfare Correspondence • 1943: (4)

Brandon, William F.

Correspondence • 1962: (1)

Brandt, J. G. W. Bioscript

Brannon, Peter A. Alabama State Archives, Montgomery, Director

Correspondence • 1961: (2) • 1963: (2) • 1963: (0) Filed with letter to Lois Chambers Stone

Branth, Jacob Severin Deichmann (1831-1917) Plant collector

Biofile

Brasher, Rex Botanical Album

Brass, Leonard John Botanical Album

Braun, Emma Lucy (1889-1971) American taxonomist, plant ecologist

Biofile $\bullet$ Bioscript $\bullet$ Botanical Album

Correspondence 1946 : (1)

Braunton, Ernest C. (1867-1954) English-born plant collector, U. S. A.

Bioscript • Botanical Album Correspondence • 1929-1933: (2) • 1936:

Braxton, John Correspondence $\bullet$ 1975: (1)

Bray, D. W. Correspondence 1941 : (1) U. of Colorado, Boulder. Dept. of Biology

Bray, Dillard Correspondence $\bullet$ 1943: (1)

Bray, Edmund C. Philadelphia, PA; nickname "Ned" Botanical Album

Correspondence • 1982: (1) • 1983: (2) • 1984: (2) • 1989: (2)

Bray, Martha Husband is Edmund C. Bray Correspondence $\bullet$ 1966: (1) $\bullet$ 1981: (2) $\bullet$ 1982: (3) $\bullet$ 1984: (4) • 1985: (1) • 1986: (1) • 1992: (3)

Brayer, Herbert O. State Historical Society of Colorado Correspondence $\bullet$ 1943: (2)

Bredemeyer, Franz (1758-1839) Gardener at Schonbrunn Bioscript

Breedlove, Dennis E. (1939-) Bioscript $\bullet$ Botanical Album

Breen, Ruth Schornhert (1905-1987) American bryologist, taxonomist; Florida State U., Tallahassee, Division of Botany Correspondence $\bullet 1960$ : (4)

Brehm, C. L. Bioscript

Breintnall, Joseph Bioscript

Breitung, August Johann (1913-1987) Canadian plant collector and taxonomist

Botanical Album

(continued on next page) 
Breitung, August Johann (1913-1987) (continued) Correspondence • 1941: (2) • 1947: (1) • 1953: (2) Delphinium • 1954: (4) • 1957: (3) "Rocky Mountain Vegetation"; Delphinium; Species

Bremer Bioscript

Bremer, Fredrika Bioscript

Brenan, John Patrick Micklethwait (1917-1985) British botanist

Botanical Album

Brenchley, Julius Lucius (1816-1873) English traveler and writer Bioscript

Brenchley, Winifred Elsie (1883-1984) English botanist; Rothamsted Experimental Station, Harpendon, England Botanical Album

Brenckle, Jacob Frederick (1875-1958) American physician, Amateur botanist Correspondence • 1943: (1)

Brendel, Frederick (1820-1912) German-born botanist, physician Biofile $\bullet$ Botanical Album

Brennan, Samuel, Jr. Bioscript

Brenner, Walter M. The Academy of Natural Sciences of Philadelphia

Correspondence $\bullet$ 1952: (1)

Brent, Francis Bioscript

Brenton, Mary E. Bioscript

Brereton, John A. Bioscript

Bretnor, Helen H. Bancroft Library, Reference Librarian Correspondence $\bullet$ 1960: (1)

Breton, Gerard Ville du Havre, Museum d'histoire Naturelle, Directeur, Professor Correspondence • 1979: (1)

Bretschneider, Emilii Vasil'evich (1833-1901) Russian botanical bibliographer, physician Bioscript

Bretting, Peter Koarad (1953-) American economic botanist; Economic Botany, Book review editor Bioscript • Botanical Album

Correspondence • 1975: (9) • 1976: Missouri Botanical Garden • 1977: (13) - 1978: (3) - 1979: (9) Letters to J. F. M. Cannon and Mr. J. Lewis $\bullet$ 1980: (8) $\bullet$ 1980: (0) Letter filed under Jane Haskett Bock $\bullet$ 1981: (9) $\bullet$ 1982: 1987: (3) 1987: (3) Letters to and from Peter Raven $\bullet 1990$ : (5) 1991: (3) - 1992: (1) • 1993: (1)

Breutel, Johann Christian (1788-1875) German Moravian bishop and botanist Bioscript Brewer, Thomas Mayo (1814-1880) American physician
and ornithologist Biofile $\bullet$ Bioscript

Brewer, Wiliam Henry (1828-1910) American botanist, plant collector Bioscript • Botanical Album
Brewster, Grace B. Montrose, CO Correspondence • 1949: (2)

Brewster, J., Mrs. Bioscript

Brewster, Lacy G. T. Bioscript

Brewster, William (1851-1919) Ornithologist Bioscript

Brey, Claire La Jolla, CA Botanical Album Correspondence • 1979: (1)

Brian, Ray California Academy of Sciences, Librarian Correspondence • 1965: (3) • 1968: (4) • 1978: (1) Letter to R. S. Cowan • 1980: (5) • 1981: (1) • 1982: (1)

Brichford, Maynard U. of Illinois at Urbana-Champaign, Archivist

Correspondence • 1991: (0) Filed under Emanuel David Rudolph

Brickell, John (1749-1809) of Savannah Irish-born botanist and physician Bioscript

Brickell, John (n. 1730-1740) of Edenton, NC American physician, natural history writer Biofile • Bioscript

Bricker, Victoria Tulane U., New Orleans Correspondence • 1982: (1) • 1986: (1)

Bridenbaugh, Carl U. of California, Berkeley, Dept. of History

Correspondence • 1965: (2)

Bridges, Thomas Charles (1807-1865) English-born plant collector

Bioscript

Bridson, Gavin D. R. Botanical Album

Correspondence • 1975: (1) • 1979: (1) • 1980: (1) • 1981: (1) • 1983: (1) • 1985: (2) • 1990: (3) • 1991: (4) • 1992: (1)

Briggs, Barbara Gillian (1934) Royal Botanic Gardens and National Herbarium, Sydney, Australia Correspondence • 1974: (9) Barton, Benjamin Smith, 1766-1815 • 1977: (1) • 1979: (1) • 1980: (1)

Briggs, L. A. Correspondence $\bullet$ 1935: (2)

Brigham, Clarence S. American Antiquarian Society, Worcester, Massachusetts

Correspondence • 1958: (1)

Bright, John (1872-1952) Plant collector Botanical Album Correspondence • 1938: (1)

Brightman, Frank Correspondence • 1991: (2)

Brill, E. J. Flora and Fauma Publications Correspondence • 1986: (1)

Brill, Grace St. Louis, MO Botanical Album Correspondence • 1988: (3) $\bullet$ 1989: (2) $\bullet$ 1990: (2) •
1992: (1)

Brinkley, David NBC News, Washington, D.C. Correspondence $\bullet 1969$ : (2) 
Brinkman, Alfred Henry (1873-1945) British-born Canadian bryologist

Bioscript

Brinton, D. G. Bioscript

Briquet, John Isaac (1870-1931) Swiss taxonomist Bioscript $\bullet$ Botanical Album

Brisky, O. Bookseller, Micanopy, FL Botanical Album

Brissot de Warville, J. P. Bioscript

Bristol, Mel U. of Hawaii Correspondence • 1967: (1)

Bristowe, W. S. Correspondence • 1967: (2)

British Columbia Botanical Association Bioscript

British Museum (Natural History) Bioscript $\bullet$ Botanical Album Correspondence • 1951: (1) Pursh, Frederick, 1774-1820 - 1956: (1) - 1960: (1) Banister, John, 1650-1692 • 1961: (3) Banister, John, 1650-1692 • 1962: (1) • 1964: (1) • 1965: (2) - 1981: (1) • 1990: (1)

Britten, James (1846-1924) English taxonomist, bryologist Bioscript

Britton, Elizabeth Gertrude (1858-1934) American bryologist, taxonomist Biofile

Britton, J. P. American Optical Co. Correspondence • 1947: (1)

Britton, Nathaniel Lord (1859-1934) American taxonomist, geologist; New York Botanical Garden Biofile $\bullet$ Bioscript $\bullet$ Botanical Album Correspondence 1 1929-1933: (1)

Brittonia New York Botanical Garden Correspondence • 1965: (1) • 1983: (2) • 1991: (3) Correspondent is Barbara M. Thiers, Assoc. Editor

Broach, E., Jr. Meridian, MS Correspondence • 1970: (4)

Broadbent, Leonard (1916-) English plant virologist; U. of Bath, Professor of Horticulture, Pro-Vice-Chancellor Correspondence $\bullet$ 1978: (2) $\bullet$ 1979: (2)

Broaddus, Mabel Jones Daughter of Marcus E. Jones Correspondence • 1935: (2) • 1945: (2)

Broadway, Walter Elias (1863-1935) English-born taxonomist, horticulturist in Trinidad Bioscript

Brocchi, G. B. Biofile

Brock, Helen U. of Glasgow, Scotland, Dept. of History of Science Correspondence $\bullet$ 1977: (2)

Brockenbrough, Austin Bioscript

Brockman-Jerosch, Heinrich (1879-1939) Swiss phytogeographer Bioscript • Botanical Album

Brockway, U. H., Mrs. Correspondence $\bullet 1963:$ (2)

Brockway, Waldo E. Correspondence • 1943: (1)
Brode, William E. Copiah-Lincoln Junior College, Wesson, MS

Correspondence $\bullet$ 1955: (5)

Brodhead, Michael J. U. of Nevada-Reno, Dept. of History

Bioscript

Correspondence $\bullet$ 1971: (1) • 1972: (2) • 1978: (3) • 1980: (7) • 1981: (1) • 1983: (2) • 1984: (1) • 1985: (1) • 1987: (3) • 1989: (4) • 1993: (9)

Brodie, William A. Bioscript

Broek, Jan O. M. Geographer Biofile

Bromfield, William Arnold (1801-1851) British botanist, botanical collector Bioscript

Brongniart, Adolphe Théodore (1801-1876) French botanist and paleobiologist Bioscript - Botanical Album

Brongniart, Alexandre (1770-1847) French paleobiologist Bioscript

Bronson, __, Miss Correspondence $\bullet$ 1967: (1)

Brookhaven Press La Crosse, WI Correspondence • 1979: (1)

Brooklyn Botanic Garden Correspondence • 1958: (1) • 1979: (1) Thomas J. Delendick

Brooks, Carol U. of Alabama, Dept. of Biology Correspondence $\bullet$ 1971: (2)

Brooks, F. G. Correspondence • 1945: (1)

Brooks, Frank Gray Botanical Album

Brooks, James H. McNeese State U., Lake Charles, LA, Dept. of Biology

Correspondence $\bullet$ 1969: (1) $\bullet$ 1973: (1)

Brooks, Leon E. Descendant of A. J. Grayson Correspondence • 1977: (0) Letters filed under Lois Chambers Stone

Brooks, Ralph Edward (1950-) U. of Kansas, Lawrence Correspondence • 1976: (2) • 1977: (2) • 1982: (2)

Broome, Carmen Rose (1939-) Duke U. Correspondence - 1971: (1)

Brosse, Verneal Chalmette, LA Correspondence $\bullet$ 1980: (1)

Brouard, Arsène Gustave Joseph (1867-1928) See also Arsène, Brother Gerfroy Bioscript

Broughton, William Robert ( -1798) Bioscript

Brouillet, Luc (1954 ) Flora of North America, Regional Director Correspondence $\bullet$ 1988: (4)

Brown (Broune), Bioscript

Brown, Clair Alan (1903-) American taxonomist; Louisiana State U., Baton Rouge Botanical Album $\bullet$ Bioscript (continued on next page) 
Brown, Clair Alan (1903-) (continued)

Correspondence - 1953: (1) • 1959: (7) • 1961: (7) • 1962: (3) • 1964: (2) • 1965: (2) • 1968: (3) • 1971: (1) 1973: (2) • 1975: (2) • 1978: (2) • 1983: (1)

Brown, Clara D. Husband is Clair Alan Brown Correspondence $\bullet 1982:$ (1)

Brown, , Dr. Correspondence $\bullet$ 1953: (1)

Brown, Frederick Martin (1903-) American physiologist, entomologist; Fountain Valley School, Colorado Springs, $\mathrm{CO}$; nickname is "Brownie" Bioscript Correspondence • 1949: $(9) \bullet 1965:(7) \bullet 1967:(1) \bullet$ 1968: (2) • 1970: (1)

Brown, J. Mercer Correspondence $\bullet 1951:$ (2)

Brown, Jack H., Mrs. Greensboro, NC Correspondence • 1958: (2)

Brown, John Physician Bioscript

Brown, Lucille Husband is Harry W. Brown Correspondence $\bullet 1951:(2)$

Brown, Maurice Botanical Album

Brown, Nicholas Edward (1849-1934) English botanist Bioscript $\bullet$ Botanical Album

Brown, Otway Hores (1877-1946) American naturalist Botanical Album

Brown, P. F. Correspondence $\bullet 1968$ : (2)

Brown, Ralph M. Tulsa, OK Correspondence $\bullet 1951$ : (3)

Brown, Rick Grandson of W. H. Fries Correspondence • 1972: (0) Filed under Waldemar H. Fries

Brown, Robert (1773-1858) Scottish botanist; British Museum (Natural Science), Keeper of Botany Biofile • Bioscript • Botanical Album

Brown, Robert (1842-1895) Botanical Album

Brown, Roland Wilbur (1893-1961) American paleobotanist Biofile • Bioscript

Brown, Rose Duke U., Dept. of Biology Correspondence - 1973: (2)

Brown, Russel G. Dept. of Biology Correspondence $\bullet$ 1973: (1)

Brown, Ruth E. Academy of Natural Science, Librarian Correspondence • 1971: (5) • 1972: (2) • 1973: (2) • 1974: (1) - 1981: (1) • 1982: (2) - 1985: (1)

Brown, Sanborn C. Smithsonian Institution, Joseph Henry Papers and Massachusetts Institute of Technology Correspondence • 1974: (1)

Brown, Stewardson (1867-1921) American taxonomist, botanist, ornithologist Biofile $\bullet$ Bioscript

Brown, W. Burlie Tulane U., Dept. of History Correspondence • 1953: (1)

Brown, W. H. Royal Botanic Gardens, Kew, Librarian Correspondence $\bullet 1964$ : (3)

Brown, William Bioscript
Brown, Wm C., Company Publishers Correspondence $\bullet 1965:$ (1)

Browne, John Ross (1821-1875) American travel writer, illustrator Biofile

Browne, Patrick Bioscript

Browne, Thomas Bioscript

Browning, M. Ralph U. S. National Museum, National Fish and Wildlife Laboratory

Correspondence $\bullet 1974$ : (1)

Brownle, Richard S. State Historical Society of Missouri Correspondence • 1965: (2)

Brozek, Arthur Botanical Album

Bruce, Archibald (1777-1818) American minerologist Biofile • Bioscript

Bruce, C. C., Mrs. Bioscript $\bullet$ Botanical Album

Bruce, David T. (1833-1903) Painter of frescoes and butterflies, book dealer

Bioscript

Correspondence • 1949: (2) Correspondence about David Bruce

Brues, Charles Thomas (1879-1955) American entomologist

Bioscript

Bruhl, Jeremy Correspondence • 1990: (1) • 1991: (2) • 1993: (2)

Brumback, Florence May (1884) Plant collector; Colorado Botanical Album

Bruner, Lawrence (1856-1937) American entomologist Bioscript

Bruner, W. E. State Teachers College, Kearney, NE Correspondence • 1948: (2)

Brunfels, Otto (1488-1534) German botanist, herbalist Bioscript

Brunn, Anton Frederik (1901-1961) Danish ichthyologist and oceanographer Biofile

Brunquist, Ernest H. Denver, CO Biofile Correspondence • 1940: (6) • 1941: (6) • 1942: (2) • 1943: (4) • 1945: (2) • 1954: (1) • 1965: (1)

Bruscate, Bazile, Mrs. New Orleans, LA Correspondence $\bullet$ 1961: (1)

Brute, Joseph U. of Georgia Press Correspondence • 1975: (1)

Bry, Johann Theodor de (1561-1623?) Biofile

Bryan, Charles F. American historian; Mercantile Library, St. Louis, MO

Correspondence $\bullet$ 1987: (3) $\bullet$ 1988: (2)

Bryan, George Smith (1879-1958) American plant morphologist, cytologist; U. of Wisconsin, Madison, Professor of Botany Correspondence $\bullet$ 1949: (4)

Bryant, Farold Child (1886-) Bioscript

Correspondence • 1929-1933: (1) 
Bryant, Walter [Pierce] E. (1861-1905) Bioscript

Bryarly, Wakeman Bioscript

Bryn Mawr College James Tanis, Director of Libraries Correspondence • 1973: (1)

Buchanan, George (1763-1808) Bioscript

Buchanan, Herbert Earl Correspondence 1964 : (1)

Buchenau, , Dr. Botanical Album

Buchheim, Günther (1924-) German-born American taxonomist, botanical bibliographer; John Carter Brown Library, Brown U., Providence Botanical Album Correspondence - 1989: (6) Letter to Buchheim fr. Dale Johnson, MBG • 1991: (3) • 1993: (2)

Buchheister, Carl W. Biofile

Buchholz, John Theodore (1888-1951) American botanist, plant morphologist, taxonomist; U. of Illinois, Urbana Bioscript

Buchner, Bioscript

Buchner, Hollingworth Husband is Will Buchner Correspondence • 1958: (5)

Buchtel, Henry Trail and Timberline, Editor; Colorado botanical explorer

Correspondence • 1941: (5)

Buchtien, Otto (1859-) German taxonomist, phytogeographer Correspondence • 1934: (3)

Buchwald, Niels Fabritius (1898-) Danish taxonomist, plant pathologist Biofile

Buck, Margaret Warriner Bioscript

Buck, Nancy Harvard U., Cambridge, Farlow Reference Library Correspondence • 1958: (1)

Buck, Paul H. Harvard Correspondence • 1942: (3)

Buck, Roger C. National Science Foundation, Program Director, History and Philosophy of Science Correspondence • 1963 : (2) Lyon, John, 1764-1814 • 1965: (1)

Buck, William Russell (1950-) New York Botanical Garden Correspondence $\bullet$ 1982: (2) 1990: (1)

Buckhaltar, Gertrude Piney Woods Country Life School, Piney Woods, MS

Correspondence $\bullet$ 1951: (1) $\bullet$ 1952: (2)

Buckland, William (1784-1856) Bioscript

Buckley, Samuel Botsford (1809-1884) American botanist Biofile $\bullet$ Bioscript

Buckman, Thomas R. U. of Kansas, Lawrence, Director of Libraries

Correspondence • 1964: (2) • 1966: (5) • 1967: (1) • 1976: (1)
Buckowitz, Georgia L. Forest Park, Saint Louis, MO Correspondence $\bullet$ 1976: (1)

Buczacki, S. T. National Vegetable Research Station Correspondence 1975 : (3)

Buddle, Adam (c. 1660-1715) English botanist Bioscript

Buell, Murray Fife (1905-) American plant ecologist Botanical Album

Correspondence • 1966: (1) Harper, Roland McMillan, 1878-1966 • 1967: (2) • 1968: (3)

Buffon, George Louis Leclerc de (1707-1788) French naturalist Bioscript $\bullet$ Botanical Album

Buffum, Burt C. (1868-1944) American botanist, agriculturist Bioscript $\bullet$ Botanical Album

Buffum, Maude S. Husband is Burt C. Buffum Correspondence • 1949: (4)

Bugnon, F. Correspondence • 1954: (1)

Buist, Robert (1805-1880) Scottish-born American gardener, seedsman, florist

Bioscript

Buitenzorg, Jardin Botanique de Botanical Album

Bull, Elias B. Charleston, SC Correspondence • 1969: (2)

Buller, Arthur Henry Reginald (1879-1944) Canadian mycologist Bioscript $\bullet$ Botanical Album

Buller, Roderic E. The Rockefeller Foundation, Assistant Agronomist Correspondence • 1954: (2) Van Schaack, George Booth, 1903-1983

Bulletin of the Torrey Botanical Club New York State U., College at Brockport, Brockport Correspondence • 1981: (1)

Bullock, William (1773-1849) English natural history collector

Biofile $\bullet$ Bioscript

Bulmer, Ralph N. J. U. of Auckland, New Zealand, Dept. of Anthropology Correspondence • 1974: (6) • 1976: (1) • 1979: (3) Rocky Mountain Naturalists • 1988: (1)

Bumpus, Hermon C. Botanical Album

Bumstead, Freeman Josiah (1826-1879) Plant collector, U. S. A.

Bioscript

Bunbury, Charles James Fox (1809-1886) Plant explorer, collector

Bioscript • Botanical Album

Bunbury, Edward Bioscript

Bunting, Arthur Hugh (1917- ) South African-born plant physiologist and ecologist; U. of Reading, England, Dept. of Agriculture

Correspondence $\bullet$ 1967: (5) $\bullet$ 1968: (3)

Burbank, Luther (1849-1926) American horticulturist, plant breeder

Biofile $\bullet$ Bioscript $\bullet$ Botanical Album 


\section{Guide to the Ewan Papers}

Burch, John Q. Importer and Dealer in Natural History Books and Specimen Shells, Los Angeles, CA Correspondence • 1951: (2) • 1954: (2) • 1956: (1) • 1967: (1)

Burchell, William John (1781-1863) Brazilian plant collector Bigfile • Bloscripi

Burclhalter, Robert U. of Alabama, College of Arts and Sciences, Tuscaloosa, Dept. of Biology Correspondence • 1989: (1)

Burdet, Hervé Maurice (1939-) Bibliothèque du Conservatoire Botanique, Genève, Conservateur Correspondence • 1976: (4)

Burean, Ed Bloscript

Bureau of Widllife and Sperts Fisheries C. Edward Carison, Regional Director Correpondence • 1969: (2)

Burger, William Carl (1932-) American taxonomist, botanical artist; Field Museum of Natural History, Dept. of Botany Correpondence • 1974: (2) • 1987: (1) • 1988: (1)

Burgess, Edward Sandford (1855-1928) American axonomist, bocanical historian Bioscript

Burgess, Robert Lewis (1931-) American plant ecologist, botanist; College of Environmental Science and Forestry, Syracuse, NY

Biofile

Correspondence • 1961: (2) • 1966: (1) • 1967: (2) • 1971: (1) • 1972: (2) • 1976: (5) • 1978: (1) See also correspondence to Dr. Burgess, filed under Ecology • 1979: (6) - 1979: (0) Letter from John Fogg • 1980: (3) • 1982 (1) • 1983: (1) • 1985: (2) • 1987: (1) • 1991: (1)

Burgehaus, Fred Hope (1869-1921) Blascript

Burk, S. G. Twin Lakes, CO Correspondence $\bullet$ 1940: (2)

Burk, W. H. Plant collector Botanical Album

Burkart, Arturo (1906-1975) Argentine taxonomist, botanical artist Bicfile $\bullet$ Botanical Album Correspondence • 1939: (2) • 1940: (2) • 1941: (1) 1948: (1) • 1953: (1)

Burke, Horace R. Texas A. \& M. University, College Station, Dept of Entomology, Professor Correspondence • 1988: (4) Book collectors

Burke, J. Bioscript

Burke, Joseph (1812-1873) British nanural history collector Bicfile • Bioscript

Burkearoad, Martin D. Correspondence • 1948: (6) • 1954: (1)

Burkett, George Correspondence $\bullet 1992:(1)$

Burkhalter, James R. Pensacola, FL Correspondence • 1980: (1)

Burkhardt, Bloscript
Burkhardt, Frederick H. Bennington, VT; The Collected Letters of Charles Darwin

Bioscript

Correspondence $\bullet$ 1976: (3) $\bullet$ 1984: (2) $\bullet$ 1990: (2)

Burkhardt, Richard, Jr. U. of Ilinois, Urbana Correspondence $\bullet$ 1989: (1)

Burkholder, Paul Rufus (1903- ) American microbiologist; Botanical Society of America Botanical Album Correspondence $\bullet$ 1941: (1)

Burkill, Isaac Henry (1870-1965) English economic botanist Botanical Album

Burleigh, Edward G. New Orleans, LA Correspondence $\bullet$ 1949: (1)

Burlew, Fred E.

Bioscript

Correspondence $\bullet$ 1935: (2)

Burlingame, M. G. Montana State College, Bozeman, Dept. of History

Correspondence $\bullet$ 1970: (1)

Burman, Alan Chelsea Physic Garden Correspondence $\bullet$ 1983: (1)

Burman, Alasdair Graham (1942- ) Correspondence • 1985: (3)

Burman, John [Johannes] Barnhart (1706-1799) Dutch physician, botanist Bioscript

Burnell, Lynette Bioscript

Burnham, John Chynoweth (1929- ) American science historian; Ohio State U.

Correspondence $\bullet$ 1982: (1)

Burnham, Stewart Henry (1870-1943) American bryologist Bioscript

Burns, George Plumer (1871-1953) American botanist, forester; U. of Vermont, Burlington Correspondence • 1939: (1) • 1943: (1)

Burrage, Severance B. U. of Colorado Correspondence $\bullet$ 1943: (2)

Burrill, Thomas Jonathan (1839-1916) American mycologist

Bioscript

Burrington, Dave Correspondence $\bullet$ 1976: (1)

Burrison, Henry K. (f. 1888-1919) Entomologist Bioscript

Burroughs, John (1837-1921) American naturalist, conservationist Bioscript $\bullet$ Botanical Album

Burstyn, Harold L. Reston, VA, U.S. Geological Survey Correspondence $\bullet$ 1977: (1)

Burt, Edward Angus (1859-1939) American librarian, mycologist

Bioscript

Burton, David (?-1792) English architect Biofile

Burton, Decimus English statesman and naturalist Biofile

Burton, E. Milby Charlestown Museum, Charleston, SC, Director

(continued on next page) 
Burton, E. Milby (continued)

Correspondence $\bullet$ 1952: (8) $\bullet$ 1953: (2) $\bullet$ 1958: (1) $\bullet$

1961: (2) • 1963 : (1) Lyon, John, 1764-1814 • 1965: (2)

- 1966: (4) • 1968: (4) • 1969: (3) Bartram, William, 1739-1823 - 1970: (4)

Burton, Richard Francis (1821-1890) British archeologist, explorer

Biofile

Burton, Robert E.

Correspondence • 1974: (2) Rocky Mountain Naturalists

Burtt, Edward $\mathbf{H}$.

Correspondence • 1992: (1)

Burtt-Davy, Joseph (1870-1940) English plant collector, taxonomist, forester

See Davy, Joseph Burtt

Burwell, Lewis

Bioscript

Bury, Priscilla Susan Falkner (fl. 1820's-1860's) British botanical artist; husband is Edward Bury

Bioscript

Buse, Lodewijk Heudrik (1819-1888) Plant collector Bioscript

Buser, $\mathbf{R}$.

Bioscript

Bush, A. E., Mrs. Bioscript

Bush, Benjamin Franklin (1858-1937) American plant collector Bioscript

Bush, J. E. New Orleans City Park Improvement Assn. Correspondence • 1956: (2) • 1958: (1) • $1963:$ (1)

Bute, Georg Bioscript

Bute, Earl of See Stuart, John, Earl of Bute

Butler, Alford Augustus (1845- ) American minister Bioscript

Butler, Bertram Theodore (1872- ) American geologist, botanist

Bioscript

Butler, Dr. Correspondence $\bullet$ 1939: (1)

Butler, George Dexter (1850-1910) American naturalist, plant collector Bioscript $\bullet$ Botanical Album

Butler, Joseph T. Historic Hudson Valley, Tarrytown, NY, Curator and Director of Collections Correspondence - 1988: (1)

Butler, W. L. Correspondence 1935 : (1) Book collectors

Butner, Jim Archer, FL Correspondence $\bullet$ 1977: (1)

Butters, Frederic King (1878-1945) American botanist Bioscript $\bullet$ Botanical Album

Buttle, John Bioscript

Byard, Frank Automobile Club of Southern California Correspondence • 1929-1933: (1)
Bye, Robert Arthur, Jr. (1947- ) American economic botanist

Bioscript

Correspondence 1975: (2) • 1976: (1) • 1977: (3) $\bullet$ 1978:

(4) - 1979: (1) • 1980: (2) • 1985: (1) • 1990: (2)

Byers, Janet L. Dictionary of Scientific Biography, New York, NY

Correspondence $\bullet$ 1977: (0) Letter filed under Charles C. Gillispie

Byers, William Newton (1831-1903) American mountaineer, historian Bioscript

Byington, Edwin Lewis ( -1891) Educator, plant collector in Colorado Bioscript

Bynum, Flora Ann Old Salem, Inc., Winston-Salem, NC; husband is Zachary T. Bynum

Bioscript $\bullet$ Botanical Album (Filed under Zachary Bynum) Correspondence $\bullet$ 1973: (3) • 1974: (4) • 1976: (3) 1979: (2) • 1984: (2) • 1989: (2) • 1990: (2) • 1991: (1) 1992: (3)

Bynum, W. F. U. of London, Sub-dept. of the History of Medicine

Correspondence $\bullet$ 1977: (2)

Bynum, Zachary

Botanical Album

Byram, James

Correspondence $\bullet$ 1965: (1)

Byrd, William, II (1674-1744) Virginian statesman and naturalist

Biofile

Byrd, William, I (1652-1704) Bioscript

Byrne, Marie Bancroft Library, U. of California, Berkeley Correspondence $\bullet$ 1986: (1)

\section{$c$}

Caan, Celia W.

Correspondence 1950: (1)

Cabanis, Jean Louis (1816-1906) Plant collector, S. E.United States Bioscript

Cabell, James Lawrence (1813-1889) Bioscript

Cabell, P. H. Bioscript

Cabot, Francis H.

Correspondence • 1991: (1)

Cabot, Samuel, Jr. (1815-1885) American physician, surgeon, ornithologist Biofile

Cabrillo, Juan Rodriquez Bioscript

Cadbury, Henry J. Correspondence • 1966: (1) • 1967: (1)

Cadet, Charles Louis (1789-1861) French botanist Bioscript

Cadzow, Jack New Orleans, LA Correspondence • 1981: (1) 
Caen, Herb

Botanical Album

\section{Cagle, Fred Ray}

Bloscript • Botenical Album

Correspondence • 1949: (0) Filed under Italia Graham •

1950: (0) Filed under Robert A. Vines • 1952: (2) $\bullet 1954$ :

(2) British Museum (Natural History). Dept. of Botany,

Banks, Joseph, Sir, 1743-1800 • 1955: (1) • 1958: (1) See also Joseph L. Ruhl • 1961: (2) • 1963: (1) BiologyInstruction and study

Cain, Stanley Adair (1902-) American plant ecologist, biologist

Bioscript • Botanical Album

Correspondence $\bullet$ 1952: (2)

Cain, Stephen

Botanical Album

Calcutta Royal Botanic Garden

Botanical Album

Calder, James Aleconder (1915-1990) Canadian

phytogeographer, taxonomist; Canada Dept. of Agriculture, Division of Botany and Plant Pathology, Ottawa

Biofile • Botanical Album

Correspondence $\bullet 1949$ : (1)

Caldesi, L.

Bioscript

Caldwell, Charles

Bioscript

Caldwell, June Columnist for Arizona Daily Star, husband is Johnson Caldwell

Correspondence • 1957: (3) • 1960: (3)

Caldwell, Otis William (1869-1947) American botanist, plant morphologist

Botanical Allown

California Academy of Science San Francisco Botanical Album Correspondence • 1976: $(0)$

California Botanical Society Correspondence • 1938: (1) • 1944: (1) • 1946: (1) • 1986: (1)

California Native Plant Society Correspondence $\bullet$ 1992: (1)

California State Library Allan R. Otiley, California Section Librarian

Correspondence • 1953: (2)

Call, Richard Ellsworth (1856-1917) American naturalist Botanioal Album

Callaghan, Nancy M. New York Botanical Garden, Secy. to T. H. Everett Correspondence $\bullet 1960$ : (1) Answer to letter addressed to
Everet

Callahan, Mary V. C. American Antiquarian Society Correspondence • 1978: (0) Filed under American Antiquarian Society

Callan, Edward McC. Imperial College of Tropical Agriculture, St. Augustine, Trinidad

Botanical Album

Correspondence • 1945: (5) • 1946: (1) • 1952: (5) • 1989: (1) • 1992: (1) • 1993: (1)

Callana,

Bioscript
Callaway, Cason (18941961) American industralist; Pine Mountain, GA; founder of Callaway Gardens Biofile

Callaway, Nellie L. National Agricultural Library, U.S.D.A., Nursery and Seed Trade Catalog Section Correspondence • 1968: (1) Bartram, William, 1739-1823

Callender, Wilson S. New Orleans, LA

Correspondence 1961 : (15) Taylor, George, Sir, 19041962: (2) 1963 : (4) Missouri Botanical Garden • 1964:

(2) Horticultural Council, written by Alvin Lichtentag, Secretary

Callery, Bernadette G. Hunt Institute for Botanical Documentation, Librarian

Correspondence • 1979: (1) • 1983: (1)

Calvert, Philip Powell (1871-1961) American entomologist; U. Pennsylvania Biofile Correspondence $\bullet$ 1950: (1)

Cambridge University Librarian Correspondence • 1964: (2) $\bullet$ 1977: (1)

Cambridge University. Clare Hall M. Loewe, Fellowship Committee

Correspondence $\bullet$ 1980: (2)

Cambridge University Press

Correspondence • 1965: (0) Letters filed under George Booth Van Schaack; signed by Ronald Mansbridge

Cameron, Hector Charles Author of a Banks biography Correspondence $\bullet$ 1956: (1)

Cammack, Horace C. Bioscript

Camp, Charles Lewis (1893-1975) American vertebrate paleontologist, historian

Biofile $\bullet$ Bioscript $\bullet$ Botanical Album

Correspondence • 1961: (3) $\bullet$ 1964: (1)

Camp, Earl D. Texas Technological College, Lubbock, Dept. of Biology Correspondence $\bullet$ 1965: (1)

Camp, John $\mathbf{H}$. Bioscript

Camp, Wendell Holmes (1904-1963) American botanist Biofile

Correspondence • 1938: (2) - 1941: (1) Cockerell, Theodore Dru Alison, 1866-1948 • 1946: (1) • 1947: (1) - 1948: (3) • 1950: (1) - 1951: (2) - 1952: (5) Pennell, Francis Whittier, 1886-1952 • 1954: (1) • 1956: (1)

Campbell, Berry (1912-1985) American neurobiologist Biofile $\bullet$ Bioscript $\bullet$ Botanical Album Correspondence $\bullet$ 1929-1933: (5) Munz, Philip Alexander, 1892-1974 • 1954: (4) • $1963:(0)$ Filed under Irene Campbell • 1978: (1) • 1979: (5) • 1981: (2) Delphinium

Campbell, Catherine U. of California, Santa Barbara Correspondence $\bullet$ 1950: (4) Book collectors $\bullet 1963$ : (0) Filed under Irene Campbell

Campbell, D. H. S. Southern Forest Experiment Station, New Orleans, LA

Correspondence 1952: (1)

Campbell, Douglas Houghton (1859-1953) American plant morphologist and taxonomist; Stanford U.

Biofile $\bullet$ Bioscript $\bullet$ Botanical Album

Campbell, E. J. F.

Botanical Album 
Campbell, Howard

Correspondence $\bullet$ 1982: (9) Letter from Roger Conant • 1983: (2)

Campbell, Irene

Correspondence • 1937: (1) - 1938: (2) • 1939: (4) •

1940: (3) - 1941: (1) • 1954: (0) Filed under Berry

Campbell 1963 : (1) $\bullet$ 1978: (0) Filed under Berry

Campbell $\bullet$ 1983: (1) $\bullet$ 1985: (3) $\bullet$ 1986: (1) 1 1990: (2)

Campbell, Robert Samuel (1904-) American botanist; U. S. D. A. Southern Forest Service Station

Botanical Album

Correspondence $\bullet$ 1948: (1) $\bullet$ 1949: (2) $\bullet$ 1951: (1) $\bullet$

1953: (2) Book collectors $\bullet$ 1956: (1) • 1959: (4) • 1963: (1)

Campbell, Rosemarie Wells Colorado College, Colorado Springs, Library, Special Collections

Correspondence • 1976: (1) Rocky Mountain Naturalists

Campbell, Vera Harvard U., Gray Herbarium Correspondence $\bullet$ 1957: (2)

Campbell, W. M. U. of Colorado, Denver Correspondence • 1941: (1) • 1942: (1) See also Frances Hutchison

Canada-Trees

Botanical Album

Canadian Pulp and Paper Correspondence $\bullet$ 1956: (1)

Canberra, Australia XVI. Congressus Internationalis Ornithologicus

Correspondence $\bullet$ 1974: (1)

Canberra Botanic Gardens

Bioscript

Canby, Carol

Botanical Album

Canby, Margaret Leslie (1904-) . American botanist; Southern California Academy of Sciences

Bioscript

Canby, William Mariott (1831-1904) American botanist, plant collector, businessman

Biofile - Bioscript $\bullet$ Botanical Album

Candolle, Alphonse de (1806-1893) Swiss botanist Biofile $\bullet$ Bioscript $\bullet$ Botanical Album

Candolle, Augustine de (1778-1844)

Bioscript

Candolle, Casimir (1836-1918)

Bioscript

Canfield, C. S.

Bioscript

Canfield, Charles Adelbert

Bioscript

Canfield, Charles E. (1865-)

Bioscript

Canfield, Colbert Austin

Bioscript

Canne, Judith M. U. of Guelph, Guelph, Ont., Dept. of Botany and Genetics

Correspondence - 1979: (1)

Cannell, Peter F. American Museum of Natural History Correspondence $\bullet$ 1983: (2)

Canner, J. S. Library Booksellers

Correspondence $\bullet$ 1956: (2)
Cannon, Evelina

Bioscript

Cannon, George Lyman (1860-1922) Paleontologist, high school teacher

Bioscript

Cannon, John Francis Michael (1935- ) British botanist, taxonomist; British Museum (Natural History), Dept. of Botany, Keeper of Botany

Botanical Album

Correspondence $\bullet$ 1967: (1) $\bullet$ 1970: (2) $\bullet$ 1971: (2) •

1977: (2) • 1978: (4) Bartram, William, 1739-1823

- 1979: (1) • 1980: (4) • 1982: (1) • 1983: (1) • 1986: (1)

- 1987: (1)

Cannon, Paul Son of J. F. M. Cannon

Correspondence $\bullet$ 1970: (0) Letter filed under John Francis Michael Cannon

Cannon, W. B.

Bioscript

Cannon, William Austin (1870-1958) American botanist Bioscript • Botanical Album

Canright, James Edward (1920-) American paleobotanist, plant morphologist; Indiana U., Bloomington Correspondence 1958 : (1)

Cantelow, Ella Dales Bioscript $\bullet$ Botanical Album

Correspondence $\bullet$ 1954: (8) Eastwood, Alice, 1859-1953

Species • 1956: (6) Eastwood, Alice, 1859-1953

Cantelow, H. C.

Correspondence • 1957: (2)

Cantrell, Andrea U. of Arkansas, Fayetteville; U. Libraries, Special Collections Dept. Correspondence $\bullet$ 1989: (2)

Cantrell, Buddy U. of Alabama, Tuscaloosa, Dept. of Biology, Ecology and Systematics Section Correspondence $\bullet$ 1979: (2)

Capen, Noreen S. The Herbarist, Bedford, NY, Editor; husband is Oliver B. Capen Correspondence $\bullet$ 1959: (2)

Cappon, Lester J. Institute of Early American History and Culture, Williamsburg, VA, Director

Correspondence 1961 : (2) Banister, John, 1650-1692

Cárdenas, Hermosa Martín (1899-1973) Bolivian plant taxonomist

Botanical Album

Carey, J. Bioscript

Carey, Mathew (1760-1839) American publisher; Philadelphia, PA Biofile

Carleton, Mark Alfred (1866-1925) American plant pathologist, agronomist; Peru Bioscript

Carleton, Robert H. Science Teacher, Washington, D.C. Correspondence $\bullet$ 1960: (1)

Carlitz, Bernard Conewell Correspondence $\bullet$ 1973: (1)

Carlquist, Sherwin (1930-) American taxonomist, plant anatomist; Rancho Santa Ana Botanic Garden, Claremont, CA

Botanical Album

(continued on next page) 


\section{Guide to the Ewan Papers}

Carlquist, Sberwin (1930-) (continued)

Correspondence • 1958: (1) • 1967: (2) • 1968: (8) • 1971: (2) • 1976: (1) • 1984: (2)

Carlson, Robert E. West Chester State College, West Chester, PA Correspondence $\bullet 1982:(5)$

Cariton, E. C. Bioscript

Carlton, Jeffrey M. State of Florida, Dept. of Natural Resources Correspondence $\bullet$ 1975: (1)

Carman, B. F. Bioscript

Carman, Lincoln, Mrs. Correspondence $\bullet$ 1941: (1)

Carmichael, Gertrude Imperial College of Tropical Agriculaure, Trinidad, B.W.I. Correspondence • 1945: (3) • 1946: (2)

Carmichael, Leonard National Geographic Society, Washington, D.C., Director of Committee for Research \& Exploration Correspondence • 1962: (2)

Carnegie Book Sthop New York, NY Correspondence $\bullet$ 1951: $(0)$ filed with Edith Scamman

Carnegie Institute of Technology Botanical Album

Carnegle Museum of Natural History Correspondence • 1976: (1)

Carothers, Zame Bland (1924 ) American botanist; U. of minois, Urbana, Dept. of Botany Correspondence $\bullet 1963:$ (2)

Carpenter, Dana Stedman (1863-1940) American botanist, naturalist Botanical Album

Carpenter, Louis George (1861-1935) American meteorologist, irrigation and artesian well engineer Bioscript

Carpenter, Mathilde M. Librarian Biascript Correspondence $\bullet$ 1949: (4)

Carpenter, William C. (19th c.) Plant collector Bloscript

Carpenter, William Lewis (-1898) American entomologist on Hayden Survey Bioftle • Biascript

Carpenter, Wmliam Marbury (1811-1848) Physician and scientist: New Orleans, LA Biofile - Bloscript

Carr, Alfred B. Cinchona Section, General Cormmodities Division, Foreign Economic Administration Correspondence • 1945: (1)

Carr, Archie U. of Florida, Gainesville, Zoology Correspondence • 1984: (1) • 1985: (1)

Carr, D. J. Botanical Album

Carr, Dorothy G. Joumal of the West Correspondence $\bullet 1967$ : (2)

Carr, Jefirey U. of Leeds Correspondence $\bullet$ 1974: (1) Banister, John, 1650-1692 Carr, Mr.

Correspondence $\bullet 1950$ : (I)
Carr, Robert Bioscript

Carr, S. G. M. Australian National U., Research School of Biological Sciences Correspondence $\bullet$ 1982: (2)

Carriger, Henry Ward Bioscript

Carrington, E. C., Jr. Bioscript

Carroll, Arthur Metairie, LA Correspondence • 1969: (1)

Carroll, Dorothy G. New Orleans, LA Correspondence $\bullet$ 1958: (2) Species

Carroll, Margaret Missouri Botanical Garden, Herbarium Secretary

Correspondence • 1958: (1)

Carroll, Mary Bioscript

Carroll-Horrocks, Elizabeth American Philosophical Society, Philadelphia, PA Botanical Album Correspondence • 1983: (1) • 1992: (1) • 1993: (3)

Carruth, Gordon McGraw Hill Publishing Company Correspondence • 1967: (0) Letter filed under George Booth Van Schaack

Carruthers, William Bioscript

Carson, Joseph (1808-1876) American physician, botanist Bioscript

Carstens, Ilse (f. 1973)

Botanical Album

Carter, Alma Tulane U., New Orleans, LA Correspondence $\bullet 1954$ : (1)

Carter, Annetta Mary (1907-1991) American plant taxonomist; U. of California, Berkeley, Dept. of Botany Biofile $\bullet$ Bioscript $\bullet$ Botanical Album

Correspondence $\bullet$ 1938: (1) Setchell, William Albert, 1864-1943 • 1944: (1) Delphinium • 1946: (3) • 1947: (4) Delphinium • 1948: (2) • 1949: (4) Vismia • 1950: (5) Science-Instruction and study $\bullet$ 1951: (1) $\bullet$ 1952: (4) $\bullet$ 1953: (4) Jepson, Willis Linn, 1867-1946 • 1955: (4) • 1957: (3) • 1958: (5) • 1959: (2) - 1960: (1) - 1962: (2) 1963: (4) Banister, John, 1650-1692; Lyon, John, 1764-1814; Langman, Ida Kaplan $\bullet$ 1965: (2) 1966 : (9) 1967: (3) • 1971: (1) - 1975: (1) • 1978: (5) • 1979: (7) • 1980: (7) - 1982: (1) • 1983: (2) • 1984: (4) • 1985: (2) • 1989: (3) • 1990: (2)

Carter, Arthur N. Correspondence • 1942: (4)

Carter, C. B. Bioscript

Carter, Charles H. Tulane U., Dept. of History, Professor of History Correspondence $\bullet$ 1976: (2) $\bullet$ 1977: (1)

Carter, Edward C., II (Ted) Librarian; American Philosophical Society, Papers of Benjamin Henry Latrobe Correspondence • 1974: (2) • 1975: (11) • 1976: (1) • 1977: (6) - 1980: (3) Banks, Joseph, Sir, 1743-1800 1982: (1) • 1984: (2) • 1985: (1) • 1986: (1) • 1987: (3) • 1988: (4) • 1991: (5) • 1992: (2) • 1993: (1) 
Carter, Edwin (1812-1900) American gold-miner and amateur naturalist Biofile - Botanical Album

Carter, George F. Johns Hopkins U., Dept. of Geography Correspondence $\bullet$ 1950: (5) $\bullet$ 1953: (2)

Carter, Harold B. Yeo Bank, Congresbury, England; British Museum (Natural History), London, England, also from Bristol, England

Correspondence • 1979: (1) Banks, Joseph, Sir, 1743-1800 - 1989: (2)

Carter, Harry, Mrs. Correspondence • 1929-1933: (1)

Carter, Joel Jackson (1843-1912) Plant collector Botanical Album

Carter, Richard Vanderbilt U., Dept. of General Biology; Valdosta State College, GA, Dept. of Biology, Curator of the Herbarium, Professor Correspondence $\bullet$ 1980: (1) $\bullet$ 1990: (4) • 1991: (6)

Cartes, A. Bioscript

Cartwright, George Bioscript

Caruel, T. Bioscript

Carus, J. V. Bioscript

Caruso, John Correspondence $\bullet$ 1986: (2)

Carver, George Washington (1864-1943) American educator, plant breeder, taxonomist Bioscript $\bullet$ Botanical Album

Carver, Jonathan (1732-1780) Bioscript

Carver, William W. Stanford U. Press, Executive Editor Correspondence - 1977: (2) Correspondence with D. E. Stone • 1979: (2) Rocky Mountain Naturalists; letter to R.W. Kiger

Cary, John Bioscript

Cary, Merritt (1880-1918) Mammalogist Bioscript

Case, Eliphalet Lewis (1843-1925) American amateur botanist Biofile

Case, F. M. Bioscript

Casey, J. P. Bioscript

Cashner, Robert C. U. of New Orleans, Dept. of Biological Sciences Correspondence $\bullet$ 1976: (1)

Caspary, R. Bioscript

Cass, Charles L. San Diego, CA Correspondence $\bullet$ 1951: (5)

Cassedy, James H. National Library of Medicine, History of Medicine Division Correspondence • 1973: (3) • 1985: (2) • 1988: (2) Barton, Benjamin Smith, 1766-1815
Cassidy, Frederic G. Dictionary of American Regional English, Director-Ed.; U. Wisconsin, Madison, Dept. of English

Correspondence • 1979: (2)

Cassidy, James (1844-1889) Agronomist Bioscript

Cassie, Brian Ibis Books Correspondence $\bullet$ 1984: (1)

Cassin, John (1813-) American ornithologist Bioscript

Cassini, A. H. Bioscript

Cassino, Harold L. Publisher Naturalists' Directory Correspondence $\bullet$ 1956: (1)

Cassino, Samuel Edson Bioscript

Castagne, $\mathbf{L}$. Bioscript

Castagnetti, John P. Correspondence - 1975: (1)

Castañeda, Manuel Dept. of Botany, Mexico Correspondence - 1940: (1)

Castellaños, Alberto (1896-1968) Argentine botanist; Fundación Miguel Lillo, Buenos Aires, Argentina Correspondence • 1945: (1) • 1946: (2) • 1948: (1) • 1952: (1)

Castelnau, Bioscript

Castetter, Edward Franklin (1896-1978) American ethnobotanist; U. of New Mexico, Albuquerque, Head of Dept. of Biology

Bioscript

Correspondence • 1949: (3)

Castiglioni, Luigi (1757-1832) Italian botanist Biofile $\bullet$ Bioscript

Castracane Degli, A. F. Bioscript

Caswell Orchid Garden Botanical Album

Catanzariti, John Princeton U., Library; The Papers of Thomas Jefferson Correspondence $\bullet$ 1988: (0) Filed under Jefferson, Thomas, Papers of

Catesby, Mark (1682-1749) English naturalist in America Biofile $\bullet$ Bioscript

Cathcart, Nancy Duane Correspondence • 1975: (2) Bartram, William, 1739-1823

Catlett, Stephen American Philosophical Society, Assistant Manuscripts Librarian

Correspondence • 1978: (0) Filed under American Philosophical Society $\bullet$ 1982: (2) 1 1984: (1) Barton, Benjamin Smith, 1766-1815 • 1986: (1)

Catley, $\mathbf{H}$. Bioscript

Catlin, George (1796-) American portrait painter Biofile $\bullet$ Bioscript

Cattell, Jacques American Men of Science, Editor Botanical Album Correspondence - 1940: (6) American Fern Society; letters to Charles A. Weatherby $\bullet$ 1949: (1) $\bullet$ 1950: (1) 


\section{Guide to the Ewan Papers}

Cattell, James McKeen (1860-1944) American psychologist, scientist

Biofile

Correspondence $\bullet 1938:(2) \bullet 1941:(2)$

Cavanilles, A. J.

Bioscript

Caxton Printers, Ltd. Caldwell, ID; Gordon Gipson, publisher

Correspondence • 1980: (1) Rocky Mountain Naturalists

Cayler, R. L. Dela State Teachers College, Cleveland, MS

Correspondence • 1950: (1) $\bullet$ 1951: (1)

Cayouette, Richard (1914-1993) Canadian agronomist and botanist

Biofile

Correspondence $\bullet 1970:(1)$

Celarier, Robert P. (1921-1959) A \& M College of Texas, College Station, TX, Genetics Dept.

Correspondence $\bullet 1951$ : (2)

Celis, John

Correspondence $\bullet$ 1990: (2)

Cels, $\mathbf{F}$.

Bioscript

Center for Field Research Watertown, MA; Earthwatch Correspondence • 1987: (1) • 1989: (1)

Cerf, ___ Miss Louisiana State Museum, New Orleans, Librarian

Correspondence • 1949: (1)

Ceroni, Luigi (1883-1951) Italian botanist Biofile

Cervantes, $\mathbf{v}$. Bioscript

Cesati, V. Bioscript

Chabran, Rafael Louisana State U., Baton Rouge; Whirtier College, Whittier, CA Correspondence • 1984: (11) • 1985: (6) • 1986: (2) • 1987: (1)

Chace, Fenner Albert, Jr. Zoologist; Smithsonian Correspondence $\bullet$ 1965: (1)

Chadbourne, Paul Ansel (1823-1883) American naturalist, theologian

Bioscript

Chadwich, Mr. \& Mrs.

Bioscript

Chaillet, Jean Frédérick (1749-1839) Swiss soldier and plant collector

Bioscript

Chaix, Dominique (1730-1839)

Bioscript

Chalmers, Lionel

Bioscript

Chalmers-Hunt, J. M. West Wickham, Kent, England Correspondence $\bullet 1977:$ (2)

Chamberlain, Charles Joseph (1863-1943) American plant morphologist and cytologist Botanical Album Chamberlain, Frederic Morton (1867-1921) American
fishery biologist Biofile
Chamberlin, Ralph Very (1879-) American zoologist Bioscript

Chambers, Douglas Trinity College, Toronto, Ontario, Canada

Correspondence • 1989: (2) $\bullet$ 1990: (3)

Chambers, John Southwestern Louisiana U., Lafayette Correspondence $\bullet$ 1968: (2)

Chambers, Kenton Lee (1929-) American taxonomist; Oregon State U., Corvallis, Herbarium, Curator Correspondence • 1981: (5) Pursh, Frederick, 1774-1820 Species

Chambers, Lois Cooper Ornithological Club See also Lois Chambers Stone Correspondence $\bullet$ 1929-1933: (1)

Chambers, Willie Lee Cooper Ornithological Club Bioscript

Correspondence • 1936: (3) • 1950: (2) • 1952: (1) • 1954: (1) • 1962: (3)

Chamisso, Adelbert Ludwig von See Chamisso, Louis Charles Adelaide (1781-1838)

Chamisso, Louis Charles Adelaide (1781-1838) Frenchborn poet and naturalist in Germany

Biofile $\bullet$ Bioscript

Champagn, G. L. Correspondence $\bullet$ 1953: (1)

Champion, H. G. Botanical Album

Champneys, Benjamin (1774-1814) Bioscript

Chancellor, John Paget Kew Book Bioscript $\bullet$ Botanical Album

Correspondence • 1973: • 1974: (3) • 1975: (1) • 1977:

(1) Letter from J. Ewan with postscript for George Gifford - 1980: (5) Book collectors - 1981: (1) - 1989: (4) Book collectors - 1990: (3)

Chandler, Albert

Correspondence • 1946: (1)

Chandler, Harley Pierce (1875-1918) American taxonomist Bioscript

Chandra, Lokesh Advancing Frontiers of Plant Sciences, Editor

Correspondence • 1968: (1)

Chandra, Sudhire Kumann U., India, Botany Dept. Correspondence $\bullet$ 1983: (1)

Chaney, Ralph Works (1890-) American geologist, paleobotanist; U. of California, Berkeley, Dept. of Paleontology

Bioscript $\bullet$ Botanical Album

Correspondence $\bullet$ 1937: (5)

1955: (1) Chang, Leslie Villard Books (div. of Random House), New
York, NY

Correspondence $\bullet$ 1992: (0) Filed under Peter Bernhardt

Channell, Robert Bennie (1924) American taxonomist; Vanderbilt U., Dept. of Biology

Correspondnce $\bullet$ 1958: (1) 1959: (3) • 1960: (1)

1961: (5) • 1962: (6) • 1963: (2) • 1964: (5) $\bullet$ 1966: (1)

1967: (6) • 1969: (1) • 1973: (1) Channing, Edward Tyrrel (1791-1856) American

Biofile 
Channing, Walter (1786-1876)

Bioscript

Chapin, Edward A. National Museum, Division of Insects Correspondence • 1954: (1)

Chapin, James Paul (1889-)

Bioscript

Chapline, William Ridgely (1891-1956) American forester Biofile • Bioscript

Chapman, Alvan Wentworth (1809-1899) American physician, botanist, plant collector; Florida

Biofile $\bullet$ Bioscript $\bullet$ Botanical Album

Chapman, Frank Michler (1864-1945) American ornithologist; American Museum of Natural History, Curator-in-Chief

Bioscript $\bullet$ Botanical Album

Correspondence $\bullet$ 1929-1933: (1)

Chapman, Nathaniel Bioscript

Chapman, Russell Leonard Louisiana State U., Baton Rouge, Dept. of Botany

Botanical Album

Correspondence $\bullet$ 1981: (1) $\bullet$ 1992: (3)

Chappe d'Auteroche

Bioscript

Chardon, Carlos Eugenio

Botanical Album

Charette, Leopold A. U. S. Air Force, Staff Sergeant Correspondence - 1951: (6) Pursh, Frederick, 1774-1820 • 1952: (2) • 1953: (3) Delphinium; Pursh, Frederick, $1774-1820$

Charles Scribner's Sons Publishers Correspondence • 1977: (1) - 1978: (2)

Charleston Tea Plantation Charleston, SC Correspondence $\bullet$ 1992: (3)

Chase, Elizabeth W. J. Philadelphia College of Pharmacy and Science, Philadelphia, PA

Correspondence • 1972: (2)

Chase, J. Smeaton

Bioscript

Chase, Laura

Correspondence $\bullet$ 1989: (1)

Chase, Mary Agnes (Merrill) (1869-1963) American agrostologist; Smithsonian Institution

Biofile $\bullet$ Bioscript $\bullet$ Botanical Album

Correspondence • 1929-1933: (1) - 1937: (1) - 1938: (5) • 1941: (1) • 1942: (2) • 1943: (9) • 1949: (8) • 1951: (4) • 1952: (3) • 1956: (2) • 1958: (4)

Chase, Virginius Heber (1876-1966) American naturalist and $R R$ telegrapher; Peoria Academy of Science, IL Biofile • Botanical Album

Correspondence $\bullet$ 1944: (1) $\bullet$ 1945: (3) $\bullet$ 1946: (6) $\bullet$ 1947: (3) • 1948: (3) • 1949: (2) • 1950: (6)

Chastellux, Francois-Jean, Marquis de (1734-1788) Bioscript

Chater, Arthur Oliver (1933-) Scottish-born taxonomist in England

Correspondence • 1983: (1)

Chatin, A.

Bioscript
Chatters, Roy Milton (1908-) Washington State U., Pullman

Correspondence $\bullet$ 1966: (2)

Chavannes, E. Bioscript

Chaw, Shu-Miaw (1954) Academia Sinica, Institute of Botany

Botanical Album

Correspondence $\bullet$ 1986: (1)

Cheatham, Norden (Dan) U. of California, Berkeley Correspondence $\bullet$ 1974: (1)

Cheeseman, Thomas Frederic (1846-1923) British-born New Zealand botanist

Bioscript

Cheesman, Ernest Entwisle (1898-) East Malling Research Station, Kent, England, formerly of Imperial College of Tropical Agriculture, Trinidad Correspondence • 1949: (2)

Cheesman, Lucy Evelyn (1881-1969) English entomologist and explorer Biofile $\bullet$ Botanical Album

Chenery, E. M. Covington, LA Correspondence • 1981: (1)

Cheney, Donald P. (1945-) U. of South Florida, Tampa, Dept. of Biology Correspondence $\bullet$ 1974: (1)

Cheng, Hsien-yu Correspondence • 1981: (1)

Chenoweth, Mary North Somers Point, NH Correspondence $\bullet$ 1980: (3)

Cherry, Hilde Correspondence $\bullet$ 1974: (1)

Chesnick, Barney Ridgway Library, Philadelphia, PA Correspondence $\bullet$ 1950: (2)

Chessid, Estelle The Book Chest Correspondence • 1976: (2) • 1977: (1) • 1985: (1)

Chester, Edward W. U. of Tennessee, Knoxville Correspondence • 1965: (2) • 1966: (1) • 1968: (1)

Chester, Kenneth Starr (1906?-) American plant pathologist Biofile

Chestnut, Victor King (1867-1938) American biochemist, botanist Bioscript

Cheston, Edward M. Botanical Album

Cheston, Emily Read ( -1982) Ambler, PA Bioscript Correspondence $\bullet$ 1972: (2)

- 1973: (11) Harper, Francis, 1886-1972; Bartram, William, 1739-1823 • 1974: (5) • 1975: (2) • 1976: (3) • 1977: (1) - 1980: (1)

Chiappelli, Fredi U. of California, Berkeley, Center for Medieval and Renaissance Studies Correspondence $\bullet$ 1973: (4) • 1974: (9) • 1975: (5) Copy of corres. with Thomas W. Whitaker $\bullet$ 1976: (1)

Chicago Academy of Sciences Librarian Correspondence 1950 : (1)

Chicago Museum of Natural History Director Correspondence • 1951: (1) • 1959: (1) Vismia 


\section{Guide to the Ewan Papers}

Chickering, J. W.

Bioscript

Child, H. S.

Biascript

Children, John George (17m-1852)

Bioscript

Childs, Heary E. Cerritos Junior College, Norwalk, CA

Correspondence $\bullet 1958$ : (3)

Childs, John Lewis (1856-1921)

Bloscript • Botanical Album

Childs, L. J

Bloscript

Childs, Martha A. U. of Georgia, Athens, Herbarium Asst.

Correspondence $\bullet$ 1971: (4)

Childs, Stacy J. Tulane U., New Orleans, student Correspondence • 1968: (1)

Chiltoskey, Mary

Correspondence $\bullet 1978:$ (1)

Chinard, Gilbert (1881-1972)

Biofile

Correspondence • 1957: (2) • 1958: (7) • 1959: (4) •

1960: (3) Tulane U., Dept. of Botany $\bullet$ 1967: (1)

Chipman, Robert K. American zoologist; nickname "Chip" Correspondence • 1959: (1) • 1960: (1) • 1961: (2) • 1963: (2) • 1970: (4) • 1971: (4) • 1975: (2)

Chippendall, L. K. A.

Botenical Album

Chisbolm, Carol H.

Correspondence • 1976: (0) Filed under American Institute of Biological Sciences

Chisholm, Maude Louise (1872-) American botanist, pteridologist

Correspondence • 1946: (1)

Chittenden, Frank Hurlbut (1958-1929) American coleopterist

Bigfile • Bioscript

Chittenden, Russell Fenry (1856-1943) American biochemist

Biofile • Bioscript

Chodat, Robert (1885-1934)

Bioscript

Choisy, J. D.

Bioscript

Choisy, Jacques Denis (1799-1859) Swiss philosopher, clergyman, botanist

Botanical Album

Chourard, Pierre (1903-1983) Conservatoire National des Arts et Metiers, Paris

Correspondence • 1953: (1) • 1954: (1)

Christ, Arthur (1906-1991) American amateur botanist, science teacher

Correspondence • 1946: (1)

Christ, Hermann

Botanical Album

Christ, John Heary (1896-). Plant collector of Idaho;
Portland, OR

Biofile • Bioscript
Christ, John Henry (1896-) (continued) Correspondence • 1937: (3) - 1945: (2) - 1946: (2) $\bullet$ 1947: (6) Delphinium • 1948: (1) • 1949: (4) Delphinium - 1950: (1)

Christensen, Carl Frederik Albert (1872-1942) Danish botanist

Biofile $\bullet$ Bioscript $\bullet$ Botanical Album

Christian Science Monitor Book Review Editor

Correspondence • 1943: (1)

Christiansen, M. Skytte Botanisk Centralbibliotek, Denmark

Correspondence $\bullet$ 1956: (2)

Christie, Manson \& Woods International New York, NY, Stephen C. Massey

Correspondence • 1977: (1)

Christy, John A. Milwaukee Public Museum, Asst.

Curator of Botany

Correspondence • 1981: (1) • 1982: (1) • 1989: (2)

Christy, W.

Bioscript

Chrysler, Mintin Asbury (1871-1963) Torrey Botanical Club; Rutgers U.

Botanical Album

Correspondence - 1935: (6) Delphinium • 1936: (6)

Delphinium • 1937: (5) • 1946: (6)

Chung, Young-Cheul

Botanical Album

Correspondence $\bullet$ 1992: (2)

Church, John Camphor

Bioscript

Churchill, David Maughan (1933-) Botanist; Royal Botanic Gardens and National Herbarium, Victoria, Australia

Correspondence $\bullet$ 1979: (1)

Churchill, Joseph Richmond (1845-1933) American judge Bioscript

Churgin, Sylvia Librarian; Smithsonian Tropical Research Institute

Correspondence • 1985: (4)

Cibula, William Q.

Correspondence $\bullet$ 1979: (0) New Orleans Mycological

Society Newsletter, June 1979

Ciencias Museum (Museo de Ciencias Naturales) Caracas, Venezuela

Correspondence • 1951: (1) $\bullet$ 1953: (1)

Cirillo, Domenico Mana Leoni (1739-1799) Italian statesman and botanist

Bioscript

Cirker, Hayward Dover Publications, President

Correspondence $\bullet$ 1973: (0) Letter filed under George Booth Van Schaack • 1976: (2)

Cisso, C.

Bioscript

Citizen Ambassador Program Spokane, WA Correspondence • 1992: (2)

Clancy, Keith

Correspondence • 1992: (1)

Clapp, A.

Bioscript

Clarion, J.

Bioscript 
Clark, Correspondence • 1938: (1) Cockerell, Theodore Dru Alison, 1866-1948

Clark, Andrew H. (1911-1975) Canadian-born geographer Biofile

Clark, Arthur B., Company Publishers \& Booksellers, Glendale, CA; Robert A. Clark Correspondence - 1980: (2) Rocky Mountain Naturalists

Clark, Arthur H., Company Correspondence $\bullet$ 1945: (3)

Clark, Austin Hobart (1880-1954) American lepidopterist Biofile

Clark, Grace Correspondence $\bullet$ 1979: (1) $\bullet$ 1981: (0) Filed under Margaret Boetjer $\bullet$ 1982: (1) $\bullet$ 1984: (2)

Clark, H. Jack Correspondence $\bullet$ 1974: (1)

Clark, Henry James Bioscript

Clark, Howard Lamar (1941- ) American taxonomist, botanist Correspondence $\bullet$ 1968: (3) $\bullet$ 1970: (2) $\bullet$ 1972: (4) Most letters from wife Katherine $\bullet$ 1973: (1) $\bullet$ 1977: (3) $\bullet$ 1990: (1)

Clark, Howard Walton (1870-1941) Bioscript

Clark, John Henry (1830- ) Naturalist, surveyor Bioscript

Clark, Joseph Bioscript

Clark, Kim Correspondence $\bullet$ 1943: (1)

Clark, Leila F. Smithsonian, Librarian Correspondence $\bullet$ 1947: (3)

Clark, Lovell U. of New Brunswick, Fredericton Correspondence $\bullet$ 1963: (1)

Clark, Neata Bioscript

Clark, Pauline P. St. Louis County Library Correspondence $\bullet$ 1952: (2)

Clark, Walter Bosworth Bioscript

Clark, William (1770-1838) American statesman, botanical explorer, plant collector Bioscript

Clark, William Bullock (1860-1917) American geologist, paleontologist

Bioscript

Clarke, Alfred (1848-1925) British chemist and mycologist Biofile

Clarke, Benjamin (1813-1890) British botanist Bioscript

Clarke, Borden Book dealer Correspondence • 1948: (1)

Clarke, Charles Baron (1832-1906) English botanist Botanical Album

Clarke, F. L. Bioscript

Clarke, George Bioscript
Clarke, L. Floyd U. of Wyoming, Laramie; Head, Dept. of Zoology and Physiology

Correspondence • 1959; (1)

Clary, Marjorie D.

Correspondence - 1929-1933: (16) Delphinium; U. of California, Berkeley. Dept. of Botany $\bullet$ 1935: (2) $\bullet 1936$ : (1) $\bullet$ 1949: (1)

Clary, R. E., Capt. Bioscript

Classey, E. W. Bookseller and publisher of natural history Correspondence • 1964: (4) • 1965: (2) • 1978: (1)

Clausen, Jens Christian (1891-1969) Danish-born American taxonomist; Carnegie Institution of Washington; Stanford U., Dept. of Plant Biology

Botanical Album

Correspondence • 1939: (1) 1957: (6)

Clausen, Robert Theodore (1911-1981) American botanist; Cornell U., New York State College of Agriculture, Ithaca, NY

Botanical Album

Correspondence • 1938: (1) $\bullet$ 1939: (7) American Fern Society $\bullet$ 1940: (14) American Fern Society $\bullet$ 1941: (11) American Fern Society $\bullet$ 1942: (7) American Fern Society - 1944: (1) • 1951: (3) • 1952: (2) • 1958: (1)

Claussen, $\mathbf{P}$. Bioscript

Clayton, John Botanical Album

Clayton, John (1686-1773) Clerk, Gloucester Co., VA Bioscript

Clayton, John (16941773) British-born American botanist and physician Biofile

Clayton, John, Rev. (1657-1725) Rector of Jamestown Biofile $\bullet$ Bioscript

Cleaton, Joe Mary Botanical Society of New Orleans, Secretary

Correspondence $\bullet$ 1952: (1)

Cleaver, Isaac Bioscript

Cleburne, William Collector of plants and fossils Bioscript

Cleland, Ralph Erskine (1892-1971) American cytologist, plant geneticist Correspondence • 1947: (1) • 1976: (5)

Clemenceau, Pierre Horticultural Council, Chairman Steering Committee Correspondence $\bullet$ 1961: (2)

Clemens, Joseph Strong (1862-1936) English-born American plant collector Botanical Album

Clemens, Mary Knapp Strong (1873-1968) American-born plant collector; husband is Joseph Strong Clemens Bioscript • Botanical Album (Filed under Joseph Strong Clemens)

Clements, Edith Gertrude (nee Schwartz) (1877- ) American ecologist, botanical artist; husband is Frederic Edward Clements Bioscript - Botanical Album Correspondence - 1946: (3) U. of Colorado, Boulder Dept. of Biology $\bullet$ 1949: (3) $\bullet$ 1953: (5) Tulane U., Dept. 
Clements, Edith Gertrude (nee Schwartz) (1877-) (continued)

of Botany "Rocky Mountain Vegetation" - 1955:(1) •

1956: (1) • 1957: (1) • 1958: (1) • 1960: (11) Cockerell,

Theodore Dru Alison, 1866-1948; La Jolla, CA stationary pictures her home $\bullet$ 1961: (29) $\bullet$ 1963: (3) $\bullet 1964$ : (4) $\bullet$ 1965: (7) • 1966: (9) • 1967: (3) One letter filed under Gene M. Gressley $\bullet$ 1968: (1) Letter written by C. Johns for Edith Clements

Clements, Frederic Edward (1874-1945) American biologist, plant ecologist, conservationist Biofile • Bioscript • Botanical Album Correspondence • 1939: (1) • 1942: (4) • 1943: (7)

Clements Library U. of Michigan, Ann Arbor Correspondence $\bullet 1977:$ (2)

Cleveland, Daniel (1838-1929) American lawyer, plant collector Bloscript • Botanical Album

Cleveland, Parker

Bioscript

Clewell, André François (1934) American taxonomist; Florida State U., Tallahassee, Dept. of Biological Science Bioscript Correspondence • 1963: (2) • 1968: (2)

Cinflord, Bloscript (friend of Rafinesque)

Cliflord, George Bioscript • Botanical Album

Clinton, DeWitt Bioseript

Clinton, George W. Biofile

Clive, Robert Bioscript

Clokey, Ira Waddell (1879-1950) American mining engineer, botanist, plant collector Biofile $\bullet$ Bioscript $\bullet$ Botanical Album Correspondence $\bullet$ 1935: (2) • 1936: (2) • 1938: (3) • 1939: (4) • 1941: (2) • 1945: (2) • 1949: (3)

Clokie, Hermia Newman Oxford U., Dept. of Botany Bloscript • Botanical Album Correspondence $\bullet$ 1954: (2) • 1955: (8) • 1956: (9) • 1957: (2) • 1958: (2) $\bullet 1961:$ (1) $\bullet$ 1964: (3) $\bullet 1965:$ (3) 1966: (1) $\bullet$ 1969: (2) $\bullet$ 1971: (3) $\bullet 1975:$ (1) $\bullet 1979:$ (1) 1981: (8) • 1982: (1) • 1985: (1) $\bullet$ 1986: (1) $\bullet$ 1987: (1) 1989: (1) $\bullet$ 1992: (2) $\bullet$ 1993: (1)

Clokite, James Winchester, England Correspondence • 1977: (3) Letters with Hermie Clokie Chusius, Carolus (1526-1609) Biofile • Bioscript

Clute, Willard Nelson (1869-1950) American botanical author, pteridologist Biofile • Bloscript Correspondence $\bullet$ 1929-1933: (2)

Clutton, George, Sir British historian; London, England Correspondence $\bullet$ 1969: (2) $\bullet 1970$ : (5)

Coats, Alice Margaret (1905-1978) English author; Birmingham, England English horticultural Bioscript • Botanical Album

Correspondence $\bullet 1964:(2) \bullet 1965:(3) \bullet 1966:(6) \bullet$
$1969:(9) \bullet 1970:(10) \bullet 1971:(6) \bullet 1972:(6)$
Coats, Alice Margaret (1905-1978) (continued) Benjamin Smith, 1766-1815 • 1973: (6) • 1974: (5) • 1975: (2) • 1976: (2) • 1977: (10) • 1978: (4)

Cobb, Theodore R. R. Bioscript

Cobb, Verma Correspondence • 1942: (1)

Cobbett, William Bioscript

Cochran, Phil St. Norbert College, De Pere, WI, Division of Natural Sciences Correspondence $\bullet$ 1990: (1)

Cockayne, Leonard (1855-1934) English-born botanist of New Zealand Biofile $\bullet$ Bioscript

Cocke, Charles Bioscript

Cocke, J. H. Bioscript

Cocke, James Bioscript

Cocke, John Bioscript

Cockerell, Douglas (1870-1945) English bookbinder Biofile $\bullet$ Bioscript

Cockerell, Theodore Dru Alison (1866-1948) English-born American naturalist

Biofile $\bullet$ Bioscript $\bullet$ Botanical Album

Correspondence $\bullet$ 1938: (18) Letter from LeRoy Abrams - 1939: (3) Eastwood, Alice, 1859-1953; letter from T. D. A. Cockrell to George T. Moore of the Missouri Botanical Garden; letter to T. D. A. Cockrell from LeRoy E. Detling - 1940: (4) • 1941: (15) • 1942: (15) • 1943: (17) • 1945: (6) Delphinium $1946:$ (7)

Cockerell, Wilmatte Porter (1871-1957) Naturalist, teacher, public lecturer; Mary Holmes Junior College, MI; husband is T. D. A. Cockerell Bioscript $\bullet$ Botanical Album

Correspondence $\bullet$ 1938: (8) $\bullet$ 1944: (1) $\bullet$ 1945: (1) $\bullet$ 1947: (2) • 1948: (10) • 1949: (5) Species $\bullet$ 1950: (4) • 1951: (5) - 1952: (8) $\bullet$ 1953: (6) $\bullet$ 1954: (18) Cockerell, Theodore Dru Alison, 1866-1948

Cocks, Reginald Somers (1863-1926) British botanist, taxonomist

Biofile $\bullet$ Bioscript $\bullet$ Botanical Album

Codrescu, Andrei Author Correspondence $\bullet$ 1989: (1)

Cody, William James (1922-) Canadian pteridologist; taxonomist; Dept. of Agriculture, Division of Botany and Plant Pathology, Ottawa, Ontario, Canada Correspondence • 1952: (3) Delphinium • 1966: (1)

Coelln, John von Bioscript Cogniaux, Célestin Alfred (1841-1916) Belgian botanist
Biofile $\bullet$ Bioscript

Cohen, Barry Mendel U. of Texas-Austin; biographer of Nikolai Ivanovich Vavilov Correspondence $\bullet$ 1987: (2) 
Cohen, I. Bernard Harvard U., Cambridge, Dept. of History of Science, Professor

Bioscript

Correspondence $\bullet$ 1972: (4) • 1974: (2) • 1984: (1)

Cohen, Joseph Newcomb College

Correspondence $\bullet$ 1975: (1) $\bullet$ 1983: (1)

Cohen, L. Argosy Book Stores Correspondence - 1956: (3) Barton, Benjamin Smith, 1766-1815

Cohn, Essie White Colorado-Wyoming Academy of Science Correspondence • 1938: (3) • 1941: (3)

Cohn, Ferdinand Bioscript

Cohn, Pauline Bioscript

Coiter, Volcher (1534-1576) Dutch anatomist, ornithologist Biofile

Coker, William Chambers (1872-1953) American plant morphologist, mycologist; U. of North Carolina, Chapel Hill, Dept. of Botany

Bioscript $\bullet$ Botanical Album

Correspondence • 1940: (3) • 1949: (3)

Colby, William E.

Botanical Album

Colden, Cadwallader (1668-1776) American botanist, statesman

Biofile • Bioscript

Colden, Jane (1724-1766) American botanist

Biofile • Bioscript

Correspondence 1963: (1) Colden Botanic Mss.

Cole, F. J. Bioscript

Cole, Fred Tulane U., New Orleans, College of Arts and Sciences, Dean

Correspondence $\bullet$ 1947: (6) $\bullet$ 1948: (5) • 1949: (2) $\bullet$ 1950: (4) • 1951: (4) • 1952: (4) • 1953: (1) - 1954: (1) • 1957: (1) • 1958: (1) • 1977: (2)

Cole, Fred C. (1912?-1986) American historian Biofile

Cole, Lamont C.

Botanical Album

Coleman, Arthur Philemon (1852-1939) Canadian geologist Bioscript

Coleman, Bob Bob Coleman photography, New Orleans; took photos of Ewans for promotion of book on John

Banister

Correspondence • 1970: (3)

Coleman Company Wichita Falls, KS Correspondence $\bullet$ 1949: (1)

Coleman, Eunice Fine Arts Club, New Orleans Correspondence $\bullet$ 1963: (1)

Coleman, James Robert (1934 ) U. of Georgia, Dept. of Biology Correspondence $\bullet$ 1973: (1)

Colenso, William Bioscript

Colglayer, Merle Correspondence • 1942: (1)

Colla, Luigi Bioscript
Collander, Paul Runar (1894 ) Helsinki, Kauppiaank; author of History of Botany in Finland 1828-1918

Botanical Album

Correspondence • 1969: (1)

College of Physicians of Philadelphia Correspondence $\bullet$ 1983: (2)

College of William and Mary Botanical Album

Correspondence $\bullet$ 1972: (12) Martha B. Armstrong and Thomas A. Graves, Jr., President • 1973: (1) • 1974: (2) • 1976: (1) • 1980: (3) - 1981: (1) • 1982: (1) • 1983: (1) John D. Haskell, Jr. Assoc. Librarian, Earl Gregg Swem Library • 1985: (1)

Collie, Alexander Bioscript

Collignon, Jean Nicholas (1762-1788) Bioscript

Collin, Nicholas Swedish-born American Lutheran pastor Biofile

Collin, Patsy Correspondence $\bullet$ 1958: (1)

Collins, Joseph L. U. of Tennessee, Knoxville, Dept. of Botany Correspondence • 1981: (2) • 1982: (1)

Collins, $\mathbf{L}$. Botanical Album

Collins, Timothy P. J. U. College, Galway, Eire, Library Correspondence • 1979: (1) • 1982: (1) • 1986: (2)

Collins, Zaccheus Bioscript

Collinson, Peter (1694-1768) English botanist Biofile $\bullet$ Bioscript

Collis, R. M. B. F. Stevens \& Brown Ltd., London, booksellers Correspondence $\bullet$ 1960: (1)

Colmeiro, Miguel Don (1816-1901) Spanish botanist Bioscript

Colonial Williamsburg Correspondence • 1960: (2) Lynette Adcock, Research Archivist \& Thad W. Thwaite, Assistant Director for Research - 1973: (8)

Colorado Agricultural College Correspondence $\bullet$ 1941: (1)

Colorado Associated U. Press U. of Colorado, Boulder; John T. Schwartz Correspondence • 1976: (1) Rocky Mountain Naturalists • 1977: (5) Rocky Mountain Naturalists • 1983: (1)

Colorado College Colorado Springs, CO Correspondence • 1973: (2) • 1976: (1) • 1980: (1)

Colorado Magazine State Historical Society, Denver; Cathryne Johnson, Ed.

Correspondence • 1977: (4) Cockerell, Theodore Dru Alison, 1866-1948

Colorado Mountain Club Correspondence • 1943: (1)

Colorado State U., Fort Collins Thelma J. Potts Correspondence • 1976: (1)

Colton, Kenneth E. Des Moines, IA, Dept. of History and Archives

Correspondence $\bullet$ 1941: (2) • 1942: (1) 


\section{Guide to the Ewan Papers}

\section{Columbia University Press}

Corrempondence • 1980: (1) Vicki P. Raeburn, Associate Erecutive Editor - 1992: (1) Edward E. Lugenbeel

Columbus, Christopher Italian explorer Blofile

Cohwell, Robert

Correspondence $\bullet 1984$ :

Coman, Edwin T. U. of California, Riverside, Library Correspondence • 1965: (0) Letters filed under George Booth Van Schaack

Combe, David A. Tulane U. Law Librarian and Professor of Law; member of New Orleans Book Collectors Cornespondence • 1987: (0) Filed under New Orieans Book Collectors Club

Comean, Nancy

Correspondence $\bullet$ 1957: (1)

Comean, Yasmin S. National Herbarium of Trinidad and

Tobago, Dept. of Plant Science and Biochernistry

Correppondence • 1985: (1) • 1986: (1)

Commerford, L. E. U. S. National Museum, Washington,

D.C., Cuief of Division of Publications

Correppondence • 1949: (I)

Commersen, $\mathbf{P}$.

Bloscript

Commoner, Barry American ecologist; American Assoc. for the Advancement of Sciences

Biascript

Correspondence $\bullet$ 1956: (2)

Commons, Albert

Bloscript • Botanical Album

Compton, Heary (1632-1717) English clergyman, author, berticulturist

Bioscript

Compton, Robert Harold (1866-) English-born South African botanist

Bloscript

Comstock, Anna Botsford (1854-1930) Bloscript

Comstock, John Adams (1833-1970) American entomologist; Los Angeles County Museum of History. Science and Art; Southern California Academy of Sciences Biofile Correspondence • 1935: (6) • 1941: (3) • 1942: (7) •
1949: (5)

Comstock, John Henry (1849-1931) Bioscript

Cometock, John Lee Bloscript

Comstock, Theodore Bryant (1849-1915) Bloscript

Conant, Roger Philadelphia Zoological Garden Correppondence • 1964: (2) • 1965: (3) • 1978: (1) 1982: (0) letters filed under Howard Campbell $\bullet$ 1983: (2)

Conard, Henry Shoemaker (1874-1971) American botanist; Grinnell College Biofle - Bioscript - Botenical Album Correspondence • 1939: (1) Conbeer, Geraldine State Teachers College, West Chester,
PA, Assistant Librarian Correspondence • 1951: (1)
Conde, José Alvarez

Bioscript

Condit, Ira Judson (1883- ) American horticulturist, botanist Bioscript

Condor

Correspondence • 1966: (1) James R. King, Editor $\bullet 1983$ :

(2) Peter Stettenheim

Cone, Keith G. General Commodities Division, Cinchona Section

Correspondence $\bullet$ 1944: (1)

Congdon, Joseph Whipple (1834-1910) Plant collector Bioscript

Conklin, Edwin G. Princeton U., American Philosophical Society

Correspondence $\bullet$ 1942: (5)

Conn, Harold Joel (1886-) American botanist

Correspondence $\bullet$ 1973: (2)

Connell, Dixon Louisiana State U., Alexandria Correspondence $\bullet$ 1962: (1)

Connolly, Glory Wade Bookseller; Connolly and Wade

Correspondence • 1991: (2)

Connolly, Henry

Bioscript

Conover, Samuel Forman

Bioscript

Conrad, John \& Co.

Bioscript

Conrad, Salomon [sic] White Bioscript

Conrad, Timothy A. Bioscript

Conradt, Leopold Bioscript

Conrotto, Eugene L. Desert Magazine, Inc. Correspondence $\bullet$ 1964: (1)

Constance, Lincoln (1909-) American botanist; U. of California, Berkeley, Dept. of Botany

Bioscript • Botanical Album

Correspondence • 1934: (3) Jepson, Willis Linn,

1867-1946 • 1935: (3) • 1936: (7) Jepson, Willis Linn,

1867-1946 • 1937: (3) • 1938: (6) Jepson, Willis Linn,

1867-1946 • 1939: (3) Jepson, Willis Linn, 1867-1946;

Species; Eastwood, Alice, 1859-1953 • 1940: (8) Jepson,

Willis Linn, 1867-1946 - 1941: (2) • 1942: (3) - 1946: (1)

- 1947: (6) Jepson, Willis Linn, 1867-1946 - 1948: (5) 1949: (3) • 1950: (3) • 1951: (1) • 1952: (6) - 1953: (5)

U. of California, Berkeley. Dept. of Botany; Delphinium; Species • 1955: (2) • 1956: (1) • 1957: (4) • 1958: (2) • 1959: (1) - 1962: (2) • 1964: (3) • 1965: (2) • 1967: (2) • 1969: (1) - 1973: (3) Setchell, William Albert, 1864-1943 - 1974: (2) • 1975: (2) • 1976: (3) Merrill, Elmer Drew, 1876-1956 • 1977: (1) • 1978: (1) - 1979: (5) 1980: (3) - 1981: (2) Pursh, Frederick, 1774-1820 1985: (2) • 1986: (2) • 1988: (3) • 1993: (2)

Contemporary Authors New York, NY, Linda Hubbard Correspondence $\bullet$ 1977: (1) Conway, Jill K. American Antiquarian Society, Worchester,
President

Correspondence $\bullet$ 1988: (0) Filed under American

Antiquarian Society 
Conway, William E. William Andrews Clark Memorial Library, Los Angeles, Librarian

Correspondence 1 1975: (2)

Conyngham, Redmond Bioscript

Cook, Barbara U. of Newcastle Correspondence $\bullet$ 1974: (1)

Cook, George A. Cleveland, $\mathrm{OH}$ Bioscript Correspondence $\bullet$ 1921-1928: (7) Letter from Carl Krebs $\bullet$ 1929-1933: (1)

Cook, James (1728-1779) (Capt.) English explorer Biofile $\bullet$ Bioscript $\bullet$ Botanical Album

Cook, Orator Fuller (1867-1949) American botanical explorer

Biofile $\bullet$ Bioscript $\bullet$ Botanical Album Correspondence 1949: (1)

Cook, Paul Bookseller; Seattle, WA Correspondence - 1983: (1)

Cook, Robert C. Son of Orator Fuller Cook Correspondence $\bullet$ 1949: (3)

Cooke, Amy W. Bioscript

Cooke, Caleb (1836-1880) American biologist Biofile $\bullet$ Bioscript

Cooke, John Esten Bioscript

Cooke, John F., Jr. (1927-1970) American botanist Biofile

Cooke, John Warren Correspondence $\bullet 1963$ : (0) Filed under Edmund Berkeley

Cooke, Mordecai Cubitt (1825-1914) English mycologist Biofile $\bullet$ Bioscript $\bullet$ Botanical Album

Cooke, Wells Woodbridge (1858-1916) American ornithologist Bioscript

Cooke, William Bridge (1908-) Mycologist, taxonomist Botanical Album Correspondence $\bullet$ 1940: (1) • 1941: (3) • 1946: (2) • 1976: (2)

Cooks Travel Organization Freda G. Batchelor, Asst. Manager Correspondence • 1967: (2)

Cookson, Sarah Sir George Taylor's grand-daughter Correspondence $\bullet 1980$ : (1)

Cooley, George Ralph (1896-1986) American botanist Biofile $\bullet$ Bioscript $\bullet$ Botanical Album Correspondence 1956 : (10) Tulane U., Dept. of Botany - 1957: (16) Letter to William B. Reese - 1958: (9) 1959: (3) 1962: (2) Letter from Harold N. Moldenke is attached $\bullet$ 1963: (2) 1 1964: (1) $\bullet$ 1968: (3) 1 1973: (1)

Cooley, Robert Allen (1873-1968) American entomologist Bioscript

Coon, Nelson Correspondence • 1976: (3)

Coons, Irene M. Colorado A \& M College, Fort Collins, Reference Librarian Correspondence • 1949: (3)

Cooper, Alice Cecilia (1895-) Writer, teacher in Los Angeles high school Bioscript
Cooper, Arthur Wells (1931- ) American plant ecologist; American Association for the Advancernent of Science, Section on Botanical Sciences, Secretary Correspondence $\bullet$ 1968: (5)

Cooper, Donald B. Tulane U., New Orleans Correspondence • 1966: (2) • 1968: (4) • 1972: (1) • 1982: (1) 1985: (3)

Cooper, Ellwood (1829-1918) American horticulturist Bioscript

Cooper, Ellwood, Mrs. ( -1908) Santa Barbara Bioscript

Cooper, G. Arthur Washington Academy of Science Correspondence $\bullet$ 1943: (1)

Cooper, Gil The Blakiston Company, Philadephia, Publishers of Books in Medicine and Science Correspondence $\bullet$ 1951: (3)

Cooper, James Graham (1830-1902) American physician, zoologist Biofile $\bullet$ Bioscript $\bullet$ Botanical Album

Cooper, Jean E. Former student Correspondence $\bullet$ 1942: (1)

Cooper, Joyce K. Pennsylvania Hospital Correspondence • 1973: (2) Barton, Benjamin Smith, 1766-1815

Cooper, Martha Riser Ellicott City, MD Correspondence • 1971: (2)

Cooper Ornithological Society Correspondence • 1964: (1) • 1980: (1) • 1981: (1) • 1983: (1)

Cooper, Robert Cecil (1917-) American plant morphologist Botanical Album

Cooper, Samuel Bioscript

Cooper, Thomas Bioscript

Cooper, William (1798-1864) Bioscript

Cooper, William Skimner (1884-1978) American plant ecologist, geomorphologist; U. of Minnesota, MN Bioscript • Botanical Album Correspondence • 1936: (2) • 1949: (2)

Cope, Edward Drinker (1840-1897) American zoologist, paleontologist

Bioscript

Cope, Thomas D. U. of Pennsylvania, Philadelphia, Randal Morgan Laboratory of Physics Correspondence $\bullet$ 1951: (3)

Cope, Thomas P. (1800-1851) Bioscript

Copeland, Donald Eugene Tulane U., New Orleans; nickname "Gene" Botanical Album Correspondence • 1972: (3) • 1974: (1) • 1985: (1)

Copeland, Edwin Bingham (1873-1964) American pteridologist Biofile • Botanical Album Correspondence • 1929-1933: (4)

Copeland, Herbert Faulker (1902-1968) American taxonomist Botanical Album Correspondence • 1937: (2) 


\section{Guide to the Ewan Papers}

Copeland, Mary C. Husband is D. Eugene Copeland Correspondence $\bullet$ 1984: (1)

Copeland, Patsy Tulane U., New Orleans, Medical Center, Rudolph Matas Medical Library

Correspondence • 1989: (1)

Corbet, Jack, Mrs. Correspondence $\bullet 1951$ : (2)

Corda, August Joseph (1809-1849) Bioscript

Cordua, Theodor (1796-1857) German-born California pioneer Biofile • Bioscript

Core, Earl Lemley (1902-1984) American taxonomist, botanist: West Virginia U., Morgantown, Dept. of Biology, Chairman Botanical Album

Correspondence - 1942: (4) Biology-Instruction and study - 1945: (1) • 1946: (2) • 1947: (3) • 1948: (2) • 1951: (1) - 1952: (1) • 1956: (1) • 1957: (2) • 1958: (1) • 1962: (1) - 1966: (1) • 1968: (2) • 1982: (1)

Corcy, H. Irene Correspondence $\bullet 1939:(1)$

Corgan, James X. Austin Peay State U., Clarksville, TN Correspondence $\bullet 1974$ : (2)

Coriden, Guy E. Dept. of State, Bureau of Educational and Culmural Affairs

Correspondence • 1968: (1)

Cork, Henry Natural History Bookseller Correspondence $\bullet$ 1954: (1) • 1956: (1)

Cormack, William Epps (1796-1868) Bioscript

Cornelius, Elias Bioscript

Cornell Laboratory of Ornithology Correspondence $\bullet$ 1973: (1)

Cornell Plantations Cornell U., Ithaca, NY Correspondence • 1983: (1)

Cornell U. Botanical Album Comespondence $\bullet 1992$ : (1)

Cornell U. Press Correspondence • 1983: (2)

Corner, Edred John Henry (1906-) U. of Cambridge, England, Botany School Botanical Album Correspondence $\bullet 1964:(2) \bullet 1977:$ (3)

Corner, George W., Mrs. Correspondence $\bullet$ 1971: (1)

Corner, George Washington (1889-1981) American Philosophical Society, Philadelphia, PA, Executive Officer Botanical Album • Bioscript Correspondence • 1956: (1) • 1960: (1) • 1961: (1) • 1962 (21) Attached is letter from H. Radclyffe Roberts -1963 (9) Bartram, William, 1739-1823 • 1964: (8) Bartram, William, 1739-1823 • 1965: (21) Bartram, William, 1739-1823; letters from/to James F. Barneth, Harold Hugo, B. H. Harley • 1966: (11) Bartram, William, 1739-1823 1967: (30) Bartram, William 1739-1823 • 1968: (11)• 1969: (13) Taylor, George, Sir, 1904- 1970: (8) 1971 :
Corner, George Washington (1889-1981) (continued) (19) - 1972: (4) - 1973: (3) Barton, Benjamin Smith, 1766-1815 • 1974: (5) Species • 1975: (4) • 1976: (4) • 1978: (5) Bartram, William, 1739-1823 • 1980: (5)

Cornu, Maxime (1843-1901) German botanist, plant pathologist Biofile

Cornut, J. Bioscript

Correa A., Mireya D.

Correspondence $\bullet$ 1980: (0) Flyer announcing a symposium signalling the completion of The Flora of Panama

Correa de Serra, José Francisco (1751-1823) Portuguese botanist, clergyman Biofile • Bioscript

Correll, Donovan Stewart (1908-1983) Taxonomist; U. S. Dept. of Agriculture, Specialty Crop Introductions; Texas Research Foundation, Renner Botanical Library Biofile $\bullet$ Botanical Album

Correspondence $\bullet$ 1949: (2) $\bullet$ 1950: (4) $\bullet$ 1959: (1) $\bullet$ 1960: (4) Banister, John, 1650-1692 - 1961: (2) Banister, John, 1650-1692 • 1967: (8) Delphinium • 1968: (3) • 1975: (1) • 1976: (2) • 1981: (1) Pursh, Frederick, 1774-1820 • 1983: (1)

Correll, Helen Husband is Donovan Stewart Correll Correspondence $\bullet$ 1983: (2)

Correns, Carl Franz Joseph Erich (1864-1933) German physician, plant geneticist Botanical Album

Corry, Thomas Hughes (1859-1883) Bioscript

Cortes, Santiago (1854-1924) Colombian botanist Bioscript

Cory, Victor Louis (1880-1964) American botanist; Texas Agricultural Experiment Station, Range botanist Botanical Album Correspondence • 1941: (2) • 1944: (2) Delphinium • 1945: (4)

Cosentino, F. Bioscript

Cosson, $\mathbf{E}$. Bioscript

Costa, Emanuel Mendes da (1717-1791) English conchologist Biofile

Costa, Hipolito Jose da Bioscript

Cota, F. M. Correspondence - 1929-1933: (5) Munz, Philip Alexander, 1892-1974 • 1934: (1) • 1943: (2)

Cotta, Heiarich von (1763-1844) German forester Bioscript

Cottam, Walter Pace (1894 ) American botanist, ecologist U. of Utah, Salt Lake City Bioscript Correspondence • 1941: (1) • 1943: (3) • 1949: (5)

Cottle, James E. Bioscript 
Cotton, Arthur Disbrowe (1879-1962) English botanist, horticulturist; Royal Botanic Gardens, Kew, Surrey Botanical Album

Correspondence • 1934: (3) • 1937: (1) • 1944: (2) Delphinium

Cotton, J. S.

Bioscript

Couch, Darius Nash (1822-1865) American naturalist Biofile

Couch, Harvey C. Tulane Law School, New Orleans, LA Correspondence • 1989: (2)

Couch, John Nathaniel (1896-1986) American mycologist; U. of North Carolina, Chapel Hill, Dept. of Botany Correspondence - 1952: (3) Pennell, Francis Whittier, 1886-1952 - 1960: (2)

Coues, Elliott (1842-1899) American ornithologist Biofile $\bullet$ Bioscript $\bullet$ Botanical Album

Coulter, E. M. Georgia Historical Quarterly, Athens, GA Correspondence • 1970: (1)

Coulter, John Merle (1851-1928) American botanist Biofile $\bullet$ Bioscript $\bullet$ Botanical Album

Coulter, Merle Crowe (1894 ) American botanist; U. of Chicago Correspondence $\bullet$ 1942: (1)

Coulter, Thomas (1793-1843) Irish botanist Biofile - Bioscript

Coulton, Harold S. Biofile

Council on Botanical and Horticultural Libraries Correspondence $\bullet$ 1990: (3) Book collectors $\bullet$ 1992: (2) Letter from Ronald L. Stuckey, executor of the estate of Emanuel D. Rudolph

Countiss Travels Countiss Travels, New Orleans, LA Correspondence • 1979: (2) • 1981: (1)

Couper, J. Hamilton Bioscript

Courtis, William Munroe (1842-1922) American mining engineer Bioscript

Courset, George Louis Marie Dumont de See Dumont de Courset, George Louis Marie (1746-1824)

Cousens, Michael I. U. of West Florida, Pensacola, Dept. of Biology Correspondence • 1979: (3)

Cousins, Norman Saturday Review, New York, NY, Editor Correspondence • 1962: (1)

Cousteau, Jacques Biofile

Couthouy, Joseph Pitty (1808-1864) American ship captain, conchologist, plant collector Bioscript • Botanical Album

Covel, Paul F. Bioscript

Coville, Frederick Vernon (1867-1937) American botanist Biofile $\bullet$ Bioscript $\bullet$ Botanical Album Correspondence $\bullet$ 1935: (2)

Cowan, Jacob Hover (fl. 1895) Botanist Bioscript

Cowan, Richard Summer (1921-) American taxonomist, plant explorer, botanist; Smithsonian Institution, Washington, D.C.
Cowan, Richard Summer (1921-) (continued) Correspondence $\bullet$ 1958: (2) $\bullet$ 1960: (2) $\bullet$ 1961: (4) $\bullet$ 1965: (2) • 1967: (1) • 1968: (8) • 1969: (3) • 1970: (2) • 1972: (1) • 1974: (2) Stafleu, Frans Antonie, 1921- • 1975: (12) • 1976: (2) • 1977: (5) • 1978: (4) • 1980: (13) Rocky Mountain Naturalists • 1981: (10) 1 1982: (2) • 1983: (4) • 1987: (1) • 1988: (1)

Cowles, Henry Chandler (1869-1939) American plant ecologist

Biofile $\bullet$ Bioscript $\bullet$ Botanical Album

Correspondence 1983: (2)

Cowles, Raymond B. U. of California, Los Angeles, Dept. of Zoology

Correspondence • 1947: (1) • 1957: (2)

Cowley, Abraham (1618-1667)

Bioscript

Cox, Clare Francis (1888- ) Botanical Album

Cox, Eleanor U. of Texas, Austin Correspondence $\bullet$ 1964: (1)

Cox, Hiden T. American Institute of Biological Sciences Correspondence $\bullet$ 1954: (1)

Cox, Jacob Dolson (1828-1900) Canadian-born American politician and microscopist Bioscript

Cox, Lucy Reeves Bridgeton, NJ Correspondence • 1921-1928: (4) • 1929-1933: (6)

Cox, Stephen D. U. of Nebraska Press, Lincoln Correspondence • 1979: (1) Rocky Mountain Naturalists

Coxe, John Redman Bioscript

Coy, Owen D. U. of Southern California, Los Angeles Correspondence • 1956: (1)

Crabb, Edward Drane (1890-) American zoologist; U. of Colorado, Boulder Correspondence $\bullet$ 1945: (1)

Crabbe, James Albert (1914 ) British pteridologist; British Museum (Natural History), Dept. of Botany

Botanical Album

Correspondence • 1955: (3) British Museum (Natural History), Dept. of Botany 1956 : (7) Banks, Joseph, Sir, 1743-1800 • 1957: (2) • 1958: (9) • 1959: (2) • 1964: (8) - 1965: (2) • 1966: (6) - 1970: (3)

Cragin, Francis Whittemore (1857-1937) American biologist, paleontologist Bioscript

Craig, Elberta Louise Bioscript

Craig, Eric Botanical Album

Craig, Gordon U. of Edinburgh, Edinburgh, Scotland Correspondence • 1981: (1) Barton, Benjamin Smith, 1766-1815

Craig, Gordon A. Stanford U., Dept. of History Correspondence $\bullet$ 1974: (3)

Craig, Thomas Theodore (1905-) Bioscript

Craine, Eugene R. Wright State U., Dayton, $\mathrm{OH}$ Chairman, Dept. of History Correspondence 1972: (3) Species 


\section{Guide to the Ewan Papers}

\section{Cramer, Carl Eduard (1831-1901)}

Biofile

Cramer, Charies

Bioscript

Cramer, J. German bookseller

Botanical Album

Correspondence $\bullet$ 1966: (2) • 1967: (3) • 1970: (1) •

1971: (7) • 1979: (11) Letter from E. F. Elmendorf •

1980: (6) Pursh, Frederick, 1774-1820 • 1982: (2)

Crandall, Charles Spencer (1852-1929) American horticularist

Bioscript • Botanical Album

Crandall, Ernest Lindsley (1870-1950) American wildflower photographer

Biofile

Crandall, Matilda California Polytechnic; husband is B. R. Crandall

Correspondence • 1929-1933: (1)

Crane, Linda Los Angeles, CA Correspondence $\bullet$ 1921-1928: (1)

Cranefield, Paul Rockerfeller U. Correspondence • 1967: (17) Bartram, William, 1739-1823 - 1968: (2)

Cratsley, Mrs. Correspondence $\bullet 1974$ : (1)

Crawe, Ithamar Bingham (1792-1847) American physician Bioscript

Crawford, Albert Cornelius (1869-1921)

Bioscript

Crawford, Daniel J. (1942-) Ohio State U., Herbarium Correspondence • 1991: (0) Filed under Ronald Lewis Stuckey

Crawford, David P. F. Collier \& Son Corporation, New York, NY

Correspondence $\bullet$ 1960: (2)

Crawford, David Livingston (1889-)

Bioscript

Crawford, William H. (1812-1888) Biofile

Cree, John (c. 1738-1816)

Bioscript

Creighton, Harriet Baldwin (1909-) American plant morphologist, plant geneticist, botanist; Wellesley College, Wellesley, MA

Botanical Albun

Correspondence • 1950: (2) • 1956: (2) • 1964: (4) • 1967: (3)

Crépin, François (1830-1903) Belgian botanist, taxonomist Biofile • Bioscript

Cresson, Exra Townsend, Sr. (1838-1926) American entomologist, clerk Bioscript

Creuzfeldt, F. (-1853) German-born gardener Bioscript

Crevecouer, Bioscript

Cribb, Alan Bridson (1925-) U. of Queensland, Brisbane, Australia

Correspondence • 1956: (1) Banks, Joseph, Sir, 1743-1800

Crisp, Katharine Maria Bruderlin

Correspondence • 1947: (1)
Crispus, Benedictus (c. 725 A.D.) Italian medical poet, Archbishop of Milan

Biofile

Critchfield, William Burke (1923-1989) Harvard U., Maria Moors Cabot Foundation, Cambridge, MA

Correspondence • 1958: (1) • 1965: (1)

Croasdale, Hannah T. (1905-) Dartmouth College, Dept. of Biological Sciences

Correspondence $\bullet$ 1976: (3)

Croat, Thomas Bernard (1938-) American botanist; Missouri Botanical Garden, St. Louis, MO, Curator of Phanerogams

Correspondence • 1972: (2) Vismia • 1973: (2) • 1989: (2)

Crocker, William

Bioscript

Crockett, Anthony van

Correspondence • 1985: (1)

Crockett, Edith Horticultural Society of New York, New York, NY, Acting Librarian

Correspondence $\bullet$ 1970: (5)

Crockett, Lawrence J. City U. of New York and the Torrey Botanical Club

Biofile $\bullet$ Bioscript $\bullet$ Botanical Album

Correspondence • 1967: (9) • 1969: (1) • 1970: (0) Filed under Edith Crockett $\bullet$ 1975: (2) $\bullet$ 1976: (2) 1980 : (4) $\bullet$ 1984: (4) • 1985: (5) • 1986: (4) • 1987: (2) • 1989: (1) 1990: (2) 1992: (1)

Croghan, George

Bioscript

Croizat, León Camille Marius (1894-1982) Italian-born American amateur botanist; Museo de Ciencias Naturales, Caracas, Venezuela Bioscript $\bullet$ Botanical Album Correspondence • 1951: (1) • 1952: (2)

Crompton, A. W. Museum of Comparative Zoology, The Agassiz Museum, Professor of Biology Correspondence $\bullet$ 1970: (3)

Cronholm, Lois S. U. of Louisville, School of Medicine, Dept. of Microbiology Correspondence • 1969: (3)

Cronkite, Walter CBS, New York, NY Correspondence • 1969: (1)

Cronquist, Arthur John (1919-1992) American taxonomist, botanist; New York Botanical Garden

Bioscript $\bullet$ Botanical Album

Correspondence • 1943: (1) • 1947: (1) - 1948: (3) •

1949: (3) $\bullet$ 1950: (2) • 1957: (2) $\bullet$ 1967: (1) • 1972: (4)

- 1974: (1) • 1976: (1) • 1978: (2) • 1987: (1)

Crook, Z. E.

Correspondence $\bullet$ 1943: (1)

Crooke, John J. (1824-1911) American plant collector Bioscript

Crooks, Donald Mundell (1902-) Correspondence • 1937: (3) • 1939: (0) Filed under Lyman David Benson

Croom, Edward M. U. of Mississippi, Assistant Professor Correspondence - 1983: (2) Species Croom, Hardy Bryan (1797-1837) American lawyer, plant
explorer

Biofile $\bullet$ Bioscript 
Crosby, Alfred W. U. of Texas, Austin, American Studies \& American Civilization Programs

Correspondence • 1977: (1)

Crosby Arboretum Botanical Album

Crosby, Marshall Robert (1943-) American bryologist, botanical bibliographer; Missouri Botanical Garden Botanical Album

Correspondence $\bullet$ 1978: (1) $\bullet$ 1979: (1) $\bullet$ 1980: (1) Missouri Botanical Garden - 1981: (1) Missouri Botanical Garden • 1986: (13) Missouri Botanical Garden • 1987: (3) Book collectors $\bullet$ 1988: (6) $\bullet$ 1990: (1) $\bullet$ 1991: (0) Filed under Emanuel David Rudolph • 1991: (2) • 1992: (2) 1993: (6)

Crosby, Rena R. Correspondence • 1929-1933: (1)

Cross, Frank Clay (1893- ) American journalist, naturalist; The Explorers League Correspondence $\bullet$ 1941: (2) $\bullet$ 1949: (3) $\bullet$ 1951: (2)

Crossette, George National Geographic Magazine Correspondence • 1956: (4) • 1957: (2) • 1958: (1)

Crossman, Muriel C. (1913-) American botanical librarian; Massachusetts Horticultural Society, Librarian Correspondence $\bullet$ 1966: (1) • 1967: (1) • 1968: (2) • 1970: (3) • 1971: (4) • 1972: (4) • 1973: (1) • 1974: (1) Book collectors • 1976: (2) • 1978: (3) • 1979: (4) • 1981: (6) $\bullet$ 1982: (5) • 1984: (2)

Crosswhite, Carol Botanical Album

Crosswhite, Frank Samuel (1940-) Boyce Thompson Southwestern Arboretum, U. of Arizona, Superior Biofile - Bioscript $\bullet$ Botanical Album Correspondence $\bullet$ 1964: (1) $\bullet$ 1976: (1) $\bullet$ 1979: (2) $\bullet$ 1990: (3)

Crotz, Keith D. American bookseller; American Botanist, Booksellers, Chillicothe, IL

Botanical Album

Correspondence $\bullet$ 1988: (18) Book collectors $\bullet$ 1989: (13) Copy of letter to D. Keith Crotz from James A. Servies 1990: (15) • 1991: (14) Book collectors $\bullet$ 1992: (1) $\bullet$ 1993: (7)

Crovetto, Raoul Martínez (1921- ) Div. of Plant Exploration, Buenos Aires, Argentina Correspondence - 1946: (1)

Crow, Elizabeth Davenport Bioscript

Crowdis, Donald Nova Scotia Museum of Science Correspondence - 1956: (1)

Crowfoot, Grace Mary Hood Bioscript

Crown, Rick Correspondence • 1986: (2) • 1988: (0) Filed under Georgia Botanical Society

Crown, Treadwell $\mathbf{R}$. Correspondence • 1983: (3)

Crownenshield, Jacob (1770-1808) Bioscript

Crum, Ethel Katherine (1886-1943) Biofile • Bioscript $\bullet$ Botanical Album Correspondence • 1929-1933: (1) • 1936: (1) • 1940: (1) • 1942: (3)
Crum, Howard Alvin (1922-) American-born bryologist; Canada; Stanford U., CA Correspondence $\bullet$ 1952: (1)

Crump, James L. Holly Bluff Gardens, Bay St. Louis, MS

Botanical Album

Correspondence • 1951: (2) $\bullet$ 1952: (4) Correspondence with A. J. Sharp - 1953: (6) Biology-Instruction and study; Royal Botanic Gardens, Kew $\bullet$ 1956: (2) • 1957: (4) - 1960: (1)

Crump, Oeta M. Husband is James L. Crump Correspondence - 1961: (10) Tulane U., Dept. of Botany; Letters from M. Nearly, H. Longenecker, C. Scheps

Crutchfield, D. M. Westvaco Corp., Summerville, SC, Research Center Leader Correspondence • 1977: (2)

Cryder, J. Bioscript

Cuatrecasas, José (1903-) Spanish-born American botanist, taxonomist; National Herbarium, Washington, D.C.

Bioscript $\bullet$ Botanical Album

Correspondence • 1954: (2) • 1972: (1) • 1989: (1)

Cuerrier, Alain U. of Montreal

Correspondence $\bullet$ 1991: (1)

Culberson, William Louis (1929- ) American bryologist, lichenologist; Duke U., Dept. of Botany; editor of The Bryologist

Correspondence • 1964: (6) • 1968: (2) • 1969: (3) • 1991: (1)

Culbertson, Myra Housing Bureau, Newcomb College Correspondence $\bullet$ 1947: (2)

Culbertson, Thaddeus A. ( -1850) Natural history collector Bioscript

Cullen, Charles T. Newberry Library, Chicago, IL Correspondence • 1989: (1)

Cullen, William Bioscript

Cullison, William R. Tulane U. Library, Howard-Tilton, Curator, Prints \& Drawings Correspondence • 1979: (0) Filed under James R. Reed • 1983: (1) • 1984: (2) • 1985: (1) • 1990: (1)

Cuming, Hugh (1791-1865) English plant explorer; Chile, Pacific area

Biofile $\bullet$ Bioscript

Cummings, Clara Eaton (1855-1906) American educator, botanist, lichenologist

Bioscript

Cummings, Mary E. Bioscript

Cummins, Harold (1893-1976) Tulane U., New Orleans, Anatomy Dept.

Biofile $\bullet$ Bioscript $\bullet$ Botanical Album

Correspondence • 1948: (2) • 1949: (1) • 1953: (1) • 1962: (1) • 1970: (3) • 1973: (3) • 1975: (1)

Cunningham, Allan (1791-1839) English botanist, plant explorer in Australia Biofile $\bullet$ Bioscript $\bullet$ Botanical Album

Cunningham, R. R. Lambert Landscape Co., Dallas, TX Correspondence $\bullet$ 1961: (2)

Cunninghame-Graham, Robert Bontine Bioscript 


\section{Guide to the Ewan Papers}

Curie, Friedrich Peter Bioscript

Curran, Hugh MeCullum (1875-1920) American-born forester, collector Botanical Album

Curran, Mary Katherine (18441920) See also Mary Katherine Brandegee Bioscript

Currier, Alice Correspondence • 1942: (3)

Curry, Mary G. VTM, Engineers Planners Environmental Scientists, Metairie, LA Correspondence • 1976: (0) Filed under Louisiana Forestry Commission

Curtin, Leonora S. M. Husband is Thomas E. Curtin Botanical Album

Correspondence • 1939: (1) Letter from John Thomas Howell, California Academy of Sciences $\bullet$ 1940: (10) 1941: (7)

Curtis, Carlton Clarence (1864-1945) American plant physiologist

Biofile $\bullet$ Bioscript $\bullet$ Botanical Album

Curtis, John Thomas (1913-1961) American botanist, plant ecologist; U. of Wisconsin, Madison, Dept. of Botany, Professor of Botany

Correspondence • 1954: (1) • 1955: (3)

Curtis, Moses Ashley (1808-1872) American teacher, priest, botanist; Hillsborough, NC

Biofile • Bioscript • Botanical Album

Curtis, Samuel (1779-1860) British florist, botanical publisher Biofile

Curtis, William (1746-1799) English apothecary, naturalist Biofile • Bioscript

Curtiss, Allen Firam (1845-1907) American plant collector, West Indies Bioscript $\bullet$ Botanical Album

Curtiss, Asahel H. Botanical Album (filed under Allen Hiram Curtiss)

Curwen Press Ltd. B. H. Harley, Plaistow, London, England Correspondence • 1965: (1) • 1972: (1)

Cusack, M. E., Mrs. Bioscript

Cushing, Frank Hamilton (1857-1900) American ethnologist, archeologist Bioscript

Cushing, Harvey (1869-) Physician Biofile • Bioscript

Cushman, Helen Correspondence $\bullet$ 1982: (1)

Cushman, Joseph Augustine (1881-1949) American paleontologist Bioscript

Cushman, R. R. Bookseller; Westfield, NJ Correspondence $\bullet$ 1982: (1)

Cusick, Frank U. of Aberdeen, Aberdeen, Scotland Botanical Album Correspondence • 1985: (1) • 1986: (1)
Cusick, William Conklin (1842-1922) American plant collector, botanist, taxonomist

Bioscript $\bullet$ Botanical Album

Custis, George Washington Parke (1781-1857) Bioscript

Custis, Peter American botanist, physician Biofile $\bullet$ Bioscript

Cutak, Ladislaus (1908-1973) Austrian-born American horticulturist; Missouri Botanical Garden Biofile

Cuthbert, Alfred Bioscript

Cutler, Hugh Carson (1912-) American taxonomist, economic botanist; Missouri Botanical Garden, St. Louis, MO

\section{Bioscript • Botanical Album}

Correspondence • 1957: (1) 1958: (1) Missouri Botanical Garden • 1960: (1) • 1964: (3) • 1971: (2) • 1975: (1)

Cutler, Manasseh (1742-1823) American clergyman, botanist; Jenkintown, PA

Biofile • Bioscript

Cutright, Paul Russell Bioscript

Correspondence $\bullet$ 1966: (7) • 1968: (2) • 1970: (2) • 1976: (3)

Cuvier, Georges Léopold Chrétien Frédéric (Baron) de (1769-1832) French naturalist

Bioscript • Botanical Album

\section{D}

D'Acquin, Mary Louise Tulane U., Dept. of Botany Correspondence $\bullet$ 1951: (3)

D'Arcy, Nancy Missouri Botanical Garden, St. Louis, MO Correspondence - 1990: (1)

D'Arcy, William Gerald (1931-) Canadian-born plant taxonomist; Missouri Botanical Garden, St. Louis, MO Correspondence • 1980: (1) • 1989: (1)

D'Orbigny, Bioscript

Dachnowski, Alfred Botanical Album

DaCosta, Emanuel Mendes (1717-1791) Bioscript

DaCosta-Pereira, Hyppolito Bioscript

Dahlstedt, Hugo (1856-1934) Swedish taxonomist Biofile

Daiker, Virginia Library of Congress, Reference Dept., Prints and Photographs Division Correspondence $\bullet$ 1951: (2) Barton, Benjamin Smith, 1766-1815

Dailey, Betty Moore Correspondence • 1985: (2) $\bullet$ 1988: (2)

Daily, Eva Fay Kenoyer (1911-) American taxonomist, plant ecologist; Butler U., Indianapolis, Dept. of Botany Correspondence $\bullet$ 1953: (5) Pursh, Frederick, 1774-1820 - 1954: (2)

Daingerfield, $\mathbf{H}$. P. Bioscript 
Dakan, Albert Attorney, amateur naturalist; Longmont, CO Correspondence • 1941: (2) • 1943: (2)

Dalcrona, Gabriel Bioscript

Dale, Edward E., Jr. U. of Arkansas, Fayetteville Correspondence • 1986: (1)

Dale, Ivan Robert (1904-1963) Botanical Album

Dale, Samuel (1659-1739) English physician, apothecary, botanist Biofile

Dale, Thomas Bioscript

Daley, Richard H. Missouri Botanical Garden, St. Louis, MO, Eco Services Div.

Correspondence - 1977: (3) Prospectus for National Park Service exhibit at Arch

Dall, William Heale (1845-1927) American paleontologist Bioscript

Dallas Costume Company Correspondence $\bullet$ 1942: (1)

Dalziel, Bruce W. Graham Arader III, Seller of Books and Maps Correspondence $\bullet$ 1983: (1)

Dampf, Alfonso Bioscript

Dampier, William (1652-1715) English amateur botanist, author, explorer, plant collector; Australia Bioscript

Dana, James Dwight (1813-1895) American geologist Bioscript

Dance, S. Peter British malacologist; British Museum (Natural History), Dept. of Zoology

Biofile $\bullet$ Bioscript

Correspondence $\bullet$ 1964: (2) $\bullet$ 1968: (1) Banister, John, 1650-1692

Dandridge, Joseph (1644-1746) English naturalist, entomologist Biofile

Dandy, James Edgar (1903-1976) English taxonomist, hptanist; British Museum (Natural History), Dept. of Botany Biofile $\bullet$ Bioscript

Correspondence $\bullet$ 1957: (3) $\bullet$ 1960: (2) $\bullet$ 1961: (4) $\bullet$ 1963: (1) 1963: (0) Letter filed under Averil Margaret Lysaght $\bullet$ 1964: (2) • 1965: (1) Bartram, William, 1739-1823 - 1969: (1)

Daniel, Henry (c. 1315-1320 to 1385?) English physician, gardener, botanist Biofile

Daniel, Rachel The Times-Picayune Publishing Co., New Orleans, LA Correspondence $\bullet$ 1955: (2) $\bullet$ 1960: (1) $\bullet$ 1961: (5) $\bullet$ 1962: (3) • 1963: (3) • 1964: (2) • 1965: (2)

Daniels, Francis Potter (1869-1947) American botanist, minister Bioscript

Daniels, Gilbert Samuel (1927-) American botanist, taxonomist, botanical bibliographer; Carnegie-Mellon U., Hunt Institute
Daniels, Gilbert Samuel (1927- ) (continued) Correspondence • 1970: (2) • 1971: (2) • 1972: (0) Filed under David Bruce Lellinger 1972 : (7) American Fern Society $\bullet$ 1973: (7) • 1974: (2) • 1976: (1)

Dann, Kevin Correspondence $\bullet$ 1986: (1)

Dansereau, Pierre Mackay (1911-) Canadian-born plant taxonomist, ecologist, phytogeographer Correspondence $\bullet$ 1968: (3)

Dant, Robert E. Correspondence $\bullet$ 1986: (1)

Dapprich, Emil (1841-1903) German-born American naturalist, physician

Biofile • Bioscript

Darby, John Bioscript

Darby, William Bioscript

Darling, Bethel Correspondence • 1954: (1) American Fern Society

Darling, Louise Librarian; U. of California, Los Angeles, Biomedical Library

Bioscript $\bullet$ Botanical Album Correspondence $\bullet$ 1938: (1) • 1939: (5) • 1960: (2) • 1961: (1) • 1963: (1) • 1964: (3) • 1969: (1) • 1970: (3) • 1972: (2) • 1975: (1) • 1978: (4) • 1979: (1) • 1980: (2) • 1982: (1) • 1983: (2) • 1985: (1) • 1987: (2) • 1990: (2)

Darlington, Cyril Dean (1903-) English plant geneticist, plant ecologist; U. of Oxford, Dept. of Botany Correspondence $\bullet$ 1955: (1)

Darlington, Henry Townsend (1875-1964) American botanist Biofile

Darlington, Philip J. Botanical Album

Darlington, William (1782-1863) American physician and botanist Biofile $\bullet$ Bioscript $\bullet$ Botanical Album :

Darlington, William McCullough Biofile $\bullet$ Bioscript

Darnell, Rexneat M. Marquette U., Milwaukee, WI Correspondence $\bullet$ 1958: (1)

Darrow, Robert Arthur (1911-) American plant ecologist; U. of Arizona, Tucson

Correspondence $\bullet$ 1936: (3) • 1938: (1) • 1942: (1)

Dart, Elisabeth Kilbourne Correspondence $\bullet$ 1984: (2)

Darton, Nelson Horatio (1865-1948) American geologist Bioscript

Darts, E. C. American Rhododendron Society, Convention Treasurer Correspondence - 1979: (0) Filed with L. Keith Wade

Dartt, Mary Bioscript

Darwin, Charles Robert (1809-1882) English naturalist Biofile $\bullet$ Bioscript $\bullet$ Botanical Album Correspondence - 1988: (1) Charlotte Bowman, Assistant Editor, The Correspondence of Charles Darwin

Darwin, Erasmus (1731-1802) English physician, philosopher Biofile • Bioscript 
Darwin, Steven P. (1949-) Tulane U., New Orleans, Dept. of Ecology and Organismal Biology

Bioscript • Botanical Album

Correspondence $\bullet 1977$ : (6) • 1978: (4) • 1979: (1) • 1980: (1) Filed under Billie Lee Turner 1982 : (9) See also Leonard J. Utral $\bullet$ 1981: (8) • 1983: (2) See also Lois Chambers Stone • 1984: (19) • 1985: (5) • 1986: (3) • 1987: (13) • 1988: (7) • 1989: (17) • 1990: (16) • 1991: (11) - 1992: (9) See also Anton Hinrichs • 1993: (6)

Dascombe, James Bioseript

Dash, John Sydney (1857-) Georgetown Botanic Gardens, Georgetown, British Guiana Correspondence $\bullet$ 1939: (3)

Daubenmire, Rexford F. (1909-) American plant ecologist; State College of Washington, Pullman, WA, Dept. of Botany

Bioscript $\bullet$ Botanical Album Correspondence $\bullet$ 1951: (2) • 1979: (2)

Daubeny, Charles Bioscript

Dauber \& Pine Book Shops, Inc. Correspondence • 1963: (1)

Daudin, F. M. Bioscript

Daum, Andreas German historian; Institut für Neuere Geschichte der Universităt Munchen Correspondence $\bullet$ 1992: (2) $\bullet$ 1993: (3)

Daunoy, Harry Louis (1889-1958) Bioscript

Daunoy, Marie Husband is Harry L. Daunoy Correspondence $\bullet 1962$ : (1)

Dautun, H. Bioscript

Davenport, Demorest (1911-) American zoologist, entomologist; U. of California, Santa Barbara, Dept. of Biological Sciences

Correspondence • 1963: (4) • 1989: (2) • 1990: (3)

Davenport, George $\mathbf{E}$. Bioscript • Botanical Album

Davenport, H. A. Stain Technology, Biological Stain Commission, Editor, Itasca, II Correspondence • 1973: (2)

Daveaport, Larry Stanford U., Dept. of Biology Correspondence • 1988: (2)

Davenport, W. W. Correspondence $\bullet$ 1943: (1)

David, Armand (1826-1900) Bioscript

David, Charles W. Longwood Library, Director Correspondence • 1965: (1)

Davidse, Jeany Missouri Botanical Garden, St. Louis, MO, Symposium Secretary Correspondence • 1989: (2)

Davidson, Anstruther (1860-1932) Scottish-born American physician, botanist Bioscript • Botanical Album Correspondence - 1929-1933: (1)

Davidson, George (1825-1911) American geographer
Biofile • Bioscript
Davidson, James Fraser Tulane U., New Orleans, LA, Newcomb College, New Orleans

Correspondence • 1976: (2)

Davidson, John Fraser (1911- ) Scottish-born American plant taxonomist; U. of Nebraska, Dept. of Botany Correspondence $\bullet$ 1967: (1)

Davidson, Merritt T. Correspondence $\bullet$ 1929-1933: (1)

Davidson, Robert A. (1927-) American taxonomic botanist; U. of Iowa, Iowa City, Dept. of Botany; U.S.D.A. New Crops Research Branch; The Catholic U. of America, Washington, D.C., Dept. of Biology, Asst. Prof. and Curator Correspondence $\bullet$ 1960: (5) • 1962: (3)

Davies, Gordon L. Bioscript

Davies, R. E. Correspondence $\bullet$ 1985: (1) • 1986: (2)

Davis, Alva Raymond (1887- ) American plant physiologist Biofile $\bullet$ Bioscript $\bullet$ Botanical Album

Davis, Arthur C. Automobile Club of Southern California Correspondence • 1929-1933: (1)

Davis, Carol Correspondence $\bullet$ 1976: (1)

Davis, Celia D. C. Heath and Company, Publishers Correspondence $\bullet$ 1957: (1)

Davis, Charles Tulane U., New Orleans, Dept. of History Correspondence • 1964: (2) • 1973: (1)

Davis, Earl Editor of the Seminar Museum Correspondence • 1942: (1) - 1944: (1)

Davis, Edward Lyon (1929- ) American botanist; U. of Massachusetts, Amherst, Dept. of Botany Correspondence • 1957: (3) • 1959: (3)

Davis, Harlan Davis, CA Correspondence $\bullet$ 1979: (1)

Davis, Helen Burns U. of Vermont, Burlington Correspondence • 1939: (1)

Davis, James Botanical Album

Davis, John Henry, Jr. (1901-) American plant ecologist; U. of Florida, Gainesville, Dept. of Botany Correspondence • 1952: (9) - 1953: (10) 1954 (2) 1961: (1) "Rocky Mountain Vegetation" 1 1965: (2) 1970: (1)

Davis, Julia Finette Colonial Williamsburg, VA Correspondence • 1972: (3) Barton, Benjamin Smith, 1766-1815 - 1973: (2) Letter from Edwin F. Steffek • 1975: (1) • 1976: (2)

Davis, Kathryn Tulane U., New Orleans, Office of the President Correspondence $\bullet$ 1976: (1)

Davis, Lambert U. of North Carolina Press, Chapel Hill, Director Correspondence • 1961: (0) Banister, John, 1650-1692; Letter filed under Brita Stina Norden-Petterson

Davis, Mervyn T. Victoria, Australia Correspondence $\bullet$ 1974: (5) • 1977: (1) • 1978: (1)

Davis, P. Hancock Museum, Newcastle-upon-Tyne Correspondence • 1986: (1) 
Davis, Peter Hadland (1918-1992) English taxonomist, plant collector; U. of Edinburgh, Royal Botanic Garden Correspondence $\bullet$ 1955: (2)

Davis, Ray Joseph (1895-1984) U. of Idaho, Pocatello, Herbarium

Correspondence $\bullet$ 1938: (4) Epling, Carl Clawson, 1894-1968 - 1938: (0) Filed under Carl Clawson Epling • 1939: (6) • 1940: (1) • 1941: (4) • 1942: (1) • 1943: (4) • 1949: (1) 1960: (1)

Davis, Richard Beale U. of Tennessee, Knoxville, Dept. of English

Botanical Album

Correspondence • 1952: (14) - 1953: (7) Pursh, Frederick, 1774-1820 • 1954: (5) • 1955: (3) • 1956: (2) • 1957: (1) - 1959: (1) • 1960: (3) Banister, John, 1650-1692 • 1961: (3) Banister, John, 1650-1692 • 1962: (3) • 1963: (6) • 1965: (5) • 1966: (9) Banister, John, 1650-1692 • 1975: (8) $\bullet$ 1977: (1)

Davis, Richard C. The Encyclopedia of American Forest and Conservation History, Editor Correspondence $\bullet$ 1980: (2)

Davis, Richard H. U. of Arkansas, Fayetteville. Dept. of Botany and Bacteriology Correspondence $\bullet$ 1977: (2) Delphinium

Davis, Richard L. Correspondence • 1976: (1)

Davis, William Morris (1850-1934) American physiographer, meteorologist Bioscript

Davis, William Thompson (1862-1945) Botanical Album

Davison, Sara E. Rutgers U., graduate student in botany; Philadelphia Botanical Club; The Nature Conservancy; student of Ewan Correspondence • 1978: (2) • 1979: (2) • 1986: (2)

Davy, Humphry, Sir (1778-1829) English chemist Biofile

Davy, Joseph Burtt (1870-1940) English plant collector, taxonomist, forester Bioscript $\bullet$ Botanical Album

Davy, Norman Bioscript

Dawe, Morley Thomas (1880-1943) English forester, horticulturist Bioscript

Dawes Arboretum Correspondence $\bullet$ 1982: (9)

Dawes, C. Burr The Dawes Arboretum, Newark, OH, Chairman-Director

Botanical Album Correspondence • 1970: (4) • 1972: (3) Species • 1978; (2) $\bullet$ 1983: (1)

Dawson, Charles ( -1916) Bioscript

Dawson, Elmer Yale (1918-1966) American marine biologist

Biofile $\bullet$ Bioscript $\bullet$ Botanical Album

Correspondence • 1949: (1) • 1950: (2) • 1951: (4) • 1952: (4) • 1953: (2) • 1955: (1) • 1956: (7) • 1957: (4) • 1965: (2)
Dawson, Glen Dawson's Book Shop, Los Angeles, CA Correspondence • 1962: (1) • 1983: (3) • 1989: (1) • 1992: (1)

Dawson, Jackson Thornton (1841-1916) English-born American horticulturist, administrator, U. S. A. Biofile

Dawson, Mary R. Carnegie Museum of Natural History, Botany Search Committee Correspondence $\bullet$ 1976: (0) Filed under Carnegie Museum of Natural History

Dawson, W. R. Dawson's Book Shop Correspondence • 1966: (5) Book collectors

Dawson, Warren R. (1889-1968)

Bioscript

Correspondence • 1956: (4) • 1957: (8) • 1958: (15)

Banks, Joseph, Sir, 1743-1800 • 1959: (3) Banks, Joseph, Sir, 1743-1800 • 1964: (9) Banks, Joseph, Sir, 1743-1800 - 1965: (9) • 1968: (3)

Dawson, William Leon American ornithological author Biofile

Dawson's Book Shop Antiquarian Booksellers, Los Angeles, CA

See also Glen Dawson; Warren R. Dawson Correspondence • 1959: (1) • 1988: (1)

Day, Mary Anna (1852-1924) American botanical librarian and botanist Biofile $\bullet$ Bioscript $\bullet$ Botanical Album

Dayton, William Adams (1885-1958) American forester; U.S. Dept. of Agriculture, Forestry Service Biofile $\bullet$ Botanical Album

Correspondence • 1945: (3) • 1947: (1) • 1949: (6) • 1950: (6) • 1951: (1) • 1952: (6) Delphinium • 1953: (12) Vismia; Harper, Francis, 1886-1972; Maxon, William Ralph, 1877-1948 • 1955: (4) • 1956: (2) • 1957: (4) • 1958: (1)

DeAbata, Juan Museo Nacional, San Jose, Costa Rica Correspondence $\bullet$ 1960: (1) $\bullet$ 1967: (1)

Deam, Charles Clemon (1865-1953) American taxonomist, forester; author of Flora of Indiana Bioscript Correspondence $\bullet$ 1948: (4)

Deam, Hubert W. Correspondence • 1960: (0) Filed under George Booth Van Schaack

Dean, Bashford (1867-1928) American zoologist Bioscript

Dean, Paul M. Correspondence $\bullet$ 1938: (1)

Dean, R. E. Royal Botanic Gardens, Port of Spain, Trinidad Botanical Album Correspondence • 1939: (3) • 1945: (1)

Deane, Walter (1848-1930) American botanist and ornithologist Bioscript $\bullet$ Botanical Album

Dearborn, Ned H. American Committee for Democracy and Intellectual Freedom Correspondence $\bullet$ 1941: (2)

Dearness, John (1852-1954) Canadian botanist Biofile 
DeBeer, Gavin Rylands, Sir (1899-) Zoologist; British Muscum (Nanural History), Director Correspondence • 1959: (1)

deBerneaud, Arsenne Thiebaut Blascript

Delirahm, John G. W. Bloscripi

DeBrahm, William Biascript

DeBruhl, Marshall Dictionary of Scientific Biography Correspondence • 1967: (4) • 1976: (4) Merrill, Elmer Drew, 1876-1956 - 1976: (0) Filed under Dictionary of Scientific Biography $\bullet 1977$ : (0) Letter filed with Charles C. Gillispie • 1978: (1) • 1980: (1)

DeBuhr, Larry Eugene (1948-) Correspondence $\bullet 1977$ : (2)

Decalisne, J. Bioscript

DeCandolle, Alphonse See Candolle, Alphonse de (1806-1893)

DeCaro, J. New York Botanical Garden, Sec. to Boris Alexander Krukoff Correspondence - 1943: (0) Filed with Boris Alexander Krukoff

Decker, Jane MeLaughlin Wood anatomist; Wesleyan U., $\mathrm{OH}$

Correspondence • 1982: (4)

Decker, John Botanical Album

Dee, E. M. McGraw Hill Book Company Correspondence $\bullet 1947$ : (1)

Deener, David R. Tulane U., New Orleans, Dean; U. Council on Research, Chairman Correspondence • 1967: (2) • 1968: (1) • 1969: (1) • 1972: (1) • 1973: (2)

Dees, Bowen C. National Science Foundation, Division of Scientific Personnel \& Education, Program Director for Fellowships

Correspondence • 1953; (3)

Dees, Sarah S. Science, American Assoc. for the Cavancement of Science, Washington, D.C., Book Reviews Correspondence 1958: (2) • 1961: (10) • 1962: (15)

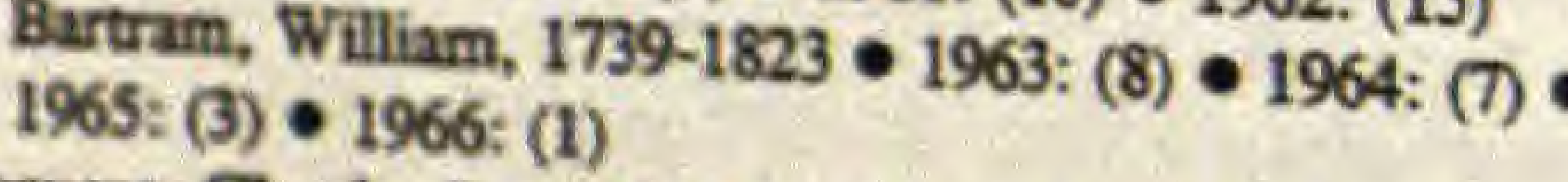

Deevers, Charles Lee (1900-) Mississippi College, Clinton
Botanical Album Correspondence • 1956: (2)

DeFilipps, Robert Anthony (1939) American plant Corservation Unit Bioscript

Correspondence • 1984: (1) • 1989: (4) Blascript

DeFiorest, Howard (1877-1946) American plant ecologist, plant physiologist; U. of Southern California ecologist, Correspondence • 1939: (1)

Degener, Otto (1899-1988) American botanist Biofile • Bioscript $\bullet$ Botanical Album Correspondence $\bullet 1971$ : (1) • 1974: (3)
DeGurse, John, Jr. Smithsonian Institution Correspondence $\bullet$ 1963: (1)

Deichmann, Elisabeth Harvard, Museum of Comparative Zoology, Div. of Marine Invertebrates Correspondence $\bullet$ 1957: (1)

Deignan, H. G. Biological Society of Washington, U. S. National Museum, Washington, D.C. Correspondence $\bullet$ 1951: (1)

Deiro, Paul, Mrs. Correspondence $\bullet$ 1951: (2)

Deiss, William A. Smithsonian Institution Archives Correspondence • 1984: (4) • 1985: (1) • 1987: (1) • 1989: (2) • 1990: (1)

DeKay, James E. Bioscript

Delacour, Jean T. (1890-) French zoologist Biofile

Delafield, Engene L.

Correspondence • 1950: (1) • 1951: (5) Barton, Benjamin Smith, 1766-1815

Delafield, John Correspondence $\bullet$ 1972: (1)

Delafield, Violetta Susan Elizabeth A. (1875-) Bioscript

Delassize, Nicolas Bioscript

Delattre, Pierre Adolphe (1805-1859) Bioscript

Delavay, (Abbe) Pierre Jean Marie (1834-1895) French missionary and plant collector in China Bioscript

Delcourt, Hazel $\mathbf{R}$. Correspondence $\bullet$ 1973: (2)

Delendick, T. J. Brooklyn Botanic Garden Correspondence • 1984: (1)

Delessert, Benjamin (1773-1847) French banker, amateur naturalist Biofile • Bioscript

Delessert, François Marie (1780-1868) Bioscript

Delevoryas, Theodore (1929- ) American taxonomist, plant morphologist; Yale Universtiy, Dept. of Zoology Correspondence • 1967: (2)

Delgado, Isaac, Museum of Art, New Orleans Correspondence • 1964: (2)

Delile, Alire Raffeneau (1778-1850) French botanist and physician Bioscript • Botanical Album

Dellenbaugh, Frederick Samuel (1853-1935) American explorer, artist Bioscript

Delo, David M. Scientific Manpower Branch, War Dept. Correspondence 1 1947: (1)

Delorme, S. Centre International de Synthese, Paris Correspondence $\bullet$ 1968: (1)

Delpino, $\mathbf{F}$. Bioscript

DelRio, Andres Manuel Bioscript 
Demaree, Delzie (1889-1987) American botanist, plant ecologist; Arkansas State College, Monticello

Biofile $\bullet$ Bioscript

Correspondence • 1938: (1) • 1940: (1) • 1941: (2) 1944: (1) • 1946: (1) • 1948: (1) • 1952: (2) Delphinium - 1955: (1) • 1960: (2) • 1963: (4) $\bullet$ 1964: (14) 1965 : $(27) \bullet 1966:(11) \bullet 1967:$ (3) $\bullet$ 1968: (8) • 1970: (2) • 1974: (1) • 1976: (1) - 1978: (1)

Demetrio, Charles Herman (1845-1936) German bryologist, clergyman

Bioscript

DeMilt, Clara M. Tulane U., New Orleans, Newcomb College

Correspondence • 1949: (1) • 1953: (1)

Demonte, Etienne Botanical Album

Demonte, Rosália Botanical Album (Filed under Etienne Demonte)

Demonte, Yvonne Botanical Album (Filed under Etienne Demonte)

Dempster, Everett Ross (1903-) American geneticist; U. of California, Berkeley; Orinda, CA; wife is Lauramay Tinsley Dempster

Botanical Album

Correspondence $\bullet$ 1937: (0) Filed under Lauramay Tinsley Dempster $\bullet$ 1938: (2) $\bullet$ 1949: (2) $\bullet$ 1950: (2) $\bullet$ 1955: (2) - 1957: (2) • 1970: (0) Filed under Lauramay Tinsley Dempster • 1979: (0) Filed under Lauramay Tinsley Dempster

Dempster, Lauramay Tinsley (1905-) American plant taxonomist; U. of California, Berkeley; husband is Everett Ross Dempster

Bioscript

Correspondence $\bullet$ 1937: (4) $\bullet$ 1939: (3) $\bullet$ 1940: (2) 1941: (2) • 1945: (1) • 1948: (1) • 1955: (5) $\bullet$ 1956: (2) 1963: (1) • 1964: (4) • 1965: (1) • 1970: (1) • 1973: (4) • 1974: (1) - 1977: (1) • 1978: (1) 1979: (3) • 1980: (1) • 1981: (1) - 1983: (1) • 1984: (1) • 1985: (1) • 1987: (1) 1989: (1) • 1993: (3)

Demsey, $\mathbf{M}$. Correspondence $\bullet$ 1972: (2)

Denison, Charles Botanical Album

Denisse, Étienne (fl. 1843-1846) French artist Bioscript

Denke, Christian Frederick (1775-1838) Moravian-born clergyman and botanist of Canada Biofile $\bullet$ Bioscript

Denman, N. S. Correspondence $\bullet$ 1963: (1)

Dennes, G. E. Bioscript

Dennie, Joseph Bioscript

Dennstedt, August Wilhelm (1776-1826) German physician, merchant, botanist Biofile

Denny, Margaret Correspondence • 1954: (4) • 1955: (1) • 1956: (1)

Denslow, W. W. Bioscript
Denton, Melinda Fay (1944) American Society of Plant Taxonomists, President

Correspondence $\bullet$ 1991: (0) Filed under Emanuel David Rudolph

Denver, Colorado Botanical Album

Denver Botanic Garden Correspondence - 1976: (3) Rocky Mountain Naturalists

Denver Museum of Natural History Correspondence • 1976: (3) Rocky Mountain Naturalists

Denver Public Library Correspondence $\bullet$ 1941: (1)

Denver University Denver; Librarian Correspondence • 1976: (1) Rocky Mountain Naturalists

DePoe, Charles E. Northeast Louisiana State College, Monroe Correspondence $\bullet$ 1966: (2) • 1967: (2) • 1970: (1)

Depp, Bonnie U. of Illinois Press, Urbana, Assistant Editor Correspondence • 1970: (13) Banister, John, 1650-1692

Deppe, Ferdinand (1794-1867) Bioscript

Deramus, Rebecca (1939-) American taxonomist; Old Dominion College Correspondence $\bullet$ 1968: (1)

Derbes, Vincent J. Tulane U., New Orleans, School of Medicine Biofile Correspondence $\bullet$ 1953: (4) $\bullet$ 1954: (2) 1 1955: (1) $\bullet$ 1957: (2) • 1961: (1) • 1962: (3) • 1971: (2) • 1974: (1) • 1975: (2) • 1979: (2) • 1982: (2)

Derby, Edward Smith, 13th Earl (1775-1851)

English biologist; Merseyside, England

Biofile • Bioscript

Correspondence $\bullet 1977:$ (3)

Derby, George Horatio (1823-1861) Bioscript

Derby, J. Bioscript

Derry, C. W. Bioscript

Desborough, L. O. Victoria U., Wellington, New Zealand, Registrar Correspondence $\bullet$ 1970: (3)

Deschamps, L. A. Bioscript

Descole, Horacio Raúl (1910-) Miguel Lillo Institute, Tucumán, Argentina

Botanical Album

Correspondence $\bullet$ 1940: (4) • 1941: (4) $\bullet$ 1942: (2) $\bullet$ 1945: (1) • 1947: (2) • 1948: (1)

Descourtilz, Michael Étienne (1775-1836) French physician, traveller, naturalist Bioscript

DeSelm, Henry Rowle (1924-) American plant ecologist; U. of Tennessee, Knoxville, Dept. of Botany; nickname "Hal"

Correspondence $\bullet$ 1964: (1) $\bullet$ 1968: (1) $\bullet$ 1977: (2) $\bullet$ 1986: (2)

Desert Magazine Correspondence $\bullet$ 1942: (3) 
Desfontaines, R. L.

Bioscript

Deshler, D.

Bioscript

Desmond, Betty Husband is Ray Desmond

Correspondence $\bullet 1978$ : (0) Correspondence filed under Ray Desmond

Desmond, Margaret Artist, architect Correspondence $\bullet 1970$ : (1)

Desmond, Ray Royal Botanic Gardens Kew, Librarian Bioscript • Botanical Album

Correspondence $\bullet$ 1964: (6) • 1965: (2) • 1968: (3) •

1969: (0) Letter filed under Clutton, George, Sir $\bullet 1969$ :

(10) Book collectors; $\bullet 1970$ : (12) Book collectors;

Lawrence, George Hill Mathewson, 1910-1978 • 1971: (10)

- 1972: (7) Lawrence, George Hill Mathewson, 1910-1978

- 1973: (4) • 1974: (5) • 1975: (5) • 1976: (4) • 1977: (8)

- 1978: (9) • 1979: (2) • 1980: (17) • 1981: (9) • 1982:

(5) • 1983: (11) • 1984: (10) • 1985: (3) • 1986: (13) •

1987: (3) - 1988: (6) Barton, Benjamin Smith, 1766-1815

- 1989: (9) • 1989: (0) Letter filed under Emanuel David

Rudolph • 1990: (11) • 1991: (9) • 1991: (0) Letter filed under Emanuel David Rudolph • 1992: (6) • 1993: (8)

DeSoto, Hernando (ca. 1500-1542)

Bioscript

Despard, Caroline G. Smithsonian, Pictures Editor Correspondence • 1984: (1) Bartram, William, 1739-1823

Desvaux, A. N. Bioscript

DeTerra, Hellmut (1900-) German-born geologist; Columbia U., New York, Dept. of Geology Botanical Album Correspondence • 1958: (3) - 1961: (1)

Detling, LeRoy Ellsworth (1898-1967) American taxonomist; U. of Oregon, Eugene Correspondence • 1935: (1) - 1936: • 1939: (5) Species: letter to T. D. A. Cockerell $\bullet$ 1941: (4) $\bullet$ 1943: (1)

DeToni, Giuseppe (1907-1950) Italian algologist Biofile

Detweiler, J. M.

Botanical Album

Detwiller, Henry (1795-1887)

Biascript

Deval, Gyorgy L. Kosuth U., Hungary, Ecological Intitute Correspondence $\bullet$ 1986: (1)

DeVille, Winston New Orleans, LA Correspondence $\bullet$ 1964: (1) • 1965: (1) • 1977: (3)

Devlin, Luella U. of Colorado, Boulder, Dept. of Biology Correspondence • 1944: (1)

DeVolls, James Correspondence • 1976: (1)

Devore, James K. Hematologist; American Rhododendron Society Meeting: Langston Medical Group, Oklahoma City,
OK

Correspondence $\bullet 1979$ : (2)

Dewar, D.

Bioscript

Dewees, William P.

Bioscript

Dewey, Chester

Bioscript
Dewey, Harry National Agricultural Library Correspondence $\bullet$ 1984: (0) Letter filed under Ray Desmond

DeWolf, Gordon Parker, Jr. (1927-) American taxonomist Biofile $\bullet$ Bioscript $\bullet$ Botanical Album Correspondence $\bullet$ 1949: (4) $\bullet$ 1950: (8) $\bullet$ 1951: (9) 1952: (16) - 1953: (14) Pennell, Francis Whittier, 1886-1952; "Rocky Mountain Vegetation"; BiologyInstruction and study $\bullet$ 1954: (3) $\bullet$ 1956: (2) $\bullet$ 1957: (7) $\bullet$ 1958: (12) • 1959: (4) Species $\bullet$ 1960: (2) $\bullet$ 1961: (7) $\bullet$ 1962: (5) • 1963: (4) • 1964: (2) • 1967: (3) Eastwood, Alice, 1859-1953 • 1968: (2) • 1969: (2) • 1970: (2) • 1977: (1) • 1982: (1)

Dexter, Ralph Warren (1912-) American natural historian; Kent State U., Kent, OH, Dept. Biology

Botanical Album

Correspondence $\bullet$ 1970: (4) 1 1974: (2) Harper, Francis, 1886-1972 • 1979: (1) • 1982: (7) • 1986: (1) • 1988: (4) - 1989: (1) • 1990: (1)

Dibble, Alison C. U. of Maine, Orono, Dept. of Botany and Plant Pathology

Correspondence $\bullet$ 1990: (2)

Dibner, Bern Burndy Library, Norwalk, CT Correspondence $\bullet$ 1984: (2) Letter from Frans Verdoorn

Dick, Everett Botanical Album

Dick, Hugh G. U. of California, Los Angeles, Dept. of English Correspondence $\bullet 1961:$ (2)

Dick, William (ca. 1820-1830) Bioscript

Dickerman, Carolyn U. of New Mexico, Albuquerque, Library Correspondence $\bullet$ 1983: (1)

Dickerman, M. B. Forest Service Correspondence $\bullet$ 1975: (1)

Dickerson, Bettie C. Arkansas Polytechnic College Correspondence $\bullet$ 1975: (2)

Dickerson, George W. Correspondence $\bullet$ 1965: (2)

Dickey, Parker A. Carter Oil Company, Tulsa, OK; Head of Geological Research Correspondence $\bullet$ 1952: (2)

Dickinson, Lenore M. Librarian; Harvard U., Arnold-Gray Herbarium, Harvard Correspondence $\bullet$ 1977: (2) • 1979: (6)

Dickinson, Samuel D. Translator of Bossu's Nouveaux Voyages; Prescott, AK Correspondence • 1980: (15) • 1981: (8) • 1982 (10) 1983: (4) • 1984: (4)

Dickinson, Vanessa Arizona Game and Fish Dept. Correspondence $\bullet$ 1993: (1)

Dickmann, Donald I. Plant Physiologist; Institute of Forest Genetics, Rhinelander, WI Correspondence • 1970: (1)

Dickson, Alexander Bioscript

Dickson, James (1738-1822) Bioscript 
Dictionary of American Biography Cambridge, MA; Edward T. James, editor Correspondence • 1969: (3) • 1972: (2) • 1973: (5)

Dictionary of American History Charles Scribner's Sons Correspondence $\bullet$ 1974: (2)

Dictionary of American Regional English The American Dialect Dictionary; Frederic G. Cassidy, Director-Editor "DARE"

Correspondence $\bullet$ 1980: (1)

Dictionary of Scientific Biography Charles Scribner's Sons, New York, NY; Charles C. Gillispie, Editor-in-chief; Marshall De Bruhl

Correspondence $\bullet 1968$ (12) $\bullet$ 1969: (11) $\bullet$ 1970: (9) 1971: (15) - 1972: (3) Pursh, Frederick, 1774-1820; Setchell, William Albert, 1864-1943 - 1973: (6) - 1974: (10) - 1976: (6) Merrill, Elmer Drew, 1876-1956

Dieck, Georg (1847-1925) German botanist Bioscript

Diefenbacher, Marguarite H. Fairfield, NY Correspondence $\bullet$ 1969: (0) Letter filed under Richard Sumner Cowan

Dieffenbach, Kenneth Correspondence $\bullet$ 1980: (1)

Diehl, I. E. Editor and printer, plant collector; Salt Lake City, UT Bioscript

Diehl, Katherine Correspondence - 1982: (3)

Diehl, William Webster (1891- ) American mycologist, taxonomist; Smithsonian Institution; U. S. Dept. of Agriculture Botanical Album Correspondence • 1946: (1) • 1948: (3) • 1949: (6)

Diels, Ludwig (1874-1945) German botanist; Universităt Berlin

Bioscript • Botanical Album Correspondence - 1939: (4) Species

Diemer, M. E. Diemer Photographic Laboratory Correspondence $\bullet$ 1935: (2) • 1936: (2)

Diener, Richard L. U. S. Air Force Correspondence $\bullet$ 1951: (1) • 1952: (10) $\bullet$ 1956: (2)

Dietrich, F. G. Bioscript

Dietrich, Werner (1938- ) Botanisches Institut, Dusseldorf, Germany Correspondence • 1978: (0) Letter filed under Peter Hamilton Raven

Diffenderfer, W. L. Surgeon and naturalist; Pope's expedition Bioscript

Diggs, Martin Lee-Jackson School, Mathews, VA Correspondence $\bullet$ 1958: (2)

Dike, David H. U. of Southwestern Louisiana, Lafayette, graduate student Correspondence $\bullet$ 1968: (2)

Dill, Joyce Correspondence - 1990: (1)

Dillen, Jon Jacob (1684-1747) See Dillenius, John James

Dillenius, See Dillenius, John James
Dillenius, J. J.

See Dillenius, John James

Dillenius, John James (Johann Jakob) German-born artist, illustrator

Biofile $\bullet$ Bioscript $\bullet$ Botanical Album

Dillon, Richard H. Librarian, author; Sutro Library, A

Branch of the California State Library, San Francisco, CA; U. of Hawaii

Biofile $\bullet$ Bioscript $\bullet$ Botanical Album

Correspondence - 1955: (1) 1960 : (2) Banks, Joseph,

Sir, 1743-1800 • 1961: (5) • 1962: (17) • 1963: (7) •1964:

(5) $\bullet$ 1965: (3) • 1966: (5) • 1967: (6) • 1968: (1) • 1969:

(3) $\bullet$ 1970: (6) • 1971: (5) • 1972: (1) • 1973: (3) • 1974:

(4) $\bullet$ 1975: (6) • 1976: (2) $\bullet$ 1978: (12) $\bullet$ 1980: (6) $\bullet$

1981: (6) 1982: (2) • 1985: (6) • 1986: (11) • 1987: (4)

- 1988: (9) • 1989: (5) • 1990: (3) • 1991: (1)

Dillwyn, Lewis Weston (1778-1855)

Bioscript

Diment, Judith A. British Museum (Natural History);

Botany Librarian

Correspondence • 1979: (4) • 1980: (7) • 1981: (3) • 1984: (12) • 1985: (4) • 1986: (7)

Dimond, Albert Eugene (1914 ) American plant pathologist; XI International Botanical Congress, Inc., Treasurer

Correspondence • 1967: (3) Letter to H. G. Baker - 1968: (5) 1969 : (3) One letter from Dimond is to George W. Fischer

Dingle, Herbert Bioscript

Dinter, Curt (1868-1945) German phytogeographer, taxonomist; S. W. Africa Bioscript

Dinwiddie, Evelyn Sanderson Bioscript

Ditmars, Raymond Lee (1876-) American zoologist Botanical Album

Dixmier, Edith Correspondence • 1968: (1)

Dixon, Henry Horatio (1869-1953) Irish plant physiologist; Trinity College, Dublin, School of Botany Correspondence • 1942: (1)

Dixon, Vern Correspondence $\bullet$ 1951: (2)

Dixon, William Bioscript

Doak, Clifton Childress (1895- ) American biologist Correspondence • 1968: (2)

Doak, Esther Larsen See Larsen, Esther Louise

Doak, Kenneth D. (1903-) American plant pathologist; wife is Esther Larsen Doak Correspondence • 1985: (2)

Doane, Rennie Wilbur (1871-1942) American zoologist Bioscript

Dobbie, Lucie E. N. U. of California Press, Executive Editor Correspondence • 1956: (2)

Dobbs, Arthur (1689-1765) Governor of North Carolina Biofile 
Dobbs, Raymond Joseph (1883-) Miami, FL Bioscript

Correspondence • 1938: (9) • 1942: (2) • 1962: (2) • 1963: (3) - 1964: (3)

Dobes, Martha E. Correspondence • 1983: (2)

Dobie, Jim Correspondence • 1962: (1)

Dobszhansky, Theodosius (1900-) Russian-born biologist; U. of California, Davis Bioscript • Botanical Album Correspondence • 1940: (3) • 1972: (2)

Dodds, Gideon Stanhope (1880-) American zoologist, anatomist Bioscript

Dodds, Robert E. Correspondence • 1954: (3)

Dodge, Bernard Ogilvie (1872-1960) American mycologist, plant pathologist Biofile

Dodge, Carroll William (1895-1988) American mycologist Biofile • Bioscript

Dodge, Charles Richards (1847-1918) American botanist Biofile

Dodge, Edgar A. ( -1933) American entomological collector Bioscript

Dodge, Ernest Stanley Peabody Museum of Salem, Director, Salem, MA Correspondence • 1960: (1) • 1974: (4)

Dodge, Henry Bioscript

Dodge, John V. Encyclopaedia Britannica, Chicago, II Correspondence $\bullet$ 1958: (1)

Dodgson, Charles Lutwidge Botanical Album

Dodson, Calaway H. (1928-) Botanical Album Correspondence • 1969: (2) Biology-Instruction and study
- 1970: (1)

Dodson, Carolyn Campbell U. of New Mexico, General Library

Correspondence • 1986: (1) • 1990: (3) • 1991: (10) • 1992: (1)

Dodson, Wulliam Rufus (1867-) American botanist, agronomist Bioscript

Dole, Elizabeth Hanford Dept. of Transportation Correspondence • 1985: (1)

Dombey, Joseph

Bioscript

Dommick, L. N.

Bioscript

Domning, Daryl P. U. of California, Berkeley, Dept. of Paleontology; Manaus, Brazil; Howard U.

Bioscript - Botanical Album

Correspondence • 1968: (3) • 1969: (2) • 1970: (2) • 1973: (1) • 1976: (2) • 1977: (1) • 1981: (2) Barton, Benjamin Smith, 1766-1815 • 1983: (1) • 1992: (1)

Don, David Bioscript
Don, George

Biofile $\bullet$ Bioscript

Donaldson, J.

Botanical Album

Donarelli, C. Bioscript

Donat, Arturo (1893-1937) Biofile

Donelan, E. A. William A. Gray Company (Insurance) Correspondence $\bullet$ 1945: (1)

Donk, Marinus Anton (1908-1972) Taxonomist, mycologist; Netherlands

Botanical Album

Donkin, Robin Jesus College Correspondence • 1991: (3)

Donn, James Bioscript

Donnelley, Gaylord Lakeside Press Correspondence $\bullet$ 1966: (1)

Donovan, William A. Correspondence $\bullet$ 1951: (2)

Donzelot, Pierre Services du Consieller Culturel, Ambassade de France Correspondence $\bullet$ 1956: (3)

Dore, William George (1912-) Canadian agronomist, taxonomist; Plant Research Institute, Experimental Farm, Ottawa, Ontario Bioscript • Botanical Album Correspondence • 1969: (2) • 1970: (8) • 1972: (1)

Dormon, Caroline (1888-1971) American botanical artist, botanist, horticulturist; Saline, LA

Biofile $\bullet$ Bioscript $\bullet$ Botanical Album

Correspondence $\bullet 1955$ (1) $\bullet$ 1957: (21) $\bullet$ 1958: (9) $\bullet$ 1959: (3) • 1960: (6) • 1962: (5) • 1963: (6) • 1963: (0)

Letters filed under Paul Bruce Dowling $\bullet$ 1964: (8) 1965 :

$(9) \bullet 1966:(1) \bullet 1967:$ (1) • 1968: (2) • 1969: (2) • 1971: (2) $\bullet$ 1976: (1)

Dorogostaiskaya, Eugenia Leningrad, Russia Botanical Album Correspondence • 1975: (1) • $1976 \bullet$ 1977: (2) • 1978: (2)

Dorogostaïsky, V. C. Bioscript

Dorr, Laurence J. (1953- ) American botanist; Smithsonian Institution, National Museum of Natural History; nickname "Larry"

\section{Biofile $\bullet$ Bioscript $\bullet$ Botanical Album}

Correspondence • 1983: (4) • 1984: (5) • 1985: (1) 1986: (1) • 1987: (10) • 1988: (9) • 1989: (6) Letter to Randoph S. Klein on Dorr • 1990: (0) Filed under Rudolph - 1990: (3) • 1991: (15) • 1992: (13) • 1993: (9)

Dorr, Sullivan (1778-1858) Bioscript

Dorsey, Maxwell Jay (1880-) American pomologist; U. of Illinois, Dept. of Horticulture, Professor of Horticulture, Emeritus Correspondence • 1959: (3)

Dorson, Richard M. U. of Indiana, Dept. of History Correspondence - 1963: (3) Letter from Francis Harper

Doty, Carol Correspondence • 1976: (1) 
Doty, Meng S. Honolulu, HI Correspondence $\bullet$ 1974: (2)

Dougan, Robert O. Librarian; Henry E. Huntington Library

Correspondence $\bullet$ 1960: (3) Book collectors $\bullet$ 1961: (2) Henry E. Huntington Library and Art Gallery

Doughty, Severn Horticulturist; United States Dept. of Agriculture; New Orleans

Correspondence • 1977: (1) • 1978: (3)

Douglas, Charles Ray Correspondence $\bullet$ 1974: (2)

Douglas, David (1799-1834) Scottish botanical explorer Biofile - Bioscript - Botanical Album

Douglas, Evalin F. U. of Kentucky Press, Lexington, Assistant Editor Correspondence • 1961: (1) Vismia

Douglas, Gina Librarian; Linnean Society of London Correspondence • 1985: (1) • 1987: (3) • 1988: (1) • 1989: (2) • 1990: (9) • 1991: (4) • 1993: (4)

Douglas, James Botanical Album

Douglas, Pete United States Fish and Wildlife Correspondence • 1978: (1)

Douglas, Robert (1813-1897) Bioscript

Douglas, William Bioscript

Douglass, Andrew Ellicott (1867-1962) American scientist, teacher Biofile

Douglass, David Bates (1790-1849) American plant collector on Cass' expedition to upper Mississippi Biofile

Douglass, Earl (1862-1931) Bioscript

Douglass, George Bioscript

Dovaston, John Freeman Milward (1782-1854) English poet, ornithologist Biofile

Dove, Lewis Correspondence $\bullet$ 1963: (2)

Dover Publications New York, NY Correspondence • 1951: (1) • 1971: (2) • 1976: (7) • 1989: (5)

Dowden, Anne Ophelia Todd See Todd, Anne Ophelia (1907-)

Dowler, Bennet (1797-1878) Biofile

Dowling, Paul Bruce Nature Conservancy, Washington, D. C., Assistant to Director Correspondence $\bullet 1962$ : (5) Letter from Dowling to Dormon, Caroline $\bullet$ 1963: (2) Letters from Caroline Dorman

Downie, Timothy Campbell (1830-1875) Bioscript

Downing, A. J. Bioscript

Downs, Arthur Channing, (1930- ) Newton Square, PA Correspondence $\bullet$ 1987: (2)
Downs, Hugh National Broadcasting Co., The Today Show

Correspondence • 1969: (0) Correspondence filed with George Wald

Downton, George ( -c. 1895) Plant collector; Venezuela Bioscript

Dozy, Franz (1807-1856) Dutch physician and botanist Bioscript

Drake, Daniel (1785-1852) American botanist Biofile • Bioscript

Drake, Francis, Sir (1540-1596) British administrator and navigator Biofile - Bioscript

Drake, J. Francis (1868-1937) Bioscript

Drake, Robert J. Bioscript

Drapalik, Donald Joseph (1934 ) Georgia Southern College, Statesboro

Correspondence • 1976: (1)

Draper, Benjamin California Academy of Sciences, San Francisco Correspondence • 1949: (4)

Drayton, Charles ( -1844) Bioscript

Drayton, John N. U. of Oklahoma Press Bioscript • Botanical Album Correspondence • 1992: (1)

Drayton, William Bioscript

Dreer, Henry Augustus (1818-1873) Bioscript

Drège, Carl Friedrich (1791-1867) German plant collector in South Africa Bioscript

Drège, Jean François (1794-1881) German plant collector in South Africa

Bioscript

Drent, Harry (1861?-) Dutch sailor, plant collector Bioscript

Drescole, H. R. Universidad Nacional de Tucumán Correspondence $\bullet$ 1954: (1)

Dresher, Barbara Biology student Correspondence 1948 : (1)

Dressler, Kerry Bioscript

Dressler, Robert Louis (1927-) American taxonomist, plant morphologist

Correspondence • 1968: (1) - 1983: (1) • 1990: (1)

Drew, Elmer Reginald (1865-1930) American physicist and plant collector Bioscript

Drew, Frank M. Amateur ornithologist Bioscript

Drew, William Brooks (1908-) American plant ecologist, plant pathologist, taxonomist; Michigan State U., East

Lansing

Botanical Album

Correspondence $\bullet$ 1941: (7) $\bullet$ 1942: (2) $\bullet$ 1943: (2) $\bullet$ 1945: (3) • 1946: (1) • 1947: (2) • 1948: (1) • 1956: (2) • 1957: (3) 


\section{Guide to the Ewan Papers}

Drealer, C. Bioscript

Dreyfous, George A.

Correspondence $\bullet$ 1955: (1)

Drinker, H. S. Academy of Natural Sciences of Philadelphia, Trustee

Correspondence $\bullet$ 1952: (0) Academy of Natural Sciences of Philadelphia; letter filed under Francis Harper

Drinkers, Mary E. Frontiers: A Magazine of Natural History, Editor

Correspondence $\bullet 1960$ : (4)

Drout, Francis Elliott (1907-1982) American taxonomist, plant collector

Botanical Album

Correspondence $\bullet 1958:$ (3) $\bullet 1975$ : (1)

Drowne, Solomon (1753-1834) American physician, agriculturist

Bioscript

Druce, George Claridge (1850-1932) English pharmacist, taxonomist, botanist

Biofile • Bioscript

Drummond, Thomas (1780-1835) Scottish botanist, bryologist

Biofile • Bioscript

Drury, Aubrey Save the Redwoods League Correspondence $\bullet$ 1942: (2)

Drury, Dru (1725-1803) British entomologist Biofile • Bioscript

Dryander, Jonas Carlsson (1748-1810) Swedish-born English botanist; librarian to Joseph Banks Bioscript

Dublin Botanic Garden Botanical Album

DuBois, Lyla U. of Wisconsin Press Correspondence $\bullet 1963:$ (1)

Dubriel, E. Bioscript

DuChaillu, Paul Belloni (1831-1903) Bioscript

Ducie Bioscript Ducke, Walter Adolpho (1876-1959) Italian-born Brazilian
botanist Biofile

Ducker, Sophie Charlotte (1909-) German-born taxonomist, phycologist; Australia Bioscript

Dudley, Benjamin W. Bioscript

Dudley, Paul (1673-1751) American jurist Bioscript Dudley, Wiliam Russel (1849-1911) American taxonomist,
botanist

Bioscript • Botanical Album

Duellman, William

Correspondence $\bullet$ 1991: (1)

Duerinek, Jean-Baptiste (1809-1 collector in America (1809-1857) Belgian-born plant Biofile - Bioscript
Duff, C. V. Cooper Ornithological Club, Hollywood, CA, Business Manager Correspondence $\bullet$ 1952: (2)

Duff, Lucille Correspondence • 1936: (4) Species

Duffield, A. J. Bioscript

Duffy, John Tulane U., New Orleans, LA, History of Medicine

Botanical Album

Correspondence • 1963: (2) • 1966: (1) Book collectors; letter from John Bowers • 1968: (1) • 1970: (3) Barton, Benjamin Smith, 1766-1815 • 1972: (1) • 1992: (3)

DuFlot de Mofras, Eugène (1810-1884) Bioscript

Dufour, J. M. Bioscript

Dufresne, Louis Bioscript

Dugand, Armando (1906-1971) Plant collector, Colombia; Institute de Ciencias Naturales, Bogotá, Colombia Bioscript • Botanical Album

Correspondence • 1945: (2) • 1946: (1) • 1947: (5) •

1948: (8) Letter from J. Ewan to Jane Waggeman 1949 : $(1) \bullet 1950:(3) \bullet 1952:(10) \bullet 1959:(3)$

Dugee, Alfred

Bioscript

Duggar, Benjamin Minge (1872-1956) American mycologist, physiologist

Bioscript

Duhamel du Monceau, Henri Louis (1700-1782) Bioscript

Duke, James A. (1929- ) American economic botanist Bioscript

Correspondence • 1961: (3) Van Schaack, George Booth, 1903-1983

Duke University Durham, NC Correspondence • 1959: (1)

Duke University Library Correspondence $\bullet$ 1952: (1) $\bullet 1981$

Dukeshire, William Correspondence $\bullet$ 1980: (1)

Dulau \& Company Ltd. Dealer in rare books and prints, London Correspondence - 1939: (1)

Dulberger, Rivka Tel-Aviv U., Israel, Dept. of Botany Correspondence $\bullet 1971$ : (1)

Dumbarton Oaks Washington, D.C. Correspondence $\bullet$ 1977: (1) $\bullet$ 1980: (1)

Dumont, Claudot Bioscript

Dumont de Courset, George Louis Marie (1746-1824) French agronomist Bioscript

Dumont D'Urville, Jules Sébastien César (1790-1842) Bioscript

Dumahoe, Dorothy H. National Research Council Correspondence • 1947: (1)

Dunal, M. F. Bioscript 
Dunant de Gallatin

Bioscript

Dunaway, Reginald American bookseller; St. Louis, MO Correspondence $\bullet$ 1990: (2) $\bullet$ 1993: (1)

Dumbar, Gary S. Author; U. of California, Los Angeles, Dept. of Geography

Biofile

Correspondence • 1959: (3) Banister, John, 1650-1692 •

1963: (8) • 1964: (5) Lyon, John, 1764-1814 • 1966: (2) • 1967: (5) • 1971: (4) • 1973: (3) • 1974: (3) • 1975: (1) • 1976: (3) Book collectors $\bullet$ 1977: (3) • 1978: (3) $\bullet$ 1983:

(2) $\bullet$ 1984: (1) • 1985: (4) • 1987: (1) • 1988: (1) • 1990: (2) $\bullet$ 1992: (3)

Dumbar, Viola Polytechnic Institute of Puerto Rico Botanical Album

Correspondence • 1942: (1)

Dumbar, William

Bioscript

Duncan, Carl U. of Georgia, Athens Correspondence $\bullet$ 1950: (2)

Duncan, F. G., Mrs. Arabia, LA Correspondence $\bullet$ 1974: (1)

Duncan, Frank, Mrs. New Orleans, LA

Correspondence • 1970: (2) Bartram, William, 1739-1823

Duncan, Marian

Correspondence $\bullet$ 1983: (1)

Duncan, Thomas

Correspondence • 1989: (1) • 1990: (1)

Duncan, Wilbur Howard (1910-) Taxonomist, botanist; U. of Georgia, Athens, Dept. of Botany

Botanical Album

Correspondence $\bullet$ 1951: (1) $\bullet$ 1952: (7) $\bullet$ 1955: (1) $\bullet$ 1956: (2) • 1957: (4) • 1958: (2) • 1961: (5) • 1962: (6) 1963: (3) • 1965: (5) $\bullet$ 1966: (7) Harper, Roland

McMillan, 1878-1966; Species • 1967: (8) • 1968: (4) • 1971: (3) • 1973: (4) • 1974: (2) • 1975: (1) • 1976: (1) • 1977: (7) • 1983: (1) • 1988: (1)

Dundas, Frederic Winn (1911- ) Bioscript

Dundas, James ( -1865)

Bioscript

Dundee, Dee Saunders

Bioscript

Correspondence $\bullet$ 1963: (1)

Dundee, Harold A. Tulane U., Dept. of Biology, Emeritus Botanical Album

Correspondence • 1982: (1) - 1989: (8) Barton, Benjamin Smith, 1766-1815; letters from Dundee to Flores included • 1993: (2)

Dunham, W. J.

Correspondence $\bullet$ 1968: (2)

Dunkle, Meryle Byron (1888-) Bioscript

Dunlap, Elizabeth F. Book and print dealer; St. Louis, MO

Correspondence - 1989: (1) 1992: (1)

Dunlap, William (1766-1839) Historian Biofile
Dumn, David Baxter (1917-) U. of Missouri, Columbia, Dept. of Botany, Herbarium Curator

Bioscript $\bullet$ Botanical Album

Correspondence $\bullet$ 1959: (1) • 1960: (3) • 1962: (1) • 1982: (1)

Dunn, George Washington (1814-1905) American plant collector

Bioscript

Dunn, Lynn Husband is Murray H. Dunn Correspondence $\bullet$ 1968: (3)

Dunn, Mary Eubanks Southern Methodist U., Dallas, TX Correspondence • 1975: (2) • 1976: (1) • 1977: (1)

Dunn, Michael Correspondence • 1975: (1) • 1976: (1)

Dumn, Murray H. Alhambra, CA Botanical Album

Correspondence • 1962: (1) • 1965: (1) • 1993: (1)

Dumn, Sterling New Orleans Botanic Garden Foundation, Acting President

Correspondence • 1962: (1)

Dunthorne, Gordon

Bioscript

DuPetit-Thouars, [L. M.] A

Bioscript

DuPont, Eleuthere Irene

Botanical Album (Filed under Pierre S. DuPont)

DuPont, Pierre S.

Botanical Album

Duprat, Gabrielle

Botanical Album

DuPratz, Le F.

Bioscript

Dupree, Anderson Hunter U. of California, Berkeley, Dept. of History

Bioscript

Correspondence • 1951: (1) - 1952: (4) Nuttall, Thomas, 1786-1859 • 1957: (3) • 1959: (1) • 1965: (4) • 1966: (1) Jepson, Willis Linn, 1867-1946

DuPriest, Margaret Donovan Antiquarian bookseller Botanical Album

Correspondence • 1969: (4) • 1970: (2) Book collectors • 1973: (2) • 1975: (1) • 1987: (4) • 1988: (3)

Duran, Victor Gershon (1897-) English-born plant collector in California; Berkeley, CA

Bioscript $\bullet$ Botanical Album

Correspondence 1949: (1)

Durand, Elias (1794-1873) French botanist, chemist Biofile $\bullet$ Bioscript $\bullet$ Botanical Album

Durando, Z. Bioscript

Dureau, Lionel D. Botanical Society of New Orleans Correspondence 1956: (1)

DuReitz, Gustaf Einar Botanical Album

Duren, William Larkin, Jr. (1905- ) American mathematician: Tulane U., New Orleans, Dept. of Mathematics

Correspondence $\bullet$ 1953: (1) $\bullet$ 1954: (1)

Durkan, Rena Amherst College, Amherst, MA Correspondence $\bullet$ 1950: (3) 
Durkee, Haroid Grinnell College

Correrpondence $\bullet 1992$ : (1)

Durket, Lenore T. Grinnell College

Correspondence $\bullet$ 1993: (1)

Durkin, William Brooklyn Botanic Garden Correspondence • 1950: (2) American Fern Society

Durner, Spence L.A. Dept. of Forestry Comerpondence • 1929-1933: (1)

Durnford, Elias Btascript

Durrell, Laurence Wood (1888-1970) American botanist, plass pathologist, Colorado Agricultural College Bloscript Corrempondence • 1941: (1)

Dusen, Karl Hjalmar (1855-1926) Swedish botanist Blofite

DuShane, Graham Science, Editor Correspondence • 1956: (2) • 1958: (3) • 1959: (2)

DuSimitiere, Pierre Eugene (1737-1784) Swiss-born American historical conservationist Biofile $\bullet$ Bloscript

Dusterdieck, F. W. Operkasit, Inc. Correspondence • 1961: (1)

Dutcher, Basil Hicks (1871-1922) Colonel, U. S. Army medical corps

Biascript

Duthie, Rena P. Bloscript

Dutilly, Arthime Antoine (1896-) Canadian-born botanist, biologist, clergyman; Catholic U., Arctic Institute Correspondence $\bullet$ 1943: (1)

Dutton, Clarence Edward (1841-1912) American geologist Bioscript

Dutton, Joan Colonial Williamsburg, Williamsburg, VA Correspondence • 1971: (3)

Dutton, Wulliam S. Correspondence • 1975: (1)

DuvalJouve, Joseph (1810-1883) French botanist Bioscript

Duvall, Allen J. Biological Society of Washington, Washington, D.C.

Correspondence • 1949: (4)

Dverhell, I. E. Bioscript

Dwight, Edward H. Museum of Art, Munson-WilliamsProctor Instiate, Utica, NY, Director Correspondence • 1961: (6) - 1962: (4) • 1963: (3) • 1964: (4) $\bullet 1965:(3) \bullet 1971:(4) \bullet 1976$ : (0) Filed under
Kathryn Proby

Dwight, $\mathbf{H}$. R. Bioscript

Dwyer, Joha Dumean (1915-) American taxonomist; Saint Louis U., St. Louis, MO Correspondence • 1958: (2) Dwyer, William G. Gulf-Park College, Gulfport, MS,
President Correspondence • 1956: (2)

Dyce, James Wood (1905) ) Scotrish-born pteridologist. England; British Pteridological Society Correspondence $\bullet 1965$ : (1) • 1970: (1)
Dyche, Lewis Lindsay (1857-1915) American zoologist Bioscript

Dyde, W. F. U. of Colorado, Boulder

Correspondence $\bullet 1946:$ (1)

Dyer, Frances T. Science Club, Newman School, New Orleans, LA

Correspondence $\bullet$ 1952: (1)

Dyer, John P. Tulane U., New Orleans Correspondence $\bullet$ 1953: (1) $\bullet$ 1954: (1)

Dyer, Margaret H. Husband is Edward M. Dyer Correspondence • 1968: (2) • 1969: (1)

Dyer, Robert Allen (1900-1987) South African botanist, taxonomist, plant ecologist Bioscript $\bullet$ Botanical Album

Dymond, John (1867-1932) Bioscript

Dyson, James Librarian; Loyola U. Correspondence $\bullet$ 1963: (1)

\section{E}

Eames, Arthur Johnson (1881-1969) American botanist, plant morphologist Botanical Album

Eames, Katherine Correspondence $\bullet$ 1941: (1)

Earle, Esther Louise Husband is Thomas Theron Earle Correspondence $\bullet$ 1972: (2) • 1986: (2)

Earle, Franklin Sumner (1856-1929) American mycologist, horticulturist, agronomist Biofile $\bullet$ Bioscript $\bullet$ Botanical Album

Earle, Ralph S. Counsellor at Law Correspondence $\bullet$ 1950: (2)

Earle, Thomas Theron Tulane U., New Orleans, Dept. of Botany; nickname "Tom"; wife is Esther Louise Earle Bioscript $\bullet$ Botanical Album Correspondence $\bullet$ 1949: (0) Letter filed under Wray M. Bowden • 1950: (3) • 1951: (1) • 1952: (6) • 1953: (1) Arnold Arboretum - 1954: (2) Royal Botanic Gardens, Kew; British Museum (Natural History), Dept. of Botany $\bullet$ 1955: (T) - 1958: (3) - 1960: (4) One letter filed under W. A. Feuillan, Jr. - 1961: (0) One letter filed under William Campbell Steere $\bullet$ 1961: (1) $\bullet$ 1962: (1) $\bullet$ 1962: (0) Letter filed under R. O. Flagg• 1963: (2) • 1964: (3) $\bullet$ 1965: (1) - 1967: (3) • 1969: (1) • 1972: (5) • 1974: (4) • 1978:

Earle, Thomas Theron (continued) (1) 1979: (1) • 1980: (3) • 1981: (2) Van Schaack, George Booth, 1903-1983 • 1982: (6) • 1983: (11) • 1984: (12) $\bullet 1985:$ (9)

Early American History and Culture, Institute of Williamsburg, VA Correspondence • 1981: (1)

Early, Eleazar Bioscript

Earthwatch See Center for Field Research

East, Edward Murray (1879-1938) American biologist, plant morphologist Biofile 
Eastern Oklahoma State Teachers College Ada, OK; Dept. of Biology

Correspondence • 1951: (1)

Eastman, J. S. Physician; Sante Fe, NM Bioscript

Eastman, William D. MacMillan Company, Executive Editor

Correspondence $\bullet$ 1970: (0) Filed under MacMillan Publishing Company, Inc.

Eastwood, Alice (1859-1953) Canadian-born American botanist; California Academy of Sciences

Biofile $\bullet$ Bioscript $\bullet$ Botanical Album

Correspondence $\bullet$ 1929-1933: (3) $\bullet$ 1935: (1) $\bullet$ 1936: (2) $\bullet$ 1940: (1) • 1941: (4) - 1942: (3) • 1943: (7) Species • 1946: (1) - 1949: (2) • 1950: (1)

Eaton, Amos (1776-1842) American naturalist and teacher Biofile • Bioscript

Eaton, Amos Beebe Bioscript

Eaton, Daniel Cady (1834-1895) American botanist Bioscript $\bullet$ Botanical Album

Eaton, Hezekiah Hulbert (1809-1832) American botanist Biofile - Bioscript

Eaton, J. H.

Bioscript

Eaton, Mary Emily (1873-1961) Botanical artist; U. S. A., England

Bioscript • Botanical Album

Eaton, Richard J. The New England Botanical Club Correspondence • 1939: (1)

Eawes, Katherine $\mathbf{H}$. Correspondence $\bullet$ 1940: (1)

Ebeling, Christoph Daniel (1741-1817) Biofile

Ebeling, Ora May Los Angeles, CA Correspondence • 1979: (1)

Eberhart, Sylvia Science Correspondence $\bullet$ 1966: (2)

Eberle, John (1788-1838) Bioscript

Echeverria, Atanasio Mexican botanical artist Bioscript

Eckenwalder, James E. (1949-) U. of California, Berkeley, Dept. of Botany Correspondence $\bullet$ 1976: (1)

Eckhardt, Carl C. U. of Colorado, Boulder, Dept. of History Correspondence - 1942: (2) $\bullet$ 1943: (1)

Eckhardt, Elroy W. New Orleans Friends of Music Correspondence $\bullet$ 1986: (1)

Ecklon, C. F. Bioscript

Ecology Ecological Society of America, Oak Ridge, TN Correspondence • 1977: (2) • 1978: (1)

Economic Botany Correspondence $\bullet$ 1978: (1)

Eden House London Guest house Correspondence $\bullet 1975:(1)$
Edgar, Ellen L. Biological Abstracts, Literature Resources Dept., Book Review Editor

Correspondence • 1970: (0) Filed under Biological Abstracts

Edgar, Robert Correspondence • 1989: (1)

Edgell, D. P. Correspondence • 1968: (2)

Edinburgh See Royal Botanic Garden, Edinburgh

Edlstein, Ludwig (1902-1965) German-born medical historian

Biofile

Edmondson, Charles Howard (1876-1970) American zoologist

Bioscript

Edmondson, John Liverpool Museum, National Museum and Galleries on Merseyside, Keeper of Botany Botanical Album

Correspondence • 1987: (1) • 1988: (5) • 1992: (3)

Edmundson, J. R. American Optical Company Correspondence - 1947: (1) Letter to John Beard

Edson, Carroll E. Physician; Denver, CO Botanical Album

Educators Travel Club Correspondence $\bullet$ 1978: (3)

Edwards, Edwin Governor of Louisiana Correspondence • 1973: (1) • 1976: (1) Bartram, William, 1739-1823

Edwards, Geniana R. Washington, D.C. Correspondence • 1946: (1)

Edwards, George (1694-1773) English zoological illustrator Biofile • Bioscript

Edwards, Henry (1830-1891) English-born American entomologist, tragedian Bioscript

Edwards, John K. Rocky Mountain Biological Laboratory; Sioux Falls College Correspondence • 1939: (5)

Edwards, L. A. Bioscript

Edwards, Phylis Irene (1916-1984) Librarian; British Museum (Natural History), Dept. of Botany

Bioscript $\bullet$ Botanical Album Correspondence • 1955: (1) • 1956: (2) $\bullet$ 1959: (2) • 1960: (1) • 1961: (3) Banks, Joseph, Sir, 1743-1800 • 1964: (3) • 1965: (7) • 1966: (8) Bartram, William, 1739-1823 • 1967: (7) • 1969: (3) • 1970: (6) Banister, John, 1650-1692 • 1971: (5) • 1974: (2) Bartram, William, 1739-1823 • 1976: (3) Barton, Benjamin Smith, 1766-1815 • 1977: (7) • 1978: (3) • 1979: (3) • 1980: (7) - 1981: (2) • 1982: (2) • 1983: (5) • 1984: (1)

Edwards, V. Robert Williams and Wilkins, Publishers Correspondence • 1949: (1)

Edwards, William Henry (1822-1909) American entomologist Biofile

Edwin, Gabriel (1926-) American taxonomist; U. S. National Arboretum Herbarium Correspondence $\bullet$ 1951: (1) 


\section{Guide to the Ewan Papers}

Egan, Edward C. American Rhododendron Society, Quarterly Bulletin, Editor Correspondence • 1980: (0) Filed under American Rhododendron Society

Ege, Vilhelm (1887-1962) Danish ichthyologist Biofile

Egerton, Frank N. III U. of Wisconsin, Madison, Dept. of History of Science

Correspondence • 1963: (7) Science-Instruction and study - 1965: (1) • 1966: (1) • 1970: (1)

Eegers, Heinrich Framz Alexander von (1844-1903) German plant collector, taxonomist Bioscript • Botanical Album

Eegler, Dorothy Asheville, NC Correspondence - 1976: (0) Correspondence filed with Willis Alexander Eggler $\bullet$ 1979: (0) Correspondence filed with Willis Alexander Eggler

Eegler, Willis Alecander (1904-1988) Tulane U., New Orleans, Dept. of Botany

Bloscript • Botanical Album

Correspondence $\bullet$ 1950: (3) • 1951: (2) • 1955: (1) • 1958: (1) Species • 1961: (1) • 1962: (1) Letter from Ben W. Smith attached • 1963: (3) • 1964: (3) • 1965: (1) • 1966: (1) Morrison, Benjamin Yoe, 1891-1966 • 1967: (3) - 1970: (10) Copy of correspondence with William C. Ashby • 1971: (1) • 1972: (2) • 1973: (3) • 1975: (1) • 1976: (2) • 1979: (1) • 1982: (2) • 1983: (3)

Egyleston, Willard Webster (1863-1935) American agriculurist

Bioscript • Botanical Album

Egler, Frank Edwin (1911-) American plant ecologist; Norfolk, CT

Correspondence • 1959: (3) "Rocky Mountain Vegetation" - 1962: (2) • 1969: (1)

Egolf, Donald R. (1928-) American plant breeder; United States Dept. of Agriculure, National Arboretum Correspondence 1 1970: (3)

Ehlers, A. E. Botanical Album

Ehlers, John Henry (1878-) American taxonomist, phytogeographer Botanical Album Correspondence • 1943: (4)

Elurenberg, C. G. Bioscript

Ehrenberg, Ralph Library of Congress, Geography and Map Division, Assistant Chief Correspondence $\bullet 1990$ : (0) Correspondence filed with Library of Congress

Ehrendorfer, Eva Vienna, Austria Correspondence $\bullet 197$ : (1)

Ehrendorfer, Friedrich E. (1927-) American taxonomist; U. of Vienna, Austria, Institute of Systematic Botany Bioscript • Botanical Album Correspondence • 1952: (10) • 1953: (1) • 1954: (2) • 1955: (3) • 1965: (1) • 1969: (1) • 1973: (1) • 1975: (2) Ehrenzeller, Jacob (1757-1838) American physician
Biofile Ehret, Georg Dionysius (1708-1770) German plant
illustrator

Biofile • Bioscript
Ehrhart, Friedrich Biofile • Bioscript

Ehrlich, Paul R. Bioscript $\bullet$ Botanical Album

Eichler, August Wilhelm (1839-1886) German botanist Biofile • Bioscript

Eichler, Hans Joerg (1916-) Canberra, Australia, Division of Plant Industry Bioscript $\bullet$ Botanical Album Correspondence $\bullet$ 1974: (5) $\bullet$ 1975: (1) $\bullet$ 1977: (3) $\bullet$ 1980: (1)

Eigenmann, Rose Smith (1859-1947) American ichthyologist

Bioscript

Eigenmann, Carl H. (1863-1927) German-born American zoologist, ichthyologist

Biofile - Bioscript

Einstein, Albert Biofile $\bullet$ Bioscript

Eiseley, Loren C. Ettinger Program for Creative Writing Correspondence • 1964: (1)

Eisen, Gustavus Bioscript

Eisenberg, John F. Correspondence $\bullet$ 1982: (1)

Eisendrath, Erna Rice (1909-1985) Washington U., St. Louis, MO

Bioscript Correspondence • 1964: (1) • 1976: (1)

Eisenhart, Luther P. American Philosophical Society Correspondence 1950 : (10) $\bullet$ 1951: (11) 1 1952: (10) $\bullet$ 1953: (1) - 1954: (4) Verdoorn, Frans Antonie, 1906-1984; Chronica Botanica; "Rocky Mountain Vegetation"; Geiser, Samuel Wood, 1890- • 1955: (1) • 1956: (1) • 1957: (6) • 1958: (3)

Eisenmann, Eugene Bioscript

Eiten, George Instituto de Botanica São Paulo, Brasil Correspondence $\bullet 1960$ : (1)

Ekman, Erik Leonard (1883-1931) Plant collector, botanist Biofile $\bullet$ Bioscript

Ela, W. D.

Correspondence • 1949: (2)

Elder, Joseph D. Harvard U. Press, Science Editor Correspondence • 1965: (8) Nuttall, Thomas, 1786-1859 • 1966: (5) Banister, John, 1650-1692

Elderfield, R. C. U. of Michigan, Ann Arbor, Dept. of Chemistry Correspondence $\bullet$ 1959: (1)

Eldridge, Inman Fowler Botanical Album

Eleutherian Mills Historical Library Greenville, DE Correspondence • 1979: (1) Barton, Benjamin Smith, 1766-1815 • 1980: (1)

Elias, M. K. U. of Nebraska Correspondence $\bullet$ 1943: (0) Letter filed under T.D.A. Cockerell

Elias, Thomas Sam (1942-) Rancho Santa Ana Botanic Garden, Director

Bioscript

Correspondence 1989 : (3) 
Eliot, Jared Bioscript

Elison, Joseph ( -1884) American naturalist Biofile

Elkins, William (1882-1947) American book collector Biofile

Ellacombe, Canon Bioscript

Ellender, Allen United States, Senator Correspondence $\bullet$ 1970: (3) $\bullet$ 1971: (2)

Ellicott, Andrew Bioscript

Elliot, Kenneth W. U. of Kentucky Press, Lexington, Production Manager Correspondence $\bullet$ 1961: (4)

Elliott, Clark A. Archivist; Harvard U., Pusey Library, Archives Correspondence • 1982: (2) • 1986: (1) • 1989: (1)

Elliott, Stephen (1771-1830) American botanist Biofile - Bioscript

Ellis, Carrie Scott Denver, CO; husband is Erl Hubert Ellis; nickname "Scotty"

Bioscript

Correspondence $\bullet$ 1976: (0) Correspondence filed with Erl Hubert Ellis $\bullet$ 1977: (0) Correspondence filed with Erl Hubert Ellis $\bullet$ 1978: (0) Correspondence filed with Erl Hubert Ellis $\bullet$ 1979: (0) Correspondence filed with Erl Hubert Ellis • 1984: (1)

Ellis, Charlotte Cortlandt (1874 ) Nurse, amateur botanist Bioscript

Correspondence • 1949: (5) Rocky Mountain Naturalists; Cockerell, Theodore Dru Alison, 1866-1948

Ellis, Erl Hubert U. of Denver, Denver; Attorney, Pueblo, $\mathrm{CO}$

\section{Bioscript $\bullet$ Botanical Album}

Correspondence $\bullet$ 1940: (10) $\bullet$ 1941: (65) $\bullet$ 1942: (16) $\bullet$ 1943: (28) • 1944: (7) • 1945: (2) • 1946: (1) • 1947: (1) - 1948: (6) Rocky Mountain Naturalists • 1949: (5) - 1950: (5) • 1951: (5) — 1953: (1) "Rocky Mountain Vegetation" - 1954: (5) • Rocky Mountain Naturalists; "Rocky Mountain Vegetation" $\bullet$ 1955: (1) - 1956: (1) • 1961: (6) Banister, John, 1650-1692; Banks, Joseph, Sir, 1743-1800; National Science Foundation • 1962: (1) - 1963: (10) Letters to and from Robert P. McIntosh $\bullet$ 1964: (3) - 1965: (6) • 1966: (1) • 1967: (2) • 1968: (3) • 1970: (6) • 1972: (4) - 1973: (2) - 1974: (4) Rocky Mountain Naturalists • 1975: (6) • 1976: (10) • 1977: (5) Missouri Botanical Garden; Rocky Mountain Naturalists 1978 (8) 1979 : (4) • 1981: (4) • 1982: (1) • 1983: (9) • 1984: (1)

Ellis, Frederick S. Baton Rouge, LA, Court of Appeal Correspondence $\bullet$ 1978: (4)

Ellis, John (1714-1776) English naturalist Biofile • Bioscript

Ellis, L. Tuffly Texas State Historical Association, Austin, TX

Correspondence 1981 : (3)

Ellis, Max Mapes (1887-)

Bioscript
Ellis, Ralph

Biofile

Ellis, Robert

Bioscript

Ellis, William (1794 ) English plant collector, minister, missionary

Biofile

Ellis, William H. Austin Peay State College, Clarksville, TN Correspondence $\bullet$ 1967: (2)

Ellison, Laurel Botanical Album

Ellison, Lincoln (1907- ) American plant ecologist, forester; Great Basin Branch Experiment Station; nickname is "Linc" Bioscript • Botanical Album

Correspondence • 1929-1933: (6) • 1942: (4) • 1943: (9) • 1945: (3) • 1951: (1) • 1953: (0) Correspondence filed under Samuel Wood Geiser

Ellsworth, Gretchen Gayle Smithsonian Institution Correspondence $\bullet$ 1983: (1) $\bullet$ 1985: (3)

Ellsworth, Ralph Librarian; U. of Colorado Correspondence • 1941: (1)

Ellwood, Christine V. York U., Science Subject Librarian Correspondence $\bullet$ 1990: (2)

Elmendorf, George F. Bookseller with Latinos Libros, Books of Latin America, Santa Monica, CA \& Redlands, CA

Correspondence • 1979: (0) Book collector; letter filed under J. Cramer

Elmer, Adolph Daniel Edward (1870-1942) American botanist Bioscript • Botanical Album Correspondence • 1941: (1)

Elmore, Harry M. Correspondence • 1929-1933: (1)

Elrod, Morton John (1863-1953) American zoologist Bioscript Correspondence $\bullet$ 1949: (1)

Elsevier Antiquarian Dept. Bookseller Correspondence $\bullet$ 1983: (1)

Elwes, A. L. Bioscript • Bioscript

Emboden, William A. California State U., Northridge; Hortulus Aliquando, Editor Correspondence - 1978: (2) Barton, Benjamin Smith, 1766-1815; Bartram, William, 1739-1823 • 1979: (3) • 1983: (2)

Emerson, Alfred E. Bioscript Correspondence $\bullet$ 1940: (2)

Emerson, Barbara H. U. of N. Carolina, Chapel Hill Correspondence • 1973: (2) • 1974: (1) • 1976: (12) • 1991: (1) • 1992: (1)

Emerson, E. W. Bioscript

Emerson, Fred Wilbert (1886-) American plant ecologist; New Mexico Highlands U.

Bioscript Correspondence - 1947: (1) Letter to William Penfound 1953: (0) Correspondence filed under Samuel Wood Geiser 


\section{Guide to the Ewan Papers}

Emerson, G. B. Bioscript

Emerson, Ralph Waldo Bioscript • Botanical Album

Emerson, W. K. American Museum of Natural History Correspondence $\bullet$ 1957: (1)

Emerson, William Otto (1856-1940) Bioscript

Emery \& Kaufmann, Ltd, Insurance Correspondence • 1951: (1)

Emmons, Ebenezer (1799-1863) American geologist Btoscript

Emory, David L. Georgia Botanical Society, Tipularia, Interim Editor Correspondence $\bullet 1992:(2)$

Emory, Kenneth P. Correspondence • 1967: (1)

Emory, Sally Palmetto, CA Correspondence $\bullet 1988$ : (1)

Emory, William Helmsley (1811-1887) American topographical engineer Biofile $\bullet$ Bioscript $\bullet$ Botanical Album

Encyclopedia Americana Correspondence • 1961: (2) • 1967: (2)

Encyclopedia Britarnica Chicago, II Correspondence • 1960: (1) • 1967: (1)

Encyclopedia of Southern Culture Correspondence • 1982: (2) • 1983: (1)

Endeavour A serial publication of Chemical House, London, England

Correspondence 1949: (2) • 1955: (1) • 1959: (2) • 1971 . (2)

Enders, Robert K. Swarthmore College, Swarthmore, PA, Dept. of Biology and Rocky Mountain Biological Lab Correspondence $\bullet$ 1958: (1) $\bullet$ 1962: (2) $\bullet$ 1965: (3) 1966: (2)

Endlicher, S. L. Bioscript

Engelmann, George (1809-1884) German-born American botanist, physician Biofile $\bullet$ Bioscript $\bullet$ Botanical Album

Engelmann, George Julius (1847-1903) American physician; St. Louis, MO; son of George Engeimann Bioscript

Engelmann, Henry (1831-1899) German-born American geologist, meterologist Bioscript

Engeimann, Wilhelm Botanical Album

Engle, Ardis Western Reserve U., Cleveland, $\mathrm{OH}$ Correspondence • 1958: (1)

Engle, Irvin A. St. Mark's Church Correspondence • 1929-1933: (2) Englekirk, Jack U. of California, Los Angeles, Dept. of
Spanish and Portuguese Correspondence $\bullet$ 1975: (1) • 1976: (1)

Engler, Adolph (1844-1930) Bioscript • Botanical Album

English, Carl S. Correspondence 1947 : (1)
English, Mary P. Bristol, England Correspondence $\bullet$ 1987: (3)

Enkelem, Gosanius Bioscript

Enoch Pratt Free Library Baltimore, MD Correspondence • 1951: (1) Pursh, Frederick, 1774-1820 - 1977: (1)

Ensign, Edgar T. Forest commissioner, State of CO Bioscript

Ensign, Margaret R. (1920-) Bioscript

Enslen, Aloysius Bioscript

Entrikin, J. Nicholas Humanist, geographer Biofile

Enviromental Defense Fund New York, NY Correspondence $\bullet$ 1990: (1)

Epling, Carl Clawson (1894-1968) American botanist, taxonomist; U. of California, Los Angeles, Dept. of Botany; nickname "Eppy"

Biofile $\bullet$ Bioscript $\bullet$ Botanical Album

Correspondence 1 1929-1933: (2) Jepson, Willis Linn, 1867-1946; Setchell, William Albert, 1864-1943 • 1934: (8) - 1935: (11) Delphinium • 1936: (11) Jepson, Willis Linn, 1867-1946 • 1937: (12) • 1938: (8) Pennell, Francis Whittier, 1886-1952; Setchell, William Albert, 1864-1943; letter to Ray J. Davis • 1939: (0) Letter filed under Ray J. Davis • 1939: (8) • 1940: (8) • 1941: (8) Delphinium; letter to A.L. Mehlquist • 1942: (8) • 1943: (9) • 1945: (3) Jepson, Willis Linn, 1867-1946; Delphinium • 1946: (4) • 1947: (5) • 1948: (1) • 1949: (3) • 1951: (1) • 1953: (1) - 1954: (2) National Science Foundation; British Museum (Natural History). Dept. of Botany - 1956: (8) Merrill, Elmer Drew, 1876-1956 • 1957: (1) • 1958: (1) • 1961: (2) $1966:$ (3) $\bullet 1967:$ (3)

Epp, Allen D. Portland Community College Correspondence • 1974: (1)

Erdtman, Gunnar Bioscript

Erickson, Martin E. Louisiana State U., Baton Rouge, Dept. of Foreign Languages Correspondence • 1960: (1)

Erlanson, Carl Botanical Album

Ernet, Detlef U. of Graz, Austria, Institute of Systematic Botany Correspondence • 1969: (0) Filed under Friedrich E. Ehrendorfer

Ernst, Adolfo Bioscript

Ernst, Wallace Ray (1928-1971) Taxonomist; Smithsonian Institution Correspondence • 1965: (1)

Ertter, Barbara Botanical Album

Ervendberg, L. C. Bioscript

Erxleben, J. C. P. Bioscript 
Escalante, Silvestre Velez de (f. 1768-1779) Spanish Franciscan missionary, explorer Bioscript

Escallon, Bioscript

Escher, Henry (1776-1853) Bioscript

Escherich, $\mathbf{K}$. Bioscript

Eschscholtz, Johann Friedrich (1793-1831) Bioscript

Eschweiler, F. G. Bioscript

Eshbaugh, William Hardy (1936-) Southern Illinois U., Carbondale

Correspondence $\bullet$ 1966: (2) $\bullet$ 1967: (1)

Eshleman, Elizabeth Correspondence $\bullet$ 1984: (1)

Espinosa, Reinaldo U. of Loja, Ecuador Botanical Album Correspondence • 1949: (1)

Essig, Edward Oliver American entomologist; U. California, Berkeley

Botanical Album Correspondence • 1938: (2)

Estes, Frederich Earle (1902-) Bioscript

Estes, J. Worth Boston U. School of Medicine, MA, Asst. Prof. of Pharmacology Correspondence • 1971: (3)

Estes, James Russell (1937- ) Taxonomist; U. of Oklahoma Correspondence $\bullet$ 1992: (3)

Estill, Botanical Album

Ethridge, Richard U. of Michigan, Ann Arbor, Museum of Zoology Correspondence $\bullet$ 1952: (3)

Eustis, Kate Longue Vue Gardens; husband is Laurence Eustis Correspondence • 1976: (1) • 1980: (2)

Evans, A. Murray (1932- ) U. of Tennesee, Knoxville, Curator Correspondence $\bullet$ 1965: (1) $\bullet$ 1970: (2) $\bullet$ 1972: (1) $\bullet$ 1974: (1)

Evans, Alexander William (1868-1959) American taxonomist, lichenologist; Yale U.

Bioscript $\bullet$ Botanical Album

Correspondence - 1938: (1)

Evans, Clifford The Association for Tropical Botany, Inc., Secretary-Treasurer

Correspondence $\bullet$ 1976: (0) Correspondence is filed under Association for Tropical Biology, Inc.

Evans, David Seller of fine bird books Correspondence 1972: (1)

Evans, Elizabeth Piedmont, CA; husband is Elliot A. P. Evans

Correspondence $\bullet$ 1969: (0) Letter filed under Elliot A. P Evans • 1974: (1) • 1977: (0) Letter filed under Elliot A. P. Evans
Evans, Elliot A. P. U. of California, Santa Barbara College, Chairman, Dept. of Art; The Society of California Pioneers; wife is Elizabeth Evans

Botanical Album

Correspondence • 1940: (1) • 1942: (5) • 1945: (2) •

1946: (1) • 1947: (3) • 1948: (1) • 1949: (7) • 1950: (3) •

1951: (5) • 1952: (6) • 1953: (9) • 1954: (4) • 1955: (3) • 1957: (11) • 1958: (6) • 1959: (3) • 1960: (2) Jepson, Willis Linn, 1867-1946 • 1961: (1) • 1962: (6) • 1964: (2) - 1965: (14) • 1966: (6) • 1967: (5) • 1968: (2) • 1969:

(3) $\bullet$ 1971: (7) $\bullet$ 1972: (2) • 1973: (6) • 1974: (4) • 1977:

(8) • 1978: (4) • 1979: (2) • 1980: (3) • 1984: (1) • 1987: (2)

Evans, Gareth Bevan (1935-1966) Bioscript

Evans, Griffith

Bioscript

Evans, Harry Correspondence • 1967: (1)

Evans, Henry (1918-1990) Printmaker, San Francisco, CA: St. Helena, CA; The Porpoise Bookshop

Bioscript $\bullet$ Botanical Album

Correspondence • 1956: (2) • 1969: (1) • 1970: (1) •

1971: (1) • 1972: (1) • 1990: (2) • 1990: (0)

Correspondence filed under Walter Middelmann

Evans, Herbert McLean (1882-1971) American anatomist. embryologist, bibliophile; U. of California, Berkeley

Biofile • Bioscript

Correspondence • 1948: (3)

Evans, Hugh (1874-1960) English-born American plantsman; Evans and Reeves Nurseries Biofile • Bioscript Correspondence $\bullet$ 1936: (3) $\bullet$ 1953: (2)

Evans, I. B. Pole South African botanist; Union of S. Africa, Bureau of Plant Industry Botanical Album

Evans, John (1812-1861) American geologist, paleobotanist Biofile

Evans, Keith W. U. of Manitoba, Winnipeg, Dept. of Entomology Correspondence $\bullet$ 1970: (2)

Evans, Lewis (1700-1756) Welsh-born American cartographer, geographer, naturalist Biofile $\bullet$ Bioscript

Evans, Margaret W. John Bartram Association, Philadelphia, PA

Correspondence • 1975: (3) • 1976: (2) Bartram, William, 1739-1823 • 1977: (5) Bartram, William, 1739-1823 • 1980: (2) • 1981: (1) Bartram, William, 1739-1823 • 1990: (2)

Evans, Melvin Gulf Oil Corp. Correspondence $\bullet$ 1966: (2)

Evans, Morgan Walt Disney World Co. Correspondence • 1970: (2) Royal Botanic Gardens, Kew

Evans, __, Mrs. LA, member of the Bot. Soc. Correspondence • 1959: (1)

Evans, Pansy Alice (1890-) Botanist; husband is A. E. Inch Bioscript

Evans, U. B. Haphazard Plantation, Ferriday, LA Correspondence • 1957: (1) 
Eve, Anthony Books For Naturalists, Taunton, Somerset, England

Correspondence • 1971: (6) • 1972: (4) • 1975: (2) • 1977: (5) • 1979: (4)

Evelyn, Jobn

Bioscript • Botanical Album

Everett, Betty Correspondence $\bullet$ 1970: (1) $\bullet$ 1972: (1)

Everett, P. C. Correspondence $\bullet 1938$ : (2)

Everett, Thomas Henry (1903-) Horticulturist; New York Botanical Garden

Biofile - Bioscript

Correspondence • 1960: (0) Filed under Nancy M.

Callaghan $\bullet 1967$ : (1)

Evermann, Barton Warren (1853-1932) American ichthyologist

Bioscript • Botanical Album

Ewan, Ada Nesta Dunn (1908-)

Botanical Album (Filed under Joseph Andorfer Ewan)

Ewan, Caroline Correspondence • 1968: (1)

Ewan, Charles Correspondence • 1929-1933: (1)

Ewan, Clara

Correspondence $\bullet$ 1929-1933: (1) Correspondence to Clara and Gil Ewan

Ewan, Dorothy Daughter of Joe and Nesta Ewan; nickname "Dord"

Correspondence • 1959: (1) • 1960: (1) • 1963: (2) • 1964: (5) • 1965: (3) • 1979: (1)

Ewan, Joseph Andorfer (1909-) Botanical Album

Ewan, Marjorie Daughter of Joe and Nesta Ewan; nickname "Marg"

Correspondence • 1961: (3) • 1962: (5) • 1965: (4) • 1966: (6) • 1969: (1) • 1970: (4) • 1971: (1) • 1973: (1) • 1974: (1) • 1978: (1) • 1982: (2) • 1983: (1)

Ewan, Maurice A. Clinton, IA Botarical Album

Correspondence • 1959: (1) • 1964: (2) • 1970: (1) • 1978: (1) • 1984: (1) Ewan, N. R. Camden County Historical Society, Camden, Correspondence • 1950: (2)

Ewan, Stacy Newcomb, Jr., (1900-) Westfield Public Schools, Westfield, NJ, Superintendent Correspondence • 1959: (2)

Exell, Arthur Wallis (1901-) English-born taxonomist, plant collector; British Museum (Natural History)

Botanical Album

Correspondence • 1939: (3) Species

Exposition Press

Correspondence $\bullet 1968$ : (1)

Eyde, Lorraine Husband is Richard H. Eyde Correspondence $\bullet$ 1990: (1)

Eyde, Richard Husted (1928-) American plant anatomist, paleobotanist; Smithsonian Institution, Dept, of Botany; wife
is Lorraine Eyde

Bioscript • Botanical Album

Correspondence • 1965: (3) • 1966: (5) • 1968: (0) Filed
Eyde, Richard Husted (1928-) (continued)

under Frans Stafleu $\bullet$ 1968: (12) $\bullet$ 1976: (2) 1 1982: (7) $\bullet$ 1983: (5) • 1984: (3) Barton, Benjamin Smith, 1766-1815

- 1985: (5) • 1986: (2) • 1988: (1) • 1990: (1)

Eyerdam, Walter J.

Botanical Album

Correspondence $\bullet$ 1940: (2) $\bullet$ 1941: (2)

\section{$\boldsymbol{F}$}

Faber, Friedrich Carl von (1880-1954) Dutch-born Austrian plant physiologist

Botanical Album

Fabre, J. H. French entomologist Biofile

Fabricius, Jean-Chrétien See Fabricius, Johann Christian (1745-1808)

Fabricius, Johann Christian (1745-1808) Danish entomologist Biofile - Bioscript

Fabris, Humberto Antonio (1924-1976) Museo de la Plata, La Plata, Argentina Botanical Album Correspondence • 1950: (1) • 1951: (3) • 1952: (1) • 1953: (1) • 1970: (8)

Faden, Robert Smithsonian, Dept. of Botany Correspondence • 1982: (1) • 1984: (3) • 1985: (2) • 1987: (2)

Faegri, Knut Botanical Album

Fagel Bioscript

Fagley, Frederick L. Correspondence • 1945: (1) American Fern Society $\bullet$ 1946: (10) American Fern Society - 1947: (16) American Fern Society $\bullet$ 1949: (3) American Fern Society $\bullet$ 1950: (3) - 1969: (0) Correspondence filed with Dr. Shaffer

Fagley, Thomas F. Tulane U., Chemistry; Sigma XI Correspondence • 1966: (1) $\bullet$ 1969: (1)

Faherty, William Barnaby (1914-) American historian and Roman Catholic priest; Saint Louis U., St. Louis, MO, Prof. Emeritus

Correspondence • 1984: (1) • 1986: (1) • 1987: (0) Letters filed under Peter Hamilton Raven $\bullet$ 1988: (2)

Fairbrothers, David E. Rutgers U., New Brunswick, Dept. of Botany, Prof. of Botany Correspondence $\bullet 1971:$ (2)

Fairchild, David (1869-1954) American horticulturist, plant explorer Biofile $\bullet$ Bioscript $\bullet$ Botanical Album Correspondence $\bullet$ 1947: (1)

Fairchild Tropical Garden Botanical Album Correspondence • 1966: (1)

Fairie, James Bioscript

Falconer, Thomas (1805-1882) Bioscript

Famitz, __, Mr. Physician, professor of botany; Naples, Italy Bioscript 
Farber, Paul Lawrence Oregon State U., Corvallis, Dept. of General Science Correspondence $\bullet$ 1979: (2) • 1980: (3)

Farley, Elizabeth B. Barnes Foundation, Merion Station, PA

Correspondence $\bullet$ 1977: (0) Correspondence filed under John Milton Fogg, Jr. $\bullet$ 1991: (1)

Farlow, Friends of the Harvard U., Cambridge, Farlow Reference Library and Herbarium of Cryptogamic Botany Botanical Album

Correspondence $\bullet$ 1980: (1) • 1985: (2) • 1986: (3) 1989: (5) - 1990: (1) • 1991: (1)

Farlow, William Gilson (1844-1919) Mycologist, taxonomist

Biofile $\bullet$ Bioscript $\bullet$ Botanical Album

Farmer, David Southern Methodist U., DeGolyer Library Correspondence $\bullet$ 1987: (3)

Farmer, Donald S. U. of Washington, Dept. of Zoology Correspondence $\bullet$ 1972: (2)

Farnum, Jessica L. Library of Congress Correspondence $\bullet$ 1935: (1)

Farr, Edith May (1864 ) Bioscript

Farr, Marie L. U. S. Dept. Agriculture, Crops Protection Research Branch, Beltsville, MD Correspondence $\bullet$ 1964: (2) Species $\bullet$ 1965: (2)

Farr, Robin M. McGill U. Press, Montreal, President Correspondence • 1961: (1) Banks, Joseph, Sir, 1743-1800; (0) Correspondence filed under Averil Margaret Lysaght 1 1962: (4) • 1963: (6) Banks, Joseph, Sir, 1743-1800 • 1965: (1) • 1966: (1)

Farrand, Beatrix Jones Bioscript

Farrar Library Correspondence 1990: (1)

Farrington, John Botanical Album

Farris, Charles Bioscript

Farrow, Jane Portland, OR; husband is Claud Farrow, Jr. Correspondence • 1969: (2)

Farwell, Oliver Atkins Bioscript • Botanical Album

Fassett, Norman Carter (1900-1954) American plant taxonomist; U. of Wisconsin, Madison Biofile $\bullet$ Bioscript $\bullet$ Botanical Album Correspondence • 1938: (3) • 1941: (3) $\bullet$ 1945: (3) $\bullet$ 1946: (1) • 1947: (5) • 1949: (3) • 1950: (2) • 1951: (2) • 1952: (3) • 1953: (10) • 1954: (6) • 1955: (1) Memorial committee

Fauna Zoological Magazine Correspondence • 1972: (2) $\bullet$ 1974: (1)

Faurie, Abbé Urbain Plant collector Biofile

Faust, Ernest Carroll Correspondence $\bullet$ 1955: (1)

Faust, Leicester B. Missouri Botanical Garden, St. Louis, MO, Trustee

Correspondence • 1964: (1) Missouri Botanical Garden
Faust, Mildred Elizabeth (1899-) Syracuse U., Syracuse, NY

Bioscript

Correspondence • 1954: (2) American Fern Society • 1958: (1) American Fern Society • 1959: (1)

Favret, Gervais F. New Orleans, LA Correspondence • 1971: (1)

Fawcett, Priscilla K. S. (1932-) Botanical artist; Missouri Botanical Garden, Research Associate; Waterloo, IL; husband is Knut Norstog

Correspondence $\bullet$ 1989: (1) Letter to P. Raven signed by Fawcett and Knut Norstog

Fawkes, Clayton Golden Fossil Tree Society, Inc., President Correspondence • 1983: (2)

Faxon, Charles Bioscript

Faxon, Edwin (1823-1898) American bryologist, industrialist Biofile $\bullet$ Bioscript

Fay, Jean Bradford Anna Head School Correspondence • 1951: (1)

Fearing, Olin S. Trinity U., San Antonio, TX, Dept. of Botany Correspondence $\bullet$ 1962: (2)

Featherman, Americus (1822-1880) Bioscript $\bullet$ Botanical Album

Featherstonhaugh, George William Bioscript

Feay, William T. (1807-1879) American educator, plant explorer Bioscript

Fechner, Gilbert Henry (1922-) American plant morphologist; Colorado State U., Fort Collins Correspondence $\bullet$ 1964: (2) Species

Fée, Antoine Laurent Apollinaire (1789-1874) French botanist, physician, pteridologist

Biofile $\bullet$ Bioscript

Feger, Franz C. New York, NY Correspondence • 1946: (1)

Feibleman, James K. Correspondence $\bullet$ 1984: (1)

Feilner, John Bioscript

Feingold, Rose Evelyn (1910?-1986) Longue Vue Garden, New Orleans, LA

Biofile

Correspondence • 1976: (1) • 1977: (2) • 1980: (1) • 1983: (1) 1986: (1)

Feins, Claire Klein Correspondence • 1953: (1) Pursh, Frederick, 1774-1820 - 1956: (1)

Fejka, Michael Correspondence $\bullet$ 1952: (2)

Feldner, Josef Correspondence $\bullet$ 1992: (2)

Felger, Alva Howard Bioscript

Fell, Egbert W. Correspondence • 1966: (1) 
Fendler, Augustus (1813-1884) Prussian-born American botanist, plant collector

Biofile $\bullet$ Bioscript $\bullet$ Botanical Album

Fenneman, Nevin Melancthon (1865-1945) American geomorphologist Bioscript

Fenton, William N. Smithsonian Institution Biofile Correspondence $\bullet 1947$ : (1)

Fenzl, Edouard (1808-1879) Austrian botanist, taxonomist Bioscript

Fergus, Charles L. Correspondence • 1949: (2) Answering for Lee O. Overholts

Ferguson, Joan P. S. Librarian; Royal College of Physicians, Edinburgh Correspondence $\bullet$ 1972: (2) • 1973: (1)

Ferguson, John, III Monumental Printing Company Correspondence $\bullet 1944$ : (1) Book collectors • 1945: (3)

Ferguson, Margaret Clay (1863-1951) American botanist Biofile

Ferguson, William Bioscript

Ferland, J. B.

Bioscript

Fernald, Merritt Lyndon (1873-1950) American phytogeographer, taxonomist; Harvard U., Gray Herbarium, Editor-in-Chief of Rhodora

Biofile • Bioscript

Correspondence • 1938: (4) • 1941: (2) Delphinium • 1942: (4) • 1943: (5) Delphinium • 1944: (3) • 1947: (2) - 1948: (3)

Fernandes, Abilio (1906) Portuguese cytotaxonomist; Universidade De Coimbra, Coimbra, Portugal Correspondence $\bullet 1971$ : (1)

Fernow, Bernhard Eduard (1851-1923) German-born Canadian forester, dendrologist Bioscript

Ferreira, A. Castano, Mrs. Botanical Album

Ferreira, H. Amerim Academia das Ciências, Lisbon, Portugal Correspondence $\bullet 1971$ : (1)

Ferren, Wayne R., Jr. (1948-) U. of California, Santa Barbara, Dept. of Biological Sciences; Madroño, Editor Correspondence • 1986: (4) • 1987: (2) • 1988: (1) Jepson, Willis Linn, 1867-1946 • 1992: (1)

Ferreniea, Viki Greenville, NH Correspondence $\bullet$ 1973: (1)

Ferreyra, Ramón Alejandro (1912-) Peruvian taxonomist Botanical Album

Ferril, Alice Mac Harg Husband of William Columbus Ferril Correspondence • 1949: (6) Rocky Mountain Naturalists Ferril, William Columbus (1855-1939) American journalist,
naturalist Bioscript Ferris, Gordon Floyd (1893-1958) American entomologist;
Stanford U.

Correspondence • 1948: (2)
Ferris, Roxana Judkins Stinchfield (1895-1978) American taxonomist, plant collector; Stanford U., Dudley Herbarium Bioscript $\bullet$ Botanical Album

Correspondence - 1929-1933: (6) - 1934: (2) Delphinium - 1935: (5) 1936 (1) • 1938: (2) • 1948: (2) • 1950: (2) - 1956: (1) • 1961: (3) • 1964: (1)

Ferriss, James H. (1849-1926) American naturalist, newspaperman Bioscript

Fessenden, Jane Marine Biological Laboratory Library, Woods Hole, MA

Correspondence • 1966: (1) • 1967: (6) • 1971: (1)

Feudge, John B. Correspondence $\bullet$ 1939: (2)

Feudge, V. B. Bioscript

Feuillan, W. A., Jr. Loyola U., New Orleans, Office of Development

Correspondence $\bullet$ 1960: (3) Letter to Thomas T. Earle referred to Ewan

Fewkes, Jesse Walter (1850-1930) American ethnologist, marine zoologist Bioscript

Fiduccia, C. S. Correspondence $\bullet$ 1949: (1)

Fiedler, Henry G. Bookseller Correspondence 1929-1933: (1)

Field Museum of Natural History (Chicago, IL) Botanical Album Correspondence $\bullet$ 1975: (1) • 1980: (1)

Field, Rob Correspondence $\bullet$ 1983: (3)

Fielding, Henry Barron (1805-1851) Bioscript

Fiennes, O. W. Rector of Lambeth, London Correspondence • 1967: (1)

Fiering, Norman S. Institute of Early American History and Culture

Correspondence • 1972: (3) • 1973: (2) • 1974: (1) • 1977: (1) 1978: (3) Copy of correspondence to Toni Pace included $\bullet$ 1980: (5) $\bullet 1982$ : (1)

Figgins, Jesse Dade (1867-1944) American paleontologist, ornithologist Bioscript

Figueiroa, Silvia F. de $\mathbf{M}$. Correspondence • 1992: (1)

Filgueiras, Tarcisco Correspondence $\bullet$ 1993: (1)

Filmer, Michael M. Filmer Brothers Press Correspondence $\bullet$ 1955: (2) $\bullet$ 1956: (2)

Finch, Vernor Clifford (1883-1959) American geographer Biofile

Findal, J. Kr. (1871-1938) Biofile

Findley, William Lovell Botanical Album

Fingerman, Milton Tulane U., New Orleans, Dept. of Zoology

Correspondence - 1965: (2) • 1966: (1) • 1975: (1) • 1978: (2) • 1980: (4) • 1981: (2) • 1982 (1) 
Fingerman, Stephen

Correspondence $\bullet$ 1972: (1)

Fink, Bruce (1861-1927) American lichenologist Bioscript

Finlay, G. R., Mrs. Bioscript

Finlayson, C. P. U. of Edinburgh, Library Correspondence $\bullet$ 1972: (1)

Finley, William Lovell (1876-1953) American ornithologist Biofile $\bullet$ Bioscript

Finn, Bernard S. Managing Editor, ISIS Correspondence $\bullet$ 1973: (1)

Finn, Melvin Florida Nature Conservancy, Miami Correspondence - 1962: (3) Copy of letter to E. Mcllhenny from $\mathrm{H}$. E. Longenecker

Fiorini-Mazzanti, Elisabetta C. (1799-1879) Italian botanist

Bioscript

Firby, John Bioscript

Fisch, Max H. Western Reserve U. Correspondence - 1943: (6) Biology-Instruction and study

Fischer, Ann Tulane U., New Orleans, School of Medicine, Dept. Public Medicine Correspondence $\bullet$ 1960: (1)

Fischer, Cecil Ernest Claude (1874-1950) Entomologist, botanist in India, England Biofile

Fischer, F. L. Bioscript

Fischer, George William (1906-) XI International Botanical Congress, Inc., Executive Director Correspondence • 1968: (1) • 1969: (0) Letter filed under Albert E. Dimond

Fischer, Gustav Botanical Album

Fish, Les Correspondence $\bullet$ 1942: (1)

Fisher, Albert Kenrick (1856-1948) American ornithologist Bioscript

Fisher, Bruce Crane Correspondence $\bullet$ 1963: (1) $\bullet$ 1965: (1)

Fisher, Clemency Liverpool Museum, Dept. of Zoology Correspondence 1992 : (2)

Fisher, Clyde Bioscript

Fisher, George Lewis (1868-1953) Canadian-born American botanist

Biofile $\bullet$ Bioscript $\bullet$ Botanical Album

Correspondence $\bullet$ 1938: (2)

Fisher, Nicholas (1786-1844) Bioscript

Fisher, Peter Thomas Correspondence $\bullet$ 1953: (1)

Fisher, Ronald A. (1890-1962) Biofile

Fisher, T. Richard Ohio State U., Columbus, Dept. of Biology Correspondence $\bullet$ 1964: (4)

Fisher, Walter Kenrick (1878-) Bioscript
Fishman, Charlyn U. of California, Davis, Agricultural History Center, Editorial Assistant Correspondence • 1968: (2)

Fisk, Erma J. Bioscript

Fitch, A., Rev. Bioscript

Fitch, Asa Entomologist Biofile

Fitch, H. M., Mrs. Bioscript

Fitch, H. P. Bioscript

Fitch, Laura A. Bioscript

Fitch, Walter Hood (1817-1892) English botanical artist Biofile

Fitz, Kurt Naturhistorisches Museum, Wien Correspondence • 1956: (1) - 1959: (1)

Fitzgerald, Shaunagh, Mrs. Shaunagh Fitzgerald Ltd., London, England

Correspondence • 1988: (0) Filed under Elsie Margaret Stones

Fitzgerald, W. Norman, Mrs. Correspondence $\bullet$ 1951: (2)

Fitzhugh, William English farmer Biofile

Fitzpatrick, Harry Morton (1886-1949) American mycologist, plant pathologist Botanical Album

Fitzpatrick, Joe F. U. of South Alabama, Mobile Correspondence $\bullet$ 1982: (1) $\bullet$ 1985: (4)

Fitzpatrick, John T. Bioscript

Fitzpatrick, Thomas Jefferson (1868-1952) American mycologist, botanical historian, bibliophile

Biofile $\bullet$ Bioscript $\bullet$ Botanical Album Correspondence $\bullet 1929-1933:$ (1) • 1949: (1)

Flagg, R. O. Blandy Experimental Farm, Boyce, VA Correspondence $\bullet$ 1962: (3)

Flahault, Charles (1852-1935) Bioscript

Flanagan, John Royal Botanic Gardens, Kew, Library, Deputy Librarian

Botanical Album

Correspondence • 1987: (2) • 1988: (1) • 1989: (4) • 1990: (6) $\bullet$ 1992: (2)

Flaxman, Lois Phelps Shoe Company Correspondence • 1963: (4)

Fleming, Charles Alexander (1916-) Geologist; New Zealand Geological Survey, Dept. of Scientific and Industrial Research Biofile Correspondence • 1974: (3) Beaglehole, John Cawte • 1975: (5) - 1976: (1)

Fleming, E. McClung Winterthur Museum Correspondence • 1966: (1)

Fleming, Guy L. Bioscript

Fleming, J. Correspondence • 1967: (1) 
Fleming, Lindsay Aldwick Grange, Bognor Regis, Sussex, England Botanical Album

Correspondence • 1964: (11) • 1965: (3) • 1966: (3)

Fleming, Pegat M. S. Correspondence • 1977: (1)

Fleming, Richard H. U. of Washington, Seattle, Dept. of Oceanography Correspondence $\bullet$ 1954: (1)

Fleming, V. Biographer; husband is William Borrer Correspondence • 1967: (0) Letter filed under George Hill Mathew Lawrence

Fletcher, Harold Roy (1907-1978) Taxonomist; Royal Botanic Garden, Edinburgh

Biofile Correspondence • 1955: (2)

Fletcher, I. M. London, England; husband is H. M. Fletcher Correspondence • 1964: (1)

Fletcher, James (1852-1908) Canadian entomologist, biologist

Biofile

Fletcher, Lazarus Bioscript

Fletcher, W. E. U. of S. W. Louisiana Correspondence • 1977: (1)

Fletcher, William Whigham (1918-) U. of Strathclyde, Glasgow, Biology Dept.

Botanical Album Correspondence • 1978: (1) • 1979: (2) • 1986: (4) • 1988: (1) • 1992: (1) •

Flett, John Tacoma, WA Correspondence $\bullet$ 1958: (1)

Flett, John Bakkie (1858-) Bioscript

Flinders, Captain Bioscript

Flinders, Matthew (1774-1814) Australian explorer Biofile $\bullet$ Bioscript

Flinn, Richard A. (1841-1924) Bioscript

Flint, Edward C. Bioscript

Floderus, B. Gustav Oscar (1867-1941) Correspondence $\bullet$ 1929-1933: (4)

Flodman, Julius Hjalmar (1859-) Swedish-born American naturalist

Bioscript

Flores, Dan Louie (1948-) ) Texas Tech U., Dept. of History Correspondence • 1982: (2) • 1984: (4) • 1985: (4) • 1987: (2)

Flores, Lucyle J. B. Correspondence • 1956: (1) • 1961: (2) • 1962: (1)

Florida Department of Environmental Regulation Correspondence • 1985: (1) Karen L. Wenzel

Florida Development Commission Tallahassee, FL Correspondence $\bullet$ 1964: (2)

Florida State U., Tallahassee Correspondence • 1966: (1) Barton, Benjamin Smith, 1766-1815

Florida State U. Press Tallahassee, FL Correspondence • 1981: (2) Bartram, William, 1739-1823
Florke, Bioscript

Florke, H. G. Bioscript

Florschütz, Peter Arnold (1923-197?) Botanical Album

Flowers, Seville (1900-1968) Taxonomist, plant morphologist; U. of Utah, Salt Lake City, Dept. of Botany Bioscript $\bullet$ Botanical Album Correspondence • 1949: (2) Rocky Mountain Naturalists • 1951: (1)

Floyd, Fred G. Correspondence • 1934: (3)

Flugge, J. Bioscript

Flynn, John E. Biological Absracts, Editor Correspondence • 1947: (6) Letter from E.H. Walker • 1949: (2)

Foard, Susan Lee U. Press of Virginia, Editor Correspondence • 1984: (1)

Fobb, F. C. (Miss) Louisiana Iris Garden Society Correspondence $\bullet$ 1953: (1)

Fogg, Helen B. Merion Station, PA; husband is John Milton Fogg, Jr.

Correspondence • 1977: (0) Letters filed under John Milton Fogg, Jr. • 1983: (9) • 1984: (1) • 1986: (1) • 1988: (2) • 1989: (2) • 1990: (1)

Fogg, John Milton, Jr. (1898-1982) U. of Pennsylvania, Philadelphia, Morris Arboretum; The Barnes Foundation, Merion Station, PA, Director Biofile $\bullet$ Bioscript $\bullet$ Botanical Album Correspondence $\bullet$ 1950: (6) $\bullet$ 1958: (1) $\bullet$ 1959: (2) 1966: (5) Species • 1967: (1) • 1968: (1) • 1970: (5) Pursh, Frederick, 1774-1820; copy of correspondence to F. G. Meyer • 1971: (8) • 1972: (1) • 1973: (18) • 1974: (12) - 1975: (9) • 1976: (2) • 1977: (13) Letters from Helen Fogg \& Elizabeth B. Farley $\bullet$ 1978: (18) $\bullet 1979$ : (10) Letter to Fogg from Robert Burgess $\bullet$ 1980: (14) 1981: (11) • 1982: (8) • 1985: (1)

Fogle, Richard U. of North Carolina, Dept. of English Correspondence • 1967: (1) • 1968: (1) • 1969: (1)

Foiret, F. J., Mrs. McGill U., Montreal, Botany Dept. Correspondence • 1959: (1)

Folkerts, George W. Auburn U., AL Correspondence • 1990: (0) Filed under Steven P. Darwin

Folsom, George Bioscript

Foltz, Frederic Bioscript

Folwell, Nathan Wright (1805-1879) American plant collector Biofile Bioscript

Fondrin, James Tulane U., New Orleans, Dept. of Physical Plant Correspondence • 1965: (6) Tulane U., Dept. of Botany

Fong, W., Company Correspondence • 1939: (3) Species

Fontana, Felice Bioscript

Fontenette, Bioscript 
Forbes, Edward (1815-1854)

Biofile $\bullet$ Bioscript $\bullet$ Botanical Album

Forbes, Eric G. U. of Edinburgh, History Dept.; Secy, XV Intl. Bot. Congress of the History of Science (1977) Correspondence $\bullet$ 1976: (2) $\bullet$ 1977: (1)

Forbes, F. B. Bioscript

Forbes, H. C. Bioscript

Forbes, H. $\mathbf{O}$. Bioscript

Forbes, Hekna M. L. South African mycologist; Botanic Gardens, Durban Botanical Album

Forbes, Margaret Maude Correspondence $\bullet$ 1945: (2)

Forbes, Paul Tulane U., New Orleans, LA, School of Business Correspondence • 1984: (1)

Forbush, William B. American Philosophical Society Correspondence 1981 (2) $\bullet$ 1983: (3)

Ford, A. J. T. Macquarie U., North Rick, Australia, Registrar Correspondence $\bullet$ 1970: (3)

Ford, Alice Botanical Album

Ford, Charles E. St. Louis U., St. Louis, MO Correspondence $\bullet$ 1964: (2)

Ford, Charlotte A. Archivist; American Philosophical Society and Georgeia Southern, Asst. Prof. of History Correspondence $\bullet$ 1984: (2)

Ford, Gill Miscellaneous Commodities Division, Foreign Economic Administration Correspondence $\bullet$ 1944: (1)

Ford, Henry Chapman Bioscript

Ford, Ebraw (Judge) Bioscript

Ford, W. T. Correspondence $\bullet$ 1951: (2)

Fordyce, George L. Bioscript

Fordyce, Gul. Bioscript

Forel, François Alphonse (1841-1912) Biofile

Forero-Gonzalez, Enrique (1942-) Taxonomist, educator; Missouri Botanical Garden, St. Louis, MO, Director of Research Bioscript Correspondence • 1988: (1) 1991: (0) Letters filed under David A. White

Forest History Society, Inc. Yale University Correspondence • 1966: (2) Filed under Yale University Press

Foret, James Aloysius (1921-) U. of Southwestern Louisiana, Lafayette; Louisiana Society for Horiticultural Research

Correspondence $\bullet$ 1973: (1) • 1978: (1)
Forman, Jonathan International Correspondence Society of Allergists, Director-General

Correspondence - 1954: (0) Filed under Vincent J. Derbes

Forman, L. Ronald Audubon Zoological Garden, New Orleans, Director

Correspondence • 1980: (1)

Formwalt, Lee W. Thronateeska Heritage Foundation; The Papers of Benjamin Henry Latrobe, American Philosophical Society Library Correspondence $\bullet$ 1980: (1) • 1982: (3)

Forshner, John Gordon Correspondence $\bullet$ 1957: (1)

Forsskảl, Peter Bioscript

Forster, Ann Correspondence $\bullet$ 1992: (1)

Forster, Georg Reinhold (1693-1753) Mayor of Dirschan; father of Johann Reinhold Forster; grandfather of Johann Georg Adam Forster Bioscript

Forster, Johann Georg Adam (1754-1794) Author, traveller Biofile $\bullet$ Bioscript $\bullet$ Botanical Album

Forster, Johann Reinhold (1729-1798) Naturalist; father of Johann Georg Adam Forster Biofile - Bioscript

Forsyth, Jane L. Ohio Journal of Science, Editor Botanical Album Correspondence $\bullet$ 1967: (1) $\bullet$ 1972: (2)

Forsyth, John Bookseller; The Old Fort Correspondence • 1952: (1)

Forsyth, William Bioscript

Forsyth, William [son] Bioscript

Fortune, Robert (1812-1880) Botanist, plant explorer, China Biofile • Bioscript

Forwood, William Henry (1838-1914) American surgeongeneral, U. S. Army Bioscript

Fosberg, Francis Raymond (1908-1993) American botanist; Smithsonian Institution, Dept. of Botany; U. S. Dept. of the Interior, Pacific Vegetation Project and The Nature Conservancy

Bioscript $\bullet$ Botanical Album

Correspondence • 1929-1933: (6) Species; letter to Harry M. Elmore • 1934: (3) Jepson, Willis Linn, 1867-1946; Setchell, William Albert, 1864-1943; U. of California, Berkeley, Dept. of Botany; Delphinium - 1935: (6) Munz, Philip Alexander, 1892-1974 • 1936: (5) Munz, Philip Alexander, 1892-1974; Jepson, Willis Linn, 1867-1946 • 1937: (10) Setchell, William Albert, 1864-1943; Species • 1938: (2); • 1939: (5) • 1941: (7) • 1942: (1) Delphinium - 1943: (2) - 1944: (10) - 1946: (7) Delphinitum 1947: (15) - 1948: (9) - 1949: (14) Rocky Mountain Naturalists - 1950: (6) • 1951: (3) • 1952: (4) • 1953: (2) • 1954: (3) National Science Foundation; "Rocky Mountain Vegetation"; Verdoorn, Frans Antonie, 1906-1984 • 1955: (1) - 1957: (13) Beaglehole, John Cawte $\bullet$ 1959: (1) • 1960: (1) - 1961: (3) • 1962: (6) • 1963: (3) $\bullet 1966:$ (1) • 
Fosberg, Francis Raymond (1908-1993) (continued) 1968: (1) • 1970: (2) Banister, John, 1650-1692 • 1972:

(2) $\bullet 1974:$ (8) • 1975: (1) • 1976: (2) • 1977: (3) • 1978:

(2) $\bullet$ 1979: (3) $\bullet$ 1980: (1) Letter to Ruth Lefevre $\bullet$ 1981:

(1) $\bullet$ 1986: (1) $\bullet$ 1990: (1)

Foshay, EMla Millbank Columbia U., New York, NY;

$\mathrm{Ph}$.D. student in art history

Correspondence $\bullet$ 1976: (7)

Foslie, Mikael Heggelund (1885-1909) Biofile

Foster, Fannie R. Elmer, NJ; mother of Mulford Bateman Foster Correspondence • 1929-1933: (6)

Foster, Gertrude B. Herb Grower Magazine; husband is Philip Foster Correspondence • 1968: (4)

Foster, Michel Bioscript

Foster, Mulford Batemen (1888-) Botanist; Orlando, FL Biofile $\bullet$ Botanical Album

Correspondence • 1937: (1) • 1946: (7) • 1948: (1) • 1959: (0) Change of address notice $\bullet 1969$ : (9) • 1970: (6) Lawrence, George Hill Mathewson, 1910-1978

Foster, Racine Sarasy Husband is Mulford Bateman Foster Correspondence • 1947: (1) • 1948: (1) • 1969: (0) Letters filed with Mulford B. Foster $\bullet 1970$ : (0) Correspondence filed with Mulford B. Foster • 1974: (1) • 1979: (1) • 1980: (2) • 1982: (1) • 1988: (1) • 1990: (1)

Foster, Robert Crichton (1904-1986) American taxonomist; Harvard U., Gray Herbarium, Cambridge, MA Biofile

Correspondence • 1945: (1) • 1946: (1) • 1950: (1) • 1952: (2)

Foster, Steven Botanical and Herb Reviews, Eureka Springs, AR

Correspondence • 1989: (3) Barton, Benjamin Smith, 1766-1815

Fothergill, Charles

Bioscript

Fothergill, John Bioscript

Fothergill, Mark Botanical Album

Fougeroux de Bondaroy, Auguste Denis (1732-1789) Biofile

Fountain, Paul (1847-1921) Sobriquet for Louis de Rougemont Bioscript

Fowier, Catherine S. U. of Nevada, Reno, Dept. of Anthropology; Northern Nevada Native Plant Society (NNNPS) Correspondence 1990 : (5)

Fowler, Jacob (1765-1850) Explorer-trader Bioscript

Fowler, James Bioscript Fowler, Robert L. Delta National Wildlife Refuge, Venice,
LA Correspondence • 1937: (1) • 1938: (4) • 1950: (3) 1951: (9) - 1952: (5) • 1965: (5)
Fowler, Samuel Biofile

Fox, Anne Correspondence $\bullet$ 1991: (1)

Fox, Dorothy Arlington, VA Correspondence $\bullet$ 1958: (1)

Fox, Gretchen Washington, D. C. Correspondence • 1977: (2)

Fox, Richard Colorado College, Colorado Springs, Dept. of Zoology Correspondence 1950: (3)

Fox, Richard M. Carnegie Museum, Dept. of Entomology Correspondence $\bullet$ 1949: (1)

Fox, William Basil (1915-1952) North Carolina State, Raleigh, College of Agriculture and Engineering Correspondence $\bullet$ 1952: (3)

Frame, Robert D. Kenner, LA Correspondence $\bullet$ 1973: (4) $\bullet$ 1977: (1)

Frampton, C. E. Louisiana State Museum, New Orleans Correspondence $\bullet$ 1961: (2)

Franc, Isadore Botanical Album

Franceschi, F. Bioscript

Franceschi, Francesco (1843-1924) Italian botanist, horticulturist Biofile

Franchet, A. Bioscript

Francillon, John Bioscript

Francis, John W. Bioscript

Franck, Daniel H. U. of California, Berkeley, Dept. of Botany Correspondence • 1972: (1)

Frank, Bioscript

Frank, G. A. Bioscript

Frank, Joseph C. (1782-1835) German physician, botanist Biofile $\bullet$ Bioscript

Frank, Joseph P. Bioscript

Frank, Robert G. U. of California, Los Angeles, Medical History Division Correspondence • 1972: (1)

Franke, Paul L. Bioscript

Franklin, Benjamin Biofile $\bullet$ Bioscript $\bullet$ Botanical Album

Franklin, John Bioscript

Franklin, John (Sir) Bioscript

Franklin, Lillian Nickname "LuAnne" Correspondence $\bullet$ 1990: (1)

Frankton, Clarence (1906-) Ninth International Botanical Congress, Ottawa Correspondence • 1958: (1) 
Frans, Edward A. St. Albans, NY Correspondence $\bullet$ 1946: (1)

Fransen, Kathryn J. Brigham Young U. Press Correspondence $\bullet$ 1980: (0) Letter filed under W. O. Kupsch

Fraser, Donald Alexander (1918-) Petawawa Forest Experiment Station, Chalk River, Ontario Botanical Album Correspondence $\bullet$ 1953: (3) $\bullet$ 1961: (1)

Fraser, Erika Husband of Donald Alexander Fraser Correspondence • 1953: (0) Filed under Donald Alexander Fraser

Fraser, John, Jr. Bioscript

Fraser, John, Sr. (1750-1811) Botanist, nurseryman, Scotland Biofile • Bioscript

Fraser, Susan New York Botanical Garden Library Correspondence $\bullet$ 1992: (3)

Frazer, James Bioscript

Frederick, John W. Ohio State U., Columbus, Dept. of Botany

Correspondence • 1982: (7) • 1983: (1) • 1986: (1) 1987: (3) • 1988: (1) • 1993: (1)

Frederick, William Heisler, Jr. (1926-) Millcreek Nursery Correspondence $\bullet$ 1974: (2)

Fredman, Caroline Correspondence $\bullet$ 1969: (1) Postcard from Caroline and Lionel Fredman

Fredman, Lionel E. International House, New York, NY; U. of Newcastle, New South Wales, Australia, Dept. of History

Botanical Album

Correspondence • 1962: (2) • 1966: (4) • 1968: (4) • 1969: (1) • 1974: (10) • 1975: (4) • 1976: (3) • 1977: (1) - 1980: (2) • 1981: (1) • 1982: (1) • 1984: (3) • 1988: (1)

Freeberg, John A. U. of Massachusetts, Boston, Chairman of Biology Correspondence $\bullet$ 1972: (2)

Freedley, Samuel (1799-1885) Bioscript

Freeman, E. G. Correspondence $\bullet$ 1943: (1)

Freeman, Elsa S. U.S.D.A., Office of the Librarian Correspondence • 1948: (5) - 1950: (2)

Freeman, John Daniel (1941-) Vanderbilt U.; Auburn U., AL, Dept. of Botany Correspondence • 1967: (1) • 1972: (1) • 1976: (1)

Freeman, M. J. U. of Chicago Correspondence • 1939: (1)

Freeman, O. M. Botanical Album

Freeman, Richard B. U. College, London, Dept. of Zoology Correspondence • 1980: (5) $\bullet$ 1981: (3)

Freeman, Sandra New Orleans, LA Correspondence $\bullet$ 1981: (1)

Freeman, Stuart I. Botanical Album

Freeman, $\mathbf{T}$. Bioscript
Freiauff, Eugen Bioscript

Freiberg, Harry A. Biofile

Freiling, Loretta U. Wisconsin-Madison, Dept. History of Science, Secretary Correspondence - 1959: (0) Filed under Robert C. Stauffer

Frémont, John Charles (1813-1890) American explorer. cartographer Biofile • Bioscript $\bullet$ Botanical Album

French, George Hazen (1841-1935) American entomologist, plant pathologist

See also $H$. G. French

Bioscript • Botanical Album

French, George Henry

Bioscript

French, H. G.

See also George Hazen French

Bioscript

French, John Homer (18241888) Bioscript

Frey, David G. Indiana U., Bloomington, Dept. of Zoology, Professor Correspondence $\bullet$ 1960: (1)

Freye, Harriet Botanical Album

Friberg, Bengt $\mathbf{F}$. Correspondence • 1965: (2)

Frick Art Reference Library Correspondence • 1951: (1) Barton, Benjamin Smith, 1766-1815

Frick, George F. U. of Delaware, Dept. of History, Newark, DE

Correspondence $\bullet$ 1961: (4) Correspondence to Frick and Raymond P. Stearns • 1962: (1) • 1963: (4) • 1967: (1) • 1968: (3) • 1970: (1) • 1971: (2) • 1974: (2)

Friedlaender, Elizabeth Correspondence - 1951: (1) American Fern Society

Friedman, Herbert Smithsonian Institution, Curator, Division of Birds; Proc. Biological Soc. Washington, Editor Correspondence $\bullet$ 1950: (3) $\bullet$ 1951: (1) $\bullet$ 1952: (4)

Friend, Douglas J. C. U. of Hawaii at Manoa Correspondence • 1980: (1)

Fries,

Bioscript

Fries, Elias Magnus Bioscript

Fries, Elizabeth Providence, RI; husband is Waldemar H. Fries

Correspondence $\bullet 1977$ : (0) Letters filed under Waldemar H. Fries $\bullet 1979$ : (0) Letters filed under Waldemar H. Fries - 1986: (2)

Fries, Magnus Stockholm, Sweden Correspondence $\bullet$ 1981: (1)

Fries, Oscar Robert (1840-1908) Biofile

Fries, Robert E. Botanical Album

Fries, T. M. Bioscript

Fries, Waldemar H. (1889-) Providence, RI; nickname "Pat;" Author of "The Double Elephant Folio: the story of Audubon's Birds of America" 
Fries, Waldemar H. (1889-) (continued)

Botanical Album

Correspondence $\bullet$ 1958: (4) $\bullet$ 1959: (12) $\bullet$ 1960: (6) Book collectors $\bullet$ 1961: (20) Copy of corres. to Homer L. Webb - 1962: (19) • 1963: (33) - 1964: (24) • 1965: (10) • 1966: (10) Book collectors • 1967: (6) • 1968: (17) • 1969: (16) Book collectors; one letter to Fries is from Richard A. Gray $\bullet 1970$ : (7) Book collectors; Bartram, William, 1739-1823 • 1971: (9) • 1972: (18) Letter from Sally H. Spofford • 1973: (9) Harper, Francis, 1886-1972 - 1974: (8) • 1975: (15) • 1976: (4) • 1977: (12) • 1978: (4) - 1979: (5) • 1980: (9) • 1981: (4) • 1982: (4) • 1983: (1)

Frings, Carl U. of Hawaii, Honolulu, Dept. of Zoology Correspondence • 1971: (1)

Frisse, Mark F. Washington U. School of Medicine, Directory - Library Correspondence • 1993: (1)

Fritchey, Bioscript

Frittchie, Charles Correspondence $\bullet 1984$ : (2)

Fritz, Emanuel U. of California, Berkeley, School of Forestry

Bioscript

Correspondence • 1949: (1) • 1965: (6)

Fritz, Percy U. of Colorado, Boulder, Dept. of History Correspondence $\bullet 1943$ : (1)

Frodin, David G. The Philadelphia Botanical Club; Academy of Natural Sciences, PA, President Botanical Album

Correspondence • 1987: (1) • 1988: (1) • 1990: (2) • 1992: (1)

Froebel, J. Bioscript

Froen, Bob American Consulate, Ecuador Correspondence • 1949: (1)

Frolich, J. A. Bioscript

Frontiers Published by Academy of Natural History of Philadelphia; Kitty Ash, editor Correspondence $\bullet$ 1952: (1)

Frost, Albert Henry (1851-1912) American ornithologist Bioscript

Frost, Charles C. Bioscript Frost, Frederick Hazard (1902-) American paleontologist
Biofile

Fruge, Angust U. of California Press, Berkeley, CA Correspondence • 1968: (4) • 1971: (1) • 1973: (6)

Fry, Edward (Sir) Bioscript

Fry, Joel T. U. of Pennsylvania, Philadelphia, Graduate Student in American Civilization

Correspondence 1 1992: (4) Bartram, William, 1739-182

Fry, Mary Isabel Henry E. Huntington Library and Art Gallery, Registrar

Correspondence • 1960: (4) • 1969: (1)

Fry, Susann

Botanical Album
Frye, Harriet

Correspondence • 1991: (1) Banister, John, 1650-1692 • 1992: (4) Letter to Emanuel Rudolph and his reply

Frye, Theodore Christian (1869-1962) American botanist; U. of Washington

Biofile $\bullet$ Bioscript $\bullet$ Botanical Album

Correspondence $\bullet$ 1937: (1) $\bullet$ 1941: (1)

Fryling, Charles F., Jr. Louisiana State U., Baton Rouge; Baton Rouge Audubon Society

Correspondence $\bullet$ 1975: (1) - 1976: (4) • 1977: (1)

Fryxell, Fritiof Melvin (1900-) American geomorphologist; Rock Island, IL Correspondence • 1964: (1)

Fryxell, Paul A. Research geneticist; U.S.D.A., Texas Agricultural Experiment Station, College Station Correspondence • 1969: (2) • 1980: (2)

Fuchs, Carl P. Arnold (1927- ) Bioscript

Fuchs, Hans P. Smithsonian Institution Correspondence $\bullet$ 1958: (3) $\bullet$ 1959: (1)

Fuchs, Leonhard (1501-1566) Bavarian physician Biofile $\bullet$ Bioscript

Fuertes, Louis Agassiz (1874-1927) Biofile $\bullet$ Bioscript $\bullet$ Botanical Album

Fulbright, J. William Botanical Album

Fulbright Program Charles C. Bryan, Program Officer Correspondence 1972: (2)

Fulbright-Hays Act Correspondence • 1973: (1)

Fulford, Margaret Hannah (1904 ) U. of Cincinnati, Dept. of Botany

Correspondence $\bullet$ 1951: (1) $\bullet$ 1964: (1)

Fulkerson, Billie C. Crowley, LA Correspondence $\bullet$ 1959: (2) $\bullet$ 1960: (1)

Fuller, Albert Morse Milwaukee Public Museum Botanical Album

Correspondence - 1949: (3) Rocky Mountain Naturalists; letter from Mathilde Prokosch

Fuller, George Damon Botanical Album

Fullerton, Dorothy Hay Correspondence • 1941: (2) • 1947: (1) • 1949: (1)

Fullertone, John C. Agriculture Experiment Station, Wooster, $\mathrm{OH}$ Correspondence • 1939: (5)

Fulling, E. H. Correspondence $\bullet$ 1940: (2)

Fullmer, June Z. Tulane U., New Orleans, Newcomb College; Ohio State U., Columbus Correspondence • 1958: (2) • 1959: (3) Banks, Joseph, Sir, 1743-1800 • 1961: (2) - 1963: (2) • 1965: (2) • 1966: (3) 1 1967: (1) - 1968: (2) • 1969: (1) $\bullet$ 1972: (2) $\bullet 1978$ : (1) 1979: (3) Barton, Benjamin Smith, 1766-1815

Fulton, John Yale, Historical Library Correspondence • 1950: (4)

Fulton, Robert Bioscript

Fultz, Dessa M. Husband of Francis Marion Fultz Correspondence $\bullet$ 1950: (3)

Fultz, Francis Marion (1857-) Bioscript • Botanical Album 
Funck, H. C.

Bioscript

Funk,

$$
\text { Bioscript }
$$

Funston, Frederick (1865-1917) American botanist; U. S. Army

Bioscript

Furber, Robert

Bioscript

Furbish, Kate American botanist, artist Biofile - Bioscript

Furcron, A. S. Georgia Geological Survey, Atlanta Correspondence • 1956: (3)

Furlow, John J. Michigan State U., Dept. of Botany and Plant Pathology, East Lansing

Correspondence $\bullet$ 1971: (1) $\bullet$ 1983: (1)

Furman, Farish Carter (1846-1883) American agriculturist Biofile

Furneaux, Tobias Explorer; Tasmania Biofile

Furniss, George B.

Correspondence $\bullet$ 1935: (3) - 1936: (1) Species

Furnrohr, A. E.

Bioscript

Fursling, Bioscript

Fusonie, Alan E. National Agricultural Library, Beltsville, MD, Coordinator; Associates Journal, Associates of the National Agricultural Library, Inc.

Botanical Album

Correspondence • 1976: (2) • 1983: (2) • 1984: (4) • 1986: (1)

Fussell, George Edwin (1889-) American agricultural historian

Biofile $\bullet$ Bioscript

Correspondence $\bullet$ 1968: (2) $\bullet$ 1969: (1)

Fussell, L. E.

Correspondence $\bullet$ 1967: (1)

Fye, W. Bruce

Correspondence $\bullet$ 1984: (2)

G

[G D. M] fl. 1896 (Denis Gale?)

Bioscript

Gabb, William More (1839-1878)

Bioscript

Gabel, Mark L. Iowa State U., Dept. of Botany and Plant Pathology

Correspondence 1979: (2) Rocky Mountain Naturalists

Gack, $\mathbf{M}$.

Correspondence • 1967: (1)

Gaddy, L. L. Aspiring graduate student Correspondence 1975: (4)

Gärtner, C. F.

Bioscript

Gaertner, Erika Eva (1921-)

Botanical Album

Gaertner, Joseph (1732-1791) German botanist Biofile

Gage, Thomas C. (1602/03-1656) Dominican explorer Biofile
Gager, Charles Stuart

Botanical Album

Gahagan, Steven W.

Correspondence $\bullet$ 1970: (1) Copy of correspondence with Marta Lamar

Gaigs, Helen U. of Michigan Correspondence • 1929-1933: (2)

Gail, Floyd W. U. of Idaho Correspondence • 1937: (3)

Gaillard, A. Bioscript

Gaillard de Charantonneau, Bioscript

Gairdner, Meredith (1809-1837) British physician Bioscript

Gaiser, Lulu Odell (1896-1965) Harvard U., Biological Laboratories Botanical Album

Correspondence $\bullet$ 1949: (6) $\bullet$ 1950: (5) $\bullet$ 1951: (4) $\bullet$ 1952: (3) • 1961: (4) Banister, John, 1650-1692; British Museum (Natural History), Dept. of Botany

Galbraith, I. C. J. British Museum (Natural History) Correspondence $\bullet$ 1957: (1)

Gale, Denis (1828-1905) Bioscript

Gale, Leonard Dunnell Bioscript

Galeotti, Henri Bioscript $\bullet$ Botanical Album

Galish, E. J. Royal Botanic Gardens, Kew Correspondence • 1945: (1)

Galissonnière, de la Biofile

Gallatin, Frederic, Jr. Bioscript

Galloway, Beverly Thomas (1863-1938) American horticulturist Biofile $\bullet$ Bioscript

Galloway, Carolyn I. New Orleans, LA Correspondence • 1976: (1)

Galphin, George

Bioscript

Galston, Arthur William Botanical Album

Galton, Francis Bioscript

Galyon, Willa Love Bioscript

Gambel, William (1821-1849) Naturalist Bioscript

Gambill, William G., Jr. Denver Botanic Garden, Director Biofile - Botanical Album Correspondence • 1973: (1) • 1974: (1) • 1976: (0) Filed under Denver Botanic Garden • 1978: (3) • 1987: (2)

Gamble, Robert A. Correspondence • 1967: (3) Letter from John H. Gribbin

Gambold, Amna Rosina (1762-1821) Biofile - Bioscript

Gambold, Elisabeth Bioscript

Game, Douglas M. McGraw-Hill Correspondence $\bullet$ 1941: (1) 
Gamiz, Norma Barrero Librarian; National Botanical Library, Mexico

Correspondence $\bullet$ 1982: (1)

Gander, Frank F. Natural History Museum, San Diego, CA Correspondence • 1936: (3) Delphiniun • 1940: (1) • 1941: (1) • 1942: (4)

Gandevia, Bryan Prince Henry Hospital Correspondence $\bullet$ 1966: (2)

Gandhi, K. N. Texas A \& M U., Dept. of Range Science Correspondence $\bullet 1987$ : (2)

Gandoger, Biofile

Gannett, Henry (1846-1919) American geographer Bioscript

Ganong, William Francis (1864-1941) Botanical Album

Gans, Carl Babcock and Wilcox Co.; U. of Florida, Gainesville Correspondence • 1955: (2) • 1958: (3)

Garaventa, H. Agustin (1911-1981) Correspondence • 1939: (1) • 1940: (4) • 1941: (4)

Garber, Abram Paschal (1838-1881) Blofile • Bioscripr $\bullet$ Botanical Album

Garber, J. D. Merck \& Co. Inc. Research Labs, Rahway, NJ

Correspondence • 1956: (2)

Garber, Janet

Correspondence • 1993: (0) Letter filed under Dale Edwin Johnson

Garcia-Aldrete, Allonso mlinois State U., Normal, Dept. of Biological Sciences

Correspondence • 1970 (1) • 1971: (1)

Garcilasso, Ynca de la Vega (1530-1568) Biofile

Garden, Alecander Biofile $\bullet$ Bioscript

Garden Club of America New York, NY Correspondence • 1959: (2) Grace A. Barker, Secretary, Medal Award Committee - 1971: (3) Correspondent is Dorothy G. Carroll, Horticulture Committee, G.C.A., New Orleans, LA • 1977: (2) Correspondence with Mary A. Hommans $\bullet$ 1978: (5)

Ganden Design Correspondence • 1983: (2)

Gardener, William North Colchester, Essex, England; biographer John Lindley \& Robt. Fortune (Gard. Chron. $11 / 27 / 65)$

Botanical Album

Correspondence • 1965: (3) • 1966: (4) • 1967: (1) • 1968: (1) • 1969: (13) • 1971: (7) • 1978: (1) • 1979:

Gardiner, C. Harvey Southern Illinois U. Press, Carbondale Correspondence • 1965: (4)

Gardiner, Dorothy Correspondence • 1948: (4)

Gardiner, James T. Bioscript

Gardiner, John Scotish-born American naturalist Botanical Album

Gardiner, John (1861-1900) Bioscript
Gardiner, Lawrence The Catskills Quarterly, Inc., Prattsville, NY

Correspondence $\bullet$ 1976: (1)

Gardiner, , Mr.

Correspondence • 1979: (1) Book collectors

Gardiner, Silvester

See Hosack, David (1769-1835)

Biofile

Gardiner, W. J.

Bioscript

Gardiner, William (1808-1852) Scottish naturalist, poet Biofile

Gardner, Alfred Smithsonian Institution, Natural History Museum

Correspondence • 1988: (3) Barton, Benjamin Smith, $1766-1815$

Gardner, Eliza Bioscript

Gardner, George Biofile $\bullet$ Bioscript

Gardner, Henry (1809-1890) Bioscript

Gardner, James Linton (1901-) American botanist Bioscript

Gardner, Leon L. Bioscript

Gardner, Nathaniel Lyon (1864-1937) Bioscript

Garman, Samuel W. (1843-1927) American zoologist Bioscript

Garner, Murvel R. Earlham College Correspondence 1957: (1)

Garovaglio, Santo (1805-1882) Biofile

Garret, James (Garrettus) (fl. 1585) Bioscript

Garrett, Albert Osbun (1870-1948) American botanist, high school teacher

Bioscript $\bullet$ Botanical Album

Correspondence $\bullet$ 1941: (10) $\bullet$ 1942: (1) $\bullet$ 1943: (3) 1949: (1)

Garrett, Kimball Natural History Museum of Los Angeles County

Correspondence • 1992: (3)

Garruth, James H. (1807-1896) American botanist Biofile

Garry, Charlene Basilisk Press Limited, London, England Bioscript

Correspondence • 1977: (1) • 1987: (0) Flyer for lecture Friends of Tulane Library

Garry, Nicholas (1782?-1856) English; deputy governor of Hudson Bay Co. Biofile $\bullet$ Bioscript

Garst, Jonathan (1893-1973) American geographer Biofile

Gartner, John Hawthorn Press, Melbourne, Australia Correspondence $\bullet$ 1975: (2)

Garvey, Eleanor Correspondence $\bullet$ 1977: (0) Note filed under George Edmond Gifford, Jr.

Garvitt, Bioscript 
Gasbury, Jerry

Correspondence • 1974: (2) American Fern Society

Gashwiler, Keturah Iowa State U., Ames, Dept. of Botany and Plant Physiology

Correspondence • 1960: (1) Letter from Don Stone

Gasparrini, de Guglielmo

Biofile

Gasser, Adolph

Correspondence • 1975: (1)

Gaston, Robert W., Jr. Baton Rouge, LA

Correspondence $\bullet$ 1962: (2)

Gastony, Gerald Joseph (1940-) Indiana U., Bloomington, Dept. of Botany

Botanical Album

Correspondence $\bullet$ 1966: (5) $\bullet$ 1969: (2) $\bullet$ 1970: (4)

1971: (4) • 1973: (1) - 1975: (2)

Gates, Burton N. Clark U., Worcester, MA Correspondence • 1950: (1)

Gates, Charles M. Pacific Northwest Quarterly, U. of Washington

Correspondence 1942 : (3)

Gates, David Murray

Botanical Album

Gates, Hezekiah

Bioscript

Gates, Reginald Ruggles (1882-1962) American botanist Botanical Album

Gattinger, Augustin (1825-1903) German-born American botanist and author

Biofile $\bullet$ Bioscript $\bullet$ Botanical Album

Gaulther,

Bioscript

Gautier, Bioscript

Gay, Claudio (Claude)

Biofile $\bullet$ Bioscript $\bullet$ Botanical Album

Gazin, Charles Lewis Smithsonian Institution; Washington Academy of Sciences

Correspondence • 1947: (2) • 1968: (1)

Gearhart, Toby Historical Society of Pennsylvania, Research Assistant

Correspondence • 1992: (0) Letter filed under David W. Johnston

Geary, Sheila Connor Arnold Arboretum Correspondence 1982: (1)

Gebhardt, Glen L.

Correspondence • 1942: (1) Letter to Robert Stearns

Geddings, Dr. Herpetologist

Bioscript

Geddy, John C. Bioscript

Gegenbaur, Carl (1826-1903) German naturalist Biofile

Geis, Helen D.

Bioscript

Geisar, Gerald Princeton U., Dept. of History Correspondence $\bullet$ 1972: (1)

Geiser, Samuel Wood (1890-) Southern Methodist U., Dallas

Bioscript $\bullet$ Botanical Album

Correspondence • 1939: (4) Species • 1942: (1) • 1945:

(1) $\bullet$ 1946: (8) • 1947: (4) • 1948: (6) • 1949: (3) • 1950:

(1) 1951: (3) Cockerell, Theodore Dru Alison,
Geiser, Samuel Wood (1890- ) (continued)

1866-1948; filed under William Alfred Weber • 1952: (5) • 1953: (1) • 1954: (1) • 1955: (3) • 1956: (7) • 1957: (12) - 1958: (1) • 1959: (12) • 1960: (6) • 1961: (2) Banister, John, 1650-1692 • 1962: (1) • 1963: (11) • 1964: (8) Rocky Mountain Naturalists • 1965: (5) • 1967: (1) • 1968: (12) • 1969: (4) • 1970: (3) • 1971: (6) • 1977: (1)

Gellar, Esther Elgen Books Correspondence • 1980: (1) • 1983: (1)

Geneve, Ville Conservatoire et Jardin Botaniques Correspondence • 1962: (1)

Genovese, Anthony J. Poet; Gretna, LA Correspondence • 1975: (2) • 1981: (2)

Gentry, Alwyn (1945-1993) Missouri Botanical Garden Bioscript $\bullet$ Botanical Album Correspondence • 1976: (1)

Gentry, Harold Denison Senior High School, TX, biology teacher; private herbarium of 500 sheets Correspondence $\bullet 1960$ : (2)

Gentry, Howard Scott (1903-) American phytogeographer, agronomist; Desert Botanical Garden, Phoenix, AZ Biofile $\bullet$ Bioscript

Correspondence $\bullet$ 1948: (2) • 1949: (1) • 1950: (4) • 1975: (1) • 1977: (1)

Geological Society of America Correspondence • 1981: (1)

George, Alexander Segger Botanical Album

George Robertson \& Sons, Inc. Florists; Philadelphia, PA; Correspondence $\bullet$ 1977: (3)

George S. MacManus Co. Correspondence $\bullet$ 1963: (0) Filed under Lloyd Herbert Shinners

George, William J. Tulane U. School of Medicine, Dept. of Pharmacology, Professor

Correspondence $\bullet$ 1979: (0) Science-Instruction and study; filed under Tulane University Pharmacology Dept.

George's Bristol, England; booksellers since 1847, R. Haynes, Director Correspondence $\bullet$ 1969: (3)

Georgia Botanical Society Correspondence • 1987: (1) • 1988: (1)

Georgia Historical Society Lilla M. Hawes, Director Correspondence • 1972: (2) Bartram, William, 1739-1823

Gerard, Louis (1733-1819) French physician, botanist Biofile

Gerard, John Bioscript - Botanical Album

Germain, Louis Muséum National D'Histoire Naturelle Correspondence • 1939: (1) Species

Germana, Violet Holland Botanical Album

Gerry, Eloise (fi. 1947) American forester Biofile

Gersdorff, Hans von (fl. 1517) German surgeon Biofile

Gerth Van Wijk, Hugo Leonardus Dutch botanical lexicographer Biofile

Gervais, John Lewis Bioscript

Gesner, Conrad (1516-1565) Swiss physician, scientist Biofile • Bioscript 
Geyer, Charles (Karl or Carl) Andreas (1809-1853)

German-born American plant collector

Bioscript • Botanical Album

Geyer, Paulo

Correspondence $\bullet$ 1985: (1)

Ghafoor, Abdul U. of Karachi, Pakistan, Herbarium Correspondence • 1975: (1) • 1976: (1)

Giacomini, Valerio (1914-1981) U. of Pavia, Istituto ed Orto Botanico

Correspondence $\bullet$ 1954: (2) • 1955: (1)

Gibb, James

Bioscript

Gibbes, Lewis $\mathbf{R}$.

Bioscript

Gibbin, John

Correspondence $\bullet$ 1966: (1)

Gibbons, Henry (1808-1884)

Bioscript

Gibbons, __, Miss

Correspondence $\bullet 1954:$ (1)

Gibbons, Walliam P.

Bioscript

Gibbs, George (1815-1873) Ethnologist, philologist, geologist, lawyer

Bioscript

Gibbs, R. W. McGill U., Montreal Correspondence • 1951: (1)

Gibbs, Ronald Darnley (1904) Plant physiologist, Canada; McGill U., Montreal, Dept. of Botany

Correspondence • 1956: (2) • 1960: (0) Letter filed under Irving William Knobloch $\bullet 1970$ : (4)

Gibbs, Rowan Smith's Bookshop, Ltd. Correspondence - 1988: (2) Book collectors

Gibson, Dennis A. U. of Southwestern Louisiana, Lafayette Correspondence $\bullet$ 1974: (1)

Gibson, James York U., Toronto, Dept. of Geography Correspondence • 1971: (1)

Gieger, Marie Jones Co. Junior College, Ellisville, MS Correspondence • 1986: (2)

Gienapp, John C. Correspondence * 1968: (3)

Gier, L. J. Correspondence • 1968: (2)

Gifford, George Edmund, Jr. ( -1981) Psychiatrist, book collector; Watertown, MA

Bioscript $\bullet$ Botanical Album

Correspondence • 1962: (9) • 1963: (6) • 1964: (3) • 1965: (11) • 1966: (5) • 1967: (7) • 1968: (5) • 1969: (7) 1970: (12) - 1971: (4) - 1972: (9) 1973: (9) 1974: (3) $\bullet$ 1975: (12) $\bullet 1976:(6) \bullet 1977:(8) \bullet 1978:(1) \bullet$ 1979: (3) • 1980: (3) • 1981: (1)

Gignac, Solange G. Huggins Librarian, Denver Botanic Garden

Correspondence - 1976: (0) Filed under Denver Botanic Garden - 1986: (2)

Gignilliat, Charles N. Correspondence • 1974: (2)

Gilbert, Benjamin Davis (1835-1907) Biofile • Bioscript

Gilbert, Charles Thibodanx, LA Correspondence • 1958: (3) - 1959: (2)

Gilbert, Charles Henry (1859-1928) Bioscript
Gilbert, G. K.

Bioscript

Gilbert, Henry National Agricultural Library

Correspondence $\bullet$ 1975: (3)

Gilbert, John P.

Bioscript

Gilbert, Pamela British Museum (Natural History),

Entomology Library

Correspondence $\bullet$ 1991: (1) • 1992: (3)

Gilden,

Bioscript

Giles, Norman H., Jr. Yale U. Correspondence • 1942: (2)

Gilfillan, F. A. Dean, Corvallis, OR

Botanical Album

Correspondence $\bullet$ 1978: (1)

Gilg, Eruss Friedrich (1867- )

Bioscript

Gilkey, Helen Margaret (1886-1972) Taxonomist, botanical artist; Oregon State College, Corvallis; nickname "Hazel" Botanical Album

Correspondence • 1943: (4) Delphinium $\bullet$ 1946: (2) $\bullet$ 1949: (2) Jepson, Willis Linn, 1867-1946 • 1951: (1) 1953: (1)

Gill, F. B. The Academy of Natural Sciences, Philadelphia, PA, Scientific Publications, Acting Editor Correspondence -1978: (2)

Gillean, Grace New Orleans, LA Correspondence $\bullet$ 1960: (2)

Gillespie, Doris Kildale Botanical Album

Gillespie, Emily C. Sigma XI, Secretary Correspondence $\bullet$ 1958: (1)

Gillespie, John Tulane U., Assistant Dean Correspondence $\bullet$ 1951: (1)

Gillespie, John Wynn ( -1932) American plant taxonomist Biofile - Bioscript

Gillespie, Richard U. of Pennsylvania, Philadelphia, ISIS, Editorial Assistant Correspondence $\bullet$ 1980: (1)

Gillett, George W. U. of Hawaii, Honolulu Correspondence 1966: (0) Filed under Noel Price Kefford - 1974: (2)

Gillett, Jon McGraw-Hill, Inc., Marketing Director Correspondence 1970: (0) Filed under McGraw-Hill, Inc.

Gillette, Clarence Preston (1859-1941) American entomologist

Bioscript

Gillick, Printing, Inc. Correspondence $\bullet$ 1967: (1)

Gillies, John

Bioscript

Gillis, William Thomas (1933-1979) Taxonomist; Hope College, Holland, MI, Dept. of Biology

Botanical Album

Correspondence • 1969: (5) • 1970: (2) • 1971: (3) • 1972: (1) • 1973: (1) • 1974: (2) • 1975: (1)

Gillispie, Charles C. Dictionary of Scientific Biography Correspondence • 1973: (3) - 1977: (3)

Gilliss, James Melville (1811- ) American navel scientific officer

Biofile 
Gillman, Henry (1833-1915) Bioscript

Gilman, D. C. Bioscript

Gilman, Marshall French (1871-1944) American ornithologist

Bioscript

Gilmartin, Amy Jean Guayaquil, Ecuador Correspondence $\bullet$ 1964: (2)

Gilmer, Francis (1790?- ) American naturalist and lawyer Biofile

Gilmore, Raymond M.

Bioscript

Correspondence • 1949: (1)

Gilmour, John Scott Lennox (1906-1986) British horticulturist; Royal Botanic Gardens, Kew; Cambridge Botanic Garden, Director

Biofile $\bullet$ Bioscript $\bullet$ Botanical Album

Correspondence • 1937: (1) • 1938: (1) • 1945: (1) • 1955: (1) • 1958: (2) 1 1962: (0) Letter filed under George Booth Van Schaack • 1969: (2)

Gimarc, Jerry Dell Quarterly Review of Biology Correspondence $\bullet$ 1963: (2)

Ginsburg, Frieda U. of Michigan, Ann Arbor, Dept. of Botany

Correspondence $\bullet$ 1948: (1)

Giorni Libri e Stampe occasioni Correspondence • 1961: (1)

Gipson, Gordon Publisher; Caxton Printers Ltd. Caldwell, ID

Correspondence • 1946: (1) • 1980: (1) Letter to Peter Hamilton Raven

Girard, Charles Bioscript

Girard, Joseph Basil ( -1918) French-born American botanical collector (Arizona) and army surgeon Biofile

Giraud, T. Bioscript

Giseke, Paul Dietrich (1741-1796) German physician and botanist Biofile

Givens, Florence Montgomery (1933-1990) Irish-born American botanist

Biofile

Correspondence $\bullet$ 1976: (1)

Gladney, W. Frank John James Audubon Foundation Correspondence • 1970: (1)

Glasgow, Clyde Correspondence - 1949: (3) Rocky Mountain Naturalists

Glasnevin National Botanic Gardens, Dublin, Ireland, Library Correspondence $\bullet$ 1990: (1)

Glass, Hiram Bentley (1906-) Quarterly Review of Biology Botanical Album

Correspondence - 1946: (1) • 1954: (4) - 1955: (3) • 1956: (2) • 1961: (1) • 1962: (3) • 1963: (3) • 1964: (1) • 1967: (1) • 1968: (1) • 1972: (1) • 1975: (1) • 1976: (1) • 1976: (0) Filed under Quarterty Review of Biology $\bullet$ 1981: (1) 1983: (3)

Glaziou, F. M. Bioscript
Gleason, Henry Allan (1882-1975) American ecologist; New York Botanical Garden; American Society of Plant Taxonomists

Biofile $\bullet$ Botanical Album

Correspondence • 1936: (4) • 1937: (1) • 1939: (1)

Delphinium • 1940: (1) • 1941: (4) Letter to Francis Ramaley • 1943: (2) • 1946: (1) • 1947: (2) • 1951: (3)

Gleich, Peter A.

Botanical Album

Glenbow-Alberta Institute The Library

Correspondence • 1970: (1) Rocky Mountain Naturalists

Glenk, Robert

Correspondence • 1949: (1)

Glenn, Frank Bookseller

Correspondence 1 1958: (1) 1961 : (1) Book collectors

Glenn, Reva A. H. \& A. W. Reed, Publishers, Wellington, New Zealand

Correspondence $\bullet$ 1952: (1)

Glick, Thomas F. Boston U., Dept. of History

Correspondence $\bullet 1977$ : (1)

Gloag, John (1896-) Society of Architectural Historians of Great Britain; author of Short Dictionary of Furniture Botanical Album

Correspondence • 1955: (6) • 1956: (3) • 1957: (5) Letter to Robert W. Hill $\bullet$ 1958: (6) $\bullet$ 1959: (1) $\bullet$ 1962: (1) $\bullet$ 1963: (7) • 1964: (1) • 1965: (3) • 1967: (2) • 1968: (1) • 1969: (4) • 1970: (6) Verdoorn, Frans Antonie, 1906-1984 - 1974: (3) - 1977: (4) Letter to John and Mim • 1980: (2)

Gloag, Mim Husband is John Gloag Correspondence • 1976: (2) Rocky Mountain Naturalists; Barton, Benjamin Smith, 1766-1815

Gloster Arboretum Gloster, MS Correspondence • 1978: (1)

Glover, Thomas (f. 1676) Bioscript

Gloyd, Howard K. Correspondence $\bullet$ 1950: (5) • 1951: (10) • 1952: (2) • 1966: (2)

Gluck, H.

Botanical Album

Gmelin, J. F. Bioscript

Gochfeld, Michael Correspondence $\bullet$ 1973: (4)

Goddard, David U. of Pennsylvania, Philadelphia Correspondence $\bullet$ 1974: (2)

Goddard, Pliny Earle (1869-1928) Bioscript

Godfrey, Robert Kenneth (1911-) Taxonomist; Florida State U., Tallahassee, Dept. of Biology; National Science Foundation, Dept. of Biological Sciences

Botanical Album

Correspondence • 1961: (4) Tulane U. Dept. of Botany • 1962: (2) • 1965: (3) • 1967: (3) • 1969: (3) • 1975: (1) • 1988: (1)

Godley, Eric John (1919-) Plant Breeder, New Zealand Dept. of Scientific and Industrial Research, Botany Division Correspondence • 1974: (4) • 1977: (2) • 1978: (1)

Godman, John Davidson (1794-1830) Bioscript

Godwin, H. Botany School, Cambridge, MA Correspondence - 1944: (1) 
Godwin, Lucia S. Thomas Jefferson Memorial Foundation Correspondence $\bullet 1980$ : (4)

Goethe, C. M.

Biofile $\bullet$ Botanical Album

Correspondence $\bullet$ 1958: (1)

Goethe, Johann Wolfgang von (1749-1832) German author and naturalist

Biofile • Bioscript

Goff, Richard A. U. of Oklahoma, Norman

Correspondence $\bullet$ 1958: (1)

Goheen, Dr.

Correspondence • 1959: (1)

Goldberg, Aaron (1917-) Brooklyn, NY; Taxonomist Botanical Album

Goldblatt, Peter (1943-) Missouri Botanical Garden, B.A. Krukoff Curator of African Botany

Correspondence • 1976: (1) • 1992: (1)

Golden Press

Correspondence $\bullet$ 1965: (1)

Goldie, John (1793-1886) Canadian botanist Biofile • Bioscript

Goldman, Edward Alphonso (1873-1946) Bioscript

Goldschmidt, Richard B. U. of California, Berkeley Botanical Album

Correspondence $\bullet$ 1953: (1)

Goldstein, Cindy Wantagh, NY; high school senior interested in plant geography

Correspondence $\bullet$ 1978: (0) Letters filed under Hilbert Garrison Wilkes

Goldstein, Cynthia Tulane U., Matas Library Correspondence $\bullet$ 1988: (2)

Goldstein, Daniel American historian Correspondence $\bullet$ 1992: (3)

Goldstein, Ron Delaware Valley Fern Society, President, Cochranville, PA

Correspondence $\bullet$ 1976: (3)

Gómez, Luis Diego Correspondence $\bullet$ 1980: (2)

Gómez-Pompa, Arturo (1934) Instituto Nacional de Investigationes sobre Recursos Bioticos, Jalapa, Mexico Bioscript

Correspondence • 1981: (1)

Gonor, Jeff

Correspondence $\bullet$ 1954: (1)

Gonsoulin, Gene East Tennessee State U., Johnson City Correspondence $\bullet$ 1974: (1) • 1975: (2)

Good, John Mason Bioscript

Good, Ralph E.

Botanical Album

Correspondence $\bullet 1977$ : (1)

Goodale, Alfred Shepard (1876-) Amherst College, MA, Dept. of Botany Correspondence $\bullet 1977$ : (1) Goodale, George Lincoln (1839-1923) Physician, botanist,
plant physiologist Biofile • Bioscript

Goodding, Leslie Newton (1880-1967) American botanist Bioscript $\bullet$ Botanical Album

Correspondence • 1949: (2) Rocky Mountain Naturalists

Goode, J. Paul American geographer Biofile
Goodenough, Commodore J. G. (1830-1875)

Bioscript

Goodman, George Jones (1904 ) American plant taxonomist; Iowa State College; U. of Oklahoma, Norman Bioscript $\bullet$ Botanical Album

Correspondence • 1929-1933: (3) • 1934: (1) • 1935: (3) • 1940: (4) • 1941: (2) • 1942: (1) • 1943: (2) • 1944: (1) • 1945: (2) • 1948: (3) Species • 1949: (1) • 1950: (2) 1953: (3) - 1954: (1) - 1961: (4) • 1963: (1) • 1975: (1) • 1988: (1) • 1989: (1) • 1990: (2)

Goodman, Marcia U. of Oklahoma, Norman, DeGolyer Library

Correspondence $\bullet$ 1966: (1) $\bullet$ 1969: (2) • 1980: (1)

Goodman, Philip I. WYES TV, Channel 12 Correspondence $\bullet$ 1970: (2)

Goodman, Roy American Philosophical Society Correspondence • 1983: (1) • 1991: (4) • 1992: (1)

Goodman, Stephen P. Tulane student Correspondence $\bullet$ 1976: (1) Biology-Instruction and study

Goodrich, S. G. Bioscript

Goodrich, Sara Frances (1851-1917) Bioscript

Goodridge, A. Bioscript

Goodridge, J. Bioscript

Goodson, Orr Field Museum of Natural History, Acting Director Correspondence • 1942: (2) • 1943: (3) • 1944: (2)

Goodspeed, , Dr. Correspondence $\bullet$ 1936:

Goodspeed, Thomas Harper (1887-1966) Botanical Album

Goodwin, George H. American Museum of Natural History, Librarian

Correspondence $\bullet$ 1961: (1)

Goodyer, John (fl. 1592) Bioscript

Gordon, Alexander (1813-1873?) Scottish botanical collector Bioscript

Gordon, Armistead C., Jr. Staunton, VA; wrote account of John Banister for the Dictionary of American Biography Correspondence • 1959: (1) Banister, John, 1650-1692

Gordon, D. H. Bioscript

Gordon, Joseph E. Tulane U., New Orleans, Dean Correspondence $\bullet$ 1967: (1) $\bullet$ 1968: (2) $\bullet$ 1971: (1) • 1974: (1) • 1977: (1) • 1982: (1) • 1984: (1)

Gordon, Norma U. of Maryland, College Park, McKeldin Library

Correspondence • 1979: (0) Letter filed under William Louis Stern

Gordon, Pauline Correspondence - 1942: (2)

Gordon, Richard L. Phi Alpha Theta, Honorary History Society, Pres.; Louisiana Tech U., Dept. of History, Ruston, LA

Correspondence • 1978: (2) 
Gordon, Robert Edward Northeast Louisiana State College; American Midland Naturalist, Notre Dame U. Bioscript $\bullet$ Botanical Album Correspondence $\bullet$ 1957: (3) • 1958: (6) 1960 : (1) $\bullet$ 1961: (4) • 1962: (1) • 1963: (2) • 1964: (1) • 1965: (1)

Gordon, Ted Philadelphia Botanical Club Correspondence • 1991: (1)

Gordon, William Bioscript

Gordon-Cumming, Constance Frederica (1837-1924) Bioscript

Gore, Kenneth Bookseller, England Correspondence • 1983: (1)

Gorham Company Correspondence $\bullet$ 1967: (1)

Gorman, Martin Woodlock (1853-1926) American amateur botanist, taxonomist Bioscript

Gorse, George L. Pomona College, Dept. of Art Correspondence 1991 : (4)

Gorter, David de (1717-1783) Botanist, physician, agriculturist Biofile

Goschen, Beatrice Correspondence - 1954: (2)

Gosney, E. S. The Human Betterment Foundation Correspondence $\bullet$ 1939: (2)

Goss, Nathaniel Stickney (1826-1891) American ornithologist Bioscript

Goss, Rod The Natural History Bookcase Correspondence $\bullet$ 1980: (2)

Gosse, Philip Henry (1810-1880) Biofile • Bioscript

Gouan, Antoine Botanical Album

Gould, Augustus Addison (1805-1866) American physician, conchologist Biofile • Bioscript

Gould, Don B. Colorado-Wyoming Academy of Science Correspondence - 1943: (1)

Gould, Edwin (1933-) American zoologist; Smithsonian Institution, National Zoological Park Biofile $\bullet$ Bioscript $\bullet$ Botanical Album Correspondence $\bullet$ 1961: (1) $\bullet$ 1962: (9) $\bullet$ 1963: (2) $\bullet$ 1964: (5) • 1965: (2) • 1966: (1) • 1967: (5) • 1968: (1) • 1970: (6) • 1971: (1) • 1972: (4) • 1973: (3) • 1975: (7) • 1976: (0) Correspondence filed with Stuart Bamforth $\bullet$ 1977: (3) • 1979: (2) • 1980: (2) • 1982: (3) • 1983: (1) • 1985: (2) • 1986: (3) • 1987: (3) • 1988: (4) • 1991: (2) Barton, Benjamin Smith, 1766-1815 • 1992: (2)

Gould, Frank Walton (1913-1981) Compton Junior College; U. of Arizona, Tucson; Texas A \& M College, College Station, Herbarium

Botanical Album

Correspondence • 1943: (3) • 1946: (2) • 1948: (1) Delphinium • 1949: (2) • 1950: (4) Delphinium • 1963: (1) $\bullet$ 1965: (2) • 1967: (2) • 1974: (2)

Gould, George Bioscript
Gould, Harley Zoologist; Tulane U. Correspondence • 1951: (1)

Gould, John

Bioscript

Gould, John (18041881) English-born bird illustrator Biofile

Gould, Joseph Edward (1866-1945) Georgia zoologist Biofile

Gould, Stephen Jay American paleontologist Bioscript

Gould, Sydney Ward (1866-1945) Madison, CT Botanical Album

Correspondence • 1963: (1) • 1966: (2) • 1969: (3) • 1970: (2)

Gould-Linne, Elizabeth Ewan's St. Louis landlord Correspondence • 1986: (5) • 1991: (3)

Goulding, Michael American zoologist Correspondence $\bullet$ 1985: (5) • 1986: (4) • 1988: (1) • 1992: (4)

Grabham, Michael (1866-1938) Portuguese physician, botanist; Trinidad, Madeira

Bioscript

Grace, Charles J., Mrs. Grace \& Way, Inc. Correspondence • 1951: (2)

Gradle, Roy S. Riflesmith-Custom Stock Maker Correspondence $\bullet$ 1950: (2) $\bullet$ 1951: (2)

Gradstein, S. R. Correspondence • 1987: (0) Filed under James Leonard Luteyn

Graenicher, Sigmund (1855-1937) American naturalist, entomologist Biofile

Graf, J. E. Smithsonian Institution Correspondence • 1945: (1) • 1946: (5)

Graff, M. M. Bioscript

Graham, Edward Harrison (1902-1966) American botanist; Carnegie Museum Correspondence • 1936: (9) Delphinium $\bullet$ 1938: (2)

Graham, H. L. Correspondence - 1940: (1) letter from Francis Ramaley

Graham, Italia Husband is L. L. Graham Correspondence - 1949: (1) letter to Fred Cagle

Graham, James Duncan (1799-1865) American topographical engineer, soldier, naturalist Bioscript

Graham, L. L. Correspondence $\bullet$ 1950: (2)

Graham, Mayme Boulder City Clerk Correspondence $\bullet$ 1941: (1)

Graham, Robert (1819-1845) Scottish botanist Biofile

Graham, Shirley A. Kent State U. Correspondence • $1964 \bullet$ 1974: (3) Species

Graham, Thomas (-1822) Bioscript

Graham, William L. U. of Michigan, Ann Arbor,

Herbarium Correspondence • 1971: (2)

Gram, Ernest Botanical Album 
Grant, Adele Lewis (1881-1969) American plant morphologist, taxonomist, plant collector

Bioscript • Botanical Album

Correspondence • 1929-1933: (8) U. of California, Los

Angeles. Dept. of Botany; Jepson, Willis Linn, 1867-1946

Munz, Philip Alexander, 1892-1974 • 1934: (11) Jepson,

Willis Linn, 1867-1946 • 1935: (2) • 1936: (4) Jepson,

Willis Linn, 1867-1946 • 1937: (7) Jepson, Willis Linn,

1867-1946 • 1960: (4) • 1962: (1) • 1963: (1) • 1964: (2)

Grant, Barry Loleta, CA; classmate of Ewan at Berkeley Correspondence • 1938: (2) • 1951: (1)

Grant, Chapman Bioscript

Grant, Dorothy S.

Botanical Album

Correspondence • 1944: (1) • 1986: (3) • 1991: (1) • 1993: (3)

Grant, George Bernard (1849-1917) American amateur botanist, mining engineer

Bioscript • Botanical Album

Grant, J. D. Save the Redwoods Correspondence $\bullet 1941$ : (1)

Grant, J. M.

Bioscript • Botanical Album

Grant, Martin Lawrence (1907-1968) Iowa State Teachers College, Cedar Falls, Dept. of Science

Bioscript • Botanical Album

Correspondence $\bullet 1945:$ (1) • 1952: (2)

Biology-Instruction and study $\bullet$ 1957: (3) • 1959: (1)

Grant, Ronald Albury, England Correspondence $\bullet$ 1951: (1)

Grant, Samuel Berry

Bioscript

Correspondence • 1968: (1)

Grant, Verne Edwin (1917-) Botanist, taxonomist, plant geneticist; Rancho Santa Ana Botanic Garden

Botanical Album

Correspondence $\bullet$ 1950: (2)

Graustein, Jeannette Flizabeth (1892-) U. of Delaware,

Newark, Dept. of Biological Sciences

Bioscript • Botanical Album

Correspondence • 1952: (2) Nuttall, Thomas, 1786-1859

Pursh, Frederick, 1774-1820 • 1953: (1) • 1956: (1)

Nutull, Thomas, 1786-1859 • 1957: (2) • 1958: (3)

Nuttall, Thomas, 1786-1859 - 1959: (3) Nuttall, Thomas,

1786-1859 • 1960: (2) Nuttall, Thomas, 1786-1859 •

1961: (2) Nuttall, Thomas, 1786-1859 • 1962: (3) Nuttall,

Thomas, 1786-1859 • 1963: (1) Nuttall, Thomas,

1786-1859; Letter filed under George W. White $\bullet 1964$ :

(12) Nuttall, Thomas, 1786-1859 • 1965: (4) • 1966: (3)

1967: (4) • 1969: (4) • 1971: (4) • 1972: (1) • 1975: (2)

Gravatt, Annie R.

Correspondence $\bullet$ 1947: (1) Grave, Benjamin Harrison (1878-1949) American zoologist
Bioscript

Graves, Arthur Harmount (1879-1962) Plant pathologist;
Brooklyn Botanic Garden

Botanical Album

Correspondence • 1950: (1) • 1951: (1) • 1952: (2)
Graves, Charles Burr

Botanical Album

Graves, Gary

Bioscript

Correspondence $\bullet$ 1985: (1)

Graves, Jas. A.

Botanical Album

Graves, Thomas A., Jr. College of William and Mary, Williamsburg, VA, President

Correspondence $\bullet$ 1971: (2) • 1972: (1) • 1973: (2) • 1981: (1) • 1982: (1)

Gray, Asa (1810-1888) American botanist, plant explorer, taxonomist, physician Biofile $\bullet$ Bioscript $\bullet$ Botanical Album

Gray, Christopher Bioscript

Gray, F. C. Bioscript

Gray, George Robert Bioscript

Gray, Henry M. Bioscript

Gray Herbarium Harvard U., Arnold Arboretum Correspondence $\bullet$ 1962: (0) Postcard filed under Albion Reed Hodgdon

Gray, Jane Loring Bioscript

Gray, John Edward, Derby, 13th Earl of (1800-1875) British algologist, herpetologist, zoologist Bioscript • Botanical Album

Gray, Peter U. of Pittsburgh Correspondence $\bullet$ 1958: (2)

Gray, Richard A. American Library Association editor, Chicago Correspondence 1969: (0) One letter to W. H. Fries is from Richard A. Gray $\bullet$ 1970: (0) Letter from Gray to Edward Riccuiti; letters to Tim Berra from Gray

Grayson, Andrew Jackson (1819-1869) American wildlife artist, ornithologist

Biofile $\bullet$ Bioscript $\bullet$ Botanical Album

Grear, John Wesley (1937- ) U. of Toronto, Dept. of Botany, Toronto, Canada

Botanical Album

Correspondence • 1968: (1) • 1970: (2) $\bullet$ 1971: (5) $\bullet$

1972: (2) • 1973: (3) • 1975: (2) • 1976: (4) • 1977: (4)

Tulane U., Dept. of Botany $\bullet$ 1979: (3) $\bullet$ 1984: (2)

Greata, Louis A. (1857-1911) Botanist: USA Bioscript

Green, Jacob (fl.1818) Biofile $\bullet$ Bioscript

Green, James N. Library Company, Philadelphia, PA Correspondence • 1985: (3) • 1988: (2) • 1992: (1)

Green, Virginia Chapman Biographer of Alvan Wentworth Chapman; Hueytown, AL; husband is Paul E. Green Correspondence • 1985: (3) • 1986: (1) • 1987: (1)

Greene, Abby Maria Mother of E. L. Greene Bioscript

Greene, Benjamin D. (1793-1862) American lawyer, physician, patron, plant collector; U. S. A Biofile $\bullet$ Bioscript 
Greene, D. B. Boston, MA

Botanical Album

Greene, Earl Rosenbury Ornithologist

Botanical Album

Correspondence $\bullet$ 1959: (4) $\bullet$ 1967: (1) • 1968: (1) $\bullet$

1969: (3) - 1972: (3) - 1975: (3) Written by Terry S.

Moore, Secretary/Treasurer of The 600 Club

Greene, Edward Lee (1843-1915) American botanist, clergyman, plant collector, taxonomist, editor Biofile - Bioscript $\bullet$ Botanical Album

Greene, Ellen U. of Connecticut, Storrs, Dept. of History; husband is John C. Greene

Correspondence $\bullet$ 1982: (1) • 1988: (0) Filed under John C. Greene

Greene, John C. U. of Connecticut, Storrs, Dept. of History

Bioscript $\bullet$ Botanical Album

Correspondence • 1959: (2) Barton, Benjamin Smith, 1766-1815 • 1962: (2) • 1965: (9) • 1966: (9) Van Schaack, George Booth, 1903-1983 - 1967: (4) • 1968: (5)

- 1969: (4) • 1970: (4) • 1971: (4) • 1972: (1) • 1973: (1) - 1974: (5) - 1976: (4) Barton, Benjamin Smith, 1766-1815 • 1977: (6) • 1978: (1) • 1979: (1) • 1980: (6) - 1981: (6) • 1982: (7) • 1983: (1) • 1988: (3) Barton, Benjamin Smith, 1766-1815 • 1993: (1)

Greene, Wilhemina Freeman (1906-) Botanical artist Bioscript

Greenewalt, Crawford Bioscript

Greenman, Jesse More (1867-1951) American taxonomist; Missouri Botanical Garden, St. Louis, MO; Washington U., St. Louis, MO

Bioscript $\bullet$ Botanical Album

Correspondence $\bullet$ 1929-1933: (1) • 1934: (5) $\bullet$ 1935: (2) • 1937: (2) • 1938: (2) 1 1940: (2) $\bullet$ 1941: (5) $\bullet$ 1942: (2) $\bullet$ 1943: (8) Delphinium • 1948: (2) • 1950: (1) $\bullet$ 1951: (2)

Greenwald, Margaret Librarian; Academy of Natural Sciences Correspondence $\bullet$ 1963: (2) $\bullet$ 1965: (2)

Greenway, James (1703-1793[4]) Bioscript

Greenwood, Barbara D. Merseyside Country Museums, Liverpool, England, Keeper of Botany

Correspondence • 1978: (2) • 1979: (6) Barton, Benjamin Smith, 1766-1815 1980: (2)

Greenwood, Eric F. Merseyside County Museum, Liverpool, England, Asst. Director Correspondence $\bullet$ 1983: (2)

Greenwood, O. M. Correspondence $\bullet$ 1929-1933: (1)

Greenwood, Robert W. (Bob) Tulane U., New Orleans, Howard-Tilton Memorial Library Botanical Album Correspondence - 1964: (1) - 1969: (2) Book collectors • 1973: (1) • 1975: (3) • 1976: (1) • 1977: (8) - 1978: (1)

Greenwood, Thelma State Teachers College, West Chester, PA

Correspondence $\bullet$ 1950: (2)

Greer, Alice Mary

Correspondence $\bullet$ 1956: (1)
Gregg, Amos

Bioscript

Gregg, Clifford C. Field Museum of Natural History Correspondence • 1938: (2) • 1941: (3) • 1946: (1)

Gregg, Josiah (1806-1850) American plant collector, pioneer trader, explorer Biofile • Bioscript

Gregg, Maxcy

Bioscript

Gregg, Raymond Rocky Mountain National Park, Naturalist Botanical Album

Correspondence • 1942: (1)

Gregg, Robert Edmond U. of Colorado, Boulder Correspondence • 1946: (1) • 1968: (2) • 1971: (1) • 1972: (1)

Gregorie, Henri (1750-1831)

Bioscript

Gregory, Emily Lovira (1841-1897) Botanist Bioscript

Gregory, Margaret P. Tennessee Polytechnic Institute Correspondence • 1941: (2) • 1942: (1)

Gress, E. M. Correspondence • 1929-1933: (1)

Gressley, Gene M. U. of Wyoming. Laramie, Western History Research Center

Correspondence • 1967: (3) Letter from Edith Clements • 1978: (3) • 1979: (4) • 1982: (1) • 1983: (3)

Greulich, W. W. Bioscript

Greville, Robert Kaye (1794-1866) Scottish botanist and landscape painter Biofile • Bioscript

Grew, Bioscript

Grew, Nehemiah Biofile

Gribben, John H. Tulane U., New Orleans, Howard-Tilton Memorial Library

Correspondence • 1967: (6) letter to Robert A. Gamble; letter from Nada Kramar • 1968: (4) • 1971: (3)

Gridley, Daniel B. Sierra Club, San Francisco, CA Correspondence $\bullet$ 1978: (1)

Gries, George A. U. of Arizona, Tucson Correspondence $\bullet 1965:(1) \bullet 1967:$ (1)

Griffin, Alfred A. Bioscript

Griffin, Dana Grove, III Correspondence $\bullet$ 1983: (1)

Grifin, Jeanne Correspondence $\bullet$ 1984: (1)

Grifin, William D. St. John's U., Jamaica, NY, Dept. of History Correspondence $\bullet 1977$ : (1)

Griffith, Consuelo Garza Tulane U., New Orleans, Howard-Tilton Memorial Library; nickname "Connie" Botanical Album

Correspondence • 1966: (1) • 1967: (1) • 1972: (1) • 1973: (1) letter to Florence Wagner $\bullet 1974$ : (1) 1989 : $(0)$ Invitation to dedication of portrait of Griffith, Emeritus Librarian, Spec. Coll, Tulane U.

Griffith, Dr.

Bioscript 
Griffith, Edward I. Metairie, LA

Correspondence • 1960: (1)

Griffith, William Tulane U., New Orleans, Howard-Tilton Memorial Library

Bioscript

Correspondence $\bullet 1964$ : (1)

Grifith, William (1810-1845) English-born botanist, physician in India

Biofile

Griffiths, Amelia

Bioscript

Griffiths, David (1867-1935) Welsh-born American agronomist, plant scientist

Bioscript

Griffiths, Mark

Botanical Album

Griggs, Anthony Reporter; Chicago Daily Defender Correspondence $\bullet$ 1973: (2)

Griggs, Robert Fiske (1881-1962) Plant ecologist Bioscript

Grimes, James U. of Texas at Austin Correspondence • 1983: (2)

Grimes, John (1781-1816)

Bioscript

Grindon, Leopold Hartley (1818-1904) British botanist, businessman

Biofile

Grinnell, Fordyce, Jr.

Bioscript

Grimell, George Bird (1849-1938) American naturalist, ethonologist

Bioscript

Grinnell, Hilda W. Cooper Ornithological Club Correspondence • 1936: (1)

Grinnell, Joseph (1877-1939) American vertebrate zoologist; U. of California, Berkeley, The Condor Biofile $\bullet$ Bioscript $\bullet$ Botanical Album Correspondence • 1921-1928: (2) • 1935: (4) • 1937: (2) • 1938: (1)

Grinnell, Mary E. Army Medical Library, Washington D.C., Head, Reference Section Correspondence • 1951: (1) Barton, Benjamin Smith, 1766-1815

Grisebach, August Heinrich Rudolf (1814-1879) Bioscript

Grissom, B. R.

Correspondence $\bullet$ 1963: (2)

Groh, Herbert

Botanical Album

Correspondence • 1956: (3)

Grolier Club of New York, The Correspondence $\bullet 1977$ : (1)

Gronovius, Johannes Fredericus (1611-1671) Bioscript

Gronovius, L. T. Bioscript

Gronweg, William E. New Orleans, LA Correspondence • 1962: (3) Letter from Gronweg to Miss Daniel

Groover, Robert D. Tulane U. New Orleans, Dept. Biology, Asst. Prof.

Correspondence $\bullet$ 1969: (0) Correspondence filed under Richard Alden Howard
Gross, C. A. Bioscript

Gross, Samuel

Bioscript

Grout, Bioscript

Grout, Abel Joel (1867-1947) American bryologist, taxonomist Biofile $\bullet$ Bioscript $\bullet$ Botanical Album

Grout, Robert C. Botanical Album

Grout, Selma U. Correspondence • 1943: (1) • 1953: (1) "Rocky Mountain Vegetation" - 1954: (2) "Rocky Mountain Vegetation"

Grover, Edwin Osgood Mead Botanical Garden; brother of Frederick Grover Botanical Album Correspondence $\bullet$ 1949: (1)

Grover, Frederick Orville (1868-1964) Oberlin College Correspondence • 1938: (2) • 1942: (1) • 1949: (8) Rocky Mountain Naturalists $\bullet$ 1950: (3)

Groves, , Dr. Bioscript

Groves, Edwin O. Correspondence • 1957: (2)

Groves, Eric William (1923-) British Museum (Natural History), Dept. of Botany, London, England

Bioscript $\bullet$ Botanical Album

Correspondence • 1956: (1) • 1957: (4) • 1958: • 1962;

(1) 1 1963: (2) • 1964: (5) $\bullet 1965:(6) \bullet 1967:$ (4) $\bullet 1968$ :

(5) Letter from Fred Meyer $\bullet$ 1969: (2) 1 1970: (1) Banks, Joseph, Sir, 1743-1800 • 1971: (1) • 1973: (1) • 1974: (2)

- 1975: (1) • 1977: (2) • 1978: (4) • 1979: (4) • 1981: (8)

- 1982: (7) • 1983: (3) • 1984: (3) • 1985: (5) • 1986: (2)

- 1988: (1) • 1990: (2) • 1991: (8) • 1992: (16) Letter

from Frank A. Lang to Jo Alexander • 1993: (1)

Grube, George

Correspondence $\bullet$ 1987: (1)

Gruber, Ferdinand (1830-1907) Bioscript

Gruell, George E. United States Dept. of Agriculture, Forest Service, Teton National Forest, Wildlife Biologist Correspondence 1970: (3)

Gruenberg, Benjamin Botanical Album

Grueningen, J. P. von Crosby-Ironton Junior College Correspondence $\bullet$ 1948: (1)

Grunder, Henry College of William and Mary, Williamsburg, VA, Earl Gregg Swem Library Correspondence • 1968: (2)

Grunwald, Claus Illinois Natural History Survey, Urbana; Head, Section of Botany and Plant Pathology Correspondence • 1976: (2)

Gruson, Edward S. Correspondence $\bullet$ 1973: (1)

Guarraia, Betty Correspondence • 1991: (1)

Guba, Emil Frederick Correspondence $\bullet$ 1967: (1)

Guberlet, John Earl (1887-1940) American parasitologist; student of T.D.A. Cockerell Biofile 
Gudger, Eugene Willis (1866-1956) American Museum of Natural History, Dept. of Fishes and Aquatic Biology, Honorary Associate

Correspondence $\bullet$ 1951: (2)

Guerlac, Henry Correspondence $\bullet$ 1962: (1)

Guerriero, Henry Correspondence $\bullet$ 1948: (2) $\bullet$ 1949: (1)

Guilding, Lansdown (1798-1831) West Indian clergyman and naturalist Biofile • Bioscript

Guillarmod, A. Jacot Rhodes U., Dept. of Botany and Microbiology, Grahamstown, C.P., South Africa Correspondence $\bullet$ 1971: (1)

Guiney, Grace School of Forestry, Oxford, England Correspondence 1973 : (1) Letter also signed by Ruth Guiney • 1977: (2) • 1980: (1)

Guiney, Ruth Oxford, England Botanical Album Correspondence $\bullet$ 1968: (3) $\bullet$ 1969: (1) $\bullet$ 1971: (1) • 1976: (1) - 1978: (1) • 1979: (2)

Guirado, Francisco Bioscript

Gulf Coast Research Laboratory Ocean Springs, MS Correspondence 1977 : (1)

Gulf Islands National Seashore Correspondence • 1974: (1)

Gumbleton, William Edward (1840-1911) Irish bibliophile, horticulturist Biofile $\bullet$ Bioscript

Gunckel, James Eugene (1914 ) Rutgers U., Dept. of Botany, Current Topics in Plant Science, Editor Correspondence • 1969: (3)

Gunn, Charles Robert (1927- ) Iowa State U., Ames; Plant Industry Station, Beltsville, MD; Nickname "Bob"

Botanical Album

Correspondence • 1963: (2) $\bullet$ 1968: (1) $\bullet$ 1973: (1) $\bullet$ 1974: (1) Biology-Instruction and study $\bullet$ 1975: (2) • 1976: (2) • 1977: (1) • 1978: (1) • 1987: (3)

Gunning, Dolores Tulane U., New Orleans, Dept. of Biology

Bioscript $\bullet$ Botanical Album (Filed under Gerald Eugene Gunning)

Correspondence $\bullet$ 1982: (2) $\bullet$ 1984: (2) Barton, Benjamin Smith, 1766-1815 • 1986: (3) • 1988: (2)

Gunning, Edith Dunn

Botanical Album (Filed under Fred Gunning)

Gunning, Fred

Botanical Album

Gunning, Gerald Eugene (1932-1991)

Bioscript $\bullet$ Botanical Album

Gunnison, John Williams (1812-1853) American military engineer

Bioscript - Botanical Album

Gunther, Erica (1896-)

Bioscript

Gunther, R. T. Botanical Album

Gunthrop, Horace Horace Gunthrop \& Sons, Scientific Literature, San Diego, CA

Correspondence • 1943: (1) Book collectors $\bullet$ 1944: (6)

Gurney, James (1831-1920) Bioscript
Gussone, Giovanni (1787-1866)

Bioscript

Gustafson, A. H. Williams College

Correspondence - 1942: (3) Biology-Instruction and study

Gustafson, Robert Natural History Museum, Los Angeles County

Correspondence $\bullet$ 1980: (1)

Gustavson, Russell G. U. of Colorado, Boulder, Dean; Colorado Chapter Sigma XI

Correspondence • 1941: (1) • 1942: (1) • 1943: (8) U. of Colorado, Boulder. Dept. of Biology • 1944: (5) • 1956: (2)

Gutowski, Robert U. of Delaware, Longwood Program Correspondence • 1984: (2) • 1985: (1)

Gutzkow, F. Bioscript

Guyana Correspondence $\bullet$ 1985: (1)

Guyénot, E. Université de Genève Correspondence • 1948: (1)

Guyot, Arnold Henry (1807-1884) Swiss-born American geologist, geographer Bioscript

Guyot, C. A. Educational Publishers, Inc. Correspondence $\bullet$ 1961: (1)

Gwyn, Ann Tulane U., New Orleans, Howard-Tilton Memorial Library, Librarian; Program Chairman of Friends Correspondence $\bullet$ 1977: (3)

\section{$\boldsymbol{H}$}

Haarstad, Vernon B. Tulane U. Medical School, New Orleans, LA

Correspondence $\bullet$ 1968: (3) $\bullet$ 1971: (1)

Haas, Anna L. Botanical Society of New Orleans; Benjamin Franklin Senior High School, New Orleans Correspondence • 1948: (1) - 1962: (1)

Haas, W. H. Bioscript

Haberer, J. V. Bioscript

Haberlandt, Gottlieb Botanical Album

Haberle, C. C. Bioscript

Habersham, James Bioscript

Hackman, Gwen Correspondence • 1980: (1)

Hackney, Julie Ewan's student; later grad student UNC, Chapel Hill Correspondence • 1969: (1) • 1969: (0) Letter filed under Bassett Maguire • 1970: (1)

Hackney, Nancy Husband is Sheldon Hackney Correspondence $\bullet$ 1972: (2)

Hackney, Sheldon Tulane U., President; later U. of Pennsylvania, Philadelphia, President

Botanical Album

Correspondence • 1976: (4) • 1977: (2) • 1980: (3) • 1982: (2)

Hadden, Elizabeth McGill U., Montreal Correspondence • 1955: (1) 
Hadley, James

Bioscript

Hadsall, Leo F. Botanical Album

Haenke, Thaddaeus Peregrinus Xaverius (1761-1817) Bohemian botanist Biofile • Bioscript

Hafen, LeRoy R. (1893-) American historian; State Historical Society of Colorado; Brigham Young U. Biofile Correspondence • 1941: (2) • 1943: (1) • 1947: (1)

Hafner Press Correspondence • 1973: (9) • 1974: (3)

Hagelstein, Robert (1870-) Botanical Album

Hagen, H. A. Bioscript

Hagenah, Dale J. (1908-1971) American botanist Biofile

Hagerup, Olaf (1889-1961) Universitetes Botaniske Museum, Kobenhavn, Denmark Correspondence $\bullet$ 1951: (2)

Hahn, Bioscript

Hahn, Eduard (1856-1928) German economic geographer Biofile

Hahn, Roger U. of California, Berkeley Correspondence $\bullet 1963$ : (0) Filed under U. of California, Berkeley

Haines, Adelbert Lee (1915-) Wife is Katherine Haines Bioscript

Correspondence • 1936: (1) 1954: (1) • 1983: (1) • 1988: (3)

Haines, Henry Haselfoot (1867-1945) British-born Indian forester Biofile

Haines, Katherine Kneen Topanga, CA; husband is Lee Haines Bioscript • Botanical Album

Correspondence • 1940: (4) • 1969: (1) • 1974: (1) 1978: (1) • 1985: (1) • 1988: (0) Filed under Adelbert Lee Haines

Haines, Kay

Correspondence $\bullet 1980$ : (1) Letter to Betty Sprague

Halcrow, A. Trinity College Library, Sub-Librarian Correspondence $\bullet$ 1969: (0) Filed under Trinity College

Haldane, J. B. S. Bioscript

Haldeman, Julius Emanuel (1889-) Bioscript

Haldeman, Samuel S. Bioscript

Hale, Josiah Bioscript - Botanical Albwn

Hale, Mason Ellsworth (1928-1990) Smithsonian Institution, Dept. of Botany Correspondence • 1979: (2) • 1985: (1) • 1986: (1)

Hale, Sarah Josepha (1788-1879) Bioscript

Hale, T. J. Botanical Album

Hales, Stephen, Rev. Bioscript
Hall, Alfred Daniel, Sir (1864-1942) John Innes Horticultural Institution, London, England, Director Botanical Album

Hall, Basil

Bioscript

Hall, C. F. Bioscript

Hall, Carlotta Case (1880-1949) Bioscript Correspondence • 1936: (2) • 1949: (2)

Hall, Charles Prentice-Hall Pub. Correspondence • 1957: (1)

Hall, E. Raymond (1902-1986) American mammologist Biofile

Hall, Elihu (1820/22-1882) American botanist Bioscript $\bullet$ Botanical Album

Hall, Elizabeth Cornelia (1898-1989) New York Botanical Garden Librarian; later Horticultural Soc. of NY

Bioscript • Botanical Album

Correspondence $\bullet$ 1941: (4) $\bullet$ 1950: (4) $\bullet$ 1951: (2) Pursh, Frederick, 1774-1820 • 1952: (4) • 1953: (1) • 1955: (4) • 1956: (10) • 1958: (6) • 1959: (2) • 1960: (1) • 1967: (2) - 1968: (2)

Hall, Leonard (1899/1900-1992) American writer and conservationist; Possum Trot Farm, Caledonia, MO; wife is Virginia (Ginnie) Hall Correspondence 1959 : (2) 1962 : (16) Attached letter to Hall from Michel, R., Audubon Society Librarian $\bullet$ 1963:

(12) • 1964: (1) • 1965: (2) • 1966: (5) • 1967: (2) • 1969: (1) - 1970: (1) • 1972: (1) • 1974: (1) • 1976: (2) - 1978: (1) • 1980: (3) • 1983: (2) • 1986: (1) • 1987: (2) - 1988: (2)

Hall, Marion Trufant (1920- ) Morton Arboretum, Lisle, II Botanical Album Correspondence • 1971: (4) • 1972: (1)

Hall, O. F. Louisville Free Public Library Correspondence • 1950: (1)

Hall, Richard Willmott Bioscript

Haller, Albrecht. van [the Elder] (1758-1823) Bioscript

Haller, Albert Bioscript

Halley, Edmond (1656-1742) English astronomer and cartographer Biofile

Hallier, Hans Bioscript • Botanical Album

Hallinen, Joseph E. Bioscript

Hallowell, Edward Bioscript

Halsey, Abraham (1790-1857) Bioscript

Halsted, Byron David (1852-1918) American botanist Biofile $\bullet$ Bioscript $\bullet$ Botanical Album

Haltgren, Matthew Bioscript

Halverson, Richard F. Historic Hudson Valley, President Correspondence • 1988: (1) Barton, Benjamin Smith, 1766-1815 
Halway, Edward W. D. (1853-1923) Bioscript

Hamann, Skee Madrone Audubon Wildlife Refuge, Middleton, CA

Correspondence - 1977: (3)

Hamel, Paul B. Clemson U., Clemson, SC, Dept. of Zoology Correspondence $\bullet$ 1978: (4)

Hamilton, Claudius (fl. 1699) Bioscript

Hamilton, Egbert Botanical Album

Hamilton, Francis (once Buchanan) Biofile

Hamilton, Jean Indiana U., Library Correspondence • 1969: (1)

Hamilton, Lowell S. Overbrook House Correspondence • 1991: (1)

Hamilton, Thomas Bioscript

Hamilton, William (1745-1813) American landscape gardener Biofile $\bullet$ Bioscript

Hamilton, William (1788-1856) Irish botanist and plant collector of the West Indies Biofile

Hamlin, Elijah L. (1800- ) American physician and minerologist Biofile

Hammer, Jeanne M. U. of Virginia Library, Charlottesville Correspondence $\bullet$ 1993: (3)

Hammett, Lisa Reynolds Madison-Morgan Culural Center, Madison, GA

Correspondence $\bullet$ 1991: (1)

Hammond, John Fox (1820-1886) American Army surgeon Bioscript

Hampe, $\mathbf{E}$. Bioscript

Hanbury, Daniel Bioscript

Hance, Henry F. Bioscript

Hanchey, _, Prof. Correspondence • 1962: (1)

Hancock, Allan Botanical Album

Hancock, Charles D. Science Education Council Correspondence $\bullet$ 1957: (2)

Hand, Dean W. Correspondence $\bullet$ 1992: (3)

Hand, Wayland D. U. of California, Los Angeles Correspondence • 1975: (4)

Handel-Mazzetti, Heinrich (1882-1940) Bioscript

Handy, Hastings Bioscript

Haney, Alan W. U. of Illinois, Urbana, Dept. of Botany Correspondence • 1969: (1)

Hanna, F. W. Correspondence $\bullet$ 1935: (1)

Hanna, G. Dallas (1887-1970) Bioscript
Hanna, Leo Arthur (1893-) American botanist; Custer County Junior College and High School, Miles City, MT Biofile

Correspondence • 1953: (3) "Rocky Mountain Vegetation"; Chronica Botanica

Hanna, Wilson C.

Correspondence • 1972: (1)

Hansell, Dorothy Ebel (1900-) New York Botanical Garden; The Garden Journal, Editor Correspondence $\bullet$ 1950: (3) • 1951: (4) • 1952: (3) • 1958: (2)

Hansen, Emil Christian (1842-1909) Swedish microbiologist Biofile

Hansen, Eric Steen (1943-) Bioscript

Hansen, George Bioscript

Hansen, Georgia Norway; husband is Jacob L. Hansen; an art historian and friend of Elliot A. P. Evans Correspondence $\bullet$ 1951: (1)

Hansler, Hilde Correspondence • 1968: (1)

Hanson, Bea Bromeliad Society of New Zealand Correspondence • 1974: (4)

Hanson, Craig Alfred Correspondence $\bullet$ 1956: (2)

Hanson, Herbert Christian (1890-1962) American plant ecologist, conservationist; Biological Society of Washington Correspondence • 1948: (1)

Hanson, Mr.

Correspondence $\bullet$ 1966: (1)

Hantel, John L. Duke and Porterie, New Orleans, LA Correspondence $\bullet$ 1977: (1)

Hapeman, Harry (1858-) Amateur botanist, physician; Minden, NE

Bioscript • Botanical Album

Correspondence $\bullet$ 1937: (3) $\bullet$ 1938: (9) $\bullet$ 1939: (6)

Species • 1940: (3) • 1941: (4) • 1942: (8) • 1942: (0) Species; letter filed under Walter Biggler Kiener • 1943: (4) - 1944: (2) • 1945: (1) • 1946: (2)

Harada, Takeshi U. of Hawaii Correspondence • 1974: (1)

Harbour, J. P. (fl. 1862) Bioscript

Harbour, Jefferson Lee, Mrs. Correspondence • 1949: (1) Rocky Mountain Naturalists

Hardaway, Daniel Bioscript

Harden, D. Bioscript • Botanical Album

Harden, John Macpherson Barrier (1810-1848) Bioscript

Hardie, Ruth Correspondence $\bullet$ 1970: (3)

Hardin, James Walker (1929-) U. of Georgia, Athens; North Carolina State College, Raleigh Correspondence $\bullet$ 1953: (1) • 1962: (2) • 1969: (1) • 1986: (1)

Hardwicke, Thomas (1756-1835) English general and zoological artist

Biofile 
Hardy, Claire New Orleans, LA

Correspondence $\bullet 1962$ : (1)

Hardy, Clive U. of New Orleans, Archivist Bioscript

Correspondence $\bullet$ 1974: (2) • 1979: (1) • 1982: (1)

Hardy, Ernest Bookseller; Church Court Bookshop, Books Old and Modern, Dorset, England Correspondence • 1955: (1) • 1958: (2) • 1961: (1)

Hardy, George A. Botanist; Provincial Museum, Victoria, B. C. Correspondence $\bullet$ 1941: (7) $\bullet$ 1942: (2) Species $\bullet$ 1943: (7) Delphinium • 1944: (2) • 1945: (2)

Harford, William George Washington (1825-1911) Bioscript

Hargrave, Lyndon Lane (1896-1978) American archeologist

Biofile

Hargrove, Carolyn Correspondence $\bullet 1992$ : (1)

Hariot, Thomas (1560-1621) English painter and geographer

See also Thomas Harriot Bioscript

Haritonidou, Pauline (1920-1981) Greek botanist, born in New Zealand Biofile

Harkness, Bernard Emerson (1907-) Highland Park Herbarium, Rochester, NY Correspondence $\bullet 1964$ : (4)

Harkness, Harvey Willson (1821-1901) Bioscript

Harkness, Hilda J. Ontario Dept. of Mines and Resources; National Museum of Canada, Ottawa Correspondence $\bullet$ 1943: (2) Delphinium $\bullet$ 1958: (1)

Harlan, Jack Rodney (1917-) U. of Illinois, Urbana Bioscript Correspondence $\bullet$ 1976: (1)

Harlan, Richard (1796-1843) American paleontologist Biofile • Bioscript

Harley, Annette Correspondence • 1983: (2)

Harley, Basil H. Curwen Press, London; later Harley Books, Natural History Publishers, England Botanical Album

Correspondence - 1965: (0) Filed under Curwen Press Ltd. - 1969: (3) • 1970: (1) • 1973: (2) • 1977: (1) • 1978: $(12) \bullet 1979:(1) \bullet 1980:(2) \bullet 1981:$ (4) • 1982: (1) • 1984: (6) • 1986: (1) • 1992: (1)

Harley, Madeline M. Royal Botanic Gardens, Kew, Palynology Unit, The Herbarium Correspondence $\bullet 1991$ : (1)

Harlow, Deborah Lawrence Marine Biological Laboratory, Woods Hole, MA Correspondence • 1956: (1)

Harned, Joseph Edward Botanical Album

Harper and Brothers Correspondence • 1940: (1)

Harper and Row Correspondence 1974: (1)

Harper, Edward Thompson (1857-1921) American mycologist Bioscript
Harper, Francis (1886-1972) American author, naturalist, and editor; Mount Holly, NJ; later Chapel Hill, NC Biofile $\bullet$ Bioscript $\bullet$ Botanical Album

Correspondence • 1950: (2) • 1951: (8) • 1952: (9) Academy of Natural Sciences of Philadelphia; Bartram, William, 1739-1823; Pennell, Francis Whittier, 1886-1952; letters to M. Albert Linton, J. Percy Moore, H. S. Drinker - 1953: (6) Bartram, William, 1739-1823; National Science Foundation - 1954: (11) Langman, Ida Kaplan; Bartram, William, 1739-1823; Barton, Benjamin Smith, 1766-1815; British Museum (Natural History). Dept. of Botany 1955 : (8) Peattie, Donald Culross, 1898-1964 • 1956: (12) Bartram, William, 1739-1823 • 1957: (8) Bartram, William, 1739-1823 • 1958: (11) Bartram, William, 1739-1823 • 1959: (10) Banister, John, 1650-1692 • 1960: (4) Harper, Roland McMillan, 1878-1966 • 1961: (4) Bartram, William, 1739-1823 • 1962: (8) Bartram, William, 1739-1823 • 1963: (0) Letter filed under Richard M. Dorson • 1963: (14) • 1964: (9) • 1966: (1) Harper, Roland McMillan, 1878-1966 • 1967: (1) • 1968: (5) Harper, Roland McMillan, 1878-1966

Harper, Jean Sherwood (1896-1990) Husband is Francis Harper

Biofile

Correspondence • 1953: (2) Harper, Francis, 1886-1972 • 1973: (1) - 1975: (8) Harper, Francis, 1886-1972;

Bartram, William, 1739-1823; letter to Ralph Palmer • 1976: (1) Harper, Francis, 1886-1972

Harper, Lucy Daughter of Francis Harper Correspondence $\bullet$ 1955: (2)

Harper, Mary Sue Gulfport, MS; husband is Roland Harper Correspondence • 1959: (1) • 1966: (8) Harper, Roland McMillan, 1878-1966 • 1967: (1)

Harper, Roland McMillan (1878-1966) American botanist, geographer; U. of Alabama, Tuscaloosa; brother of Francis Harper

Biofile $\bullet$ Bioscript $\bullet$ Botanical Album

Correspondence $\bullet$ 1950: (3) $\bullet$ 1951: (5) $\bullet$ 1952: (15)

Cockerell, Theodore Dru Alison, 1866-1948; Pennell, Francis Whittier, 1886-1952 - 1954: (8) • 1955: (3) • 1956: (3) 1957: (9) - 1958: (5) • 1959: (5) • 1960: (0) letter filed with Francis Harper - 1960: (10) Species $\bullet$ 1961: (4) • 1963: (3) • 1964: (8) • 1965: (2) • 1966: (1) Harper, Roland McMillan, 1878-1966

Harrar, E. S. Duke U., Acting Dean Correspondence $\bullet$ 1956: (2)

Harrell Books Correspondence $\bullet$ 1992: (2)

Harriman, Edward Henry (1849-1909) Bioscript

Harriman, Neil A. (1938-) Vanderbilt U., Nashville, TN; U. of Wisconsin-Oshkosh Correspondence • 1964: (1) - 1985: (5) • 1986: (3) • 1989: (2) Barton, Benjamin Smith, 1766-1815 • 1992: (1)

Harrington, Harold David (1903-1981) Colorado State A \& M College, Fort Collins

Botanical Album

Correspondence • 1936: (1) • 1943: (2) • 1944: (4) • 1946: (1) • 1948: (4) • 1949: (4) Rocky Mountain Naturalists • 1951: (1) - 1952: (2) • 1956: (1) - 1958: (3) Delphinium • 1962: (4) • 1963: (1)

Harrington, John Peabody (1884) Ethnologist Bioscript 
Harrington, Mark Walrod (1848-1926) Bioscript

Harriot, Thomas Bioscript

Harris, Charles M. Papers of William Thornton, editor Correspondence • 1982: (5) • 1983: (11) • 1984: (2) • 1988: (2)

Harris, Edward Bioscript

Harris, James G. Botanical Album

Harris, Kathleen Ewan Daughter of Joseph and Nesta Ewan; nickname "Kada"; husband is Richard "Dick" Harris Botanical Album

Correspondence • 1958: (1) • 1959: (2) • 1963: (3) • 1964: (3) $\bullet$ 1965: (2) • 1966: (3) • 1967: (2) $\bullet$ 1968: (2) • 1969: (3) • 1971: (2) • 1974: (5) • 1975: (6) • 1976: (1) $\bullet$ 1977: (1) - 1979: (1) Book collectors • 1980: (2) • 1982:

(4) $\bullet$ 1984: (6) $\bullet$ 1986: (1) $\bullet$ 1987: (1) $\bullet$ 1988: (3) $\bullet$ 1989: (1) $\bullet$ 1991: (1) $\bullet$ 1993: (1)

Harris, Lena Scott Bioscript

Harris, Lucile Desert Magazine Correspondence • 1943: (1)

Harris, Melinda Woolf Botanical Album (Filed under James G. Harris)

Harris, Moses Bioscript

Harris, Richard U. S. Navy Commanding Officer, USS Saginaw; wife is Kathleen Ewan Harris; nickname is "Dick" Botanical Album

Correspondence - 1959: (0) Letter to both filed under Kathleen Ewan Harris $\bullet$ 1962: (1) $\bullet$ 1973: (2) $\bullet$ 1976: (0) Letter filed with Kathleen Ewan Harris • 1989: (3) • 1988: (1) $\bullet$ 1990: (3)

Harris, Richard U. of Michigan, Herbarium, Ann Arbor, MI

Correspondence $\bullet$ 1978: (1)

Harris, Rufus C. Tulane U., President (1948-1959); Mercer U., Macon, GA (1964-1983)

Bioscript

Correspondence • 1948: (2) • 1949: (2) $\bullet$ 1951: (1) $\bullet$ 1952: (1) • 1953: (2) • 1954: (1) • 1956: (0) Letter filed under George Ralph Cooley • 1956: (2) • 1959: (1) Letter from Harris to Miss Harriett Joor $\bullet 1964$ : (1) $\bullet$ 1983: (1)

Harris, Thaddeus William (1795-1856) American entomologist and physician Biofile • Bioscript

Harris, Tucker Bioscript

Harris, Van T. Bureau of Sport Fisheries and Wildlife, Wildlife Research Biologist Correspondence • 1959: (2)

Harris, William Bioscript

Harris, Wilson Park (1869-1929) American forester Bioscript

Harrison, Bertrand Fereday (1908-) American plant physiologist; Brigham Young U. Correspondence • 1939: (2) • 1940: (2)

Harrison, John C. Correspondence • 1970: (1) Rocky Mountain Naturalists
Harrison, John P. Bioscript

Harry, Robert Rees Academy of Natural Sciences of Philadelphia Correspondence $\bullet$ 1953: (2)

Harshberger, John William (1869-1929) American botanist Bioscript

Hart, Helen (1900-1971) American plant pathologist Biofile

Hart, John Hinchley (1847-1911) Bioscript

Hart, Melinda B. Université de Paris, Departement de Langues Appliquées, Instructor in Legal English Correspondence • 1979: (3)

Hartig, Theodor (1805-1880) Bioscript

Hartley, Carl Pierce (1887-1968) American plant pathologist

Bioscript Correspondence - 1949: (1) Rocky Mountain Naturalists

Hartman, Bioscript

Hartman, Ronald Lee (1945- ) Bioscript

Hartog, M. M. Bioscript

Hartweg, F. Bioscript

Hartweg, Karl Theodor (1812-1871) Bioscript

Hartwell, John B. (1878-1957) American surgeon and botanist; Colorado Springs, CO

Biofile • Botanical Album

Correspondence $\bullet$ 1942: (1) • 1943: (5) • 1944: (3) • 1946: (1) • 1948: (6) Delphinium; Species • 1949: (1) 1955: (1)

Harvard College Library Cambridge, MA Correspondence • 1937: (1) • 1964: (1)

Harvard U. Bioscript $\bullet$ Botanical Album

Harvard U. Houghton Library Cambridge, MA Correspondence $\bullet$ 1964: (1)

Harvard U. Press Cambridge, MA Correspondence • 1964: (3) • 1969: (1)

Harvard U. History of Science Dept. Correspondence $\bullet$ 1974: (2)

Harvey, Barbara New Orleans, LA \& Metaire, LA; husband is Theodore H. Harvey, Jr. Correspondence • 1971: (2) • 1974: (2) • 1976: (1)

Harvey, Burwell Towns (1892-) American chemist Bioscript

Harvey, F. L. Bioscript

Harvey, H. Thomas San Jose State U., San Jose, CA, Dept. of Biological Sciences Correspondence $\bullet 1977$ : (2)

Harvey, Harriet U. of Oklahoma, Norman Correspondence • 1953: (6) National Science Foundation • 1955: (2)

Harvey, John H. Historian of horticulture, registered architect; Frome, Somerset, England Biofile - Botanical Album 
Harvey, John H. (continued)

Correspondence $\bullet$ 1972: (2) • 1975: (5) • 1977: (8) • 1979:

(3) $\bullet$ 1980: (2) • 1981: (1) • 1984: (3) $\bullet$ 1985: (4)

Harvey, Leroy E. U. of Montana, Dept. of Botany Correspondence • 1970: (1) Rocky Mountain Naturalists

Harvey, Marian H. U. S. Dept. of Agriculture Correspondence • 1948: (1)

Harvey, , Mrs. Former student Correspondence • 1973: (1)

Harvey, Theodor H., Jr. Harvey Press, Kenner, LA; wife is Barbara Harvey

Correspondence - 1976: (1) Biology-Instruction and study

Harvey, William Henry (1811-1866) Biofile • Bioscript

Harvill, Alton M., Jr. Longwood College, Farmville, VA Bioscript Correspondence $\bullet$ 1970: (1) • 1975: (5) $\bullet$ 1978: (2) • 1980: (2) Pursh, Frederick, 1774-1820

Harwood, Robert Daniel (1899-) Bioscript

Haselton, Scott E. Abbey Garden Press, Pasadena, CA; Cactus and Succulent Journal Correspondence • 1947: (1) • 1949: (0) Rocky Mountain Naturalists; letter filed under Boyd L. Sloane

Haskett, Jane U. of California, Berkeley Correspondence $\bullet$ 1963: (2)

Haslett, Philip H. Weybridge, Surrey, England Correspondence • 1980: (2) • 1981: (2)

Hasse, Hermann Edward (1836-1915) Bioscript

Hassler, Emil Botanical Album

Hassler, F. A. (A. 1912-1919) Bioscript

Hassler, Mildred Esther Mathias See Marthias, Mildred Esther Hassler (1906-1995)

Hastings, George T. Torreya, Torrey Botanical Club Correspondence • 1934: (2) • 1935: (5) • 1936: (3) • 1939: (3) • 1940: (5)

Hatch, Melville H. American entomologist; U. of Washington, Seattle

Bioscript

Correspondence • 1951: (2) • 1970: (1) Rocky Mountain Naturalists

Hatch, Peter Thomas Jefferson Memorial Foundation Correspondence • 1993: (3)

Hathaway, Edward Sturtevant ( -1984) Tulane U., Dept. of Biology; Louisiana Mosquito Control Association (1961. 1967)

Biofile • Bioscript • Botanical Album

Correspondence $\bullet$ 1947: (15) Tulane U., Dept. of Botany

- 1949: (1) Tulane U., Dept. of Botany 1950 : (6)

Tulane U., Dept. of Botany $\bullet$ 1952: (1) $\bullet$ 1961: (2) 1962

(2) • 1963: (1) • 1966: (1) • 1967: (2) • 1969: (1) 1975

(3) Letters to and from Harry J. Bennett

Hathaway-Henderson, Marcia Daughter of Edward Hathaway, Tulane Prof.

Correspondence - 1984: (8) Tulane U., Dept. of Botany; letters to and from Warren A. Johnson

Hauck, Ferdinand Botanical Album

Haught, Oscar Smithsonian Institution, Dept. of Botany Bioscript • Botanical Album
Hauke, Richard Louis (1930- ) U. of Michigan, Ann Arbor Correspondence • 1959: (2)

Haussknecht, C. Biofile

Havard, Valery (1846-1927) Surgeon U. S. Army, botanist; Compiègne, France

Bioscript

Havell, Robert Bioscript

Haverford College, Librarian Correspondence $\bullet$ 1975: (1)

Havy, Rene Just Bioscript

Hawkes, Jack G. U. of Birmingham, England Correspondence • 1991: (4) • 1992: (2)

Hawkeswood, Trevor J. (1956- ) Correspondence 1988 : (1)

Hawkins, Benjamin Bioscript

Hawkins, Murray Correspondence • 1992: (1) • 1993: (1)

Hawksworth, Frank Goode (1926-1993) Rocky' Mountain Forest and Range Experiment Station, Fort Collins, CO, Correspondence • 1976: (2)

Hay, Bioscript

Hay, P. R. Bioscript

Hayata, Bunzo Botanical Album

Hayden, Ferdinand Vandeveer (1829-1887) American geologist

Biofile $\bullet$ Bioscript $\bullet$ Botanical Album

Hayden, Horace $\mathbf{H}$. Bioscript

Hayek, August von (1871-1928) Austrian physician and botanist

Biofile

Hayes, A. L. Bioscript

Hayes, Sutton ( -1863$)$ Bioscript

Haygood, Tamara Miner U. of New Mexico, Dept. of Radiology, resident See also Tamara A. Miner Correspondence • 1989: (2)

Hayne, F. G. Bioscript

Haynes, Parke G. U. S. Dept. of Agriculture Correspondence • 1949: (1) Rocky Mountain Naturalists

Haynes, Robert Ralph (1945- ) Louisiana State U., Shreveport Correspondence • 1974: (1)

Haynes, William G. Ashantilly Press Correspondence • 1973: (1)

Hazelbaker, N. D. Arkansas State College Correspondence $\bullet$ 1965: (2)

Head, Henry (1861-1940) English physician, poet Biofile • Bioscript

Hearn, Lafcadio (1850-1904) Journalist, writer on Japan Biofile • Bioscript 
Hearne, Samuel (1745-1792) Bioscript

Hearne, Thomas (1678-1735) Bioscript

Heartman, Charles F. (fl. 1911-1964) German-born American book dealer; New Orleans, LA Biofile - Bioscript Correspondence $\bullet$ 1964: (1)

Heartman, Martha E. Book dealer; Southern Library Service, New Orleans; husband is Charles F. Heartman Correspondence $\bullet$ 1958: (3) $\bullet$ 1959: (1) $\bullet$ 1964: (1)

Heath \& Company Correspondence 1975: (1)

Heath, Harold (1868-) Bioscript

Heath, Victoria Piedmont, CA Correspondence $\bullet$ 1952: (2)

Hebard, Morgan (1887-1946) American entomologist Bioscript

Correspondence • 1949: (0) Letter filed under James A. G. Rehn

Hebert, Leo P. U.S. Sugarcane Field Station, Houma, LA Correspondence $\bullet$ 1956: (4) 1 1957: (2)

Hecht, Adolph Botanical Society of America, Inc.; Plant Science Bulletin

Correspondence $\bullet$ 1966: (2)

Hecht, Selig (1892- ) Austrian-born American zoologist Biofile

Hecht, William Robert American bookseller; St. Louis, MO, falconry specialist; nickname "Bob"

Correspondence • 1951: (17) $\bullet$ 1952: (4) • 1953: (3) Book collectors $\bullet$ 1961: (7) $\bullet$ 1962: (5) Letter from Hecht to George H. Penn • 1963: (2) • 1964: (1) • 1968: (2) • 1972: (2) • 1975: (2) • 1979: (1) • 1982: (1) • 1986: (1) • 1990: (2) - 1991: (1) Book collectors

Heckard, Lawrence Ray (1923-1991) U. of California, Berkeley, Jepson Herbarium; nickname "Larry" Correspondence • 1963: (1) • 1966: (1) • 1967: (6) Jepson, Willis Linn, 1867-1946 • 1972: (2) • 1979: (2) • 1981: (1) • 1985: (2) • 1986: (4)

Heckewelder, John (1743-1823) Moravian-American missionary, linguist, archaeologist Biofile - Bioscript

Heckewelder, Wallace Bioscript

Hector, James (1834-1907) Scottish-born geologist, explorer, administrator of New Zealand Biofile - Bioscript

Hedberg, Inga M. M. (1927- ) Institute of Systematic Botany, U. of Uppsala, Sweden Correspondence $\bullet$ 1972: (1) • 1974: (1)

Hedberg, Nils Instituto Ibero-American Correspondence • 1958: (1)

Hedgcock, George Grant (1863-1946) American phytopathologist

Bioscript Correspondence 1949: (1) Rocky Mountain Naturalists

Hedge, Ian Charlesson (1928-) Royal Botanic Garden Edinburgh Correspondence • 1986: (2) • 1991: (1)

Hedge, Thompson Bioscript
Hedgpeth, Joel W. (1911-) American marine biologist, author; Pacific Marine Station, Oregon State U., Newport, OR; U. of the Pacific, Dillon Beach, CA.; Society for the Prevention of Progress, Santa Rosa, CA

Biofile $\bullet$ Botanical Album

Correspondence $\bullet$ 1978: (3) $\bullet$ 1988: (8) Book collectors • 1990: (2) - 1991: (1) Barton, Benjamin Smith, 1766-1815; Nuttall, Thomas, 1786-1859 • 1992: (1) • 1993: (1)

Hedin, Carl Stockholm; brother of Sven Hedin Correspondence • 1934: (1) • 1935: (2) • 1936: (1) • 1937: (1) • 1938: (1) • 1939: (1) • 1958: (2)

Hedin, Sven (1865-1952) Swedish explorer, Near and Far East; brother of Carl Biofile $\bullet$ Botanical Album

Hedrick, Ulysses Prentiss (1870-1951) American plant breeder and horticulturist Biofile • Bioscript

Hedwig, Johann (1730-1799) Bioscript

Hedwig, Romanus Adolf (1772-1806) Bioscript

Heer, $\mathbf{O}$. Bioscript

Heerfordts, Christopher Biofile

Heerman, Lewis Botanical Album

Heermann, Adolphus Lewis (1827-1865) American ornithologist, surgeon-naturalist

Bioscript

Heim, Roger Jean Botanical Album

Heimans, Bioscript

Heimerl, Anton Botanical Album

Heine, $\mathbf{F}$. Correspondence $\bullet$ 1940: (1)

Heinisch, 0. Botanical Album

Heiser, Charles Bixler (1920-) American taxonomist, botanist; Indiana U., Bloomington; nickname "Charlie" Biofile $\bullet$ Botanical Album Correspondence $\bullet$ 1948: (1) • 1949: (3) • 1952: (2) • 1953: (7) $\bullet$ 1954: (13) $\bullet$ 1956: (2) $\bullet$ 1957: (3) $\bullet$ 1963: (1) - 1967: (3) • 1968: (11) • 1970: (1) • 1974: (5) - 1975: (1) 1 1976: (3) $\bullet$ 1977: (2) $\bullet$ 1978: (5) $\bullet$ 1980: (0) Stuessy to Heiser in Bretting corr. -1985 : (2) $\bullet$ 1993: (1)

Heller, Amos Arthur (1867-1944) American botanist; Chico State Teachers College

Bioscript • Botanical Album

Correspondence • 1943: (2)

Heller, Ben A. Ohio State U., Columbus, Dept. Spanish and Portuguese Correspondence • 1991: (2) • 1991: (0) Filed under Bruce Holst

Heller, Edmund (1875-1939) American zoologist Bioscript

Heller, Eugene Twelfth Naval District, Committee of Deans Correspondence $\bullet$ 1944: (1) 
Heller, John Lewis (1906-) ～U. of Ilinois, Urbana Botanical Album

Correspondence • 1960: (2) • 1962: (3) • 1964: (1) • 1965: (1) • 1969: (1) • 1970: (2) • 1973: (5) • 1974: (1) • 1980: (2) • 1986: (4)

Hellman, C. Doris Xth International Congress of History of Science, Cornell U.

Correspondence • 1962: (3) Banister, John, 1650-1692

Hellpach, Willy Universität Heidelberg Botanical Album

Helman, Florence S. Bibliographer; Library of Congress Correspondence $\bullet$ 1943: (3)

Helms, Judith S. Correspondence $\bullet 1980$ : (2)

Helwing, Edwin R. U. of Colorado, Boulder, Dept. of Biology Correspondence $\bullet$ 1966: (3)

Helyar, L. E. James U. of Kansas Library, Lawrence Correspondence • 1967: (1)

Hemming, John Royal Geographic Society of Great Britain Correspondence • 1989: (1) • 1990: (1)

Hemphill, Henry (1830-1914) Bioscript

Hemphill, M. L.

Correspondence $\bullet$ 1977: (0) Correspondence filed under Ellis L. Yochelson $\bullet 1977$ : (1)

Hemsley, W. B. Bioscript

Henderson, Mrs. Apalachicola, FL Correspondence $\bullet$ 1952: (1)

Henderson, Gladys S. Rollins College Correspondence • 1949: (1)

Henderson, Junius (1865-1937) American zoologist Biofile • Bioscript

Henderson, Louis Fourniquet (1853-1942) American botanist and plant collector Biofile $\bullet$ Bioscript $\bullet$ Botanical Album Correspondence $\bullet$ 1934: (1)

Henderson, Norlan C. U. of Missouri, Kansas City, Dept. of Botany Correspondence • 1967: (3)

Henderson, Randall Desert Magazine Correspondence • 1942: (1) • 1943: (1) • 1959: (1)

Hendler, Richard Correspondence $\bullet 1977$ : (1)

Hendricks, Donald U. of New Orleans, Librarian Correspondence • 1981: (1) • 1982: (2) • 1984: (1)

Hendricks, Paul Washington State U., Pullman Correspondence $\bullet$ 1992: (1)

Hendricks, Sterling B. U.S.D.A. Mineral Nutrition Laboratory, Beitsville, MD Correspondence • 1967: (3) • 1968: (7)

Hendrickson, Walter B. American historian; MacMurray College, Jacksonville, II Correspondence • 1963: (6) Missouri Botanical Garden; Van Schaack, George Booth, 1903-1983 • 1969: (1)

Hendrix, Ben, Mrs. Correspondence • 1977: (1)

Hendrix, Ben R. Gloster, MS; potential donor Correspondence • 1976: (1) Tulane U., Dept. of Botany

Hendry, Andrew N. Davis, CA Correspondence • 1968: (3) • 1969: (1)
Hendry, G. M.

Botanical Album

Henfrey, $\mathbf{A}$.

Bioscript

Henke, Rose St. Louis, MO; volunteer at Missouri Botanical Garden Library; student, Ewan's seminar at Boulder Correspondence • 1976: (1)

Hennedy, Roger Biofile (Filed under John Scouler)

Hennepin, Father Louis Bioscript

Henrey, Blanche Elizabeth Edith (1906-1983) British Museum (Natural History), Botany Library

Bioscript $\bullet$ Botanical Album

Correspondence $\bullet$ 1955: (4) • 1956: (1) • 1976: (1) • 1977: (5) • 1979: (3)

Henry, Augustine (1857-1930) Scottish-born Irish plant collector in China, physician, dendrologist Biofile

Henry, Caroline Husband is Augustine Henry Bioscript

Henry E. Huntington Library Correspondence $\bullet$ 1943: (1)

Henry Foundation for Botanical Research Gladwyne, PA; Angus Paxton Heeps Correspondence $\bullet$ 1975: (2)

Henry, Joseph (1797-1878) American experimental physicist Biofile $\bullet$ Bioscript

Henry, Josephine de Nancrede (1911-) Gladwyne, PA Botanical Album Correspondence $\bullet$ 1952: (2) - 1979: (1)

Henry, Mary Gibson (1884-1967) American horticulturist Bioscript

Henry, R. D. Western Illinois U., Macomb, Biological Sciences Correspondence • 1979: (2)

Henry, Roemol Transylvania College, Lexington, Kentucky, Frances Carrick Thomas Library Correspondence • 1967: (0) Filed under Robert Vosper

Henry, Samuel (fl. 1814) Bioscript

Henry, Thomas Charlton (1825-1877) American ornithologist, surgeon, U. S. Army Bioscript

Henry, Tina Pennsylvania Horticultural Society Correspondence $\bullet$ 1968: (2)

Henry, William Arnon (1850-1932) American agriculturist Bioscript

Henshall, Hester Stansbury Botanical artist; husband is J. A. Henshall Bioscript

Henshall, James Alexander (1844-1925) American ichthyologist Bioscript

Henshaw, Henry Weatherbee (1850-1930) American naturalist, entomologist Biofile • Bioscript

Henshaw, Julia Wilmotte (1869-1937) English-born Canadian botanist; husband is Charles Grant Henshaw Bioscript

Henshaw, Samuel (1833-1907) Bioscript 
Hensley, Richard G. Boston Public Library

Correspondence $\bullet$ 1951: (1)

Henslow, John Stevens (1796-1861)

Bioscript • Botanical Album

Henssen, Aino (1925-) U. of Marburg, West Germany, Dept. of Botany

Botanical Album

Correspondence $\bullet$ 1963: (1)

Hentz, Caroline Lee Bioscript

Hentz, Nicholas Marcellus (1797-1856) American naturalist, entomologist Biofile $\bullet$ Bioscript

Hepburn, James (1811-1869) British-born California ornithologist Biofile • Bioscript

Hepper, F. Nigel Botanical Album

Herbert, Diane Correspondence • 1956: (2)

Herbert, Henry Nicholas Bioscript

Herbst, Bioscript

Herff, Ferdinand Ludwig Johann Arnold von (1820-1912) Bioscript

Herholdt, A de V U. of the Witwatersrand, Johannesburg, South Africa, Registrar Correspondence $\bullet$ 1975: (1)

Hering, Constantine (1800-1880) Bioscript

Herman, Jan K. U. S., Dept. of the Navy Correspondence $\bullet$ 1984: (1)

Hermann, Carolyn Correspondence • 1975: (1)

Hermann, Dory Correspondence $\bullet$ 1950: (1)

Hermann Éditeurs des Sciences et des Arts Paris, France Correspondence $\bullet$ 1964: (1)

Hermann, Frederick Joseph (1906-1987) U.S.D.A. Plant Industry Station, Beltsville, MD

Botanical Album

Correspondence • 1942: (3) • 1949: (6) - 1950: (14) • 1951: (2) • 1952: (1) • 1953: (0) Filed under Vincent J. Derbes - 1953: (4) Rocky Mountain Naturalists; "Rocky Mountain Vegetation"; Verdoorn, Frans Antonie, 1906-1984 • 1954: (3) • 1955: (2) • 1956: (3) • 1957: (3) - 1960: (6) • 1963: (2) • 1964: (5) Banister, John, 1650-1692 - 1967: (3) - 1968: (1) • 1970: (2) Rocky Mountain Naturalists • 1975: (2) $\bullet$ 1977: (1)

Hernández, Francisco Bioscript

Héroux, Roy Botanical Album

Herr, William Silver City, NM Correspondence $\bullet$ 1979: (1) Book collectors

Herre, Albert William Christian Theodore (1868-1962) American naturalist Biofile $\bullet$ Bioscript $\bullet$ Botanical Album

Herre, Hans German-born South African botanist; University of Stellenbosch Botanical Garden Botanical Album
Herrera, Fortunato $L$. Correspondence $\bullet$ 1934: (1)

Herrman, I. Gottlb. Bioscript

Herschell, Bioscript

Herschman, Arthur American Association for the Advancement of Science Correspondence - 1975: (0) Filed under Hilbert Garrison Willkes

Hersey, Jane American Scientist, book review editor Correspondence • 1982: (1)

Hershey, A. L. New Mexico State College; Colorado State College, Greeley Correspondence • 1939: (2) Delphinium • 1949: (1) Rocky Mountain Naturalists

Hershkovitz, Philip Field Museum of Natural History, Mammal Division, Chicago Correspondence • 1967: (2) • 1985: (1) • 1989: (2) • 1992: (3)

Herter, Wilhelm Gustav Franz (1884-1958) GermanUraguayan botanist; Jefe del Jardin y Musée Botánico Biofile Correspondence • 1939: (1)

Hertrich, William Huntington Botanical Garden Botanical Album Correspondence • 1942: (3)

Hervey, Annette (1920-) American botanist, mycologist; New York Botanical Garden Correspondence • 1969: (2) • 1976: (2) • 1979: (1)

Hesler, Lexemuel Ray (1888-1977) American mycologist Biofile

Hess, Gertrude D. American librarian; American Philosophical Society Library, Philadelphia, PA Correspondence • 1958: (10) Bartram, William, 1739-1823 - 1959: (8) • 1960: (2) • 1961: (8) Lyon, John, 1764-1814; Pursh, Frederick, 1774-1820 • 1962: (11) • 1963: (15) • 1964: (2) Bartram, William, 1739-1823 • 1965: (10) • 1966: (10) • 1967: (3) • 1968: (3) • 1969: (6) - 1970: (3) • 1971: (3)

Hesse, Vesta Florence (1901-1982) American plant collector Biofile

Hesselins, Andreas Bioscript

Hester, J. Pinckney (I. 1943) Bioscript

Heston, Charles Bioscript

Hettrich, A. L. Bioscript

Hetzel, Frederick A. Institute of Early American History and Culture, Williamsburg, VA Correspondence • 1960: (5) Banister, John, 1650-1692 • 1961: (4) Banister, John, 1650-1692; National Science Foundation

Heuer, Kenneth Charles Scribner's Sons Publishers Correspondence • 1969: (0) Filed under Charles Scribner's Sons Publishers

Hevrck, Henry Ferdinand van (1838-1909) Plant morphologist, taxonomist Bioscript

Heward, H. Bioscript 
Hewett, Edgar Lee (1865-1946) American archeologist Bioscript

Hewson, Thomas T. Bioscript

Heywood, Vernon Hilton (1927-) U. of Reading, Professor of Botany Botanical Album

Hiatt, Otis Bioscript

Hibbard, Margaret E. McGill U., Montreal, Wood Library Ornithology Correspondence • 1956: (8)

Hibbs, Richard G., Jr. Correspondence $\bullet 1972$ : (1)

Hicken, Cristóbal María (1875-1933) Biofile

Hickman, J. B. Bioscript

Hickman, James Craig (1941-) Madroño, Editór; U. of California, Berkeley, Jepson Herbarium Botanical Album Correspondence • 1978: (6) • 1979: (4) • 1981: (4) 1993: (0) Letter filed under Dale Edwin Johnson

Hicks, Arthur James (1938-) American taxonomist; U. of Georgia, Dept. of Botany Correspondence $\bullet$ 1975: (1)

Hicks, Charles Henry ( -1941) Entomologist Bioscript

Hiern, W. F. Bioscript

Hiesey, William McKinley (1903-) California Botanical Society Correspondence $\bullet$ 1941: (2)

Hiffell, Chr. G. Bioscript

Fliggins, Ethel Bailey (1866-1963) Natural History Museum, San Diego, CA

Bioscript

Correspondence • 1942: (1) • 1945: (1) • 1946: (5) • 1947: (1) • 1948: (1) • 1949: (1) • 1957: (13) • 1958: (4) - 1959: (4)

Higham, C. F. W. U. of Otago, New Zealand, Dept. of Anthropology Correspondence • 1982: (1)

Hilair, Jean Baptiste Bioscript

Hilldebrand, Samuel F. (1883-1949) Ichthyologist and herpetologist Biofile

Hildreth, Bioscript Contemporary of B. S. Barton

Hilend, Martha (1902-) U. of California, Los Angeles, Botany instructor Bioscript

Hilgard, Eugene Woldemar (1833-1916) Bavarian-born American soil scientist, stratigrapher Bioscript

Hilgard, T. C. Bioscript

Hill, Albert F. New England Botanical Club, Rhodoro Botanical Album Correspondence $\bullet$ 1948: (2)
Hill, Andrew

Bioscript

Hill, Arthur M. Tulane U., New Orleans, Mechanical Engineering

Correspondence $\bullet$ 1954: (1)

Hill, Arthur William (1875-1941) British botanist, horticulturist; Royal Botanic Gardens, Kew

Botanical Album

Correspondence • 1936: (2) • 1937: (2) • 1938: (2) • 1940: (1)

Hill, Bradford M. Boston Public Library

Correspondence - 1957: (1)

Hiil, Ellsworth Jerome (1833-1917) American clergyman and botanist

Biofile $\bullet$ Botanical Album

Hill, John, Sir (1707-1775) English apothecary, physician Biofile $\bullet$ Bioscript

Hill, Joseph Correspondence • 1921-1928: (1)

Hill, Robert T. Bioscript

Hill, Robert W. New York Public Library, keeper of manuscripts

Correspondence $\bullet$ 1957: (0) Letter filed under John Gloag 1957: (2) 1961: (1)

Hill, Steven Richard (1950- ) Texas A \& M U., College Station, Dept. of Biology Correspondence $\bullet$ 1977: (1)

Hill, Thomas Bioscript

Hill, Virginia Millsaps College, Jackson, MS Correspondence $\bullet$ 1980: (2)

Hiil, Walter $\mathbf{R}$. Bioscript

Hillebrand, William (Wilhelm) (1821-1886) Prussian-born American physician, botanist, plant collector in Hawaii Biofile $\bullet$ Bioscript $\bullet$ Botanical Album

Hilliard, Sam B. Louisiana State U., Baton Rouge, LA Correspondence $\bullet$ 1978: (1)

Hillman, F. H. Bioscript

Hilton, Edward W. National Science Foundation Correspondence $\bullet$ 1958: (1)

Hinchcliffe, Robert National Science Foundation Correspondence $\bullet$ 1963: (2)

Hinckley, L. C. U.S.D.A. Soil Conservation Service Correspondence $\bullet$ 1945: (2)

Hincks, William Bioscript

Hinds, Richard Brinsley (1812-1847) Bioscript

Hinkle, George Bioscript

Hinrichs, Anton Correspondence $\bullet$ 1992: (1)

Hinton, George Book (1882-1943) English-born metallurgist and plant collector in Mexico and S. W. United States Biofile $\bullet$ Bioscript $\bullet$ Botanical Album

Hinton, J. Bioscript

Hipp, Carl Friedrich (1763-1838) German mathematician, philosopher Biofile 
Hisaw, Frederick Lee (1891-) American vertebrate embryologist

Bioscript

Historic New Orleans Collection Correspondence $\bullet$ 1985: (2)

Historical Society of Pennsylvania Philadelphia Correspondence $\bullet$ 1952: (1) $\bullet$ 1959: (1)

History of Science Society Correspondence • 1984: (1) • 1986: (1)

Hitchcock, Albert Edwin (1898-) Boyce Thompson Institute, Yonkers, NY; son of Albert Spear Hitchcock Correspondence $\bullet$ 1949: (1) • 1974: (2)

Hitchcock, Albert Spear (1865-1935) American agrostologist; Smithsonian

Biofile $\bullet$ Bioscript $\bullet$ Botanical Album Correspondence - 1935: (5) Species • 1974: (3) Letters with Clark D. Kingsbury, re: A. S. Hitchcock

Hitchcock, Charles Leo (1902-1986) American taxonomist; Southern U. of Montana; U. of Washington, Seattle; nickname "Hitchy"

Bioscript $\bullet$ Botanical Album

Correspondence $\bullet$ 1935: (1) $\bullet$ 1936: (6) $\bullet$ 1937: (3) $\bullet$ 1938: (4) - 1939: (13) Delphinium • 1940: (2) • 1941: (4) - 1942: (5) - 1943: (3) Delphinium • 1944: (1) - 1945: (2) $\bullet$ 1946: (7) $\bullet$ 1948: (5) $\bullet$ 1951: (2) 1 1966: (2) Delphinium; Species • 1972: (2)

Hitchcock, Edward Bioscript

Hitchcock, Henry III (1906-) Missouri Botanical Garden, St. Louis, MO, Board of Trustees, President Correspondence - 1964: (4) Missouri Botanical Garden • 1965: (3) Missouri Botanical Garden

Hoare, Michael Edward (1941-) Australian Academy of Science; Royal Society of NZ, Wellington

Botanical Album

Correspondence $\bullet$ 1974: (2) $\bullet$ 1975: (1) $\bullet$ 1976: (5) $\bullet$ 1977: (2) • 1978: (2) • 1979: (5) • 1980: (3) • 1981: (2) • 1982: (4)

Hobbs, Morris Henry (1893?- ) New Orleans artist; bromeliad enthusiast Botanical Album

Hobbs, W. H. Bioscript

Hobson, William Bioscript

Hoch, Gloria Missouri Botanical Garden Correspondence 1992: (1)

Hochderffer, George Bioscript

Hochreutiner, Bénédict Pierre Georges (1873-1959) Conservatoire et Jardin Botanique, Geneva Botanical Album Correspondence 1938: (4) Species • 1939: (2) Species $\bullet$ 1940: (1)

Hochschwender, Herman (Ted) Tulane U., Architecture; Nashua, NH and Akron, OH; illustrator for book on John Banister Correspondence $\bullet$ 1970: (3) $\bullet$ 1971: (3)

Hochstetter, Christian Ferdinand (1787-1860) Bioscript

Hochstetter, Ferdinand Ritter von (1825?- ) Austrian-born geologist of New Zealand Biofile
Hock, Alfred Quarterman Publications Correspondence • 1973: (1)

Hocker, Sarah Haines American librarian; U. of Kansas, Lawrence, Kenneth Spencer Research Library, Special Collections Correspondence • 1978: (1)

Hocking, Brian U. of Alberta, Edmonton, Dept. of Entomology Correspondence $\bullet$ 1970: (1) Rocky Mountain Naturalists

Hocking, George M. Alabama Polytechnic Institute, Auburn; Auburn U., School of Pharmacy Correspondence • 1945: (1) • 1959: (1) • 1964: (3) Rocky Mountain Naturalists • 1970: (1) Harper, Roland McMillan, 1878-1966 • 1988: (1) • 1992:

Hockizaki, Barbara Joe Correspondence • 1964: (1)

Hodgdon, Albion Reed (1909-1976) American taxonomist, plant ecologist; U. of New Hampshire, Durham; Rhodora, editor-in-chief Correspondence $\bullet$ 1962: (5) • 1968: (4)

Hodge, Benjamin Bioscript

Hodge, Bobbie Husband is Walter Henricks Hodge Correspondence $\bullet$ 1963: (1)

Hodge, Walter Henricks (1912-) American economic botanist, photographer, administrator, phytogeographer Botanical Album

Correspondence • 1938: (1) • 1947: (8) • 1948: (4) • 1949: (4) • 1950: (6) Pursh, Frederick, 1774-1820; Verdoorn, Frans Antonie, 1906-1984 • 1952: (6) Pursh, Frederick, 1774-1820 • 1957: (1) • 1961: (3) • 1962: (1) • 1964: (2) • 1965: (6) • 1966: (2) • 1970: (2) • 1971: (2) • 1974: (2) • 1976: (2) Barton, Benjamin Smith, 1766-1815 - 1978: (1) • 1979: (4) • 1982: (1) • 1983: (2) • 1984: (4) - 1985: (1) • 1987: (1) • 1989: (6) • 1989: (0) Filed under Steven P. Darwin • 1990: (2) 1 1993: (2)

Hodges, Harry G. Angus \& Robertson, Ltd., Booksellers and Publishers, Sydney, Australia Correspondence $\bullet$ 1951: (1)

Hodgson, James G. American librarian; Colorado A \& M, Fort Collins Correspondence $\bullet$ 1941: (1) $\bullet$ 1949: (1)

Hodgson, Matthew U. of North Carolina Press, Chapel Hill Correspondence $\bullet$ 1972: (2)

Hodgson, Ruth Sec. to Edwin B. Williams of U. of Pennsylvania Correspondence • 1942: (1)

Hodson, Elmer Reed (1875-) Bioscript

Hoefer, Jean Chrétien Ferdinand (1811-1878) Bioscript

Hoek, Ray van de Sylmar, CA Correspondence • 1986: (1) • 1987: (1)

Hoffiman, __ Wrote paper on John Clayton for Ethnohistory, Winter 1964 Correspondence $\bullet$ 1976: (1)

Hoffman, Bernard G. Correspondence $\bullet$ 1965: (1)

Hoffman, G., Fr. Bioscript

Hoffman, James I. National Bureau of Standards Correspondence - 1947: (1) Jepson, Willis Linn, 1867-1946 
Hoffman, Richard L. Virginia Museum of Natural History Correspondence • 1991: (2) • 1992: (2)

Hoffman, Walter James (1846-1899) American ornithologist, ethnologist, physician Bioscript

Hoffmann, Fritz L. U. of Colorado, Boulder, Dept. Spanish; on leave in Buenos Aires 1943 Correspondence • 1943: (3) • 1948: (2) • 1949: (1) 1952: (3) Book collectors

Hoffmann, Ralph (1870-1932) American scientist; Santa Barbara Museum of Natural History, Director Bioscript - Botanical Album Correspondence • 1921-1928: (1)

Hoffmannsegg, Johann Centurius, Graf von (1766-1849) Nanuralist

Bioscript

Hoffy, Alfred M. American horticulturist, artist Bioscript

Hofrath von Martius Bioscript

Hogan, Jane Correspondence • 1983: (2)

Hogan, Sharon A. American librarian; Louisiana State U. Libraries, Baton Rouge

Botanical Album

Correspondence $\bullet 1984:$ (2) $\bullet$ 1986: (2)

Hogan, William R. American historian; Tulane U. Correspondence • 1957: (1) • 1958: (3) • 1960: (1) • 1964: (1) • 1969: (2)

Hogg, Thomas (1771-1841) British-born American nurseryman, florist Bioscript

Hogue, Charles Leonard Natural History Museum of Los Angeles County Correspondence • 1990: (1) • 1991: (3)

Hohenacker, R. F. Bioscript

Hohenheim, Francisca Theresa, Countess, Duchess of Wurtemberg Bioscript

Hohman, Henry Bioscript

Hohn, Janet E. U. of Washington, Seattle, Dept. of Botany Correspondence • 1970: (2) • 1971: (2)

Holkkaido Imperial U. Botanical Album

Holboell, Carl Peter Bioscript

Holboell, Bioscript Governor of South Greenland

Holbrook, John Edwards (1794-1871) American herpetologist Biofile • Bioscript

Holden, E. S. Bioscript

Holden, J. B. Bioscript

Holder, William Bioscript

Holdridge, Leslie Botanical Album
Holgate, Jeanne (1920- ) English-born American botanical artist Biofile

Holland, Leicester B. Library of Congress Correspondence $\bullet$ 1943: (1)

Holland, Nathaniel Littleton Bioscript

Holland, S. B., Mrs. Tulane U., Howard-Tilton Memorial Library, Tulane U.

Correspondence $\bullet$ 1951: (1) $\bullet$ 1953: (0) Filed with B. W. Lucas letters

Holland-Germany, Violet Tulane U., Dept. Biology Botanical Album

Correspondence $\bullet$ 1972: (2) • 1974: (2) 1 1975: (2) • 1977: (4) • 1978: (5) • 1981: (3) • 1982: (1) • 1983: (1) • 1986: (2) • 1990: (1)

Hollenbach, C. B. Albright College, Reading, PA, Dept. Biology

Correspondence • 1949: (1)

Hollick, Charles Arthur (1857-1933) American paleobotanist Bioscript

Holliday, I. Bioscript

Hollister, J. M. Correspondence • 1945: (1) • 1949: (1) American Fern Society

Hollister, Mabel Botanical Album

Hollister, Ned (1876-1924) American zoologist; Smithsonian Institution, Mammology Bioscript

Hollmann, Sylvia H. U. of Chicago Press Correspondence $\bullet$ 1948: (1)

Hollombe, David S. Biographical reseacher; Los Angeles, CA

Bioscript

Correspondence $\bullet$ 1990: (5)

Holloway, Mark Turnstile Press, London, England Correspondence $\bullet$ 1951: (2)

Holm, Herman Theodor (1854-1932) Danish-born American botanist

Bioscript $\bullet$ Botanical Album

Holman, Manuel Correspondence $\bullet$ 1943: (1)

Holmes, A. F. Physician Bioscript

Holmes, Andrew Fernando (1797-1860) Spanish-born Canadian botanist Biofile

Holmes Book Co. Oakland, ? Correspondence $\bullet$ 1962: (1)

Holmes, E. M. Bioscript

Holmes, Ezekiel Bioscript

Holmes, Oliver Wendell Bioscript $\bullet$ Botanical Album

Holmes, Walter C. (1944) Northwestern State U. of Louisiana, Natchitoches, Dept. Bio. Sciences Correspondence • 1976: (3) • 1977: (2) 
Holmes, William Henry (1846-1933) American artist, geologist, archeologist Biofile $\bullet$ Bioscript

Holmgren, Arthur Hermann (1912-1992) American plant taxonomist; Utah State Agricultural College, Logan Correspondence $\bullet$ 1942: (3) $\bullet$ 1943: (3) Delphinium $\bullet$ 1944: (3) • 1945: (3) • 1947: (2) • 1948: (3) Delphinium

Holmgren, Patricia (1940-) American plant taxonomist; New York Botanical Garden; Brittonia, editor Correspondence • 1973: (6) - 1976: (2) Merrill, Elmer Drew, 1876-1956 - 1984: (3) Stafleu, Frans Antonie, 1921- 1985: (1) • 1986: (2) • 1988: (1)

Holmskjold, Theodor (1732-1794) Bioscript

Holmstrand, Carolyn U. of Illinois Press, Urbana Correspondence $\bullet$ 1964: (3)

Holst, Bruce Ohio State U., Columbus, Dept. of Spanish and Portuguese

Botanical Album

Correspondence $\bullet$ 1991: (1)

Holt, M. B. City Club of Denver Correspondence $\bullet$ 1942: (1)

Holthuis, L. B. Botanical Album

Holton, Isaac Farwell (1812-1874) Plant explorer, plant collector

Bioscript

Holton, Raymond William (1929-) American plant physiologist, phycologist; U. of Tennessee, Knoxville Correspondence • 1974: (1) • 1979: (4) • 1983: (4)

Holttum, Richard Eric (1895-1990) English-born botanist, pteriodologist; U. of Malaya, Singapore

Biofile • Bioscript $\bullet$ Botanical Album

Correspondence $\bullet$ 1951: (1) • 1952: (1) • 1975: (1)

Holzinger, John Michael (1853-1929) German-born American bryologist

Bioscript $\bullet$ Botanical Album

Home, $\mathbf{E}$. Bioscript

Home of the Old Settler Bookshop Correspondence $\bullet$ 1966: (1)

Home, R. W. U. of Melbourne, Parkvile, Victoria, Australia

Correspondence $\bullet$ 1974: (8)

Hommersand, Max Hoyt (1930-) Plant morphologist; U. of North Carolina, Chapel Hill

Correspondence • 1961: (1)

Honeger, Walter Botanical Album

Hood, James N. Correspondence $\bullet$ 1974: (1) $\bullet$ 1975: (1)

Hood, M. Tulane U., Dept. of History Correspondence • 1974: (1)

Hood, Robert Bioscript

Hoogstraal, Harry Correspondence $\bullet$ 1938: (5)

Hooker, Charles Bioscript

Hooker, Joseph Dalton (1817-1911) British botanist Biofile $\bullet$ Bioscript $\bullet$ Botanical Album

Hooker, William Jackson (1785-1865) British botanist Biofile • Bioscript • Botanical Album
Hoopes, Josiah (1832-1904) American nurseryman, ornithologist

Bioscript

Hoopes, Thomas (1834-1925) Bioscript

Hoover, John Neal American rare book librarian; Mercantile Library, St. Louis, MO

Biofile $\bullet$ Botanical Album

Correspondence • 1988: (3) Rocky Mountain Naturalists • 1990: (1) • 1991: (1)• 1992: (1)

Hoover, Jume Correspondence $\bullet$ 1985: (3)

Hoover, M. Scott Coronation Farm, Williamstown, MA; collector for Missouri Botanical Garden and Gray Herbarium Correspondence $\bullet$ 1976: (1)

Hoover, Robert Francis (1913-1970) U. of California, Berkeley, research assistant to Jepson (1936- ); California State Polytechnic College, San Luis Obispo Botanical Album

Correspondence $\bullet$ 1937: (7) Jepson, Willis Linn, 1867-1946 • 1938: (12) Jepson, Willis Linn, 1867-1946 • 1939: (4) Delphinium • 1940: (1) • 1942: (2) Setchell, William Albert, 1864-1943 • 1943: (1) • 1946: (1) Delphinium • 1948: (2) Delphinium • 1954: (2) Delphinium $\bullet$ 1962: (1)

Hope, John (1725-1786) Bioscript

Hopkins, L. S. Botanical Album

Hopkins, Milton (1906-) U. of Oklahoma, Norman Botanical Album Correspondence • 1942: (2) • 1943: (3) Delphinium • 1944: (1) Delphinium $\bullet$ 1950: (2)

Hopley, Catherine C. Bioscript

Hoppe, D. H. Bioscript

Hopwood, D. L. Scenic Photograph Correspondence - 1953: (2) "Rocky Mountain Vegetation"

Horan, C. U. of Queensland, Australia, Dept. of Zoology Correspondence $\bullet 1970$ : (1)

Horavka, Frantisek Universita Karlova Correspondence - 1948: (0) Letters filed under Frans Antonie Verdoorn and William Webster Diehl • 1948: (1) Verdoorn, Frans Antonie, 1906-1984

Horberg, Carl Leland (1910-1955) American geologist Biofile

Horchderffer (?), see Hochderffer, George H. Bioscript

Horine, Emmet Field, M. D. Brooks, KY Correspondence • 1962: (2) - 1963: (2)

Horn, Cland Office of Foreign Agricultural Relations Correspondence • 1947: (1)

Horn, George H. Bioscript

Horn, T. G. Bioscript

Hornaday, William Temple (1854-1937) American zoologist Biofile $\bullet$ Bioscript

Hornemann, Jens Wilken (1770-1841) Danish botanist Biofile 
Horner, Gustavus Richard Brown (18041892) Bioscript

Horner, Harry Theodore, Jr. (1936- ) Iowa State U., Ames, Dept. of Botany and Plant Pathology Correspondence $\bullet$ 1963: (3) • 1964: (1) Species

Horner, Nell C. (1889-1976) Missouri Botanical Garden, Librarian and Editor of Publications Correspondence • 1935: (2) Species • 1938: (2) - 1939: (3) $\bullet$ 1940: (4) • 1942: (2) $\bullet$ 1943: (6) $\bullet 1944:$ (1) Delphinium • 1947: (3) • 1948: (3) • 1949: (2) Rocky Mountain Naturalists • 1950: (3) • 1952: (1) - 1953: (1) Bartram, William, 1739-1823; Harper, Francis, 1886-1972; British Museum (Natural History). Dept. of Botany; Missouri Botanical Garden

Hornschuch, C. F. Bioscript

Horowitz, Rich Academy of Natural Sciences of Philadelphia Correspondence $\bullet 1992:$ (1)

Horr, Worthie Flarold U. of Kansas, Lawrence, Dept. of Botany

Botanical Album

Correspondence • 1940: (5) • 1949: (1) • 1953: (5)

Horrocks, Elizabeth A. Carroll See Carroll-Horrocks, Elizabeth

Horsfield, Thomas (1773-1859) American naturalist and explorer; student of B. S. Barton Biofile $\bullet$ Bioscript

Horsford, Frederick Hinsdale Bioscript

Horticulture Boston, MA Correspondence • 1971: (1) • 1973: (1) • 1978: (1)

Horton, Minnie G. Husband is H. L. Horton Correspondence • 1942: (1)

Hortulus Aliquando Grant Dahlstrom, Inc., The Castle Press, Pasadena, CA Correspondence - 1978: (2)

Hosack, David (1769-1835) American physician and botanist Biofile • Bioscript

Hoskins, Barbara New York Botanical Garden Correspondence • 1945: (1)

Hotchkiss, Neil (1901-) Petuxent Wildlife Research Center, Laurel, MD

Correspondence • 1948: (3) Species • 1958: (1) • 1965: (1) $\bullet 1971:(3) \bullet 1972:(2)$

Houckgeest, André Everard van Braam (1739-1801) Dutch representative of East India Company to Chin Biofile

Hougas, Robert Wayne (1918 ) Plant geneticist Bioscript

Hough, Franklin B. Bioscript

Hough, Lawrence P. Correspondence • 1941: (3) - 1942: (2)

Houghton, Douglass American physician and plant collector

Houk, William G. St. Lawrence U. Correspondence • 1943: (2) Species

Hounsell, Robert W. Canada Dept. of Agriculture, Research Branch

Correspondence • 1968: (1) Species
House, Homer Doliver (1878-1949) American botanist; State Musuem, Albany, NY

Bioscript • Botanical Album Correspondence • 1946: (1)

Housman, A. E. Bioscript

Houston, C. Stuart Saskatoon, Saskatchewan Correspondence • 1981: (1) Rocky Mountain Naturalists

Houston, Clifford U. of Colorado, Boulder Correspondence 1940: (2) U. of Colorado, Boulder, Dept. of Biology $\bullet$ 1942: (1)

Houstoun, William (1695-1733) Botanist Biofile - Bioscript

Houttuyn, Maarten (1720-1798) Dutch naturalist and physician; Amsterdam Bioscript

Hover, G. W. Fidelity Van \& Storage, Los Angeles, CA Correspondence $\bullet$ 1939: (1)

Hovey, Charles Mason (1810-1887) American horticulturist Biofile

Howard, Alice Q. U. of California, Berkeley, Dept. of Botany, Herbarium Correspondence • 1969: (1)

Howard, Elise New Orleans, LA; husband is Harry Turner Howard, Jr.

Correspondence • 1969: (2) • 1972: (3) • 1973: (6) • 1973: (0) Letter filed under William Sheldon Stewart • 1975: (1) • 1977: (2)

Howard, Harry Turner, Jr. (1886-1972) Self-trained natural historian, ichthyologist; New Orleans, LA; nicknames "Henri" or "Henry"; wife is Elise Howard Bioscript $\bullet$ Botanical Album Correspondence $\bullet$ 1951: (1) $\bullet$ 1959: (7) $\bullet$ 1961: (6) $\bullet$ 1962: (12) • 1963: (15) • 1964: (4) • 1965: (10) $\bullet 1966:$ (3) $\bullet$ 1967: (9) $\bullet$ 1968: (16) $\bullet$ 1969: (4) $\bullet$ 1970: (1) $\bullet$ 1971: (1)

Howard, Orson W. (1853-1923) American zoologist, physician; U. of Utah, Professor Bioscript

Howard, R. Palmer American physician and historian of medicine; U. of Oklahoma Medical Center, Oklahoma City Correspondence $\bullet$ 1968: (0) Letter filed under Joseph E. Gordon • 1969: (5) 1970: (4)

Howard, Richard Alden (1917-) American plant taxonomist, plant collector, economic botanist; Amold Arboretum

Bioscript $\bullet$ Botanical Album $\bullet$

Correspondence $\bullet$ 1948: (2) $\bullet$ 1954: (2) $\bullet$ 1956: (3) $\bullet$ 1965: (2) • 1967: (2) • 1969: (7) • 1970: (4) $\bullet 1971:(5) \bullet$ 1972: (5) • 1974: (1) • 1979: (6) • 1980: (4) • 1981: (1) • 1982: (3) • 1984: (3) • 1986: (3) • 1990: (1) • 1991: (4) • 1992: (4) • 1993: (3)

Howard-Tilton Memorial Library See Tulane University, Howard-Tilton Memorial Library

Howard, William Bioscript

Howard, Winslow J. Jeweler, collector of natural history specimens Bioscript

Howarth, O. J. R. Bioscript

Howe, Ernest (1875-1932) Bioscript 
Howe, Henry Van Wagenen (1896-1973) American geologist

Biofile

Howe, Marshall Avery (1867-1936) American botanist, taxonomist

Biofile

Howell, Arthur Holmes (1872-1940) American ornithologist, self-made naturalist

Bioscript

Howell, Hannah J. Frick Art Reference Library, New York, NY, Librarian

Correspondence $\bullet$ 1951: (1)

Howell, Jesse V. (1891-) American geologist; Tulsa, OK Correspondence • 1959: (2) • 1960: (2) • 1961: (1)

Howell, John Thomas (1903-1994) American plant taxonomist; California Academy of Science, San Francisco; nickname "Tom"

Bioscript $\bullet$ Botanical Album

Correspondence • 1935: (1) • 1936: (4) • 1937: (4) •

1938: (8) Eastwood, Alice, 1859-1953; Species • 1939:

(10) Delphinium $\bullet$ 1939: (0) Letter filed under Leonora S. M. Curtin • 1940: (2) • 1941: (6) • 1942: (9) Species • 1943: (8) Delphinium; Jepson, Willis Linn, 1867-1946 • 1945: (3) • 1946: (1) • 1948: (6) • 1949: (4) • 1950: (5) • 1951: (4) • 1952: (1) • 1953: (16) • 1954: (8) • 1957: (4) Jepson, Willis Linn, 1867-1946; Eastwood, Alice,

1859-1953 • 1959: (3) • 1960: (1) • 1961: (3) • 1966: (1)

- 1967: (4) • 1970: (2) • 1972: (1) • 1974: (2) • 1975: (1)

- 1977: (2) • 1979: (2) • 1982: (3) • 1984: (1) • 1985: (2)

1988: (1) • 1989: (1)

Howell, Thomas Jefferson (1842-1912) American botanist, plant collector, taxonomist

Biofile $\bullet$ Bioscript $\bullet$ Botanical Album

Howell, Thomas Raymond (1924 ) U. of California, Dept. of Zoology, Ornithologist, Los Angeles, CA Correspondence $\bullet$ 1951: (1) $\bullet$ 1971: (3)

Howell, Timothy California Academy of Science, San Francisco Correspondence $\bullet$ 1968: (3) Book collectors

Howell, Warren R. John Howell - Books, San Francisco Correspondence $\bullet$ 1967: (5) Book collectors $\bullet$ 1968: (1) Book collectors • 1970 (1) • 1978: (5) • 1981: (1)

Howes, Nick Alton, IL Correspondence • 1987: (0) Letter filed under Sue Gibson Strommen

Howgate, James C. Bookseller Correspondence • 1963: (2)

Hoy, Philo Romayne Bioscript

Hoy, Thomas Bioscript

Hoyem, Andrew Bioscript

Hoyt, Mary E. Colorado School of Mines, Librarian Correspondence • 1949: (1)

Hrdlicka, Ales (1869-1943) Czechoslavakian physical anthropologist; Smithsonian Institution Biofile Botanical Album

Hu, Charles Y. Botanical Album

Hu, Hsen-Hsu (1894-1968) Chinese taxonomist, plant collector, phanerogamist Botanical Album
Hu, Shiu Ying, (1910-) Chinese-born American plant taxonomist; Arnold Arboretum Correspondence • 1976: (1) Merrill, Elmer Drew, 1876-1956

Huang, Tseng-Chieng (1931-) National Taiwan U., Taipei Botanical Album

Correspondence • 1965: (2)

Huartes, Helen de U.S.D.A. Bureau of Plant Industry, Div. of Rubber Investigations

Correspondence • 1952: (1) Filed with Richard Evans Schultes

Hubbard, Dorothy J. Academy of Natural Sciences of Philadelphia

Correspondence $\bullet$ 1976: (0) Filed under Academy of Natural Sciences of Philadelphia

Hubbard, Frederick Tracy (1875-1962) American plant collector Biofile

Hubbard, John R. Tulane U.; U. of Southern California Correspondence • 1976: (0) TLS filed under Ronald Lockmann

Hubbard, Ralph U. of Michigan, Museum of Zoology; U. of California, San Diego

Correspondence • 1944: (4) • 1954: (1) • 1966: (2)

Hubby, Frank W. Bioscript

Huber, _, Mr. Correspondence • 1948: (1)

Huber, Leonard V. Correspondence $\bullet 1973$ :

Huber, Neil M. U. of California, Berkeley Correspondence • 1968: (2) Harper, Roland McMillan, 1878-1966

Huber, Otto Scientia Guaianae, editor; Gerencia de Estudios y Projectos, Caracas, Venez., research associate Botanical Album

Correspondence • 1984: (1) • 1985: (1) • 1989: (3) • 1990: (1)

Hubert, Joseph French agriculturist, botanist; Mauritius Biofile

Hubner, A.

Bioscript

Hubner, Jacob (1761-1826)

Bioscript

Hubrict, Russell

Bioscript

Hudson Bay Company

Bioscript

Correspondence • 1956: (1)

Hudson, Charles Bradford (1865-) Bioscript

Hudson, Jack W. Cornell U. Correspondence $\bullet$ 1972: (1)

Hudson, William Henry (1841-1922) English writer, field naturalist Bioscript • Botanical Album

Huebner, Thurman $\mathbf{M}$. Correspondence • 1958: (1)

Hueffel, Bishop Bioscript

Huestis, William (fl. 1904) Plant collector, Colorado Bioscript 
Huffel, Christian Gottlieb (1762-1842) Bioscript

Huffiner, Mary A. Smithsonian Libraries, Acting Director Correspondence • 1966: (1)

Huft, Michael J. (1949 ) American botanist; Field Museum, Chicago

Correspondence • 1983: (1)

Huggins, __, Miss California Historical Society Correspondence $\bullet$ 1956: (1)

Huggins, Solange G. See Gignac, Solange G. Huggins

Hughes, Edan Milton San Francisco, CA Correspondence $\bullet$ 1987: (1)

Hughes, G. G. Southwestern Louisiana Institute Correspondence $\bullet$ 1954: (1)

Hughes, Griffith Bioscript

Hughes, William (n. 1652-1683) Bioscript

Hugo, Harold Meriden Gravure Company, publisher Correspondence • 1965: (0) Filed under George Washington Corner $\bullet$ 1965: (12) Bartram, William, 1739-1823 • 1966: (19) Bartram, William, 1739-1823 • 1967: (0) Filed under George Washington Corner $\bullet 1967$ : (6) Bartram, William, 1739-1823

Hull, John (1761-1843) British botanist Bioscript

Hult, Ragnar (1857-1899) Finnish phytogeographer; Helsingfors, Finland Biofile

Hultén, Eric Oskar Gumnar (1894-1981) Swiss phytogeographer, taxonomist, plant collector; Naturhistoriska Riksmuseet Botaniska Avdelningen, Stockholm

Botanical Album

Correspondence • 1953: (2) • 1954: (2) • 1955: (1) • 1972: (1) - 1973: (1)

Humbert, Jean Henri (1887-1967) French botanist of Madagascar; Museum of Natural History, Paris Correspondence • 1936: • 1939: (1) • 1954: (1)

Humboldt, Alexander von (1769-1859) German naturalist and explorer

Biofile $\bullet$ Bioscript $\bullet$ Botanical Album

Humbolt, F. A. Bioscript

Hume, Alexander (n. 1730) Bioscript

Hume, Hardrada Harold (1875-1965) Canadian-born horticulturist; U. S. A. Bioscript

Humes, Elizabeth D. Correspondence $\bullet$ 1974: (1)

Hummel, David Medical officer, whaling factory Pelagos; Lidingo, Sweden

Bioscript • Botanical Album

Correspondence $\bullet$ 1958: $(0)$ Letter filed under Warren Smith 1 1958: (8) • 1959: (4) • 1960: (1) • 1961: (2) 1963: (1) • 1964: (3) • 1965: (4) • 1971: (4) • 1972: (17) - 1973: (3) • 1974: (2) - 1975: (3) 1976: (4) 1977 : (3) 1978: (1) Rocky Mountain Naturalists • 1979: (5) 1980: (6) • 1981: (5) • 1982: (2) • 1983: (3)

Humphrey, Derek National Hemlock Society, Eugene, OR
Humphrey, George (?1745-c. 1830) Bioscript

Humphrey, Harry Baker (1873-1955) American plant pathologist

Bioscript • Botanical Album $\bullet$

Humphrey, Herbert N. Oakland, CA Correspondence $\bullet$ 1971: (2)

Humphrey, Philip S. U. of Kansas, Museum of Natural History, Assoc. of Systematics Collections Correspondence $\bullet$ 1973: (1)

Humphreys, A. A. Bioscript

Humphreys, C. E. Correspondence $\bullet$ 1949: (1)

Humphreys, Clarence John (1882-) Bioscript

Humphries, Jill Oxford, Dept. of Plant Sciences Correspondence $\bullet$ 1991: (2)

Hungarian Academy of Sciences Botanical Album

Hunger, Friedrich Wilhelm Tobias (1874-1952) Historian of botany Biofile

Hunkin, Ruth Husband is J. W. Hunkin; Oxford, England Correspondence $\bullet$ 1964: (3) $\bullet$ 1965: (1) $\bullet$ 1977: (2)

Hunnemann, J. Bioscript

Hunnewell, Horatio Hollis (1810-1902) American horticulturalist, industrialist, benefactor Biofile

Hunt Botanical Library See Hunt Institute of Botanical Documentation

Hunt, Charles Butler (1906-) American geologist Bioscript

Hunt, Everett Swarthmore College, PA, Dean Correspondence $\bullet$ 1953: (3)

Hunt Institute for Botanical Documentation CarnegieMellon U., Pittsburgh, PA

Botanical Album

Correspondence • 1973: (3) - 1978: (2) Lawrence, George Hill Mathewson, 1910-1978 - 1979: (2) - 1980: (1) $\bullet$ 1981: (1) • 1982: (1) • 1983: (2) • 1984: (3) • 1987: (0) Invitation to preview for art exhibit $\bullet 1988$ : (1)

Hunt, Lowry W. Correspondence $\bullet$ 1983: (1)

Hunt, Mandy Graduate student at Tulane Correspondence $\bullet$ 1972: (1)

Hunt, Peter Francis (1936- ) Taxonomist Correspondence $\bullet$ 1977: (3)

Hunt, Rachel McMasters Miller (1882-1963) Botanical historian Biofile

Hunt, Richard United States Army Corps of Engineers, Colonel

Correspondence • 1972: (0) Filed under United States Army Corps of Engineers

Hunt, Robert Bioscript

Hunt, Roy A. Hunt Botanical Library Correspondence • 1958: (1) 
Hunt, William L. The Botanical Garden Foundation, Inc., Chapel Hill, NC, Chairman Executive Committee Bioscript • Botanical Album Correspondence • 1977: (1)

Hunter, A. Bioscript

Hunter, C. L. Bioscript

Hunter, Dard Institute of Paper Chemistry Correspondence $\bullet$ 1961: (3)

Hunter, Fred Correspondence $\bullet$ 1990: (1)

Hunter, Mary Bartram Husband is Frank P. Hunter Correspondence $\bullet$ 1985: (3) $\bullet$ 1986: (1)

Huntington, Henry E. Bioscript

Huntington Library Library, Art Gallery, Botanical Gardens; San Marino, CA; Robert O. Dougan, Librarian Correspondence • 1965: (1) • 1967: (1) • 1977: (1)

Huntington, Selina (Countess of) Bioscript

Hurd, Albert (1823-) Chemist Biofile

Hurley, Homer Appalachian State U., Boone, Dept. of Biology Correspondence $\bullet$ 1970: (1)

Hurlock, D(?) (or Joseph) Bioscript

Husseini, Mohammed Abdullah Nazimabad, Karachi, Pakistan Correspondence 1977: (3)

Hustis, Jabez Bioscript

Huston, McCready Academy of Natural Sciences; Frontiers, Editor Correspondence $\bullet$ 1952: (5) Academy of Natural Sciences of Philadelphia; Nuttall, Thomas, 1786-1859 • 1954: (3)

Hutchings, James Mason (1818-1902) Bioscript

Hutchins, Lee M. Cacao Center, Inter-American Institute of Agricultural Sciences, Turialba, Costa Rica Correspondence 1960: (1)

Hutchins, Ross E. Mississippi State College, Dept. of Biology Correspondence - 1959: (1)

Hutchins, Thomas (of Army) Bioscript

Hutchins, Thomas (of Hudson's Bay Co.) Bioscript

Hutchinson, C. E. Bioscript

Hutchinson, Isobel Bioscript

Hutchinson, Jay G. United States, Forest Service, International Forestry Correspondence $\bullet$ 1970: (2)

Hutchinson, John (1884-1972) English botanist, taxonomist, botanical artist Biofile • Botanical Album

Hutchinson, Susan Whipple American amateur botanist; Glendale, CA; Ojai, CA; Nickname "Suz"; husband is William Hutchinson Bioscript • Botanical Album
Hutchinson, Susan Whipple (continued) Correspondence • 1934: (4) • 1935: (8) Species; Delphinium; Jepson, Willis Linn, 1867-1946 • 1936: (6) • 1937: (10) Species • 1938: (4) • 1939: (2) • 1941: (6) • 1942: (8) Eastwood, Alice, 1859-1953 - 1943: (2) - 1949: (1) - 1951: (1) • 1952: (6) Delphinium; Book collectors • 1953: (1) • 1954: (8) • 1956: (3) • 1957: (2) • 1958: (1) • 1959: (6) • 1960: (4) • 1962: (1) • 1965: (1) • 1969: (1)

Hutchison, Frances Sec. to W, M. Campbell, U. of Colorado Correspondence $\bullet$ 1942: (1)

Huttleston, Donald Grunert (1920- ) Brooklyn Botanic Garden and Longwood Gardens; American Fern Society Correspondence • 1955: (1) • 1956: (2) • 1960: (2) • 1962: (1) - 1964: (1)

Hutton, Mary L. M. Tulane U., School of Medicine, Librarian Correspondence $\bullet$ 1948: (1)

Hutton, Wiliam (1798-1860) British paleobotanist; Durham Bioscript

Hutzel, J. M. American Assoc. of Advancement of Science Correspondence - 1946: (2) American Fern Society

Huxley, Anthony Botanical Album

Huxdey, Julian Sorrel (1887-1975) British biologist, eugenicist; London Bioscript • Botanical Album Correspondence • 1944: (1) Species

Huxley, Thomas Henry (1825-1895) Biofile • Bioscript $\bullet$ Botanical Album

Hyams, Edward Las Palmas, Canary Islands Correspondence • 1969: (1)

Hyams, M. E. Bioscript

Hyatt, Alpheus (1838-1902) American zoologist, paleontologist Biofile

Hyde, Gene, Mrs. Tulane U., Order Librarian Correspondence • 1960: (0) Letter filed under William R. Hogan

Hyde, Richard Smithsonian Institution, Division of Botany Correspondence $\bullet$ 1969: (1)

Hyder, Clyde K. U. of Kansas Press, Lawrence Correspondence • 1965: (10)

Hyland, Fay (1900-) Taxonomist, forester; U. of Maine, Orono, Botany \& Entomology Correspondence $\bullet$ 1952: (1)

Hyland, Howard Lewis (1907-) Agronomist Bioscript

Hylander, Clarence John (1897-) American botanist Botanical Album

Hyman, Libbie Henrietta (1888-1869) Bioscript

Ibis Books Brian E. Cassie Correspondence • 1983: (2)

Ichida, Jann Paulette (1941-) American botanist, bacteriologist; Ohio Wesleyan, Botany Dept.; husband is Allen A. Ichida Correspondence $\bullet$ 1982: (1) 
Iddenden, James R. Holly Bluff On-the-Jordan, Holly Bluff Gardens, Bay St. Louis, MI, Head Gardener Correspondence $\bullet$ 1953: (2)

Ide, F. P. U. of Toronto, Dept. of Zoology, Associate Professor

Correspondence • 1960: (10) Banks, Joseph, Sir, 1743-1800 • 1961: (1) Beaglehole, John Cawte • 1962: (3)

Idler, Mary Jane Richie Denver, CO Correspondence • 1943: (1) • 1946: (2)

Idrobo, Jesus M. (1917-) Instituto De Ciencias Naturales, Bogatá, Colombia

Correspondence $\bullet$ 1948: (1) • 1952: (1)

Iliffe, J. J. Liverpool Museums Correspondence 1954: (2)

Ilinger, Carl (1775-1813) Bioscript

Illingworth, James Franklin Bioscript

minois State Teachers College Correspondence • 1949: (2)

Ittis, Hugh Helmutt (1925-) American botanist; U. of Wisconsin, Madison

Biofile $\bullet$ Bioscript $\bullet$ Botanical Album

Correspondence • 1953: (4) • 1954: (3) • 1955: (1) • 1956: (4) • 1957: (10) $\bullet$ 1958: (1) $\bullet$ 1959: (6) Van Schaack, George Booth, 1903-1983 - 1961: (6) Banister, John, 1650-1692; Science-Instruction and study $\bullet 1962$ : (7) Bartram, William, 1739-1823 • 1964: (10) Banister, John, 1650-1692 • 1965: (4) • 1966: (2) Banister, John, 1650-1692 • 1967: (7) • 1968: (4) • 1970: (4) • 1971: (2) - 1973: (1) - 1974: (3) Missouri Botanical Garden • 1976: (2) 1 1977: (4) • 1978: (2) • 1979: (1) • 1980: (1) $\bullet 1982$ : (2) $\bullet$ 1983: (5) • 1984: (3) • 1985: (4) • 1986: (1) • 1987: $(2) \bullet$ 1989: (3) $\bullet$ 1990: (5) $\bullet 1992:(1) \bullet 1993:$ (4)

Im Thurn, Everard Bioscript

Imler, Ralph H. U. S. Fish and Wildlife Service Correspondence $\bullet 1940$ : (1) • 1942: (3)

Independent Research Libraries Association Huntington Library, San Marino, CA Correspondence $\bullet$ 1981: (1)

Indiana Historical Society Indianapolis, IN Correspondence • 1951: (2)

Indiana Magazine of History Assistant Editor, Lorna Lutes Sylvester Correspondence $\bullet$ 1969: (2)

Indiama U., Bloomington Correspondence • 1970: (1)

Information Please New York City Correspondence • 1943: (1) • 1945: (1)

Ingalls, Thomas Russel Bioscript

Ingen-Housz, Bioscript

Ingersoll, Ermest (1852-1946) American naturalist, journalist, editor Bioscript

Ingram, Marie Correspondence • 1992: (1) • 1993: (2)

Innis, Harold Adams (1894-1952) Canadian geographer
Biofile
INQUA Travel Support Program National Academy of Science

Correspondence $\bullet$ 1973: (1)

International Association for Plant Taxonomy Correspondence • 1992: (1)

International Botanical Congress, Inc. George W. Fischer, Seattle, WA

Correspondence • 1969: (4)

International Congress of the History of Science Correspondence $\bullet$ 1966: (1)

International Exchange of Persons, Committee on Elaine A. Harris and Francis A. Young

Correspondence • 1967: (4)

International Expedition, Inc. Correspondence $\bullet$ 1988: (1)

Iowa State Board of Education Iowa State College Correspondence 1948 : (1)

Iowa State Journal of Research Correspondence $\bullet$ 1985: (1)

Iowa State U. Press Correspondence $\bullet$ 1984: (9)

Iredale, Tom (1880-1972) English ornithologist in New Zealand and Australia Biofile

Irénée-Marie, Frère (1898-1960) Canadian botanist and clergyman Biofile

Irish Garden Plant Society Glasnevin, Dublin, Ireland Correspondence 1990: (1)Irving, Joan Philadelphia Area Consortium of Special Collections Libraries Correspondence • 1986: (1)

Irving, Washington Bioscript

Irwin, Howard Samuel, Jr. (1928-) New York Botanical Garden, President

Botanical Album

Correspondence • 1976: (2) • 1976: (0) Filed under Peter Hamilton Raven $\bullet$ 1977: (4)

Irwin, Margaret C. Correspondence $\bullet$ 1937: (1)

Isaman, $\mathbf{S}$. G. Bioscript

Ise, Walter J. Colorado State Forestry Asociation Correspondence • 1942: (1) • 1944: (1)

Isely, Duane (1918- ) American taxonomist, economic botanist, seed technologist; Iowa State U. Dept. of Botany and Plant Pathology, Ames

Botanical Album

Correspondence • 1953: (2) • 1971: (3) • 1982: (3) • 1984: (1) • 1991: (3)

Isely, Frederick Benjamin (1873-1947) American orthopterist Biofile $\bullet$ Bioscript

Isennock, Mary R. Educators Travel Club Correspondence $\bullet$ 1978: (0) Filed under Educators Travel Club

Isenstein-Froomer, Marcia Correspondence $\bullet$ 1977: (3)

Ish-Shalom, Naomi U. of Miami, Coral Gables, Dept. of Biology

Correspondence $\bullet$ 1991: (1) 
ISIS

Correspondence • 1963: (2) • 1967: (3) $\bullet$ 1968: (1) • 1972: (1) • 1973: (3) • 1975: (2) • 1976: (1) • 1982: (3) • 1987: (4) - 1988: (1) Verdoorn, Frans Antonie, 1906-1984 - 1989: (1)

Isnard, Antoine-Tristan Danty d' (1663-1743) French botanist; Jardin de Roi, Paris

Bioscript

Ivanov, A. I. Botanical Album

Ives, Eli (1779-1861) Bioscript

Ives, Joseph Christmas (1828-1868) American explorer, officer, U. S. Army topographical engineers Bioscript

Ives, Ronald L. Cornell Aeronautical Lab, Buffalo, NY; U. of Colorado Museum in Boulder, 1937-1944 Correspondence • 1953: (3) "Rocky Mountain Vegetation" Ives, Sidney Correspondence $1982:(1)$

Ivker, Barry Dillard U., New Orleans, LA, Division of the Humanities

Correspondence • 1969: (2)

\section{$J$}

Jack, John George (1861-1949) American largely selftaught dendrologist; Arnold Arboretum Bioscript $\bullet$ Botanical Album

Jackson-Arnold, Ann Husband is Ian Jackson Correspondence - 1986: (5)

Jackson, Donald U. of Illinois Press, Urbana; The Papers of George Washington, Alderman Library, U. Virginia, Charlottesville, Editor

Correspondence • 1964: (6) • 1965: (2) • 1967: (5) Banister, John, 1650-1692 • 1968: (4) Banister, John, 1650-1692 • 1969: (13) • 1970: (14) • 1970: (0) Filed with letters to Bonnie Depp • 1972: (2) • 1973: (1) • 1977: (2) - 1981: (3) Rocky Mountain Naturalists • 1983: (4) Barton, Benjamin Smith, 1766-1815 • 1985: (2)

Jackson, Helen Hunt (1830-1885) American author, poet, philanthropist

Bioscript

Jackson, Henry (1778-1840) Bioscript

Jackson, Ian American bookseller, author; Hortulan Books, Berkeley, CA

Biofile $\bullet$ Bioscript $\bullet$ Botanical Album

Correspondence - 1979: (0) Letter filed under Berry Campbell $\bullet$ 1979: (18) Book collectors $\bullet$ 1980: (10) $\bullet$ 1981: (10) • 1982: (11) • 1983: (6) • 1984: (10) • 1985: (9) $\bullet$ 1986: (14) • 1987: (11) • 1988: (21) • 1989: (35) Book collectors $\bullet$ 1989: (0) Book collectors; Filed under Sylvia Metzinger • 1990: (59) $\bullet$ 1991: (17) $\bullet$ 1992: (20) Taylor, George, Sir, 1904-; Book collectors; Jepson, Willis Linn, 1867-1946 • 1993: (6)

Jackson, John (1748-1821) Bioscript

Jackson, L. O., Mrs. Correspondence • 1949: (3) Rocky Mountain Naturalists

Jackson, Lawrence Oscar (1890-1946) Entomologist Bioscript
Jackson, Peter (1921-) English collector Biofile

Jackson, R. C. U. of Kansas, Lawrence. Dept. of Botany Correspondence • 1962: (1) Delphinium

Jackson, Samuel Bioscript

Jackson, William Henry (1843-1942) American photographer, artist, explorer Biofile $\bullet$ Bioscript

Jackson, William M. Bioscript

Jacquemont, Victor (1801-1832) French explorer and naturalist Bioscript $\bullet$ Biofile

Jacquin, Nicolaus Joseph, Baron von (1727-1817) Dutchborn Austrian botanist Bioscript $\bullet$ Botanical Album

Jaeger, Benedict (1789-1869) Entomologist Biofile

Jaeger, Edmund Carroll (1887-1983) American conservationist, zoologist; Riverside Municipal Museum, Zoology Dept., Riverside, CA Biofile $\bullet$ Bioscript $\bullet$ Botanical Album Correspondence • 1936: (2) • 1937: (1) • 1942: (2) • 1961: (4) Book collectors $\bullet$ 1962: (8) Rocky Mountain Naturalists • 1963: (1) • 1964: (3) • 1967: (1) • 1974: (4) - 1975: (1) • 1976: (1) • 1978: (3) • 1982: (1) • 1983: (2) - 1984: (5)

Jaeger, Eugene Correspondence • 1943: (2)

Jaeger, Willard Correspondence $\bullet$ 1943: (2)

Jagendorf, Andre Tridon (1926-) American plant physiologist Botanical Album

Jahn, Alfredo (1867-1940) Venezuelan botanist Botanical Album

Jahn, Theodore L. U. of California, Los Angeles, Dept. of Zoology Correspondence $\bullet$ 1958: (1)

Jamaica See Natural History Society of Jamaica

James, Charles Mason Philadelphia, PA Botanical Album Correspondence $\bullet$ 1972: (2) • 1974: (2) • 1983: (1)

James, Douglas A. American ornithologist; U. of Arkansas, Fayetteville Botanical Album

James, , Dr.

Bioscript

Correspondence $\bullet$ 1963: (1)

James, Edward T. Notable American Women 1607-1950, Radcliffe College

Correspondence $\bullet$ 1964: (1) • 1965: (2) • 1966: (3) • 1967: (7) - 1968: (3)

James, Edwin (1797-1861) American surgeon, botanist, geologist

Biofile • Bioscript

James, Francis G.

Correspondence $\bullet 1981$ : (1)

James, George Wharton (1858-1923)

Bioscript • Botanical Album 


\section{Guide to the Ewan Papers}

James, Janet Wilson Notable American Women, A Biographical Dictionary; husband is Edwin T. James Correspondence $\bullet$ 1970: (1)

James, Maurice Theodore (1905-) American entomologist; Colorado State College Correspondence • 1938: (5)

James, Stephen Louis Bioscript

James, Thomas Potts (1803-1880) American bryologist Biofile • Bioscript

James, William U. of Georgia, Athens Correspondence $\bullet$ 1952: (2)

Jameson, Dean Camden, AK Correspondence $\bullet 1976$ : (1)

Jameson, Robert Bioscript

Jameson, William Bioscript

Jamestown Society Thomas Connell Correspondence $\bullet$ 1973: (1)

Jansen, Bob Ohio State, Columbus, Botany Correspondence $\bullet$ 1982: (1)

Jansma, Harriet Correspondence • 1990: (0) Filed under Rudolph • 1990: (13)

Janssen, Paul L. Southern Forest Experiment Station, New Orleans, LA

Japan Correspondence $\bullet$ 1953: (2) • 1955: (1)

Botanical Album

Jaques, Harry Edwin Iowa Wesleyan College, Professor of Biology

Bioscript • Botanical Album

Jardel, Benjamin Bioscript

Jardine, William (1800-1874) Scottish naturalist Bioscript • Botanical Album

Jarratt, Devreaux (1773-1801) Bioscript

Jarrel, Nancy Correspondence - 1981: (2)

Jarrett, Henry Resources for the Future, Inc. Washington, D.C. Correspondence $\bullet$ 1956: (1)

Jarvis, Peter U. of Birmingham, England Correspondence • 1974: (1)

Jaster, Frank Tulane U., Graduate School of Business Administration, New Orieans, LA Correspondence $\bullet 1977$ : (2)

Jean, F. C. Colorado State College of Education Correspondence • 1939: (1)

Jeanplong, Joseph Universitas Scientiarum Agrariarum, Godollo, Hungaria Correspondence $\bullet 1974$ : (2)

Jefferson, Howard B. American Antiquarian Society Correspondence • 1976: (0) Filed under American Antiquarian Society

Jefferson, Thomas (1743-1826) American horticulturist, statesman, President of the U. S. A. Biofile $\bullet$ Bioscript $\bullet$ Botanical Album
Jefferson, Thomas, Papers of John Catanzariti, Princeton U. Library Correspondence • 1988: (3)

Jefferys, Thomas Bioscript

Jeffrey, John Bioscript

Jeffrey, John (1826-1854) Scottish botanical explorer of the American northwest Biofile

Jeffreys, Thomas Bioscript

Jeffries, Theodore W. Lorain County Community College, Elria, $\mathrm{OH}$, Assoc. Prof. of Science

Correspondence • 1974: (2)

Jekyll, Gertrude (1843-1932) British horticulturalist, landscape architect Bioscript

Jelliffe, Derrick B. Botanical Album

Jelliffe, Richard B. Tulane School of Tropical Medicine and Public Health; nickname "Dick" Correspondence $\bullet$ 1956: (1) • 1957: (8)

Jellison, Richard M. Miami U., Oxford, OH, Assoc. Prof. History Correspondence 1964 : (2)

Jenkins, Anna Eliza (1886-1973) Canadian-born American mycologist; U. S. Dept. of Agriculture, Bureau of Plant Industry

Botanical Album

Correspondence • 1948: (2) Verdoorn, Frans Antonie, 1906-1984; Maxon, William Ralph, 1877-1948 • 1949: (7) - 1953: (5) Pennell, Francis Whittier, 1886-1952 • 1954 (2)

Jenkins, Charles F. Bioscript

Jenks, J. W. L. Bioscript

Jenne, Trudy Vanderbilt U. Correspondence $\bullet 1966$ : (1)

Jenner, Edward Bioscript

Jennings, Edward H. Ohio State U., Columbus, President Correspondence • 1986: (1) • 1988: (0) Filed under Ohio State University

Jennings, Herbert Spencer (1868-1947) American geneticist, physiologist

Biofile - Bioscript

Jennings, Jack Correspondence $\bullet$ 1992: (1)

Jennings, Mark United States Dept. of the Interior, Fish and Wildlife Service, Fishery Biologist, Los Banos, CA Correspondence $\bullet$ 1988: (3) $\bullet$ 1989: (1)

Jennings, Otto Emery (1877-1964) American botanist; Carnegie Museum, Pittsburgh Correspondence $\bullet$ 1941: (1)

Jennings, William F. Consulting Mining Engineer Correspondence • 1983: (2)

Jensen, Dr.

Correspondence $\bullet$ 1959: (0) Letter from David Hummel, medical officer of the whaling ship "Pelagos" to Professor Jensen is filed under Hummel 
Jensen, Vera E. U.S.D.A. Graduate School

Correspondence $\bullet$ 1975: (3)

Jepson, Glenn Lowell (1903- ) American vertebrate paleontologist

Botanical Album

Jepson Herbarium

Botanical Album

Jepson Herbarium, Friends of Correspondence $\bullet$ 1986: (1)

Jepson, Willis Linn (1867-1946) American botanist; U. of California, Berkeley

Biofile - Bioscript $\bullet$ Botanical Album

Correspondence 1934 : (6) $\bullet$ 1935: (2) Delphinium; Royal Botanic Gardens, Kew $\bullet$ 1936: (4) • 1937: (3) • 1938: (4)

- 1939: (6) Missouri Botanical Garden - 1940: (3) • 1941:

(3) 1942: (6) Written by Jon Estecourt and A. C. Carison - 1945: (2) - 1946: (3)

Jermy, Anthony Clive (1932-) British taxonomist; British Museum (Natural History)

Correspondence $\bullet$ 1979: (3)

Jespersen, Beryl Olive Schreiber (1911-) American taxonomist; husband is Holger J. Jespersen Botanical Album

Jespersen, Holger J. American plant collector; wife is Beryl Olive Schreiber Jesperson

Botanical Album

Jessen-Hansen, Hans (1860-1934) Danish agriculturist, food scientist

Biofile

Jeter, Marvin D. Anthropologist; Arkansas Archeological Survey

Bioscript $\bullet$ Botanical Album

Correspondence • 1986: (1) • 1987: (1) • 1988: (1) • 1990: (2) • 1991: (2) • 1993: (4)

Jewett, Adams (1807-1875) Plant explorer Bioscript

Jewett, William Dunbar (1873-1926) Artist Biofile

Jha, Pramoa K. Botanical Album

Jiménez, Otén (1895-) Costa Rican taxonomist Correspondence $\bullet$ 1939: (1)

Johannessen, Carl L. U. of Oregon, Eugene, Dept. of Geography

Correspondence • 1963: (1) • 1976: (1) • 1978: (1) • 1979: (2)

Johansen, Donald Alexander (1901-) American plant collector; California Botanical Materials Co., Pomona Correspondence • 1929-1933: (18) Munz, Philip Alexander, 1892-1974 • 1939: (1) Species • 1961: (1) 1962 : (0) Letter filed under Anne Ophelia Todd $\bullet$ 1962: (2)

Johansen, Wilhelm (1857-1927) Danish botanist; Copenhagen, Denmark Biofile

Johanson, Teresa The Kelmscott Bookshop Correspondence $\bullet$ 1992: (1)

John Howell-Books Correspondent is Jeffrey Kearns Correspondence • 1970: (1) See also Howell, Warren R.

John Bartram Association Correspondence - 1982: (1) - 1983: (1) • 1984: (5)

John Carter Brown Library Brown U., Providence Correspondence • 1966: (1)
John Simon Guggenheim Memorial Foundation G.

Thomas Tanselle, Vice President, New York, NY

Correspondence $\bullet$ 1978: (2) • 1979: (2) • 1985: (1) • 1990: (3) 1992: (1) Letter signed by Peter H. Raven

Johnson, Albert G. U. of Minnesota, St. Paul, Dept. of Horticulture

Correspondence $\bullet$ 1961: (2)

Johnson, Albert S.

Bioscript

Johnson, Arthur Monrad (1878-1943) Botanical artist, taxonomist

Correspondence $\bullet$ 1935: (2)

Johnson, B. F.

Bioscript

Johnson, C. E. Bioscript

Johnson, Dale Edwin (1949-) American botanist, botanical bibliographer, editor; Missouri Botanical Garden Library; Timber Press

Bioscript $\bullet$ Botanical Album

Correspondence $\bullet$ 1975: (1) • 1978: (4) Book collectors $\bullet$ 1979: (1) • 1980: (3) • 1986: (11) • 1987: (2) Book collectors • 1988: (3) - 1989: (0) Letters filed under Günther Buchheim and Frederick Gustav Meyer $\bullet$ 1990: (2) - 1991: (1) Stafleu, Frans Antonie, 1921- - 1992: (0) Letter filed under Ronald Lewis Stuckey $\bullet$ 1992: (31) • 1993: (24)

Johnson, Duncan Starr (1867-1937) American botanist Biofile

Johnson, Edna Louise (1887-1991) American plant physiologist; U. of Colorado, Boulder

Bioscript $\bullet$ Botanical Album

Correspondence $\bullet$ 1940: (2) $\bullet$ 1942: (1) $\bullet$ 1943: (1) $\bullet$ 1945: (2) • 1946: (4) • 1947: (2) • 1949: (2) • 1950: (1) • 1976: (1) • 1977: (3) • 1979: (1)

Johnson, Edward Summer Twelfth Naval District, Committee of Deans

Correspondence $\bullet$ 1944: (1)

Johnson, Faith Central Michigan College of Education, Dept. of Biology

Correspondence $\bullet$ 1944: (2)

Johnson, Frank William (1867-1934) American publisher, botanist, plant collector Bioscript

Johnson, George Correspondence $\bullet$ 1972: (2)

Johnson, H. MeClure, Mrs. Correspondence $\bullet$ 1942: (1)

Johnson, Harry Bioscript

Johnson, J. E. Bioscript - Botanical Album

Johnson, Jerah U. of New Orleans Correspondence 1992: (1)

Johnson, Jesse Harlan (1892-) American paleontologist, geologist; Colorado School of Mines Museum and U. S. Geological Survey, Denver

Botanical Album Correspondence • 1940: (2)

Johnson, John John Johnson Natural History Books, North Bennington, VT

Correspondence • 1952: (0) Filed under Roland MclMillan Harper • 1960: (0) Letter filed under Gertrude D. Hess • 
Johnson, John (continued)

1961: (1) • 1964: (2) • 1978: (I) • 1980: (3) • 1981: (1) • 1982: (4) - 1983: (3) Book collectors • 1983: (1) Book collectors • 1984: (3) Book collectors • 1985: (2) - 1986: (3) $\bullet$ 1987: (4) $\bullet$ 1988: (1) $\bullet$ 1989: (5) Barton, Benjamin Smith, 1766-1815; Book collectors • 1990: (3) • 1991: (3) Book collectors $\bullet$ 1992: (1) $\bullet$ 1993: (5) Book collectors

Johnson, Karl Richard (1907-) American plant ecologist Botanical Album Correspondence • 1949: (2)

Johnson, Lee H. Tulane U. Council on Research and School of Engineering

Correspondence • 1956: (2) • 1958: (2) • 1961: (2) • 1962: (3) • 1963: (1) • 1964: (3) • 1965: (1) • 1979: (2)

Johnson, Lewis Correspondence • 1991: (1)

Johnson, Marion Alvin (1901-1964) American taxonomist, plant ecologist; Rutgers U., New Brunswick, NJ Correspondence • 1964: (1) Rocky Mountain Naturalists

Johnson, Martha

Correspondence • 1983: (1)

Johnson, Orson Bennett (1848-1917) American naturalist Biofile

Johnson, Raymond A.

Correspondence • 1967: (1) • 1971: (1) • 1974: (3)

Johnson, Raymond Roy (1932-) American taxonomist, cytogeneticist; Prescott College, Prescott, AZ, Center for Anthropological Studies, Assoc. Prof. Biology

Correspondence • 1966: (3) • 1969: (2)

Johnson Reprint Corp. Publishers, NY Correspondence • 1971: (3) • 1983: (1) Book collectors

Johnson, Richard L. Saline, LA Correspondence • 1975: (3) • 1978: (2) • 1981: (1)

Johnson, Samuel Botanical Album

Johnson, Walter J. Bookseller; Scientific Periodicals, New York, NY

Correspondence $\bullet$ 1953: (1) $\bullet$ 1956: (1)

Johnson, Warren A. Tulane U., Director of Development Correspondence $\bullet$ 1980: (2) $\bullet$ 1984: (0) Letters filed under Marcia Hathaway-Henderson, Vice-Pres. of Development, Tulane

Johnson, William D. Bioscript

Johnson, William Savage U. of Kansas, Lawrence, English professor Biofile

Johnston, David W. Ornithological Monographs Correspondence • 1988: (6) - 1989: (6) Barton, Benjamin Smith, 1766-1815 • 1990: (1) • 1991: (3) • 1992: (3) Barton, Benjamin Smith, 1766-1815

Johnston, Edith Farrington (1888-) American botanical artist Botanical Album

Johnston, Faith Central Michigan College of Education Correspondence 1943: (2)

Johnston, G. W. Mississippi State U., State College, MS Correspondence $\bullet$ 1962: (3)

Johnston, Harry Bioscript
Johnston, Ivan Murray (1898-1960) American botanist; Arnold Arboretum

Biofile $\bullet$ Bioscript $\bullet$ Botanical Album

Correspondence 1929-1933: (3) Jepson, Willis Linn, 1867-1946; Setchell, William Albert, 1864-1943 • 1935: (4) Species • 1936: (4) Delphinium $\bullet$ 1937: (2) • 1938: (6) • 1939: (4) Species; Arnold Arboretum • 1940: (1) • 1948: (5) $\bullet$ 1950: (2) $\bullet$ 1951: (1)

Johnston, J. Bennett United States Senator, U.S. Senate, Washington, D.C.

Correspondence $\bullet$ 1976: (0) Filed under Louisiana Trails Advisory Council $\bullet$ 1976: (1) $\bullet$ 1978: (2) $\bullet$ 1980: (2)

Johnston, Lawrence Waterbury (1871-1958) English horticulturist Biofile

Johnston, Marshall Conring (1930-) American botanist; Sull Ross State College, Alpine, TX, Dept. of Bio.; U. Texas, Austin, Plant Research Institute Correspondence • 1956: (2) • 1960: (4) • 1961: (2) • 1974: (2) • 1976: (2) Delphinium

Jokela, Paavo Sigrid (1896-1971) Physician in Finland Correspondence $\bullet$ 1954: (2)

Jollie, Malcolm Correspondence $\bullet$ 1943: (1)

Jolly, Donald H. McMaster University, Cornell U. Correspondence $\bullet$ 1938: (3)

Jonassen, Hans B. Tulane U., Dept. of Chemistry Correspondence $\bullet$ 1963: (2)

Jones, Correspondence $\bullet$ 1937: (1)

Jones, Almut Gitter (1923- ) U. of Illinois, Herbarium, Dept. Botany

Botanical Album

Correspondence • 1968: (1) • 1979: (2) • 1980: (2) Species

Jones, Burle Jackson Bioscript

Jones, Charles Richardson (1879-) American entomologist

Bioscript

Jones, Clyde Harold (1902-) Ohio State U., Columbus, Botany Dept.

Correspondence • 1946: (3) American Fern Society • 1950: (3)

Jones, Eric Northwestern U., Evanston, IL, Dept. of Economics

Correspondence 1974 : (2)

Jones, Frank Morton (1869- ) American naturalist Biofile $\bullet$ Bioscript $\bullet$ Botanical Album

Correspondence • 1951: (4) Jepson, Willis Linn, 1867-1946 • 1954: (6) • 1955: (1) • 1956: (3)

Jones, George (1800-1870) Bioscript

Jones, George Neville (1903-1970) American botanist; U. of Illinois, Urbana

Biofile $\bullet$ Bioscript $\bullet$ Botanical Album

Correspondence • 1943: (0) Filed under Patterson, Florence Anne (Mrs. H. N.) • 1948: (2) • 1949: (3) Rocky Mountain Naturalists • 1951: (1) - 1952: (3) • 1953: (3) • 1954: (2) • 1957: (2) • 1958: (2) • 1959: (3) • 1960: (1) 1962: (4) • 1963: (7) • 1966: (1) • 1967: (3) • 1968: (4) • 1972: (1) 
Jones, Horace Wife is Alice Gwen Botanical Album

Jones, Hugh (d. 1701) Bioscript

Jones, J. Thad Bioscript

Jones, Joseph Merrick Jones, Walker, et al., Attorneys at law; Administrators of the Tulane Educational Fund Correspondence • 1959:(3) Tulane U., Dept. Of Botany • 1961: (0) Letter filed under Oeta M. Crump

Jones, Joseph L., Col. Newbern, TN Correspondence $\bullet$ 1977: (2)

Jones, Katherine Davies (1860-1943) Bioscript

Jones, Kenneth R. New Orleans, LA Correspondence $\bullet$ 1976: (1)

Jones, Lewis Ralph (1864-1945) American plant pathologist Biofile

Jones, Lillian C. Academy of Natural Sciences of Philadelphia, Librarian Correspondence • 1963: (1)

Jones, Marcus Eugene (1852-1934) American professional plant collector, botanist, explorer, and mining consultant in California; Pomona College

Biofile $\bullet$ Bioscript $\bullet$ Botanical Album

Correspondence $\bullet$ 1929-1933: (7)

Jones, Sam U. of Georgia, Athens Correspondence $\bullet$ 1985: (1)

Jones, William Albert (1841-1914) American engineer, U. S. Army Bioscript

Jones, William L. Bioscript

Jones, Wyatt Wagner (1879-) American economic entomologist, plant pathologist Bioscript

Joor, Harriet Lafayette, LA; daughter of Joseph Joor Correspondence • 1954: (3) • 1955: (1) • 1959: (0) Letter filed under Rufus C. Harris

Joor, Joseph Finley (1848-1893) New Orleans physician and plant collector

Bioscript • Botanical Album

Joppien, Rüdiger Kunstgewerbemuseum, Koln, West Germany

Correspondence $\bullet$ 1983: (1)

Jordan, Alma U. of St. Augustine, West Indies, Librarian Correspondence $\bullet$ 1985: (4)

Jordan, David Starr (1851-1931) American naturalist, ichthyologist, educator; Stanford U.

Biofile $\bullet$ Bioscript

Jordan, Edwin Oakes (1866-1936) American bacteriologist Biofile

Joslyn, Marcia H. Wellesley College, Wellesley, MA Correspondence $\bullet$ 1965: (2)

Josslyn, Charles

Bioscript

Jost, Ludwig (1865-1947) German plant pathologist Botanical Album

Journal of American History Richard Blackett, Indiana U., Bloomington, Associate Editor

Correspondence • 1987: (1)
Journal of the History of Biology Harvard U. Press; Reidel Publishing Company

Correspondence • 1970: (1) • 1979: (1)

Journal of the History of Collections Oxford U. Press, Oxford, England; Jane Parker, Journal Publicity Manager

Correspondence $\bullet$ 1989: (1)

Joumal of the History of Medicine and Allied Sciences Correspondence • 1969: (4) • 1978: (3)

Journal of the West

Correspondence $\bullet$ 1966: (1) $\bullet$ 1967: (2)

Juday, Chancey (1871-1944) American limnologist; U. of WI

Bioscript

Correspondence • 1939: (1)

Judd, Carolus Hubbard Botanical Album

Judd, Orange (1822-1874) Bioscript

Judd, Ruina Wallace Starwood Publishing Correspondence - 1991: (1) Book collectors

Judd, Walter Stephen (1951-) U. of Florida, Gainesville Bioscript

Jun, Yao Bi See Yao, Bi-Jun

Junak, Steve Santa Barbara Botanic Garden, Herbarium Curator Correspondence • 1990: (4) Eastwood, Alice, 1859-1953; Jepson, Willis Linn, 1867-1946

Junge, Carlos (fl. 1931-1941) Chiloe Castro Correspondence $\bullet$ 1939: (1)

Jürgensen, $\mathbf{C}$. Bioscript

Jussien, Adrien Henri Laurent de (1797-1853) French botanist; son of A. L. Jussieu Biofile $\bullet$ Bioscript

Jussieu, Antoine Laurent de (1748-1836) French botanist Biofile • Bioscript

Jussieu, Bernard de (1699-1775) French botanist, uncle and teacher of A. L. de Jussieu

Bioscript

Jussieu, Joseph de (1704-1779) French botanist and explorer; brother of Antoine and Bernard de Jussieu Bioscript

Just, Ernest E. Botanical Album

Just, Theodore Karl (1904-1960) Austrian-born botanist and paleontologist in America; Field Museum of Natural History, Chicago, IL; American Midland Naturalist Biofile

Correspondence • 1929-1933: (1) • 1935: (10) Species • 1936: (5) • 1937: (5) • 1938: (7) • 1939: (8) Species $\bullet$ 1941: (5) Species - 1942: (0) Letter from and filed under Erl Hubert Ellis • 1942: (3) - 1944: (5) Delphinium • 1945: (1) • 1946: (8) • 1952: (1) Vismia • 1957: (1) Delphinium

Justice, Clive Vancouver, B.C Correspondence $\bullet 1979$ : (2)

Juul, Ole

Bioscript 
$\mathbf{K}$

Kaeding, Henry Barroilhet (1877-1913) Ornithologist Bioscript

Kämpfer, Engelbert (1651-1716) German physician and traveller in Japan

Biofile • Bioscript

Kagan, Jacques U. of Mlinois, Chicago Circle Correspondence • 1968: (2) Species

Kahn, Robert L. U. of Wisconsin, Dept. of Germanic Languages and Literature

Correspondence $\bullet$ 1959: (7)

Kaiser, Margaret

Botanical Album

Kalk, Daniel S. (1938-) Librarian Correspondence • 1969: (3) • 1970: (3) • 1980: (4)

Kallunki, Jacquelyn A. New York Botanical Garden Correspondence • 1993: (1)

Kalm, Peter (1715-1779) Finnish naturalist, botanist in America

Biofile • Bioscript

Kalmbach, Edwin Richard (1884-1972) American ornithologist, mammalogist, artist; U. S. Dept. of the Interior, Fish and Wildlife Research Lab, Boulder, CO; wife is Kathryn Kalmbach

Bioscript • Botanical Album

Correspondence • 1942: (28) Species • 1943: (0) Species; Filed under Kathryn Kalmbach • 1944: (7) • 1948: (6) • 1949: (5) • 1952: (0) Filed under Kathryn Kalmbach • 1956: (6) • 1959: (1) • 1960: (0) Filed under Kathryn Kalmbach • 1961: (3) • 1962: (2) • 1963: (2) • 1967: (1) - 1968: (1) • 1969: (1) • 1972: (1)

Kalmbach, Kathryn Denver, CO; Boulder, CO; husband is Edwin Kalmbach

Correspondence • 1940: (2) • 1941: (11) • 1943: (25) Species • 1945: (10) • 1946: (2) • 1947: (1) • 1948: (0) Filed under Edwin Richard Kalmbach $\bullet 1950$ : (1) 1951 : (2) - 1952: (11) - 1952: (0) Letter filed under Elmer Drew Merrill $\bullet$ 1953: (0) Filed under Elmer Drew Merrill $\bullet$ 1953: (12) Book collectors • 1954: (7) • 1955: (5) • 1957: (8) Cockerell, Theodore Dru Alison, 1866-1948 1958 : (4) $\bullet$ 1960: (6) • 1961: (0) Filed under Edwin Richard Kalmbach

Kamel, George Joseph (1661-1706) Moravian-born Jesuit (lay brother) missionary and pharmacist in the Philippines Bioscript

Kamen-Kaye, Dorothy Harvard, Botanical Museum, Research Associate, Ethnobotany Botanical Album Correspondence $\bullet$ 1985: (3)

Kampf, Louise F. Colorado College, Colorado Springs, Librarian

Correspondence • 1949: (4) Rocky Mountain Naturalists • 1950: (3)

Kampman, Bioscript

Kane, George Robert Correspondence • 1986: (1)

Kane, Herman Tulane U. Correspondence • 1966: (1)

Kane, Michal Husband is Norman Kane Correspondence • 1968: (16) Species • 1976: (1) • 1985:
Kane, Norman American bookseller; The Americanist: literary and Historical Americana, Pottstown, PA Correspondence • 1967: (4) • 1969: (1) • 1970: (3) Book collectors $\bullet$ 1971: (6) $\bullet$ 1975: (2)

Kanouse, Mabel C. Correspondence $\bullet$ 1942: (1)

Kaplan, Donald Robert (1938- ) American plant morphologist Correspondence • 1974: (1)

Kaplan, Lawrence (1926- ) American botanist; Roosevelt U., Chicago, Dept. of Biology Correspondence • 1960: (1) 1990: (1)

Karakawa, James Arata U. of California, Berkeley Botanical Album

Correspondence • 1936: (1) - 1937: (7) Jepson, Willis Linn, 1867-1946 • 1938: (1) • 1939: (3) Delphinium • 1940: (1) • 1949: (1)

Karel, John American historian; Tower Grove Park, Director

Botanical Album

Correspondence $\bullet$ 1989: (1) • 1990: (1) • 1992: (3)

Karel, Leonard Correspondence $\bullet$ 1970: (3)

Karling, John Sidney (1898-) American algologist and mycologist; Columbia U., Dept. of Botany; Botanical Society of America Correspondence $\bullet$ 1947: (2)

Karno, Howard Bookseller Correspondence $\bullet$ 1980: (1) $\bullet$ 1986: (1)

Karnes, Tom Tulane U. Correspondence $\bullet$ 1966: (2)

Karr, Dorothy G. Journal of the West Correspondence • 1968: (1)

Karten, David Correspondence $\bullet$ 1958: (2)

Karwinski von Karwin, Wilhelm Friedrich Freiherr (c. 1780-1855)

Biofile

Kaser, David Vanderbilt U., Librarian Correspondence $\bullet$ 1993: (2)

Kaskell, Ralph L., Jr. New Orleans Book Collectors Club, New Orleans; Deutsch, Kerrigan \& Stiles, lawyer Correspondence $\bullet$ 1979: (0) Book collectors; filed under New Orleans Book Collectors Club • 1980: (1) • 1983: (1) Book collectors

Kaspiew, B. Adelaide, Australia Correspondence • 1952: (2) • 1956: (1) • 1962: (1)

Kasteil, Annamarie Correspondence • 1984: (2)

Kastner, Joseph Correspondence $\bullet$ 1977: (7)

Kauffman, Calvin Henry (1869-1931) American mycologist; U. of Michigan, Ann Arbor Bioscript

Kaufman, Albert L. Inventor, bookseller; Eugene, OR; wife is Thelma Kaufman

Botanical Album

Correspondence • 1981: (3) - 1982: (4) Van Schaack, George Booth, 1903-1983 • 1983: (4) • 1984: (3) - 1985: $(1) \bullet 1986:(2) \bullet 1987:$ (1) $\bullet$ 1991: (1) • 1992: (1) $\bullet 1993$ : (3)

Kay, E. Alison U. of Hawaii, Honolulu Correspondence • 1974: (1) 
Keam, Thomas Varker (1842-1904) American ethnologist Bioscript

Keane, Susie B. Tulane U., New Orleans, LA, Archivist Correspondence $\bullet$ 1950: (3) $\bullet$ 1952: (2) $\bullet$ 1955: (2) 1956: (1) • 1957: (2) • 1966: (1)

Kearney, Thomas Henry (1874-1956) American taxonomic botanist; U.S.D.A. Bureau of Plant Industry; California Academy of Sciences, San Francisco

Biofile $\bullet$ Bioscript $\bullet$ Botanical Album

Correspondence 1936: (4) Delphinium • 1938: (2)

Delphinium • 1939: (8) Delphinium; Species • 1941: (11) - 1942: (3) • 1946: (2) Delphinium; Species • 1947: (1) • 1948: (1) Delphinium • 1949: (4) • 1950: (2) • 1954: (1)

Kearns, Denis M. American taxonomist, book collector Botanical Album

Kearns, Jeffrey John Howell - Books Correspondence • 1970: (0) Filed under Warren R. Howell

Kearsley, John Bioscript

Keating, Richard Clark (1937- ) American plant anatomist Correspondence • 1991: (1)

Keating, W. K. Bioscript

Keating, William Hypolitus (1799-1840) American minerologist and geologist Bioscript

Keay, Ronald William John (1920- ) British plant ecologist, dendrologist Correspondence • 1991: (1)

Keck, David Daniels (1903-1995) Carnegie Institution of Washington at Stanford; New York Botanical Garden; National Science Foundation; Medford, OR Botanical Album

Correspondence - 1929-1933: (21) Munz, Philip Alexander, 1892-1974 • 1934: (4) • 1935: (2) Species • 1936: (4) • 1937: (1) • 1938: (7) Species; Delphinium • 1939: (4) Missouri Botanical Garden • 1942: (2) • 1946: (1) - 1947: (1) Jepson, Willis Linn, 1867-1946 • 1948: (4) Species • 1949: (2) • 1950: (2) • 1953: (2) • 1954: (2) $\bullet$ 1955: (2) • 1956: (4) • 1957: (1) • 1958: (2) $\bullet$ 1960: (3) $\bullet$ 1961: (1) Banks, Joseph, Sir, 1743-1800; National Science Foundation • 1965: (1) • 1966: (8) Munz, Philip Alexander, 1892-1974 - 1967: (1) • 1970: (3) Beaglehole, John Cawte • 1972: (2) • 1974: (5) • 1977: (2) • 1978: (1) - 1979: (2) - 1982: (3) - 1983: (3) Van Schaack, George Booth, 1903-1983 • 1984: (4) • 1986: (2) • 1987: (5) • 1989: (1) • 1990: (5) • 1993: (1)

Keeler, Charles Bioscript

Keeley, Jon E. Occidental College, Dept. of Biology, Associate Prof.

Correspondence $\bullet$ 1987: (1)

Keeley, Sterling C. U. of Georgia, Athens, Dept. of Botany Correspondence • 1976: (4)

Keen, Morris Suffolk Academy of Medicine, Hauppauge, NY

Correspondence $\bullet$ 1974: (1)

Keen, Myra Stanford Correspondence • 1967: (1)

Keener, Carl Samuel (1931-) Pennsylvania State U., U. Park, Dept. of Biology

Correspondence • 1970: (2) Delphinium • 1974: (2) Delphinium
Keener, Gladys M. Science and The Scientific Monthly, Washington, D.C., Executive Editor Correspondence • 1950: (4) • 1951: (3)

Keep, Josiah (1849-1911) Bioscript

Keerl, F. W. Bioscript

Keever, Catherine (Nancy) Botanical Album

Kefford, Noel Price (1927-) Australian-born American plant morphologist; U. of Hawaii, Honolulu, Dept. of Botany; nickname "Ned"

Correspondence • 1966: (9) • 1971: (5)

Biology-Instruction and study • 1973: 12) • 1974: (3) • 1975: (3) • 1976: (1) Biology-Instruction and study $\bullet$ 1976: (0) Filed under Charles H. Lamoureux • 1980: (1)

Keidel, George C. Bioscript

Keil, David John (1946-) California Polytechnic State U., San Luis Obispo, Bio. Sciences Dept.

Correspondence • 1984: (1) • 1987: (1) • 1988: (1)

Keillor, Garrison (c. 1942-) American humorist, author Biofile

Keilman, Gustavus Frederic Bioscript

Keith, Freeman Correspondence • 1979: (0) Postcard filed under Stinehour Press

Keith, Henry Newcomb College Correspondence • 1967: (2)

Keith, Hugh U. of Washington, Seattle, Dept. of Botany Correspondence • 1968: (4)

Keith, Paul Gardener; Audubon Park Conservatory, New Orleans, LA

Bioscript

Correspondence • 1966: (1) • 1973: (1) • 1974: (1) • 1975: (1) - 1980: (1)

Kellam, Donald S., Jr. Physician; Charlotte Orthopedic clinic, Charlotte, NC; American Rhododendron Society Correspondence $\bullet$ 1979: (7) $\bullet$ 1981: (1) • 1984: (2)

Keller, Frank L. Tulane U., College of Commerce Correspondence • 1952: (1) • 1957: (1) • 1966: (1)

Kellerman, Stella Victoria Dennis (1855-1936) American botanist, horticulturist Biofile

Kellerman, William Ashbrook (1850-1908) American botanist, mycologist

Biofile $\bullet$ Bioscript $\bullet$ Botanical Album

Kelley, Augustus M. Theophrastus Reprints, Little Compton, RI; descendant of Bartrams Correspondence - 1976: (4) Rocky Mountain Naturalists • 1977: (2)

Kelley, Lynwood J. Bioscript

Kelloff, Carol L. Smithsonian Institution, Biological Diversity of the Guianas Correspondence $\bullet$ 1991: (2)

Kellogg, Bioscript

Kellogg, Albert Bioscript

Kellogg, Hariette S. Botanical Album 


\section{Guide to the Ewan Papers}

Kellogg, Remington Smithsonian, Director Botanical Album

Correspondence • 1949: (2) • 1957: (1)

Kellogg, Vernon Lyman (1867-1937) American entomologist, ornithologist

Bioscript

Kelly, Eamon M. Tulane U., President Correspondence $\bullet$ 1982: (3) $\bullet$ 1983: (2) $\bullet$ 1984: (3) $\bullet$ 1985: (1) • 1986: (1) • 1987: (1)

Kelly, George W. Colorado Mountain Club; Colorado Forestry and Horticulture Association; Cottonwood Garden Shop, Lituleton, CO

Botanical Album

Correspondence $\bullet$ 1941: (2) • 1942: (2) • 1947: (1) • 1964: (2)

Kelly, Harry C. National Science Foundation Correspondence • 1961: (0) Letter filed under Albert C. Spaulding

Kelly, Howard Atwood (1858-1943) American physician, botanist, bibliophile Biofile

Kelly, Matthias Joseph Bioscript

Kelly, Suzanne U. of Notre Dame Correspondence $\bullet 1968$ : (1)

Kelsey, Francis Duncan (1845-1905) American clergyman, botanist Bioscript $\bullet$ Botanical Album

Kelso, Henrietta Zobel See Zobel, Henrietta $L$.

Kelso, Leon Hugh (1907-) American botanist, ornithologist; Washington, D.C.

Bioscript • Botanical Album

Correspondence • 1943: (2) • 1970: (1) • 1971: (2) • 1972: (2) • 1973: (3) • 1974: (1) • 1976: (1) Rocky Mountain Naturalists • 1980: (10) • 1981: (1) Rocky Mountain Naturalists

Kelso, Louis D. City Club of Denver Correspondence • 1942: (3)

Kemanorusla, Vera Correspondence $\bullet$ 1971: (1)

Kemp, Jack Harvie Guest House, Edinburgh Correspondence $\bullet$ 1972: (2)

Kempner, Daniel W. Correspondence $\bullet 1950$ : (1)

Kendall, William Converse (1861-1939) Bioscript

Kendeigh, S. Charles Dept. of Zoology, U. of Mlinois Correspondence $\bullet$ 1954: (1)

Kendrick, James Bioscript

Kennedy, George Golding (1841-1918) Bioscript • Botanical Album

Kennedy, Irene M. Bioscript

Kennedy, Patrick Beveridge (1874-1930) Scottish-born American agronomist Biofile $\bullet$ Bioscript $\bullet$ Botanical Album

Kennedy, Patrick George (1881-) Bioscript

Kennedy, Peggy Reily Graduate student with Duncan Porter Correspondence $\bullet$ 1979; (1)

Kennedy, W. L. Bioscript
Kennerly, Caleb Burwell Rowan (1829-1861) Naturalist, surgeon, U. S. Army

Bioscript

Kenney, $\mathbf{O}$. Bioscript

Kennicott, Robert (1835-1866) American naturalist, explorer Biofile $\bullet$ Bioscript

Kent, Anne Kentfield, CA Correspondence $\bullet$ 1981: (2)

Kent, Sherman Washington, D.C.; Relative of Mary Elizabeth Parsons

Botanical Album

Correspondence $\bullet$ 1980: (3) $\bullet$ 1981: (2)

Kentish, Anne Correspondence $\bullet$ 1955: (4)

Kephart, L. W. Cereal Crops and Diseases, Senior Agronomist in Charge of Weed Investigations Correspondence • 1947: (1)

Kephart, Susan R. Correspondence $\bullet$ 1976: (1)

Kerber, E. Bioscript

Kern, Frank Dunn (1883-1973) American mycologist, taxonomist Bioscript

Kern, Richard Hovenden (1821-1853) American artist on Fremont's 4th expedition Bioscript

Kerner, J. S. von Bioscript

Kersey, R. E. Great States Corp., President Correspondence $\bullet$ 1980: (1)

Kerz, Marcia M. Missouri Botanical Garden, Director of Development

Correspondence • 1990: (0) Filed under Missouri Botanical Garden

Kessel, Edward L. U. of San Francisco, California Academy of Science Correspondence $\bullet$ 1956: (3)

Kessler, Robert J. Whittier, CA Correspondence - 1934: (1) Letter to Louis Cutter Wheeler - 1935: (1) Species • 1959: (0) Filed under Louis Cutter Wheeler correspondence $\bullet$ 1959: (1)

Kessler, Robert M. Bioscript

KETC Nine St. Louis, MO, Public Broadcast Station Correspondence $\bullet$ 1990: (1)

Ketchum, Dana G. Johns Hopkins U. Correspondence $\bullet$ 1983: (1)

Ketz, Louise B. Dictionary of Scientific Biography, Administrative Editor Correspondence • 1976: (0) Letter filed under Dictionary of Scientific Biography

Keuken, Willy (1926- ) Dutch botanist; International Association of Plant Taxonomy, Secy. Correspondence • 1978: (0) Letter filed under Frans Antonie Stafleu

Kevles, Daniel Jerome California Institute of Technology, Division of Humanities and Social Sciences, Pasadena, CA Correspondence $\bullet$ 1977: (2)

Keynes, Geoffrey (1887-1982) Physician, author Biofile • Bioscript 
Keyser, Leander Sylvester (1856-1937) American clergyman

Bioscript

Khopf, Bioscript

Kiaerskou, Hjalmar Frederick Christian (1835-1900) Danish botanist and high school teacher Biofile

Kibbe, Alice Lovina (1881- ) American taxonomist, plant morphologist; Carthage College, Carthage, II Correspondence • 1954: (6) "Rocky Mountain Vegetation" Kibbe, Doug Correspondence • 1985: (1)

Kibbee, James R. Tulane University Howard-Tilton Memorial Library

Correspondence • 1970: (0) Filed under Tulane University Howard-Tilton Memorial Library

Kibre, Pearl Hunter College Correspondence $\bullet$ 1956: (1)

Kidd, John Limestone County, Texas, County Clerk Correspondence - 1966: (1)

Kidd, Joseph Bartholomew (1808-1889) Artist; Scotland Biofile

Kidd, Mary Maytham (1914 ) South African botanical artist Botanical Album

Kidman, Roy L. American Librarian; Tulane, Howard Tilton Memorial Library; U. of California, San Diego Correspondence $\bullet$ 1960: (3) $\bullet$ 1965: (2)

Kiener, Walter Bigler (1894-1959) Swiss-born American plant ecologist, lichenologist, mountaineer; U. of Nebraska, Lincoln

Bioscript $\bullet$ Botanical Album Correspondence $\bullet$ 1942: (10) • 1943: (1) • 1944: (2) • 1946: (2) • 1949: (2) Rocky Mountain Naturalists • 1953: (1) "Rocky Mountain Vegetation"

Kiger, Robert William (1940-) Hunt Institute for Botanical Documentation, Pittsburgh, PA

Botanical Album

Correspondence • 1967: (1) • 1971: (2) • 1973: (1)

1976: (2) Merrill, Elmer Drew, 1876-1956 • 1977: (6) •

1978: (2) Rocky Mountain Naturalists • 1979: (9) • 1980:

(0) Filed under Bernard Boivin $\bullet$ 1980: (14) Rocky

Mountain Naturalists $\bullet$ 1981: (3) $\bullet$ 1982: (4) $\bullet$ 1983: (2) $\bullet$ 1990: (2)

Kilbourne, Frederick

Bioscript

Killeen, Timothy J. Missouri Botanical Garden, Research Assoc.

Correspondence • 1993: (1)

Killip, Ellsworth Paine (1890-1968) American taxonomist, plant collector; Smithsonian Institution; nickname "Buddie" Botanical Album

Correspondence - 1938: (4) • 1941: (4) • 1942: (3) • 1946: (1) - 1947: (10) • 1948: (12) Species; Vismia 1949: (2) Vismia • 1950: (8) • 1951: (20) $\bullet$ 1952: (11) Pennell, Francis Whittier, 1886-1952 • 1953: (9) - 1954: (1) • 1955: (3) • 1956: (4) • 1957: (2) • 1962: (1) • 1965: (2)

Kilpatrick, Elihu Bioscript
Kimball, Laura F.

Bioscript

Correspondence $\bullet$ 1936: (1)

Kimnach, Myron William (1922-) American botanist, bookseller; Huntington Botanical Gardens Correspondence • 1965: (1) • 1986: (5)

Kincaid, Marjorie A. Correspondence • 1992: (1)

Kincaid, Trevor Charles Digby (1872-) Canadian entomologist, naturalist, zoologist Biofile

Kinch, Michael P. Oregon State U., Corvallis, Science-Technology Librarian Correspondence • 1980: (6) • 1984: (2) - 1987: (4) Barton, Benjamin Smith, 1766-1815 - 1988: (2) Barton, Benjamin Smith, 1766-1815 • 1989: (2) • 1991: (1)

Kinch, R. C. Dept. of Agriculture and Inspection Correspondence $\bullet 1946$ : (2) Species

King, Alan G. New Orleans Orchid Society Correspondence • 1962: (2) Letter from King to DeWolf, Gordon P., Jr.

King, Arden Tulane U., Latin American Anthropology; wife is Isabel King

Bioscript $\bullet$ Botanical Album

King, Charles C. Ohio Biological Survey, Executive Director

Correspondence • 1976: (2) • 1980: (1) • 1982: (1)

King, Clarence Rivers (1842-1901) American geologist, explorer

Bioscript

King, Dale Stuart Dale Stuart King, Publisher Correspondence $\bullet$ 1970: (2)

King, Duncan Bioscript

King, Henry St. Louis, MO Bioscript

King, Isabel New Orleans, LA; husband is Arden King Botanical Album (filed under Arden King) Correspondence $\bullet 1950$ : (2)

King, J. A. Bioscript

King, Lawrence J. Ohio Agricultural Experiment Station, Wooster; Plant Lore, Geneseo, NY

Bioscript Correspondence $\bullet$ 1945: (2) • 1978: (1)

King, Louisa Yeomans Bioscript

King, Marjorie Jefferson Parish School Board, Grema, LA Correspondence $\bullet 1966:$ (1)

King, Richard Bioscript

King, Robert Merrill (1930-) American taxonomist; Smithsonian Correspondence $\bullet$ 1973: (2)

King's College Prof. of Biology, E. A. Bell Correspondence • 1975: (3)

Kingdon-Ward, Francis (1885-1958) English explorer, horticulturist; nickname "Frank" Biofile $\bullet$ Bioscript $\bullet$ Botanical Album

Kingery, Elinor Eppich Colorado Mountain Club; Editor of Trail and Timberline; husband is H. M. Kingery Botanical Album Correspondence 1942 : (4) • 1943: (6) • 1944: (1) 
King's Manor Hotel Edinburgh, Scotland Correspondence $\bullet$ 1981: (1)

Kingsley, Karen Correspondence $\bullet$ 1983: (2)

Kingsolver, John M. Ag. Research Service of the U.S.D.A., Systematic Entomology Lab., c/o U.S. National Museum, Washington, D.C., Research Entomologist Correspondence • 1978: (1)

Kinkead, _, Mr. Correspondence • 1969: (1)

Kinman, Guy $\mathbf{M}$. Correspondence $\bullet$ 1954: (6)

Kinn, Mathias ( -1826 ) Bioscript

Kinney, Abbot (1850-1920) American popular writer on botany Bioscript

Kinney, John M. Correspondence $\bullet$ 1942: (1)

Kinsey, Alfred Charles (1894-1956) American zoologist, entomologist Botanical Album

Kinsey, E. B. Texas Ornithological Society, Burnet, TX Correspondence $\bullet$ 1971: (2)

Kinsey, Martha Hilend Botanical Album

Kinsky, Fred National Museum, Wellington, New Zealand Correspondence • 1974: (1)

Kippist, Richard (1812-1882) British librarian, botanist Bioscript

Kirchwey, Freda The Nation, Editor Correspondence • 1942: (1) • 1943: (1)

Kirk, John, Sir (1832-1922) Scottish explorer, plant collector, naturalist in east Africa Biafile

Kirk, Thomas (1828-1898) British-born taxonomist, botanist in New Zealand Biofile

Kirkbridge, Joseph Washington, D.C. Correspondence • 1976: (1) Book collectors

Kirkpatrick, C. M. Purdue U., Forestry Dept., Lafayette, IN

Correspondence • 1951: (2)

Kirkwood, Hughes

Botanical Album

Kirkwood, Joseph Edward (1872-1928) American forester, ecologist

Bioscript

Kirtland, Jared Potter (1793-1877) American physician, naturalist, horticulturist

Biofile • Bioscript

Kirtley, Charles L. Bioscript

Kish, George (1914 ) U. of Michigan, Ann Arbor, Dept. of Geography

Bioscript

Correspondence $\bullet 1977:$ (2) • 1980: (3) • 1982: (1) • 1984: (2)

Kissig, Leonard, Mrs. Daughter of George Cook Correspondence • 1929-1933: (2)

Kitaibel, Pál 1757-1817) Hungarian botanist, physician, taxonomist Bioscript
Kitchell, Mary Correspondence • 1939: (1)

Kitchen, Herman W. HWK Books Correspondence • 1988: (4) Book collectors $\bullet 1990$ : (1)

Kjeldahl, Johan Gustav Christoffer Thorsager (1849-1900) Danish biochemist Biofile

Klaiber, Gustav A. (1870- ) American horticulturist; Denver, CO Bioscript

Klášterský, Ivan (1901-1979) Austrian-born Czech taxonomist; Narodni Museum V Praze, Czechoslovakia Correspondence $\bullet$ 1947: (1)

Klatt, F. W. Bioscript

Klauber, L. M. Natural History Museum, San Diego, CA Bioscript

Klein, J. Perry, Mrs. Correspondence $\bullet$ 1929-1933: (1)

Klein, Janet E. Morris Arboretum; husband is William M. Klein; American Historical Print Collection Society Bioscript • Botanical Album Correspondence • 1982: (1) • 1988: (1)

Klein, Randolph S. American Philosophical Society Correspondence • 1984: (2) • 1985: (13) • 1986: (3) 1987: (0) Letters filed under Laurence J. Dorr and Jean O'Neill • 1987: (1) • 1989: (2)

Klein, Richard M. American plant physiologist; Plant Science Bulletin; U. of Vermont, Burlington,, Dept. of Botany

Correspondence • 1975: (1) • 1976: (5) • 1979: (3) 1987: (2)

Klein, William McKinley (1933-) American plant taxonomist and plant ecologist; Morris Arboretum, Philadelphia, PA; wife is Janet E. Klein

Bioscript

Correspondence • 1982: (1) - 1989: (1) Barton, Benjamin Smith, 1766-1815 • 1990: (1) Barton, Benjamin Smith, 1766-1815

Kleinpell, Robert M. Bioscript

Klinger, Martin Manitou Springs, CO Correspondence • 1943: (2) • 1944: (1) Delphinium

Klippart, John H. (1823-1878) Bioscript

Kloepfer, H. Warner Tulane U., Dept. of Anatomy Correspondence • 1962: (1)

Kloot, P. M. U. of Adelaide, Waite Agricultural Research Institute, Glen Odmond, S. Australia Correspondence $\bullet$ 1979: (1)

Klote, Alexander Barrett (1903-) American entomologist; American Museum Natural History, NY

Correspondence • 1949: (1) • 1968: (1) Rocky Mountain Naturalists

Klotsch, J. F. Bioscript

Kluge, Charles Bioscript

Knaebel, Ernest (1872-1947) American lawyer, amateur botanist, ornithologist

Bioscript

Knapp, M. A. Bioscript 
Knaus, Mabel U. of Colorado, Assistant Dean of Women Correspondence - 1942: (1)

Knieskern, Peter D. (1798-1871) American plant collector, physician Bioscript

Kniffen, Fred B. Louisiana State U., Baton Rouge, Dept. of Geography

Biofile

Correspondence $\bullet$ 1960: (7)

Knight, Charles Robert (1874-1953) American artist Biofile

Knight, Thomas Andrew (1759-1838) British plant physiologist, horticulturist

Bioscript

Knight, Wllbur Clinton (1858-1903) American geologist, ornithologist

Bioscript

Knights Templar Educational Foundation Committee Correspondence • 1940: (1) Recommendation for William A. Nies

Knobloch, Eric Roger ( -1979) Patio Planters, New Orleans, LA; Mary Plantation, Braithwaite, LA

Bioscript $\bullet$ Botanical Album

Correspondence $\bullet$ 1950: (2) $\bullet$ 1951: (3) $\bullet$ 1952: (2)

1955: (1) • 1956: (5) • 1958: (1) • 1961: (1) • 1962: (2)

1965: (3) • 1966: (2) • 1967: (2) • 1969: (1) • 1972: (1) • 1976: (2)

Knobloch, Irving William (1907-) American plant cytologist; Michigan State U., East Lansing; American Fern Society

Bioscript $\bullet$ Botanical Album

Correspondence - 1939: (3) American Fern Society • 1940: (3) • 1954: (2) • 1955: (3) $\bullet$ 1957: (3) $\bullet$ 1958: (3) • 1959: (14) • 1960: (3) • 1961: (4) • 1967: (2) • 1968: (2) - 1969: (1) • 1981: (2) • 1982: (2) • 1983: (2)

Knobloch, Marge Husband is Eric Roger Knobloch Correspondence • 1976: (0) Correspondence filed under Eric Roger Knobloch

Knoche, Edward Louis Herman, (1870-1945) American taxonomist, plant collector

Biofile $\bullet$ Bioscript $\bullet$ Botanical Album

Knollenberg, B. W. F. G. Ludwig, Inc. Correspondence $\bullet$ 1954: (1)

Knopf, Garry N. Texas Tech. College, Lubbock, Texas, Dept. of Biology

Correspondence $\bullet$ 1959: (1)

Knowles, Matilda Cullen (1864-1933) Irish botanist, lichenologist Biofile

Knowlton, Frank Hall (1860-1926) American botanist, paleontologist Bioscript - Botanical Album

Knox, Kenneth A. The Tree Doctor Correspondence - 1983: (2)

Knuckles, Gath Santa Fe, NM Correspondence $\bullet$ 1977: (1)

Knuckles, Leonard Santa Fe, NM Correspondence 1977: (0) Filed under Gath Knuckles

Knudsen, Noreen Correspondence $\bullet$ 1971: (2)

Knüpffer, Helmut Akademie der Wissenschaften der DDR, Zentralinstitut für genetic und Kulturpflanzen.. Correspondence • 1979: (1)
Kny, L. Bioscript

Kobayashi, Jane B. Correspondence $\bullet$ 1968: (2)

Kobuski, Clarence Emmeren (1900-1963) American botanist; Harvard, Gray Herbarium

Biofile • Bioscript

Correspondence • 1956: (3) • 1957: (5) • 1958: (7)

Koch, Albert C. (1804) Paleontologist Bioscript

Koch, Elers (1880-1954) American forester; U. S. Forest Service, Missoula, MT

Biofile

Correspondence $\bullet$ 1966: (1)

Koch, Emilie Sister of Minna F. Koch Correspondence $\bullet$ 1977: (1)

Koch, Karl Heinrich Emil (1809-1879) German physician, plant explorer Bioscript

Koch, Leo Francis (1916-) Tulane U., New Orleans, Dept. of Botany

Biofile $\bullet$ Bioscript $\bullet$ Botanical Album

Correspondence • 1952: (1) • 1953: (1) • 1954: (5) •

1955: (10) - 1956: (3) • 1957: (4) Tulane U., Dept. of Botany $\bullet$ 1958: (4) $\bullet$ 1959: (2)

Koch, Minna Frotscher Bioscript $\bullet$ Botanical Album

Koch, __, Miss Correspondence • 1985: (1)

Koch, Peter (1844-1917) Danish-born American banker, book collector, diarist Biofile $\bullet$ Bioscript

Koch, Richard Architect; New Orleans, LA; donor for Minna Koch Library of Botany at Tulane Correspondence • 1958: (1) - 1966: (2) • 1969: (2)

Koch, Rudy Garner (1939-) American taxonomist; Wisconsin State U., Superior, Dept. of Biology Correspondence $\bullet$ 1969: (3)

Koch, Stephen Collegio de Postgraduades, Rama de Botánica, Chapingo, Mexico Botanical Album

Koch, Walo (1896-1956) Eidg. Technische Hochschule Correspondence $\bullet$ 1939: (1)

Koch, Wilhelm Daniel Joseph (1771-1849) Taxonomist, botanist

Bioscript

Kochel, Ludwig von (1800-1877) Bioscript

Kocher, A. Lawrence Williamsburg, VA Correspondence • 1963: (1) • 1964: (3) Banister, John, $1650-1692$

Koehne, E. Bioscript

Koek-Noorman, Jifke State U. of Utrecht, Institute of Systematic Botany Correspondence • 1986: (3) • 1987: (0) Letter filed under James Leonard Luteyn

Koelbing, w.

Bioscript Koenig, Myron L. American Embassy, London Correspondence $\bullet$ 1954: (2) • 1955: (1) 
Koerner, Harold Elton (1905-) American vertebrate paleontologist; Lafayette College; Longmont, $\mathrm{CO}$ Bioscript Correspondence $\bullet$ 1943: (3)

Koerner, Margaret S. Longmont, CO; husband is Harold Koerner Correspondence • 1943: (0) Filed under Harold Elton Koerner

Kofoid, Charles Atwood (1865-1947) American algologist and zoologist

Biofile • Bioscript

Correspondence $\bullet$ 1937: (2)

Kohlepp, Peg Tulane U., New Orleans, Bookstore Correspondence • 1983: (1)

Kohler, Piotr S. U. of Jagellonicae, Hortus Botanicus, Krakow

Correspondence • 1991: (3) • 1991: (0) Letter filed under Emanuel David Rudolph

Kohlmeister, Benjamin Gottlieb (1775- ) Bioscript

Kohn, David (1941-) Collected Letters of Charles Darwin, Cambridge, England Correspondence $\bullet$ 1981: (2)

Kohn, Joseph Gustave (1837-1906) American naturalist Biofile

Koie, Mogens Engell (1911-) Botanical Album

Kokawa, Shohei Osaka City University, Osaka, Japan, Professor of paleobotany

Botanical Album

Correspondence $\bullet$ 1985: (1)

Koler, G. L.

Bioscript

Kollock, L.

Bioscript

Kologiski, Russell L. North Carolina State U., Raleigh, Botany Dept., graduate student

Correspondence $\bullet$ 1976: (1)

Kolstad, Ole Kearney State College, Kearney, NE Correspondence • 1982: (1)

Komarov, W. L. Akademii Nauk, Botanitscheskij Institut Correspondence - 1939: (1) Species

Komissarav, Boris N. Leningrad State U., Leningrad, USSR; Historical Faculty

Correspondence $\bullet$ 1977: (1) • 1979: (5)

Komodore, Bill

Botanical Album

Konig,

Bioscript

König, Carl Bioscript

Koonce, William W. Ambrosia Chocolate Company, Milwaukee, WI Correspondence - 1956: (1)

Koponen, Timo (1939) ) Finnish phytogeographer, plant ecologist; U. of Helsinki, Dept. of Botany Correspondence - 1989: (2) Correspondence with Peter Raven

Kores, Paul Tulane U. Correspondence • 1991: (1) Korthals, Pieter Willem (1807-1892) Dutch plant collector
Bioscript $\bullet$ Botanical Album
Kosanke, Robert M. Illinois Geological Survey, Urbana Correspondence $\bullet$ 1950: (1)

Kosmos Editora LTDA Bookshop

Correspondence $\bullet$ 1991: (1)

Koster, Joséphine Thérèse (1902-1986) Dutch taxonomist, phycologist

Botanical Album

Correspondence $\bullet$ 1955: (1) $\bullet$ 1956: (2)

Koster, Rudy

Correspondence • 1972: (1)

Koster, Ruth

Correspondence • 1963: (1)

Kotschy, Carl Georg Theodor (1813-1866) Austrian botanist, clergyman

Biofile $\bullet$ Bioscript

Kotzebue, Otto von (1787-1846) Bioscript

Kowalski, John Macmillan Publishing Co. Correspondence $\bullet$ 1974: (1)

Koyama, Harriet H. U. of Hawaii Correspondence $\bullet$ 1967: (5)

Koza, Barbara Babcock U. of Chicago Press Correspondence $\bullet$ 1968: (3)

Kozloff, Eugene N. American zoologist; Oklahoma A. \& M. College Correspondence • 1943: (1)

Kraak, William Landscape Architect Correspondence $\bullet$ 1959: (1)

Krager, Joan U. of Wisconsin Press, Madison Correspondence • 1962: (7) Banister, John, 1650-1692 • 1963: (8) Banister, John, 1650-1692

Kral, Robert (1926- ) American taxonomist; Northern Louisiana State College; Va. Polytechnic Institute, Blacksburg; Louisiana Polytechnic Institute, Ruston; Vanderbilt U., Nashville, TN

Correspondence $\bullet$ 1959: (5) $\bullet$ 1960: (1) $\bullet$ 1962: (1) $\bullet$

1963: (4) • 1964: (5) Arnold Arboretum • 1965: (1) • 1966: (2) • 1967: (3) • 1969: (1) • 1970: (4) • 1977: (1) • 1980: (1)

Kramar, Nada Natural History Bookseller, Washington, D.C.

Correspondence • 1942: (1) Book collectors - 1943: (1) Book collectors • 1947: (1) - 1948: (1) - 1949: (1) - 1950:

(4) Book collectors $\bullet$ 1954: (4) Book collectors $\bullet 1957$ : (1) - 1958: (2) • 1959: (3) • 1961: (5) Banister, John, 1650-1692; Banks, Joseph, Sir, 1743-1800 1966 : (2) 1967: (8) Letter to John H. Gribbin filed under Gribbin • 1969: (4)

Kramer, Angust Henry Bioscript

Kramer, Gerhardt American preservation architect, St. Louis, MO Correspondence • 1989: (1)

Kramer, Karl Ulrich (1928- ) American pteridologist; Botanisch Museum, Utrecht, Netherlands Botanical Album

Correspondence • 1961: (1) • 1964: (1) • 1965: (2) • 1967: (1)

Kramsch, Samuel Gottlieb (1756-1834) German mycologist in America

Biofile • Bioscript 
Kranzberg, Melvin Society for the History of Technology, Cleveland, $\mathrm{OH}$

Correspondence • 1962: (1)

Krapfl, Stephen Bioscript

Krasheninnikov, S. Bioscript

Kraus, Correspondence $\bullet$ 1950: (1)

Kraus, Ezra Jacob (1895-1960) American economic botanist, plant geneticist; U. of Chicago, Dept. of Botany Botanical Album

Correspondence • 1939: (4) • 1942: (5) • 1943: (2) 1944: (2)

Kraus, Joe Tulane U., New Orleans, LA, Howard-Tilton Memorial Library

Correspondence - 1951: (1)

Kraus, Michael College of the City of New York, Dept. of History

Correspondence $\bullet$ 1951: (3) $\bullet$ 1953: (1)

Krauss, Beatrice H. (1903-) American plant physiologist, ethnobotanist; U. of Hawaii, Honolulu Biofile Correspondence $\bullet$ 1974: (1)

Krebs, Carl Cleveland, $\mathrm{OH}$ Correspondence • 1921-1928: (1)

Kreeger, M. M. Correspondence $\bullet$ 1963: (2)

Krelage, Ernest Heinrich (1869-) Dutch horticulturist, nurseryman, book collector Biofile

Krenz, Mathilde Hannah (c. 1900) Botanist Biofile

Krider, John Bioscript

Krieg, David, ( -1712) Bioscript

Krieger, Louis Charles Christopher (1873-1940) American mycologist, botanical artist Biofile

Krieger,

Correspondence • 1960: (1)

Krieger, Robert E. Correspondence • 1963: (2)

Krieger, William Botanical Album

Krivanek, Jerome O. U. of South Florida, Tampa Botanical Album

Correspondence $\bullet$ 1968: (1) $\bullet$ 1973: (1) $\bullet$ 1974: (2)

Kruckeberg, Arthur Rice (1920- ) American taxonomist; U. of Washington, Seattle Correspondence $\bullet$ 1956: (1) $\bullet$ 1968: (1)

Krukoff, Boris Alexander (1898-1983) Russian-born American botanical explorer; New York Botanical Garden Botanical Album

Correspondence $\bullet$ 1942: (4) • 1943: (13) • 1944: (11)

Kruschef, S. Correspondence $\bullet$ 1971: (1)

Kruska, Dennis Correspondence $\bullet$ 1982: (5)

Kruta, Vladislav O. U. of Brne, Brno, Czechoslavakia, Dept. of Physiology

Correspondence $\bullet 1971$ :
Küchler, August Wilhelm (1907-) German-born American phytogeographer, plant ecologist; U. of Kansas, Lawrence, Dept. of Geography

Botanical Album

Correspondence • 1963: (1) 1984: (1)

Kuck, Loraine E. Botanical Album

Kucyniak, James (1919-1962) Canadian bryologist Correspondence • 1956: (1)

Kuhn, Adam (174l-1817) Bioscript

Kühnel, Josef, (1886-) Biographer of Thaddeus Haenke Correspondence • 1955: (1)

Kuijt, Job (1930-) Dutch-born Canadian plant anatomist: U. of Lethbridge, Alberta, Canada, Dept. of Bio. Sciences Botanical Album

Correspondence $\bullet$ 1987: (1) • 1991: (2)

Kumlien, $\mathbf{T}$. Botanical Album

Kunkel, Günther W. H. (1928-) German taxonomist, Canary Islands Biofile - Botanical Album

Kunth, Karl Sigismund (1788-1850) German botanist Bioscript

Kuntze, Carl Ernest Otto (1843-1907) German taxonomist Biofile $\bullet$ Bioscript

Kunze, Gustav (1793-1851) German physician, mycologist, pteridologist

Bioscript

Kupsch, W. O. U. of Saskatchewan, Saskatoon Correspondence • 1980: (2)

Küster, Ernst (1874-1953) German botanist, plant pathologist Botanical Album

Kützing, Friedrich Traugott (1807-1893) German pharmacist Biofile • Bioscript • Botanical Album

Kylin, Johan Harald (1879-1949) Swedish phycologist Biofile

\section{$\boldsymbol{L}$}

L'Héritier de Brutelle, Charles Louis (1746-1800) Botanist

Bioscript

L'Herminier, Felix Louis (1779-1833) Botanist Bioscript

La Gasca, Mariano (1776-1839) Spanish botanist Biofile

Laage, R. J. C. V. ter Correspondence $\bullet$ 1965: (1)

Laan, Cyril R. C. New Orleans, LA Correspondence 1981 : (1)

LaBarre, Weston Duke U., Dept. of Sociology and Anthropology Correspondence • 1959: (2)

Labillardière, Jacques Julien Houtou (1755-1834) French physician, botanist, plant explorer Biofile • Bioscript 
Laborde, Ellis P. Board of Commissioners of City Park, New Orleans, LA, General Manager Correspondence - 1953: (1) Letter to Laborde is with Bernice Schubert letters

Lacaita, Charles Carmichel (1853-1933) British amateur botanist Bioscript $\bullet$ Botanical Album

Lacépède, Bernard Germain Étiennede la Ville (Conte) de (1756-1825) Naturalist Bioscript

Lacey, Trammel C., Mrs. Correspondence $\bullet$ 1961: (1)

Lacher, T. V. Correspondence $\bullet$ 1962: (2)

Lack, David Edward Gray Institute of Ornithology, Oxford Correspondence • 1956: (3)

Lacordaire, Jean-Théodore (1801-1870) Biofile

Lada-Mocarski, Laura K. Correspondence $\bullet$ 1971:

Lada-Mocarski, Valeria (1899-1971) Yale U., New Haven, Library, Advisor on Russian Book Collections

Biofile • Botanical Album

Correspondence • 1967: (2) • 1970: (2) 197

Lademann, Otto Botanical Album

Laden, Milton American Rock Garden Society, Delaware Valley Chapter

Correspondence • 1985: (3) • 1986: (1) • 1991: (1)

Laetsch, Watson

Botanical Album

Laevlor, Kay Carmel Bookshop, CA Correspondence $\bullet$ 1965: (1)

Lafayette Natural Fistory Museum Allison H. Pena, Curator of Exhibits

Correspondence • 1984: (3) • 1985: (1)

Lafier, Hugh U. of North Carolina, Chapel Hill, Dept. of History

Correspondence $\bullet$ 1967: (2)

Lafiresnaye, Frederic de (1783-1861) Bioscript

LaGardie, Lady Eve Contess de Bioscript

LaGasca y Segura, Mariano Bioscript

Laglaige, Leon Bioscript

Lahshmama U. of Toronto, Dept. of Botany Correspondence $\bullet$ 1965: (1)

Laishley, Richard (1815-1897) American naturalist, artist Biofile

Lakanal, Joseph (1762-1845) Bioscript

Lakes, Arthur (18441917) English-born American paleontologist, mining engineer Bioscript

Lakey, Joy Correspondence $\bullet$ 1980: (1) Laking, Leshe Royal Botanical Gardens, Hamilton, Canada,
Director Correspondence $\bullet 1971$ : (1) • 1978: (4)
Lam, Herman Johannes (1892-) Taxonomist; Rijksherbarium, Leiden, Director

Botanical Album

Correspondence • 1956: (1) • 1962: (1)

Lamar, Marta Television talk show host; New Orleans, LA Bioscript $\bullet$ Botanical Album Correspondence • 1953: (1) • 1954: (2) • 1955: (2) • 1956: (2) • 1957: (7) • 1959: (1) • 1960: (1) • 1962: (3) • 1963: (7) • 1965: (5) • 1966: (2) • 1968: (1) • 1969: (2) • 1970: (2) Correspondence between Steven W. Gahagan and Marta Lamar • 1972: (1) • 1975: (1) • 1976: (6) • 1977: (2)

Lamarck, Jean Baptiste Antoine Pierre de Monnet de (1744-1829) French biologist, author Biofile $\bullet$ Bioscript $\bullet$ Botanical Album

Lamare-Piquot, Christophe Augustin Biofile

Lamas, Gerardo Museo de Historia Natural, Universidad Nacional Mayor de San Marcos, Lima, Peru, Director Correspondence • 1992: (2)

Lamb, Chester Converse Bioscript

Lamb, Edgar Botanical Album

Lamb, Franklin Bruce (1913-) Forester Correspondence $\bullet$ 1956: (5)

Lamb, Ivan Mackenvie (1911-1990) Mycologist, phycologist; National Museum of Canada, Ottawa, National Herbarium; Harvard U., Farlow Herbarium Bioscript Correspondence $\bullet 1952$ (1) $\bullet$ 1955: (3)

Lambert, Alymer Bourke (1761-1842) British botanist, taxonomist Biofile $\bullet$ Bioscript

Lambeth Palace Librarian; London, England Correspondence • 1966: (2) Banister, John, 1650-1692

Lamboll, Thomas Bioscript

Lamborn, Robert Henry (1835-1895) American metallurgist

Bioscript

Lammers, Thomas G. Ohio State U.; Field Museum of Natural History

Correspondence • 1983: (1) • 1991: (2)

Lamond, Jennifer M. (1936-) Royal Botanic Garden, Edinburgh

Correspondence 1972: (1) • 1975: (2)

Lamontagne, Roland Historian; U. of Montreal, Montreal, Canada

Correspondence • 1962: (4) • 1964: (1)

LaMotta, Jacob de Bioscript

Lamoureux, Charles H. (1933-) U. of Hawaii Correspondence • 1967: (1) • 1974: (1) • 1976: (1)

Lamouroux, Jean Vincent Félix (1779-1825) Naturalist, botanist

Bioscript

$\underset{\text { Bioscript }}{\text { Lamson, }}$, Prof. 
Lamson-Scribner, Frank (1851-1938) American agrostologist Bioscript

Lan, Richard M. Martayan Lan Inc., Rare Books and Maps Correspondence $\bullet$ 1985: (1)

Lancaster County Historical Society James B. McMullin, Program Chairman Correspondence - 1984: (4) Barton, Benjamin Smith, 1766-1815

Landa, Alfons B. Law offices of Davies, et al. Correspondence • 1945: (1)

Landacre, Paul Hambleton (1893-1963) Artist Bioscript Correspondence $\bullet$ 1983: (1)

Landreth, David (1802-1880) (the Younger) Bioscript

Landreth, David, Sr. (1752-1836) Bioscript

Landrieu, Moon Correspondence 1974 : (1) Letter written by Nesta to Mayor of New Orleans

Landrum, Leslie Roger (1946- ) Arizona State U., Tempe, Dept. of Botany/Microbiology Correspondence $\bullet$ 1988: (2)

Landry, Garrie Paul (1951-) Louisiana State U., Baton Rouge, Dept. Botany

Bioscript

Correspondence • 1976: (2) Letter to Roland Treubig • 1977: (2) • 1985: (1)

Landry, Stuart Louisiana State U., New Orleans Correspondence - 1961: (2)

Lane, Alfred C. Correspondence $\bullet$ 1935: (1)

Lane, Carolina H. Atlanta, GA Correspondence 1 1978: (2)

Lane, Charles J. Correspondence $\bullet$ 1958: (1)

Lane, Charles J. Company New York, NY Correspondence - 1966: (1)

Lane, Irwin E. (1926-) Harvard U., Biological Laboratories, Cambridge, MA Correspondence $\bullet$ 1951: (2)

Lane, Meredith A. (1951-) U. of Colorado, Boulder, EPO Biology; ASPT secretary Correspondence $\bullet$ 1987: (2)

Lane, Mills, IV Bee Hive Press, Savannah, GA Botanical Album Correspondence $\bullet$ 1972: (10) 1 1973: (19) $\bullet$ 1974: (8) $\bullet$ 1975: (4) Letter to H. K. Swann and Eric Lundberg 1976: (2) • 1977: (6) • 1978: (9) • 1979: (12) Letter from C. Freeman Keith, Stinehour Press $\bullet$ 1980: (3)

Lane, Richard D. Correspondence • 1970: (1)

Lang, Frank Alexander (1937-) Taxonomist; Southern Oregon State College, Ashland Correspondence $\bullet$ 1992: (0) Filed with Eric William Groves

Lang, L. M. Barnes and Noble Correspondence $\bullet$ 1940: (2)

Langbelle, Thomas Bioscript
Lange, Axel Edward (1871-1941) Horticulturist, botanical historian

Biofile

Lange, Carla (Charlotte) Missouri Botanical Garden Library

Correspondence $\bullet$ 1970: (3) $\bullet$ 1971: (4) $\bullet$ 1973: (2) $\bullet$ 1975: (2) - 1976: (7) Rocky Mountain Nanuralists • 1977: (3) • 1978: (1) • 1980: (1) • 1982: (2) Van Schaack, George Booth, 1903-1983 • 1984: (1)

Lange, Erwin F. Portland State Extentsion Center, OR Bioscript Correspondence $\bullet$ 1952: (2) $\bullet$ 1953: (2)

Lange, J. Bioscript

Lange, Johan Martin Christian (1818-1898) Biofile • Bioscript

Langford, Nathaniel Pitt (1832-1911) American conservationist, banker Bioscript

Langille, H. D. Bioscript

Langlassé, Eugène (c. 18641900) French botanical and horticultural collector Biofile

Langlois, Auguste Barthélemy (1832-1900) French theologian, plant collector Biofile $\bullet$ Bioscript

Langman, Ida Kaplan (1904) Academy of Natural Sciences of Philadelphia Bioscript $\bullet$ Botanical Album Correspondence - 1947: (2) • 1949: (3) Rocky Mountain Naturalists • 1950: (5) • 1951: (5) • 1952: (11) Pennell, Francis Whittier, 1886-1952 • 1953: (12) Pennell, Francis Whittier, 1886-1952 • 1954: (13) • 1955: (4) Merrill, Elmer Drew, 1876-1956 • 1957: (5) • 1958: (8) • 1959: (8) 1960: (7) Academy of Natural Sciences of Philadelphia; Harper, Francis, 1886-1972 - 1961: (14) Banister, John, 1650-1692; National Science Foundation • 1962: (13) • 1963: (25) • 1964: (13) Missouri Botanical Garden • 1965: (12) • 1966: (13) • 1967: (7) • 1968: (5) - 1969: (5) • 1970: (6) • 1971: (13) - 1972: (3) • 1973: (11) 1974: (20) Letter from Ralph S. Palmer $\bullet$ 1975: (12) - 1976: (14) • 1977: (10) • 1978: (6) • 1979: (10) Book collectors - 1980: (13) - 1981: (6) - 1982: (12) - 1983: $(2) \bullet$ 1984: (5) • 1986: (3) • 1987: (7) • 1988: (2) $\bullet 1989$ : (3)

Langman, Oscar Wife is Ida Langman Bioscript

Langsdorff, Georg Heinrich (1774-1852) Naturalist, physician Bioscript

Langstroth, Lorenzo Lorraine (1810-1895) American bee culturist Biofile

Laning, E. Bioscript

Lanjouw, Joseph (1902-1984) Botanical Museum and Herbarium, Utrecht, Netherlands

Correspondence - 1937: (2) Chronica Botanica - 1938: (1) $\bullet$ 1947: (1) • 1948: (2) • 1951: (1) - 1953: (1) $\bullet 1954:$ (2) 1955: (1) - 1963: (2) Verdoorn, Frans Antonie, 1906-1984 


\section{Guide to the Ewan Papers}

Lansing, Dorothy I. Physician of Obstetries and Gynecology; husband is Robert Penman

Bioscript • Botanical Album (Filed under Penman as well as Lansing)

Correspondence • 1967: (10) • 1968: (18) • 1969: (8) • 1970: (20) Missouri Botanical Garden $\bullet$ 1971: (8) • 1972:

(6) • 1973: (1) • 1974: (3) • 1975: (1) • 1977: (1) • 1978:

(3) $\bullet$ 1979: (3) $\bullet$ 1980: (1) $\bullet$ 1982: (5) $\bullet 1983:$ (1) $\bullet 1984:$

(1) $\bullet$ 1985: (1) • 1986: (1) • 1991: (3) Bartram, William, 1739-1823 • 1992: (1)

Lanszweert, L. Bioscript

Lantz, Walter D. Bookseller Correspondence • 1941: (2)

LaPérouse, Jean-Francois de Galaup comte de (1741-1788) French cartographer Biofile $\bullet$ Bioscript

Lapeyrouse, Philippe Picot de (1744-1848) Bioscript

Lapham, Increase Allen (1811-1875) Bioscript • Botanical Album

LaPidus, Jules B. Ohio State U., Columbus Correspondence • 1980: (1)

LaPylale, Bachelot Bioscript

Large, Alix Service National Agricole Haiti, Horticulturist Correspondence • 1941: (1) • 1942: (1)

Largen, Malcolm J. Merseyside Museum, Liverpool Botanical Album

Correspondence • 1991: (2)

Larisey, Mary Maxine Medical College of the State of South Carolina, Charleston, School of Pharmacy, Assoc. Professor

Bioscript • Botanical Album Correspondence • 1953: (1)

Larison, Lorraine L. U. of Massachusetts, Boston Correspondence $\bullet$ 1973: (3)

Larner, John W. Penn State U., Altoona, Asst. Prof. History; Ed., The Papers of Joseph Trimble Rothrock Correspondence $\bullet$ 1989: (6)

LaRocque, Aurele Ohio State U., Columbus Correspondence • 1964: (3) • 1969: (1)

Larsen, Esther Louise (1901-) American botanical historian, taxonomist; husband is Kenneth Davis Doak Bioscript

Correspondence • 1948: (1) • 1949: (2) • 1953: (1)

Larson, Edward

Correspondence • 1988: (1)

Lasègue, Antoine (1793-1873) Bioscript

Lashma, __, Miss Correspondence • 1963: (1)

Laska, Toni Correspondence $\bullet 1974:$ (1)

Laskowski, Chester Walter (1941-) U. of Michigan Herbarium, Ann Arbor Correspondence • 1964: (2)

Lasser, Tobias (1911-) Taxonomist, phytogeographer; Ministerio De Agricultura Y Cria, Institute Botanico No. B-3, Caracas, Venezuela Correspondence • 1951: (1) - 1952: (4)

LaTaste, $\mathbf{L}$. Bioscript
Latham, John Bioscript

Lathrop, Barbour (1846-1927) Amateur botanist, patron Bioscript

Latour, Maurice-Quentin de Botanical Album

Latourette, Mark Antoine Claret (1729-1793) Bioscript

Latreille, Pierre Andre Bioscript

Latrobe, Benjamin Henry (1764-1820) English-American architect, navigator Biofile $\bullet$ Bioscript $\bullet$ Botanical Album

Latrobe, Benjamin Henry, The Papers of Maryland Historical Society, Baltimore; Lee W. Formwalt, Research Assistant

Correspondence • 1976: (1) • 1981: (1) Species

Latrobe, Peter (1795-1863) Bioscript

Latvijas U. Botanical Album

Laubenfels, David J. de Taxonomist, geographer Biofile

Lauener, Lucien Andre Royal Botanic Garden, Edinburgh Botanical Album

Correspondence • 1955: (1) • 1956: (1) • 1970: (3) Rocky Mountain Naturalists • 1977: (1) • 1984: (1) • 1988: (1) Lauerhass, Ludwig, Jr. U. of California, Los Angeles, Latin American Center Correspondence $\bullet$ 1990: (1)

Laurens, Henry Bioscript

Laurent, Linda Correspondence $\bullet$ 1970: (1)

Lavallee, Andre (1936-) Bioscript

Lavelle, Alice M. U. of Pennsylvania, Philadelphia, Dept. of English Correspondence $\bullet 1971:$ (1)

LaViolette, Forrest Gresham, OR Correspondence $\bullet$ 1981: (7)

Law, Barbara W. Natural History Museum, Stanford, Secretary Correspondence - 1949: (1)

Law, Elizabeth A. Husband is John M. Law; niece of Andrew Allison (1879-1960) Correspondence • 1965: (4)

Law, , Mrs. Correspondence • 1929-1933: (1)

Law, J. S. Bioscript

Law, John Eugene (1877-1931) American bird collector, banker, ornithologist Biofile

Lawrence, Francis L. Tulane U., New Orleans Correspondence • 1982: (3) • 1983: (1) • 1984: (1) • 1986: (1)

Lawrence, George Hill Mathewson (1910-1978) American botanist, botanical historian; Hunt Botanical Library Biofile • Bioscript $\bullet$ Botanical Album Correspondence • 1938: (3) - 1939: (1) Delphinium Species • 1948: (3) • 1949: (2) • 1952: (1) • 1955: (2) 1956: (4) - 1958: (13) Henry E. Huntington Library and 
Lawrence, George Hill Mathewson (1910-1978) (continued)

Art Gallery • 1959: (7) • 1961: (2) Banister, John, 1650-1692; Banks, Joseph, Sir, 1743-1800 - 1962: (9) Banister, John, 1650-1692 • 1963: (28) Harper, Roland McMillan, 1878-1966 • 1964: (15) Missouri Botanical Garden; Van Schaack, George Booth, 1903-1983 • 1965: (32) Harper, Roland McMillan, 1878-1966 • 1966: (14) • 1967: (15) Bartram, William, 1739-1823 • 1968: (21) Biology-Instruction and study $\bullet$ 1969: (14) • 1970: (13) Banister, John, 1650-1692 • 1973: (1) • 1974: (1) • 1976: (4) $\bullet$ 1977: (2)

Lawrence, George N. Bioscript

Lawrence, Jenny Natural History, Editor Correspondence • 1992: (3)

Lawrence, John T. William Morrow \& Co. Correspondence • 1959: (1)

Lawrence, Matthew U. of North Carolina, Chapel Hill Correspondence $\bullet$ 1991: (1)

Lawrence, William (1783-) English physician Biofile

Lawson, George (1827-1895) Bioscript $\bullet$ Biofile

Lawson, John Bioscript

Lawson, Marmaduke Alexander (1840-1896) Bioscript

Lawson, Peter Scottish seedsman, nurseryman Biofile

Lawson, Wilfrid J. E. M. Lawson \& Co., Rare Books \& Manuscripts

Correspondence $\bullet$ 1968: (1) Book collectors $\bullet$ 1984: (1) Book collectors

Lawton, Bioscript

Lawton, Marcy F. U. of Alabama, Huntsville, Dept. of Biology

Correspondence • 1990: (1)

Laxalt, Robert U. of Nevada Press Correspondence $\bullet$ 1965: (0) Filed under Olga Augusta Wuertz Reifschneider

Laxmann, Erik Gustavovich (1737-1796) Bioscript

Lay, George Tradescant Bioscript

Lay, Koko Missouri Botanical Garden Botanical Album Correspondence • 1949: (6) • 1950: (5) Delphinium

Le Doux, M. Correspondence 1963 : (1)

Le Faivre, Carol N. American Philosophical Society Correspondence $\bullet$ 1987: (1)

Lea and Febiger Correspondence $\bullet$ 1940: (1)

Lea, Isaac Bioscript

Lea, John S. U. S. National Museum, Editor Correspondence • 1960: (1) Vismia

Lea, T. G. Bioscript

Lea, Thomas G. Charophyte collector Biofile
Leach, David Goheen Botanical Album

Leach, Lilla

Bioscript Leaman, William G., Jr. Bioscript

Leandri, Jacques Désiré (1903-1982) Museum National d'Histoire Naturelle, Paris

Correspondence • 1954: (1) • 1967: (1)

Lear, Edward (1872-1888) Biofile $\bullet$ Bioscript $\bullet$ Botanical Album

Learn, Clarence Delbert (1875-1935) American mycologist Bioscript

Leavenworth, Melines Conkling (1796-1862) Biofile • Bioscript

LeBeau, Francois (f. 1775) Physician, botanist Biofile

LeBlanc, Katherine Tulane U., New Orleans, Biology secretary

Correspondence • 1972: (3) • 1973: (1) • 1975: (2)

Lebreton, Adrien (1662-1736) Bioscript

Lebron-Luteyn, Maria New York Botanical Garden Correspondence • 1984: (4) Rocky Mountain Naturalists

Lechler, Willibald Bioscript

Leconte, John (1739-1822) Bioscript

Leconte, John Eatton (1784-1860) American botanist, botanical artist, topographical engineer Biofile • Bioscript

Le Conte, John Laurence (1825-1883) American entomologist Bioscript

Le Conte, Joseph (1823-1901) American geologist; ornithologist Biofile $\bullet$ Bioscript $\bullet$ Botanical Album

Le Counte, Louis (1782-1838) Bioscript

Lecoyer, M. J. Bioscript

Ledebour, Bioscript

Ledin, R. Bruce U. of Florida, Sub-Tropical Experiment Station, Homestead, FL Correspondence • 1951: (1) • 1952: (13) • 1953: (3)

Ledlie, Patricia Natural History Books Correspondence • 1991: (3) Book collectors

Lee, Arthur Bioscript

Lee, Cayee $\mathbf{R}$. Correspondence $\bullet$ 1967: (1)

Lee, Clermont H. Bartram Trail Bicentennial Committee Botanical Album Correspondence • 1972: (1) • 1973: (5)

Lee, E. J. State Forester, CO Correspondence - 1949: (1)

Lee, Ed (1914-1979) American amateur botanist Biofile

Lee, James Bioscript

Lee, Sarah Wallis Bowditch Botanical Album 
Leech, James H. U. of Texas, Austin Correspondence • 1967: (1) • 1968: (0) Filed under University of Texas

Leeds, Arthur Newlin (1870-1939) American botanist, pteridologist

Biofile • Botanical Album

Leeper, Pat Louisiana State Library Correspondence • 1976: (2)

Lees, Cariton B. (1924) ) Massachusetts Horticultural Society

Correspondence • 1965: (4) • 1967: (5) • 1968: (1)

Bartram, William, 1739-1823 • 1970: (2) • 1972: (5) • 1973: (1) • 1975: (7) • 1976: (1) • 1977: (2) • 1987: (1)

Lees, J. Cardwell Bioscript

Leeuwenhoek, Anton van (1632-1723) Zoologist Biofile $\bullet$ Bioscript $\bullet$ Botanical Album

LeFaivre, Carole N. American Philosophical Society, Assistant to the Editor

Correspondence • 1980: (1) • 1986: (1)

LeFanu, W. R. Correspondence $\bullet$ 1982: (2)

Lefebvre, J. J. Archivist, Old Court House, Montreal, Quebec Correspondence • 1951: (1)

Lefevre, Ruth U. of Oregon, Eugene, Dept. of Geography Correspondence $\bullet$ 1980: (3) Letter from Ray Fosberg

Lefler, Hugh T. U. of North Carolina, Chapel Hill, NC Correspondence $\bullet$ 1966: (2)

Lefroy, John Henry (1817-1890) Bioscript

Leggett, William Henry (1816-1882) Bioscript

Lehman, Arthur Bioscript

Lehmann, Friedrich Carl (1850-1903) Bioscript

Lehmann, Johann George Christian (1792-1860) Bioscript

Leiberg, John Bernhard (1853-1913) Bioscript $\bullet$ Botanical Album

Leichhardt, Friedrich Wilhelm Ludwig (1813-1848) Prussian ichthyologist Biofile

Leichtlin, Charles Bioscript

Leichtlin, Maximilian (1831-1910) Horticulturist, plant collector Bioscript

Leidendeker, Anne Los Angeles Public Library Correspondence • 1936: (1)

Leidy, Joseph (1823-1891) American naturalist Biofile $\bullet$ Botanical Album

Leighton, Ann (1902-1985) See also Isadore Leighton Luce Smith Bioscript

Leighton, M. M. Illinois Geological Survey Correspondence - 1950: (1)

Leighton, William Allport (1805-1889) Bioscript
Leinbach, Philip E. Tulane U., New Orleans, Howard Tilton Memorial Library

Correspondence • 1982: (1) • 1983: (3) Barton, Benjamin Smith, 1766-1815 • 1984: (6) • 1985: (2) • 1986: (3) • 1988: (2)

Leisinger, Albert H. National Archives and Records Service Correspondence $\bullet$ 1954: (2)

Leisman, Gilbert Arthur (1924) Paleobotanist; Kansas State Teachers College, Emporia, Associate Professor of Biology Correspondence $\bullet$ 1960: (1)

Leisure, Harold Bookseller; New Orleans Correspondence • 1956: (1) • 1957: (1) • 1958: (1) • 1964: (3) • 1965: (2) • 1966: (1) • 1969: (1) • 1970: (1) Royal Botanic Gardens, Kew

Leith-Ross, Prudence Wimbledon, England Correspondence • 1979: (4)

Leitner, Edward Frederick (1812-1838) Botanist Biofile $\bullet$ Bioscript

Lekisch, Barbara Librarian; Sierra Club Correspondence $\bullet$ 1986: (1)

Lellinger, David Bruce (1937- ) Pteridologist, taxonomist; Smithsonian Institution, Assoc. Curator Correspondence $\bullet$ 1965: (0) Letter filed under Conrad Vernon Morton - 1968: (3) American Fern Society • 1970: (1) American Fern Society • 1972: (5) American Fern Society; letter to Gilbert S. Daniels • 1973: (6) • 1974: (1) - 1975: (5)

Lelong, Michel Georges (1932-) Taxonomist, agrostrologist; Northwestern State College, Natchitoches, LA

Correspondence $\bullet$ 1960: (1) • 1966: (1) • 1969: (2)

LeMaire, $\mathrm{C}$. Bioscript

Lemaire, Robert Joseph (1921- ) Biologist; United States, Dept. of the Interior, Fish and Wildlife Service Correspondence $\bullet 1960:(1) \bullet 1961:(8)$

Lemann, Correspondence • 1973: (1)

Lemann, Charles Morgan Bioscript

Lemann, Thomas B. Monroe \& Lemann, Attorneys and Counsellors at Law

Correspondence $\bullet$ 1970: (3)

Lembert, John Baptist (1840-1896) Bioscript

Lemke, Paul Botanical Album Correspondence $\bullet 1964$ : (2)

Lemman, Bernard Tulane U., School of Architecture, Office of the Dean Correspondence $\bullet$ 1969: (1)

Lemmon, John Gill (1832-1908) Plant collector Biofile $\bullet$ Bioscript $\bullet$ Botanical Album

Lemoine, Steven Attorney, New Orleans, LA Correspondence 1977 : (1)

Lemon Hill-Philadelphia Bioscript

Lemonnier, Louis-Guillaume (1717-1799) French botanist, physician Biofile 
LeMoyne, Jacques Bioscript

Lempriere, Thomas James (1796-1852) Tasmanian naturalist Biofile

Lems, Kornelius (1931-1968) Botanical Album Correspondence $\bullet$ 1961: (2)

Leningrad Botanical Album

Lennon, Florence Becker Correspondence $\bullet$ 1945: (1)

Lenoir, Timothy U. of Arizona, Tucson Correspondence • 1983: (1) Barton, Benjamin Smith, 1766-1815

LeNormand, Rene Bioscript

Lenz, Lee Wayne (1915-) Rancho Santa Ana Botanic Garden, Claremont, CA

Bioscript

Correspondence $\bullet$ 1949: (3) $\bullet$ 1968: (1) $\bullet 1977:$ 1981: (5) • 1985: (1) • 1986: (3)

León, Blanca Universidad Nacional, Museo de Historia Natural, Lima, Peru Correspondence $\bullet$ 1987: (1)

Léon, Hermano (Sauget-Barbier, Joseph Sylvestre) Botanical Album

Leon, Solomon de Bioscript

Leonard, Emery Clarence (1892-1968) Taxonomist; Smithsonian Institution, Dept. of Botany, Assoc. Curator Bioscript $\bullet$ Botanical Album Correspondence • 1950: (1) $\bullet$ 1951: (1) $\bullet$ 1952: (5) - 1953: (1)

Leonard, M. D. Correspondence $\bullet$ 1947: (2)

Leopold, Aldo (1886-1948) American environmentalist, author Biofile

Leopold, Wilma W. Many, LA; descendant of John Bartram Correspondence $\bullet$ 1979: (2) $\bullet$ 1980: (2)

Lepechin, Bioscript

Lequin, Frank Rijksuniversiteit te Leiden Bioscript Correspondence $\bullet$ 1987: (1) $\bullet$ 1988: (3)

Leroy, André (1801-1875) Bioscript

Leroy, Jean-Francois Pierre (1915-) Museum National D' Histoire Naturelle, Paris, Director Correspondence $\bullet$ 1956: (13)

Leschenault de la Tour, Jean Baptise Louis Théodore (1773-1826) Natural historian, plant explorer Bioscript

Leslie, Charles Robert Bioscript

Lesquereux, Charles Leo (1806-1889) Swiss paleobotanist Biofile • Bioscript

Lester, Malcolm Davidson College, Davidson, NC Correspondence • 1978: (1) • 1979: (2)
Lester, Oliver C. U. of Colorado, Dean

Correspondence 1 1940: (2) • 1941: (3) U. of Colorado, Boulder. Dept. of Biology $\bullet$ 1942: (5)

Lesueur, Charles Alexandre (1778-1846) French artist, naturalist in America Biofile • Bioscript

LeSueur, Harde Botanical Album

Letcher, Beverly (1864-1905) Bioscript

Letterman, George Washington (1841-1913) American teacher, plant collector

Bioscript $\bullet$ Botanical Album

Lettsom, John Coakley (1744-1815) English physician, botanist

Biofile $\bullet$ Bioscript

Lettunich, Mateo Pacific Tropical Botanical Garden, New York, NY, President

Correspondence $\bullet$ 1977: (2)

Leurs, Gloria Stichting Surinaams Museum Correspondence $\bullet$ 1985: (4)

Léveillé, Augustin Abel Hector Botanical Album

Léveillé, J. H.

Bioscript

LeVeque, Norma (1891-1967) U. of Colorado, Boulder, Dept. of Biology Biofile $\bullet$ Bioscript Correspondence $\bullet$ 1939: (1)

Lever, John Ashton (1729-1788) English naturalist Biofile • Bioscript

Levette, G. M. Physician; Indianapolis, IN Bioscript

Levin, Ashton Bioscript

Levin, Donald A. (1939-) Taxonomist, biosystematist; U. of Illinois, Chicago Correspondence $\bullet$ 1964: (3)

Levin, Louis National Science Foundation, Regulatory Botany, Program Director Correspondence • 1953: (2) • 1954: (1)

Levine, Abby Archivist; Hunt Botanical Library Correspondence $\bullet$ 1972: (2)

Levine, Emanuel Newark College of Engineering, NJ, Asst. in the History of Science Correspondence $\bullet$ 1950: (1)

Levine, Michael Howard (1886-1952) American botanist Biofile

Levine, Michele Tri Beta Correspondence $\bullet$ 1967: (1)

Levine, Neil Marshall Miami, FL, teacher; Tulane U., student Correspondence • 1969: (4)

Levine, Robert M. U. of Miami, South Eastern Latin Americanist Correspondence $\bullet$ 1985: (2)

Lewis and Clark Biofile

Lewis, C. Bernard The Institute of Jamaica, Kingston, Jamaica, B.W.I., Director Correspondence $\bullet$ 1950: (5) $\bullet$ 1951: (1) $\bullet$ 1953: (5) $\bullet$ 1954: (1) • 1957: (2) • 1958: (1) • 1966: (1) 
Lewis, Clarence McK. New York, NY Correspondence • 1950 (3) • 1951: (11) American Fern Society

Lewis, Frank Harlan (1919) Taxonomist Bioscript

Correspondence • 1951: (6)

Lewis, John

Botanical Album

Lewis, Meriwether (1774-1809) American explorer, naturalist

Biofile $\bullet$ Bioscript $\bullet$ Botanical Album

Lewis, Walter Hepworth (1930-) American ethnobotanist Biofile $\bullet$ Bioscript $\bullet$ Botanical Album

Correspondence • 1957: (3) • 1959: (1) • 1960: (2) • 1961: (6) • 1965: (3) • 1967: (1) • 1968: (1) Missouri Botanical Garden • 1969: (2) Missouri Botanical Garden • 1972: (1) • 1990: (1) • 1991: (0) Filed under Carlton B. Wood • 1992: (1)

Lewitus, $\mathbf{v}$. Correspondence $\bullet$ 1937: (2)

Leyden Botanical Garden Botanical Album

Leyendecker, Philip J. Iowa State College, Ames Correspondence • 1940: (1) • 1946: (2) • 1959: (1)

Lezermes, Biofile (I. 1788) French arborist

Lhotzky, John Bioscript

Lhwyd, Edward (1660-1709) English botanist, paleontologist Biofile

Li, Hui-Lin (1911-) Cytotaxonomist; U. of Pennsylvania, Morris Arboretum Bioscript $\bullet$ Botanical Album Correspondence • 1953: (4) • 1963: (2) • 1966: (1) • 1974: (2) • 1975: (4)

Libbey, William (1855-1927) American geologist, meteorologist Bioscript

The Library Company of Philadelphia Andrea J. Tucher, Natural History Bibliographer, Philadelphia, PA Correspondence $\bullet$ 1978: (1)

Library of Congress James H. Hutson, Chief Correspondence $\bullet$ 1983: (2) $\bullet$ 1990: (2) Correspondence between Martha Riley, Missouri Botanical Garden and Ralph Ehrenberg, Library of Congress

Libros Latinos George F. Elmendorf, bookseller; books of Latin America, Santa Monica CA \& Redlands, CA Correspondence $\bullet$ 1979: (5) Book collectors

Lichtenstein, $\mathbf{H}$. Bioscript

Liebmann, Frederick Michael (1813-1856) Bioscript

Lieftinck, G. I. Academy Library, Leiden, Netherlands Correspondence $\bullet$ 1956: (1)

Liesner, Ronald L. (1944-) Botanist; Missouri Botanical Garden Correspondence • 1988: (1)

Lieux, Meredith Hoag (1939-) Bioscript

Lievens, Alan Botanist Correspondence $\bullet$ 1992: (1) • 1993: (1)
Lifschiz, Alexander Correspondence • 1962: (3)

Lifschiz, Leland Correspondence • 1963: (3) - 1964: (1)

Liggett, William E. University City, MO Correspondence $\bullet$ 1946: (3) American Fern Society $\bullet$ 1951: (2)

Light, J. E., Mrs.

Bioscript

Lightfoot, John (1735-1788) Bioscript

Lightner, Amelia B. Upperville, VA; husband is Gordon H. Lightner Correspondence $\bullet$ 1953: (2)

Lighty, Richard William (1933-) Plant geneticist, horticulturist; U. of Delaware, Newark, Longwood Program in Ornamental Horticulture, Coordinator; Mt. Cuba Botanical Garden

Bioscript

Corresondence • 1976: (1) • 1984: (3) • 1988: (2) • 1989:

(2) Book collectors $\bullet$ 1991: (1) Barton, Benjamin Smith, 1766-1815

Ligon, James Stokley (1879-) American ornithologist Correspondence $\bullet$ 1963: (2)

Ligon, Richard Bioscript

Liliencrona, W. v.(on?) Bioscript

Lill, Althea U. of Colorado, Librarian Correspondence • 1940: (1)

Lillie, Frank Rattray (1870-1947) Zoologist Biofile

Lillienthal, Bioscript

Lillo, Miguel (1862-1931) Botanist Biofile

Limbaugh, Conrad (1925-1960) American scuba diver Biofile

Limbert, R. W. Bioscript

Limestone Press Correspondence $\bullet$ 1975: (1)

Lincoln, Almira Bioscript

Lincoln, F. C Correspondence $\bullet$ 1936: (1)

Lincoln Park Conservatory Correspondence $\bullet$ 1942: (1)

Lind, Jens Wilhelm August (1874-1939) Mycologist Biofile

Linda Hall Library Correspondence $\bullet$ 1982: (1)

Lindberg, Sextus Otto (1835-1889) Bryologist; Sweden, Finland Bioscript

Linden, Jean Jules (1817-1898) Botanist; Venezuela Biofile

Lindheimer, Ferdinand Jacob (1801-1879) German plant collector; U. S. A. Biofile $\bullet$ Bioscript $\bullet$ Botanical Album

Lindley, John (1799-1865) English botanist Biofile $\bullet$ Bioscript $\bullet$ Botanical Album 
Lindman, Carl Axel Magnus (1856-1928) Taxonomist, plant ecologist, botanist

Botanical Album

Lindroth, Sten Uppsala universitet, Institutionen for idé-och lärdomshistoria, Uppsala

Correspondence • 1977: (1) • 1978: (2)

Lindsay, George Edmund (1916-) Taxonomist, plant ecologist, plant collector

Bioscript

Correspondence • 1943: (5)

Lindsay, John English botanist Biofile

Lindsay, W. L.

Bioscript

Lindsey, Alton Anthony (1907-) Plant ecologist; Purdue U.

Correspondence $\bullet$ 1984: (2)

Lindsey, Arthur Ward (1894-1963) American zoologist Biofile

Ling, Yeou-Renn (1937- ) South China Institute of Botany, Guangzhou, China

Botanical Album

Correspondence $\bullet$ 1987: (2)

Lingelbach, William E. American Philosophical Society Correspondence • 1956: (1) • 1958: (1)

Link, Conrad Barnett (1912-) Horticulturist, botanist; American Horticultural Society

Correspondence $\bullet$ 1950: (2)

Link, George Konrad Karl (1888-) Plant pathologist Correspondence • 1974:

Link, Johann Heinrich Friedrich (1767-1851) Plant physiologist, taxonomist Bioscript

Linnaeus, Carl (1701-1778) Swedish naturalist Biofile $\bullet$ Bioscript $\bullet$ Botanical Album

Linne, Larry Paul Centerville, Ohio Correspondence • 1987: (1)

Linnean Society of London Botanical Album

Correspondence • 1939: (1) Species • 1976: (3) • 1977:(3) - 1978: (1) - 1983: (2) • 1985: (1) • 1993: (1)

Linscheid, Chester H. New Mexico College of Agriculture, Librarian

Correspondence $\bullet$ 1949: (3)

Linsdale, Jean M. U. of California, Berkeley Correspondence • 1936: (1) $\bullet$ 1938: (2)

Lintilhac, Philip M. (1940-) Plant morphologist; U. S. A.; U. of Vermont, Burlington, Dept. of Botany Correspondence 1989: (2) Biology-Instruction and study; Book collectors

Linton, Albert M. Academy of Natural Sciences of Philadelphia, President Correspondence • 1952: (2) Letters from Francis Harper

Lipman, Charles B.

Correspondence 1936: (1) U. of California, Berkeley. Dept. of Botany $\bullet$ 1937: (1)

Lipman, L. A. Montgomery Ward Correspondence - 1947: (1)

Lippman, Monroe Tulane U., Dept. of Theatre and Speech, Head

Correspondence $\bullet$ 1960: (2)

Lippmann, Denise

Correspondence • 1972: (1)
Lippold, Hans (1932-1980)

Biofile

Lipps, Lewis U. of Tennessee, Knoxville, Dept. of Botany Correspondence • 1956: (3) • 1957: (1)

Lipschitz, Sergej Julievitsch (1905-1983) Komarov Botanical Institute, Academy of Sciences of the USSR, Leningrad, Russia

Correspondence $\bullet$ 1971: (1)

Lipscomb, Bernard L. (Barney) Southern Methodist U. Bioscript $\bullet$ Botanical Album

Correspondence • 1978: (4) • 1980: (2) • 1981: (1) •

1982: (3) • 1989: (5) • 1990: (3) • 1991: (4) • 1992: (2)

Letter from George B. Ward $\bullet$ 1993: (2)

Liskow, Cullen R. Liskow \& Lewis, Attorneys at Law, Lake Charles, LA

Correspondence $\bullet 1960$ : (2)

Lisney, Arthur A.

Correspondence • 1963: (3) Banister, John, 1650-1692

List, George M. Colorado Agricultural College Correspondence • 1949: (1) Rocky Mountain Naturalists • 1950: (1)

Lister, Martin (1638-1712) Biofile $\bullet$ Bioscript

Litsinger, Elizabeth C. Enoch Pratt Free Library, Baltimore, MD Correspondence $\bullet$ 1951: (2)

Little, Elbert Luther, Jr. (1907-) Dendrologist, taxonomist; U.S.D.A. Forest Service

Botanical Album

Correspondence - 1939: (2) American Fern Society • 1945: (1) • 1947: (1) Vismia • 1951: (1) • 1953: (1) • 1954: (2) • 1959: (1) • 1960: (3) • 1963: (1) $\bullet 1966:$ (1) $\bullet$ 1971: (5) • 1972: (2) • 1975: (2) • 1978: (3) • 1979: (3) • 1980: (1) • 1981: (1) • 1985: (3) • 1989: (2)

Little, Henry Bioscript

Little, Ruby Husband is Elbert Little Correspondence $\bullet$ 1945: (1)

Little, Silas Botanical Album

Little, Stephen Bioscript

Littleboy, Sheila Oxford U. Correspondence $\bullet$ 1955: (1)

Littler, Diane S. Botanical Album

Littler, Mark Masterson (1939-) Algologist; Smithsonian Institution Correspondence $\bullet$ 1983: (2) $\bullet$ 1984: (1)

Liu, Shungho U. of Alberta, Dept. of Botany Correspondence - 1991: (1)

Livingston, Burton Edward (1875-1948) American botanist Biofile $\bullet$ Bioscript $\bullet$ Botanical Album

Livingston, Robert Blair (1911-) Plant ecologist Correspondence - 1939: (2) Species

Livingston-Little, D. E. Journal of the West, Los Angeles, CA Correspondence $\bullet$ 1966: (2) $\bullet$ 1967: (1)

Livsey, Rosemary Earnshaw Los Angeles Public Library Correspondence - 1936: (1)

Ulano, George Albert (1911-) Lichenologist

Bioscript $\bullet$ Botanical Album (continued on next page) 
Llano, George Albert (1911-) (continued) Correspondence • 1984: (3) • 1985: (1) • 1986: (1) • 1988: (3) $\bullet$ 1989: (13) Book collectors $\bullet$ 1990: (3) $\bullet$ 1991: (3) • 1992: (1) • 1993: (1)

Lloyd, C. E. Bioscript

Lloyd, Curtis Gates (1859-1926) American mycologist Biofile • Bioscript $\bullet$ Botanical Album

Lloyd, Daniel Lloyds of Kew, Booksellers Correspondence • 1986: (1)

Lloyd, Francis Ernest (1868-1947) Cytologist; Canada, U.S. A. Biofile • Botanical Album

Lloyd, John Uri (1849-1936) Pharmacist, chemist Biofile • Bioscript $\bullet$ Botanical Album

Lloyd Library Botanical Album

Lloyd, Robert Michael (1938-) Biologist, taxonomist; Ohio U., Dept. of Botany, Professor Correspondence • 1990: (2) Barton, Benjamin Smith, $1766-1815$

Lloyd, Sophia Webster (1820-1903) Mother of John Uri Lloyd and Curtis Gates Lloyd

- Biofile

Lloyd, William Bioscript

Lloyd, William (f. 1880-90) British-American natural history collector Biofile

Lloyd's Bank London, England; Sir Jeremy Morse, Chairman Correspondence $\bullet$ 1989: (1)

Lloyd-Binns, Blodwen U. of Malawi, Limbe, Malawi Botanical Album

Correspondence • 1964: (8) • 1965: (2) • 1966: (5) • 1967: (2) • 1968: (2) • 1969: (2)

Lobb, William (1809-1863) English plant collector Biofile • Bioscript

Lobel, Mathias (1538-1610) Bioscript

Lochee, Bioscript

Loeke, John (1792-1856) American physician, botanist, geologist

\section{Biofile • Bioscript}

Locke, Michael U. of Western Ontario, London, Canada, Dept. of Zoology Correspondence • 1971: (1)

Locke, _, Mrs. Correspondence • 1936: (1) Letter from Herbert L. Mason

Lockington, William Neale (1842?-1902) Bioscript

Lockmann, Ronald F. Bioscript • Botanical Album Correspondence $\bullet$ 1969: (2) • 1970: (1) • 1976: (2) 1978: (4) • 1979: (6) • 1980: (2) • 1981: (2) • 1990: (1) 1991: (4) • 1992: (2)

Loddiges, Conrad (1738-1826) Bioscript

Lodewick, Kenneth Correspondence • 1982: (1)
Lodwick, Larry Texas Parks and Wildlife Dept., Austin Correspondence $\bullet$ 1975: (1)

Loeb, Leo Correspondence $\bullet$ 1942: (1)

Loepel Bioscript

Loew, Oscar [Karl Benedict] (1844 ) German chemist, mineralogist

Bioscript

Loewer, Peter Botanical Album Correspondence $\bullet$ 1992: (3)

Logan, Bari M. Royal College of Surgeons, London, Dept. of Anatomy, Prosector Correspondence • 1986: (9)

Logan, James Bioscript

Lokken, Roy N. East Carolina U., Greenville Correspondence • 1974: (3) • 1977: (1)

Lomax, _, Messrs. Correspondence $\bullet$ 1950: (1)

London, George (-1713) Bioscript

London, Jack Author, adventurer Biofile • Bioscript

Lone Star Cement Corporation Correspondence $\bullet$ 1958: (0) Filed under Otto Wiesener A.

Long, Bayard Henry (1885-1969) Bioscript • Botanical Album

Long, Charles Robert (1936-1986) Botanist, librarian Botanical Album

Correspondence $\bullet$ 1970: (2) • 1971: (4) • 1972: (2) • 1974: (1) • 1976: (5) Merrill, Elmer Drew, 1876-1956 • 1977: (7) • 1978: (3) Eastwood, Alice, 1859-1953 1980 : (3) $\bullet$ 1985: (1)

Long, Frances Louise (1885-1946) American plant physiologist

Bioscript

Correspondence $\bullet$ 1943: (2)

Long, Horace M. Explorer Bioscript

Long, Michael Animator, historian; St. Louis, MO Correspondence $\bullet$ 1987: (1)

Long, Robert William (1927-1976) U. of South Florida, Tampa, Dept. of Botany and Bacteriology Botanical Album Correspondence • 1969: (6) • 1970: (15) • 1971: (9) • 1972: (3) • 1973: (4) • 1974: (2) • 1975: (6)

Long, Russell B. United States Senate, Senator Correspondence • 1970: (3) • 1971: (2) • 1978: (2) 1980: (3)

Long, Stephan Harriman (1784-1864) American explorer Biofile $\bullet$ Bioscript $\bullet$ Botanical Album

Long, Timothy $\mathbf{P}$. Correspondence 1993: (1)

Longenecker, Herbert E. Tulane U., New Orleans, President Botanical Album

Correspondence $\bullet$ 1961: (1) 1961 : (0) Letters filed under Oeta M. Crump • 1962: (2) • 1965: (1) • 1966: (4) • 1968: (2) • 1969: (1) • 1971: (2) • 1977: (2) 
Longley, Melvin (fl. 1991) American farmer, grass expert Biofile

Longue Vue Gardens New Orleans

Correspondence - 1970: (7) • 1971: (1) • 1972: (1) • 1974: (3) • 1975: (10) • 1976: (3) • 1977: (2) • 1978: (1) - 1979: (0) Flyers and reports $\bullet$ 1980: (1)

Longwood Gardens

Botanical Album Correspondence $\bullet$ 1974: (11)

Longyear, Burton Orange (1868-1969) American forester, pharmacist, plant collector Bioscript

Loomis, Harold Frederick (1896- ) Agronomist Biofile • Bioscript

Correspondence $\bullet$ 1950: (1) $\bullet$ 1951: (0) Filed under Egbert Hamilton Walker

Loomis, Leverett Mills (1857-1927) Bioscript

Looser, Gaulterio (1898-1982) Chilean taxonomist, phytogeographer Botanical Album

Correspondence 1929-1933: (2) Epling, Carl Clawson, 1894-1968 • 1935: (2) • 1937: (1) • 1938: (2) • 1939: (2) - 1940: (1) • 1943: (1) • 1945: (1) • 1948: (1) • 1953: (1) 1957: (1)

López Guillén, Julio E. (1914-) Universidad Nacional, Instituto de Botánica, Lima, Peru, Director Correspondence $\bullet$ 1969: (1)

Lord, John Keast (1818-1872) British ornithologist Bioscript

Lord, __, Mrs. Bioscript

Lord, V. S. Industrial Service Laboratories Correspondence • 1943: (2)

Lorentz, Paul Günther (1835-1881) Botanist; Uruguay Bioscript

Lorenz, Konrad Austrian zoologist Biofile

Lorey, Hanri Bioscript

Lorimer, George $\mathbf{H}$. Bioscript

Loring, John Alden (1871-1947) American mammalogist, ornithologist Bioscript

Lorquin, Pierre Joseph Michel (1797-1873) Bioscript

Los Angeles Public Library Correspondence $\bullet$ 1950: (1)

Los Angeles State and County Arboretum Botanical Album

Lotsy, John Paul (1867-1931) Dutch biologist, plant geneticist, biological historian Bioscript • Botanical Album

Lottinville, Savoie U. of Oklahoma Press, Norman, OK Correspondence • 1966: (7) Banister, John, 1650-1692 • 1967: (9) • 1980: (4) • 1981: (2)

Loud, Frank Herbert (1852-1927) American astronomer, meteorologist, botanist Bioscript
Loudon, Jane C. (1807-1858) English gardener, horticultural author; husband is John C. Loudon Biofile • Bioscript

Loudon, John Claudius (1783-1843) English horticulturist, landscape gardener, author Biofile $\bullet$ Bioscript $\bullet$ Botanical Album

Loughman, Deirdre A. Correspondence • 1990: (0) Filed under Oxford University Press

Louis-Marie, __, Father (1896-1978) Canadian taxonomist, plant geneticist Correspondence • 1929-1933: (3) • 1934: (1)

Louisiana Miscellaneous Botanical Album

Louisiana Cooperative Extension Service Louisiana State U. and Agricultural and Mechanical College, Metairie, LA Correspondence $\bullet 1977$ : (1)

Louisiana Division of Outdoor Recreation Baton Rouge Correspondence - 1978: (1) Bartram, William, 1739-1823

Louisiana Criminal District Court Parish of New Orleans, LA Correspondence $\bullet$ 1976: (1)

Louisiana Department of Fighways Correspondence $\bullet$ 1973: (1)

Louisiana Forestry Commission Roland J Treubig-Staff Forester Correspondence $\bullet$ 1975: (3) • 1976: (13)

Louisiana History Louisiana State U., Baton Rouge Correspondence $\bullet 1968$ : (1)

Louisiana Landmarks Society Correspondence $\bullet$ 1983: (1)

Louisiana. Revenue and Taxation Correspondence • 1978: (3) Book collectors

Louisiana Society for Horticultural Research Marjorie Cook, Chairman of Membership; Barbara F. Nelson, President; Lafayette, LA Correspondence $\bullet$ 1976: (2)

Louisiana State Anatomical Board Correspondence $\bullet$ 1980: (1)

Louisiana Trails Advisory Council Sandra S. Thompson, Chairman Correspondence • 1976: (9) Bartram, William, 1739-1823

Louisiana State University Baton Rouge Botanical Album Correspondence $\bullet 1974:(1) \bullet 1983:(2) \bullet 1984:$ (3) 1986: (1)

Louisiana State University New Orleans Correspondence • 1964: (2) Letter of recommendation re: Donald Clive Hardy

Louisiana State U. Press Baton Rouge, LA; Margaret Fisher Dalrymple, Editor-In-Chief Correspondence • 1989: (1) - 1992: (3) ourteig, Alicia (1913-) Taxonomist; Museum National D'Histoire Naturelle, Laboratoire de Phanerogamie, Paris. France Botanical Album Correspondence • 1955: (2) • 1956: (4) • 1968: (4) Species • 1969: (1) • 1975: (4) Lyon, John, 1764-1814 Pursh, Frederick, 1774-1820 • 1976: (4) • 1977: (1) • 1979: (1) 
Löve, Áskell (1916-) Cytogeneticist, taxonomist; Canada, U. S. A. Bioscript

Löve, Doris Benta Maria (1918-)

Cytogeneticist, taxonomist; U. of Manitoba, Winnipeg, Canada

Correspondence $\bullet$ 1955: (1) $\bullet$ 1956: (3)

Lovejoy, Thomas E. Bioscript $\bullet$ Botanical Album

Correspondence • 1984: (1) • 1985: (3) • 1986: (1) • 1987: (3) Book collectors • 1988: (1) • 1989: (3) • 1990: (2) - 1991: (1)

Lovell, Phebe Susan Biofile

Lovell, Phoebe Husband is Preston Spencer Botanical Album

Lovell, Robert Bioscript

Lowe, Cheryl U. of Delaware, Newark, Longwood Graduate Fellow Correspondence $\bullet 1990$ : (2)

Lowe, Frank E. Correspondence • 1949: (2) American Fern Society

Lowe, Rachel L. Harrison, ME Correspondence • 1952: (1)

Lowe, Willoughby Patrick (1872-1949) British ornithologist

Bioscript

Lowell, John Amory (1798-1881) Patron, plant collector Bioscript

Lowery, George H. Louisiana State U. Correspondence • 1957: (1) • 1958: (1) • 1961: (1) • 1961: (0) Filed under Waldo Lee McAtee • 1963: (4) • 1973: (1)

Lowman, Charles LeRoy Orthopedic Surgeons Correspondence $\bullet$ 1939: (3)

Lownes, Albert Calbert Edgar Providence, RI Botanical Album

Correspondence • 1968: (3) $\bullet 1971$ : (2)

Lowry, Edward D. Trinity College, Washington, D.C., Dept. of English, Professor Correspondence • 1979: (3)

Lowry, John S. Bioscript

Loyola U. New Orleans, LA Correspondence • 1966: (1)

Lozieres, Bandry des Bioscript

Lu Tu, Frank Eugene (1879-1943) Bioscript

Lubbock, John, Sir (1834-1913) British banker, naturalist Bioscript

Lubrecht, Harry D. Hafner Publishing Co., NY; Lubrecht \& Cramer, Booksellers \& Publishers, Monticello, NY Bioscript - Botanical Album Correspondence $\bullet$ 1951: (2) • 1956: (1) • 1958: (2) • 1959: (4) - 1961: (3) • 1963: (3) Letter from George Booth Van Schaack • 1964: (16) Book collectors $\bullet$ 1965: (18) Letter from George Booth Van Schaack • 1966: (20) 1967: (21) Letters from William D. Reese and F. A. Stafleu - 1968: (8) Book collectors • 1969: (34) Copy of correspondence with F. A. Stafleu $\bullet$ 1970: (23) Book
Lubrecht, Harry D. (continued)

collectors; Nuttall, Thomas, 1786-1859 1971: (32)

1972: (13) • 1973: (8) • 1974: (11) • 1975: (5) • 1976: (8)

Book collectors $\bullet$ 1977: (6) - 1978: (6) Pursh, Frederick,

1774-1820 • 1979: (13) Pursh, Frederick, 1774-1820 •

1980: (8) • 1981: (3) • 1982: (4) • 1983: (6) • 1984: (4) •

1985: (2) • 1986: (4) • 1987: (2) • 1989: (3) • 1991: (2) • 1992: (6) - 1993: (1)

Lucas, B. W. B. W. Lucas College Book Co., Berkeley, CA

Correspondence • 1941: (1) • 1953: (3)

Luckingham, Brad Correspondence $\bullet$ 1965: (1)

Lüders, Friedrich, G. I. (1814-1904) German botanist Bioscript

Ludlow, William (1843-1901) American U. S. Army engineer

Bioscript

Ludwig, C. G. Bioscript

Ludwig, F. G., Inc. Correspondence $\bullet$ 1961: (1)

Ludwig - of Leipzig Bioscript

Lueth, Francis X. State of Alabama, Dept. of Conservation Correspondence $\bullet$ 1947: (2)

Luggins, Dorothy H. California Historical Society Correspondence $\bullet$ 1943: (1)

Lull, Robert W. Newburyport, MA Correspondence $\bullet$ 1951: (2)

Lülmann, I. A. Bibliothek des Botanischen Gartens und Museums, Berlin-Dahlem, Königin-Luisestr.

Correspondence $\bullet$ 1951: (1)

Lumiansky, Robert M. Dean, Tulane Correspondence $\bullet$ 1956: (1) $\bullet$ 1971: (2)

Lumsden, Jennifer Grandaughter of A. Erling Porsild Correspondence $\bullet$ 1986: (3)

Lumsden, Richard D. Tulane U., The Graduate School, New Orleans, LA, Dean Correspondence $\bullet$ 1977: (1)

Lund, $\mathbf{P}$. W. Bioscript

Lundberg, Eric Antiquarian bookseller; Walpole, NH Bioscript

Correspondence $\bullet$ 1953: (1) $\bullet$ 1958: (1) $\bullet$ 1960: (2) Book collectors • 1961: (1) • 1962: (2) • 1963: (1) • 1964: (5) Book collectors • 1965: (1) • 1966: (1) • 1967: (3) • 1968: (7) Book collectors $\bullet$ 1969: (2) • 1971: (3) • 1972: (4) $\bullet$ 1973: (2) • 1974: (4) • 1975: (3) • 1976: (1) Rocky Mountain Naturalists; Book collectors

Lundell, Cyrus Longworth (1907-1994) Taxonomist, economic botanist, plant ecologist; Southern Methodist U. Bioscript • Botanical Album Correspondence $\bullet$ 1946: (4)

Lundgren, J. H. Bioscript

Lunnell, Joel Botanical Album

Lunzer, Alois Bioscript 
Lustig, Lawrence K. Arete Publishing Company, Inc., Editor-in-Chief

Correspondence $\bullet$ 1978: (0) Filed under Arete Publishing Company, Inc.

Luteyn, James Leonard (1948-) Botanist; New York Botanical Garden; Taxon, Associate Editor Correspondence • 1987: (3) One letter signed Gradstein, Koek-Noorman, Maas

Lutrell, Estelle Correspondence $\bullet$ 1935: (2)

Luzenberg, Charles A. Bioscript

Ly-Tio-Fane, Marie Madelene (1928-) Librarian, author Correspondence $\bullet$ 1992: (2)

Lyall, David (1817-1895) Scottish botanist Bioscript

Lycett, C. V. L. Royal Horticultural Society, Secretary Bioscript $\bullet$ Botanical Album Correspondence $\bullet$ 1955: (2)

Lyell, Charles (1797-1875) English geologist, botanist, paleontologist Biofile $\bullet$ Bioscript $\bullet$ Botanical Album

Lyell, Katherine Murray (1817-1915) Bioscript

Lyle, James A. Auburn U., Auburn, AL Correspondence $\bullet$ 1967: (1)

Lynas, Lothian Librarian; New York Botanical Garden Correspondence • 1978: (1) Eastwood, Alice, 1859-1953

Lynch, F. C. C. Dept. of Mines and Resources, Canada Correspondence • 1941: (1) • 1943: (1)

Lynch, John Fish and Wildlife Service, Abbeville, LA Correspondence $\bullet$ 1949: (1) • 1952: (1) • 1953: (1) Copy of letter to Clyde B. Terrell

Lynes, Wilson Correspondence $\bullet$ 1961: (3)

Lynn, Eleanora V. Enoch Pratt Free Library, Baltimore, MD Correspondence $\bullet$ 1953: (2)

Lynn, John S. Lilly Endowment, Inc. Correspondence • 1967: (1)

Lyon, Brad Correspondence $1992(0)$ Letter under Elizabeth Woodburn

Lyon, Harold Lloyd (1879-1957) Agriculturist, forester, horticulturist; Hawaiian Sugar Planters AssociationBotanical Album Correspondence $\bullet$ 1948: (1)

Lyon, John (1765-1814) Bioscript

Lyon, William Scrugham Bioscript

Lyonet, Pierre (1706-1789) Swiss lawyer, entomologist Biofile

Lyons, James Bioscript

Lysaght, Averil Margaret (1905-1981) English biologist, artist, botanical historian; British Museum (Natural History), Bird Room Biofile $\bullet$ Bioscript $\bullet$ Botanical Album Correspondence • 1955: (6) • 1956: (10) Banks, Joseph, Sir, 1743-1800; Cockerell, Theodore Dru Alison, 1866-1948
Lysaght, Averil Margaret (1905-1981) (continued) - 1957: (15) Banks, Joseph, Sir, 1743-1800 • 1958: (7) • 1959: (16) Banks, Joseph, Sir, 1743-1800 • 1960: (7) British Museum (Natural History). Dept. of Botany; Banks, Joseph, Sir, 1743-1800; letter from Beaglehole to Lysaght enclosed - 1961: (8) Banks, Joseph, Sir, 1743-1800; British Museum (Natural History). Dept. of Botany $\bullet$ 1962: (15) Banks, Joseph, Sir, 1743-1800 • 1963: (13) Banks, Joseph, Sir, 1743-1800

Lysenko, Trofim D. Soviet geneticist Biofile

\section{$M$}

M. Barrows \& Co., Inc. New York, NY; publishing house Correspondence $\bullet$ 1959: (1)

Maas, Paulus Johannes Maria (1939-) See Luteyn, James Leonard (1948-)

Correspondence $\bullet$ 1987: (0)

Mabberley, David John (1948-) English plant taxonomist; U. of Oxford, Oxford, England Biofile $\bullet$ Bioscript $\bullet$ Botanical Album Correspondence $\bullet$ 1981: (1) • 1988: (4) • 1990: (6) Barton, Benjamin Smith, 1766-1815 • 1991: (7) Barton, Benjamin Smith, 1766-1815 • 1992: (2)

Macadam, John Bioscript

MacBride, George McCutcheon U. of California, Los Angeles, Geography Correspondence $\bullet 1938$ : (2)

Macbride, James Francis (1892-1976) American plant collector, taxonomist Bioscript $\bullet$ Botanical Album Correspondence • 1938: (1) • 1961: (2)

MacBride, Thomas Huston Botanical Album.

MacBryde, Bruce (1941-) Taxonomist; U. of British Columbia, Vancouver, Assoc. Editor, Flora of B.C. Correspondence • 1974: (2) • 1976: (1) • 1985: (2)

MacClatchie, Alfred James Botanical Album

MacDonald, Malcolm M. U. of North Carolina Press Correspondence $\bullet$ 1975: (2) $\bullet 1977:$ (2)

MacDougal, Daniel Trembly (1865-1958) American botanist, plant physiologist Bioscript $\bullet$ Botanical Album

MacDougal, Jan Correspondence • 1992: (1)

MacDougal, John Bioscript Correspondence $\bullet$ 1992: (1)

MacDougall, Elisabeth B. Dumbarton Oaks, Trustees for Harvard U. Correspondence $\bullet$ 1978: (2)

MacDougall, Thomas Baillie (1895-1973) Bioscript

Mace, Stuart Botanical Album

MacElwee, Alexander (1869-1923) Bioscript - Botanical Album 


\section{Guide to the Ewan Papers}

MacFadden, Fay A. (1888-1964) American plant collector, bryologist, botanist

Bioscript • Botanical Album

Correspondence • 1929-1933: (9) • 1934: (1) • 1935: (1)

- 1951: (2)

MacFarland, F. M. California Academy of Sciences

Correspondence • 1942: (2) Eastwood, Alice, 1859-1953

- 1943: (6) Species

Macfarlane, John Muirhead (1855-1943) Botanist,

clergyman, plant collector

Bioscript

Correspondence • 1935: (1)

MacGillivray, John (1822-1867) Scottish-born, Australian naturalist, plant collector, explorer Biofile

MacGillivray, William (1792-1852)

Bioscript

Macgowan, D. J. Bioscript

Mack, George Correspondence • 1942: (1)

Mackaness, Faith Corbett, OR; husband is Frank G. Mackaness

Correspondence $\bullet$ 1951: (3)

Mackaness, Frank G. Wife is Faith Mackaness Correspondence - 1985: (2) Royal Botanic Gardens, Kew $\bullet$ 1986: (1) - 1987: (1) Royal Botanic Gardens, Kew - 1988: (0) Filed under Philip E. Leinbach

Mackay, J. T. Bioscript

Mackay, James Townsend (1775-1862) Irish botanist, author Biofile

MacKeever, Frank Clifford (1902-1967) American writer, horticulturist; New York Botanical Garden Correspondence $\bullet$ 1949: (1)

MacKenzie, Alexander (1755-1820) Scottish explorer Bioscript

MacKenzie, Kenneth Kent (1877-1934) American botanist, corporate attorney

Bioscript • Botanical Album

Correspondence • 1929-1933: (3)

MacKenxie, William Gregor (1904) Scottish horticulturist; Chelsea Physic Garden, Chelsea, London, England

Correspondence • 1966: (1)

Mackie, George U. of Victoria, Victoria, Dept. of Zoology Correspondence • 1970: (1) Rocky Mountain Naturalists

Mackie, William Wylie (1873-) Bioscript

Maclagan, Philip Whiteside (1818-1892) Scottish surgeon and naturalist Biofile • Bioscript

Macleod, Margaret U. of Chicago, Botanical Gozzette Correspondence • 1935: (2) Delphinium

Maclure, William (1763-1840) Scottish-born American agriculturist, geologist, patron Biofile • Bioscript

MacManus, George S., Co. Philadelphia book dealer Correspondence $\bullet$ 1954: (3)
MacMaster, J. E. Pasadena, CA Correspondence $\bullet$ 1960: (2)

MacMillan Publishing Co. Inc. Correspondence • 1970: (2) Lawrence, George Hill Mathewson, 1910-1978 • 1975: (3) John Kowalski • 1976: (2) $1981:$ (2)

MacNeil, Robert Broadcast journalist; The MacNeil Lehrer Report

Correspondence $\bullet$ 1979: (1)

Macoun, James Melville (1862-1920) Canadian botanist, ornithologist

Bioscript • Bioscript

Macoun, John (1832-1920) Irish-born Canadian ornithologist, botanist, plant collector Biofile • Bioscript

MacOwan, Peter (1830-1909) Botanist; South Africa Bioscript

MacPhail, Ian (1923- ) American botanical bibliographer, librarian; Morton Arboretum

Biofile $\bullet$ Botanical Album

Correspondence $\bullet$ 1971: (14) $\bullet$ 1972: (7) $\bullet$ 1973: (2) $\bullet$

1974: (2) • 1975: (6) Bartram, William, 1739-1823 •

1976:(7) • 1978: (3) • 1982: (6) • 1983: (10) • 1984: (7)

Barton, Benjamin Smith, 1766-1815 • 1989: (2) • 1990: (5)

- 1991: (8) Barton, Benjamin Smith, 1766-1815 • 1992:

(8) - 1993: (3)

Macrae, James

Bioscript

Macrae, W. F.

Bioscript

MacRoberts, D. T. Louisiana State U., Shreveport Correspondence • 1985: (1) • 1992: (2)

MacRoberts, , Dr.

Correspondence $\bullet$ 1980: (1)

MacRoberts, Michael H. Correspondence $\bullet$ 1988: (2)

Madden, Edward H. San Jose State College, San Jose, Philosophy Dept.

Correspondence $\bullet$ 1963: (6)

Madden, Henry Librarian; Fresno State College, Fresno, CA

Botanical Album

Correspondence $\bullet$ 1956: (3)

Madden, Margaret H. Tulane U., New Orleans, LA Correspondence $\bullet$ 1966: (1)

Maddox, Alex Bookbinder Correspondence $\bullet$ 1962: (1)

Maddox, Anne Atlanta, GA; husband is Alex Maddox Correspondence $\bullet 1965:(1) \bullet 1966:(2)$

Madiana

Bioscript

Madison, James Catesby Son of Bishop James Madison Bioscript

Madison, James Fourth President of United States Biofile

Madison, James C.

Bioscript

Madison-Morgan Cultural Center Madison, GA Correspondence • 1990: (1) 
Madroño

Correspondence $\bullet$ 1976: (2) $\bullet$ 1977: (3) $\bullet$ 1979: (0) Filed under James Craig Hickman, Editor $\bullet$ 1983: (1) • 1986: (1) - 1989: (2)

Mady, Christopher Columbia U., New York, NY Correspondence $\bullet$ 1952: (1)

Maempel, George Zammit Bioscript

Maeser, Karl G. Bioscript

Magdalen College, Oxford G. L. Harris, Librarian; N.R. Ker, Librarian Correspondence • 1969: (1)

Magellan, Ferdinand Explorer Biofile

Maggiori, Gloria Smithsonian Tropical Research Institute, Panama Correspondence $\bullet$ 1985: (5)

Maggs Brothers, Ltd. London, England; Rare Books, Manuscripts and Old Maps

Correspondence • 1959: (4) • 1962: (8) • 1990: (1) Book collectors

Maggs, Ernest Botanical Album

Maggs, John F. London rare book dealer Correspondence - 1954: (3) Book collectors $\bullet$ 1955: (1) - 1956: (6) - 1957: (5) Book collectors • 1958: (3) • 1960: (6) • 1961: (2) • 1963: (7) • 1965: (7) $\bullet$ 1966: (1) - 1979: (2) - 1980: (3) • 1981: (4) Book collectors - 1983: (1) - 1993: (1) Book collectors

Magill, Robert Earle (1947- ) Bryologist; Missouri Botanical Garden, St. Louis, MO Correspondence • 1987: (1)

Magnus, $\mathbf{P}$. Bioscript

Maguire, Bassett (1904-1991) Taxonomist, plant explorer; New York Botanical Garden Bioscript • Botanical Album Correspondence $\bullet$ 1941: (8) $\bullet$ 1942: (11) $\bullet$ 1943: (4) $\bullet$ 1947: (5) American Fern Society $\bullet$ 1948: (2) Species $\bullet$ 1951: (3) • 1952: (1) Vismia • 1955: (1) - 1956: (2) $\bullet$ 1959: (2) 1 1960: (1) 1963: (1) $\bullet$ 1969: (1) Letter to Julie Hackney, Ewan's student

Mahalick, Patricia E. BioScience, Book Review Editor Correspondence $\bullet$ 1968: (1)

Mahe, George A., Jr. Physician; St. Louis, MO Correspondence $\bullet$ 1987: (1)

Maher, Louis J. Jr. U. of Wisconsin, Madison Correspondence $\bullet$ 1964: (1)

Maheshwari, Pancanan (1904-1966) India botanist; International Commission on Plant Embryology Correspondence $\bullet$ 1956: (1)

Mahler, William Fred (1930-) Taxonomist, bryologist, educator Southern Methodist U., Dallas, TX, Herbarium Correspondence • 1973: (4) • 1975: (1) • 1978: (1) - 1980: (2) • 1981: (1) • 1982: (1) • 1984: (1)

Mahurin, Carl Correspondence $\bullet$ 1943: (2) Delphinium

Maiden, Joseph Henry (1859-1925) Author, taxonomist, plant explorer, plant collector, botanical historian; Australia Botanical Album
Mailliard, John Ward (1862-1935) California businessman, zoologist

Biofile

Mailliard, Joseph (1857-1945)

Bioscript

Main, Angie Kumlien Granddaughter of Thure Kumlien Correspondence • 1942: (1) Letter from Erl Ellis

Main, Sally Newcomb College, Art Dept. Correspondence $\bullet$ 1983: (1)

Mains, Ernest Butterworth (1890-1968) U. of Michigan, Ann Arbor, U. Herbarium, Director Correspondence $\bullet$ 1951: (3)

Maire, René Charles Joseph Ernest (1878-1949) Frenchborn mycologist, phytogeographer, plant collector, taxonomist in U. S. A. Biofile

Maitland, A. Gastair British Consulate General, New Orleans Correspondence • 1961: (1)

Maitland, Louise The Royal Empire Society Correspondence • 1955: (1) • 1956: (2)

Maitland, Thomas Douglas (1885-1976) Correspondence • 1962: (3) • 1963: (2)

Major, Jack (1917- ) Plant ecologist; U. of California, Berkeley, Div. of Soils Correspondence $\bullet$ 1951: (2) • 1952: (1)

Makower, Ben Botanical Album Correspondence • 1968: (1) • 1969: (1)

Makower, Rachel (Uhvits) Husband is Ben Makower Bioscript Correspondence $\bullet$ 1977: (1)

Malaspina Expedition (c. 1789) Biofile

Malcolm, William Bioscript

Malinowski, Robert F. Gillis, Ellis \& Baker, Inc., Insurance Correspondence $\bullet$ 1982: (1)

Mallet, J. W. Bioscript

Mallinson, George G. School Science and Mathematics, Editor Correspondence $\bullet$ 1960: (1)

Maloney, James Tulane U., New Orleans, LA, Dept. of Spanish and Portuguese Correspondence - 1977: (2)

Malpighi, Marcello (1628-1697) Italian botanist, physician, plant anatomist Bioscript $\bullet$ Botanical Album

Malter, Jeffry L. U. of Temnessee, Knoxville, Dept. of Botany Correspondence 1977 : (1)

Mammerly, Betty California Academy of Science, San Francisco Botanical Album

Manard, John P. Correspondence $\bullet$ 1972:

Mandal, Richard Tulane, Personnel Office Correspondence - 1975: (1) 
Mandon, Gilbert Gustav (1799-1866) Plant collector in Bolivia, Madeira, and the Canary Islands Bioscript

Manetti, Giuseppe (fl. 1831-1858) Italian botanist, head gardener of the Royal Garden at Monza Bioscript

Mangelsdorf, Paul Christoph (1899-1989) Economic botanist, plant geneticist; Bussey Institution, Harvard Bioscript Correspondence $\bullet$ 1967: (2)

Manitoulin Expositor Little Current, Ontario; W. J. Patterson, Ed. Correspondence $\bullet$ 1959: (1)

Manks, Dorothy St. John (1898-) American horticultural historian, librarian; Massachusetts Horticultural Society Correspondence • 1959: (2) • 1960: (2) • 1962: (3) - 1966: (15) • 1967: (12)

Mann, Horace, Jr. (1844-1868) Student of Asa Gray; son of Horace and Mary Mann Bioscript

Mann, M. D.

Correspondence • 1951: (6) • 1952: (1) • 1953: (0) Filed under Ralph Curtis Benedict

Mann, W. F. United States Dept. of Agriculture, Southern Forest Experiment Station, Project Leader Correspondence $\bullet$ 1970: (2)

Mann, William M. (1886-) Bioscript

Manning, H. M., Mrs. Botanical Album

Manning, Wayne Eyer (1899-) Taxonomist, plant morphologist; Bucknell U.

Botanical Album

Correspondence • 1957: (1) • 1958: (1) • 1968: (0) Filed under Donald Eugene Stone • 1969: (1) • 1977: (2)

Manson, James Bioscript

Mantell, Gideon AIgernon (1790-1852) Bioscript

Manton, Irene (1904-1988) English cytologist, cytotaxonomist Botanical Album Correspondence • 1955: (8) • 1957: (1)

Manuel Del Rio, Andres Bioscript

Many, Amna E. Newcomb College, New Orleans, LA Correspondence $\bullet$ 1952: (1)

Many, Leonce Husband is J. L. Many Correspondence $\bullet$ 1963: (1)

Manza, Artemio V. (1896-1964) Philippine agriculturist, dendrologist Botanical Album

Maples, Robert S. McNeese State College, Dept. of Biology

Correspondence • 1973: (1)

Marbut, Curtis F. Botanical Album

Marcgrave, Georg (1610-1644) German-born physician and naturalist of Brazil Biofile $\bullet$ Bioscript
Marcou, Jules (1824-1898) French geologist Bioscript

Marcus, Esther K. Correspondence • 1977: (2)

Marcy, Oliver (1820-1899) Bioscript

Marcy, Randolph Barnes (1812-1887) American explorer and topographical engineer, U. S. Army Biofile • Bioscript

Mardersteig, Giovanni (1892?- ) German-born Italian printer Biofile

Margadant, Willem Daniel (1916- ) Dutch botanist; Biohistorisch Institut, Utrecht; Index Biologicorum Botanical Album Correspondence • 1977: (2)

Marie-Victorin, Joseph Louis Conrad, Frère (1885-1944) French-Canadian clergyman Botanical Album $\bullet$ Biofile

Marietta College Sandra B. Neyman, Head, Readers Services, Dawes Memorial Library, Marietta, OH Correspondence • 1978: (1)

Marin M., Felipe Universidad del Cuzco, Peru, Prof. Correspondence $\bullet$ 1951: (2)

Marine Biological Lab Woods Hole Oceanographic Institution, Librarian Correspondence $\bullet$ 1956: (1)

Mark, Clara Gould Bioscript

Mark, Frank R. Correspondence $\bullet$ 1942: (1)

Marker, R. E. Pennsylvania State College Correspondence $\bullet$ 1941: (3)

Markgraf, Friedrich (1897-1987) German taxonomist, phytogeographer; Botanischer Garten und Institut für Syst. Botanik, Universität Zürich Correspondence $\bullet$ 1965: (2)

Markham, Clements Robert (1830-1916) English plant explorer, phytogeographer, economic botanist Bioscript

Markham, Leo Thomas Correspondence $\bullet 1940$ : (1)

Markley, Klare S. Southern Regional Laboratory, New Orleans, LA Correspondence $\bullet$ 1952: (1)

Marks, Barbara Correspondence • 1949: (2)

Marks, Edwin P. Washburn U., Topeka, KS Correspondence • 1958: (1)

Marks, Lester Correspondence • 1967: (1) 1974: (3)

Marland, Frederick C. Virginia Polytechnic Institute Correspondence $\bullet$ 1966: (3)

Marple, Richard Marple Apicultural Graphics Archives Correspondence • 1988: (1)

Marr, John Winton (1914) American alpine ecologist Botanical Album

Marret, Léon Botanical Album 
Marriage, Kathleen N. O'Neill (1882-1958) Irish-born American educator, horticulturist, nurseryman; husband is George Reynolds Marriage; nickname "Molly"

Biofile $\bullet$ Botanical Album

Correspondence $\bullet$ 1938: (2) $\bullet$ 1941: (1) $\bullet$ 1942: (6) $\bullet$

1943: (6) Species $\bullet$ 1944: (4) $\bullet$ 1945: (2) • 1946: (3)

- 1948: (2) • 1955: (1)

Marsden, Ernest (1889-1970) English-born New Zealand physicist, educator, administrator Biofile

Marsden-Jones, Eric Marsden Botanical Album

Marsh, Charles H. Taxidermist, ornithologist Bioscript

Marsh, George Perkins Lawyer, diplomat, collector of natural history specimens Biofile

Marsh, Othniel Charles (1831-1899) American paleontologist Bioscript

Marsh, Thompson George American legal educator and amateur ornithologist

Biofile

Marshall, Alexander (1639?-1682) English entomological and botanical artist

Biofile $\bullet$ Bioscript

Marshall, Amy Gould Correspondence • 1936:

Marshall, C. C. Bioscript

Marshall, Humphrey (1722-1801) American botanist and collector

Biofile • Bioscript

Marshall, James (f. 1695-1705) Bioscript

Marshall, Joe T. Smithsonian Institution, Fish and Life Service

Correspondence • 1988: (1)

Marshall, John Correspondence • 1980: (1)

Marshall, John Braybrooke (1913-) National Research Council, Canada Correspondence • 1958: (1)

Marshall, Linda W. Hortulus Correspondence $\bullet$ 1982: (1)

Marshall, Margaret The Nation, Literary Editor Correspondence - 1942: (1)

Marshall, Mary Louise Tulane U. Correspondence • 1948: (2)

Marshall, Moses Bioscript

Marshall, W. P. Bioscript

Marshall, William Emerson (1872-1937) Bioscript

Marten, Humphry (b. 1729?) Bioscript

Marter, Franz Joseph Bioscript
Martin, Ben F. Louisiana State U., Alexandria, Division of Sciences, Asst. Prof. Correspondence • 1976: (0) Filed under Louisiana Forestry Commission

Martin, C. E. Bioscript

Martin, Floyd Leonard (1909-) Correspondence $\bullet$ 1949: (2)

Martin, Foster N. Botanical Album Correspondence • 1950: (1) • 1952: (2) • 1958: (6)

Martin, George Willard (1885-1971) American botanist, mycologist, ecologist Botanical Album

Martin, H. Bradley Bioscript

Martin, James Bland, Mrs. Correspondence $\bullet$ 1970: (3)

Martin, Joseph Bioscript

Martin, Lloyd M. Correspondence • 1929-1933: (1)

Martin, Louis B. Brooklyn Botanic Garden Correspondence • 1970: (0) Filed under George Sherman Avery $\bullet$ 1972: (1)

Martin, Murray Simpson The Pennsylvania State U., University Park, Associate Dean of Libraries Correspondence $\bullet 1977$ : (2)

Martin, Robert Franklin (1910-) Botanical Album Correspondence $\bullet$ 1936: (1)

Martin, Robert S. Louisiana State U., Hill Memorial Library, Asst. Director Correspondence $\bullet$ 1985: (3) $\bullet$ 1986: (3)

Martin, William Bioscript

Martindale Collection Mrs. Elsasser, U. S. National Arboreum Correspondence $\bullet 1972$ : (1)

Martindale, Isaac Comly (1842-1893) American collector of Lepidoptera, plant collector Biofile $\bullet$ Bioscript

Martínez Crovetto, Raoul See Crovetto, Raoul Martinez (1921 -)

Martinez de Hoz, Saturnino W. (fl. 1943) Bookseller Biofile

Martinez, Esteban Bioscript

Martínez, Maximino (1888-1964) Mexican botanical explorer; Trabajos de la Comision Botánica Exploradora del Estado de México Biofile $\bullet$ Botanical Album Correspondence $\bullet$ 1957: (1)

Martiniere, de Boissieu lac Bioscript

Martius, Charles Frédéric Philippe von (1794-1868) German botanist and entomologist, author, physician, plant explorer, taxonomist Biofile • Bioscript

Martyn, John (1699-1768) British botanist and physician Biofile 
Martyn, Thomas (1735-1825) British botanist, taxonomist Bioscript

Correspondence $\bullet$ 1983: (1)

Martyr, Peter

Bioscript

Maryland, Natural History Natural History Society of Maryland, Baltimore

Correspondence • 1951: (1) Pursh, Frederick, 1774-1820

Marynick, Sam P. Book collector; former Tulane student Correspondence • 1968: (2) Bartram, William, 1739-1823 - 1969: (1) • 1971: (3) • 1975: (2) • 1977: (1)

Masi, Peter L. Bookseller Correspondence • 1986: (1) 1992: (1)

Maslin, Paul T. U. of Colorado, Boulder, Dept. of Biology Correspondence $\bullet$ 1962: (1)

Mason, Alexandra Librarian; U. of Kansas, Lawrence Spencer Research Library, Special Collections Correspondence • 1966: (3) • 1967: (2) • 1975: (2) • 1977: (1) • 1980: (1) • 1981: (1) • 1984: (5) • 1985: (1) - 1992: (1) • 1993: (2)

Mason, Charles Thomas, Jr. (1918-) Cytotaxonomist Biofile Correspondence $\bullet$ 1954: (1)

Mason, David T. Bellingham, WA Correspondence • 1987: (0) Filed under Herbert Louis Mason

Mason, Georgia U. of Oregon, Eugene, Museum of Natural History

Correspondence • 1964: (2) Delphinium • 1967: (4) Delphinium

Mason, Henry L. Correspondence $\bullet$ 1966: (1)

Mason, Herbert Louis (1896-1994) American botanist, phytogeographer, taxonomist, plant collector

Biofile • Bioscript $\bullet$ Botanical Album Correspondence • 1929-1933: (2) • 1936: (3) • 1939: (3) • 1941: (3) • 1943: (3) • 1944: (2) Delphinium • 1951: (1) letter to Mrs. Locke $\bullet$ 1952: (1) $\bullet$ 1957: (3) • 1961: (1) • 1979: (1) • 1987: (1)

Mason, Joseph

Bioscript

Massachusetts Horticultural Society

Botanical Album

Correspondence • 1966: (4) • 1992: (2)

Massart, Jean

Botanical Album

Massey, A. B.

Bioseript

Massey, Jimmy R.

Bioscript

Correspondence • 1970: (2) • 1972: (1) • 1973: (2)

Massie, Thomas

Bioscript

Masson, Francis (1741-1805) Scottish-born botanist, plant collector, botanical illustrator; South Africa Biofile - Bioscript

Masters, Maxwell Tylden (1833-1907) English physician, botanist, horticulturist, editor

Bioscript
Matas, Rudolph (1860-1957) Louisiana surgeon, physician

Biofile $\bullet$ Bioscript $\bullet$ Botanical Album

Correspondence 1949: (1)

Matheson, William Washington U. Libraries, Chief; Library of Congress, Rare Books

Correspondence - 1964: (1) Van Schaack, George Booth, 1903-1983 • 1975: (3) • 1977: (2) • 1985: (2) • 1988:

(1) Missouri Botanical Garden; Van Schaack, George Booth, 1903-1983

Mathew, M. V. Royal Botanic Garden, Edinburgh, Librarian

Correspondence $\bullet$ 1972: (1) $\bullet$ 1983: (1)

Mathews, Andrew (1801?-1841) English horticulturist and plant collector in Peru

Biofile

Mathews, Gregory Macalister (1876-1949) British ornithologist in Australia, book collector Biofile

Mathias, James F. Guggenheim Foundation, Assoc. Sec. Correspondence • 1954: (1) • 1955: (1) • 1963: (2) • 1965: (4) • 1969: (4) Banister, John, 1650-1692 • 1971: (3)

Mathias, Mildred Esther Hassler (1906-1995) American plant taxonomist; husband is Gerald L. Hassler Biofile $\bullet$ Bioscript $\bullet$ Botanical Album

Correspondence • 1929-1933: (2) - 1934: (2) Species • 1936: (1) • 1937: (13) • 1938: (1) • 1939: (7) Species; Delphinium • 1948: (1) • 1949: (1) • 1950: (5) • 1951: $(2) \bullet$ 1952: (1) • 1954: (4) $\bullet$ 1955: (2) $\bullet$ 1957: (2) • 1959: (4) • 1960: (4) • 1961: (4) • 1963: (2) Stafleu, Frans Antonie, 1921- 1968: (1) • 1976: (1) • 1980: (3) - 1985: (1) • 1988: (5) • 1991: (1) • 1992: (1)

Matlack, Bennett K. Bridgeton, NJ Botanical Album Correspondence • 1951: (2) • 1958: (5)

Matson Lines San Francisco, CA Correspondence $\bullet$ 1966: (1)

Matthes, B. Bioscript

Matthes, Francois Emile (1874-1948) Dutch-born American geologist Biofile

Matthews, Washington (1843-1905) Archeologist, ethnobotanist Bioscript

Matthiesen, Diana U. of Florida, Gainesville, Dept. of Zoology, Brodkorb Collections

Correspondence • 1990: (2)

Mattioli, Pietro Andrea (1501-1577) Italian physician, naturalist, herbalist Biofile $\bullet$ Bioscript

Mattison, Joel Correspondence $\bullet$ 1984: (2) $\bullet$ 1985: (2)

Mattoon, Wilbur Reed (1875-1941) American forester Bioscript

Matzke, Edwin B. (1902-) Plant morphologist Correspondence - 1949: (2) Rocky Mountain Naturalists; letter from Ray Fosberg 
Maupertuis, Pierre-Louis Moreau de (1698-1759) French scientist and philosopher Biofile

Maury, Matthew Fontaine (1806-1873) American naval officer and scientist Biofile

Maverick, Samuel (1772-1852) Bioscript

Maxfield, Alice Newcomb Women's Center Correspondence $\bullet$ 1982: (1)

Maximilian, Alexander Philip, Prince of Neuwied (1782-1867) German naturalist, scientist, plant explorer Biofile • Bioscript

Maximowicz, Carl Johann Iwanovich (1827-1891) Russian botanist, plant collector Bioscript

Maxon, Mary Correspondence • 1948: (1) Maxon, William Ralph, 1877-1948

Maxon, William Ralph (1877-1948) American pteridologist; taxonomist, plant collector; Smithsonian Institution, U. S. National Herbarium Bioscript $\bullet$ Botanical Album Correspondence - 1929-1933: (7) American Fern Society - 1934: (2) • 1936: (2) Species • 1937: (4) • 1938: (6) Species • 1939: (5) Species $\bullet$ 1941: (6) American Fern Society $\bullet$ 1942: (5) American Fern Society $\bullet$ 1943: (15) Species • 1944: (8) American Fern Society $\bullet$ 1945: (1) $\bullet$ 1946: (3) American Fern Society $\bullet$ 1947: (7) American Fern Society

Maxwell, I. C. M. Inter-U. Council for Higher Education Overseas, London

Correspondence $\bullet$ 1958: (2)

Maxwell, Martha Ann Dartt (1831-1881) Bioscript

Maxwell, Richard Howard (1926-) Southern Illinois U., Carbondale Correspondence • 1967: (2)

Maxwell, Ruth N. Correspondence $\bullet$ 1949: (1)

May, J. Thomas Nickname "Tommy" Correspondence • 1966: (1) • 1967: (2) • 1968: (1) • 1970: (1) • 1971: (2) • 1972: (1)

Maynard, Charles Johnson (1845-1929) Bioscript

Mayo, Robert Bioscript

Mayr, Ernest Zoologist Bioscript • Botanical Album

Mazzeo, Peter M. National Arboretum, research assistant Correspondence $\bullet$ 1969: (3) $\bullet$ 1981: (1)

McAdoo, Richard Editor; Harper and Brothers, Publishers Correspondence • 1954: (2) Pursh, Frederick, 1774-1820

McAfee, A. J. Bioscript

McAllister, Frederick (1876-1949) American cytologist, educator Biofile

McArdle, H. J., Mrs. Librarian; Oxford, Botany School Correspondence $\bullet 1970$ : (0) Filed under Oxford University Botany School
McArthur, James M. Orleans Parish School Board Correspondence • 1948: (3) • 1949: (1)

McAtee, Robert Correspondence $\bullet$ 1962: (1)

McAtee, Waldo Lee (1883-1962) Bioscript $\bullet$ Botanical Album Correspondence • 1952: (5) • 1956: (4) • 1958: (3) - 1961: (5)

McAvoy, Blanche (1885-1976) Plant ecologist Bioscript

McBerty, P. A. Patio Planters Correspondence $\bullet$ 1958: (1)

McBride, George McCutcheon Ecuador-Peru Boundary Demarcation Commission Botanical Album Correspondence • 1937: (2) • 1943: (5) • 1946: (1) - 1948: (2)

McBryde, Felix Webster Botanical Album

McCale, T. T. Botanical Album

McCaleb, Harvey C. W. H. Freeman and Company, Publishers Correspondence $\bullet$ 1957: (2) Book collectors $\bullet$ 1958: (1) - 1962: (1) • 1979: (1)

McCall, Edwin LeRoy Bioscript

McCall, Harry, Jr. Chaffe, McCall, Phillips, Toler \& Sarpy, New Orleans, LA Correspondence $\bullet$ 1979: (1) Letter from J. White to McCall

McCalla, Margaret A. Correspondence $\bullet$ 1964: (1)

McCalla, William (1814-1849) Irish phycologist Biofile

MeCalla, William Copeland (1872-1962) Canadian school teacher, amateur botanist, plant collector Biofile $\bullet$ Bioscript $\bullet$ Botanical Album Correspondence • 1942: (6) Delphinium • 1943: (3) • 1947: (1) 1949: (9) Rocky Mountain Naturalists $\bullet 1951$ : (6) $\bullet 1954:(1)$

McCallum, G. A. San Jose State College, San Jose, CA, Dept. of Biological Sciences Correspondence $\bullet$ 1959: (2)

McCallum, John (1843-1918) Bioscript

McCan, Martha Nelson California Botanic Garden Correspondence • 1921-1928: (1)

McCann, Charles Correspondence $\bullet$ 1948: (2) • 1949: (1)

McCart, Wiliam L. North Texas State College, Denton Correspondence • 1958: (1)

McCarthy, Charles S. Taxidermist Bioscript

McCarthy, Gerald Bioscript

McCarty, Edward Clayton Bioscript

McChesney, James D. Indiana U., Bloomington, Dept. of Chemistry Correspondence $\bullet$ 1965: (3) 


\section{Guide to the Ewan Papers}

McClatchie, Alfred James (1861-1906) Phycologist, mycologist

Bioscript

McClelland, Joe U. of Arizona, Tucson Correspondence • 1967: (2)

McClintock, Barbara (1902-1992) Bioscript - Botanical Album

McClintock, Elizabeth May (1912-) Taxonomist, horticulturist; California Academy of Sciences, Dept. of Botany, associate curator

Bioscript • Botanical Album

Correspondence • 1943: (1) • 1944: (2) • 1945: (3) • 1946: (2) U. of California, Berkeley. Dept. of Botany $\bullet$ 1947: (1) • 1949: (5) • 1953: (1) • 1954: (1) • 1961: (3) - 1962: (1) • 1966: (1) - 1967: (3) - 1968: (4) Species - 1969: (2) • 1970: (1) • 1972: (2) • 1973: (1) - 1974: (3) $\bullet$ 1975: (3) • 1976: (1) $\bullet$ 1977: (2) $\bullet$ 1978: (3) $\bullet$ 1980: (11) • 1984: (5) • 1987: (1) • 1989: (2) • 1991: (2)

McClung, Clarence Erwin (1870-1946) American zoologist, cytologist Biofile

McClung, William J. U. of California Press Correspondence • 1975: (1)

MeClure, Charles R. Librarian; U. of Oklahoma, Western History Collection

Correspondence • 1972: (2)

McClure, Floyd Alonzo (1897-1970) American taxonomist, plant collector

Biofile $\bullet$ Bioscript $\bullet$ Botanical Album

Correspondence $\bullet$ 1968: (1) Species

McColl, W. R. (1855-1933)

Correspondence • 1929-1933: (7)

McComb, Fran

Correspondence • 1955: (1) • 1956: (1)

McComb, John C.

Correspondence • 1962: (1) • 1983: (1) • 1984: (1)

McConnell, Burt R. U.S.D.A. Wenatchee Research Center, Wenatchee, WA

Correspondence • 1963: (2) "Rocky Mountain Vegetation"

McConnell, John W.

Correspondence • 1947: (1)

MeConnell, Virginia F.

Botanical Album

Correspondence • 1968: (1)

McCook, Lucile U. of New Orleans, New Orleans Correspondence • 1980: (2) • 1982: (1) • 1991: (1)

MeCord, Paul P.

Correspondence $\bullet$ 1942: (1)

MeCorison, Marcus A. American historian and librarian; American Antiquarian Society, Worcester, MA

Biofile • Bioscript

Correspondence • 1973: (4) • 1975: (2) • 1976: (2) • 1978: (1) • 1979: (2) • 1981: (4) Pursh, Frederick, 1774-1820 • 1983: (1) • 1984: (7) Rocky Mountain Naturalists • 1985: (1) • 1986: (2) • 1988: (2) • 1989: (3) 1990: (2)

McCormick, Jack Academy of Natural Sciences of Philadelphia, Waterloo Mills Field Research Station Correspondence • 1968: (3) Species • 1970: (2)

McCouley, Charles Adam Hoke Bioscript
McCrae, W. F. Bioscript

McCullock, Thomas Bioscript

McCulloh, R. S. M. Bioscript

McCullough, James Haines, Jr. Bioscript

McCully, Bruce T. College of William and Mary Correspondence - 1968: (4) Banister, John, 1650-1692

McCutcheon, Roger P. Tulane U., New Orleans, U. Council on Research, Graduate School

Correspondence - 1948: (11) Tulane U. Dept. of Botany - 1951: (4) • 1952: (1)

McDade, Lucinda A. (1953-) Botanist Correspondence 1975: (6) Tulane U. Dept. of Botany - 1976: (1) • 1977: (2)

McDaniel, Joseph C. U. of Illinois, Urbana, Dept. of Horticulture

Correspondence $\bullet$ 1970: (3)

McDaniel, Sidney Thomas (1940-) American taxonomist Correspondence • 1958: (1) • 1962: (2) • 1963: (2) • 1966: (1) • 1967: (2) • 1977: (3) • 1991: (1)

McDermott, John Francis (1902-) American historian; Washington U., St. Louis, MO

Botanical Album

Correspondence $\bullet$ 1959: (11) • 1960: (4) • 1961: (16)

- 1962: (5) - 1963: (4) - 1964: (20) • 1965: (2) - 1966:

(1) $\bullet$ 1967: (5) • 1968: (1) • 1970: (2) • 1973: (1) $\bullet$ 1974:

(2) 1 1976: (1) $\bullet$ 1977: (1) $\bullet$ 1979: (1) $\bullet$ 1980: (3)

McDermott, Laura Frances (1882-1923) Bioscript

McDermott, Mary Stephanie Husband is John Francis McDermott

Correspondence $\bullet$ 1983: (1)

McDonald, Francis Eugene (1860-1920) American bryologist Biofile

McDonald, James Monroe (1825-1907) Bioscript

McDonald, Mamie New England Mutual Life Insurance Co. Correspondence • 1939: (1)

McDonald, Virginia Canadian Library Association, Microfilm Committee, Secretary; husband is John C. McDonald

Correspondence $\bullet$ 1951: (3)

McDougall, Walter Byron (1883-1980) American botanist, plant ecologist Bioscript

McDowell, Bart National Geographic Magazine, Senior Assistant Editor

Correspondence • 1987: (0) Filed under National Geographic Magazine

McDowell, Silas (1795-1879) American farmer, horticulturist

Biofile

McDuffie, Arthur F. (1884) Bioscript

McDunnough, James Halliday (1877-1962) Canadian entomologist; American Museum of Natural History Correspondence • 1949: (1) Rocky Mountain Naturalists 
McElvain, Samuel Marion (1897-?) American organic chemist

Biofile

McEuen, Thomas

Bioscript

McEwan Gallery Edinburgh

Correspondence 1981: (3) Book collectors

McEwan, Peter J. M.

Correspondence $\bullet$ 1980: (3)

McFarland, James Irwin U. of Colorado, Boulder, Classics Prof.

Bioscript - Botanical Album

McFarland, W. N. Cornell U., Div. of Biological Sciences Correspondence $\bullet$ 1967: (1)

McGah, Russella K. Los Angeles Arboretum, Librarian Correspondence • 1956: (1)

McGill U. R. Pennington, U. Librarian

Botanical Album

Correspondence $\bullet$ 1951: (1) $\bullet$ 1952: (1) $\bullet$ 1962: (2) •

1965: (2) - 1990: (2) Book collectors

McGourty, Frederick, Jr. Brooklyn Botanic Garden Correspondence • 1967: (6) Bartram, William, 1739-1823 - 1968: (3) Bartram, William, 1739-1823 • 1969: (3) • 1971: (5) • 1972: (2) • 1973: (1) • 1977: (2) • 1980: (1) - 1981: (1)

McGowan, Raymond E. St. Mary's Dominican College, New Orleans, LA., English Dept

Correspondence • 1977: (1) • 1986: (1) • 1989: (2)

McGraw-Hill Book Company Correspondence - 1940: (1)

McGraw-Hill, Inc. Jon Gillett, marketing manager Correspondence $\bullet$ 1970: (2)

McGregor, Ernest Alexander (1880-) Bioscript

McGregor, Marylee G. Colonial Williamsburg, research archivist

Correspondence • 1967: (2) Banister, John, 1650-1692

McGregor, Richard Crittenden (1871-1936) Australian ornithologist

Bioscript

McGregor, Ronald Leighton (1919- ) Taxonomist, bryologist, pteridologist; U. of Kansas, Lawrence, Dept. of Botany

Botanical Album

Correspondence $\bullet$ 1952: (2) $\bullet$ 1967: (1) $\bullet$ 1979: (2)

McGufin, W. C. National Museum, Ottawa, Division of Entomology

Correspondence • 1970: (1) Rocky Mountain Naturalists

McHale, Deborah Berry Gulfport, MS

Correspondence $\bullet$ 1951: (2) - 1952: (2)

Mcllhenny, Edmund Avery New Orleans, LA; Avery Island, LA

Bioscript $\bullet$ Botanical Album

Correspondence $\bullet$ 1962: Copy of letter from H. E.

Longenecker attached to letter from Melvin Finn -1973 :

(3) $\bullet 1974:$ (4) $\bullet 1975:$ (2)

McIlhenny, Virginia Garden Study Club of New Orleans Correspondence • 1976: (43)

McIllhenny, Walter S. Bioscript
McIntosh, Lachlan Bioscript

McIntosh, Robert P. U. of Notre Dame, Notre Dame, IN Correspondence • 1962: (1) • 1963: (3) • 1966: (3) • 1970: (2) Rocky Mountain Nanuralists • 1973: (1)

McKay, H. M. Johannesburg, S. Africa Correspondence • 1951: (3)

McKay, William (d. 1946) Bioscript

McKelvey, Susan Adams (Delano) (1883-1964) American botanist; Arnold Arboretum

Bioscript $\bullet$ Botanical Album

Correspondence • 1947: (1) • 1949: (3) Rocky Mountain Naturalists $\bullet$ 1950: (4) • 1951: (4) • 1953: (6) • 1956:

(4) - 1957: (2)

McKenrie, Gail Correspondence $\bullet$ 1991: (1)

McKevan, D. Keith McGill U., Quebec, Canada, Dept. of Entomology

Correspondence $\bullet$ 1978: (1)

McKinley, Daniel L. Botanical Album

Correspondence $\bullet$ 1962: (3) $\bullet 1965:(2) \bullet 1968:(1) \bullet$ 1969: (1) • 1974: (3) • 1979: (8) • 1982: (1) • 1983: (4) - 1984: (4) • 1985: (7) • 1986: (9) • 1987: (4) • 1988: (3) - 1989: (5) • 1990: (5) • 1991: (3) • 1992: (7) $\bullet$ 1993: (3)

McKinley, William W. Correspondence $\bullet$ 1944: (1)

McKinnell, Correspondence $\bullet$ 1970: (1)

McKinney, H. Lewis U. of Kansas, Lawrence, Dept. of History

Correspondence • 1969: (2) Book collectors • 1971: (3) • 1973: (2) • 1986: (1)

McKinney, Madison New Orleans, LA Correspondence • 1966: (2) • 1971: (1) • 1986: (2)

McLane, Bioscript

McLaren, John (1846-1943) Scottish-born American horticulturist, landscape gardener Biofile $\bullet$ Bioscript $\bullet$ Botanical Album

McLaughlin, Robert Penfield (1898-) U. of Tennessee, Knoxville, Dept. of Botany Correspondence $\bullet$ 1952: (2) $\bullet$ 1954: (4)

McLean County Court House (Hall of Records) Correspondence $\bullet 1949$ : (1)

McLean, Elizabeth P. Academy of Natural Sciences, Philadelphia Botanical Album Correspondence • 1981: (3) • 1982: (4) • 1983: (3)

McLean, F. P. Bioscript

McLean, Forman T. Torrey Botanical Club Correspondence - 1934: (1)

McLean, William L., III, Mrs. Wynnewood, PA Correspondence - 1978: (2)

McLellan, J. Ellis Collector of natural history specimens Biofile

McLeod, Norma Newcomb College Correspondence $\bullet$ 1966: (2) 


\section{Guide to the Ewan Papers}

McMahon, Bernard (c. 1775-1816) Philadelphia nurseryman

Biofile $\bullet$ Bioscript

MeMahon, Virginia Missouri Botanical Garden Correspondence - 1957: (1) Van Schaack, George Booth, 1903-1983

McMichael, Vicki Missouri Botanical Garden Library, library assistant

Correspondence • 1987: (2)

McMillen, Wheeler (1893-1992) American agricultural author Biofile

McMinn, Howard Ernest (1892-1963) Dendrologist, horticulturist Bioscript • Botanical Album

McMullin James B. Lancaster County Historical Society Correspondence • 1984: (1) • 1985: (2)

McMurtrie, Henry Bioscript

McNab, James (1810-1878) Botanist, plant collector Bioscript

MeNab, William (1780-1884) Scottish horticulturist Bioscript

MeNab, William Ramsay (1844-1889) Scottish-born botanist, physician

Biofile - Bioscript

McNair, A. J. U. of Colorado, Dept. of Civil Engineering Correspondence - 1953: (1) "Rocky Mountain Vegetation"

MeNair, Anna A. Husband is J. C. McNair Correspondence • 1949: (2) • 1950: (2) • 1952: (2)

McNair, J. C. Gulfport, MS Correspondence • 1951: (1)

McNair, James Birkley (1889-1967) Biochemist Bioscript

MeNair, Spencer B. Orleans Parish Medical Society, New Orleans, LA, Medical Museum Committee, Chairman Correspondence $\bullet$ 1962: (2)

McNeill, John Canadian taxonomist; U. of Ottawa, Canada, Dept. of Biology

Correspondence • 1984: (1) • 1985: (1)

McPeck, Eleanor "Eye of Thomas Jefferson Exhibition," consultant Correspondence • 1975: (4) • 1976: (1) Bartram, William, 1739-1823

McPeek, Gwynn Correspondence $\bullet$ 1955: (6)

McPherson, Gordon (1947-) Botanist Botanical Album Correspondence • 1993: (1)

McRee, James Fergus Bioscript

McReynolds, Scott W. Louisiana State U., Botany Dept., student worker Correspondence • 1976: (2) • 1980: (2)

McTaggart-Cowan, Ian Correspondence - 1981: (2) • 1982: (2)

McVaugh, Michael U. of North Carolina, Chapel Hill, Dept. of History Correspondence $\bullet$ 1986: (1)
McVaugh, Rogers (1909-) American taxonomist, botanical historian

Biofile $\bullet$ Bioscript $\bullet$ Botanical Album

Correspondence • 1942: (3) • 1943: (1) • 1946: (8) •

1947: (8) • 1948: (6) • 1949: (2) • 1950: (1) • 1951: (4)

- 1952: (3) • 1953: (8) • 1954: (2) • 1956: (12) 1 1958:

(1) 1960: (1) 1 1961: (1) Letter from Nordin-Petterson to H. Bartlett $\bullet$ 1962: (6) $\bullet$ 1963: (10) $\bullet$ 1965: (3) $\bullet$ 1966: (1) - 1969: (1) • 1973: (2) • 1974: (2) • 1975: (1) • 1976: (4) Merrill, Elmer Drew, 1876-1956 • 1979: (3) • 1980: (1) • 1983: (1) • 1985: (1) • 1987: (3) • 1988: (1) • 1990: (5) • 1991: (2) • 1992: (4) • 1993: (1)

McWilliams, Edward L. Iowa State U., Ames, Dept. of Horticulture, graduate assistant Correspondence • 1965: (2) • 1965: (2) • 1969: (1) • 1970: (1)

Mead, E. J. Louisiana Forestry Commission, Hammond Correspondence $\bullet$ 1952: (0) Letter filed under Koch, Minna Frotscher

Mead, Margaret American anthropologist Biofile

Mead, Samuel Barnum (1798-1880) Botanist Bioscript $\bullet$ Botanical Album

Mead, Theodore Luqueer (1852-1936) Entomological collector on Wheeler survey (1882), orchid hybridizer Biofile • Bioscript

Meadows, Don Botanical Album

Mearns, Barbara C. Dumphries, Scotland Correspondence - 1989: (2) Letter from J. Ewan to Andrew Richford re: Biographies for Bird Watchers • 1990: (6) • 1991: (8)

Mearns, David C. Library of Congress Correspondence $\bullet$ 1958: (2) • 1968: (1)

Mearns, Edgar Alexander (1856-1916) American naturalist, army surgeon

Bioscript • Botanical Album

Mearns, Richard Botanical Album

Correspondence • 1990: (0) Filed under Barbara C. Mearns - 1991: (4) • 1992: (6) • 1993: (5)

Mears, Helen Correspondence 1975 : (1)

Mears, James Austin (1944 ) American botanist, botanical historian; Academy of Natural Sciences in Philadelphia Biofile $\bullet$ Bioscript $\bullet$ Botanical Album Correspondence $\bullet$ 1975: (3) $\bullet$ 1977: (2) $\bullet$ 1980: (5) $\bullet$ 1981: (2) • 1982: (1) • 1983: (1) Missouri Botanical Garden

Meats, R. W. National Research Council; U.S. Dept. of Agriculture

Correspondence • 1946: (1)

Meckler Publishing Correspondence • 1985: (1)

Medikus, Fredric Kasimir (1736-1808) Bioscript

Medley, Max E. U. of Louisville Correspondence • 1991: (1)

Medway, David G. New Plymouth, New Zealand Correspondence $\bullet$ 1974: (1) $\bullet$ 1980: (1) 
Mee, Margaret Ursula (1909-1988) English-born Brazilian botanical artist; husband is McKee Mee Biofile $\bullet$ Bioscript $\bullet$ Botanical Album Correspondence $\bullet$ 1985: (5) Letters to Peter Raven

Meehan, S. Mendelson Ellis Arboretum, West Chester, PA

Correspondence $\bullet$ 1955: (2)

Meehan, Thomas (1826-1901) English-born American nurseryman, botanist, horticultural author Bioscript $\bullet$ Botanical Album

Mehlquist, Gustav A. L. (1906-) Swedish-born plant geneticist, horticulturist, cytologist; U. S. A. Bioscript Correspondence - 1939: (2) Delphinium • 1940: (1) • 1941: (4) Delphinium; letter from Carl Epling • 1942: (1)

Mehrhoff, Loyal A. Bishop Museum Correspondence • 1991: (1)

Mehring, Albert G. Pennsylvania Historical and Museum Commission Correspondence $\bullet$ 1986: (1) • 1987: (1)

Meigs, Peveril Correspondence • 1936: (2)

Meijer, Willem (1923-) Dutch bryologist, plant ecologist Correspondence • 1993: (1)

Meinkoth, Norman Botanical Album

Meisel, Blanche Miami Beach, FL; husband is Max Meisel Correspondence • 1969: (8) • 1970: (4) • 1971: (2) • 1974: (2) • 1975: (1)

Meisel, Max (1892-1969) Bibliographer Bioscript Correspondence $\bullet$ 1946: (2) $\bullet$ 1957: (1) $\bullet$ 1968: (2) Science-Instruction and study $\bullet$ 1969: (5) Letters with Mrs. Max Meisel

Meisner, Carl Friedrich (1800-1874) Swiss physician, botanist, taxonomist Bioscript

Melan, Melissa Correspondence • 1976: (0) Filed under Lori Schoen

Melhus, Irving E. (1881-1969) American plant pathologist, economic botanist Correspondence • 1937: (1)

Mellanby, Kenneth Bioscript

Mellichamp, Joseph Hinson (1829-1903) Bioscript

Melsheimer, Frederick Valentine (1749-1814) American clergyman, educator, entomologist Biofile $\bullet$ Bioscript

Melvill, James Cosmo (1845-1929) British businessman, botanist Biofile $\bullet$ Botanical Album

Menard County Courthouse Correspondence • 1949: (1)

Mencken, H. L. Author Bioscript

Mendel, Gregor Johann (1822-1884) Czech plant geneticist, clergyman Biofile • Botanical Album
Mendelsohn, Everett Harvard U., Journal of the History of Biology, Editor

Correspondence - 1968: (4) Science-Instruction and study - 1971: (5) • 1972: (2) • 1974: (3) • 1976: (1) • 1977: (3)

- 1978: (2) • 1979: (2) • 1980: (4) • 1981: (3) • 1982: (2)

- 1988: (2) • 1990: (1) • 1991: (1)

Mendenhall, John

Bioscript

Mendes da Costa, Emanuel

See Costa, Emanuel Mendes da (1717-1791)

Meneray, Jane Yeuell

Correspondence $\bullet$ 1972: (1)

Meneray, Wilbur E. Tulane U., Howard-Tilton Memorial Library, Rare Books and Mamuscripts

Botanical Album

Correspondence $\bullet$ 1973: (1) $\bullet$ 1977: (2) Letters from and to Joseph Ewan from Fred W. Hanes • 1982: - 1984: (3) - 1985: (1) • 1986: (4) • 1987: (2) • 1988: (5) • 1990: (1) $\bullet$ 1992: (1) • 1993: (1)

Mengel, Robert M. U. of Kansas, Lawrence, Dept. of Zoology

Correspondence $\bullet$ 1973: (1)

Menke, Herman F. Andrews Seed Company Shippers, Harrah, WA, entomologist and manager

Correspondence $\bullet$ 1953: (2)

Mennega, Alberta Maria Wilhelmina (1912-) Dutch wood anatomist; Botanisch Museum en Herbarium Van de Rijksuniversitei te Utrecht Botanical Album Correspondence • 1964: (3)

Menninger, Edwin A. Botanical Album

Menter, J. E. Correspondence $\bullet$ 1942: (2)

Menzies, Archibald (17541842) British naturalist, plant explorer

Biofile $\bullet$ Bioscript

Menzies, James I. Zoologist; U. of Papua and New Guinea at Boroko

Botanical Album

Correspondence • 1971: (4) • 1975: (1) • 1976: (1)

Book collectors

Mérat, François Victor (1780-1851)

Bioscript

Mercer, John (17041768) Virginia lawyer and planter Biofile

Mercer, Samuel A. B.

Correspondence • 1958: (1)

Mercier, (Marie) Philippe (1781-1831) French botanist Bioscript

Merck, Carl Bioscript

Merckling, J. O. Pan American World Airways System Correspondence $\bullet$ 1945: (2)

Merian, Maria Sibylla (1647-1717) Dutch artist in Surinam Biofile $\bullet$ Bioscript

Meriden-Stinehour, Inc.

Correspondence 1977 : (0) Announcement of merger of Stinehour Press with Meriden Gravure

Meriwether, Margaret U. of South Carolina, Library Correspondence $\bullet$ 1956: (1) 


\section{Guide to the Ewan Papers}

Merriam, Clinton Hart (1855-1942) American biologist, ethnologist Biofile $\bullet$ Bioscript $\bullet$ Botanical Album

Merriam, G. \& C. Company Correspondence $\bullet$ 1947: (3)

Merriam, John Campbell (1869-1945) Bioscript • Botanical Album

Merrill, Augusta Husband is E. D. Merrill Correspondence • 1956: (3)

Merrill, Charles Botanical Album Correspondence $\bullet$ 1972: (2)

Merrill, Elmer Drew (1876-1956) American botanist, bibliographer; Arnold Arboretum Biofile $\bullet$ Bioscript $\bullet$ Botanical Album Correspondence • 1939: (2) Arnold Arboretum $\bullet$ 1945: (2) • 1946: (1) • 1947: (2) • 1949: (1) • 1950: (5) • 1951: (4) Pursh, Frederick, 1774-1820 1952: (12) Pursh, Frederick, 1774-1820 • 1953: (7) Arnold Arboretum • 1954: (7) • 1955: (1)

Merrill, John Harvard Correspondence $\bullet$ 1942: (1)

Merrill, Luella B. Sec. to George Lawrence Correspondence • 1958: (1)

Merrill, Nettie M. Belmont High School, Los Angeles Correspondence • 1921-1928: (1) • 1943: (3) • 1950: (3) - 1952: (1) - 1967: (1) • 1968: (1) • 1970: (1) • 1976: (1)

Merrill, William L. Correspondence $\bullet$ 1973: (6)

Merriman, Paul Rossiter (1882-) American botanist Biofile

Merritt, Alice Jane Bioscript

Merry, James Bioscript

Merry, __, Miss Bioscript

Merserean, H. E. Correspondence $\bullet$ 1950: (1)

Merseyside County Council Liverpool Correspondence $\bullet 1984$ : (4)

Mertens, Franz Carl (1764-1831) German botanist Bioscript

Mertens, Robert Senckenbergisch Naturforschende Gesellschaft, Frankfurt

Correspondence • 1958: (2)

Mertz, Henry Ney (1846-1926) American botanist Bioscript

Merxmilller, Hermann (1920-1988) German botanist Bioscript

Merz, ___ Pharmazeutischen Institut der Universität, Freiburg Correspondence $\bullet 1951$ : (1)

Meserve, Mary Fleishman Botanical Album

Messmer, W. A. German physician, botanist; Debrille Chemical Corporation Correspondence • 1941: (2)

Metcalf, Bioscript
Metcalf, Franklin P. Botanical Album

Metcalf, Maurice A.

Correspondence • 1958: (1)

Metcalfe, Orrick Baylor (1879-1936) Botanist, ornithologist Bioscript

Mettenius, Georg Heinrich (1823-1866) German physician, botanist

Bioscript

Metzinger, Sylvia V. Tulane U., Howard-Tilton Memorial Library

Correspondence $\bullet$ 1982: (2) $\bullet$ 1984: (5) Book collectors - 1985: (3) • 1986: (1) • 1987: (4) Letters with Charles Nelson • 1988: (2) • 1989: (1) Book collectors

Mexia, Ynes Enriquetta J. R. (1870-1938) American botanist, plant collector in Mexico

Biofile $\bullet$ Bioscript $\bullet$ Botanical Album

Correspondence $\bullet$ 1938: (1)

Mexico, Sociedad Botánica de See Sociedad Botánica de Mexico

Meyen, Franz Julius Ferdinand (1804-1840) German physician, botanist Bioscript

Meyer, A. J. Bioscript

Meyer, C. A. Bioscript

Meyer, Carl Valentine Bioscript

Meyer, Dieter Erich (1926) German botanist Bioscript

Meyer, Ernst Heinrich Friedrich (1791-1851) Prussian botanist, botanical historian Biofile

Meyer, Frank Nicholas (1875-1918) Dutch-born plant hunter; U.S.D.A. Biofile • Bioscript

Meyer, Frederick Gustav (1917- ) American taxonomist; National Arboretum

Bioscript $\bullet$ Botanical Album

Correspondence $\bullet$ 1937: (2) $\bullet$ 1953: (1) $\bullet$ 1956: (1) $\bullet$ 1965: (1) - 1967: (3) - 1968: (5) Also see Eric William Groves • 1969: (6) • 1970: (13) also see letter filed under John Milton Fogg, Jr. $\bullet$ 1971: (2) • 1972: (13) $\bullet$ 1973: (5) - 1974: (5) • 1975: (1) • 1976: (4) • 1977: (2) • 1979: (2) - 1980: (8) • 1981: (3) Species • 1982: (1) • 1983: (4) $\bullet$ 1984: (1) • 1985: (2) • 1986: (1) • 1987: (3) • 1988: (5) • 1989: (5) Letters of Meyer with Dale Johnson included 1990: (5) • 1991: (2) • 1992: (3) • 1993: (4)

Meyer, K. F. U. of California, San Francisco, Medical Center, Director Emeritus Correspondence $\bullet$ 1970: (2)

Meyer, Marvin Clinton U. of Maine, Orono Correspondence $\bullet$ 1956: (1)

Meyer, Teodoro (1910- ) Argentine taxonomist; Instituto Miguel Lillo, Tucumán, Argentina Correspondence $\bullet$ 1967: (1) $\bullet$ 1970: (1)

Meyers, Amy R. The Huntington Library, historian Botanical Album • 1984: (3) • 1987: (3) • 1988: (5) 1989: (7) • 1990: (12) • 1991: (5) • 1992: (1) • 1993: (5) 
Meyers, George

Correspondence $\bullet$ 1981: (1)

Miall, Louis Compton (1843-1921) English botanist Biofile

Miasek, Meryl A. New York Botanical Garden, Interlibrary Loans

Correspondence • 1972: (1)

Michael, Peter William U. of Sydney, Dept. of Agronomy Correspondence $\bullet$ 1974: (2)

Michaux, André François (1746-1803) French botanist Biofile $\bullet$ Bioscript $\bullet$ Botanical Album

Michaux, François (1770-1855) French botanist; son of André Miçhaux Biofile

Michaux Memorial Arboretum Correspondence • 1979: (1)

Michel, F. A. W. Botanical Album

Michel, R. National Audubon Society, New York, NY, Librarian Correspondence • 1962: (1) • 1962: (0) Letter attached to Leonard Hall correspondence

Michels Books Correspondence 1981 : (1) Book collectors

Michener, Charles A. Bioscript

Michener, Ezra (1794-1887) American physician, natural historian, plant collector, conchologist Biofile $\bullet$ Bioscript

Michigan Botanist Correspondence $\bullet$ 1987: (1) • 1993: (1)

Mickel, John Thomas (1934-) American taxonomist, plant morphologist; Editor of Brittonia; New York Botanical Garden

Botanical Album

Correspondence • 1975: (1) • 1983: (1)

Mickey, George H. Louisiana State U., Dept. of Zoology, Chairman

Correspondence • 1959: (2)

Micro Methods Limited

Correspondence • 1964: (1)

Middelmann, Walter

Correspondence - 1990: (3)

Middlebury College Archivist

Correspondence 1983: (1) 1984: (5)

Middleton, Margaret Canton High School, Canton, IL Correspondence - 1945: (1)

Midlo, Charles (-1974) Physician, collector of book plates; New Orleans

Bioscript • Botanical Album

Correspondence • 1959: (2) • 1960: (1) • 1968: (1)

Book collectors $\bullet$ 1973: (1) $\bullet$ 1974: (1)

Midlo, Natalie Husband is Charles Midlo Correspondence • 1965 • 1972: (1) • 1974: (2)

Miers, John

Bioscript $\bullet$ Botanical Album

Mifflin, Thomas

Bioscript

Mikan, J. G.

Bioscript
Mikosch, Elisabeth

Botanical Album

Correspondence • 1986: (3)

Milam, "Georgia Tulane Medical School, Tulane U., New Orleans, LA

Correspondence $\bullet$ 1966: (2) Species

Milbert, Jacques Gérard (1766-1840) French traveller. collector of animals and plants Biofile

Miles, Oliver B.

Correspondence $\bullet$ 1963: (1)

Milius, Susan Swarthmore College Correspondence • 1975: (1)

Millar, John Randolph (1899-) Plant collector. administrator; Chicago Natural History Museum, Deputy Director

Correspondence • 1948: (1) • 1949: (1) • 1951: (1) • 1954: (3) • 1955: (1) • 1962: (1) • 1963: (2) • 1969:

(0) Filed under Richard Alden Howard

Millardet, Pierre Marie Alexis (1838-1902) French physician, botanist

Biofile

Miller, Alden Holmes (1906-1965) American vertebrate zoologist; son of Loye Holmes Miller

Bioscript $\bullet$ Botanical Album

Correspondence • 1941: (3) • 1949: (1)

Miller, Alfred A. New York, NY; nephew of Nada Kramar Correspondence $\bullet$ 1976: (2)

Miller, Annetta Correspondence • 1991: (1)

Miller, Carl F. Correspondence • 1974: (2)

Miller, Elizabeth Rachel Correspondence $\bullet$ 1940: (1)

Miller, Elliott U. of Colorado, Boulder, student Correspondence • 1940: (3) • 1941: (2) • 1942: (3) • 1943: (6) • 1945: (1) • 1945: (2) • 1946: (3)

Miller, Ethel M. Correspondence $\bullet$ 1945: (1)

Miller, Frances Correspondence $\bullet$ 1967: (2)

Miller, Gretchen Correspondence • 1983: (1) Van Schaack, George Booth. 1903-1983

Miller, Horace P. Larchmont, NY; wife is Hortense S. Miller Correspondence $\bullet 1966:$ (1) $\bullet 1971:$ (1)

Miller, Hortense S. Librarian, bibliographer Botanical Album Correspondence • 1964: (6) • 1965: (4) • 1966: (12) • 1967: (10) • 1968: (16) • 1969: (5) • 1970: (2) • 1974: (6) 1975: (2) • 1978: (2) - 1979: (2) Barton, Benjamin Smith, 1766-1815 • 1980: (2)

Miller, Kathryn N. U. of Chicago, Library Correspondence -1929-1933: (1)

Miller, Kermit Correspondence $\bullet$ 1929-1933: (1)

Miller, L. Purdue U., Lafayette, IN, Dept. of Botany Correspondence $\bullet$ 1967: (1)

Miller, Lillian Wood (1937-) Bioscript 


\section{Guide to the Ewan Papers}

Miller, Loye Holmes (1874-1970) American naturalist, botanist; U. of California, Los Angeles; nickname "Padre" Biofile - Bioscript • Botanical Album

Correspondence • 1936: $\bullet$ 1940: (4) $\bullet$ 1943: (1) $\bullet$ 1946:

(3) U. of California, Los Angeles. Dept. of Botany Species - 1967: (1) - 1968: (4) Jepson, Willis Linn, 1867-1946; Setchell, William Albert, 1864-1943 • 1969: (2) Book collectors

Miller, Philip (1691-1771) English botanist, horticulturist Biofile $\bullet$ Bioscript $\bullet$ Botanical Album

Miller, Richard F.

Correspondence • 1929-1933: (2)

Miller, Robert C. California Academy of Sciences Correspondence - 1939: (1) Eastwood, Alice, 1859-1953 - 1944: (2) • 1945: (1) • 1953: (2)

Miller, Robert R. U. of Michigan, Ann Arbor, Museum of Zoology

Correspondence • 1973: (1)

Miller, Robert Ryal California State U., Berkeley, CA, Prof. Emerius of Latin American History

Botanical Album

Correspondence • 1978: (4) • 1981: (2) • 1982: (5)

- 1983: (1) • 1984: (3) • 1986: (2) • 1987: (2) • 1988: (1) $1989:(5)$

Miller, Scott E. Santa Barbara Museum of Natural History, Santa Barbara, CA, Dept. of Invert. Zoology Correspondence • 1977: (3)

Miller, Thomas

Bioscript

Miller, William J. Geologist; U. of California, Los Angeles

Correspondence • 1929-1933: (1) • 1939: (3) Species

Milligan, Carolyn B. American Philosophical Society, Librarian

Correspondence • 1974: (4)

Millin, A. L. Bioscript

Mills, Cynthia National Portrait Gallery, Dept. of Painting and Sculpture, Research Assistant

Correspondence • 1989: (0) Filed under National Portrait Gallery

Mills, Grace S.

Correspondence • 1945: (4)

Mills, Richard R. Entomologist; Tulane U. Correspondence $\bullet$ 1965: (4)

Millspaugh, Charles Frederich (1854-1923) Botanist; Field Museum

Bioscript • Botanical Album

Milne, Edwards $\mathbf{H}$. Bioscript

Milne-Redhead, Edgar Wolston Bertram Handsley (1906-) British taxonomist Biofile

Milner, James Wood (1841-1879) Ichthyologist Bioscript

Milstead, Wayne Lavine (1932-) Taxonomist; Purdue U. Correspondence $\bullet$ 1964: (1)

Mindlin, Jose Botanical Album

Miner, Eunice Thomas New York Academy of Sciences Correspondence • 1952: (3)
Miner, Tamara A. Rice U., Houston, TX, Dept. of History, graduate student Correspondence $\bullet$ 1979: (4) $\bullet$ 1980: (4)

Minnich, D. E. U. of Minnesota, Minneapolis Correspondence $\bullet$ 1958: (1)

Minor, William Cleo (1903-) Amateur naturalist, ranchman; Fruita, $\mathrm{CO}$ Correspondence • 1949: (3) Rocky Mountain Naturalists

Minthorn, Maude (1883-1966) See Minthorn, Theodore (1886-1967)

Minthorn, Theodore (1886-1967) California nurseryman, botanist

Biofile • Bioscript

Minto, Katherine V. Botanical Album Correspondence $\bullet$ 1939: (1) Species $\bullet$ 1940: (3) • 1941: (1)

Mintz, Joy Longue Vue Gardens, New Orleans, LA Correspondence • 1979: (1) • 1981: (1)

Miquel, Friedrich Anton Wilhelm (1811-1871) Dutch physician, botanist Biofile • Bioscript

Miramon, Charles J. City of New Orleans, Health Dept., Bureau of Public Health Sanitation Correspondence $\bullet$ 1957: (1)

Mirbel, Charles Françios Brisseau de (1776-1854) French botanist Bioscript

Mirov, Nicholas Tihomitorich (1893-1980) Botanical Album Correspondence $\bullet$ 1979: (1)

Mississippi Academy of Sciences Botanical Album

Missouri Botanical Garden St. Louis Bioscript • Botanical Album Correspondence • 1976: (5) • 1977: (3) • 1978: (1) 1983: (3) • 1984: (2) • 1987: (3) • 1990: (2) $1991:$ (11)

Mitchell, Adelphia Correspondence $\bullet$ 1937: (1)

Mitchell, Elisha (1793-1857) American naturalist Bioscript

Mitchell, John Correspondence • 1971: (1)

Mitchell, John (1711-1768) Virginia-born physician, naturalist Biofile • Bioscript

Mitchell, John ( -1772) Bioscript

Mitchell, Joseph C. U. of Richmond, VA, Research Biologist and Chairman, VMNH Editorial Board Correspondence $\bullet$ 1987: (3)

Mitchell Library Sydney, Australia; Suzanne Mourot Correspondence • 1974: (2)

Mitchell, Michael Edward (1934) Lichenologist; U. College, Galway, Ireland, Dept. of Botany Botanical Album Correspondence $\bullet 1977:$ (5)

Mitchell, Palmyre Louise de Chateaudun (1880-1968) American botanist Biofile 
Mitchell, Thomas Livingston (1792-1865) Plant collector; Australia

Bioscript

Mitchill, Samuel Latham (1764-1831) American physician, naturalist

Biofile $\bullet$ Bioscript

Mitich, Larry W. State U. Station, Fargo, ND, Associate Professor Correspondence • 1969: (5) Henry E. Huntington Library and Art Gallery $\bullet 1970:(2) \bullet 1972:$ (3)

Mitten, William (1819-1906) Bryologist Bioscript

Mittkowski, Richard Correspondence • 1949: (1) Rocky Mountain Naturalists

Mivart, St. George Bioscript

Mix, Grace R. Southern California Audubon Society; husband is Arthur J. Mix

Correspondence • 1921-1928: (1)

Mixon, James E. Louisiana Forestry Commission, State Forester

Correspondence - 1956: (2)

Miyabe, Kingo (1860-1951) Japanese taxonomist, plant ecologist

Bioscript

Mizell, Merle Tulane U., Dept. of Biology Correspondence • 1992: (1)

Mizelle, John D. U. of Notre Dame, American Midland Naturalist

Correspondence • 1946: (1)

Mociño, Jose Mariano (1757-1819) Spanish botanist See also Sessé y Lacasta, Martin de Biofile

Mockel, Henry R. (1905-) German-born botanical artist; U. S. A.

Bioscript $\bullet$ Botanical Album

Moe, Henry Allen John Simon Guggenheim Memorial Foundation, New York, NY

Correspondence $\bullet$ 1953: (1) • 1954: (1) • 1955: (3) • 1956: (6) • 1957: (5) • 1958: (1) • 1959: (3) • 1962: (1) - 1963: (1)

Moe, John H. Physician; Minneapolis, MN Correspondence - 1961: (1)

Moench, Conrad (1744-1805) German botanist, chemist, pharmacist Biofile

Moffitt, James (1900-) California museum curator, ornithologist Biofile

Mofras, de See DuFlot de Mofras, Eugène (1810-1884)

Moggridge, Johann Traherne (1842-1874) English botanist, botanical artist, entomologist Bioscript

Mohlenbrock, Robert H. (1931-) American taxonomist; Southern Illinois U., Carbondale

Botanical Album

Correspondence • 1966: (2) • 1967: (1)
Mohr, Charles Theodore (18241901) German-born American botanist, chemist, pharmacist Biofile $\bullet$ Bioscript $\bullet$ Botanical Album

Correspondence $\bullet$ 1978: (2) Correspondence with Barney Lipscomb re: Mohr

Mohr, Frederick Bioscript

Mohr, Karl Theodor See Mohr, Charles Theodore (18241901)

Mohr, P. F. Bioscript

Mohr, Thekla Bioscript

Mohr, Warren David Botanical Album

Moin, Alfred K. Friends of the Cabildo; Newcomb College Correspondence • 1954: (1) • 1957: (4)

Moldenado, Manuel Instituto Panamericano de Geografia e Historia, Mexico City, Mexico

Correspondence $\bullet$ 1962: (1)

Moldenke, Harold Norman (1909-) American taxonomist, plant historian

Botanical Album

Correspondence $\bullet$ 1939: (1) Species • 1941: (1) • 1946:

(3) - 1948: (2) • 1949: (11) • 1950: (5) • 1951: (13) • 1952: (1) 1956: (1) - 1958: (1) 1960: (1) 1961: (5) - 1962: (0) Letter to Ewan included in George Ralph Cooley correspondence $\bullet 1962$ (1) • 1963: (3) • 1967: (9) • 1968: (4) Species • 1978: (3) • 1986: (1)

Molkenberg, J. H.

Bioscript

Monachino, Joseph Vincent (1911-1962) Italian-born taxonomist; New York Botanical Garden Biofile Correspondence $\bullet$ 1951: (3) • 1952: (4)

Monks, Sara Preston Bioscript

Monnet, Paul-Louis ( -1915) Bioscript

Monro, Alexander (1733-1817) Bioscript

Monroe, Henry F. Bioscript

Monroe, Robert D. U. of Washington, Seattle, Rare Book Librarian Correspondence $\bullet$ 1979: (1)

Monroe, Rose M. Longue Vue Gardens Correspondence $\bullet$ 1970: (0) Filed under Longue Vue Gardens • 1973: (1)

Monson, Alfred S. Bioscript

Monsted, Charles N. Correspondence • 1956: (2) • 1986: (1)

Monsted, Lucile S. New Orleans Town Gardeners; husband is Charles Monsted

Correspondence $\bullet$ 1957: (3)

Montague, Henry de la Bozeman, MT Correspondence 1976: (2) Merrill, Elmer Drew, 1876-1956 


\section{Guide to the Ewan Papers}

Montagne, Jean Pierre François Camille (1784-1866) French botanist, physician

Bioscript

Montgomery, B. Elwood Entomologist; Purdue U., Dept. of Entomology, Professor Emeritus

Correspondence • 1979: (2)

Montgomery, C. E. Northern Illinois State Teachers College

Correspondence $\bullet$ 1939: (2) $\bullet$ 1940: (1)

Montgomery, Frank A., Jr. Correspondence • 1939: (1)

Montgomery, Joseph W. United Fruit Company, Boston, MA

Correspondence • 1948: (0) Filed under Samuel Zemurray

Montgomery, R. B. Gypsum, $\mathrm{OH}$ Correspondence • 1946: (1) American Fern Society • 1949: (1)

Montgomery, William ISIS Correspondence $\bullet$ 1982: (1)

Montiel, Olga Martha Missouri Botanical Garden Correspondence $\bullet$ 1993: (2)

Montreal Botanical Garden Les Floralies Internationales de Montréal Correspondence $\bullet$ 1979: (2)

Montz, Glen N. U. S. Army Corps of Engineers, New Orleans, LA

Correspondence $\bullet$ 1972: (1) • 1973: (4) • 1975: (2) • 1976: (4)

Moody, James W. T. Greenville College; Lake Superior State U. Correspondence $\bullet$ 1968: (3) $\bullet$ 1992: (1)

Mooney, Harry Denver Public Library, Denver, CO Correspondence • 1979: (1) Barton, Benjamin Smith, 1766-1815

Moore, Alexander Bioscript

Moore, Caroline Correspondence • 1986: (1)

Moore, Donald E. Bioscript

Moore, Dwight Munson (1891-1985) American biologist; U. of Arkansas, Dept. of Botany

Bioscript $\bullet$ Botanical Album

Correspondence $\bullet$ 1939: (4) Delphinium $\bullet$ 1940: (2) $\bullet$ 1941: (3) • 1942: (4) • 1944: (1) • 1946: (3) American Fern Society • 1948: (1) American Fern Society • 1949 (3) 1954: (2) American Fern Society

Moore, E. G. Bureau of Plant Industry Correspondence • 1942: (2) • 1963: (0) Filed under Albert Charles Smith

Moore, E. J.S. Bioscript

Moore, Enid G. Aldus Books Limited Correspondence • 1970: (2) Pursh, Frederick, 1774-1820

Moore, Frederick G. Correspondence - 1993: (2) Book collectors

Moore, George A. T. Correspondence $\bullet 1938:(2)$
Moore, George Thomas (1871-1956) American plant physiologist, algologist; Missouri Botanical Garden

Biofile $\bullet$ Botanical Album

Correspondence • 1939: (1)

Moore, Gladys

Correspondence • 1962: (2) • 1974: (1)

Moore, Harold Emery, Jr. (1917-1980) Taxonomist, plant explorer, and collector; Cornell U., Ithaca, Bailey

Hortorium

Botanical Album

Correspondence - 1961: (1) • 1965: (2) 1967: (1) •

1971: (5) • 1972: (1) • 1974: (1) • 1977: (2)

Moore, J. Percy Academy of Natural Sciences, Philadelphia Correspondence • 1943: (2) • 1952: (0) Filed under Francis Harper

Moore, John A. U. of California, Riverside Correspondence • 1975: (2)

Moore, John Adam (1908-1962) American taxonomist; Louisiana Polytechnic Institute, Ruston, LA

Bioscript $\bullet$ Botanical Album

Correspondence • 1956: (36) • 1957: (5) • 1958: (4) • 1959: (5) $\bullet$ 1960: (3) Biology-Instruction and study - 1962: (2) • 1963: (2)

Moore, John Adam, Mrs. Correspondence $\bullet$ 1975: (1)

Moore, John Alexander Columbia University, NY, Dept. of Zoology Correspondence $\bullet$ 1965: (2)

Moore, John M. Friends Historical Association, Swarthmore College, Swarthmore, PA; wife is Margaret Moore

Correspondence - 1979: (3) Barton, Benjamin Smith, 1766-1815 • 1986: (1)

Moore, John, Mrs. Correspondence - 1968: (2)

Moore, John Preston Louisiana State U., Dept. of History Correspondence $\bullet$ 1960: (1)

Moore, Julie

Correspondence • 1972: (1) • 1973: (1) • 1974: (1)

- 1977: (1)

Moore, Lucy Beatrice (1906-1987) New Zealand botanist Correspondence $\bullet$ 1956: (3)

Moore, Margaret Claremont, CA Correspondence • 1958: (1) • 1959: (1)

Moore, Mary Meek Atkeson Husband is Blaine Free Moore Correspondence • 1942 (2) • 1943: (3) • 1945 (2) 1947 (1)

Moore, Patricia A.

Correspondence $\bullet$ 1992: (3) Letter from Martha Riley

Moore, Terry The 600 Club of bird watchers Correspondence • 1976: (1) • 1978: (1) • 1979: (2) - 1981: (1)

Moore, Thomas Bioscript

Moore, Walter G. Loyola U., New Orleans; New Orleans Academy of Sciences Correspondence $\bullet$ 1949: (2) $\bullet$ 1950: (1) $\bullet$ 1952: (4) Letter to E. C. Stakman attached 1953: (3) 1971 : (3)

Moosig, Steuerrath Moravian lichen collector Bioscript 
Moquin-Tandon, Alfred (1804-1863)

Bioscript

Mora, Alberto Manuel Brenes (1870-1945) Biofile

Moran, Reid Venable (1918- ) American taxonomist, plant collector; Natural History Museum, Balboa Park, San

Diego, CA

Bioscript - Botanical Album

Correspondence • 1943: (2) • 1946: (2) • 1949: (2)

- 1950: (5) • 1951: (5) • 1952: (1) • 1958: (3) • 1959: (2)

- 1960: (6) • 1961: (4) • 1963: (2) • 1965: (4) • 1966: (1)

- 1972: (3) • 1974: (1) • 1976: (2) • 1978: (2) • 1979: (2)

- 1981: (2) • 1982: (1) • 1983: (5) • 1984: (2) $\bullet$ 1985: (2)

- 1990: (1)

Moran, Robbin Craig (1956-) Pteridologist; Missouri Botanical Garden, St. Louis, MO

Biofile $\bullet$ Bioscript $\bullet$ Botanical Album

Correspondence • 1988: (1) • 1990: (1) • 1992: (1) • 1993: (1)

Moravian Church Archives Winston-Salem, NC Correspondence $\bullet$ 1952: (1)

More, (Moore?) , Mr. Bioscript

More, Robert E. Attorney, amateur botanist; Denver, CO Correspondence • 1940: (3) • 1942: (32) • 1943: (3) • 1944: (2) • 1945: (1)

More, Thomas Bioscript

More, Thomas, Sister, O. P. Dominican Sisters, Columbus, $\mathrm{OH}$

Correspondence $\bullet$ 1961: (1)

Morel, Vera Walker Medical artist; New Orleans Botanical Album

Correspondence • 1956: (1) $\bullet$ 1960: (2) • 1961: (4) - 1962: (2) • 1963: (3) - 1964: (2) • 1965: (7) $\bullet 1966:$ $(7) \bullet 1967:(2) \bullet 1968:(6) \bullet 1969:(2) \bullet 1970:(2) \bullet$ 1972: (1)

Moreton, C. Oscar Botanical Album

Moretti, Giuseppe (1782-1835) Italian botanist Biofile Bioscript

Morgan, B. Berry Port Gibson, MI; husband is geologist Lee Morgan Botanical Album Correspondence $\bullet$ 1965: (1) • 1966: (2)

Morgan, Charles Bioscript

Morgan, Dale Lowell U. of California, Berkeley, Bancroft Library

Biofile

Correspondence • 1964: (4) • 1965: (5) • 1966: (1) - 1968: (1)

Morgan, E. K. Husband is Henry G. Morgan Correspondence - 1964: (1)

Morgan, Edward P. Broadcast journalist; American Broadcasting Company, Washington, D.C. Correspondence - 1969: (2)

Morgan, John (1735-1789) American physician Biofile

Morgan, Katherine East Orange, NJ Correspondence • 1946: (1)
Morgan, Kathleen

Correspondence • 1949: (1)

Morgan, Kathryn Librarian Correspondence • 1986: (1) • 1992: (1) • 1993: (7)

Morgan, Lewis Henry (1818-1881) Ethnologist Bioscript

Morgan, Thomas Hunt (1866-1945) American geneticist Biofile $\bullet$ Botanical Album

Mori, Scott A. (1941- ) New York Botanical Garden, Curator Correspondence $\bullet$ 1989: (2)

Moriarity, Henrietta Maria (fl. 1806-1843?) Bioscript

Moricand, Stephano Moise Etirre (1799-1854) Swiss biologist, plant collector Bioscript

Morière, Pierre Giles (Jules) (1817-1888) French botanist, paleontologist Bioscript

Morillo, Carolyn R. Orleans Audubon Society Correspondence $\bullet$ 1970: (3)

Morin, Nancy Ruth (1948-) American botanist; Missouri Botanical Garden

Bioscript $\bullet$ Botanical Album Correspondence $\bullet$ 1978: (2) • 1982: (1) • 1984: (2) • 1989: (1) Letter from Mike Warnock to Ewan with note from Ewan to Morin

Morin, René ( -1657/8) French plantsman Biofile

Morison, Robert (1620-1683) Scottish-born physician, botanist Bioscript

Moritz, Karl Johann Wilhelm (1797-1866) German botanical explorer of the West Indies and Venezuela Biofile

Morley, Brian Derek (1943-) English botanist; National Botanic Gardens, Dublin, Ireland Correspondence $\bullet$ 1974: (2)

Morley, S. G. Correspondence $\bullet$ 1936: (2)

Morong, Thomas (1827-1894) Amateur botanist, clergyman Bioscript $\bullet$ Botanical Album

Morphos, Panos Paul Tulane U., New Orleans, French Dept. Correspondence $\bullet$ 1963: (1) $\bullet$ 1966: (3)

Morren, E. Bioscript

Morrill, Samued R. Bookseller; Edward Morrill \& Sons, Inc., Boston, MA Correspondence • 1964: (1) Book collectors $\bullet 1966$ : (1) - 1968: (1) Book collectors • 1969: (1) • 1973: (3) - 1976: (2) 1977: (2) - 1983: (1) Book collectors - 1984: (2) Book collectors $\bullet$ 1989: (1)

Morris Arboretum U. of Pennsylvania, Philadelphia Botanical Album Correspondence • 1977: (5) $\bullet$ 1983: (1) $\bullet$ 1985: (1)

Morris, Caroline Pennsylvania Hospital, Librarian/Archivist Correspondence • 1972: (0) Filed under Pennsylvania Hospital $\bullet$ 1976: (10) Barton, Benjamin Smith, 1766-1815 - 1978: (2) Barton, Benjamin Smith, 1766-1815 - 1984: 
Morris, Caroline (continued)

(8) Bartram, William, 1739-1823 • 1988: (4) Barton, Benjamin Smith, 1766-1815 • 1989: (3) • 1990: (1)

Morris, Charles Bioscript

Morris, Constance Husband is Seymour Morris Correspondence $\bullet$ 1963: (2)

Morris, D. Bioscript

Morris, Earl Halstead (1889-1956) American archeologist Correspondence $\bullet$ 1942: (1)

Morris, H. L. U. of Colorado, Boulder, Accountant Correspondence $\bullet$ 1943: (1)

Morris, J. U. of Texas, Houston, School of Epidemiology, post-doctoral fellow Correspondence $\bullet$ 1979: (1)

Morris, James Polk History of Medicine, College Station, TX

\section{Botanical Album}

Correspondence • 1970: (1) • 1972: (1) • 1973: (4)

Tulane U., Dept. of Botany; letter to Norman C. Woody; letter from James H. Cassedy $\bullet$ 1974: (7) $\bullet$ 1975: (7) • 1976: (1) • 1977: (3) • 1978: (2) • 1984: (3) • 1985: (2) - 1989: (4)

Morris, Jane Pennsylvania Hospital Correspondence • 1950: (1) Barton, Benjamin Smith, 1766-1815

Morris, John G. Bioscript

Morris, Julie Pennsylvania Horticultural Society, Horticultural Librarian Correspondence • 1973: (11) • 1974: (14) • 1975: (14) • 1976: (2) Correspondence with Julie Morris included with corres. from Ernesta Ballard $\bullet$ 1977: (1)

Morris, M. Y. Royal Medical Society, Edinburgh Correspondence • 1972: (1)

Morris, Russell The Johns Hopkins Hospital Correspondence $\bullet$ 1970: (1)

Morrison, Benjamin Yoe (1891-1966) American horticulturist, botanical artist Botanical Album

Correspondence • 1945: (5) $\bullet$ 1946: (4) • 1947: (6) • 1948: (11) Species • 1950: (3) • 1951: (3) • 1952: (1) • 1954: (3)

Morrison, Charles F. Bioscript

Morrison, De Lesseps S. Mayor, New Orleans Correspondence $\bullet$ 1960: (1)

Morrison, Florence Husband is John Morrison Correspondence - 1937: (1) Jepson, Willis Linn, 1867-1946

Morrison, Herbert Knowles (1854-1885) Entomologist Bioscript

Morrison, John Lawrence (1911- ) American taxonomist, plant ecologist, forester

Botanical Album

Correspondence $\bullet$ 1937: (4) Jepson, Willis Linn, 1867-1946 • 1938: (7) • 1939: (8) • 1940: (1) • 1942: (1) -1943: (1) • 1946: (2) • 1951: (3) • 1952: (2) • 1953: (1) - 1954: (2) • 1962: (3)
Morrow, James (1820-1865) American physician, plant collector

Biofile • Bioscript

Morse, Edward Sylvester (1838-1925) American zoological illustrator, zoologist, ethnographer

Biofile $\bullet$ Bioscript

Morse, Jedediah

Bioscript

Morse, Larry Eugene (1947-) American taxonomist; Smithsonian Institution Correspondence $\bullet$ 1968: (2)

Morton Arboretum Lisle, IL Botanical Album

Correspondence • 1971: (1) • 1972: (1) • 1993: (1)

Morton, Conrad Vernon (1905-1972) American taxonomist, pteridologist; Smithsonian Institution

Bioscript $\bullet$ Botanical Album

Correspondence • 1937: (1) • 1942: (4) • 1943: (1) • 1947: (1) - 1948: (8) American Fern Society; Maxon, William Ralph, 1877-1948 • 1949: (12) American Fern Society $\bullet$ 1950: (12) American Fern Society $\bullet$ 1951: (6) American Fern Society 1 1952: (7) • 1953: (3) Letter from Morton to Mrs. Kalmbach with Kalmbach letters - 1954:

(1) - 1955: (5) - 1956: (9) American Fern Society $\bullet$ 1957:

(6) - 1958: (9) • 1959: (4) - 1960: (11) Vismia $\bullet 1961$ :

(24) Vismia • 1962: (10) • 1963: (2) • 1964: (4) 1 1965:

(9) Letter written by David B. Lellinger $\bullet$ 1966: (9) 1967 :

(4) Banister, John, 1650-1692 • 1968: (8) • 1969: (2) • 1970: (2)

Morton, Maude E.

Correspondence $\bullet 1956$ : (1)

Morton, Samuel George (1799-1850) American physician, naturalist Biofile $\bullet$ Bioscript

Mosauer, Walter Botanical Album

Moscow, Hotel National Mail Desk for Intourist Visitors Correspondence $\bullet 1971$ : (1)

Moseley, Edwin Lincoln (1865-1948) Bioscript

Moseley, Frank Yandell Bioscript

Moseley, Hal W., Mrs. Correspondence $\bullet$ 1957: (1)

Moseley, Henry Nottidge (1844-1890) Bioscript

Moser, C. J. Bioscript

Moser, Mary San Antonio, TX Correspondence - 1952: (2)

Moser, $\mathbf{R}$. Bioscript

Moss, Charles Edward (1870-1930) Botanical Album

Moss, Charles Edward, Mrs. Correspondence • 1939: (1)

Moss, Eugenia L. Correspondence • 1962: (2) 
Moss, Ezra Henry (1892-1963) Canadian botanist, mycologist

Biofile - Bioscript

Correspondence - 1955: (2) 1 1958: (3) Delphinium

Moss, R. H. U. of Alberta, Dept. of Botany Correspondence • 1949: (3) Rocky Mountain Naturalists

Mossman, Samuel Bioscript

Mostert, Leon Boshoff Amaryllis grower; Kleinskuur, Balfour, Transvaal, South Africa

Correspondence $\bullet 1960$ : (1)

Mott, Richard Correspondence • 1986: (1)

Motte, Jean (1897-) Université de Montpellier, Montpellier Correspondence • 1955: (1) • 1956: (1)

Moufet, Thomas (fl. 1634) Biofile

Mougeot, Jean Baptiste (1776-1858) French physician, botanist Bioscript

Moulton, F. R. American Association for the Advancement of Science

Correspondence • 1939: (1) • 1943: (1) • 1948: (1)

Moulton, Gary E. U. of Nebraska, Lincoln, Journals of the Lewis and Clark Expedition Correspondence - 1992: (2)

Moulton, Mary The Morton Arboretum Correspondence • 1971: (3)

Moultrie, William Bioscript

Mount, Norris Bioscript

Moxley, Ed Correspondence - 1929-1933: (2)

Moxley, George Loucks (1871-) American Methodist clergyman, missionary in Liberia

Bioscript • Botanical Album

Correspondence • 1929-1933: $\bullet$ 1934: (2) $\bullet$ 1935: (2)

Delphinium • 1936: (5) • 1937: (4) Eastwood, Alice, 1859-1953

Moyers, Bill

See also Bill Moyer's Journal

Correspondence $\bullet$ 1974: (2) $\bullet$ 1980: (1)

Mueller, Ferdinand Jakob Heinrich von (1825-1896)

German taxonomist; Australia

Bioscript $\bullet$ Botanical Album

Mueller, J. Argov Bioscript

Mueller, Justus F. The Journal of Parasitology, New York, NY, Editor

Correspondence $\bullet$ 1962: (1)

Muenscher, W. C. Cornell U. Correspondence - 1949: (2) Rocky Mountain Naturalists

Muenscher, Walter Leopold Conrad (1891-1963) Germanborn American plant physiologist and aquatic biologist Bioscript • Botanical Album

Muenz, Mercedes U. of Notre Dame, Archives Correspondence $\bullet$ 1970: (2)

Muesebeck, Carl F. W. (1895?-) American entomologist Biofile • Botanical Album
Muffett, Thomas (-1604) English physician, naturalist Biofile

Mũhlenberg, Frederich Augustus Bioscript

Mühlenberg, Gotthilf Henrich Ernest See Mühlenberg, Henry (1753-1815)

Mühlenberg, Henry (1753-1815) American clergyman, taxonomist

Biofile $\bullet$ Bioscript $\bullet$ Botanical Album

Muhlfeld, Johann Carl Mergerle von (1765-1832) Bioscript

Muir, Andrew Forest The Journal of Southern History, The Rice Institute, Houston, TX

Correspondence $\bullet 1961$ : (3)

Muir, John (1834-1914) Scottish-born American mountaineer, naturalist

Biofile $\bullet$ Bioscript $\bullet$ Botanical Album

Mullahy, John H. Loyola U., New Orleans Correspondence • 1958: (4) • 1976: (1)

Mullens, Bioscript

Müller, Christian Bioscript

Müller, Cornelius Hermann (1909-) American plant ecologist, taxonomist; U. of California, Santa Barbara; nickname "Neil;" wife is Katherine Kinsel Müller Bioscript • Botanical Album

Correspondence • 1937: (1) • 1938: (3) • 1939: (2)

Species • 1940: (1) Jepson, Willis Linn, 1867-1946;

Species • 1953: (2) • 1968: (0) Filed under Katherine Kinsel Müller • 1974: (1) • 1979: (2) • 1978: (2) Jones, Marcus Eugene, 1852-1934 • 1980: (1) • 1981: (4) • 1982: (1) • 1986: (1) • 1993: (1)

Müller, Hermann Joseph (1890-1967) American geneticist Biofile

Müller, Katherine Kinsel Santa Barbara Botanic Garden; husband is Cornelius Hermann Müller

Botanical Album

Correspondence • 1968: (1) • 1970: (2) • 1976: (1) •

1978: (1) 1979: (1) One postcard is from Katherine $K$. Haines

Mullett, Aidan Broadmoor Garden Club Correspondence $\bullet$ 1943: (5)

Mulligan, Edward, Mrs. Belmont, CA Correspondence $\bullet$ 1946: (1)

Multhauf, Robert P. Smithsonian Institution, Washington, D.C., Editor of ISIS

Correspondence • 1971: (4) • 1972: (3) • 1975: (1)

- 1976: (1) • 1978: (2) • 1985: (1)

Mumford, A. A. Bioscript

Munby, A. N. L. Kings College, Cambridge, England; Librarian Correspondence • 1969: (2)

Munch, C. Bioscript

Munger, Robert Lexington, VA Correspondence • 1968: (1) • 1969: (2)

Muñoz-Pizarro, Carlos (1913-1976) Chilean botanist Bioscript 


\section{Guide to the Ewan Papers}

Munro, William (1818-1889) English plant collector Bioscript

Munson, Ronald U. of Missouri, St. Louis, Dept. of Philosophy

Correspondence $\bullet$ 1971: (3)

Muntyan, Miodrag U. of minois Press, Urbana, Director Correspondence • 1969: (6) Banister, John, 1650-1692; letter to Gordon Ray is included $\bullet$ 1970: (1) Banister, John, 1650-1692 • 1971: (1)

Munz, Philip Alexander (1892-1974) American taxonomist, botanist; Rancho Santa Ana Botanic Garden Biofile • Bioscript $\bullet$ Botanical Album

Correspondence • 1929-1933: (14) Delphinium • 1934: (2) Delphinium • 1935: (7) Jepson, Willis Linn, 1867-1946 - 1936: (4) • 1937: (5) Cockerell, Theodore Dru Alison, 1866-1948 • 1938: (9) Species • 1939: (2) Species; Munz, Philip Alexander, 1892-1974 • 1941: (5) Delphinium • 1942: (4) • 1943: (4) • 1945: (5) Cockerell, Theodore Dru Alison, 1866-1948 Delphiniwn • 1946: (3) - 1948: (3) Cockerell, Theodore Dru Alison, 1866-1948 • 1949: (2) Rocky Mountain Naturalists • 1951: (4) • 1952: (5) • 1953: (0) Filed under Samuel Wood Geiser, Fred Wilbert Emerson, Walter S. Phillips, and Lincoln Ellison $\bullet 1954$ : (1) • 1956: (1) • 1957: (2) • 1958: (1) • 1960: (4) • 1961: (1) - 1962: (5) $\bullet$ 1963: (4) 1 1964: (2) National Science Foundation • 1965: (2) • 1966: (5) • 1967: (1) • 1971: (1) - 1972: (1) • 1974: (1)

Murcheson, Roderick Impey (1792-1871) British geologist Botanical Album

Murchison, Geraldine Correspondence $\bullet 1972$ : (2)

Murdock, Daniel Hamilton ( -1886) American Army captain Bioscript

Murduck, Jonathan (fl. 1811) Bioscript

Murie, Olaus Johan (1889-1963) American zoologist, conservationist, artist Bioscript

Muroi, Hiroshi (1914) Kobe, Japan Correspondence • 1952: (1)

Murphy, , Dr. Correspondence $\bullet 1971:$ (1)

Murphy, Gordon U. of Oregon, Eugene, Dept. of Biology Correspondence $\bullet 1977:$ (4) • 1978: (5) $\bullet$ 1979: (2) $\bullet$ 1980: (7) • 1981: (4) Science-Instruction and study

Murphy, Guy W. Conway Chamber of Commerce, Conway, AR

Correspondence $\bullet 1975$ : (2) Nuttall, Thomas, 1786-1859

Murphy, Robert Cushman Ornithologist; The American Museurn of Natural History, NY, Dept. of Ornithology, Curator Oceanic Birds

Biofile • Bioscript $\bullet$ Botanical Album

Correspondence $\bullet 1951$ : (1) $\bullet$ 1959: (6) Banks, Joseph, Sir, 1743-1800 • 1962: (4) • 1973: (2) Harper, Francis, $1886-1972$

Murray, Andrew (1812-1878) Scottish botanist, entomologist

Bioscript
Murray, David Fletcher (1937-) U. of Alaska, Fairbanks, Herbarium

Correspondence • 1981: (1)

Murray, Edward Levittown, PA

Bioscript

Correspondence • 1968: (2) • 1969: (1)

Murray, G. R.

Bioscript

Murrill, Paul W. Louisiana State U., Baton Rouge, Chancellor

Correspondence $\bullet$ 1979: (3)

Murrill, William Alphonso (1869-1957) American botanist; Gainesville, FL

Biofile • Bioscript

Correspondence $\bullet$ 1946: (1)

Murtaugh, Marie Chicago, IL Correspondence $\bullet$ 1946: (1) Species

Musgrave, John Brent Correspondence $\bullet$ 1984: (2)

Musselman, Lytton J. (1943- ) U. of Wisconsin, Jamesville, Dept. of Botany and Zoology, instructor Correspondence • 1969: (2) • 1970: (2)

Mutis, José Celestino Bruno (1732-1808) Spanish physician, botanist Biofile • Botanical Album

Muttkowski, Richard A(nthony von) (1887- ) Bioscript

Myers, Christopher A. College of Wooster, Wooster, OH Correspondence $\bullet$ 1992: (2)

Myers, George Sprague (1905-1985) American ichthyologist

Biofile - Botanical Album

Correspondence • 1929-1933: (1) • 1971: (4) • 1975: (1) - 1979: (1) Book collectors • 1980: (3)

Myers, Kate The Huntington Library, visiting fellow Correspondence $\bullet 1990$ : (1)

Myers, Norman Botanical Album

Mykrantz, Barbara L. Missouri Botanical Garden, St. Louis, MO, Archivist

Correspondence $\bullet$ 1982: (2)

Mylechreest, Murray Worcester College of Higher Education, Worcester, England, senior lecturer in rural studies

Correspondence • 1979: (3)

Mystic Seaport Mystic CN, Gerald Morris, Librarian Correspondence • 1967: (1)

\section{$N$}

Nabersching, Grace Q. Westwego, LA; father owned Quinette Nursery

Correspondence $\bullet$ 1961: (1)

Nabokov, Vladimir (1899-1977) Russian-born American writer Biofile

Naccari, Fortunato Luigi (1793-1860) Italian naturalist Bioscript

Nadolny, Julian Natural history bookseller Correspondence • 1975: (1) 
Naeser, Charles $\mathbf{R}$.

Correspondence • 1929-1933: (3)

Nagel, Lilliam Julia

Botanical Album

Nägeli, Carl Wilhelm von (1817-1891) Swiss-born German botanist

Bioscript

Nakai, Takenoshin (1882-)

Botanical Album

Nannenga, E. T. Utrecht Correspondence $\bullet$ 1938: (1)

Nardelli, Francesco Bioscript

Narita, George U. of Chicago Press, Biological Sciences, Editor Correspondence • 1967: (1)

Nash, George Valentine (1864-1921) American botanist, horticulturist Botanical Album

Nash, H. W. Bioscript

Nathorst, Alfréd Gabriel (1850-1921) Swedish paleobotanist, phytogeographer Bioscript

Nation, The Correspondence 1940: (1)

National Academy of Science Botanical Album Correspondence $\bullet$ 1971: (1)

National Academy of Sciences Leningrad Bioscript

National Agricultural Library Beltsville, MD Correspondence • 1975: (8) • 1976: (3) • 1984: (7)

National Bank of Commerce Correspondence 1964: (1)

National Botanic Gardens Ireland Botanical Album

National Endowment for the Humanities Correspondence - 1975: (1)

National Gallery of Art Correspondence $\bullet$ 1975: (11) $\bullet$ 1976: (8) 1978: (2)

National Geographic Magazine Washington, D.C. Correspondence • 1979: (2) • 1981: (1) • 1987: (0) Bartram, William, 1739-1823; filed under Robert McCracken Peck 1990 : (1) Correspondent is Edwin W. Snider

National Portrait Gallery Dept. of Painting and Sculpture; Cynthia Mills

Correspondence • 1989: (3)

National Research Council Washington, D.C. Correspondence $\bullet$ 1952: (7) $\bullet$ 1956: (1)

National Science Foundation Washington, D.C. Correspondence • 1952: (3) • 1954: (1) • 1964: (1) • 1966: (1) • 1969: (2) • 1977: (2) • 1980: (1) • 1981: (1)

National Science Teachers Association Robert L. Silber, Exec. Director Correspondence • 1978: (2)

Nations, Claude Southern Methodist U., Dallas, TX, Dept. of Biology Correspondence • 1971: (4)
Natural History Museum (England)

Botanical Album

Natural Fistory Society of Jamaica

Correspondence • 1950: (4) • 1951: (1) • 1952: (1)

Naturalists Directory

Correspondence $\bullet$ 1986: (2)

Nature Conservancy

Botanical Album

Naudin, Charles Victor (1815-1899) French botanist Bioscript

Nault, William Field Enterprises, Inc., World Book Encyclopedia

Correspondence $\bullet$ 1956: (3)

Naylor, Richard M. Lakeland, FL Correspondence • 1953: (2)

Neal, John (f 1889-1965) American author; Portland, ME Biofile

Neal, Marie Catharine (1889-1965) American botanist; Bishop Museum, Honolulu Biofile

Nealley, George C. Bioscript

Nealley, Greenleaf Cilley (1846-1896) American botanist and plant collector of Texas Biofile $\bullet$ Bioscript $\bullet$ Botanical Album

Nearing, George Guy (1890-) American nurseryman, lichenologist Botanical Album

Nearly, M. Holly Bluff On-The-Jordon, Bay St. Louis, MS, Sec. Correspondence • 1961: (0) Letter filed under James L. Crump

Neboux, Adolphe Simon (fl. 1836-1840) Bioscript

Nebruske Alumnus U. of Nebraska, Lincoln Correspondence • 1976: (1) Rocky Mountain Naturalists

Necker, Walter Chicago Academy of Sciences Correspondence $\bullet$ 1943: (1)

Nee, Luis (fl. 1789-1794) Bioscript

Needham, Joseph Bioscript • Botanical Album

Nees von Esenbeck, Christian Gottfried (1776-1858) German botanist Bioscript

Nees von Esenbeck, Theodor Friedrich Ludwig (1787-1837) German pharmacist, taxonomist Bioscript

Negus, Norman Tulane U., Dept. of Zoology Correspondence $\bullet$ 1964: (2)

Neill, Emmett Correspondence $\bullet 1948:$ (1)

Neill, Patrick (1776-1851) Scottish botanist, horticulturist Biofile

Neilson, John (17??-1785) English drummer, conchologist Biofile

Neirn, Joseph Cook Bioscript 


\section{Guide to the Ewan Papers}

Neisler, H. M. Bioscript

Nelson, Aven (1859-1952) American systematic botanist, plant collector, educator; U. Wyoming Biofile $\bullet$ Bioscript $\bullet$ Botanical Album Correspondence • 1929-1933: (11) - 1934: (2) • 1936: (1) - 1937: (2) • 1939: (7) Delphinium; Species • 1941: (8) - 1942: (4)

Nelson, Barbara Louisiana Society for Horticultural Research, Lafayette Correspondence • 1977: (1)

Nelson, Bernard Stanley Correspondence $\bullet 1973:$ (1)

Nelson, C. 0. Correspondence $\bullet$ 1942: (1)

Nelson, Clifford M. Cabrills College, Aptos, CA; U. of California, Berkeley, Dept. of Paleontology Correspondence - 1969: (1) - 1972: (2) Rocky Mountain Naturalists • 1975: (6) • 1976: (1) • 1977: (1) • 1979: (4) Rocky Mountain Naturalists; Barton, Benjamin Smith, 1766-1815 • 1982: (1)

Nelson, David (?-1789) British garden collector and explorer Biofile

Nelson, Edward William (1853-1934) American naturalist, ornithologist, explorer of Alaska, Arctic, and Mexico with U.S.D.A. Biofile • Bioscript

Nelson, Elias Emanuel (1876-1949) Swedish-born American agriculturist Bioscript

Nelson, Erich (1897-1980) Correspondence $\bullet 1958:(1)$

Nelson, Ernest Charles (1951-) Taxonomist; National Botanic Gardens, Glasnevin, Dublin Bioscript Correspondence $\bullet$ 1975: (1) • 1976: (1) • 1977: (22) • 1978: (13) • 1979: (10) • 1980: (18) • 1981: (13) Missouri Botanical Garden • 1982: (9) • 1983: (19) • 1984: (20) • 1985: (11) • 1986: (8) • 1987: (29) Letters from U. of Alabama Press • 1988: (11) • 1989: (17) See also letters filed under Paul E. Berry • 1990: (30) - 1992: (4) - 1993: (3)

Nelson, Ira, Mrs. Correspondence • 1977: (0) Filed under Deborah C. Ziegler

Nelson, Ira Schreiber (1911-1965) American horticulturist, plant collector; U. of Southwestern Louisiana, Lafayette Bioscript

Correspondence • 1948: (0) Filed under James M. McArthur - 1963: (3)

Nelson, Mary H. New Orleans Correspondence • 1954: (3) • 1961: (1) • 1964: (1) • 1968: (1)

Nelson, Nelson Lawrence T. (1862-1932) American bryologist Biofile

Nelson, Pearl M. Oberlin Correspondence • 1942: (1)

Nelson, Richard C. Western Pacific Lumber Dealers, Inc. Correspondence $\bullet$ 1958: (1)
Nelson, Ruth Elizabeth Ashton (1896- ) Botanist; U. of Wyoming

Botanical Album

Correspondence 1940 : (3) $\bullet$ 1957: (2) • 1961: (1) • 1962: (2) 1976: (1) Rocky Mountain Naturalists

Nelson, Thurlow C. Rutgers U., New Brunswick, NJ, Dept. of Zoology

Correspondence • 1954: (1)

Nelson, Vincent Correspondence 1929-1933: (1)

Nesbitt, Paul Scottish art historian; Royal Botanic Garden, Edinburgh

Botanical Album

Correspondence • 1992: (1) • 1993: (2)

Nestler, Christian Gottfried (1778-1832)

Bioscript Correspondence $\bullet$ 1963: (4)

Nevin, Joseph Cook (1835-1913) American taxonomist, plant collector, clergyman Bioscript

Nevins, Allan Columbia U. Correspondence • 1943: (2)

Nevling, Lorin Ives, Jr. (1930-) American taxonomist; American Society of Plant Taxonomists Correspondence $\bullet 1968:(2) \bullet 1977:(1)$

New England Garden History Society Correspondence $\bullet$ 1991: (1)

New Harmony, Indiana Botanical Album

New Orleans Mayor; letter signed by Victor $\mathrm{H}$. Schiro Correspondence $\bullet$ 1965: (1)

New Orleans Academy of Sciences Correspondence $\bullet$ 1957: (1)

New Orleans Book Collectors Club Correspondence • 1979: (8) • 1986: (5) • 1987: (1)

New Orleans City Council Joseph I. Giarrusso, Councilman at Large; Sidney J. Barthelemy, Councilman at Large Correspondence $\bullet$ 1979: (3)

New Orleans Collection Dode Clabon, New Orleans, LA Correspondence $\bullet$ 1978: (1)

New Orleans. District Corps of Engineers Correspondence • 1974: (1)

New Orleans Garden Clubs Botanical Album

New Orleans Museum of Art Correspondence • 1976: (2)

New Orleans. Park Board of Commissioners New Orleans, LA

Correspondence • 1966: (1)

New Orleans. Park Office Lucien Delory Correspondence • 1973: (1)

New Orleans Philharmonic Correspondence $\bullet$ 1971: (1)

New Orleans Town Gardeners Mrs. J. Marvin Shaw Correspondence $\bullet$ 1970: (1)

New York Botanical Garden Botanical Album Correspondence • 1952: (1) • 1965: (1) • 1972: (1) • 1977: (3) 
New York College of Pharmacy

Correspondence • 1948: (1) • 19,49: (0) Filed under

Harold Norman Moldenke

New York Historical Society Dorothy C. Barck, Librarian Correspondence $\bullet$ 1952: (2)

New York Horticultural Society Botanical Album

New York Times Book review editor Correspondence • 1942: (1) • 1943: (1)

New Yorker

Botanical Album

Correspondence • 1973: (1)

New Zealand Institute of Horticulture K. J. Lemmon, secretary Correspondence $\bullet$ 1964: (3)

Newberry, John Strong (1822-1892) American botanist and paleontologist Biofile $\bullet$ Bioscript

Newberry Library

Correspondence - 1989: (2) Correspondent is Charles T. Cullen, President and Librarian

Newbould, Josephine P. U. College, London, Botany Dept. Correspondence • 1956: (3)

Newcomb, Gene Blanchard Botanist; Tulane U., New Orleans, Dept. of Biology

Botanical Album

Correspondence • 1964: (2) • 1966: (1) • 1969: (1)

Newcomb, Wesley (1808-1892) Bioscript

Newcombe, Lydia Rancho Santa Ana Botanic Garden Correspondence • 1988: (1)

Newell, J. B. Franklin High School Correspondence $\bullet$ 1921-1928: (1)

Newhoff, Bertha Correspondence $\bullet$ 1954: (4)

Newman, E. Bioscript

Newman, George Upstate Medical Center, State U. of New York, Syracuse, NY Correspondence $\bullet$ 1971: (2)

Newman, Helen C. Husband is W. L. Newman Correspondence • 1949: (3)

Newman, J. D. Correspondence $\bullet$ 1974: (1)

Newman, Vivien New Orleans Mycological Society Correspondence • 1980: (1)

Newman, William Tulane U., New Orleans, Howard-Tilton Memorial Library, Director

Correspondence $\bullet$ 1978: (1) • 1979: (1) • 1981: (1)

Newsom, Vesta Marie (1902-1958) Bioscript

Newson, Isaac Ernest (1883-1954) American veterinarian and pathologist; Colorado City, TX Bioscript

Newton, Alfred Bioscript

Newton, Leonard Eric (1936-) English-born West African biologist; U. Kumasi, Ghana, Botany School Botanical Album

Correspondence • 1964: (6) • 1969: (2) • 1970: (2)

- 1971: (1) Biology-Instruction and study
Niccolls, Robert S., Mrs. Detroit, MI Correspondence $\bullet 1951$ : (1)

Nice, William George Bioscript

Nicely, Kenneth Aubrey (1938-) American taxonomist; Virginia Polytechnic Institute, Blacksburg Correspondence • 1966: (1) Bartram, William, 1739-1823

Nicholas, J. P. Geographer; Institute Francais d'Afrique Noire, Geography Dept. Correspondence $\bullet 1960$ : (1)

Nicholls, William Henry Botanical Album

Nichols, George Elwood (1882-1939) American bryologist, economist Biofile $\bullet$ Botanical Album

Nichols, Helen Jane Correspondence $\bullet$ 1940: (1)

Nichols, Margaret Quarterly Review of Biology, editorial assistant Correspondence $\bullet$ 1958: (3) $\bullet$ 1960: (6) $\bullet$ 1962: (0) Filed under Hiram Bentley Glass

Nicholson, David Bioscript

Nicholson Francis, Gov. [Va. 1699] Bioscript

Nicholson, Mrs. Correspondence $\bullet$ 1986: (1)

Nicholson, Rosemary Botanical Album

Nicolson, Dan Henry (1933-) American taxonomist, plant collector; Smithsonian Institution Botanical Album Correspondence • 1966: (5) 1970: (0) Filed under Phyllis Irene Edwards • 1985: (2) • 1989: (2)

Niederlander, Donald $\mathbf{R}$. Botanical Album

Niedrach, Robert James (1890-1974) American ornithologist

Bioscript

Correspondence • 1941: (1) • 1942: (4) • 1943: (1) - 1949: (3)

Niehaus, Thomas Tulane U., New Orleans, Latin American Library Correspondence $\bullet$ 1980: (1) • 1981: (1) - 1982: (3) - 1984: (2) • 1985: (1) • 1986: (1)

Niering, William Albert (1924) American plant ecologist; Rutgers U., Dept. of Botany Correspondence $\bullet$ 1952: (2)

Nieuwland, Julius Arthur (1878-1936) Belgian-born American clergyman, botanist, and chemist; Notre Dame Biofile $\bullet$ Botanical Album Correspondence • 1929-1933: (1) Delphinium

Nightingale, Alice Allen Bioscript

Niles, Blair Botanical Album

Nilsson, Karen Tioga Publishing Company Correspondence • 1990: (4) 1991: (3) 


\section{Guide to the Ewan Papers}

Nilsson, Siwert (1933-) Palynologiska Laboratoriet, Stockholm, Sweden

Correspondence • 1967: (1) • 1968: (1) Species • 1969: (4)

Nisbet, Ada U. of California, Los Angeles, Dept. of English

Botanical Album

Correspondence $\bullet$ 1961: (3)

Nisbet, Gladys T. (1895-) U. of New Mexico, Albuquerque Correspondence • 1945: (2)

Nisbet, John P. Bioscript

Nishizaki, Ichire Tokyo, Japan Correspondence • 1959: (2)

Nissel, Jeremy Bookseller; J. Michaels Books Correspondence • 1982: (9) Van Schaack, George Booth, 1903-1983

Nissen, Claus Wiesbaden (1901-) German biobibliographer Bioscript • Botanical Album Correspondence $\bullet 1970$ : (1)

Nixon, E. R. U. of Pennsylvania, Vice Dean Correspondence $\bullet$ 1959: (1)

Nixon, Thomas Yale U. Press Correspondence • 1991: (4)

Noble, David School of American Research, Santa Fe, NM Assistant to the Director

Correspondence $\bullet 1977:(4) \bullet 1978:$ (4)

Noble, Levi Fatzinger (1882-1965) American geologist Botanical Album Noble, Mary

Correspondence • 1952: (1)

Nocea, Domenico (1758-1841) Italian clergyman and botanist, plant collector

Biofile • Bioscript

Nodder, F. P. Bioscript

Noerager, A. J. Cleveland, $\mathrm{OH}$ Correspondence $\bullet$ 1946: (1) Species

Noggle, Glen Ray (1914) Botanist Correspondence • 1958: (2)

Nolan, Edward J. Botanical Album

Noll, Henry R. (f. 1852) Bioscript

Noonan, Julia A. American Philosophical Society Correspondence • 1942: (1) • 1954: (1) • 1955: (1) - 1958: (2) • 1969: (1)

Nooney, G. J. G. J. Nooney and Company Correspondence $\bullet$ 1964: (1)

Norden-Petterson, Brita Stina Kungl, Vetenskapsakademiens Bibliotek, Stockholm, Sweden Correspondence - 1961: (3) Letter to H. Bartlett sent by R. McVaugh

Nordenstam, Rune Bertil (1936-) Swedish botanist; Museum of Natural History, Stockholm Correspondence $\bullet$ 1972: (1)

Nordstedt, O. C. F. Bioscript

Norland, Calvert E. Spring Valley, CA Correspondence • 1966: (1)
Norlin, George U. of Colorado, President Correspondence • 1937: (1)

Norman, Jeremy Correspondence $\bullet$ 1992: (1)

Noroña, Francisco ( -1790) Spanish physician and plant collector of Java and Madagascar Biofile

Norris, Robert A. Tall Timbers Research Station, Tallahassee, FL Botanical Album Correspondence • 1960: (1) • 1964: (1) • 1965: (3) - 1966: (1) Letter to Dr. Kent Rylander

Norris, Vivian Correspondence $\bullet$ 1959: (1)

Norstog, Knut Jonson (1921-) American biologist; Fairchild Tropical Garden, Miami, FL; wife is Priscilla Fawcett Norstog

Botanical Album

Correspondence • 1987: (2) Letters between Norstog and Peter Raven - 1988: (1) Copy of correspondence from Knut Norstog to Peter Raven, MBG Director $\bullet$ 1989: (0) Letter to P. Raven filed under Priscilla K. S. Fawcett $\bullet 1989$ : (1)

North Carolina Botanical Garden Botanical Album Correspondence $\bullet$ 1976: (1)

North, Marianne (1830-1890) British botanical artist Bioscript

Northen, Henry Theodore (1908-) Plant physiologist, plant ecologist; U. of Wyoming, Laramie Correspondence $\bullet$ 1967: (1)

Northwestern State College of Louisiana Natchitoches; Louisiana Room, Russell Library; Katherine Bridges, librarian Correspondence $\bullet$ 1969: (1)

Norton, Andrea Massena (1853-1930) Pacific Grove, CA

Bioscript $\bullet$ Botanical Album

Correspondence $\bullet$ 1953: (6)

Norton, Claire V.

Botanical Album

Correspondence $\bullet$ 1942: (5)

Norton, Gertrude P. Botanical collector Bioscript

Norton, Jesse Baker (1877-1938) American entomologist, plant breeder Biofile

Norton, Scott Tulane U., New Orleans, LA, student Correspondence • 1977: (4) • 1978: (1) • 1983: (1)

Notable American Women Barbara Sicherman, Carol Hurd Green, Radcliffe College, Cambridge, MA Correspondence • 1978: (11) Eastwood, Alice, 18591953

Notestein, F. N. Bioscript

Notre Dame University South Bend, IN, Archivist

Corre Correspondence $\bullet$ 1949: (1)

Nott, Josiah (1804-1869) Bioscript 
Nowak, John H. Delta National Wildlife Refuge, Venice, LA; responding for Ronald L. Fowler Correspondence $\bullet$ 1965: (1)

Nowak, Ronald M. U. S. Fish and Wildlife Service, Office of the Scientific Authority Correspondence • 1988: (3)

Nückolls, Gath Santa Fe, NM Botanical Album Correspondence • 1979: (2) $\bullet$ 1987: (0) Filed under Leonard A. Nückolls • 1988: (1)

Nückolls, Leonard A. Botanical Album

Correspondence $\bullet$ 1977: (1) $\bullet$ 1978: (5) • 1979: (0) Filed under Gath Nückolls • 1982: (1) • 1984: (1) - 1987: (3) • 1989: (1)

Nunenmacher, Frederick William (1870-) Bioscript

Nunes, Sally Vas Louisiana Bromeliad Society, New Orleans, LA, Secretary Correspondence $\bullet$ 1959: (1)

Nusbaum, Deric Bioscript

Nute, Grace L. Correspondence $\bullet$ 1964: (2)

Nuttall, Lawrence William (1857-1933) Plant collector Bioscript

Nuttall, Thomas (1786-1859) British botanist, ornithologist, scientific explorer of U. S.

Biofile $\bullet$ Botanical Album

Nutting, Charles Cleveland (1858-1927) American zoologist

Bioscript

Nye, Clarice Correspondence • 1929-1933: (2)

Nylander, Wilhelm (1822-1899) Finnish-born physician, botanist in France Bioscript

\section{$O$}

O'Brien, Patricia J. Kansas State U., Dept. of Anthropology Correspondence $\bullet$ 1972: (1)

O'Dette, Ralph E. National Science Foundation, Program Director for Foreign Science Information Correspondence - 1957: (3)

O'Donell, Carlos Alberto (1912-1954) Fundación Miguel Lillo, Tucumán, Argentina, Director Botanical Album Correspondence $\bullet$ 1948: (1) • 1949: (1) • 1951: (1) - 1952: (2) • 1953: (2) Vismia

O'Gara, P. J. Botanical Album

O'Gorman, Helen Lanpher Botanical Album

O'Grady, Thomas Limnean Society of London, London, England, Secretary Correspondence • 1962: (2)

O'Hara, Patrick Botanical sculptor Bioscript

Correspondence $\bullet$ 1974: (4) • 1982: (1)
O'Harra, Roberta (1920-) Husband is Robert H. Potts Bioscript

O'Malley, Theresa

Correspondence $\bullet 1984$ : (1)

O'Neal, David L. Antiquarian Booksellers, Inc. Correspondence • 1983: (1) Book collectors

O'Neal, David, Mrs. Correspondence • 1983: (1)

O'Neil, John P. Louisiana State U., Natural History Museum

Correspondence $\bullet$ 1989: (1)

O'Neil, Russel Texas Ornithological Society

Correspondence • 1968: (0) Filed under Michael Kent Rylander

O'Neil, Ted Louisiana Wild Life and Fisheries Commission, New Orleans, LA, Chief of Fur Division Correspondence $\bullet$ 1962: (2)

O'Neill, Hugh Thomas (1894-1969) Botanist, plant ecologist; Catholic U. of America

Botanical Album

Correspondence • 1929-1933: (4) • 1934: (2) • 1935:

(1) • 1937: (2) • 1940: (1) • 1943: (3) • 1951: (1)

- 1952: (2)

O'Neill, Jean

Correspondence • 1980: (1) • 1983: (2) • 1984: (1)

- 1986: (1) - 1987: (1) Letter from Ewan to Randolph

S. Klein filed under O'Neill

O'Neill, John P. Louisiana State U., Natural History Museum/Zoology, Baton Rouge

Correspondence • 1991: (1) • 1992: (2)

Oak Alley Plantation Vacherie, LA; Carol Hester Correspondence $\bullet$ 1980: (3)

Oakes, William (1799-1848) Plant collector, botanist Bioscript

Oakland, California-Fire (1991)

Botanical Album

Ober, Frederick Albion (1849-1913)

Bioscript

Ochoa, Carlos M.

Bioscript Correspondence $\bullet 1984:$ (1) $\bullet$ 1985: (1)

Ochoterena, Isaac (1885-1950)

Botanical Album

Correspondence $\bullet$ 1948: (1)

Odelberg, Wilhelm Stockholm U. Library, Head Librarian Correspondence $\bullet$ 1980: (2)

Odenwald, Neil Botanical Album

Odum, Eugene Pleasants (1913-) American ecologist, ornithologist Biofile $\bullet$ Botanical Album

Oehser, Paul H. McLean, VA; wife is Grace Oehser Bioscript

Correspondence • 1938: (2) • 1942: (1) • 1948: (4) - 1955: (1) • 1956: (1) • 1957: (1) • 1977: (4) Rocky Mountain Naturalists $\bullet$ 1978: (1) $\bullet$ 1983: (3) Barton, Benjamin Smith, 1766-1815 • 1984: (11) • 1985: (2)

Oemler, Augustus Gottlieb (fl. 1811-1840) Bioscript

Oersted, Anders Sandoe

See Örsted, Anders Sando (1816-1872) 


\section{Guide to the Ewan Papers}

Ogden, Edith B. Husband is E. C. Ogden Correspondence $\bullet$ 1949: (2)

Ogden, Eugene Cecil (1905-) American taxonomist, palynologist; Albany, NY

Botanical Album

Correspondence • 1957: (1) • 1971: (2)

Ogilvie, P. W. Minnesota Zoological Garden, Director Correspondence • 1969: (1) • 1970: (3) • 1971: (4)

Oglethorpe, James Bioscript

Obio Bookhumter

Correspondence • 1983: (1) Book collectors

Ohio State University Columbus

Correspondence $\bullet$ 1973: (2) $\bullet$ 1982: (8) • 1986: (2) - 1988: (3)

Oken, Loremz (1779-1851) German naturalist, physician Biofile • Bioscript

Olby, Robert U. of Oxford, Dept. of Biology, Librarian Correspondence • 1969: (1)

Old Authors Farm Bookseller; Morrisburg, Ontario, Canada

Correspondence $\bullet$ 1951: (2)

Old Book Corner Chapel Hill, NC; Michael Barnes, Bookseller

Correspondence • 1973: (1) Harper, Francis, 1886-1972 - 1977: (3) • 1979: (1)

Old Dominion U. Lytton J. Musselman Correspondence $\bullet$ 1975: (2)

The Old Parsonage Oxford, England Correspondence • 1969: (1) • 1971: (2)

Oldenburg, Henry (1615-1677) German naturalist, philosopher in England

Biofile • Bioscript

Oldfield, Augustus Frederick (1820-1887) English plant collector in Tasmania

Bioscript

Oldfield, __, Miss Correspondence $\bullet 1990$ :

Oleson, Olaf M. Norwegian-born American horticulturist Bioscript

Oleson, Sandy American Academy of Arts and Sciences Correspondence $\bullet$ 1976: (0) Filed under American Academy of Arts and Sciences

Olivares, Antonio (1917-1975) Colombian priest, ornithologist Biofile

Oliver, Catherine Correspondence 1980: (2)

Oliver, D. Bioscript

Oliver, J. C. Bioscript

Oliver, Walter Reginald Brook (1883-1957) Ornithologist; Dominion Museum, New Zealand Correspondence $\bullet$ 1943: (2)

Olkowski, William U. of California, Berkeley Correspondence • 1975: (2)

Øllgaard, Benjamin (1943-) U. of Ảarhus, Risskov, Denmark, Botanical Institute Correspondence $\bullet$ 1978: (1)
Olmedo, Ignacio H. Bioscript

Olmo, Harold Paul (1909-) American plant geneticist; U. of California, Davis, Dept. of Viticulture Correspondence $\bullet$ 1962: (3)

Olmsted, Charles E. (1908-1976) American ecologist Biofile

Olmsted, Frederick Law (1822-1903) American landscape architect, horticulturist Bioscript

Olney, Stephen Thayer (1812-1878) American botanist Bioscript

Olsen, C. J. Forest Service Correspondence $\bullet$ 1941: (2)

Olsen, Humphrey A. Correspondence $\bullet$ 1970: (2)

Olsen, Wallace C. Academy of Natural Sciences, Philadelphia, PA, Librarian Correspondence $\bullet$ 1962: (1)

Olson, A. B.

Correspondence $\bullet$ 1941: (1)

Olson, Earl E. Church of Jesus Christ of Latter Day Saints, Church Historian Correspondence • 1949: (4) Rocky Mountain Naturalists

Olson, George Botanical artist; College of Wooster, $\mathrm{OH}$ Botanical Album Correspondence $\bullet$ 1990: (4) • 1991: (6) • 1992: (11) • 1993: (12)

Ontario Historical Society Correspondence • 1983: (2) Barton, Benjamin Smith, 1766-1815

Oosting, Henry John (1903-1968) American botanist; Duke U., Dept. of Botany Botanical Album Correspondence • 1943: (1) • 1944: (1) • 1947: (1) - 1963: (2) • 1967: (3) • 1968: (5)

Opie, John Duquesne U., Pittsburgh, PA, Dept. of History, Professor; Ed., Environmental Review Correspondence • 1976: (6) • 1977: (4)

Oppenheimer, Jane Marion (1911-) American physiologist; Bryn Mawr College Botanical Album Correspondence $\bullet$ 1972: (2)

Oram, John F. Correspondence $\bullet$ 1992: (1)

Orbigny, Alcide Dessalines d' (1802-1857) French paleontologist, zoologist; son of Charles-Marie Dessalines d' Biofile

Orbigny, Charles-Marie Dessalines d' (1770-1850) See Orbigny, Alcide Dessalines d' (1802-1857)

Orcutt, Charles Russell (1864-1929) American botanist, horticulturist, plant collector; Haiti Bioscript

Ord, George Bioscript

Ordway, Ellen Correspondence $\bullet$ 1967: (1)

Ordway, John Morse (1823-1909) American chemist, botanist; MTT, Tulane Biofile $\bullet$ Bioscript $\bullet$ Botanical Album 
Oregon State U. Press

Correspondence • 1991: (1) Book collectors

Oren, David Museu Paraense Emilio Goeldi

Correspondence $\bullet$ 1985: (2)

Orleans Audubon Society New Orleans, LA; Carolyn R. Morillo, Program Chairman

Correspondence $\bullet$ 1970: (2)

Ornduff, Robert (1932-) American taxonomist, botanist Bioscript

Correspondence • 1956: (2) • 1963: (2) • 1964: (3)

Species • 1968: (1) • 1969: (1) • 1973: (5) 1976: (1) 1977: (4) • 1978: (2) • 1980: (1) • 1984: (1)

Ornithological Conference, $\mathbf{X X}$

Correspondence $\bullet$ 1988: (1) Correspondence to Dr. Bell

Orpet, Edward Owen

Botanical Album

Orphanides, Theodoros Georgios (1817-1886) Greek botanist, horticulturist

Bioscript

Orr, Daniel T. Bioscript

Orsini, Antonio (1788-1870) Italian pharmacist, taxonomist Bioscript

Örsted, Anders Sandö (1816-1872) Danish biologist, taxonomist

Biofile • Bioscript

Ortega, J. New Mexico Quarterly Review, Editor Bioscript

Correspondence 1949 : (2)

Orthley, Alice Maria (1882- ) Bioscript

Orton, Edward Francis Baxter (1829-1899) American geologist

Bioscript $\bullet$ Botanical Album

Orton, James (1830-1877) American explorer. naturalist; Vassar College Biofile • Bioscript $\bullet$ Botanical Album

Orton, William Allen (1877-1930) American plant pathologist, mycologist Biofile

Osbeck, Peter (1723-1805) Swedish clergyman, plant collector, botanist Biofile • Bioscript

Osborn, Fairfield (1887-1969) American conservationist, zoologist; New York Zoological Society; son of Henry Fairfield Osborn (1857-1935)

Bioscript

Correspondence $\bullet$ 1945: (1)

Osborn, Gray

Correspondence 1954 : (2)

Osborn, Henry Fairfield (1857-1935) American paleontologist, zoologist; American Museum of Natural History

Bioscript $\bullet$ Botanical Album

Osborn, Henry Fairfield, Jr. (1887-1969)

See Osborn, Henry Fairfield (1857-1935)

Osborn, Jeffrey M. Ohio State U., Columbus

Correspondence • 1991: (1)

Osborne, Michael A. U. of California, Santa Barbara, Dept. of History

Correspondence • 1989: (1) • 1990: (7)
Osculati, Gaetano

Bioscript

Osgood, Wilfred Hudson (1875-1947) American mammalogist, ornithologist

Bioscript

Oslar, Ernest John (1858-1944) British-born entomologist, professional collector

Bioscript

Osler, William (1849-1919) Canadian physician and medical educator

Biofile

Osten-Sacken, Carl Robert (1828-1906) Russian entomologist, diplomat

Bioscript

Ostenfeld, Carl Emil Hanson (1873-1931) Danish taxonomist, phytogeographer

Biofile

Osterhout, George Everett (1858-1937) American amateur naturalist, lumberman

Bioscript $\bullet$ Botanical Album

Correspondence • 1929-1933: (2)

Ottawa Public Library Public Archives, Ottawa, Ontario, Canada

Correspondence $\bullet$ 1951: (1)

Ottley, Alice Maria

Botanical Album

Otto, F. Bioscript

Overal, William L. Museu Goeldi, Brazil Botanical Album

Correspondence $\bullet$ 1985: (1)

Overholts, Lee Oras (1890-1946) American mycologist Bioscript

Overlease, William R. West Chester State College, PA, Dept. of Biology Bioscript $\bullet$ Botanical Album Correspondence • 1969: (12) • 1970: (1) • 1980: (1) - 1983: (1) • 1986: (3) • 1988: (2) • 1992: (2)

Overpeck, J. C. Correspondence • 1939: (2) Delphinium

Overstreet, Leslie Smithsonian Library Correspondence $\bullet 1985:$ (2)

Overton, Ernest (1865-1933) British-born biologist Biofile

Owen, Edgar W. San Antonio, TX Botanical Album Correspondence $\bullet$ 1977: (3) $\bullet$ 1978: (2) Book collectors - 1979: (3) Book collectors • 1980: (2)

Owen, Mirva C. Bioscript

Owen, Richard (1804-1892) British naturalist, paleontologist Bioscript $\bullet$ Botanical Album

Owen, Robert American geologist Bioscript

Owen, Robert (1771-1858) Scottish-born industrialist and utopian, philanthropist of New Harmony, IN Biofile

Owens, C. E. Oregon State Agricultural College Correspondence - 1937: (2) 


\section{Guide to the Ewan Papers}

Ownbey, Francis Marion (1910-1974) American taxonomist, plant geneticist; Washington State U., Pullman Botanical Album

Correspondence • 1938: (2) • 1940: (4) 1941: (2) Delphinium • 1943: (1) • 1947: (2) • 1948: (1) • 1954: (3) Species; Pursh, Frederick, 1774-1820 - 1955: (3)

Ownbey, Gerald Bruce (1916-) American taxonomist; U. of Minnesota, Minneapolis, Dept. of Botany Correspondence $\bullet$ 1968: (3) Species

Ownbey, Marion

See Ownbey, Francis Marion (1910-1974)

Oxford, Bed and Breakfast Lisle Court Cottage Correspondence • 1986: (1)

Oxford University Botanical Album

Oxford University. Ashmolean Library Correspondence • 1971: (5)

Oxford University. Botany School Mrs. H. J. McArdle, Librarian Correspondence $\bullet$ 1970: (1)

Oxford University Press Deirdre A. Loughman Correspondence • 1990: (1)

Oyster, John Houck (1849-1920) Kansas botanist, physician, pharmaceutical manufacturer Biofile

\section{$\boldsymbol{P}$}

P \& O Orient Lines Miami, FL Correspondence $\bullet 1966$ : (1)

Pace, Antonio (1914) Syracuse U., Syracuse, NY, Romance Languages; nickname "Toni"; translator of Castiglioni's Viaggio

\section{Biofile • Bioscript • Botanical Album}

Correspondence • 1958: (3) • 1959: (8) • 1960: (4) Henry

E. Huntington Library and Art Gallery $\bullet$ 1961: (4) Book collectors • 1962: (3) • 1964: (2) • 1966: (3) $\bullet$ 1967: (4) • 1968: (6) Species $\bullet$ 1969: (4) • 1970: (6) $\bullet 1972:(2) \bullet$ 1973: (1) • 1974: (1) • 1975: (3) • 1976: (2) • 1977: (6) • 1978: (0) Filed under Norman S. Fiering $\bullet$ 1979: (19) • 1980: (5) • 1981: (15) • 1982: (6) • 1983: (13) Barton, Benjamin Smith, 1766-1815 • 1984: (5) • 1985: (6) • 1986: (1) • 1987: (1) • 1989: (2) • 1990: (4) • 1993: (1)

Pacific Book House Honolulu, HI Correspondence $\bullet$ 1970: (0) Filed under Janet Bell letters

Pacific Science Association Correspondence • 1983: (1) • 1990: (1)

Pacific Tropical Botanical Garden Botanical Album Correspondence $\bullet 1977:$ (1)

Pack Memorial Public Library Asheville, NC Correspondence • 1964: (1) Lyon, John, 1764-1814 - 1965: (2)

Packard, Alpheus Spring (1839-1905) American zoologist, teacher Biofile • Bioscript

Packard, Fred Mallery (1913-) Conservationist Correspondence • 1942: (3) 1943: (2) • 1945: (1)

Packer, John G. U. of Alberta, Edmonton, Canada, Dept. of Botany, Associate Prof., Curator of the Herbarium Correspondence • 1969: (2) Rocky Mountain Naturalists
Paclt, Joraj (1925-) Czech biologist, plant pathologist, taxonomist; Bratislava, Czechoslovakia Botanical Album Correspondence • 1974: (1) • 1993: (2)

Paddock, Elton Farnham (1913- ) American cytogeneticist; Ohio State U., Columbus, Professor of Genetics Correspondence $\bullet$ 1976: (1)

Paddock, Johnathan Roberts Bioscript

Paddock, Wendell (1866-1953) American horticulturist Bioscript Correspondence • 1949: (2) Rocky Mountain Naturalists

Padilla, Victoria Botanical Album

Pagan, Francisco Mariano (1896-1942) Puerto Rican bryologist, plant morphologist Biofile • Bioscript

Page, Chester H. Washington Academy of Science, Editor Correspondence - 1956: (3) Merrill, Elmer Drew, 1876-1956 • 1958: (2)

Page, Clayton R. (1943 - ) Bioscript $\bullet$ Botanical Album

Page, Robert M. Stanford U., Palo Alto, CA, Biological Sciences Correspondence $\bullet$ 1968: (1)

Paillon, Peter Bioscript

Paine, Alfred W. Bookseller, Bethel, CT Correspondence • 1969: (0) Filed under Janet Bell

Paine, Carla Correspondence $\bullet$ 1972: (1)

Paine, J. A. W. Bioscript

Paine, Martyn Bioscript

Palairet, J. Bioscript

Paley, William Bioscript

Palgrave, Bioscript

Palgrave, William Bioscript

Palisi, Vincent A. Martin Behrman Senior-Junior High School, Principal Correspondence $\bullet$ 1958: (3)

Palisot De Beauvois Bioscript

Pallas, Peter Simon (1741-1811) German plant botanist, geographer, explorer of Russia Biofile $\bullet$ Bioscript $\bullet$ Botanical Album

Palliser, John (1807-1887) Irish explorer of Canada, geographer Bioscript

Palmer, Edward (1831-1911) English-born American surgeon, botanist, natural history collector Biofile • Bioscript $\bullet$ Botanical Album

Palmer, Elizabeth Day Bioscript 
Palmer, Ernest Jesse (1875-1962) English-born American botanist, plant collector

Bioscript • Botanical Album

Palmer, Howard (1883-1944) American geographer, explorer, lecturer

Bioscript

Palmer, Howard (fl. 1968)

Correspondence • 1968: (1)

Palmer in Colorado

Bioscript

Palmer, Miriam Augusta (1878-) American entomologist Bioscript

Palmer, Ralph Simon (1914 ) Ornithologist

Bioscript

Correspondence $\bullet$ 1973: (1) $\bullet$ 1974: (2) Letter to Ida Langman • 1975: (2) Letter from Jean Harper $\bullet 1978$ : (3) - 1980: (7) Harper, Francis, 1886-1972 • 1981: (8) • 1982: (1) - 1983: (2) Barton, Benjamin Smith, 1766-1815 - 1988: (5) Bartram, William, 1739-1823 • 1989: (2) Barton, Benjamin Smith, 1766-1815; Harper, Francis, 1886$1972 \bullet$ 1990: (2)

Palmer, Theodore Sherman (1868-1955) American zoologist, ornithologist; American Ornithologists' Union Bioscript • Botanical Album

Correspondence • 1921-1928: (1) • 1938: (2) • 1942: (1) - 1949: (1) • 1953: (2)

Paluka, Frank U. of lowa, Iowa City, Special Collections, Head

Correspondence • 1978: (2)

Pammel, Louis Hermann (1862-1931) American botanist, conservationist

Bioscript $\bullet$ Botanical Album

Pamphlin, William (1806-1899) Bioscript

Pan American Union Library Thomas L. Welch, Director Correspondence • 1985: (1)

Pan American World Airways System Correspondence • 1945: (1)

Panchet, Bioscript

Panza, Elisa N., de Instituto de Botánica Darwinion, Argentina

Correspondence $\bullet$ 1948: (1)

Paolillo, Dominick J. U. of Illinois, Urbana, Dept. of Botany

Correspondence $\bullet$ 1970: (1)

Papenfuss, George Frederik (1903-1981) South African phycologist, U. S. A.; U. of California, Berkeley; California Botanical Society

Correspondence • 1952: (2) • 1956: (4) • 1957: (1)

- 1972: (2) • 1977: (1)

Paracelsus, Bioscript (1493-1541)

Pardolini, A. Bioscript

Pargellis, Stanley Newberry Library, Chicago, IL Correspondence • 1961: (3)

Parham, John Correspondence • 1974: (1)

Paris, France Botanical Album
Parish, Mary B.

Correspondence • 1935: (3) • 1936: (1) • 1937: (4)

- 1938: (4) • 1939: (3) • 1943: (2) • 1945: (2)

Parish, Samuel Bonsall (1838-1928) American botanist, plant collector, rancher, fruit grower

Biofile $\bullet$ Bioscript $\bullet$ Botanical Album

Parish, William Fletcher Bioscript

Park House Hotel, London Correspondence • 1964: (1) • 1965: (1) • 1967: (2) - 1969: (1) • 1971: (5) • 1973: (2) • 1975: (1)

Park, John Rockey (1833-1900) American physician, educator

Bioscript

Park, Mungo

Bioscript

Parke, Davis \& Company Detroit, MI Correspondence $\bullet$ 1959: (1)

Parke, Thomas Philadelphia, PA Bioscript

Parker, Bruce C. Bioscript

Parker, Charles F, (1820-1883) American bookbinder, botanist

Bioscript

Parker, Charles Sandbach (-1869)

Bioscript

Parker, David Bioscript

Parker, Earl S. Georgia Southern College Correspondence $\bullet$ 1968: (2)

Parker, Hubert G. ( -1888) Bioscript

Parker, Kittie Fenley (1910-1994) George Washington U., Washington, D.C. Correspondence - 1985: (1)

Parker, William B. Geologist Bioscript

Parkinson, Sydney (1745-1771) English woolen draper, botanical artist Biofile $\bullet$ Bioscript

Parkman, F. Bioscript

Parkman, George (I. 1833) American physician Biofile

Parks, Harry Ernest (1880-1967) American mycologist, botanical explorer, salesman

Bioscript

Parks, J. Correspondence $\bullet$ 1968: (1)

Parkway Commission, New Orleans H. E. Polson Correspondence • 1969: (1)

Parkyns, Mansfield Bioscript

Parlatore, $\mathbf{P}$. Bioscript

Parmentier, André Joseph Ghislain (1780-1830) Bioscript

Parodi, Lorenzo Raimundo (1895-1966) Argentine agronomist Correspondence $\bullet$ 1939: (1) $\bullet$ 1948: (1) 


\section{Guide to the Ewan Papers}

Parris, G. Keith (1908-) Plant pathologist, U. S. A.; Mississippi State U., College of Agriculture and Agricultural Experiment Station, Professor of Plant Pathology Correspondence • 1970: (4)

Parris, James W. Bioscript

Parrish, Lewis New Orleans, LA Correspondence • 1979: (1)

Parrish, Robert Bioscript

Parrott, Annie Howard Botanical Album

Parry, Capt. Bioscript

Parry, Charles Christopher (1823-1890) English-born American plant explorer, botanist, geologist Biofile $\bullet$ Bioscript $\bullet$ Botanical Album

Parry, Victor T. H. Royal Botanic Gardens, Kew, Chief Librarian and Archivist Correspondence • 1977: (2) • 1978: (5)

Parseval-Grandmaison, Jules de (1795-1876) American botanist, pteridologist

Bioscript

Parsley, Ron Wife is Shirley Parsley Botanical Album

Correspondence • 1973: (1) • 1974: (1) • 1975: (1) - 1976: (3) • 1977: (1) • 1979: (1) • 1981: (1) • 1983: (2) 1 1984: (1) • 1988: (1)

Parson, Mary Elizabeth (1859-1947) Bioscript

Parsons, Frances Theodora (1861-1952) American writer on botany; husband is James $\mathbf{R}$. Parsons Biofile

Parsons, Thomas Smith (1873-1923) English-born American agronomist Bioscript

Partridge, Ned Tulane U., New Orleans, LA, Senate Committee on Libraries Correspondence • 1976: (1) • 1982: (1)

Paschall, Andrew (1630/1-1696) British clergyman, advocate for a universal language Biofile

Pasquale, G. A. Bioscript

Passos, Cyril F., Dos Lawyer, entomologist; Mendham, NJ Biofile Correspondence • 1951: (2) • 1956: (2) $\bullet$ 1958: (2) - 1961: (4) - 1964: (2) Geiser, Samuel Wood, 1890 -

Pasteur, Louis (1822-1895) French chemist, bacteriologist, plant morphologist Botanical Album

Pasti, George East Carolina College, Dept. of History Correspondence • 1964: (1) Banister, John, 1650-1692

Pastore, John U. S. Senate Correspondence - 1973: (1)

Paterson, Robert A. Virginia Polytechnic Institute, Blacksburg Correspondence • 1975: (1)
Patrick, Ruth Academy of Natural Sciences, Philadelphia, PA

Bioscript $\bullet$ Botanical Album

Correspondence $\bullet$ 1949: (2)

Patris, J. B.

Bioscript

Patsky, Julius Herman Bioscript

Patterson, Flora Wambough (1847-1928) American mycologist

Bioscript

Patterson, Florence Anne Husband is H. N. Patterson Correspondence - 1943: (6)

Patterson, Harry Norton (1853-1919) American botanist, printer Bioscript $\bullet$ Botanical Album

Patterson, Robert H. Librarian Correspondence • 1968: (3) • 1971: (2) • 1972: (1) - 1977: (2) Rocky Mountain Naturalists • 1978: (1) - 1979: (1) - 1981: (1) Rocky Mountain Naturalists

Patterson, Theresa Harriet Correspondence $\bullet$ 1929-1933: (1)

Paulsen, Ove Vilhelm, (1874-) Danish botanist Bioscript $\bullet$ Botanical Album

Pauly, Phillip Correspondence $\bullet$ 1982: (1)

Pavón, José Antonio (1754-1844) Spanish botanist Biofile • Bioscript

Pawlowski, Bogumil (1898-1971) U. Jagelonienne, Krakow, Poland Correspondence • 1936: (6) Delphinium

Paxton, John R. Australian Museum, Sydney, Dept. of Ichthyology, Curator of Fishes Correspondence $\bullet$ 1976: (2)

Paxton, Joseph (1803-1865) British horticulturist, editor, landscape gardener, architect Biofile

Payne, Anthony Bookseller; Bernard Quaritch, Ltd. Correspondence $\bullet$ 1986: (2) - 1990: (0) Filed under Quaritch, Bernard, Ltd. • 1991: (1)

Payne, F. O. Botanical Album

Payne, Fernandus National Research Council, Assistant Director for Biological and Medical Sciences Correspondence • 1953: (2)

Payne, Gualterus A. Botanical Album

Payne, John Howard Bioscript

Payne, Theodore (1872-1963) English-born American nurseryman, seedsman, conservationist; Los Angeles, CA Biofile $\bullet$ Bioscript $\bullet$ Botanical Album

Payne, Theodore Correspondence • 1935: (1) • 1959: (5) • 1960: (2)

Payne, Willard William (1934 ) Taxonomist; U. of Illinois, Champaign-Urbana, Dept. of Botany Correspondence $\bullet$ 1959: (2) • 1967: (2) • 1968: (5) Species • 1969: (1) • 1973: (6) • 1978: (1) 
Paynter, Raymond A., Jr. Harvard U., Cambridge, MA, Museum of Comparative Zoology

Correspondence $\bullet$ 1991: (3) $\bullet$ 1992: (2)

Payson, Edwin Blake (1893-1927) American botanist; wife is Lois Elizabeth Butler Payson

Bioscript • Botanical Album

Payson, Lois Elizabeth Butler (1895-1969) American botanical collector; husband is Edwin B. Payson Bioscript

Correspondence • 1949: (6)

Peabody, A.

Bioscript

Peabody, W. B. O

Bioscript

Peace, Rufus Bioscript

Peace, Steve California State Legislature, Subcommittee on Resources, Chairman

Correspondence • 1987: (1)

Peachey, John Bioscript

Peake, John Bioscript

Peale, Bioscript

Peale, Albert Charles (1849-1914) American geologist of the Hayden survey; grandson of Charles Willson Peale Biofile - Bioscript

Peale, Charles Willson (1741-1827) American portrait painter, amateur naturalist, museum curator Biofile - Bioscript

Peale Museum Baltimore, MD Bioscript

Peale, Rembrandt (1778-1860) Bioscript

Peale, Rubens (1784-1865) Bioscript

Peale, Titian Ramsay (1799-1885) American artist and naturalist; son of Charles Wilson Peale Biofile • Bioscript

Pearce, Kit U. of Oklahoma, Norman, Bebb Herbarium, Research Associate \& Herbarium Assistant Correspondence • 1976: (2)

Pearce, Richard Bioscript

Pearce, Robert W. Bioscript

Pearl, Raymond Bioscript $\bullet$ Botanical Album

Pearsall, Correspondence $\bullet$ 1944: (1)

Pearsall, Gordon Forest Preserve District of Cook County, II

Correspondence • 1939: (2)

Pearsall, John Bioscript

Pearse, C. Kenneth Forest Service Correspondence • 1942: (3)

Pearse, Theed Comax, British Columbia Correspondence • 1970: (1)
Pearson, Daphne Victoria, Australia Correspondence $\bullet 1984:$ (4) $\bullet 1990$ : (1)

Pearson, Henry Harold Welch (1870-1916) English-born morphologist, taxonomist, plant collector; South Africa Botanical Album

Pearson, Michael B.

Correspondence $\bullet$ 1992: (4)

Pease, Frederic Noah (1850-1933) Amateur botanist, chemist

Bioscript

Pease, H. W. Bioscript

Pease, William $\mathbf{H}$. Bioscript

Peattie, Donald Culross Bioscript • Botanical Album Correspondence $\bullet$ 1951: (1) $\bullet$ 1956: (2)

Peck, Charles Horton Biofile - Botanical Album

Peck, Clarendon (1812-1837) American physician, naturalist Biofile • Bioscript

Peck, J. B. Botanical Album

Peck, Morton Eaton (1871-1959) American botanist Biofile $\bullet$ Botanical Album Correspondence $\bullet$ 1948: (1) $\bullet$ 1949: (2) $\bullet$ 1951: (3)

Peck, Robert McCracken The Academy of Natural Sciences

Biofile $\bullet$ Bioscript $\bullet$ Botanical Album Correspondence $\bullet 1977$ : (4) • 1978: (25) Bartram, William, 1739-1823; copy of letter from Charlotte M. Porter - 1979: (9) • 1980: (6) Bartram, William, 1739-1823 • 1981: (10) Bartram, William, 1739-1823 • 1982: (4) • 1983: (16) • 1984: (9) • 1985: (7) • 1986: (6) • 1987: (8) - 1988: (11) • 1989: (9) • 1990: (12) • 1991: (10) - 1992: (13) $\bullet$ 1993: (14)

Peck, W. A. Bioscript

Peck, William Dandridge (1763-1822) American entomologist, botanist; Harvard U. Biofile • Bioscript

Peckham, Howard H. U. of Michigan, Ann Arbor, William L. Clements Library, Director Correspondence $\bullet$ 1959: (2)

Peckham, Stephen Farnum Bioscript

Pedicino, G? _, Dr. Bioscript

Peebles, Correspondence $\bullet$ 1943: (1)

Peebles, Edward Louisiana State Anatomical Board, New Orleans, LA, Secretary-Treasurer Correspondence $\bullet$ 1958: (1)

Peebles, J. Howell, Jr. Correspondence $\bullet$ 1962: (1)

Peebles, Nora Correspondence • 1978: (2) 
Peebles, Robert Hibbs (1900-1956) American botanist, plant breeder; United States Dept. of Agriculture Correspondence • 1937: (4) • 1939: (4) Delphinium • 1940: (2)

Peery, William Tulane University, New Orleans, LA, Dean Correspondence • 1956: (3) • 1957: (3) • 1958: (3)

Peirce, George James (1868-1954) Manila-born American botanist; Stanford U. Botanical Album

Peirson, Frank Warrington (1865-1951) California plant collector, realtor; Altadena, CA

Biofile $\bullet$ Bioscript $\bullet$ Botanical Album

Correspondence • 1929-1933: (48) Grant, Adele Lewis, 1881-1967; Jepson, Willis Linn, 1867-1946 • 1934: (2) • 1935: (5) Jepson, Willis Linn, 1867-1946 • 1936: (11) Species • 1937: (3) Eastwood, Alice, 1859-1953 • 1938: (10) Munz, Philip Alexander, 1892-1974 • 1940: (1) • 1942: (2) • 1944: (2) • 1945: (3) Jepson, Willis Linn, 1867-1946 • 1951: (1)

Peirson, Mabel B. Altadena, CA; sister of Frank Peirson Correspondence • 1929-1933: (1) • 1951: (2) • 1953: (4) - 1960: (1)

Pelkey, Kimberly A. History of Science Society, Philadelphia, PA Correspondence • 1987: (1)

Pemberton, Ida Hrubesky Bioscript Correspondence • 1943: (2) • 1944: (5)

Pemberton, John Bioscript

Pemberton, W. E. Correspondence $\bullet$ 1942: (1)

Penard, Eugène (1855-1954) Swiss protozoologist, naturalist, plant collector

Biofile $\bullet$ Bioscript $\bullet$ Botanical Album

Correspondence - 1948: (3) Cockerell, Theodore Dru Alison, 1866-1948

Penci, __ Stanford U., Dept. of Botany Bioscript $\bullet$ Botanical Album

Pendergrast, Garrett Elliott Bioscript

Pendle Hill Quaker Study for Study and Contemplation, Wallingford, PA

Correspondence $\bullet$ 1962: (3)

Pendleton, Lee Christiansburg, VA Correspondence • 1966: (3) Lyon, John, 1764-1814 - 1967: (2)

Pendley, Bettie Longue Vue Gardens; husband is Sylvan Pendley Bell

Correspondence $\bullet$ 1980: (2)

Penfound, William Theodore (1897-) American plant ecologist, plant collector; U. of Oklahoma, Norman, Dept. of Botany; nickname "Penny"

Bioscript $\bullet$ Botanical Album

Correspondence - 1947: (0) Letter from Fred W. Emerson - 1949: (0) Filed under Wray M. Bowden $\bullet$ 1951: (0) Letter filed under Faith Mackaness • 1954: (2) • 1961: (1) - 1966: (1) • 1975: (2)

P'eng, Ching-i Academia Sinica, Institute of Botany Correspondence • 1989: (4)
Penick, Charles New Orleans, LA, City Health Dept. Correspondence 1957: (1)

Penington, John Bioscript

Penland, Charles William Theodore (1899-1972) American botanist; Colorado College, Colorado Springs Bioscript

Correspondence • 1939: (3) • 1941: (4) • 1942: (2)

- 1948: (1) • 1949: (5) Rocky Mountain Naturalists

- 1953: (3) Inquiry from Bertel W. Peterson included

- 1954: (1) • 1980: (1) • 1981: (1)

Penland, Nyla M. Widow of William T. Penland Correspondence $\bullet$ 1982: (2) $\bullet$ 1983: (2)

Penman, Robert Physician; wife is Dorothy I. Langsford Botanical Album

Penn, George Henry Tulane U., New Orleans, LA, Dept. of Zoology

Correspondence $\bullet$ 1947: (1) • 1957: (2) • 1961: (1)

- 1962: (1)

Penn, William (16441718) English Quaker and founder of Pennsylvania

Biofile

Penn, William, The Papers of Correspondence $\bullet 1975$ : (1)

Pennak, Alberta Husband is Robert William Pennak Correspondence - 1939: (0) Filed under Robert William Pennak • 1945: (1) • 1978: (1) • 1979: (0) Filed under Robert William Pennak

Pennak, Robert William Botanical Album

Correspondence • 1939: (2) • 1940: (1) U. of Colorado, Boulder, Dept. of Biology 1941 : (1) U. of Colorado, Boulder, Dept. of Biology $\bullet$ 1942: (1) $\bullet$ 1945: (1) 1946 : (2) U. of Colorado, Boulder, Dept. of Biology $\bullet$ 1979: (2)

Pennant, Thomas (1726-1798) English zoologist, antiquarian

Biofile • Bioscript

Pennebaker, E. S. Correspondence $\bullet$ 1962: (2)

Pennell, Anne Husband is Francis Whittier Pennell Correspondence $\bullet$ 1951: (1) $\bullet$ 1952: (3) Academy of Natural Sciences of Philadelphia; Pennell, Francis Whittier, 1886-1952

Pennell, Francis Whittier (1886-1952) American botanist; The Academy of Natural Sciences of Philadelphia, PA, Curator of Plants Bioscript $\bullet$ Botanical Album Correspondence $\bullet$ 1935: (2) Academy of Natural Sciences of Philadelphia; Nuttall, Thomas, 1786-1859 • 1937: (4) Academy of Natural Sciences of Philadelphia 1938 : (10) - 1939: (13) Nuttall, Thomas, 1786-1859; Jepson, Willis Linn, 1867-1946; Species; Delphinium; Epling, Carl Clawson, 1894-1968 - 1940: (9) Species - 1941: (4) - 1942: (2) • 1943: (12) • 1944: (3) • 1945: (3) • 1946: (0) Letter from Frans Verdoorn filed under George Booth Van Schaack • 1946: (8) Delphinium • 1947: (4) Letter from Frans Verdoorn • 1948: (10) Species • 1949: (10) - 1951: (15) - 1952: (3) "Rocky Mountain Vegetation"

Pennington, Edward Bioscript 
Pennington, Richard McGill U., Montreal, Canada, Library, Redpath Library, Librarian

Correspondence • 1956: (1) • 1960: (1) Banister, John, $1650-1692$

Pennington, Terence Dale (1938- ) English taxonomist; Royal Botanic Gardens, Kew; Fielding - Druce Expedition to Ecuador

Correspondence $\bullet$ 1962: (2)

Pennock, J. Rolland

Correspondence - 1954: (2)

Pennsylvania Horticultural Society Ann Waterman, Librarian

Correspondence $\bullet$ 1961: (1) $\bullet$ 1976: (2) • 1989: (1) Correspondence from Sandra Ward, Awards Committee; Book collectors

Pennsylvania Hospital Medical Library, Caroline Morris, Librarian

Correspondence • 1972: (1) Barton, Benjamin Smith, 1766-1815

Pennsylvania Hospital Joyce K. Cooper

Correspondence $\bullet$ 1973: (2) • 1974: (6) • 1990: (1)

Pennsylvania Magazine

Correspondence - 1985: (2)

Pentland, Joseph Barclay (1797-1873) Irish naturalist, plant collector; Consul-General in Bolivia Biofile

Penzate, Joseph New Orleans, LA Correspondence • 1961: (1)

Peplow, Mrs.

\section{Bioscript}

Pepper, I.

Correspondence $\bullet$ 1965: (1) Carbon with note to Ida Langman

Pepper, J. O.

Correspondence $\bullet$ 1934: (2)

Peradeniya Campus Library, U. of Ceylon H. A. I. Goonetileke, Librarian

Correspondence • 1974: (1)

Perceval, Anne Mary (1790-1876) English botanical collector of Canada

Biofile - Bioscript

Percival, Bioscript

Percival, William Bioscript

Percy, Ann Philadelphia Museum of Art, Curator of Drawings

Correspondence • 1987: (0) Filed under Philadelphia Museum of Art $\bullet$ 1988: (5) $\bullet$ 1990: (3)

Perdue, Robert Edward, Jr. (1924) ) U. of Maryland, Dept. of Botany; Harvard U., Gray Herbarium; U. S. Geol. Survey

Correspondence $\bullet$ 1951: (1) $\bullet$ 1952: (4) • 1953: (1)

- 1956: (3) Species

Pereira, Hyppolito Da Costa (1774-1823) Bioscript

Périer, Claire Librarian; Jardin Botaniques, Genève, Switzerland Correspondence - 1956: (1)

Perino, Charles H.

Correspondence 1972: (2)
Peron,

Bioscript

Perrault, Anna Louisiana State U., Troy H. Middleton Library

Correspondence $\bullet$ 1984: (1)

Perreira-Cutinho, Antonio Xavier Bioscript

Perret, Vaughn J. Correspondence $\bullet$ 1983: (1)

Perrin, Bioscript

Perrine, Henry Bioscript

Perrottet, G. S. Bioscript

Perry, Bioscript

Perry, Bernard B. Indiana U. Press, Bloomington, IN Correspondence $\bullet$ 1966: (3)

Perry, Donald (fl. 1974-1980) American naturalist Biofile

Perry, Matthew Calbraith (1794-1858) American Navy officer and explorer Biofile • Bioscript

Persoon, Christian Hendrik (1761-1836) South African botanist, mycologist Biofile - Bioscript

Pesman, Michael Walter (1887-1962) Danish-born American landscape architect, botanist of Denver Biofile $\bullet$ Bioscript $\bullet$ Botanical Album Correspondence • 1940: (3) • 1941: (8) • 1942: (9) - 1943: (2) • 1944: (2) • 1945: (2) • 1949 (1) • 1954: (1)

Pessin, Louis Jerome (1891-) Russian-born American botanist Bioscript $\bullet$ Botanical Album

Pestell, W. A. Carnegie Institution of Washington Correspondence $\bullet$ 1939: (1)

Pestell, William D. Tulane U., New Orleans, LA, Medical School, Library

Correspondence • 1968: (1)

Peter, John Cowles Magazines, Inc., New York, NY, Home Living Editor Correspondence $\bullet$ 1952: (2)

Peter, Robert Bioscript

Peter, Susan U. of California Press Correspondence • 1973: (8)

Peter, W. Bioscript

Peters, Dewitt Clinton (1829-1876) American surgeon, U. S. Army Bioscript

Peters, T. M. Bioscript

Petersen, Otto Georg (1847-1937) Danish plant anatomist and taxonomist Biofile • Bioscript •

Petersohn, Thor Axel Edvard (1857-1916) Swedish botanist, pteridologist Biofile $\bullet$ Bioscript 


\section{Guide to the Ewan Papers}

Peterson, Barbara C. American Nature Study Society, Glen Echo, MD; husband is Roger Tory Peterson Correspondence • 1953: (0) Filed under Roger Tory Peterson

Peterson, Bertel W.

Correspondence • 1953: (0) Filed under Charles William Theodore Penland

Peterson, Edward Leslie Culver City, CA Bioscript $\bullet$ Botanical Album Correspondence • 1929-1933: (2) • 1936: (5) • 1942: (2) - 1945: (1) • 1956: (1) • 1964: (1) • 1970: (5) Royal Botanic Gardens, Kew • 1979: (4) • 1992: (1) • 1993: (4)

Peterson, Lilian

Correspondence $\bullet$ 1954: (1)

Peterson, Olaf August (1865-1933) Swedish-born American vertebrate paleontologist Bioscript

Peterson, Roger Tory (1908-) American ornithologist, artist

Biofile $\bullet$ Bioscript $\bullet$ Botanical Album

Correspondence • 1953: (5) One letter from Barbara C. Peterson

Peterson, Ronald H. Mycological taxonomist; U. of Tennessee, Knoxville, Dept. of Botany, Professor Correspondence • 1979: (2)

Petiver, James (1658-1718) British botanist, entomologist, apothecary

Biofile • Bioscript

Petre, Robert James (1712-1742), 8th Lord British horticulturist, plant collector

Biofile • Bioscript

Petrie, Donald (1846-1925) Scottish-born government official and plant collector in New Zealand Biofile

Petry, Loren Clifford (1887-1970) American paleobotanist, educator Biofile

Pettibone, Nora Bioscript

Pettingill, Eleanor Rice (1908-1977) Bioscript

Pettingill, Olin Sewall, Jr. (1907-) American ornithologist Biofile - Bioscript $\bullet$ Botanical Album Correspondence $\bullet$ 1943: (2)

Peyton, Sidney B. Cooper Ornithological Club Correspondence $\bullet$ 1936: (1)

Pfeifer, Howard William (192\& ) American plant taxonomist; U. of Connecticut, Storrs Correspondence • 1968: (3) • 1969: (1)

Pfeiffer, George Bioscript

Pfeiffer, L. Bioscript

Pfeiffer, Norma Etta (1889-1989) Bioscript

Pfister, Donald H. (1945-) Harvard U., Farlow Herbarium, Assoc. Prof. \& Assoc. Curator Correspondence $\bullet$ 1979: (4) • 1980: (6) • 1981: (4)

Phelps, Almira Hart Lincoln (1793-1884) American popular writer on botany, teacher; Troy Female Seminary Biofile • Bioscript
Phelps, J. W. Captain, U. S. Army Bioscript

Phelps, William H., Jr. Correspondence $\bullet$ 1985: (1)

Philadelphia Area Consortium of Special Collection Joan Irving, Project Coordinator Correspondence $\bullet$ 1986: (1)

Philadelphia Botanical Club Botanical Album

Correspondence • 1980: (1) 1981 : (1) • 1982: (1)

- 1983: (1) • 1985: (1) • 1987: (1) • 1989: (2)

Philadelphia Museum of Art Ann Percy, Curator of Drawings

Correspondence $\bullet$ 1987: (2)

Philadelphia Rare Books and Manuscripts Co. Philadelphia, PA

Correspondence • 1987: (1)

Philbrick, Ralph N. (1934 ) American taxonomist, plant collector; Santa Barbara Botanic Garden

Correspondence • 1970: (2) • 1979: (2) • 1982: (1) - 1984: (1)

Philip, Cornelius Becker (1900-) American entomologist; Rocky Mountain Laboratory, Director Correspondence • 1963: (2)

Philip, E. L. Bioscript

Phillips, Allan R. Correspondence $\bullet$ 1981: (2)

Phillips, Edwin Percy (1884-1967) South African taxonomist, plant ecologist, plant collector; University of South Africa

Bioscript $\bullet$ Botanical Album

Correspondence • 1929-1933: (6) Grant, Adele Lewis, 1881-1967

Phillips, John U. of Witswatersrand, Johannesburg, S. Africa

Correspondence • 1929-1933: (4) • 1938: (1) • 1939: (1) - 1940: (4)

Phillips, John Charles (1876-1938) Bioscript

Phillips, Maurice E. Academy of Natural Sciences of Philadelphia

Correspondence - 1953: (1) Academy of Natural Sciences of Philadelphia 1958 : (2)

Phillips, Raymond B. U. of California, Berkeley, Dept. of Botany

Correspondence $\bullet$ 1977: (2)

Phillips, Richard Correspondence $\bullet$ 1992: (1)

Phillips, Thomas, Sir Bioscript

Phillips, Venia T. Academy of Natural Sciences of Philadelphia, Librarian

Correspondence - 1952: (8) Pennell, Francis Whittier, 1886-1952 • 1953: (3) • 1954: (1) • 1955: (2) • 1956: (9) Book collectors $\bullet$ 1959: (1) - 1961: (1) Lyon, John, 1764-1814 • 1963: (1) • 1964: (2) Bartram, William, 1739-1823 • 1976: (2) 
Phillips, Walter Sargeant (1905-) American taxonomist, plant anatomist, plant ecologist; U. of Arizona, Tucson, Dept. of Botany and Range Ecology, Head

Correspondence $\bullet$ 1948: (1) • 1953: (1) • 1954: (3)

- 1955: (1) • 1956: (3) • 1962: (2)

Philpott, Bill Rice U., Dept. of Biology

Correspondence $\bullet$ 1973: (1)

Phipps, Constantine John Bioscript

Picado Twight, Clodomiro (1887-1944) Costa Rican biologist and natural historian

Bioscript

Pickens, Andrew

Bioscript

Pickering, Charles (1805-1878) American botanist, ethnologist

Biofile $\bullet$ Bioscript

Pickering, Timothy

Bioscript

Pickett,

Bioscript

Pickett, (Ferman Layton?)

Botanical Album

Piddington, $\mathrm{H}$.

Bioscript

Piehl, Martin Abraham (1932-) American botanist Botanical Album

Correspondence • 1961: (2) • 1972: (5) • 1974: (6)

- 1975: (3) • 1976: (7) • 1977: (2)

Piehl, Paula A. Jodrell Lab; husband is Martin Abraham Piehl

Correspondence • 1976: (1) Bartram, William, 1739-1823

Piel, Kenneth M. Union Oil Co., Palynologist Correspondence • 1968: (4) Langman, Ida Kaplan • 1969: (2) Biology-Instruction and study $\bullet$ 1970: (3)

Pierce, James Bioscript

Pierce, Joshua Bioscript

Pierce, Mary-Elizabeth Assist. to Dr. Svenson Correspondence - 1941: (3) American Fern Society - 1942: (1)

Pierce, Richard A. Queen's U., Kingston, Canada, Dept. of History Correspondence • 1975: (1) • 1977: (1) • 1979: (3) Pursh, Frederick, 1774-1820

Pierce, Wright McEwan ( -1937) Cactus fancier Bioscript

Pietro, A. San Indiana U., Bloomington, Dept. of Botany Correspondence • 1970: (0) Filed under Indiana U., Bloomington

Pijl, Leendert van der (1903/4?-1990) Dutch botanist, flower-biologist

Biofile

Pike, Eva Frances Correspondence • 1938: (1)

Pike, Jemuel W. (1828-1898) American manufacturer, popular science lecturer Biofile

Pike, Nicholas Bioscript
Pike, Zebulon Montgomery (1779-1813) American explorer, soldier Biofile • Bioscript $\bullet$ Botanical Album

Pilate, Eugene (1840-1890) Ohio naturalist, entomologist, physician, surgeon Biofile

Pilger, Robert Knuds Friedrich (1876-1953) German botanist Bioscript $\bullet$ Botanical Album

Pilgrim, R. L. C. U. of Canterbury, New Zealand, Dept. of Zoology Correspondence $\bullet 1974:(6)$

Pilous, Zdenēk (1912-) Czech bryologist; Conservator for Protection of Nature, Czechoslovakia Correspondence • 1949: (1)

Pilsbry, Henry Augustus (1862-1957) American malacologist Bioscript

Pinckney, Charles Cotesworth Bioscript

Pincus, I. Jack Beverly Hills, CA Botanical Album Correspondence $\bullet$ 1983: (1)

Pinkava, Donald John (1933-) American botanist Correspondence $\bullet$ 1964: (0) Species; correspondence with Donald E. Stone filed with T. Richard Fisher • 1987: (1)

Piper, Charles Vancouver (1867-1926) American botanist, economic botanist, agronomist Biofile $\bullet$ Bioscript $\bullet$ Botanical Album

Piper, Raymond Botanical Album

Pippen, Richard Wayne (1935-) American taxonomist; U. of Michigan, Ann Arbor, Dept. of Botany Correspondence $\bullet 1960$ : (5)

Pires-O'Brien, Maria J. Brazil Correspondence • 1993: (1)

Pirotta, R. Bioscript

Pistoia, Marilena Bioscript

Pitard, Charles-Joseph Marie (1873-1927) French botanist Biofile • Bioscript

Pitcairn, William (1712-1791) Scottish-born London physician, gardener Biofile

Pitcher, Zina (1797-1872) American physician, botanist Bioscript

Pitt, Hamilton Winsted, CT Correspondence • 1979: (4)

Pittendrigh, Colin Stephenson (1918-) Princeton U., Dept. of Biology, Professor of Biology Correspondence • 1945: (2) • 1946: (1) • 1948: (1) - 1960: (2)

Pittier, Henri François (1857-1950) Swiss botanist, civil engineer; Ministerio de Agriculura y Cría, Estados Unidos de Venezuela Bioscript • Botanical Album Correspondence • 1945: (1) • 1946: (4) Species • 1948: (1) 


\section{Guide to the Ewan Papers}

Pittman, Dora H. Botanical Album

Planchon, J. E. Bioscript

Planer, Richard (A. 1715) Bioscript

Planned Parenthood Sanky Perlowin, Director Correspondence • 1970: (2)

Plaskett, R. A. Bioscript

Plass, __ Mrs. Philadelphia, PA Correspondence $\bullet 1975$ : (1)

Plate, James B. Prentice-Hall, Inc., Associate Editor Correspondence • 1952: (1) • 1953: (9)

Platter, Felix (1536-1614) Swiss physician Biofile

Plée, Auguste (1787-1825) French traveller; Paris Museum, Histoire; West Indies Biofile • Bioscript

Pliny the Elder (29-79 A.D.) Roman naturalist Biofile

Plitt, Charles Christian (1869-1933) American bryologist Biofile

Plitt, Joh.

Bioscript

Plot, Robert Bioscript

Plukenet, Brook Son of Leonard Plukenet Bioscript

Plukenet, Leonard Bioscript

Plum, Dorothy A. Vassar College, Poughkeepsie, NY Correspondence $\bullet$ 1947: (1) • 1948: (2)

Plumier, Charles (1646-1704) French missionary explorer, plant collector Biofile • Bioscript

Plumier, P. Carolo Bioscript

Plummer, Sara Allen Bioscript

Plunkett, Orda Allen (1897-) Bioscript Correspondence • 1934: (1) • 1937: (2) • 1940: (1) - 1943: (1)

Pocock, Mary Agard (1886-) South African phycologist, plant collector Botanical Album

Poe, Edgar Allen (1809-1849) American poet Biofile • Bioscript

Poe, Ione (1901- ) Bioscript

Poe, Joe Tulane U., New Orleans, LA, Dept. of Classical Languages Correspondence • 1988: (3)

Poeppig, Edward Bioscript

Poesch, Jessie J. Newcomb College, Dept. of Art Bioscript • Botanical Album Correspondence • 1958: (3) • 1959: (14) • 1960: (2)
Poesch, Jessie J. (continued)

1961: (2) • 1963: (2) • 1964: (3) • 1965: (1) • 1978: (1) 1981: (1) • 1982: (3) • 1984: (1) • 1989: (2) • 1991: (1)

Poett, Joseph Correspondence $\bullet$ 1992: (2)

Pohl, E. Bioscript

Pohl, Johann E. Bioscript

Pohl, Marjorie Correspondence $\bullet$ 1988: (1)

Pohl, Richard Walter (1916-1995) American agrostologist; Iowa State U., Ames, Science and Technology Correspondence • 1963: (2) • 1979: (1) • 1983: (1)

Poinsett, Joel R. Bioscript

Poiret, Bioscript

Poiret, Jean Louis Marie Bioscript

Poland, William Ohio State U., Columbus, Associate Dean Correspondence $\bullet$ 1980: (1)

Polehow, Robert William Correspondence $\bullet$ 1974: (2)

Polehow, Mary Metaire, LA; husband is Robert W. Polehow Correspondence • 1975: (1) • 1977: (1)

Polites, Angeline Maryland Historical Society, Labrobe Papers, Editor

Correspondence • 1977: (0) Filed under Edward C. Carter, II

Polk, Noel Bioscript

Pollack Halls of Residence U. of Edinburgh Correspondence $\bullet$ 1981: (2)

Pollak, Paddy M. Bookseller Correspondence • 1959: (1) • 1977: (5) • 1980: (1) - 1983: (2) Book collectors - 1984: (1) Book collectors - 1985: (3) • 1986: (2) • 1989: (1) • 1990: (2) Book collectors • 1991: (4) • 1992: (2) • 1993: (2)

Pollock, Jim Correspondence $\bullet$ 1943: (1)

Polunin, Nicholas Vladimir (1909-) English plant ecologist, taxonomist, phytogeographer in Nigeria; Harvard U., Gray Herbarium Botanical Album Correspondence • 1952: (6) • 1953: (4) • 1954: (2) - 1955: (1) • 1965: (3) • 1966: (2)

Pomarède, Leon (ca. 1807-1892) American mural artist Biofile

Pomfret, John E. Henry E. Huntington Library and Art Gallery, Office of the Director Correspondence • 1960: (3) • 1963: (1) • 1965: (2)

Pomini, Luigi (1905-) Italian biologist Biofile

Pomona College Botanical Album

Ponce de León, Antonio (1867-1961) Cuban taxonomist Correspondence • 1954: (1)

Pond, Bremer Whidden Landscape Architect; Cambridge, MA and Pittsburg, NH

Correspondence $\bullet$ 1951: (2) American Fern Society 
Ponleithner, Henry U. of Illinois Press, Urbana, II Correspondence • 1966: (3) Letter to John Francis McDermott

Pool, Raymond John (1882-1967) American botanist Bioscript $\bullet$ Botanical Album Correspondence • 1939: (1) • 1949: (1)

Poole, James Plummer Dartmouth College, Hanover, NH, Jesup Herbarium, Curator Botanical Album

Correspondence • 1970: (1) Book collectors • 1971: (1) - 1979: (1) Barton, Benjamin Smith, 1766-1815

Pope, Charles Alexander (1818-1870) Bioscript

Pope, J. H. Bioscript

Popenoe, Edwin Alonzo (1853-1913) American entomologist Bioscript

Popenoe, Wilson Frederick (1892-1975) American horticulturist, pomologist, plant explorer, tropical fruit specialist Bioscript

Popham, Richard Allen (1913-1988) American plant anatomist Biofile Correspondence $\bullet$ 1971: (2)

Popham, T. W. U. S. Dept. of Agriculture, Forest Service, Assistant Biometrician Correspondence 1 1966: (2)

Pöppig, Eduard Friedrich (1798-1868) Bioscript

Porcher, Francis P. Bioscript

Porsild, Alf Erling (1901-1977) Danish-born botanist in Canada; National Museum of Natural Sciences, Ottawa, Canada

Biofile $\bullet$ Botanical Album

Correspondence • 1941: (1) • 1942: (3) • 1943: (2) - 1944: (1) - 1950: (2) "Rocky Mountain Vegetation" - 1951: (3) Pursh, Frederick, 1774-1820 - 1955: (2)

- 1957: (1) Banks, Joseph, Sir, 1743-1800 • 1958: (2) - 1959: (1) • 1964: (2) 1966: (1) 1968: (0) Filed under Muriel C. Crossman • 1969: (2) Rocky Mountain Naturalists • 1970: (2) • 1971: (2)

Portenschlag-Ledermayer, Franz E. von (1772-1822) Botanist Bioscript

Porter, Carlos E. (fl. 1897-1924) Chilean botanist, entomologist Biofile

Porter, Cedric Lambert (1905-) Pakistani-born American botanist; U. of Wyoming, Rocky Mt. Herbarium, Laramie, WY Biofile

Correspondence • 1940: (1) • 1941: (2) • 1942: (1)

- 1943: (7) • 1944: (4) Delphinium • 1948: (1) 1949:

(3) - 1951: (1) - 1952: (1) 1953: (3) - 1958: (1) 1966: (1)

Porter, Charles Lyman

Botanical Album
Porter, Charlotte M. Florida Natural History Museum Bioscript $\bullet$ Botanical Album

Correspondence • 1978: (0) Bartram, William, 1739-1823; filed under Robert McCracken Peck • 1985: (1) • 1986: (1) - 1988: (2) - 1989: (4)

Porter, Duncan MacNair (1937-) American taxonomist; Virginia Polytechnic Institution, Blacksburg

Biofile $\bullet$ Bioscript $\bullet$ Botanical Album

Correspondence • 1976: (3) • 1979: (4) • 1980: (1)

- 1981: (3) • 1982: (2) • 1984: (1) • 1985: (1) • 1986:

$(2) \bullet 1987:(3) \bullet 1988:(1) \bullet 1989:(4) \bullet 1990:(5) \bullet$ 1991: (3) - 1993: (2)

Porter, Thomas Conrad (1822-1901) American botanist, minister

Bioscript • Botanical Album

Portland, Bioscript

Portland, Duchess of Margaret Cavendish Bentinck (1715-1785) British bibliographer, collector, landscape gardener Biofile • Bioscript

Portland State University Portland, OR Correspondence • 1991: (1)

Portz, Carl Correspondence $\bullet$ 1982: (1)

Posey, Tharp H. Brain Wave Station, Charity Hospital of Louisiana; physician to Dr. John A. Stevenson Correspondence 1951: (0) Species; filed under John Albert Stevenson

Posiesek, Frank Correspondence $\bullet$ 1954: (2)

Postell, W. D. Tulane U., New Orleans, LA, Medical School Library

Correspondence $\bullet$ 1964: (1)

Poston, Muriel E. Missouri Botanical Garden, Administrative Curator Correspondence • 1980: (3)

Pott, Johann Friedrich Bioscript

Potter, Marie San Luis Ranch School, Colorado Springs, $\mathrm{CO}$; husband is Robert $\mathrm{K}$. Potter Correspondence • 1942: (3) • 1951: (3)

Pottle, Frederick A. Yale University; Papers of James Boswell Correspondence • 1975: (5) • 1975: (0) Filed under William Jacob Robbins

Potts, John Correspondence • 1945: (1) Jepson, Willis Linn, 1867-1946

Potts, Robert H. Entomologist Correspondence • 1954: (2) "Rocky Mountain Vegetation"

Potts, Thelma J. Colorado State U., Fort Collins, Assistant Reference Librarian Correspondence • 1976: (0) Filed under Colorado State U., Fort Collins

Pough, Richard Bioscript

Poulsen, Viggo Albert (1855-1919) Danish botanist, high school teacher Biofile 
Pound, Roscoe (1870-1964) American jurist, educator, botanist Botanical Album

Pourtales, L. F. Bioscript

Povey, Dorothy Liverpool U. Library, Liverpool, England, Librarian Correspondence • 1954: (3) • 1955: (6) • 1959: (1) Bartram, William, 1739-1823

Powell, Dulcie A. Smithsonian Institution, Dept. of Botany Bioscript • Botanical Album Correspondence • 1977: (4) • 1978: (4) • 1980: (2) - 1981: (1)

Powell, John Wesley (18341902) American geologist, Major, U. S. Army Bioscript

Powell, Lawrence Clark U. of California, Los Angeles, Librarian ; U. of Tucson Biofile • Bioscript $\bullet$ Botanical Album Correspondence $\bullet$ 1978: (2) • 1985: (1) • 1986: (2) - 1989: (3)

Powell, S. M. Bioscript

Powell, W. Byrd Bioscript

Powell, William S. U. of North Carolina, Chapel Hill Correspondence • 1980: (2)

Power, Elizabeth L. Botanical Album Correspondence $\bullet 1968$ (2) $\bullet$ 1969: (5) $\bullet$ 1970: (3) - 1971: (8) • 1972: (3) • 1973: (1) • 1974: (1) • 1976: (2) Book collectors $\bullet$ 1977: (2) $\bullet$ 1981: (1)

Powers, LeRoy (1902-1965) Correspondence • 1937: (1)

Powers, Thomas Bioscript

Poyser, William Aldworth Bioscript

Praeger, R. Lloyd Bioscript

Prain, David (1857-1944) Scottish-born taxonomist, plant collector Biofile

Prance, Ghillean Tolmie (1937-) English taxonomist, plant explorer in the U. S. A

Bioscript • Botanical Album

Correspondence • 1976: (1) • 1979: (4) • 1980: (8) - 1983: (4) • 1988: (1)

Prator, Ralph U. of Colorado, Alumni Assoc. Correspondence • 1941: (3)

Pratt, Anna Marie Martin

Botanical Album (Filed under Harlan K. Pratt) Correspondence $\bullet$ 1937: (1) • 1938: (4) • 1939: (3) - 1940: (6) • 1941: (4) • 1942: (5) • 1952: (1) 1975 (1) 1976: (1) • 1977: (1) • 1978: (3) • 1979: (2) -1981: (1) - 1984: (2) • 1985: (1) • 1986: (1) • 1989: (3) - 1991: (1)

Pratt, Harlan K.

Botanical Album

Correspondence • 1971: (1) • 1972: (1) • 1983: (2)
Pratt, Henry (1761-1838)

Bioscript

Pratt, Herbert W.

Correspondence • 1968: (1)

Pratt, Joe D. Naturalist Correspondence • 1981: (2) • 1991: (1)

Pratten, Henry Bioscript

Preble, Edward Alexander (1871-1957) American conservationist, ornithologist, mammalogist Biofile $\bullet$ Bioscript Correspondence • 1921-1928: (3)

Preble, George Henry Bioscript

Preece, Sherman J. U. of Utah, Dept. of Botany Correspondence • 1949: (5) • 1950: (3) Delphinium

Prentice-Hall, Inc. Russ Kamp, Customer Service Manager Correspondence $\bullet$ 1971: (2)

Prescott, Gerald Webber (1901- ) American taxonomist; Michigan State U., East Lansing, Dept. of Botany and Plant Pathology, Professor

Botanical Album

Correspondence • 1958: (1) • 1963: (9) • 1966: (2)

Presl, Karl Bioscript

Pressler, Guido Guido Pressler Verlag, Wiesbaden, Germany Correspondence • 1971: (4) • 1972: (3)

Prestele, Joseph Bioscript

Preston, Charles (1660-1711) Scottish physician and botanist; brother of George Preston Biofile

Preston, George (1665-1749) Scottish surgeon-major and botanist Biofile

Preuss, Charles (1803-1854) German-born American cartographer, artist Bioscript

Price, Derk J. deSolla Yale U., Dept. of History of Science and Medicine Correspondence $\bullet$ 1960: (2)

Price, Overton Westfeldt (1873-1914) Bioscript

Price, Rogers Correspondence $\bullet 1980$ : (3)

Price, Sarah F. Bioscript

Price, Vivian Jean (Rogers) Athens, GA; formerly Vivian Rogers Bioscript - Botanical Album Correspondence • 1979: (3) • 1981: (4) • 1982: (1) - 1983: (16) • 1984: (4) • 1985: (1)

Price, William Wightman (1871-1922) Bioscript

Pridgeon, Alec Louisiana State U., Baton Rouge, Dept. of Botany, graduate student in systematics Correspondence $\bullet$ 1976: (2)

Priest, Josiah Bioscript 
Priestley, Herbert Ingram U. of California, Bancroft Library

Correspondence - 1938: (1) - 1943: (1)

Priestley, Joseph (1733-1804) British chemist, philosopher, theologian

Biofile $\bullet$ Bioscript $\bullet$ Botanical Album

Prince, William

Bioscript

Prince, William (1766-1842) American botanist and horticulturist

Biofile $\bullet$ Bioscript

Princeton University Botanical Album

Princeton University. Dept. of Archives Correspondence - 1963: (2)

Pring, George Harry (1885-1974) English-born horticulturist, plant breeder in the U. S. A.; Missouri Botanical Garden, St. Louis, MO Bioscript

Pringle, Cyrus Guernsey (1838-1911) American botanist, collector, plant breeder Biofile $\bullet$ Bioscript $\bullet$ Botanical Album

Pringle, Elizabeth A. Trinity College, Dublin Correspondence • 1955: (1)

Pringle, James S. Taxonomist; Royal Botanic Gardens, Hamilton, Ontario

Botanical Album

Correspondence • 1962: (3) • 1964: (5) • 1965: (3)

- 1970: (2) Rocky Mountain Naturalists • 1971: (7) - 1974: (1) • 1976: (2) • 1977: (1) • 1978: (4) • 1985: (2) $1986:$ (3) $\bullet$ 1990: (2)

Pringsheim, Nathaniel (1823-1894) German botanist Bioscript

Prior, R. C. A.

Bioscript

Pritzel, Georg August (1815-1874) German botanical bibliographer, librarian, author Bioscript

Proby, Kathryn Hall (1921-) Miami, FL Bioscript $\bullet$ Botanical Album

Correspondence • 1974: (1) • 1975: (8) • 1976: (5) Copy of letter from Edward H. Dwight to Proby $\bullet 1978$ : (2) Copies of letters to Proby from Jacquelyn L. Sheehan - 1979: (1) • 1983: (6) • 1985: (3) • 1986: (1) • 1991: (2)

Proctor, George Richardson (1920-) American taxonomist, plant collector; Science Museum, Institute of Jamaica; nickname "Swampy"

\section{Biofile - Botanical Album}

Correspondence • 1947: (5) • 1948: (1) • 1950: (6) Maxon, William Ralph, 1877-1948; Species • 1951: (6) • 1952: (1) Academy of Natural Sciences of Philadelphia 1953: (3) • 1954: (5) • 1955: (3) • 1960: (2) • 1971: (1) 1974: (2) - 1975: (2) • 1976: (1) Book collectors $\bullet$ 1977: (1) $1980:$ (2) $\bullet$ 1988: (1)

Prokosch, Mathilde Correspondence $\bullet$ 1949: (0) Filed under Albert Morse Fuller

Proskaur, Johannes Max (1923-1970) German-born bryologist, taxonomist in the U. S. A. Bioscript
Prossu, Dean Bioscript

Prost, T. C. Bioscript

Provancher, Abbe Bioscript

Prudhome, Neowied Bioscript

Pruski, John Louisiana State U. Correspondence $\bullet$ 1981: (2)

Puckett, Charles Edwin Botanical Album

Pugh, Evan Bioscript

Pugsley, Herbert William (1868-1948) British botanist Biofile

Pulle, August Adriaan (1878-1955) Dutch botanist Biofile - Botanical Album

Pulling, Howard B. Wellesley College Correspondence • 1940: (1) Grant, Adele Lewis, 1881-1967

Pullman, William A.

Correspondence $\bullet 1970$ : (3)

Pulteney, Richard (1730-1801) English physician, botanist, botanical historian Bioscript $\bullet$ Botanical Albwn

Pultz, L. M. U. of Arizona, Tucson Correspondence $\bullet$ 1945: (2)

Pumpelly, Raphael (1837-1923) American geologist, explorer Bioscript

Puolanne, Mielo (1871-1941) Finnish plant collector Biofile

Purdy, Carl Bioscript

Purer, Edith A. ( -1941) Bioscript

Purkyne, J. E. (1787-1869) Austrian physiologist, physician Biofile

Purpus, Carl Albert (1851-1941) German botanical explorer of western U. S. and Mexico Biofile • Bioscript $\bullet$ Botanical Album

Purseglove, John (1912-1991) Bioscript

Pursell, William M. Berkeley, CA; member of Cooper Ornithological Society Correspondence $\bullet 1953:$ (2)

Pursh, Frederick Traugott (17741820) German botanist Biofile - Bioscript • Botanical Album

Purves, Dorothy E. Royal Botanic Garden, Edinburgh, Librarian Botanical Album Correspondence • 1953: (1) • 1954: (3) • 1956: (1) • 1969: (3) • 1970: (4) • 1972: (8) • 1973: (3) • 1975: (1) • 1977: (2) • 1980: (2) • 1981: (3) $\bullet$ 1982: (1) • 1984: (2) $\bullet$ 1985: (3) • 1989: (2) • 1990: (2) • 1991: (1) • 1992: (1)

Pusey, Nathan Harvard U., President Correspondence $\bullet$ 1955: (3)

Pusman, G. Correspondence $\bullet 1921-1928$ : 
Putnam, Frederic Ward (1839-1915) American anthropologist, naturalist Biofile • Bioscript

Putnam, G. D. Bioscript

Putnam, Joseph Duncan (1855-1881) American naturalist, entomologist Bioscript

Putz, Francis E. U. of Florida, Gainesville Correspondence • 1993: (1)

Pyeatt, Lyle U. of California, Los Angeles, Dept. of Botany Correspondence $\bullet 1961$ : (1)

Pylaie, Bachelot de la (1786-1856) Bioscript

\section{$Q$}

Quaintance, Charles W. Eastern Oregon College of Edmonton, La Grande

Correspondence • 1936: (1) • 1938: (1) • 1941: (2)

- 1943: (1) • 1946: (1) • 1958: (1)

Quaritch, Bernard, Ltd. Antiquarian booksellers; Anthony Payne

Correspondence $\bullet$ 1990: (1)

Quarterly Review of Biology

Correspondence $\bullet$ 1951: (2) • 1964: (2) • 1967: (2)

- 1971: (5) • 1973: (10) • 1975: (1) • 1976: (1) Ewan letter to Glass $\bullet 1977:(8) \bullet 1978:(1) \bullet 1979:(5) \bullet 1980$ : (2) $\bullet$ 1981: (1) • 1983: (3) $\bullet$ 1984: (4) • 1985: (2)

Quean, Patrick Travel Center, St. Louis, MO Correspondence $\bullet 1990:(1)$

Quekett, Edwin J. Bioscript

Quen, Jacques M. Correspondence $\bullet$ 1970: (2)

Quentin, L.

Correspondence • 1951: (1) Pursh, Frederick, 1774-1820

Quevedo a Traves, Marco T Varea Bioscript

Quibell, Charles Fox (1936-) American plant anatomist; California Botanical Society and Sonoma State U. Botanical Album Correspondence • 1985: (6) • 1986: (3)

Quick, Clarence Roy

Botanical Album

Correspondence • 1943: (2)

Quigley, Edward J. Hafner Press, Director Correspondence $\bullet 1974$ : (9)

Quigley, Margery Free Public Library, Montclair, NJ Correspondence • 1951: (1) • 1952: (1) • 1953: (1) - 1956: (1) • 1959: (3)

Quincy, E. S. Bioscript

Quinn, David B. U. of Liverpool, Professor of Modern History

Correspondence $\bullet 1976:$ (4) 1977: (2)

Quinn, I. T. Alabama Dept. of Game and Fisheries Correspondence $\bullet 1921-1928:$

Quito, Ecuador, Herbarium Universitatis Centralis Botanical Album

\section{$\boldsymbol{R}$}

Rachal, William M. E. Virginia Historical Society, Richmond

Correspondence • 1966: (4) Banister, John, 1650-1692

Raddi, Giuseppe

Bioscript

Radford, Albert Ernest (1918-) American taxonomist; U. of North Carolina, Chapel Hill, Dept. of Botany;

Southeastern

Flora, Executive Committee Chairman

Botanical Album

Correspondence • 1969: (3) • 1972: (3) • 1973: (2)

Radlkofer, L. Bioscript

Raffles, Thomas Stamford Botanical Album

Rafinesque, Constantin Samuel (1783-1840)

Turkish-born naturalist and author in America Biofile • Bioscript

Raggio, Miguel Mario (1926- ) Argentine plant physiologist Correspondence • 1956: (1)

Ragsdale, George Henry (1846-1895) American naturalist, ornithologist

Biofile

Raiche, Roger U. of California, Berkeley, Botanical Garden Correspondence $\bullet$ 1982: (2)

Rainbow Bridge Glen Canyon Correspondence $\bullet$ 1962: (1)

Rainey, Edward Cornell U., Dept. of Zoology Correspondence • 1963: (1) Banister, John, 1650-1692

Rainio, Aarne Jakob Pehr (1898-) Finnish botanist Biofile

Rait, A. B.

Correspondence $\bullet$ 1945: (1)

Raitt, Tom M. Ventura, CA Correspondence 1951 : (2)

Ramaley, Francis (1870-1942) American plant ecologist

Biofile $\bullet$ Bioscript $\bullet$ Botanical Album

Correspondence 1937 : (11) U. of Colorado, Boulder. Dept. of Biology; letter to E. C. Smith - 1938: (1) - 1939: (0) Filed under John Thomas Baldwin $\bullet$ 1940: (6) Letters to W. H. Horr • 1941: (1)

Ramer, Richard C. Bookseller, New York, NY Correspondence • 1985: (4) • 1993: (1)

Ramsay, Rebecca L. Massachusetts Horticultural Society, Boston Correspondence $\bullet$ 1971: (1)

Ramsbottom, John (1885-1974) British botanist Biofile $\bullet$ Botanical Album Correspondence • 1956: (1) • 1958: (1) • 1964: (1) Verdoorn, Frans Antonie, 1906-1984

Rancho Santa Ana Botanic Garden Botanical Album Correspondence • 1972: (2)

Rand, Edward Lothrop (1859-1924) American botanist and lawyer

Biofile - Bioscript

Randall, Bioscript 
Randall, Andrew Bioscript

Randolph, Th. M.

Bioscript

Randolph, Thomas Jefferson Bioscript

Rankey, George Correspondence - 1973: (1)

Rankin, David C. U. of California, Riverside, Dept. of History Correspondence $\bullet$ 1980: (2)

Rankin, Hugh Correspondence $\bullet$ 1973: (1)

Ransom, Betty Ransom Seed Laboratory Botanical Album Correspondence • 1936: (1)

Ransom, Leander (1800-1872) Bioscript

Ransome, Frederick Leslie (1868-1935) English-born American geologist Bioscript

Raphael, Sandra Oxford U., Oxford, England Correspondence $\bullet$ 1971: (1)

Rapp, William F. Doane College, Crete, NE Correspondence • 1949: (2)

Rappleye, Howard S. Washington Academy of Sciences Correspondence • 1947: (1) 1958: (1)

Rasmussen, D. Tab Anthropologist; Washington U., St. Louis, MO

Correspondence • 1992: (4)

Rasmussen, Wayne D. U. S. Dept. of Agriculture, Washington, D.C.

Correspondence $\bullet$ 1950: (5)

Rataj, Ing C. Karel Agricultural Research Station, Czechoslovakia

Correspondence • 1964: (2)

Rathbun, Mary Jane Bioscript

Rattan, Volney (1840-1915) American botanist, taxonomist, plant collector Bioscript

Rau, Eugene Abraham (1848-1932) American botanist Bioscript • Botanical Album

Rau, Nellie Lois Harris (1885- ) Bioscript

Rauch, $\mathbf{F}$. Bioscript

Rauch, William Bioscript

Raun, Gerald G. Texas Memorial Museum Correspondence • 1965: (1)

Raup, Hallock Floyd (1901-) - American geographer; Kent State U., OH, Professor of Geography Correspondence • 1966: (3) • 1967: (1) • 1968: (1)

Raup, Hugh Miller (1901-) American botanist, forest ecologist, phytogeographer Botanical Album

Rauschenberg, Roy A. Ohio U., Athens, Dept. of History (contntinue on next column)
Rauschenberg, Roy A. (continued)

Correspondence • 1965: (4) • 1968: (5) • 1969: (3) • 1971: (3) • 1972: (5) • 1973: (2) • 1974: (1) • 1977: (3) • 1978: (1) • 1983: (1)

Rausen, F. L. Taxidermist Bioscript

Raven, Charles E.

Bioscript

Raven, John Earle (1914-1980)

Bioscript

Raven, Peter Hamilton (1936-) American plant taxonomist; Missouri Botanical Garden, Director

Biofile $\bullet$ Bioscript $\bullet$ Botanical Album

Correspondence $\bullet 1961$ : (3) Nuttall, Thomas, 1786-1859;

Species • 1962: (3) Delphinium • 1963: (14) • 1965: (2)

Species; letter to R. M. Harper $\bullet$ 1966: (2) $\bullet$ 1967: (11)

Species • 1971: (9) • 1973: (1) • 1974: (10) Missouri

Botanical Garden; letter from Hugh Dtis • 1975: (12) •

1976: (25) Book collectors; letter from W. H. Adams, H.

Irwin, F. Stafleu $\bullet$ 1977: (16) Book collectors; Rocky

Mountain Nanuralists • 1978: (4) Letters from Raven to

Werner Dietrich and W. T. Stearn included $\bullet$ 1979: (4) $\bullet$

1980: (13) Rocky Mountain Naturalists • 1981: (11)

Missouri Botanical Garden • 1982: (2) • 1983: (6) Missouri

Botanical Garden • 1984: (4) • 1985: (4) Letters from

Margaret Mee • 1986: (12) Missouri Botanical Garden •

1987: (11) Letters to and from Peter Bretting, W. B.

Faherty, Martha Riley $\bullet$ 1988: (1) Copy of correspondence from Knut Norstog • 1989: (0) Filed under Timo Koponen - 1989: (5) • 1990: (0) Filed under Emanuel Rudolph • 1990: (1) Correspondence with Peter and Tamra Raven $\bullet$ 1991: (0) Filed under Frans Antonie Stafleu $\bullet$ 1992: (1) • 1993:(1)

Raven, Tamra Engelhorn (1945-) American plant ecologist Correspondence • 1988: (1) • 1990: (0) Filed under Peter Hamilton Raven

Ravenel, Henry William (1814-1887) American botanist Biofile $\bullet$ Bioscript

Ravenel, Harriot Horry Rutledge (Mrs. St. Julien) Bioscript

Ravenel, W. de Correspondence $\bullet$ 1921-1928: (1)

Ravn, Frederik Kelpin (1873-1920) Danish plant pathologist, mycologist Biofile

Rawlings, Marjorie Kinnan American author Botanical Album

Rawson, Alice B. Trail and Timberline Correspondence $\bullet$ 1948: (2)

Rawson, Donald M. Northwestern State College of Louisiana, Natchitoches, Dept. of Social Sciences Correspondence $\bullet 1964:$ (2)

Ray, Clayton E. United States Government Correspondence $\bullet$ 1984: (2)

Ray, David T. Correspondence • 1929-1933: (2)

Ray, Gordon N. John Simon Guggenheim Memorial Foundation, Secretary General (continued on next page) 
Ray, Gordon N. (continued)

Correspondence • 1963: (2) • 1968: (2) Banister, John, 1650-1692 • 1969: (4) Banister, John, 1650-1692; letter to Gordon Ray filed with Muntyan letters

Ray, James Davis (1918-) Mississippi State College Correspondence • 1957: (1)

Ray, John (1627-1705) British naturalist Biofile $\bullet$ Bioscript $\bullet$ Botanical Album

Ray, William Winfield (1909-) U. of Nebraska, Dept. of Botany Correspondence • 1967: (1)

Rayment, Tarlton National Museum, Melbourne, Australia Correspondence • 1956: (1)

Raymond, Helen Correspondence $\bullet$ 1964: (2)

Raymond, Louis Marcel (1915-) Phytogeographer; Montreal Botanical Garden Correspondence • 1955: (1) • 1956: (3) • 1972: (2)

Raymond, William James (1865-1947) American conchologist Biofile

Rayner, Douglas A. U. of South Carolina, Columbia; student

Correspondence • 1975: (2)

Raynolds, William Franklin (1820-1894) American Army officer, topographic engineer, and explorer of Yellowstone Park Biofile

Raysor, Warren Heirloom Garden Seeds Bioscript

Correspondence • 1985: (4)

Re, Filippo (1763-1817) Italian agriculturist Bioscript

Re, Giovanni Francesco (1773-1833) Bioscript

Rea, Carleton (1861-1946) British mycologist, lawyer Bioscript

Rea, J. Bryan, TX Correspondence $\bullet$ 1977: (2)

Read, Robert W. Flora of North America, Editor Correspondence $\bullet$ 1973: (1)

Reader, Richard U. of Guelph, Ontario, Canada Correspondence • 1974: (1)

Reading, John Bioscript

Reakirt, Tryon (1844-1872) Bioscript

Rechinger, Karl Heinz (1906-) Austrian phytogeographer Botanical Album Correspondence $\bullet$ 1956: (2)

Rechus, John Jacques Elisée French traveler and geographer Biofile

Record, Samuel James (1881-1945) American forester Botanical Album

Reddick, Ava R. Correspondence - 1992: (3)

Reddick, Donald (1883-1955) American mycologist, plant pathologist Botanical Album
Redfearn, Paul Leslie (1926- ) American taxonomist, bryologist, plant ecologist Bioscript

Redfield, John Howard (1815-1895) American businessman, botanist, zoologist, paleontologist Biofile $\bullet$ Bioscript $\bullet$ Botanical Album

Redfield, William C. Bioscript

Redford, James E. Case Western Reserve U., Asst. Prof. Correspondence $\bullet$ 1982: (4)

Redinger, Karl (1907-1940) Austrian lichenologist Botanical Album

Redmond, Donna Correspondence $\bullet$ 1941: (2)

Redouté, Pierre-Joseph (1759-1840) Luxembourg-born botanical artist Biofile • Bioscript

Reed, Alfred Z. Correspondence $\bullet$ 1943: (2)

Reed, Charles B., Mrs. Garden Club of Orange and Dutchess Counties Correspondence • 1957: (1)

Reed, Clarence Arthur (1880-1950) American pomologist, horticulturist Biofile

Reed, Clyde Franklin (1918-) Bioscript • Botanical Album Correspondence $\bullet$ 1945: (3) • 1946: (4) • 1948: (2) Species • 1953: (2)

Reed, Edward Looman (1873-) American plant ecologist; Texas Technological College Bioscript Correspondence $\bullet$ 1939: (2)

Reed, Eleanor Dawson's Book Shop, Los Angeles, CA Correspondence • 1953: (1) • 1957: (2)

Reed, Frederick Morris Bioscript

Reed, James R. (1944 ) Missouri Botanical Garden, Head Librarian

Botanical Album

Correspondence $\bullet$ 1976: (4) $\bullet$ 1978: (4) $\bullet$ 1979: (2) Letter to Reed from William R. Cullson, Tulane U. Library $\bullet$ 1980: (2) Missouri Botanical Garden • 1981: (1) Missouri Botanical Garden • 1982: (1) • 1983: (2) • 1984: (3) • 1986: (1)

Reed, James (the Quaker) Bioscript

Reed, John F. New York Botanical Garden, Bronx, NY, Curator of the Library Correspondence $\bullet$ 1966: (1) • 1967: (3) • 1968: (1) • 1969: (0) Filed under Mary Lee Spence • 1970: (6) • 1971: (11) • 1972: (5) • 1974: (1) • 1986: (4) • 1990: (1) • 1991: (1) • 1993: (5)

Reed, John Wilson Correspondence $\bullet$ 1980: (2)

Reed, Maud Boulder, CO Correspondence $\bullet$ 1948: (1)

Reed, William Gibbon Museum of New Mexico, Santa Fe, Library, Assistant Librarian Correspondence $\bullet$ 1950: (2) • 1951: (2) 
Reeder, Charlotte Olive (Godding) (1916-) American taxonomist; Oregon State College; husband is John Reeder Correspondence • 1942: (4) • 1948: (1)

Reeder, John Raymond (1914) ) American taxonomist; Yale U., Osborn Botanical Laboratory

Correspondence • 1953: (3) • 1956: (1) • 1957: (1) • 1966: (1) • 1967: (2) • 1970: (2) • 1976: (1) • 1980: (2) 1981: (2) Species • 1990: (1)

Rees, Abraham (1763-1825) British encyclopedist and clergyman Biofile $\bullet$ Bioscript

Rees, Maurice $\mathbf{H}$.

Correspondence 1940: (2) U. of Colorado, Boulder, Dept. of Biology $\bullet$ 1941: (1)

Reese, William Dean (1928-) American taxonomist; Southwest Louisiana Institute, Lafayette; American Bryological and Lichenological Society, Inc.

Botanical Album

Correspondence - 1957: (1) Letter from George R. Cooley - 1958: (11) — 1959: (10) - 1961: (9) • 1962: (10) - 1963: $(2) \bullet$ 1964: (15) • 1965: (14) • 1966: (8) • 1967: (0) Filed under Harry D. Lubrecht $\bullet$ 1970: (7) $\bullet$ 1971: (1) $\bullet$ 1973:

(7) $\bullet$ 1975: (2) $\bullet$ 1976: (4) $\bullet$ 1977: (1) $\bullet$ 1979: (2) $\bullet 1984$ : (1) • 1989: (1) • 1990: (1) • 1992: (65)

Reese, William S. Bookseller Correspondence $\bullet$ 1993: (2)

Reeves, Fontaine (1939-) American mycologist Correspondence $\bullet$ 1962: (1)

Reeves, John (1774-1856) British tea merchant and naturalist in China Biofile

Regel, E. Bioscript

Regenos, Graydon W. Tulane U., New Orleans, LA Correspondence 1950 : (1)

Regis, Pamela Western Maryland College, Assoc. Prof. of English Correspondence - 1993: (2)

Regnell, Anders Fredrick (1807-1884) Swedish-born physician, patron, plant collector in Brazil Botanical Album

Rehbock, Philip Fritz U. of Hawaii, Manoa, Honolulu, Dept. of History Correspondence $\bullet$ 1972: (1) $\bullet$ 1990: (2)

Rehder, Alfred (1863-1949) German-born American botanist, dendrologist, gardener Biofile $\bullet$ Bioscript $\bullet$ Botanical Album Correspondence 1939: (3) Species

Rehder, Harold A. U. S. National Museum, Washington, D.C.

Correspondence • 1950: (3) Rocky Mountain Naturalists • 1953: (1)

Rehn, James Abram Garfield (1881-1965) American entomologist, mammalogist; Academy of Natural Sciences, Philadelphia

Bioscript

Correspondence $\bullet$ 1949: (2) Responding for Morgan Hebard, Academy of Natural Sciences, Philadelphia

Reich, Stephen G. American physician, medical historian; former student of Ewan at Tulane U.

Bioscript • Botanical Album
Reich, Stephen G. (continued)

Correspondence • 1982: (12) • 1983: (21) • 1984: (18) • 1985: (13) • 1986: (26) • 1987: (25) • 1988: (24) • 1989: $(9) \bullet 1990:(4) \bullet 1991:(7) \bullet 1992:(3) \bullet 1993:$ (1)

Reichel, Charles Gotthold Bioscript

Reichenbach, Heinrich Gottlieb (1793-1879) German physician, taxonomist Bioscript • Botanical Album

Reichenback, Ludwig Bioscript

Reid, Derek A. (1927-) English mycologist; Royal Botanic Gardens, Kew, England Correspondence • 1969: (1) • 1975: (1)

Reid, Finola Correspondence $\bullet$ 1990: (1)

Reid, Horace S. Las Vegas, NM Correspondence $\bullet 1950:(2) \bullet 1951:$ (1)

Reid, Hugo (1809-1872) Scottish-American scientist, writer, educator Biofile

Reid, Mayne Bioscript • Botanical Album

Reidel, Ludwig Bioscript

Reidy, Janet Correspondence $\bullet$ 1992: (1) • 1993: (1)

Reifschneider, Olga Augusta Wuertz (1900-) American botanist; husband is J. C. Reifschneider Correspondence $\bullet$ 1965: (4) Letter from Robert Laxalt • 1966: (3) • 1967: (2) Letter from Robert Laxalt $\bullet 1968$ : (1)

Reily, Gladys Marshdown Correspondence $\bullet$ 1957: (1) • 1959: (1)

Reily, James W., Mrs. New Orleans horticulturist Correspondence • $1957 \bullet 1961$ : (2) Letter from Ewan is also to Edgar Anderson and George H. M. Lawrence 1969: (1) • 1970: (1)

Reily, Peggy Metaire, LA Correspondence $\bullet 1978$ : (1) Wedding invitation

Reimarus, J. A. H. Bioscript

Reimers, N. F. Bioscript

Reingold, Nathan National Archives Bioscript Correspondence $\bullet$ 1954: (3) $\bullet$ 1966: (2)

Reinhardt, J. C. Bioscript

Reinikka, Merle A. Botanical Album

Reinsch, P. F. Bioscript

Reinwardt, C. G. C. Bioscript

Reiter, Beth Lattimore Historical Preservation Planning Correspondence $\bullet$ 1981: (2)

Reko, Blas Pablo Botanical Album 
Rembert, David Hopkins, Jr. (1937- ) American plant morphologist; U. of South Carolina, Columbia, Biology Dept.

Bioscript

Correspondence $\bullet$ 1979: (3) • 1980: (5) • 1982: (3) • 1985: (1) • 1986: (1) • 1989: (1)

Remy, Ezechial Jules (1826-1893) French naturalist, plant explorer

Bioscript

Renard, Louis (1670(8?)-1746) French bookseller-publisher, illustrator

Biofile $\bullet$ Bioscript

Rendle, Alfred Barton (1865-1938) British taxonomist; British Museum (Natural History)

Bioscript $\bullet$ Botanical Album

Renner, Susanne S. (1954) Botanisk Institut, Aarhus Universitet, Denmark, Associate Professor

Botanical Album

Correspondence $\bullet$ 1990: (7)

Requien, Spirito [Espirit] (1788-1851) French taxonomist Bioscript

Ressler, Charlotte Institute For Muscle Disease, Inc., New York, NY, Division of Protein Chemistry

Correspondence • 1962: (1)

Retberg, Rolf Leopold von (1812-1885) Bioscript

Reuter, George F. Bioscript

Reveal, James Lauritz (1941-) American taxonomist Bioscript

Correspondence • 1967: (5) • 1968: (6) Species $\bullet$ 1969:

(5) • 1970: (2) • 1973: (1) • 1975: (1) • 1976: (2) • 1977:

(1) Rocky Mountain Naturalists • 1980: (2) • 1982: (1) • 1991: (1)

Reverchon, Julien (1834-1905) French-born botanist, gardener, plant collector, taxonomist in Texas Bioscript • Botanical Album

Rex, Millicent B.

Correspondence $\bullet$ 1957: (5)

Reynoids, George F. U. of Colorado, Dept. of English Literature

Correspondence • 1942: (3)

Reynolds, Howard Clayton (1944) American taxonomist, agrostologist; Fort Hays Kansas State College,

Hays, KS

Botanical Album

Correspondence • 1966: (1)

Reynolds, Lisa Madison-Morgan Cultural Center, Madison, GA

Correspondence • 1983: (5) • 1984: (2) • 1985: (1)

Reynolds, Mary Collins

Bioscript $\bullet$ Botanical Album

Reynolds, P. K. United Fruit Co. Correspondence $\bullet$ 1939: (1)

Reynolds, R. A. Hudson's Bay Company Correspondence $\bullet$ 1956: (2)

Reynolds, Victor U. Press of Virginia, Charlottesville, VA

Correspondence • 1966: (2) Banister, John, 1650-1692; correspondence with William M. E. Rachal, U. Press of
Rhoades, Nancy L.

Correspondence • 1982: (2)

Rhoades, Rendell

Botanical Album

Rhodes, Donald Gene (1933- ) Louisiana Tech U., Ruston, Prof. of Botany

Correspondence $\bullet$ 1989: (2)

Ricciuti, Edward R. New York Zoological Society, Curator of Publications

Correspondence $\bullet$ 1970: (0) Letter from Richard A. Gray filed under Tim Martin Berra

Rice, Herbert P. Forest Service, Rolling Fork, MS, District Ranger

Correspondence • 1958: (3)

Rice, Malcolm McKee Botanical Album

Rice, Myron Arthur Botanical Album

Rich, 0. Bioscript

Rich, Obadiah Bioscript

Rich, Patricia E. Missouri Botanical Garden, Director of Planning \& Development Correspondence • 1987: (0) Filed under Missouri Botanical Garden

Rich, William Bioscript

Rich, William (fl. 1830-1850) American botanist; Wilkes expedition Biofile

Richard, A. Bioscript

Richard, Larry G. Jefferson, LA Correspondence • 1979: (1)

Richard, Louis Claude Bioscript • Botanical Album

Richarderie, Bioscript

Richards, Charles Correspondence $\bullet$ 1942: (1)

Richards, Edward L. U. of Kansas, Lawrence, Dept. of Botany Correspondence • 1958: (3)

Richards, Horace V. Realtor, New Orleans, LA Correspondence • 1961: (2)

Richards, Marie A. American Philosophical Society, Assistant to the Editor

Correspondence • 1954: (1) • 1959: (2) • 1963: (8) Lyon, John, 1764-1814 • 1966: (7) Bartram, William, 1739-1823 • 1967: (7) Bartram, William, 1739-1823 • 1968: (25) Bartram, William, 1739-1823 • 1969: (4) Bartram, William, 1739-1823

Richardson, Alfred U. of Texas, Austin, TX, Herbarium Correspondence $\bullet$ 1971: (1)

Richardson, Annie L. U. of Illinois, Urbana, Dept. of Botany Correspondence $\bullet$ 1961: (2)

Richardson, C. Correspondence $\bullet$ 1984: (2) 
Richardson, Emma B. The Charleston Museum, Charleston, SC

Correspondence • 1943: (1) • 1944: (2) • 1950: (3)

Richardson, Eve Augusta College, Augusta, GA Correspondence • 1992: (2) • 1993: (2)

Richardson, Ida A. Bioscript

Richardson, John (1787-1865) Explorer, naturalist, England Bioscript

Richardson, L. F., II Realmarco, New Orleans, LA Correspondence • 1976: (1)

Richardson, __, Miss U. of Colorado, Library Correspondence $\bullet$ 1941: (1)

Richardson, P. Mick Correspondence $\bullet$ 1993: (1)

Richardson, Richard Bioscript

Richardson, Ricki Tulane This Month, Editor Correspondence $\bullet$ 1980: (1)

Richardson, William $\mathbf{H}$. Bioscript

Richford, Andrew Academic Press, London, England Correspondence • 1989: (0) Filed under Richard Mearns

Richmond, Charles Wallace (1868-1932) American ornithologist Biofile

Richter, Anders U. of Chicago Press, Science Editor Correspondence • 1963: (5)

Richter, Carl H. Correspondence -1929-1933: (1)

Richter, Charles F. Bioscript

Richter, Lloyd G. Correspondence $\bullet$ 1940: (1) $\bullet$ 1941: (4)

Richthofen, Ferdinand Paul Wilhelm von, (1833-1905) Bioscript

Rickabaugh, , Mrs. Daughter of Emest Braunton Correspondence $\bullet$ 1966: (0) Filed under Rimo Carlo Felice Bacigalupi

Ricker, Percy Leroy (1878-1973) Taxonomist, plant ecologist; Wild Flower Preservation Society, Washington, D.C.

Bioscript $\bullet$ Botanical Album

Correspondence 1 1948: (3) Book collectors • 1949: (6) $\bullet$ 1951: (1) • 1952: (2) Book collectors • 1958: (3) - 1959: (1) 1964: (3) Harper, Roland McMillan, 1878-1966 • 1965: (2) • 1970: (2)

Rickett, Harold William (1896-) English-born American botanist; New York Botanical Garden Bioscript $\bullet$ Botanical Album Correspondence • 1941: (9) Delphinium - 1942: (1) • 1943: (3) • 1945: (1) • 1948: (2) Delphinium • 1949: (2) - 1950: (5) - 1952: (3) • 1953: (1) • 1960: (2) Copy of letter from Rickett to Arthur M. Scott $\bullet$ 1963: (1) $\bullet 1964$ : (5)

Ricketts, Mary Lynn Missouri Botanical Garden Library, St. Louis, MO, Ewan collection volunteer Correspondence • 1987: (1) • 1988: (1) • 1990: (2) • 1991: (1) • 1992: (4)
Ricketts, Rachel

Correspondence • 1987: (1) • 1988: (1)

Rickettson, , Mrs.

Correspondence $\bullet$ 1954: (1)

Ricksecker, Lucius Edgar (1841-1913) American entomological collector

Bioscript

Riddell, John Leonard (1807-1865) American botanist, chemist, physician, inventor Biofile

Ridgely, Robert S. (1946-) Correspondence • 1989: (1)

Ridgway, Robert (1850-1929) American ornithologist Bioscript

Ridings, James (1803-1880) English-born American entomological collector Bioscript

Ridley, H. W. Bioscript

Ridley, Henry Nicholas (1855-1956) British botanist Biofile $\bullet$ Bioscript $\bullet$ Botanical Album

Riecken, Henry W. National Science Foundation, Office of Social Sciences, Head Correspondence • 1959: (3)

Riedel, F. Atherton Correspondence $\bullet$ 1940: (1)

Riehl, Nicholas (1808-1852) Bioscript

Riemer, William J. (1924) American zoologist; National Science Foundation, Acting Associate Program Director for Systematic Biology Correspondence $\bullet$ 1965: (1)

Riess, Karlem (1913-) Tulane U., Dept. of Physies; President, New Orleans Academy of Science Correspondence • 1949: (2) • 1951: (1) • 1953: (3) • 1954: (3) • 1956: (3) Setchell, William Albert, 1864-1943• 1957: (1) • 1959: • 1961: (1) • 1965: (1) • 1966: (1) • 1967: (1) • 1970: (1) • 1971: (1)

Rigby, Elizabeth Sedona, AZ Correspondence $\bullet$ 1951: (3)

Riggs, Edmond Horticulturist; St. Martinville, LA Correspondence $\bullet$ 1953: (1)

Riina, John Prentice-Hall, Inc. Correspondence $\bullet 1964$ : (2)

Riker, Albert Joyce (1894) American pathologist; U. of Wisconsin, Madison, Dept. of Plant Pathology Correspondence • 1956: (2)

Riley, Charles Valentine (1843-1895) American entomologist Bioscript

Riley, Edward M. Colonial Williamsburg, Williamsburg. VA, Director of Research Correspondence • 1962: (4) • 1963: (2)

Riley, Herbert Parkes (1904) American cytogeneticist Botanical Album

Riley, Joseph Harvey (1873-1941) American ornithologist Bioscript 


\section{Guide to the Ewan Papers}

Riley, Martha Missouri Botanical Garden, Archivist Correspondence • 1987: (0) Filed under Peter Hamilton Raven - 1987: (1) Letter to Milton Laden from Riley $\bullet$ 1990: (1) See also correspondence between Martha Riley and Ralph Ehrenberg, filed under Library of Congress • 1991: (0) Missouri Botanical Garden; see also Emanuel David Rudolph, Paul Theerman, Duane Isely $\bullet 1992$ : (0) Filed under Patricia A. Moore

Riley, N. D. British Museum (Natural History) Correspondence $\bullet$ 1963: (1)

Riley, Stephen T. Massachusetts Historical Society, Boston, MA, Director

Correspondence • 1976: (1)

Rion, Mary C. (fl. 1860) South Carolina author of Ladies Southern Florist Biofile

Ripley, Sidney Dillon (1913-) American ornithologist; Smithsonian Institution

Biofile $\bullet$ Bioscript

Correspondence -1978 : (4) Letter referred to E. F. Rivinus, S. I. Press $\bullet$ 1979: (2) Letter from Karen D. Williamson to Ewan included • 1981: (1) • 1982: (1)

Ripper, Chuck Botanical Album

Risley, _, Mr. Correspondence $\bullet$ 1955: (1)

Risser, Paul G. U. of Oklahoma, Norman Correspondence • 1975: (1)

Ritchie, Donald Bioscript

Rittenhouse, Jack D. Bookseller; Albuquerque, NM Correspondence - 1987: (2) Rocky Mountain Naturalists

Ritter, William Emerson, (1856-1944) American zoologist, naturalist Bioscript

Ritterbush, Philip C. (1936-) American natural historian; Smithsonian Institution

Correspondence • 1965: (8) Banister, John, 1650-1692

Rivera, Carmen U. of Puerto Rico Correspondence $\bullet$ 1975: (2)

Rivers, James John (18241913) English-born American entomologist

Bioscript

Rivers, William H. R. (1864-1922) British physician and anthropologist

Biofile $\bullet$ Bioscript

Rivet, Charles L.

Correspondence $\bullet$ 1963: (1)

Rivette, Louis P.

Correspondence • 1951: (1)

Rivinius, E. F. Smithsonian Institution Press, Director Correspondence • 1978: (0) Letters filed under Sidney Dillon Ripley and Lois Chambers Stone

Rixford, Gulian Pickering (1838-1930) American physiologist, horticulturist Bioscript

Rjneinea, Silvia São Paulo, Brazil Correspondence • 1991: (1)

Roach, O. Denver, CO

Correspondence • 1953: (1) "Rocky Mountain Vegetation"
Roan, Orleans , Mr. Horticulturist, Audubon Park, New Correspondence $\bullet$ 1949: (1)

Robb, Andrew D. Nickname "Sandy" Botanical Album

Correspondence • 1921-1928: (2) • 1929-1933: (3) Epling, Carl Clawson, 1894-1968 • 1935: (1) • 1936: (1)

Robb, J. Bioscript

Robb, Mary Ann Boulton (1829-1912) British gardener Biofile

Robbert, Ellis New Orleans, LA Correspondence $\bullet$ 1949: (2)

Robbins, Caroline Bryn Mawr College, Bryn Mawr, PA Correspondence • 1962: (4) • 1984: (2)

Robbins, Christine Chapman The Rockefeller Institute; husband is William Robbins Correspondence $\bullet$ 1960: (1) • 1961: (2) Banks, Joseph, Sir, 1743-1800 • 1965: (8) Barton, Benjamin Smith, 1766-1815 • 1966: (1) • 1967: (9) Pursh, Frederick, 1774-1820 • 1968: (3) • 1969: (3) • 1970: (4) • 1971: (1) - 1973: (1)

Robbins, Guy Thomas (1916-1960) U. of California, Berkeley; nickname "Tom"

Botanical Album

Correspondence • 1940: (1) - 1941: (1) • 1942: (17) • 1943: (14) • 1944: (1) • 1946: (2) • 1948: (6) Delphinium - 1949: (6) - 1950: (3) • 1951: (5) Delphinium; "Rocky Mountain Vegetation" $\bullet$ 1952: (1) $\bullet$ 1953: (5) • 1954: (2)

Robbins, J. W. Bioscript

Robbins, James Watson (1801-1879) American botanist Bioscript $\bullet$ Botanical Album

Robbins, Wilfred William (1884-1952) American botanist Bioscript $\bullet$ Botanical Album

Robbins, William Jacob (1890-1978) American botanist, plant physiologist; New York Botanical Garden Biofile $\bullet$ Bioscript $\bullet$ Botanical Album Correspondence $\bullet$ 1940: (2) - 1941: (3) Letter from Ellsworth - 1955: (1) Letters to and from Frans Verdoorn • 1956: (12) • 1957: (3) • 1958: (7) • 1959: (27) • 1960: (11) • 1962: (1) • 1968: (2) • 1975: (3) Letter to Frederick A. Pottle • 1976: (5) Merrill, Elmer Drew, 1876-1956 • 1977: (1)

Robert, L., Brother Christian Brothers Prep, St. Paul, MN Correspondence $\bullet$ 1961: (3)

Roberts, Edith Adelaide Vassar College, Dept. of Plant Sciences Botanical Album

Correspondence • 1946: (2) • 1947: (2) • 1948: (2)

Roberts, Florence D. Librarian, Longwood Gardens Correspondence • 1965: (1) - 1966: (4) Bartram, William, 1739-1823 • 1968: (3) • 1969: (3) • 1970: (2)

Roberts, Franklin

Botanical Album

Roberts, G. C.

Correspondence 1948 : (1) Cockerell, Theodore Dru

Alison, 1866-1948 
Roberts, H. Radclyffe Academy of Natural Sciences, Philadelphia, PA, Director

Correspondence $\bullet$ 1952: (3) - 1962: (0) Letter filed under George Washington Corner 1962 : (1)

Roberts, Harold De Witt Bioscript $\bullet$ Botanical Album

Correspondence 1940: (3) U. of Colorado, Boulder, Dept. of Biology $\bullet$ 1941: (4) $\bullet$ 1942: (2) $\bullet$ 1943: (2) $\bullet$ 1946: (1) • 1953: (1) • 1954: (2) • 1956: (1)

Roberts, Marvin L. Ohio State U., Columbus, Dept. of Botany

Correspondence $1972:$ (1)

Roberts, Norman C. Bioscript

Roberts, Oliver U. College, Cork, Ireland, Dept. of Botany Correspondence $\bullet$ 1977: (1)

Roberts, P. Correspondence $\bullet$ 1971: (1)

Roberts, Radclyffe See Roberts. H. Radclyffe

Roberts, Sara F. Historiographer; Lancaster County Correspondence - 1985: (1)

Roberts, William (1862-1940)

Botanical Album

Roberts, William (c. 1763)

Bioscript

Robertson, Carl T. Bioscript

Robertson, Donald Tulane U., New Orleans, LA Correspondence - 1977: (0) Filed under Marcia Isenstein-Froomer

Robertson, Elisabeth Booknoll Farm, Hopewell, NJ; See also Elisabeth $\mathrm{H}$. Woodburn (later name) Correspondence - 1970: (3) Book collectors

Robertson, Felix Randolph (1781-1865) Bioscript

Robertson, George Ross Bioscript $\bullet$ Botanical Album

Robertson, Hope E. Daughter of Elisabeth Woodburn Correspondence $\bullet$ 1992: (1)

Robertson, James (fl. 1760s-1770s) Garden plant collector Biofile

Robertson, John McB. Cooper Ornithological Club Correspondence $\bullet$ 1940: (1)

Robertson, Kenneth R. (1941-) Illinois Natural History Survey Correspondence $\bullet$ 1982: (1)

Robertson, Martha B. Tulane U., Howard-Tilton Library, New Orleans, LA

Correspondence • 1965: (2) • 1981: (1) • 1983: (1) • 1988: (2)

Robertson, William Bioscript

Robin, Charles César (fl. 1803-1805) French traveler who visited Louisiana Biofile $\bullet$ Bioscript

Robin, Jean (1550-1629) French botanist and royal gardener Biofile • Bioscript

Robins, Richard J. U. of Oxford, Dept. of Botany Correspondence • 1977: (1)
Robinson, B. B. Crops and Diseases, Division of Cotton and Other Fiber

Correspondence $\bullet 1947$ : (1)

Robinson, Benjamin Lincoln (1864-1935) American botanist

Biofile $\bullet$ Bioscript $\bullet$ Botanical Album

Correspondence • 1929-1933: (2) • 1935: (3) Species

Robinson, D. H. Glacier National Park, West Glacier, MT, Acting Chief Park Naturalist Correspondence $\bullet$ 1953: (2)

Robinson, E. S. Bioscript

Robinson, Edgar Eugene Stanford U. Correspondence $\bullet$ 1956: (2)

Robinson, Floy L. Bioscript

Robinson, Harold Ernest (1932-) American bryologist; Smithsonian Institution, Dept. of Botany, Acting Chairman Botanical Album Correspondence • 1969: (1)

Robinson, John (1846-1925) American botanist Biofile

Robinson, Michael H. National Zoological Park Correspondence $\bullet$ 1985: (2) $\bullet$ 1989: (2)

Robinson, Orrin W. Bioscript

Robinson, Rachel Correspondence $\bullet$ 1988: (1)

Robinson, William (1838-1935) British gardener, landscape designer, author Biofile $\bullet$ Bioscript

Robison, William Condit U. S. Army Topographical Labs, Fort Belvoir, VA, Commander \& Director Botanical Album Correspondence $\bullet$ 1976: (1)

Robson, Norman Keith Bonner (1928-) Scottish-born taxonomist; British Museum (Namral History), Dept. of Botany. Bioscript Correspondence $\bullet$ 1977: (1)

Robyns, Walter (1901-) American phytogeographer. taxonomist Botanical Album

Roca, Andres Fabregas El Progreso, Tuxtla Gutierrez, Mexico Correspondence • 1953: (1)

Rocha-Campos, A. C. Universidade de San Paulo, Brasil Correspondence - 1985: (3) Letter from Ellis L. Yochelson

Roche, John Linacre College, Oxford, England Correspondence - 1978: (2)

Rochel, Anton (1770-1847) Bioscript

Rochfort, David Botanical Album

Rock, Howard Francis Leonard (1925-) Duke U., Herbarium Correspondence $\bullet$ 1956: (3)

Rock, Joseph Francis Charles (1884-1962) Austrian-born American botanical explorer Biofile $\bullet$ Botanical Album 


\section{Guide to the Ewan Papers}

Rockefeller University Mabel H. Bright, Executive Assistant to the President

Correspondence • 1967: (2)

Rockie, W. A. Correspondence • 1936: (1)

Rockwood, A. P. Bioscript

Rockwood, L. P. Ichthyologist; Forest Grove, OR Correspondence • 1953: (1)

Rodeck, Hugo George (1902-) American entomologist; U. of Colorado Museum

Bioscript • Botanical Album

Correspondence • 1939: (1) • 1942: (2) • 1943: (2) • 1949: (4) • 1950: (1) - 1951: (3)

Rodgers, Andrew Denny Botanical Album

Rodgers, John Bioscript

Rodin, Robert J. California State Polytechnic College Correspondence • 1976: (1)

Rodrigues, William Antonio (1928-) Instituto Nacional de Pesquisas da Amazônia, Manaus, Chief of Centro de Pesquisas Florestais Correspondence $\bullet 1960$ : (1)

Rodriguez, Rafael Lucas (1915-) U. of California, Berkeley, Dept. of Botany Correspondence $\bullet$ 1961: (1)

Rodriguez M., Ignacio Mexico Correspondence • 1959: (2)

Rodway, James Bioscript

Roebuck, Jarvis Bioscript

Roeder, Donald R. Iowa State U., Ames, Environmental Studies, Extension Ecologist Correspondence • 1977: (2)

Roeder, Kenneth David Botanical Album

Roelofswaard, , Mr. Correspondence - 1982: (1)

Roemer, Ferdinand (1818-1891) Bioscript

Roemer, J. J. Bioscript

Roemer, R. Von Bioscript

Roemer, Theodor Ernst Martin (1883-) Correspondence • 1935: (1)

Roezl, Benedict (1824-1885) Bohemian collector of seeds and bulbs in Mexico and South America Biofile • Bioscript

Roezl, Benito See Roezl, Benedict (18241885)

Roffavier, G. Bioscript

Rogers, Carol L. American Association for the Advancement of Science Correspondence • 1975: (0) Filed under Hilbert Garrison Wilkes
Rogers, Claude Marvin (1919-) American taxonomist; Wayne State U., Detroit, MI

Correspondence • 1959: (2) • 1960: (2) • 1961: (2) • 1968: (2) • 1969: (5)

Rogers, David James (1918- ) American economic botanist, taxonomist; Allegheny College Correspondence • 1949: (4) Delphinium • 1955: (1) 1972: (2) • 1973: (3)

Rogers, Donald Philip (1908-) American cytologist, taxonomist, mycologist; U. of Illinois, Urbana, Dept. of Botany

Correspondence - 1958: (1) • 1976: (2)

Rogers, George A. Georgia Southern College, Statesboro, Dept. of History

Bioscript $\bullet$ Botanical Album

Correspondence • 1969: (1) • 1973: (9) Bartram, William, 1739-1823 • 1977: (3) • 1978: (7) Book collectors • 1979 (9) $\bullet$ 1980: (5) • 1981: (3) • 1982: (8) • 1983: (9) • 1984: (12) • 1985: (7) Lyon, John, 1764-1814 • 1986: (2) - 1987: (7) Lyon, John, 1764-1814 • 1988: (2) • 1989: (1) - 1992: (2) - 1993: (3)

Rogers, Jean Muir Ohio State U., Columbus Correspondence • 1969: (1) • 1972: (1)

Rogers, Julia Ellen Botanical Album

Rogers, Roy P. Correspondence $\bullet$ 1943: (2)

Rogers, Vivian U. of Georgia, graduate student in history; daughter of George A. Rogers Correspondence • 1977: (4) • 1978: (2) • 1979: (2)

Rohay, Ernest Correspondence $\bullet$ 1991: (1)

Rohde-Fulton, Monica Missouri Botanical Garden Correspondence $\bullet 1987$ : (1)

Rohling, Johann C. Bioscript

Rohr, Julius Philipp Benjamin von (1737-1793) Danish surveyor, agronomist, and soldier in West Indies Biofile - Bioscript

Rohrbaugh, Lewis H. U. S. Dept. of Agriculture Correspondence $\bullet$ 1948: (1)

Rohwer, George Newton Bioscript

Rohwer, Sievert Allen (1888-1951) American entomologist Bioscript Correspondence • 1949: (1) Rocky Mountain Naturalists

Rolfe, R. A. Bioscript

Rolfe, W. D. Ian U. of Glasgow, Hunterian Museum Correspondence $\bullet$ 1977: (2)

Roller, Duane H. D. U. of Oklahoma, DeGolyer Library, History of Science Collections Correspondence • 1965: (4) • 1977: (1) • 1979: (1) • 1980: (1)

Rollins College Librarian Correspondence • 1949: (1)

Rollins, Mary L. New Orleans, LA Botanical Album Correspondence • 1985: (3) 
Rollins, Reed Clark (1911-) American cytologist, taxonomist; Harvard U., Gray Herbarium; American Society of Plant Taxonomists

Biofile $\bullet$ Botanical Album

Correspondence - 1936: (3) Species • 1938: (10) Species - 1948: (4) Species • 1949: (5) • 1950: (4) • 1951: (2) • 1952: (9) - 1953: (2) See correspondence with Fassett $\bullet$ 1954: (2) • 1955: (3) • 1956: (6) • 1957: (1) • 1961: (6) • 1962: (3) • 1964: (4) • 1965: (1) • 1966: (3) • 1967: (6) • 1968: (1) See correspondence with Edward G. Voss $\bullet$ 1969: (3) • 1976: (2) • 1987: (3) • 1990: (1) • 1991: (0) Filed under Emanuel David Rudolph

Rollins, Sophie H. Gulfport, MS Correspondence • 1949: (2) • 1951: (2) • 1952: (1) • 1958: (1) • 1962: (3) • 1965: (2)

Romanes, G. J. Bioscript

Romans, Bernard (ca. 1720-1784) German-born Dutch-naturalized American patriot, surveyor, and soldier Biofile • Bioscript

Rome, Italy Botanical Album

Romell, Lars (1854-1927) Swedish mycologist, plant collector Biofile

Romer, Correspondence • 1962: (1)

Romer, Alfred S. Botanical Album

Romero, Gustavo A. (1955-) Oakes Ames Orchid Herbaria, Cambridge, MA

Correspondence • 1989: (1) • 1993: (1)

Romy, E. Jules Bioscript

Ronald Press Correspondence • 1962 : (0) Filed under George Booth Van Schaack

Rood, Almon Nicholson (1876-) Ohio plant collector; Cleveland, $\mathrm{OH}$ Biofile Correspondence • 1921-1928: • 1929-1933: (9) Letter to George A. Cook • 1936: (3) • 1937: (2) • 1939: (1) • 1940: (1)

Rookmaaker, $\mathbf{M}$.

Correspondence • 1981: (2) Book collectors

Rooney, James

Correspondence • 1962: (2)

Roosevelt, Eleanor Botanical Album

Roosevelt, Theodore (1858-1919) American president (of the U. S.), ranchman, statesman, soldier, naturalist Bioscript

Root, Charles (1863-) Bioscript

Root, Katherine U. of California, Berkeley, Library School Correspondence • 1937: (2)

Root, Nina J. American Museum of Natural History, Chief Librarian Correspondence • 1978: (2) • 1980: (1) • 1981: (1) • 1982: (1) • 1983: (2)

Röper, Johannes Bioscript
Roper, Lanning Royal Horticultural Society, London, Editorial Office Correspondence $\bullet 1960$ : (1)

Roppolo, Michael Tulane U., Assistant Dean Correspondence $\bullet$ 1975: (1)

Roscoe, William (1753-1831) British historian, botanist, banker at Liverpool

Biofile

Roscowius, D. M. Bioscript

Rose, Agnes Knowsley, Liverpool, Earl of Derby's Estate. Librarian

Correspondence • 1975: (8) Bartram, William, 1739-1823 - 1977: (1)

Rose, John Bioscript

Rose, Joseph Nelson (1862-1838) American botanist Biofile $\bullet$ Bioscript $\bullet$ Botanical Album

Rose, L. J. Bioscript

Rose, Lewis Samuel (1893-) American plant collector, taxonomist; California Academy of Sciences, San Francisco. CA

\section{Bioscript} Correspondence $\bullet 1961:(2)$

Rosen, James M. "Eminent Scholar in Art," Augusta College, GA Correspondence • 1993: (5)

Rosenbach, A. S. W. Bioscript

Rosenberg, Charles E. ISIS, Editor Correspondence $\bullet$ 1989: (1)

Rosenberg, Gary H. Correspondence $\bullet 1990:$ (2)

Rosendahl, Carl Otto (1875-1956) U. of Minnesota, Dept. of Botany, Chairman Botanical Album Correspondence $\bullet$ 1939: (7) • 1942: (3) • 1943: (2)

Rosengarten, Frederic, Jr. Princeton, NJ Bioscript $\bullet$ Botanical Album Correspondence $\bullet$ 1991: (4) 1992: (2)

Rosengurtt, Bernardo (1916-) Uruguayan agronomist, taxonomist; Uruguay Botanical Album

Rosenheim, Eddie St. Louis, MO Correspondence $\bullet$ 1989: (1)

Rosenthal, Bernard M. Botanical Album

Rosenthal, Marcia Correspondence $\bullet 1992:$ (1)

Rosenvinge, Lauritz Kolderup (1858-1939) Danish algologist Biofile

Rosier, Abbe Bioscript

Ross, B. R. Bioscript

Ross, David Bioscript

Ross, Edith A. Botanical collector Bioscript 


\section{Guide to the Ewan Papers}

Ross, Robert (1912-) English taxonomist; British Museum (Natural History), Botany Dept.

Botanical Album

Correspondence • 1965: (1) • 1966: (1) • 1967: (2) •

1969: (1) British Museum (Natural History). Dept. of Botany $\bullet 1977$ : (1)

Ross, Roland Case Los Angeles City Schools, Roland Case Ross Library for Nature Study

Bioscript

Correspondence • 1921-1928: (1) • 1965: (1) • 1984: (1) • 1985: (2)

Rossbach, George Bowyer (1910-) American biologist, taxonomist; Stanford U., Dudley Herbarium

Correspondence • 1939: (2) Species • 1940: (1)

Rosser, Effie Moira (1923-) Welsh plant anatomist, taxonomist; U. of Manchester, England, Asst. Keeper of Botany

Correspondence • 1955: (4) Pursh, Frederick, 1774-1820

Rossi-Wilcox, Susan M. Harvard U., Botanical Museum Correspondence $\bullet$ 1993: (1)

Rossmäesler, Emil Adolph (1806-1867) Biofile $\bullet$ Bioscript

Rosso, Samuel Wilford (1928-) American plant anatomist; U. of Southern Mississippi, Hattiesburg, Dept. of Biology Correspondence • 1977: (1)

Rostan, E. Bioscript

Rostrup, Emil (1831-1907) Danish botanist Biofile

Rota, Lorenzo (1819-1855) Italian physician, taxonomist Bioscript

Rotell, Thomas M. Macmillan Company, New York, Biology Editor

Correspondence $\bullet$ 1962: (2)

Rotger, Bernard C. R. (1904) Entomological collector and Roman Catholic clergyman; St. Joseph's Church, Capulin, CO

Correspondence • 1949: (3) Rocky Mountain Naturalists

Rothmaler, Werner Hugo Paul (1908-1962) German botanist

Biofile

Rothrock, Joseph Trimble (1839-1922) American surgeon, botanist, explorer, and forester

Biofile • Bioscript

Rothschild, Lionel Walter Botanical Album

Rothwell, Norman V. Indiana U., Botany Dept., graduate student

Correspondence • 1951: (6) Species

Rougemont, Louis de (Grien, Henri Louis, or Grin or Grein) (1847-1921) Literary fraud Bioscript

Rouleau, Ernest Botanical Album

Roumeguère, $\mathrm{C}$. Bioscript

Round Table Club New Orleans, LA Correspondence $\bullet$ 1975: (2)

Rounds, R. Stowell Ukiah, CA Correspondence • 1990: (5) $\bullet$ 1992: (1)
Rouse, Parke Jamestown Foundation, Williamsburg, VA Correspondence $\bullet$ 1966: (3) $\bullet$ 1969: (3)

Rousseau, George Sebastian U. of California, Los Angeles, Dept. of English Bioscript $\bullet$ Botanical Album

Correspondence • 1969: (1) $\bullet$ 1970: (6) Book collectors 1971: (3) • 1972: (2) 1974 (8) $\bullet 1975:(2) \bullet 1978:(3) \bullet$ 1980: (1) • 1983: (6) • 1984: (2) • 1986: (1)

Rousseau, Jacques (1905-1970) Canadian ethnobiologist, botanical historian; Université Laval, Quebec, Canada Botanical Album

Correspondence $\bullet$ 1966: (2) $\bullet$ 1968: (3)

Rousseau, Jean-Jaques (1712-1778) French writer and philosopher

Biofile

Rousseau-Girard, ___ Libraire-Expert-Editeur D'Art, Paris, France

Correspondence $\bullet$ 1970: (1)

Rowe, Francis T. Correspondence • 1939: (1)

Rowed, Harry Photographer Correspondence • 1954: (3) "Rocky Mountain Vegetation"

Rowland, Lloyd W. Fine Arts Club of New Orleans Correspondence $\bullet$ 1967: (5)

Rowlands, Maldwyn Jones (1918- ) British Museum (Natural History), Librarian

Correspondence • 1967: (3) • 1969: (2) • 1970: (1) • 1977: (3) • 1978: (2) • 1979: (3) • 1980: (4) • 1981: (1)

Rowley, Gordon Douglas (1921-) British taxonomist, cytotaxonomist; U. of Reading, England, Dept. of Agriculture

Biofile • Botanical Album

Correspondence • 1964: (3) $\bullet$ 1965: (1) • 1969: (8) $\bullet$ 1970: (1) • 1973: (2) • 1974: (1) • 1975: (3) • 1976: (2) • 1978: (2) Book collectors $\bullet$ 1983: (3) $\bullet$ 1992: (1)

Rowntree, Lester (1879-1979) British-born California naturalist, horticulturist, writer Biofile

Roxburgh, William (1751-1815) British botanist and physician Biofile • Bioscript

Roy, Donald E. Bioscript

Roy, William, Mrs. Bioscript

Royal Botanic Garden, Edinburgh Botanical Album Filed under Edinburgh Correspondence $\bullet$ 1948: (1) $\bullet$ 1962: (1)

Royal Botanic Gardens (Hamilton, Canada) Leslie Laking, Director; Jack Lord, Historical Researcher Correspondence • 1973: (1) • 1979: (1)

Royal Botanic Gardens, Kew Botanical Album

Correspondence - 1948: (1) - 1950: (2) • 1953: (0) Filed under Edward James Salisbury

Royal College of Physicians

Correspondence • 1964: (2) Bartram, William, 1739-1823 
Royal Horticultural Society (Great Britain) London, England

Botanical Album

Correspondence • 1958: (1) • 1961: (1) • 1964: (2) • 1969: (2)

Royal Society (Great Britain) London Correspondence 1970: (1) Beaglehole, John Cawte

Royce, Josiah (1855-1916) American philosopher and teacher

Biofile

Royen, Adriaan van (1704-1779) Plant collector Bioscript

Royen, David van (1727-1799) Plant collector Bioscript

Royen, Pieter van (1923- ) Indonesian-born taxonomist; Rijksherbarium, Leiden, Netherlands; Bernice P. Bishop Museum

Correspondence • 1967: (2) • 1970: (1) • 1971: (2) • 1974: (1)

Royer, Sue U. of Oregon, Eugene, Biol. Dept. Correspondence • 1978: (1)

Royle, J. F. Bioscript

Royle, John Forbes (1799-1858) British botanist and physician Biofile

Roze, Ernest (1833-1900) French mycologist Bioscript

Rübel, Eduard (fl. 1913) Botanical Album

Rübel, Eduard August (1876-1960) Bioscript

Rubin, Jay Lewis Correspondence $\bullet$ 1948: (2)

Rubin, Mayer U. S. Geological Survey Correspondence • 1959: (2)

Rubinstein, Joseph Correspondence $\bullet 1972:(1)$

Rubnioff, Roberta Smithsonian Institution; nickname "Bobbie"

Correspondence $\bullet$ 1985: (2)

Rubtzeff, Peter U. of San Francisco, Dept. of Botany Correspondence • 1954: (1)

Ruckdeschel, Carol Correspondence • 1982: (2)

Rudbeck, Olaus (1660-1740) Swedish naturalist, physician Bioscript

Rudd, Hugh CBS News Correspondence $\bullet$ 1976: (1)

Rudd, Velva Elaine (1910-) American taxonomist Bioscript Correspondence • 1956: (1) • 1966: (1) • 1987: (3)

Rudge, Edward Bioscript

Rudoff, Stan L. Columbia U., New York, Dept. of Chemistry

Correspondence $\bullet$ 1953: (1)

Rudolph, Ann Waterman (1934-1991) American bibliographer; Columbus, $\mathrm{OH}$; husband is Emanuel D. (Rudy) Rudolph Bioscript Correspondence • 1969: (2) • 1981: (5)
Rudolph, Emanuel David (1927-1992) American plant taxonomist, lichenologist; Ohio State U., Columbus, Dept. of Plant Biology, nickname "Rudy"

Biofile • Bioscript $\bullet$ Botanical Album

Correspondence • 1964: (1) • 1965: (1) • 1966: (10) • 1967: (13) - 1968: (14) Biology-Instruction and stady; Science-Instruction and study • 1969: (9) Lawrence, George Hill Mathewson, 1910-1978 • 1970: (9) • 1971: $(8) \bullet 1972:(6) \bullet 1973:(11) \bullet 1974:(9) \bullet 1975:(3) \bullet$ 1976: (10) Barton, Benjamin Smith, 1766-1815; Harper, Francis, 1886-1972; Book collectors - 1977: (3) Book collectors • 1978: (2) Van Schaack, George Booth, 1903-1983 - 1979: (1) • 1980: (4) Van Schaack, George Booth, 1903-1983 • 1981: (1) • 1982: (17) • 1983: (4) • 1984: (5) • 1985: (1) • 1986: (2) • 1987: (10) • 1988: (3) - 1989: (12) • 1990: (0) Filed under Harriet Jansma • 1990: (22) - 1991: (22) - 1992: (10)

Rudolph, Johann H.

Bioscript

Ruel, Jean

Bioscript

Rufin, Edmund (1794?-1865) American publisher of Farmers Register, 1833-1842, and agricultural author Biofile $\bullet$ Bioscript

Rugel, E. Bioscript

Rugel, Ferdinand Bioscript

Rugel, Ferdinand (1806-1878) German-born American botanical explorer, physician Biofile

Rugg, Harold Goddard (1883-1957) Dartmouth College, Hanover, NH

Correspondence • 1929-33: - 1946: (1) American Fern Society; letter to C. A. Weatherby $\bullet$ 1947: (1) American Fern Society

Ruhl, Joseph L. Covington Correspondence $\bullet 1958$ : (3) Letter to $\mathrm{Dr}$. Cagle referred to Ewan

Ruhle, G. C. Dept. of Interior Correspondence $\bullet$ 1936: (1)

Rule, G. H. Bioscript

Rulon Miller Rulon Bookseller Correspondence • 1986: (1)

Rumford, Benjamin Thompson Botanical Album

Rumford, Count Bioscript

Rumphins, Bioscript

Rumsey, Eric Indiana U., Bloomington, Dept. of Biology; M.A. student of C. Heiser's Correspondence $\bullet$ 1978: (2)

Rungius, Carl Clemens Morris (1869-1959) German-born American natural history illustrator Biofile

Rumnette, Evelyn Colorado Mountain Club, Secretary Correspondence • 1943: (3) • 1949: (2)

Runyon, Everett Bioscript $\bullet$ Botanical Album 
Runyon, Robert (1881-1968)

Bioscript

Ruppell, Eduard

Bioscript

Ruppert, Jeanne Florida State U. Press, Tallahassee, FL Correspondence • 1990: (1) • 1991: (1)

Ruprecht, Franz J.

Bioscript

Rusby, Henry Hurd (1855-1940) American botanist, physician, plant explorer

Biofile • Bioscript • Botanical Album

Correspondence $\bullet$ 1938: (3)

Ruschenberger, W. W. Bioscript

Rusconi, Mauro (1776-1849) Zoologist Botanical Album

Rush, Benjamin (1745-1813) American physician of Philadelphia

Biofile • Bioscript

Rush, James

Bioscript

Rush, Jane

Correspondence • 1969: (1)

Rush, William (1745-1813) American sculptor Biofile

Rusk, Hester M. Brooklyn Botanic Garden; American Fern Society

Correspondence • 1941: (5) • 1942: (2) • 1943: (1)

Russell, Bertrand London, England

Bioscript $\bullet$ Botanical Album

Correspondence $\bullet$ 1959: (2)

Russell, E. C.

Bioscript

Russell, J. L. Bioscript

Russell, Norman Hudson (1921-) American taxonomist; Grinnell College, Grinnell, IA, Botany Dept., Chairman Correspondence • 1958: (6) • 1959: (10) • 1960: (1) • 1964: (1) • 1966: (2) Bartram, William, 1739-1823 - 1967: (6)

Russell, Paul George (1889-1963) American botanist, plant collector, seed technologist; U. S. Dept. of Agriculture, Bureau of Plant Industry, Beltsville, MD Correspondence • 1953: (2) • 1955: (1)

Russell, Thomas Bioscript

Rust, Mary Olivia Husband is Styles M. Rust Bioscript

Rutgers University Botanical Album

Ruth, Albert Botarical Album

Ruth, Joel (1844-1932) American botanist and plant collector Biofile

Rutter, J. M. Bioscript
Ruxton, George Frederick Augustus (1820-1848) British traveller, ethnologist Biofile $\bullet$ Bioscript

Ryan, Don Northwestern State U., Matchitoches, LA, Mathematics

Correspondence $\bullet$ 1978: (1)

Ryan, R. M.

Correspondence $\bullet$ 1975: (1)

Ryan, Shirley The U. of Wisconsin Press, Madison, WI, Secretary

Correspondence $\bullet$ 1962: (2)

Rydberg, Per Axel (1860-1931)

Bioscript $\bullet$ Botanical Album

Rylander, Michael Kent (1935- ) Texas Tech. College, Lubbock, Dept. of Biology, Ornithology, Vertebrate morphology

Correspondence • 1965: (3) - 1966: (4) • 1968: (4) 1969: (1) • 1970: (3) • 1971: (1) • 1972: (2) - 1974: (1) 1975: (1) • 1976: (2) • 1979: (1) • 1982: (2) • 1983: (3) 1984: (1) 1986: (1)

Rzedowski, Jerzy (1926-) Polish taxonomist in Mexico; U. of San Luis Potosi, Mexico,

Institute of Desert Investigation

Correspondence • 1955: (4) • 1958: (1)

\section{$S$}

Sabin, Joseph (1821-1881) English-born American bibliopolist

Bioscript

Sabine, Edward (1788-1883) Arctic explorer, ornithologist Bioscript $\bullet$ Botanical Album

Sabine, Joseph (1770-1837) English botanist; Royal Horticultural Society

Bioscript

Saccardo, P. A. Bioscript

Sachet, Marie-Hélène (1922-1986) Smithsonian Institution, National Academy of Sciences

Botanical Album

Correspondence • 1962: (2) • 1968: (1)

Sachs, Julius von (1832-1897) German botanist, plant physiologist

Biofile

Sadler, Alfredo F. São Paulo, Brazil; nickname "Fred" Correspondence • 1985: (4) • 1986: (1)

Saenz, Gabriel

Botanical Album

Correspondence • 1945: (1)

Safford, William Edwin (1859-1926) American ethnobotanist and conchologist

Bioscript • Botanical Album

Sagard, Theodat

Bioscript 
Sagra, Ramón de la (1798-1871) Spanish-born botanical historian, naturalist; Spain, Switzerland

Bioscript

Sahlins, Marshall

Botanical Album

Saint-Pierre, Bernardin de Bioscript

Sakamaki, Shunzo U. of Hawaii, Honolulu Correspondence $\bullet$ 1966: (1)

Salbach, Carl Bioscript

Saldanha de Gamma, J. de Bioscript

Sale, G. U. of Colorado Correspondence $\bullet$ 1947: (1)

Sales, Fatima Royal Botanic Garden, Edinburgh Correspondence 1990: (1)

Salisbury, Edward James (1886-) English ecologist, administrator; Royal Botanic Gardens, Kew, Director Botanical Album

Correspondence • 1949: (1) • 1951: (1) • 1955: (1) 1956: (2)

Salisbury, R. A. Bioscript

Salle, A. Bioscript

Salloch, William Bookseller; Oswego, NY Correspondence - 1976: (2) Book collectors

Salm-Reifferscheid, Joseph, Graf zu (1773-1861) Botanical Album

Saltzmann, Phillipp Bioscript

Salvin, $\mathbf{O}$. Bioscript

Sam Jones Book Store Correspondence 1986 : (1)

Sambo, Maria Cengia (1888-1939) Italian lichenologist Biofile • Bioscript

Samples, John (fl. 1833-1838) Ohio plant collector and teacher Biofile

Sampson, Homer Cleveland Botanical Album

Sams, Mary Correspondence • 1968: (3)

Samuel, Ray New Orleans Book Collectors Club, New Orleans Correspondence $\bullet$ 1979: (2) Book collectors

Samuels, Isadora New England Mutual Life Correspondence 1 1941: (1)

Samuelsen, J. Roger U. of California, Los Angeles Correspondence $\bullet$ 1975: (1)

Samuelson, Gunnar (1885-1944) Swedish taxonomist; Natural History Museum Stockholm Botanical Album Correspondence • 1929-1933: (2) • 1934: (1) • 1938: (2) • 1939: (4) • 1940: (1)

Sancton, Seta Correspondence • 1976: (0) Filed under Tulane University Howard-Tilton Memorial Library, Librarian
Sandberg, John Herman (1849-1917) Bioscript

Sandel, Benjamin

Bioscript

Sanders, Albert E. Charleston Museum, Director Correspondence • 1982: (1) • 1983: (1) • 1989: (1) • 1993: (1)

Sanders, Albert N. Furman U., Greenville, SC, Dept. of History

Correspondence • 1971: (3)

Sanders, Dana R. Northwestern State U. of Louisiana, Natchitoches, Dept. of Biology

Correspondence $\bullet 1971$ : (2)

Sanders, W. D., Jr. Tulane U., Dept. of Psychiatry \& Neurology

Correspondence $\bullet$ 1970: (1)

Sanderson, Ruth Dexter (1891-) American librarian; Harvard U., Gray Herbarium, Librarian Correspondence • 1935: (5) $\bullet$ 1936: (1) • 1937: (3) • 1939: (1) • 1940: (1) • 1941: (7) • 1942: (1) • 1944: (4) Delphinium • 1945: (2) • 1946: (2) • 1949: (4) • 1950: (1) $\bullet 1951:(2)$

Sandig, A. Botanical Album

Sandoe, James U. of Colorado, Drama Dept. Correspondence $\bullet$ 1943: (1) $\bullet$ 1947: (1)

Sandwith, Noel Yvri (1901-1965) English taxonomist; Royal Botanic Gardens, Kew, Herbarium

Biofile • Botanical Album Correspondence • 1945: (2) Vismia • 1946: (2) Vismia • 1948: (2) • 1951: (2) Pursh, Frederick, 1774-1820 • 1956: (2) - 1962: (2) Vismia

Sandys, George (1578-1644) English-American poet, traveller, book collector Biofile

Sanford, James A. Bioscript

Sanger, Marjory Bartlett Correspondence • 1968: (3)

Sanguinetti, Pietro (1802-1868) Italian botanist Bioscript

Sanson, Norman Bethune Bioscript $\bullet$ Botanical Album Correspondence $\bullet$ 1949: (3) Rocky Mountain Naturalists

Santa Barbara Botanic Garden Botanical Album Correspondence $\bullet$ 1990: (1)

Santa Barbara Museum of Natural History Correspondence • 1968: (0) Filed under Clifton F. Smith Santa Monica Planning Commission Botanical Album

Santi, Giorgio Bioscript

Santvoort, Bioscript

Saporta, Gaston - Mg. de Bioscript

Sappa, Francesco Instituto Botanico dell'Universita Torino Correspondence $\bullet$ 1947: (2) • 1956: (1)

Sapper, Kari (1866-) Bioscript 


\section{Guide to the Ewan Papers}

Saquet, Jannette Librarian; Smithsonian Institution, Dept. of Anthropology

Correspondence • 1975: (2)

Sargent, Charles Sprague (1841-1929) American horticulturist, taxonomist, dendrologist Bioscript $\bullet$ Botanical Album

Sargent, Francis H. Amateur botanist; Picayune, MS Bioscript • Botanical Album

Correspondence $\bullet$ 1964: (11) Species • 1966: (11) Harper. Roland McMillan, 1878-1966; Species • 1967: (3) • 1968:

(2) - 1969: (4) Biology-Instruction and study $\bullet$ 1971: (1)

Delphinium • 1972: (5) • 1973: (3) • 1974: (4) • 1975: (3)

- 1976: (2) Copy of correspondence between Warren

Wagner and F. H. Sargent • 1977: (3) • 1978: (1)

Sargent, H. W.

Bioscript

Sargent, Ralph M. Haverford College, Professor Emeritus of English

Botanical Album

Correspondence • 1970: (4) • 1972: (3) Barton, Benjamin Smith, 1766-1815 • 1973: (2) Harper, Francis, 1886-1972 - 1975: (1) • 1978: (2) • 1979: (2) • 1984: (1)

Sarjeant, William A. S. Canadian geologist Biofile • Bioscript

Correspondence • 1986: (1)

Sarraxin, Michel (1659-1735) Biofile

Sarton, George ISIS, Editor Botanical Album

Correspondence • 1949: (2)

Sartori, Franz

Bioscript

Sartorius, Carlos (1796-1872) German-born Mexican farmer and botanist Biofile

Sartwell, Henry Parker (1792-1867) American botanist and physician

Biofile • Bioscript

Sass, Herbert Ravenel

Botanical Album

Satchell, Geofirey, Mrs. U. of Adelaide, Adelaide, South Australia, Dept. of Zoology

Correspondence $\bullet$ 1962: (2)

Sauer, Carl Ortwin (1889-1975) American geographer, U. of California, Berkeley, Dept. of Geography Biofile • Bioscript

Correspondence • 1937: (2) • 1959: (2) • 1960: (1)

Sauer, Gordon C. Physician, Kansas City, MO Botanical Album

Correspondence • 1983: (3) • 1984: (7) • 1989: (5) • 1992: (2) • 1993: (7)

Sauer, Jonathan Deininger (1918- ) American botanist, ethnobotanist, phytogeographer; U. of California, Los Angeles, Dept. of Geography

Bioscript • Botanical Album

Correspondence • 1952: (8) • 1953: (2) • 1967: (2) •

1974: (4) • 1975: (5) • 1978: (2) 1984: (2) $\bullet$ (2) • 1990: (2)

Saugrain, Antoine Francois Bioscript
Saul, Sara Bioscript

Saunders, Charles Francis (1859-1941) American economic botanist

Bioscript $\bullet$ Botanical Album

Saunders, De Aiton (1870-) American plant breeder, phycologist, pteridologist

Bioscript

Saunders, R. Frank

Correspondence $\bullet$ 1981: (1)

Saunders, William (1836-1914) English-born Canadian botanist and agricultural scientist; Experimental Farms, Canada, Director

Biofile $\bullet$ Bioscript $\bullet$ Botanical Album

Saunders, William Edwin (1861-1943) Naturalist Bioscript

Saunders, William Wilson (1809-1879) Bioscript

Saunier, Paul ( -1818) French-born American gardener; gardener to Michaux Biofile - Bioscript

Saurman, B. L.

Bioscript $\bullet$ Botanical Album

Savage, Jay M. U. of Southern Calif., Los Angeles, Dept. of Biological Sciences

Correspondence $\bullet$ 1975: (1)

Savage, Spencer (1886-1966) Englist botanical bibliographer; Linnean Society of London

Botanical Album

Correspondence • 1939: (1) • 1954: (3) Pursh, Frederick, 1774-1820

Savage, Thomas I.

Bioscript

Save the Dunes Council Sylvia Troy

Correspondence • 1976: (1)

Save the Redwoods League San Francisco, CA; Newton B. Drury, Secretary

Correspondence • 1959: (2)

Savi, Gaetano

Bioscript

Savi, Paolo

Bioscript

Savi, Pietro

Bioscript

Savicz, Vsevolod Pavlovich (1885-) Lithuanian lichenologist

Botanical Album

Savile, Douglas Barton Osborne (1909-) Irish-born mycologist, botanist in Canada; Dept. of Agriculture, Ottawa, Canada

Correspondence • 1954: (2)

Sawyer, Frederick Charles British Museum (Natural History)

Correspondence 1966: (2)

Sax, Karl (1892- ) American plant geneticist, plant breeder, plant cytologist

Botanical Album

Correspondence • 1968: (1) • 1969: (2)

Saxe, A. W.

Bioscript 
Say, Thomas (1787-1834) American naturalist, entomologist, zoologist, collector Biofile $\bullet$ Bioscript $\bullet$ Botanical Album

Saylor, Larry United States Dept. of the Interior Botanical Album Correspondence • 1939: (1)

Sayre, Geneva (1911-) Russell Sage College, Dept. of Biology

Botanical Album

Correspondence • 1943: (2) • 1968: (1) Rocky Mountain Naturalists • 1975: (1) • 1976: (3) • 1977: (1) • 1980: (7) - 1981: (2) • 1982: (2) • 1990: (1)

Sayre, L. E. Bioscript

Sayre, Robert H. Correspondence $\bullet$ 1944: (1)

Scacchi, Arcangelo Bioscript

Scamman, Edith Henry (1882-1967) American botanist; Harvard U., Gray Herbarium Biofile $\bullet$ Botanical Album Correspondence $\bullet$ 1949: (3) American Fern Society $\bullet$ 1950: (10) American Fern Society $\bullet$ 1951: (9) $\bullet$ 1963: (1)

Scammon, Charles Mellville Bioscript

Scandella, Giambattista (1770-1798) Venetian physician Biofile

Scannell, Maura J. P. National Botanic Gardens, Dublin, Ireland, Herbarium

Correspondence • 1964: (1) • 1969: (2) • 1978: (2)

Scarborough, John U. of Kentucky, Lexington, Prof. History of Medicine Correspondence $\bullet$ 1980: (2)

Scase, R. P. Royal Horticultural Society Garden, England Correspondence - 1964: (1)

Schaeffer, Robert L. (1917- ) American botanist, phytogeographer Botanical Album Correspondence • 1938: (2) • 1939: (2) Delphinium

Schaerer, Ludwig E. Bioscript

Schaffer, Mary T. S. Bioscript

Schaffner, John Henry (1866-1939) American botanist; Ohio State U., Columbus

Botanical Album

Correspondence • 1929-1933: (2) Species • 1935: (3) Species $\bullet$ 1936: (1)

Schaffiner, Mabel (Brockett) Bioscript

Schaffner, Wilhelm (1830-1882) German plant collector and pharmacist in Mexico Biofile • Bioscript

Schaffranek, A. Bioscript

Schallert, Paul Otto (1879-1970) Botanical Album Correspondence • 1940: (1) • 1942: (1) • 1952: (1) • 1957: (1)

Schallert, Ruth F. Smithsonian Institution Correspondence • 1975: (1) • 1985: (1)
Schath, Leonard P. Correspondence $\bullet$ 1945: (1)

Schatz, G. E. Bioscript

Schedin, L. M. Bioscript

Scheerer, Hans Correspondence • 1939: (1)

Schenck, C. Alwin Bioscript

Scheps, Clarence Tulane U., Office of Vice President and Controller

Correspondence • 1957: (1) $\bullet$ 1960: (1) • 1961: (0) Filed under Oeta M. Crump • 1963: (1) • 1965: (2) • 1971: (2)

Schery, Robert Walter (1917-1987) American agronomist, botanist, horticulturist, taxonomist Botanical Album

Scheuber, Emma Ware Bioscript

Scheuerman, Margie T. Correspondence $\bullet 1977:$ (1)

Schevermann, Milton G., Jr. Tulane U., New Orleans, School of Architecture Correspondence $\bullet 1984$ : (4) $\bullet 1985:$ (1)

Scheuler, Amy Missouri Botanical Garden, St. Louis, MO Correspondence $\bullet$ 1993: (1)

Schernayder, Vilma D. Correspondence $\bullet$ 1976: (1)

Schiede, C. J. W. Bioscript

Schiefer, Helen Pease Philadelphia, PA; husband is Dr. Manuel Roca-Garcia Botanical Album Correspondence • 1945: (2) • 1946: (2)

Schiel, Jacob Heinrich (1813-post 1879) Bioscript

Schierenberg, R. \& Sons Inc. Bookseller; Antiquariaat Junk, Lochem, Holland Correspondence • 1969: (2) Book collectors $\bullet$ 1970: (2)

Schimper, Andreas Franz Wilhelm (1856-1901) Germanborn plant explorer, phytogeographer Botanical Album

Schimper, Karl Friedrich (1803-1867) German naturalist Biofile

Schimper, Wilhelm Georg Bioscript

Schimper, Wilhelm Philipp (1808-1880) German bryologist, paleontologist

Bioscript

Schipp, William August (1891-1967) Australian-born explorer and plant collector

Biofile Correspondence $\bullet$ 1934: (1)

Schiro, Victor H. City of New Orleans, Commissioner of Public Buildings and Parks Correspondence $\bullet$ 1953: (1) $\bullet$ 1965: (1)

Corisckin, Boris Konstantinovich (1886-1963) Russian Schisckin, Boris Kontamtiner; Institute and Botanic Gardens of Acad. of Sciences Botanical Album Correspondence $\bullet$ 1939: (2) 


\section{Guide to the Ewan Papers}

Schlebecker, John T. Smithsonian, Division of Agriculture and Forest Products

Correspondence • 1967: (18) • 1968: (6) • 1969: (2) • 1984: (4)

Schlechtendal, Diederich Franz Leonard (1794-1866) German botanist Bioscript

Schleicher, J. C. Bioscript

Schlenker, Gerhard Botanical Album

Schlesinger, Thomas B. Williamsburg Garden Symposium Correspondence $\bullet$ 1972: (3) • 1973: (1) • 1977: (0) Filed under Williamsburg Colonial Foundation

Schlosser, Joseph V., Mrs. Correspondence $\bullet 1976$ : (1)

Schmalz, Lydia Huggins Longue Vue, Center for Decorative Arts, New Orleans; Louisiana Correspondence $\bullet$ 1981: (1)

Schmautz, Jack E. U. S. Dept. of Agriculture, Boise Research Center

Correspondence - 1955: (1) Letter to O. A. Beath 1956 : (4) Delphinium

Sehmid, E. Botanisches Museum und Botanischer Garten, Zurich, Switzerland

Correspondence - 1938: (1) Species

Schmid, Rudolf (1942-) American plant anatomist, plant morphologist; U. of California, Dept. of Botany; Taxon, Book Review Editor; nickname "Rudi"

Correspondence • 1987: (1) • 1988: (2) • 1990: (9) • 1991: (8) • 1992: (4) • 1993: (9)

Schmidt, Elizabeth B. Arnold Arboretum, Harvard Correspondence • 1982: (2)

Schmidt, (Ernst) Johannes (1877-1933) Danish botanist, bacteriologist and oceanographer Biofile

Schmidt, F. Bioscript

Schmidt, G. Correspondence • 1943: (1)

Schmidt, Henry D. Bioscript

Schmidt, J. E. National Association on Standard Medical Vocabulary

Correspondence • 1963: (2)

Schmidt, Johannes Bioscript

Schmidt, John Jacob Bioscript

Schmidt, Karl P. Society for the Study of Evolution Correspondence 1948: (3) Schmitt, John Arved (1925-) American mycologist; Ohio
State U., Columbus

Correspondence $\bullet$ 1972: (2) Schmitt, Martin U. of Oregon, Eugene, Library, Special
Collections

Correspondence • 1969: (2) • 1978: (2)

Schmitt, Waldo La Salle (1887-) American zoologist,
Smithsonian

Biofile • Bioscript • Botanical Album

Correspondence • 1945: (1) • 1946: (1) • 1947: (4) •
Schmitt, Waldo La Salle (1887- ) (continued)

1950: (1) • 1958: (2) • 1965: (10) Banister, John, 1650-1692 • 1966: (2) Banister, John, 1650-1692 • 1969:

(2) Banister, John, 1650-1692 • 1970: (1) • 1977: (1)

Schmitz, Edith A. Edgewater, NJ; husband is L. J. Crockett

Correspondence $\bullet$ 1969: (0) Wedding announcement and xerox of marriage certificate filed under Lawrence J. Crockett

Schmitz, Fred B. Louisiana State U., Port Sulphur Correspondence $\bullet$ 1969: (1)

Schmitz, Irene Los Angeles Audubon Society; husband is John J. Schmitz

Correspondence • 1929-1933: (1)

Schmoll, Hazel Marguerite (1891-)

Bioscript • Botanical Album

Correspondence • 1942: (2)

Schnabel, Muller

Bioscript

Schneider, Camillo Karl (1876-1951) German taxonomist, dendrologist in Austria

Bioscript

Schneider, Carl

Bioscript

Schneider, Johann G. (1750-1822)

Bioscript

Schneider, Walter $\mathbf{M}$.

Correspondence $\bullet$ 1976: (2) • 1989: (1) • 1990: (1)

Schneider, Woldemar Pharmazeutisches Institut der Universität Freiburg, Germany

Correspondence • 1951: (2) Delphinium

Schoen, Lori

Correspondence • 1976: (1) Correspondence with Lori Schoen and Melissa Melan

Schoenemann, Charles William (1821-1912)

Bioscript

Schomburgk, Robert Herman (1804-1865) German botanist and explorer

Biofile - Bioscript

Schoolcraft, Henry Rowe (1793-1864) American geologist Biofile

Schopfer, William Henri

Botanical Album

Schöpf, Johann David (1752-1800) German army physician and plant collector in American Revolutionary War

Biofile • Bioscript

Schott, A.

Bioscript

Sehott, Elliot G. F.

Bioscript

Schott, H. W. Bioscript

Schott, Heinrich Wilhelm (1784-1865) Moravian-born Austrian botanist, gardener Biofile

Schouw, J. F. Bioscript

Schrader, A. Bioscript 
Schrader, Heinrich Adolph (1767-1836) German physician, botanist

Bioscript

Schrameyer, Henry American Fern Society Correspondence - 1946: (1) American Fern Society

Schramm, Jacob Richard (1885-) American plant physiologist; U. of Pennsylvania, Philadelphia Botanical Album

Correspondence • 1939: (1) • 1945: (3) • 1956: (5) • 1957: (2) • 1966: (2) Bartram, William, 1739-1823

Schramm, Mildred W. S. Husband is J. R. Schramm Correspondence $\bullet$ 1958: (2) • 1959: (6)

Schreber, Daniel Bioscript

Schreber, Jonathan Christian Daniel von (1739-1810) Bioscript

Schroeder, Charles Arthur (1913-) American plant morphologist; U. Of California, Los Angeles; nickname "Art"

Botanical Album

Correspondence • 1950: (2) Species • 1989: (3) Species • 1990: (1)

Schroeder, Gail Animal behaviorist; Mikumi [National Park in E. Tanzania]

Correspondence $\bullet$ 1977: (1)

Schroeder, Robert S. U. of Arkansas, Fayetteville, Dept. of Chemistry

Correspondence $\bullet$ 1966: (2) Delphinium

Schröter, Carl Joseph (1855-1939) German-born phytogeographer, taxonomist; Switzerland Bioscript $\bullet$ Botanical Album

Schryver, C. D. Correspondence 1949: (3) Rocky Mountain Naturalists

Schubeler, F. C. Bioscript

Schubert, Bernice Giduz (1913-) American taxonomist; Arnold Arboretum Bioscript Correspondence • 1943: (6) Delphinium $\bullet$ 1945: (1)

Vismia • 1953: (4) • 1963: (1) - 1964: (21) $\bullet$ 1965: (16) 1966: (4) • 1967: (3) $\bullet$ 1968: (2) • 1969: (4) • 1975: (2) $\bullet$ 1976: (1) - 1978: (1)

Schuchert, Charles (1858-1942) American paleogeographer Biofile

Schueler, Fred Tulane Medical School, Dept. of Pharmacology, Head Correspondence $\bullet$ 1958: (2) $\bullet$ 1961: (2)

Schuetz, Christie U. of minois Press Correspondence • 1970: (1) • 1971: (3) • 1972: (3) Banister, John, 1650-1692

Schufle, J. A. New Mexico Highlands U., Las Vegas, NM Botanical Album

Schuhr, Klaus J. Arbeitskrels fur Mammillarien-Freund Correspondence • 1984: (1)

Schulenberg, Ray Correspondence - 1975: (2)

Schulman, Edmund U. of Arizona, Tucson Correspondence - 1956: (2) "Rocky Mountain Vegetation" Schultes, Julius Bioscript
Schultes, Richard Evans (1915-) American ethnobotanist, plant collector; Harvard, Botanical Museum Biofile $\bullet$ Bioscript $\bullet$ Botanical Album

Correspondence • 1941: (2) • 1947: (1) • 1949: (1) • 1952: (1) • 1954: (4) • 1958: (1) • 1961: (2) • 1962: (2) • 1965: (2) • 1969: (1) • 1970: (1) • 1976: (2) Merrill, Elmer Drew, 1876-1956; copy of correspondence with John W. Thieret • 1978: (1) • 1985: (1) • 1988: (4) • 1989: (7) - 1990: (10) - 1991: (1) • 1992: (2) • 1993: (5)

Schultheisz, Emil (1899-) Semmelweis U. of Medicine, Budapest, Hungary

Correspondence • 1990: (1)

Schultz, Clarence Jefferson Expansion Memorial, St. Louis Correspondence • 1963: (1)

Schultz, L. P. Smithsonian Institution Correspondence • 1945: (1)

Schultz, Wilhelm Bioscript

Schulze, George Martin ( 1909-) German taxonomist Botanical Album

Schuman, Henry Henry Schuman, Inc., Publishers Correspondence • 1950: (2) Rocky Mountain Naturalists

Schumann, Karl Bioscript

Schutz, Terry Quarterly Review of Biology, Johns Hopkins U., Dept. of Biology Correspondence $\bullet$ 1957: (4)

Schuyler, Alfred Ernest (1935-) American taxonomist; carciologist; The Academy of Natural Sciences, Philadelphia, Dept. of Botany; nickname "Ernie" Biofile • Botanical Album

Correspondence • 1965: (4) • 1966: (1) • 1968: (3) • 1971: (1) • 1972: (1) • 1974: (2) • 1975: (8) • 1976: (2) • 1977: (5) Academy of Natural Sciences of Philadelphia $\bullet$ 1978: (3) • 1979: (3) Pursh, Frederick, 1774-1820 • 1980: (3) • 1982: (3) • 1983: (5) • 1984: (12) • 1985: (8) - 1986: (1) - 1988: (12) • 1989: (8) • 1990: (10) - 1991: (10) - 1992: (14) Pursh, Frederick, $1774-1820$

Schwägrichen, Christian Friedrich (1775-1953) Bioscript

Schwarten, Lazella Harenberg (1900-) American librarian, botanical bibliographer; Harvard U., Arnold Arboretum, Gray Herbarium, Librarian

Botanical Album

Correspondence • 1942: (2) • 1946: (1) • 1948: (4) • 1952: (3) - 1957: (1) - 1958: (2) • 1959: (15) $\bullet 1961$ : (1) Banister, John, 1650-1692 • 1962: (2) • 1964: (1) • 1965: (2) • 1966: (3) • 1967: (1)

Schwartz, Diane Librarian, New York Botanical Garden Correspondence • 1975: (2) • 1976: (3)

Schwartz, Douglas W. School of American Research, Santa Fe, Director Correspondence $\bullet 1977$ : (2) $\bullet$ 1978: (2)

Schwartz, John T. Colorado Associated U. Press, U. of Colorado, Boulder Correspondence • 1976: (0) Filed under Colorado Associated U. Press

Schwartz, Karlene V. U. of Massachusetts, Boston, Dept. of Biology

Correspondence • 1976: (2) 
Schwarz, Eugene Amanders (1844-1928) Bioscript

Schwarz, Herbert F. American Museum of Natural History Correspondence • 1948: (2) Cockerell, Theodore Dru Alison, 1866-1948

Schweid, Richard Portland, OR Correspondence • 1979: (2)

Schweinfurth, Charles (1890-1970) American botanist, orchidologist

Biofile $\bullet$ Botanical Album

Correspondence • 1951: (4)

Schweinfurth, George

Bioscript

Schweinitz, Lewis David von (1780-1834) American clergyman, botanist

Biofile $\bullet$ Bioscript $\bullet$ Botanical Album

Schwöder, Ad (A. 1882)

Bioscript • Botanical Album

Science American Assn. for the Advancement of Science Correspondence • 1966: (3)

Scientific Monthly Editorial Board Correspondence $\bullet$ 1955: (1)

Scoggan, Homer John (1911-) American-born taxonomist in Canada; National Museum of Canada, Ottawa Correspondence $\bullet$ 1952: (2)

Scoggins, William

Correspondence $\bullet 1974:$ (1)

Scolay, Samuel ( -1857)

Bioscript

Scopoli, Giovanni Antonio (1723-1788) Italian naturalist, physician Biofile

Scott, Arthur Moreland (1888-1963) English-born American civil engineer and amateur phycologist Biofile • Bioscript • Botanical Album Correspondence • 1957: (1) - 1959: (1) TLS Ewan to W. J. Robbins 1960: (1) Includes copy of letter from Arthur M. Scott to H. W. Rickett $\bullet$ 1961: (1) $\bullet$ 1963: (2) Letter from George Lawrence

Scott, B. D. Northwest Missouri State U., Maryville, Dept. of Biology, Professor

Correspondence • 1976: (2)

Scott, Burnett

Botanical Album

Correspondence • 1983: (1)

Scott, Flora Murray (1891-) Scottish-born American plant anatomist; U. of California, Los Angeles, Dept. of Botany and Plant Biochemistry

Biofile $\bullet$ Bioscript $\bullet$ Botanical Album

Correspondence • 1942: (1) • 1945: (1) • 1948: (2) • 1951: (1) $\bullet$ 1965: (2) $\bullet$ 1966: (1) $\bullet 1968:(1) \bullet 1970:$ (2) $\bullet$ 1972: (3) • 1976: (1) • 1977: (1) • 1983: (1) • 1988: (1)

cott, Harold W.

Correspondence $\bullet 1977$ : (1)

Scott, Hugh

Bioscript

Scott, John R.

Correspondence $\bullet$ 1940: (2)

Scott, John W. U. of Wyoming, Laramie

Correspondence • 1939: (2)
Scott, Julian H. U. of Bristol, England

Correspondence $\bullet$ 1982: (1)

Scott, Lois S.

Botanical Album (Filed under Burnett Scott)

Correspondence • 1982: (1) • 1986: (3) • 1990: (1) • 1992: (1) • 1993: (1)

Scott, _, Mrs. Tulane U., New Orleans, LA

Correspondence $\bullet 1964$ : (1)

Scott, Nell I. Adult Education Council of Denver Correspondence 1943: (1) U. of Colorado, Boulder. Dept. of Biology - 1943: (0) Letter filed under Kathryn Kalmbach

Scott, Peter Bioscript

Scott, R. Robinson Bioscript

Scott, W. E. D. Bioscript

Scott, William Berryman (1858-1947) Bioscript

Scouler, John (1804-1871) Scottish geologist and botanist Biofile • Bioscript

Scrase, David Botanical Album

Seribner, Frank Lamsoń (1851-1938) Bioscript

Seribner's, Charles and Sons Publishers Rare Book Dept. Correspondence • 1967: (2) • 1968: (2) • 1969: (1)

Scudder, Samuel Hubbard (1837-1911) American entomologist Biofile $\bullet$ Bioscript

Scully, Arthur L. P., Jr. Architect and Architectural Historian Correspondence $\bullet$ 1970: (3) $\bullet$ 1971: (2) $\bullet$ 1991: (4) 1992: (1)

Seal, Thomas

Botanical Album

Seaman, W. H.

Bioscript

Searls, Fanny (1851-1939)

Bioscript - Botanical Album

Sears, James R. U. of Massachusetts, Amherst Correspondence $\bullet$ 1971: (1)

Sears, Paul Bigelow (1891-1990) American botanist, plant ecologist; Yale U.

Bioscript $\bullet$ Botanical Album

Correspondence • 1966: (3) • 1968: (4) • 1969: (2) • 1971: (2) • 1976: (2)

Sears, W. L.

Correspondence 1 1929-1933: (3)

Seaver, Fred Jay (1877-1970) American mycologist Biofile $\bullet$ Bioscript $\bullet$ Botanical Album Correspondence • 1949: (3) • 1951: (1)

Seaward, Mark Richard David (1938-) English bryologist, lichenologist; U. of Bradford, Dept. of Environmental Science, England

Correspondence • 1993:

Seba, Albertus

Bioscript • Botanical Album 
Seddon, George U. of Western Australia, Nedlands, Dept. of Philosophy

Correspondence $\bullet$ 1975: (3)

Seebeck, John H. Arthur Rylah Institute for Environmental Research, Heidelberg, Victoria, Australia

Correspondence - 1991: (3) Harper, Roland McMillan, 1878-1966

Seefelder, Matthias

Bioscript

Seege, T. L. Postal Telegraph-Cable Company Correspondence $\bullet$ 1942: (1)

Seeleman-Ehret Photo Engraving Co. Correspondence $\bullet$ 1943: (2)

Seemann, B.

Bioscript

Séguier, Jean-François (1703-1784) French botanist in Italy Bioscript

Seibert, Russell Jacob (1914-) American horticulturist, botanist, plant explorer; Longwood Gardens, Director Bioscript $\bullet$ Botanical Album

Correspondence $\bullet$ 1963: (0) Filed under Albert Charles Smith • 1969: (1) • 1973: (2) • 1975: (2)

Seidenberg, Charlotte Journalist, publisher; New Orleans Correspondence • 1990: (1) • 1991: (1)

Seiferth, Solis Dreyfous, Seiferth and Gibert, Architects, New Orleans, LA Correspondence • 1957: (1) • 1958: (2)

Seifriz, William, (1888-1955) Bioscript $\bullet$ Botanical Album

Seijo, Irma H. War Dept., Office of the Surgeon General, Medical Intelligence Branch Correspondence • 1946: (1) • 1947: (2)

Seiler, Harold A., Mrs. Correspondence $\bullet$ 1949: (2)

Seip, Frederic Bioscript

Selby, Augustine Dawson (1859-1924) American educator, plant breeder Bioscript

Selby Botanical Gardens Botanical Album

Selby, Marie Bioscript

Selby, Prideaux John (1788-1867) British naturalist, artist, ornithologist Bioscript

Self, Nancy Hope U. of Tulsa, Tulsa, OK Correspondence $\bullet$ 1971: (2)

Selkirk, Alexander (1676-1721) Scottish mariner, prototype Robinson Crusoe Biofile

Sellers, Charles Coleman Carlisle, PA Correspondence • 1966: (2) Bartram, William, 1739-1823 - 1977: (4) Barton, Benjamin Smith, 1766-1815 - 1978: (2)

Sellers, Janet R. Arnold Arboreum Correspondence • 1944: (1)
Selling, Olof Hugo (1917-) Swedish plant explorer, taxonomist, paleobotanist; Natural History Museum. Stockhoim

Correspondence • 1954: (2) Merrill, Elmer Drew, 1876-1956 • 1955: (6) • 1957: (1)

Sellow, Friedrich (1789-1831) Bioscript

Semenov, P. P. (of Tyan-Shan)

Bioscript

Semmes, Thomas

Bioscript

Sender, 0. W.

Bioscript

Sendter, Otto

Bioscript

Senn, Harold Archie (1912-) American borticulturist, plant physiologist; Dept. of Agriculaure, Ottawa, Canada: Principal Botanist; Head, Botany Unit Botanical Album Correspondence • 1953: (2) • 1959: (3)

Senn, Taze Leonard (1917-) American horticulturist, plant physiologist; Clemson College, Dept. of Horticulture Correspondence • 1967: (1) • 1968: (1)

Sera, J.

Bioscript

Seringe, N. C. Bioscript

Serturner, Friedrich Wilhelm Botanical Album

Servies, James A. U. of West Florida, Pensacola, John C. Pace Library, Director of Libraries

Botanical Album

Correspondence • 1979: (4) • 1980: (6) • 1984: (2) •

1985: (2) • 1986: (1) • 1987: (2) • 1988: (1) • 1989: (0)

Letter filed under Keith D. Crotz $\bullet$ 1989: (7) $\bullet$ 1990: (7) $\bullet$ 1993: (11)

Sessé y Lacasta, Martin de (1751-1808) Spanish physician, botanist

Biofile $\bullet$ Bioscript

Sessions, Kate Olivia (1857-1940) California floriculturist; San Diego Biofile • Bioscript $\bullet$ Botanical Albwm

Setchell, William Albert (18641943) American botanist, plant geographer, botanical historian; U. of California, Berkeley Biofile $\bullet$ Bioscript $\bullet$ Botanical Album Correspondence $\bullet$ 1939: (2)

Seton, Ernest Evan Thompson (1860-1946) Bioscript

Seventh International Botanical Congress Botanical Museum and Herbarium Correspondence • 1950: (0) filed under "Stockholm" Severeid, Eric Columbia Broadcasting Service Correspondence • 1969: (1) • 1970: (1) • 1972: (1)

Severy, Joseph Warren (1893-1965) Bioscript Correspondence $\bullet 1937$ : (2)

Seward, Albert Charles (1863-1941) English paleobotanist Botanical Album 


\section{Guide to the Ewan Papers}

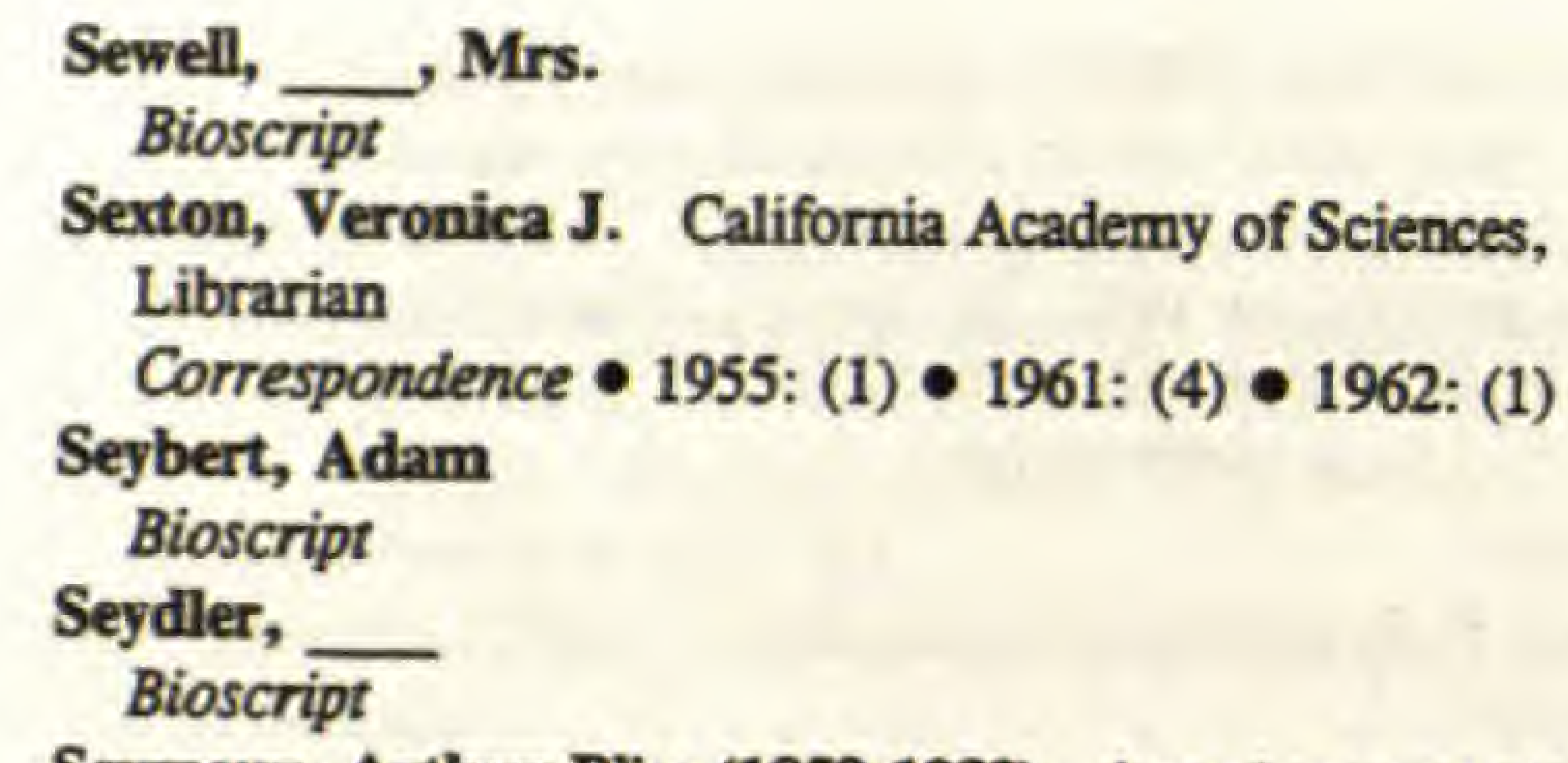

Seymour, Arthur Bliss (1859-1933) American mycologist, plant pathologist Bioscript $\bullet$ Botanical Album

Seymour, Frank Conkling (1895-1985) American taxonomist, clergyman

Botanical Album

Seymour, Samuel Botanical Album

Shacklette, Hansford Threlkeld (1914 ) American plant ecologist, soil chemist

Correspondence $\bullet$ 1949: (1)

Shafer, John Adolph (1863-1918) American pharmacist, botanist

Botanical Album

Shaffer,

Correspondence - 1969: (1) Correspondence with Drs. Shaffer and Fagley

Shaffer, Mary

Bioscript

Shaffer, __, Mrs. Patio Planters Correspondence • 1953: (1)

Shaler, N. S. Bioscript

Shallenberger, Alfred L. Office of County Clerk, Oquawka, II Correspondence • 1943: (1)

Shannon, Margaret Duluth, GA Correspondence • 1981: (4) • 1982: (13) • 1983: (3) 1984: (7) • 1987: (1) • 1988: (2)

Shantz, Homer LeRoy (1876-1958) American botanist, agronomist, soil technician Biofile • Bioscript • Botanical Album

Sharma, Indira Botanical Album Correspondence • 1989: (1)

Sharp, Aaron John (1904 ) American botanist, taxonomist, bryologist; U. of Tennessee, Knoxville; nickname "Jack" Bioscript • Botanical Album Correspondence • 1946: (1) • 1951: (1) • 1952: (5) • 1953: (2) Correspondence with James L. Crump • 1956: (1) -1962 : (3) - 1965: (1) • 1966: (2) Species $\bullet$ 1970: (4) $\bullet$ 1978: (5) - 1987: (2) • 1993: (2)

Sharp, Dallas Lore Bioscript

Sharp, John Bookseller, Williamsburg, VA Correspondence • 1970: (1)

Sharples, John T. Bioscript Sharr, Francis Librarian; State Reference Library, Perth,
West Australia Correspondence $\bullet$ 1980: (2)
Sharsmith, Carl William (1903-) American

phytogeographer, taxonomist; State College of Washington, Pullman

Bioscript $\bullet$ Botanical Album

Correspondence $\bullet$ 1929-1933: Setchell, William Albert, 1864-1943 Jepson, Willis Linn, 1867-1946 • 1938: (3) 1939: (11) Delphinium; Species • 1984: (1)

Sharsmith, Helen Katherine (1905-1982) American plant taxonomist; U. of California, Berkeley

Biofile

Correspondence • 1954: (3) Delphinium • 1957: (1) • 1958: (1) • 1967: (4) • 1983: (1)

Sharswood, William Bioscript

Shattuck, George Cheyne, Jr. (1813-1893) American physician, medical educator, philanthropist Biofile • Bioscript

Shattuck, Helen B. U. of Vermont, Library Correspondence $\bullet$ 1943: (1)

Shatzer, Robert L. American Begonia Society Correspondence $\bullet$ 1965: (1)

Shaw, Alva, Mrs. Correspondence $\bullet 1941$ :

Shaw, Charles Hugh Bioscript $\bullet$ Botanical Album

Shaw, Elizabeth Correspondence $\bullet$ 1990: (1)

Shaw, Frank U. of Chicago Correspondence $\bullet$ 1940: (2)

Shaw, George Bioscript $\bullet$ Botanical Album

Shaw, Henry (1800-1889) English-born American merchant and benefactor, founder of Missouri Botanical Garden Biofile $\bullet$ Bioscript $\bullet$ Botanical Album

Shaw, J. Marvin, Mrs. New Orleans Town Gardeners Correspondence • 1970: (0) Filed under New Orleans Town Gardeners

Shaw, Marjorie I. Zoological Society of San Diego Bioscript $\bullet$ Botanical Album

Correspondence • 1984: (2)

Shaw, Richard Shaw-Banfil Books Correspondence 1981 : (3) Book collectors

Shaw, William Thomas (1873- ) Bioscript Shear, Cornelius Lott (1865-) American mycologist, plant
pathologist

Bioscript $\bullet$ Botanical Album

Correspondence • 1949: (2) Rocky Mountain Naturalists • 1953: (6)

Shecut, John Linnaens Edward Whitridge (1770-1836) American botanist Biofile $\bullet$ Bioscript

Sheehan, Jack Steel Equipment, Bloomington, IL Correspondence $\bullet$ 1958: (1) $\bullet$ 1961: (6)

Sheehan, Jacqueline L. National Gallery of Art, Dept. Graphic Arts, Asst. Cur.

Correspondence • 1978: (0) Filed under Kathryn Hall Proby Sheehan, Marion Ruff (1923-) American botanical artist,
botanist.

Correspondence $\bullet$ 1970: (5) • 1971: (2) 
Sheehan, Robert J., Rev. U. of Notre Dame, Notre Dame, IN, Dept. of Biology, Head Correspondence • 1953: (2)

Shelby, Bioscript

Shelby, Alice Vaughan U. of Colorado; husband is William Shelby; see also Alice Vaughn Correspondence $\bullet$ 1940: (9)

Shelby, Lewis B. Correspondence $\bullet$ 1942: (1)

Sheldon, Charles Stiles (1854 ) Plant collector, educator; U. S. A. Bioscript

Sheldon, Charlotte B. Bioscript

Sheldon, Don R. Superintendant of Schools, Prescott, AZ Correspondence $\bullet$ 1943: (1)

Sheldon, William George (1858?-1943) British entomologist Biofile

Shelford, V. C. U. of IIlinois, Urbana Correspondence $\bullet$ 1939: (1)

Shelton, C. A. (-1853) 'California botanist Biofile

Shelton, Lee M. Correspondence $\bullet$ 1980: (2)

Shelton, Lennis E. Correspondence $\bullet 1980$ : (2)

Shelton, C. C. (d. 1853) Bioscript

Shenfield, Raymond Correspondence $\bullet$ 1971: (1)

Shepherd, $\mathbf{H}$. Bioscript

Shepherd, John Bioscript $\bullet$ Botanical Albun

Shepherd, William M. Correspondence $\bullet$ 1956: (2)

Sheppard, William (1783-1867) Bioscript

Shepperson, Wilbur S. U. of Nevada, Reno, Dept. of History Correspondence $\bullet$ 1970: (2)

Sheral, Juarez Instituto nacional de investigaciones sobre recursos bióticos, México D. F. Correspondence 1988 : (1)

Sherard, James Bioscript

Sherard, William (1659-1728) British botanist and lawyer, consul at Smyrna Biofile • Bioscript

Sherborn, Charles Davies (1861-1942) Bioscript

Sherff, Earl Edward (1886-1966) American taxonomist, plant ecologist, plant collector Botanical Album

Sherman, David M. National Park Service Correspondence • 1985: (2)

Sherman, John D., Jr. Botanical Album
Sherrouse, Julian M., Jr. Correspondence $\bullet 1972:$ (1)

Sherwood, Morgan U. of California, Davis, Dept. of History Correspondence $\bullet 1971:$ (5)

Shetler, Stanwyn Gerald (1933-) American taxonomist; Smithsonian Institution, Dept. of Botany

Botanical Album

Correspondence $\bullet$ 1967: (1) $\bullet$ 1970: (2) $\bullet$ 1971: (2)

Shideler, James H. Agricultural History, Periodicals Dept. Correspondence $\bullet 1968$ : (3)

Shields Date Gardens Correspondence $\bullet$ 1992: (1)

Shilstone, Cecil M. Garden Club of the Junior Leagae of New Orleans Correspondence • 1969: (5)

Shinn, Thomas S. Correspondence • 1964: (5) Lyon, John, 1764-1814

Shinners, Lloyd Herbert (1918-1971) Canadian-born American taxonomist, plant collector; Southern Methodist U., Dallas, Herbarium Biofile $\bullet$ Bioscript $\bullet$ Botanical Album Correspondence $\bullet$ 1943: (2) $\bullet$ 1946: (9) Jepson, Willis Linn, 1867-1946 • 1947: (1) • 1948: (3) • 1949: (7) • 1950: (9) Geiser, Samuel Wood, 1890- 1952 : (2) • 1953: (1) • 1954: (2) • 1955: (1) • 1956: (7) • 1957: (12) Species • 1958: (2) • 1960: (3) $\bullet$ 1961: (5) Species • 1962: (4) - 1963: (5) Letter from J. Ewan to George S. McManus Co. • 1966: (3) • 1967: (11) • 1969: (6)

Shipman, Ruth Tulane U., New Orleans Correspondence $\bullet$ 1967: (1)

Shipps, Anthony W. U. of Colorado Libraries, Boulder; Correspondence • 1965: (3) Letter from Louisa Ward Arps

Shipton, Clifford K. American Antiquarian Society, Director Correspondence $\bullet$ 1960: (0) Filed under American Antiquarian Society $\bullet$ 1965: (1)

Shivas, Molly G. U. of Leeds \& York, England, Botany Dept. Correspondence • 1953: (4) Species • 1955: (3)

Shoaf, John Botanical Album

Shoaf, Mary L. West Chester State College, PA, Chairman, Lecture Committee, West Chester Lectures in Science Correspondence $\bullet 1969$ : (0) Filed under William R. Overlease

Shockley, William Hillman (1855-1925) American plant collector Bioscript $\bullet$ Botanical Album

Shoemaker, C. Bioscript

Shoemaker, L. C. Correspondence $\bullet 1938$ : (1)

Shoop, C. Robert (1935-) Ecologist; Wellesley College Correspondence • 1962: (2) • 1963: (6) • 1964: (2) • 1965: (4) • 1985: (2)

Shope, Paul Franklin (1894-1986) Bioscript $\bullet$ Botanical Album Correspondence $\bullet$ 1939: (0) Filed under Roger Adams • 1943 (1) 


\section{Guide to the Ewan Papers}

Short, Charles Wilkins (1794-1863) American botanist, physician

Biofile $\bullet$ Bioscript $\bullet$ Botanical Album

Short, __ Mr. Garden Symposium, Colonial Williamsburg, VA

Correspondence $\bullet$ 1973: (1)

Shorthouse, J. D. Laurentian U.. Dept. of Biology, Sudbury, Canada

Correspondence • 1983: (1)

Shovlin, Mary U. of Colorado Libraries Correspondence $\bullet$ 1942: (2)

Shrawder, John Dept. of Natural Resources, Sacramento CA

Correspondence $\bullet$ 1959: (1)

Shreve, Forrest (1878-1950) American plant ecologist; Carnegie Institution of Washington, Desert Lab Bioscript • Botanical Album Correspondence • 1936: (3)

Shriver, Howard Bioscript

Shryock, Augustus Bioscript

Shryock, Richard H. American Philosophical Society, Librarian

Correspondence $\bullet$ 1959: (1) • 1960: (5) • 1961: (7) • 1963: (3) - 1964: (1) - 1965: (3) Barton, Benjamin Smith, 1766-1815

Shuck, A. L. Southwestern State College, Weatherford, OK Botanical Album

Correspondence - 1953: (4)

Shufeldt, Robert Wilson (1850-1934)

Bioscript

Shulan, __ Group Health Association, Washington, D.C. Correspondence • 1947: (1)

Shull, Charles A.

Correspondence • 1940: (2)

Shull, George Harrison ( 1874-1954) American plant geneticist, plant morphologist

Botanical Album

Shull, Leon Americans for Democratic Action, National Director

Correspondence - 1976: (0) Filed under Americans for Democratic Action

Shultz, Benjamin

Bioscript

Shuman, John R. Arkansas Polytechnic College, Russellville, Associate Professor of Biology Correspondence $\bullet$ 1952: (3)

Shumard, Benjamin Franklin (1820-1869) American geologist, paleontologist

Biofile

Shumard, G. G.

Bioscript

Shurcliff, William A. Citizens Leauge Against the Sonic Boom, Cambridge, MA

Correspondence • 1970: (2) • 1971: (2) • 1972: (2)

Shurtieff, Bill Soyfoods Center, The

Correspondence • 1981: (2)

Shuttleworth, Robert James (1810-1874) English botanist in Switzerland, Ireland

Bioscript • Botanical Album
Sibald, Robert, Sir (1611-1722) Scottish natural historian, physician

Bioscript

Sibley, Charles G. Tiburon Center for Environmental Studies, Tiburon, CA

Bioscript

Correspondence $\bullet$ 1989: (1)

Sibley, Elbridge Social Science Research Council Correspondence $\bullet$ 1947: (1)

Sibthorp, John Bioscript

Sicherman, Barbara

Correspondence • 1979: (1)

SIDA

Correspondence $\bullet$ 1990: (2) $\bullet$ 1991: (1)

Sidey, Hugh

Bioscript

Sidey, J. L.

Correspondence $\bullet$ 1949: (1)

Sidney, Cedric Louisiana Forestry Commission, Woodworth, LA; American Fern Society

Correspondence • 1951: (3) • 1953: (6) • 1954: (2)

Sieber, Franz Wilhelm

Bioscript

Siebold, Philipp Franz von (1796-1866) German physician, natural scientist and orientalist

Biofile $\bullet$ Bioscript

Siegel, Daniel M \& S Press, Inc., Weston, MA Correspondence • 1978: (1)

Siegel, Sanford $\mathbf{M}$. Botanical Album

Siegfried, I. C. Botanical Album

Siegfried, Sybil (Mrs. John D. Siegfiried)

Correspondence • 1975: (1)

Siegrist, Urban J. Saint Joseph's College, Rensselaer, IN, Dept. of Biology

Correspondence • 1970: (1)

Sierra Club

Botanical Album

Correspondence • 1972: (1) • 1988: (1)

Sierra Club Library Barbara Lekisch Librarian Correspondence $\bullet 1986$ : (7) Jepson, Willis Linn, 1867-1946

Sievers, A. F.

Correspondence • 1947: (1)

Sifton, Paul Library of Congress Correspondence • 1968: (1)

Sigma XI, Tulane Chapter Correspondence $\bullet$ 1952: (0) Filed under Wendell Meredith Stanley

Silberschmidt, Karl Martin (1903-) German plant physiologist in Brazil; Instituto Biológico, São Paulo, Brasil, Chef da Seccao de Fisiologia Vegetal

Correspondence • 1957: (1) • 1958: (1) • 1959: (1)

1960: (1) • 1969: (1)

Silkwood, Mary Former student

Correspondence $\bullet$ 1940: (2)

Sillern, William

Bioscript 
Silliman, Benjamin (1779-1864) American chemist, geologist Biofile - Bioscript

Sillman, Emmanuel I. U. of Michigan, Dept. of Zoology Correspondence $\bullet$ 1951: (2)

Silva, Paul Bioscript

Silver, Andrew L. Bioscript

Silversword Inn Maui, HI Correspondence • 1967: (2)

Silveus, W. A. Bioscript

Simitiere, Pierre Eugene du (1736-1784) Bioscript

Simmers, Richard W., Jr. Botanical Album Correspondence $\bullet$ 1976: (2)

Simmonds, A. Royal Horticultural Society, London, England, Deputy Secretary Correspondence $\bullet$ 1958: (1) $\bullet$ 1962: (6)

Simmons, Edward M. Louisiana Research Foundation, Avery Island, LA; nickname "Ned" Correspondence • 1964: (3) - 1968: (6) Species • 1969: (1) Bartram, William, 1739-1823; Banister, John, 1650-1692 - 1977: (1)

Simmons, Vicky Tulane U., School of Medicine, Dept. of Pharmacology, Secretary

Correspondence $\bullet$ 1961: (1)

Simon Fraser University D. Zarn Correspondence • 1976: (3) Merrill, Elmer Drew, 1876-1956

Simon, Jean-Pierre J. (1938- ) French biochemist, taxonomist in the U. S. A.; Claremont Graduate School, Assistant Professor of Botany Correspondence $\bullet$ 1970: (2)

Simons, Corinne Miller The Lloyd Library and Museum, Cincinnati, Librarian Correspondence $\bullet$ 1963: (1) • 1964: (1) • 1976: (1)

Simons, $\mathbf{M}$. Correspondence $\bullet$ 1962: (1)

Simonson, Emma C. Indiana U. Libraries Correspondence $\bullet$ 1980: (5) $\bullet$ 1983: (1) • 1985: (1)

Simoons, Frederick J. (1922- ) American geographer Biofile

Simpkins, Diana $\mathbf{M}$. Correspondence $\bullet$ 1983: (1)

Simpson, Beryl B. Correspondence • 1990: (0) Filed under Laurence J. Dorr and Rudolph

Simpson, Charles Torrey Bioscript

Simpson, Elizabeth Correspondence • 1950: (2) • 1954: (2)

Simpson, George (1792-1860) Bioscript

Simpson, George Gaylord Bioscript

Simpson, James Hervey Bioscript
Simpson, Joseph Herman (1841-1918) American botanical collector, plant explorer, naturalist Bioscript

Simpson, Larry W. Medical U. of South Carolina, Charleston

Correspondence $\bullet 1980:$ (1)

Simpson, Marcus B., Jr. Duke U. Medical Center Bioscript $\bullet$ Botanical Album

Correspondence • 1976: (4) Geiser, Samuel Wood, 1890. - 1982: (4) • 1983: (7) • 1984: (2) • 1985: (1) • 1988: • 1992: (6) • 1993: (12)

Simpson, Norman Douglas (1890-1974) British botanist, bibliographer Biofile

Sims, Asa C. Southern U., Baton Rouge, LA Correspondence $\bullet 1964$ : (3) $\bullet$ 1968: (2) Biology-Instruction and study

Sinclair, Andrew (1796-1861) Bioscript

Sinclair, Isabella (1842-1900) British-born botanist; New Zealand, Hawaii

Biofile

Singer, Charles Correspondence $\bullet$ 1950: (2)

Singer, Josephine M. Correspondence • 1921-1928: (2)

Singleton, W. Ralph Accokeek Foundation, Inc. Correspondence $\bullet 1966:(7) \bullet 1967:(3)$

Sinnott, Edmund Ware (1888-1968) American botanist, plant geneticist, editor; Osborn Botanical Laboratory Biofile $\bullet$ Botanical Album Correspondence • 1938: (2) • 1942: (1)

Sisson, J. H. Bioscript

Sisson, Norman F, Bioscript

Skallerup, Harry R. Missouri Botanical Garden Correspondence $\bullet$ 1952: (1)

Skelton, Botanical Album

Skinner, Francis Bioscript

Skinner, George Ure Bioscript

Skinner, Henry Thomas (1907-) English-born botanist, horticulturist, plant breeder Biofile

Skinner, Hubert Clayton (1929-) Tulane, Dept. of Geology Correspondence $\bullet$ 1958: (3) Book collectors • 1959: (1) • 1961: (1) • 1963: (2) • 1964: (2) • 1972: (2) • 1978: (2) • 1982: (5) • 1983: (2)

Skinner, Milton Philo (1879-) Bioscript

Skinner, S. A. Bioscript

Skog, Laurence Edgar (1943-) National Museum of Natural History Correspondence $\bullet$ 1990: (1) 


\section{Guide to the Ewan Papers}

Skottsberg, Carl Johann Friedrich (1880-1963) Marine

Botanical Institute of the U., Göteborg, Sweden

Bioscript - Botanical Album

Correspondence • 1961: (1)

Skutch, Alexander Frank (1904)

Bioscript

Slack, Nancy G. Russell Sage College, Troy, NY, Dept. of Biology

Bioscript $\bullet$ Botanical Album

Correspondence • 1989: (1) • 1990: (4) • 1991: (9) • 1992: (4) - 1993: (1)

Slade, Thomas Bog (1800-1882) Bioscript

Slayback, Preston Trabue Correspondence • 1929-1933: (5)

Slevin, Joseph Richard (1881-) American herpetologist, collector; California Academy of Sciences

Biofile - Bioscript

Correspondence $\bullet$ 1955: (1)

Slevin, Thomas Edwards (1871-1902) Bioscript

Sloan, Bioscript

Sloane, Boyd L. See also Scott E. Haselton Correspondence $\bullet$ 1949: (2)

Sloane, Hans (1660-1753) British physician, naturalist Biofile • Bioscript $\bullet$ Botanical Album

Sloat, Lewis W. Bioscript

Small, E. Plant Research Institute, Research Branch, Central Experimental Farm, Ottawa, Canada Correspondence $\bullet$ 1970: (1)

Small, John Alvin (1900-) American plant ecologist, plant collector; Rutgers U.

Botanical Album

Correspondence - 1950: (2) - 1952: (2) Pennell, Francis Whittier, 1886-1952 • 1963: (2) • 1967: (2) • 1968: (5) Harper, Roland McMillan, 1878-1966; Harper, Francis, 1886-1972

Small, John Kunkel (1869-1938) American botanist; New York Botanical Garden

Biofile $\bullet$ Bioscript $\bullet$ Botanical Album

Correspondence • 1929-1933: (2) $\bullet$ 1934: $\bullet$ 1935: (2)

Munz, Philip Alexander, 1892-1974 • 1947: (3)

Smalley, Alfred E. Tulane U., Dept. of Biology, Professor Botanical Album

Correspondence $\bullet$ 1972: (1) • 1974: (1) • 1977: (1) • 1981: (1) • 1989: (1) • 1991: (1)

Smallwood, Mabel S. C.

Botanical Album (Filed under William M. Smallwood) Correspondence $\bullet$ 1943: (2)

Smallwood, William Martin (1873-1949) Syracuse U. Botanical Album

Correspondence • 1942: (2)

Smart, Charles Bioscript

Smart, John Bioscript

Smeathman, Henry (1750-1787) Bioscript
Smeaton, John Bioscript

Smellie, William Bioscript

Smet, Pierre Jean de (1801-1873) Bioscript

Smiley, Frank Jason (1880-) Occidental College, Los Angeles

Botanical Album

Correspondence • 1936: (4) • 1938: (1) • 1940: (3) • 1959: (2)

Smit, Pieter Biohistorical Institute, Utrecht Bioscript $\bullet$ Botanical Album

Correspondence $\bullet$ 1965: (6) $\bullet$ 1966: (5) Verdoorn, Frans Antonie, 1906-1984 • 1968: (1) Verdoorn, Frans Antonie, 1906-1984 1980: (1)

Smith, A. W. Ipswich, MA Correspondence $\bullet$ 1970: (3) • 1971: (2)

Smith, Albert Charles (1906-) American botanist, taxonomist

Biofile $\bullet$ Bioscript $\bullet$ Botanical Album

Correspondence $\bullet$ 1943: (3) Arnold Arboretum $\bullet$ 1944: (2)

- 1945: (1) - 1946: (1) • 1948: (8) Arnold Arboretum; Species $\bullet$ 1949: (4) $\bullet$ 1950: (5) American Fern Society $\bullet$ 1951: (6) Vismia • 1952: (6) Vismia • 1955: (5) Vismia $\bullet$ 1956: (7) Vismia $\bullet$ 1957: (4) $\bullet$ 1958: (5) $\bullet$ 1959: (2) 1962: (1) • 1963: (7) • 1964: (1) • 1966: (1) • 1967: (8) • 1967: (10) Van Schaack, George Booth, 1903-1983 - 1968: (2) • 1972: (1) • 1973: (3) • 1974: (4) Species • 1975: (1) - 1976: (13) • 1977: (3) • 1978: (1) • 1979: (3) • 1980: (1) $\bullet$ 1984: (1) $\bullet$ 1985: (2)

Smith, Allen Menard County, Peterburg, IL, Clerk Correspondence • 1949: (1) Rocky Mountain Naturalists

Smith, Annie Lorrain (1854-1937) Scottish-born mycologist, lichenologist

Botanical Album

Smith, Arthur Donaldson Bioscript

Smith, Arthur M. Correspondence $\bullet$ 1948: (3)

Smith, Aubrey $\mathbf{H}$. Bioscript

Smith, Beatrice A. Correspondence $\bullet$ 1976: (7)

Smith, Benjamin Hayes Bioscript • Botanical Album

Smith, Benjamin Warfield (1913-) American plant geneticist; Professor of Genetics

Correspondence $\bullet$ 1962: (0) Filed under Willis Alexander Eggler

Smith, Budd E. Coker College, Huntsville, SC, Instructor in Biology and Curator of Botany, Correspondence $\bullet$ 1943: (2) Delphinium

Smith, Claude Earle, Jr. (1922-1987) Academy of Natural Sciences of Philadelphia; nickname "Smitty" Correspondence $\bullet$ 1953: (5) Academy of Natural Sciences of Philadelphia; Pursh, Frederick, 1774-1820 • 1954: (5) 1955: (3) • 1956: (4) - 1957: (4) • 1958: (2) • 1959: (1) Nuttall, Thomas, 1786-1859 • 1974: (2) • 1975: (2) • 1977: (3) 
Smith, Charles Eastwick (1820-1900) American amateur botanist Bioscript

Smith, Charles Piper (1877-1955) Canadian-born American botanist, educator in U. S. A.

Biofile $\bullet$ Bioscript $\bullet$ Botanical Album

Correspondence • 1929-1933: (1) • 1935: (5) Species • 1936: (1) - 1949: (4) Rocky Mountain Naturalists • 1951: (1) 1 1952: (2)

Smith, Christopher Bioscript

Smith, Claude W. Correspondence $\bullet$ 1982: (1)

Smith, Clifton F. (1920-) American taxonomist; Museum of Natural History, Santa Barbara Bioscript • Botanical Album Correspondence $\bullet$ 1959: (6) Book collectors $\bullet$ 1960: (8) Book collectors $\bullet$ 1961: (2) Book collectors $\bullet$ 1962: (1) $\bullet$ 1965: (5) • 1967: (4) • 1968: (5) • 1968: (0) Filed under Lois Chambers Stone $\bullet$ 1970: (2) • 1972: (7) • 1975: (1) 1976: (8) • 1977: (10) Book collectors • 1978: (4) • 1979: (1) Book collectors $\bullet$ 1981: (1) $\bullet$ 1985: (1) • 1986: (1)

Smith, Debra D. Lutheran Church of the Holy Trinity Correspondence • 1985: (1) Barton, Benjamin Smith, 1766-1815

Smith, E. D. Bioscript

Smith, Edgar Fahs Bioscript

Smith, Edmond Reuel Bioscript

Smith, Elmer W. Bioscript

Smith, Ernest Charles (1864 ) Colorado State College, Fort Collins

Bioscript • Botanical Album

Correspondence $\bullet$ 1937: (2) Letter from Francis Ramaley • 1939: (1) • 1940: (1) • 1941: (7) • 1942: (2) • 1943: (3) • 1949: (1) Rocky Mountain Naturalists

Smith, Eugene A.

Bioscript

Smith, Francis Bioscript

Smith, Frank K. Correspondence $\bullet$ 1949: (1)

Smith, Franklin (1857- ) Bioscript

Smith, Galen Botanical Album

Correspondence • 1949: (3) • 1992: (1)

Smith, George (1804-1882) American educator, jurist, naturalist Bioscript

Smith, H. L. Bioscript

Smith, Herbert Huntington (1851/2-1919) American botanical and zoological collector Biofile $\bullet$ Bioscript

Smith, Howard M. U. of Richmond, VA, Dept. of Biology Correspondence • 1972: (4) • 1975: (3) • 1977: (1) • 1982: (1) • 1983: (1) 1988: (1)
Smith, Huron Herbert (1883-1933) American dendrologist Bioscript • Botanical Album

Smith, Isadore Leighton Luce (1902-1985)

See also Ann Leighton

Correspondence $\bullet$ 1972: (2)

Smith, J. Alden

Bioscript

Smith, Jacob M. J. (1799- ) Arkansas murseryman Biofile

Smith, James (1771-1841) Bioscript

Smith, James Edward (1759-1828) British botanist, Nova Scotia; founded Linnean Society Biofile $\bullet$ Bioscript $\bullet$ Botanical Album

Smith, Jean Chandler Correspondence • 1986: (1)

Smith, Jean Cuvier Correspondence • 1984: (1)

Smith, Jeffrey E. Mercantile Library Correspondence • 1990: (1) • 1992: (2) • 1993: (1)

Smith, John (1798-1888) British gardener and pteridologist Biofile

Smith, John, Captain (1580-1631) Leader of Jamestown colonists Biofile

Smith, John Donnell (1829-1928) American taxonomist Bioscript $\bullet$ Botanical Album

Smith, John Jay (1798-1881) Collector Bioscript • Botanical Album

Smith, Katherine Bioemendaal, The Lewis Ginter Botanical Garden, Richmond, VA

Correspondence $\bullet$ 1988: (1)

Smith, Louisa Hutchings Bioscript

Smith, Lyman Bradford (1904) American taxonomist, plant collector; Harvard U., Gray Herbarium; Smithsonian Institution, Botany

Botanical Album

Correspondence • 1939: (1) Species • 1940: (1) $\bullet 1941$ :

$(4) \bullet 1942:(5) \bullet 1943:(1) \bullet 1947:$ (4) $\bullet$ 1952: (2) $\bullet 1955:$

(2) 1956: (2) Vismia $\bullet$ 1958: (2) $\bullet$ 1959: (1) $\bullet 1960$ : (2)

1961: (2) 1962: (2) • 1964: (4) - 1965: (4) • 1966: (2)

- 1967: (1) • 1973: (2) Barton, Benjamin Smith,

1766-1815 • 1974: (3) • 1976: (2) Merrill, Elmer Drew,

1876-1956 • 1981: (1) • 1982: (2) - 1983: (6) • 1992: (3)

Smith, Margaret H. D. Tulane U., Dept. of Medical School Correspondence • 1969: (1)

Smith, Millie M. Bioscript

Smith, Murphy D. American Philosophical Society. Philadelphia

Bioscript

Correspondence • 1971: (3) • 1977: (1)

Smith, Nigel J. H. (1949-) U. of Florida, Gainesville Correspondence $\bullet 1981$ : (1)

Smith, Nina G. (1906-) Swedish-bors botanical bibliographer in the U. S. A.; Hunt Botanical Library, Pittsburg, PA Correspondence $\bullet$ 1970: (1) • 1972: (1) • 1975: (1) • 1976: (1) 


\section{Guide to the Ewan Papers}

Smith, P.

Bioscript

Smith, Paul Bookseller; The Old Book Corner, Chapel Hill, NC

Correspondence $\bullet$ 1970: (1) • 1973: (1)

Smith, Persifor F. Bioscript

Smith, Peter Botanical Album

Smith, Ralph H. St. Petersburg, FL; Friend of Francis Harper Correspondence • 1979: (1)

Smith, Roger Cletus (1888-) Bioscript

Smith, Roger G. Kansas State College, Manhattan Correspondence $\bullet$ 1949: (2)

Smith, Samuel Bioscript

Smith, Stanley Jay (1915-) American taxonomist, agrostologist; U. of the State of NY, Albany, New York State Museum, Senior Curator

Correspondence • 1946: (1) • 1947: (2) • 1948: (2) Delphinium • 1949: (2) • 1950: (1) • 1951: (1) Pursh, Frederick, 1774-1820 • 1952: (1)

Smith, Thomas

Bioscript

Smith, Thomas M. U. of Oklahoma, History of Science Correspondence • 1966: (2)

Smith, Titus, Jr. (1768-1856) Land surveyor, geographer Biofile

Smith, Warren Lone Star Cement Corporation, New Orleans, LA

Correspondence $\bullet$ 1958: (2) Copy of letter from Dr. Hummel

Smith, William Wright (1875-1956) Scottish horticulturist, plant explorer, taxonomist; Royal Botanic Garden, Edinburgh, Scotland, The Regius Keeper

Botanical Album

Correspondence $\bullet$ 1951: (2)

Smithey, Doug U. of Virginia, Pembroke Mountain Lake Biological Station

Correspondence $\bullet 1970$ : (1)

Smithsonian Joyce McCarten, Office of Editor Correspondence • 1977: (1) Bartram, William, 1739-1823 - 1979: (1)

Smithsonian Institution

Botanical Album

Correspondence • 1952: (1) • 1965: (2) • 1966: (1) • 1978: (2) • 1983: (6) • 1984: (5) • 1985: (4)

Smitson, Harold

Correspondence • 1940: (1) • 1941: (1) • 1943: (2)

Smocovitis, Vassiliki Betty Stanford U., Stanford, CA

U. of Florida, History of Science

Botanical Album

Correspondence $\bullet$ 1989: (2) $\bullet$ 1991: (1) Copy of letter sent to J. Ewan by Emanuel Rudolph

Smyth, Lumina Cotton (Riddle) (1871-1906) Bioscript

Smyth, Robert Brough (1830-1889) British scientific administrator at Victoria, B. C

Biofile
Sneddon, Kathleen F. Auckland, New Zealand Correspondence • 1977: (1) • 1978: (1)

Sneidern, Kiell von

Botanical Album

Snell, John L. Dean, Tulane Correspondence • 1964: (1) • 1965: (1) • 1966: (1)

Snell, Walter Henry (1889-1980) American mycologist, plant pathologist, botanist; Brown U., Dept. of Botany Correspondence $\bullet$ 1950: (7) Verdoorn, Frans Antonie, 1906-1984

Snider, Edwin W. National Geographic Society Correspondence • 1976: (0) Filed under Tim Martin Berra corres. - 1990: (0) Filed under National Geographic Magazine

Snodgrass, Robert Evans (1875-1962) Bioscript

Snow, D. W. British Museum Natural History, London, England

Correspondence 1971 : (2)

Snow, Francis Huntington (1840-1908) Kansas ornithologist Biofile • Bioscript

Snow, William Appleton (1869-1899) Bioscript

Snyder, David B. Correspondence • 1983: (4) • 1984: (1)

Snyder, E. Bayne Forest Service Geneticist Correspondence 1964: (1)

Snyder, Henry L. Louisiana State U., Baton Rouge, College of Arts and Sciences, Dean

Correspondence • 1979: (9) • 1980: (1) • 1981: (1) • 1982: (5) • 1983: (2) • 1984: (4) • 1985: (6) • 1986: (4) • 1987: (3) • 1988: (1)

Snyder, James A. Bioscript

Snyder, John J. Correspondence • 1938: (1) • 1939: (1) • 1940: (2) • 1951: (1)

Snyder, John Offerbein (1867-) Bioscript

Sociedad Botánica de México Botanical Album

Society for Economic Botany Correspondence $\bullet$ 1993: (1)

Society for the Advancement of Education Correspondence $\bullet 1942$ : (1)

Society for the Bibliography of Natural History Secretary, Judith A. Diment Correspondence • 1975: (1) • 1976: (1) • 1978: (1) • 1983: (1) • 1986: (1) • 1987: (2) • 1990: (4)

Society of Friends Edward H. Milligan, Librarian Correspondence $\bullet$ 1964: (2)

Soderstrom, Thomas Robert (1936-1987) American taxonomist, agrostologist, plant collector; Smithsonian Institution, Division of Grasses, Curator Correspondence • 1967: (3) • 1970: (2) • 1985: (1)

Sodiro, Luigi (Aloysius Luis) (1836-1909) Italian-born botanist in Ecuador Bioscript $\bullet$ Botanical Album

Sodiro, R. P. Bioscript 
Sogandares, A.

Correspondence $\bullet$ 1955: (1)

Sogandares-Bernal, Francis (1931-) Tulane U., Dept. of Zoology

Botanical Album

Correspondence • 1959: (1) • 1961: (2) • 1975: (1)

Sohns, Ernest Reeves (1917- ) American taxonomist, plant morphologist; Smithsonian Institution, Associate Curator, Division of Grasses

Correspondence $\bullet$ 1952: (1)

Sokal, Michael M. Worcester Polytechnic Institute, Dept. of History; History of Science Society, Executive Secretary Correspondence • 1973: (1) • 1988: (1)

Solander, Daniel Charles (1733-1782) Swedish botanist and traveller

Biofile - Bioscript

Solander, Daniel Carl

See Solander, Daniel Charles (1733-1782)

Solheim, Stephen L. U. of Wisconsin, Madison, Dept. of Botany

Correspondence • 1971: (1) - 1991: (1)

Solheim, William Gerhard (1898-1978) Mycologist, U. of Wyoming, Laramie, Professor of Botany Correspondence $\bullet$ 1958: (2)

Sollman, Mary A. Tulane U., New Orleans, Classics Dept. Correspondence • 1959: (2) • 1961: (1) • 1974: (1)

Solms-Laubach, Hermann, Graf zu (1842-1915) German botanist Biofile - Bioscript

Solymosy, Sigmond L. (1906-1974) U. Southwestern Louisiana, Lafayette, College of Agriculture Correspondence $\bullet$ 1967: (4) $\bullet$ 1968: (2) $\bullet$ 1970: (4) $\bullet$ 1971: (9) Species

Somers, Wayne Hammer Mountain Book Halls Correspondence • 1973: (5) 1978: (1)

Sonne, Charles Frederick (1845-1913) Danish-born botanist, plant collector in the U. S. A. Bioscript $\bullet$ Botanical Album

Sonnerat, Pierre (1748-1814) Bioscript

Sonnini, Charles Sigisbert (1751-1812) Bioscript

Sorda, F. S. Bioscript

Sorenson, Conner U. of Alaska, Southeast, Juneau Bioscript Correspondence 1990 : (1)

Sorvig, Vernie U. Press of Denver Correspondence • 1950: (20) Rocky Mountain Naturalists - 1951: (1)

Soth, Blanche H. Husband is M. E. Soth Bioscript

Sotheby Parke Bernet \& Co. R. L. Davids, Director Manuscripts, London, England Correspondence • 1978: (0) Filed under Dorothy I. Lansing

Soule, Michael Bioscript

Soule, Oscar Missouri Botanical Garden Correspondence • 1970: (5) "Rocky Mountain Naturalists" Missouri Botanical Garden
Soule, Phelps U. of Pennsylvania Press

Correspondence $\bullet$ 1950: (1)

Southeastern Council of Latin American Studies George A. Bowdler

Correspondence • 1985: (1)

Southern Forest Experiment Station

Correspondence • 1965: (1)

Southern Methodist U.

Botanical Album

Correspondence • 1962: (2) • 1964: (2) Librarian

Sowell, Frederick McGee San Marcos, TX

Correspondence $\bullet$ 1970: (7)

Sowell, Lee Husband is Leon B. Sowell; Houston, TX Correspondence $\bullet 1970$ : (2)

Sowerby, James

Bioscript

Sowerby, James (1757-1822) British botanical artist Biofile

Sowerby, W.

Bioscript

Soxman, G. M.

Correspondence $\bullet$ 1941: (1) $\bullet 1942:$ (2)

Spaans, Arie L. Research Institute for Nature Management Correspondence • 1988: (6) Book collectors

Spach, Eduardo (1801-1879)

Bioscript

Spachman, W. M. U. of Colorado, Office of Public Information

Correspondence $\bullet$ 1942: (1)

Spalding, David A. E. Provincial Museum of Alberta, Edmonton, Head Curator of Natural History Correspondence • 1977: (4) "Rocky Mountain Naturalists" - 1979: (2) "Rocky Mountain Naturalists" • 1980: (2) • 1982: (2)

Spalding, Henry Harmon (1804-1874)

Bioscript

Spalding, Thomas

Bioscript

Spalding, Volney Morgan (1849-1918) Bioscript

Spallanzani, Lazzaro (1729-1799) Bioscript

Sparrman, Anders (174\&-1820) Bioscript

Sparrow, Arnold Hicks (1914) Canadian-born biologist. plant cytologist, geneticist; Brookhaven National Laboratory, Upton, NY Correspondence $\bullet 1955$ : (1)

Sparrow, Frederick Kroeber (1903-1977) American mycologist; U. of Michigan, Ann Arbor, Dept. of Botany Correspondence • 1968: (1) • 1969: (6)

Spanlding, Albert C. National Science Foundation; Program Director for History and Philosophy of Science Correspondence $\bullet 1961:(2) \bullet 1962:$ (4)

Spanlding, Randall Bioscript

Spawn, Carol M. Academy of Natural Sciences, Manuscript/Archives Librarian; husband is Willman Spawn Correspondence $\bullet$ 1987: (2) $\bullet$ 1980: (2) $\bullet$ 1982: (1) $\bullet$ 1983: (2) • 1985: (6) • 1986: (6) • 1988: (1) • 1993: (2) 


\section{Guide to the Ewan Papers}

Spawn, Willman ANSP

Biofile • Botanical Album

Correspondence - 1950: (2) Verdoorn, Frans Antonie, 1906-1984 • 1951: (8) Pursh, Frederick, 1774-1820 • 1952: (2) Pennell, Francis Whittier, 1886-1952 • 1953: (1) - 1956: (2) • 1958: (2) Missouri Botanical Garden • 1959:

(2) $\bullet$ 1960: (0) Pamphlet $\bullet$ 1961: (2) $\bullet$ 1962: (5) $\bullet$ 1963:

(6) - 1964: (3) • 1965: (3) • 1966: (1) • 1967: (2) $\bullet 1968$ :

(3) $\bullet$ 1970: (1) • 1974: (2) • 1975: (1) • 1976: (1) $\bullet$ 1982:

(2) $\bullet$ 1993: (1)

Speaker, Susan L. Editorial Assistant

Correspondence • 1988: (0) Filed under ISIS

Spear, Dorothea E. American Antiquarian Society, Worcester, MA, Assistant Librarian Correspondence • 1958: (1)

Specht, Heinz Washington Academy of Sciences,

Washington, D.C., Secretary

Correspondence • 1962: (2)

Speck, Frank Goldsmith (1881-1950) American naturalist ethnographer Biofile

Speckman, W. M. Office of Public Information, U. of Colorado, Boulder

Correspondence • 1941: (2) U. of Colorado, Boulder.

Dept. of Biology; Delphinium

Speese, Bernice Marguerite (1911-) American plant cytologist; College of William and Mary

See also John Thomas Baldwin

Correspondence • 1949: (1)

Speir, Francis, Jr.

Bioscript

Speizman, Arleen Tulane U., Dept. of Botany Correspondence $\bullet$ 1960: (1)

Spellenberg, Richard Correspondence $\bullet$ 1992: (3)

Spence, Eliza Jane (Edmondson) (1832-1917) Bioscript

Spence, John Bioscript

Spence, Mary Lee U. of Illinois Press, Urbana Correspondence - 1969: (3) Copy of correspondence from John Reed • 1972: (2) • 1973: (3)

Spence, W. Bioscript

Spencer, A. P. Agricultural Extension Service, Gainesville, FL

Correspondence • 1947: (1)

Spencer, Frank Queens College Correspondence $\bullet 1981$ : (2)

Spencer, Mary F. Bioscript

Spencer, O. H. Bioscript

Spener, Claudia Correspondence • 1992: (2)

Sperry, John J. Agricultural and Mechanical College of Texas, College Station, TX, Dept. of Biology Correspondence • 1949: (1) - 1951: (2) • 1972: (2)

Spicer, Millicent M. U. S. National Arboretum, Librarian Correspondence • 1965: (3)
Spicer, W. W. Bioscript

Spigno, Marquis de Bioscript

Spivak, Morris J. Botanical Album

Splitgerber, Frederick Lewis (1801-1845) Bioscript $\bullet$ Botanical Album

Spofford, Sally $\mathbf{H}$.

Correspondence 1 1972: (0) Filed under Waldemar H. Fries

Sponberg, Harold E. Northern Michigan College,

Marquette

Correspondence $\bullet$ 1959: (1)

Spongberg, Stephen A.

Bioscript

Sponsler, Olenus Lee

Botanical Album

Correspondence • 1940: (1)

Spooner, David M. U.S.D.A.

Correspondence • 1992: (1)

Spooner, Harry L.

Bioscript

Spragg, Frank A.

Bioscript

Sprague, Charles J. Bioscript

Sprague, Elizabeth Fern (1911-) Sweet Briar College, VA, Dept. of Biology; nickname "Betty"

Bioscript $\bullet$ Botanical Album

Correspondence • 1940: (1) • 1968: (1) • 1969: (2)

Bartram, William, 1739-1823 • 1972: (1) • 1973: (4) • 1974: (1) • 1976: (2) • 1978: (1) • 1979: (5) • 1980: (6) Letter from Kay Haynes $\bullet$ 1981: (1) 1 1982: (1) $\bullet$ 1983: (1) Letter from Nesta $\bullet$ 1984: (2) • 1985: (1) $\bullet 1986$ : (2) • 1989: (2) • 1990: (1)

Sprague, Isaac (1811-1895) American botanical and zoological artist

Biofile $\bullet$ Bioscript

Sprague, M. L., Mrs. Cheltenham, Glos. England Correspondence $\bullet$ 1960: (1)

Sprague, Thomas Archibald (1877-1958) Scottish taxonomist, bibliographer in England

Botanical Album

Correspondence • 1938: (1) Cockerell, Theodore Dru Alison, 1866-1948 - 1939: (3) Species

Spraker, Leslie J. Longwood Gardens Correspondence $\bullet$ 1974: (2)

Spreen, Orville The St. Louis Westerners, Secretary Correspondence $\bullet$ 1964: (2)

Sprengel, Curt Polycarp Joachim (1766-1833) German physician, botanist, taxonomist Bioscript

Sprengel, $\mathbf{K}$. Bioscript

Sprenger, $\mathbf{C}$. Bioscript

Springer, Frank (1848-1927) Bioscript

Springer, Jack

Correspondence • 1929-1933: (3) 
Sproul, Robert Gordon U. of California, Berkeley, Chancellor

Correspondence $\bullet$ 1944: (1)

Spruce, Richard (1817-1893) British botanist, plant collector, school master Biofile $\bullet$ Bioscript

Squibb, Robert

Bioscript

Squier, E. G.

Bioscript

Squire, Ephraim George (1821-1888)

Biofile

St. Hilaire, Auguste de (1779-1853) French naturalist, plant collector in Brazil

Biofile $\bullet$ Bioscript

St. Hilaire, Geoffroy

Bioscript

St. John (de Creve Coeur), Hector (1735-1813)

Bioscript

St. John, Harold (1892- ) American botanist; U. of Hawaii, Honolulu

Bioscript $\bullet$ Botanical Album

Correspondence $\bullet$ 1936: (1) • 1937: (2) • 1938: (1) •

1940: (3) • 1942: (1) • 1945: (2) • 1946: (7) • 1947: (2) •

1950: (1) • 1955: (1) • 1956: (2) • 1957: (2) • 1959: (1) •

1962: (3) $\bullet$ 1963: (4) Species $\bullet$ 1966: (2) $\bullet$ 1967: (5) $\bullet$

1970: (1) • 1971: (2) • 1973: (2) • 1974: (5) • 1975: (1) •

1976: (6) • 1978: (2) • 1979: (1) • 1980: (1) • 1981: (1) •

1986: (1) 1989: (1) 1990: (1)

St. John, O., Mrs.

Bioscript

St. John, Robert P. (1869-1960) American English teacher and pteridologist

Biofile

St. John-Martin, Martha Daughter of Harold St. John

Correspondence 1992: (2)

St. Louis Mercantile Library

See also John Neil Hoover

Botanical Album

Correspondence • 1986: (1) • 1987: (2) • 1989: (1) pamphlet

St. Louis Post-Dispatch

Correspondence - 1976: (1) Missouri Botanical Garden

St. Romain, B. G. Bunkie High School, Bunkie, LA, Vo-Ag instructor

Correspondence $\bullet$ 1954: (3)
Stacey, John William (1871-1943) American businessman, botanist, ornithologist

Biofile $\bullet$ Botanical Album

Correspondence - 1934: (1) Book collectors • 1935: (1) •

1936: (3) Book collectors $\bullet$ 1937: (7) Epling, Carl

Clawson, 1894-1968 1939: (4) Book Collectors • 1940:

(1) 1 1965: (2)

Stadelman, Bonnie S. Texas A \& M University

Correspondence • 1965: (1) $\bullet$ 1972: (1)

Stafleu, Frans Antonie (1921-) Dutch taxonomist,

botanical bibliographer; International Association for Plant

Taxonomy (IAPT)

Biofile $\bullet$ Bioscript $\bullet$ Botanical Album

Correspondence $\bullet$ 1954: (1) $\bullet$ 1958: (3) $\bullet$ 1963: (1) •

1965: (4) • 1966: (5) • 1967: (0) Filed under Harry D.
Stafleu, Frans Antonie (1921-) (continued)

Lubrecht • 1968: (10) Verdoorn, Frans Antonie, 1906-1984

- 1969: (15) Bartram, William, 1739-1823; Lyon, John,

1764-1814; Geiser, Samuel Wood, 1890- 1970 : (14) •

1971: (10) Verdoorn, Frans Antonie, 1906-1984 • 1972: (5)

- 1973: (11) • 1974: (5) • 1975: (21) • 1976: (6) • 1977:

(3) Harper, Roland McMillan, 1878-1966 • 1978: (5) •

1979: (5) • 1980: (4) • 1981: (16) • 1982: (8) • 1983: (12)

- 1984: (2) • 1985: (5) • 1986: (8) • 1988: (3) Book collectors $\bullet$ 1989: (3) • 1990: (5) • 1991: (4) Barton, Benjamin Smith, 1766-1815 • 1992: (2)

Stageman, P. F. Royal Horticultural Society, Library Correspondence $\bullet$ 1975: (1) $\bullet$ 1979: (1) Book collectors

Stager, Waira Correspondence $\bullet$ 1929-1933: (1)

Stail, C. T. U. S. Dept. of Agriculture Correspondence $\bullet$ 1939: (1)

Stakman, Elvin Charles (1885-1979) American plant geneticist, plant pathologist; U. of Minnesota, St. Paul Correspondence $\bullet$ 1946: (2) • 1952: (1) • 1953: (1)

Stalter, Richard (1942-) American plant ecologist, taxonomist; St. John's U., Jamaica, NY, Environmental Studies Program, Director Correspondence $\bullet$ 1977: (3)

Stamper, E. R. Agricultural Experiment Station, Baton Rouge, LA

Correspondence $\bullet$ 1956: (2)

Standard Fruit Company

Correspondence $\bullet$ 1972: (2)

Standifer, Marie Baton Rouge, LA

Correspondence $\bullet$ 1992: (2)

Standley, H. L. Photographer Correspondence • 1953: (1) "Rocky Mountain Vegetation"

Standley, Paul Carpenter (18841963) American taxonomist, plant collector; Field Museum of Natural History

Bioscript

Correspondence • 1939: (3) • 1941: (2) • 1943: (1)

Delphinium

Stanford, Ernest Elwood (1888-)

Botanical Album

Stanford University

Botanical Album

Stanford University Press

Correspondence $\bullet$ 1943: (1) 1977: (2)

Stanley, Amn F. Philadelphia, PA; works for Hui-Lin Li Correspondence $\bullet 1977$ : (3)

Stanley, Edward Smith (1775-1851) Bioscript

Stanley, G. Correspondence -1964 : (1)

Stanley, Wendell Meredith (1904) American biochemist, virologist; U. of California, Berkeley, Virus Laboratory Correspondence • 1951: (1) • 1952: (4) • 1953: (1)

Stannard, Jerry Willmert (1921-) American science historian, botanist; U. of Kansas, Lawrence, Dept. of History, History of Medicine

Botanical Album

(contimued on next page) 


\section{Guide to the Ewan Papers}

Stannard, Jerry Willmert (1921-) (continued) Correspondence • 1963: (1) • 1965: (3) • 1966: (9) • 1967: (8) • 1968: (20) • 1969: (5) Bartram, William, 1739-1823 • 1970: (2) • 1971: (6) • 1973: (2) • 1974: (1) - 1975: • 1976: (1)

Stanorov, Oskar Correspondence • 1967: (1)

Stansbury, Howard (1806-1863) Bioscript

Stansfield, Harry Liverpool Museum, Keeper of Botany Botanical Album

Correspondence $\bullet$ 1950: (2) $\bullet$ 1954: (6) $\bullet$ 1955: (5) $\bullet$ 1956: (2) • 1957: (4) • 1958: (8) • 1959: (1) • 1960: (1) • 1963: (1) • 1964: (6) • 1965: (4)

Stanton, Lucia C. Monticello, Charlottesville, VA, Director of Research

Correspondence • 1991: (4) Barton, Benjamin Smith, 1766-1815 • 1992: (1)

Stanton, Timothy William (1860-) Bioscript

Stanton, William R. U. of Pittsburgh, Dept. of History Correspondence • 1975: (1)

Stapf, Otto (1857-) Bioscript

Stapp, William F. National Portrait Gallery Correspondence $\bullet$ 1985: (1)

Stark, J. Ohio Bookhunter Correspondence • 1982: (1) • 1984: (1)

Stark, Oliver J. Botanical Album

Starks, Gilbert Dale (1933-) American taxonomist; Michigan State U., E. Lansing, Herbarium, Dept. of Botany Correspondence • 1970: (1)

Starr, S. Frederick Oberlin College Correspondence • 1980: (2) • 1991: (1)

Statesville, North Carolina Botanical Album

Stauffer, Jacob Bioscript

Stauffer, Robert C. U. of Wisconsin, Madison, Dept. of the History of Science

Correspondence • 1959: (2) Banks, Joseph, Sir, 1743-1800 - 1961: (3)

Stearn, E. R. Correspondence • 1981: (1)

Stearn, William Thomas (1911-) British botanist, antiquarian bookseller, librarian; British Museum (Natural History)

Biofile $\bullet$ Bioscript $\bullet$ Botanical Album

Correspondence • 1945: (1) • 1946: (2) • 1947: (2) • 1951: (1) - 1955: (1) • 1957: (1) • 1958: (2) • 1959: (1) • 1960: (3) • 1962: (1) • 1964: (2) • 1971: (1) • 1978: (0) Filed under Peter Hamilton Raven $\bullet$ 1979: (2) 1 1980: (2) - 1981: (2) • 1984: (1) - 1992: (1) Letter from Ronald Stuckey

Stearns, Raymond Phineas (1904-1970) U. of minois, Urbana, Dept. of History

Correspondence - 1961: (1) Correspondence between Stearns and George F. Frick • 1966: (2) Banister, John, 1650-1692
Stearns, Robert Edward Carter Bioscript

Stearns, Robert L. U. of Colorado, Boulder, President Correspondence • 1939: (2) • 1940: (1) U. of Colorado, Boulder. Dept. of Biology; Book collectors 1 1940: (0) Letter to F. Angwin $\bullet$ 1941: (1) $\bullet$ 1942: (2) $\bullet$ 1943: (5) $\bullet$ 1946: (2) • 1947: (4)

Stebbins, George Ledyard (1906-) American botanist, plant geneticist

Biofile $\bullet$ Bioscript $\bullet$ Botanical Album

Correspondence $\bullet$ 1938: (8) Species $\bullet$ 1941: (1) $\bullet$ 1942: (5) $\bullet$ 1944: (1) $\bullet$ 1945: (1)

Stechert, G. E.

Correspondence $\bullet$ 1940: (2) Book collectors

Stechert-Hafner Inc. Harry Lubrecht, bookseller Correspondence $\bullet$ 1956: (4)

Steele, Arthur Robert Botanical Album

Steele, J. E. U. of Western Ontario, London, Ontario, Dept. of Zoology Correspondence • 1971: (1)

Steenis, Cornelis Gijsbert Gerrit Jan Van (1901-1986) Dutch botanist; Oegstgeest, Holland

Biofile Botanical Album Correspondence $\bullet$ 1977: (5)

Steenis-Kruseman, M. J., Van Correspondence • 1957: (2) Delphinium; Nuttall, Thomas, 1786-1859

Steenstrup, J. S. Bioscript

Steere, Russell Ladd (1917- ) American cytologist, virologist Botanical Album

Steere, William Campbell (1907-1989) American bryologist; New York Botanical Garden Biofile • Bioscript $\bullet$ Botanical Album Correspondence 1 1945: (2) • 1949: (3) $\bullet$ 1950: (1) • 1952: (1) • 1954: (2) • 1957: (5) • 1958: (3) • 1959: (4) • 1960: (1) - 1961: (1) Letter to Steere from T. T. Earle $\bullet$ 1962: (4) - 1963: (3) Letter from George Booth Van Schaack • 1964: (1) • 1965: (4) • 1966: (12) Harper, Roland McMillan, 1878-1966 • 1969: (1) Letter from Lubrecht to Steere • 1970: (1) • 1975: (1) • 1977: (3) Biology-Instruction and study $\bullet$ 1978: (1) Book collectors - 1980: (1) • 1981: (2) Pursh, Frederick, 1774-1820

Steerman, Fritz Correspondence $\bullet$ 1954: (1)

Steetz, Joachim (18041862) Bioscript

Steffek, Edwin Francis (1912-) American horticulturist, botanical author; Horticulture, Boston, MA, Editor Correspondence • 1971: (4) Banister, John, 1650-1692 • 1973: (2)

Stehlé, Henri (1909-1983) Correspondence $\bullet$ 1945: (1)

Stein, Roy A. Ohio State U., Dept. of Zoology Correspondence • 1988: (2) • 1989: (0) Letter filed under Tim Martin Berra

Steiner, Allen M. Dorsey and Company Correspondence $\bullet$ 1964: (2) 
Steiner, Erich E. (1919- ) Swiss-born plant breeder, chemotaxonomist; Indiana U., Bloomington, IN Correspondence • 1949: (2) • 1950: (1)

Steinhauer, Daniel (1785-1852) Biofile • Bioscript

Steinhauer, Henry, Rev. (1782-1818) Bioscript

Steinmeyer, Reinhold H. Correspondence • 1963: (1)

Steitz, Brad Tulane U., New Orleans, LA Correspondence • 1979: (1)

Stejneger, Leonhard (1851-1943) Norwegian plant collector, naturalist Biofile • Bioscript

Stellati, Vincenzo (1780-1852) Italian botanist Bioscript

Steller, Georg Wilhelm (1709-1746) German naturalist in Alaska Biofile

Stenzel, Franz R.

Correspondence - 1964: (4) Copy of letter to Stenzel sent to Jessie Poesch - 1965: (1)

Stephanopoli, Gisele Hunt Botanical Library Correspondence $\bullet$ 1972: (1)

Stephens, Frank (1849-1937) Bioscript • Botanical Album Correspondence • 1921-1928: (1) • 1936: (2)

Stephens, Grover C. U. of California, Dept. of Organismic Biology

Correspondence $\bullet$ 1964: (2) $\bullet$ 1965: (1)

Stephens, Hildegard American Philosophical Society Library

Correspondence $\bullet$ 1978: (0) Filed under American Philosophical Society $\bullet$ 1983: (2)

Stephens, Lamarr Correspondence • 1978: (1)

Stephens, Lester D. U. of Georgia, Athens, Dept. of History

Correspondence • 1981: (5) • 1987: (1) Filed under E. Charles Nelson 1988 : (1)

Stephens, Stanley George (1911-) English plant geneticist; North Carolina State U., Raleigh, Emeritus Prof. of Genetics Correspondence • 1978: (6)

Stephenson, George K. Southern Forest Experiment Station, New Orleans

Correspondence • 1963: (1) Lyon, John, 1764-1814 • 1964: (2)

Stephenson, Wendell H. Tulane U., New Orleans, LA, Dept. of History Correspondence $\bullet$ 1950: (1) 1951: (3)

Stephenson, William Bioscript

Sterling, David Eugene, OR Correspondence • 1979: (3)

Sterling, E. B. Colorado plant collector Bioscript

Sterling, Keir Brooks (1934) American historian Bioscript

Correspondence • 1976: (2) $\bullet$ 1981: (2) Barton, Benjamin Smith, 1766-1815
Stern, Daniel Henry Louisiana State U., New Orleans, Dept. of Biological Sciences Correspondence $\bullet$ 1968: (2)

Stern, Edgar B. New Orleans, LA Correspondence $\bullet 1975$ : (2)

Stern, Edith New Orleans, LA; husband is Edgar Stern Correspondence • 1971: (1) • 1978: (1)

Stern, Frances Van Keuren U. of Oregon, Eugene, Assistant Professor of Ancient Art and Archaeology Correspondence $\bullet$ 1978: (2)

Stern, William Louis (1926-) American taxonomist, plant and wood anatomist

Biofile $\bullet$ Botanical Album

Correspondence • 1968: (5) • 1972: (5) • 1973: (2) • 1975: (1) - 1979: (3) Letter from Norma Gordon to WLS • 1981: (15) Bartram, William, 1739-1823 • 1982: (4) • 1983: (1) • 1985: (2) • 1988: (1) • 1989: (2) • 1990: (1) • 1991: (1) • 1993: (2)

Sternberg, Kaspar Marie (Graf) von (1761-1838) Austrian amateur botanist

Bioscript

Stetson, John Minor

Correspondence $\bullet$ 1950: (9)

Stetson, Sarah Pattee Williamsburg. VA Correspondence • 1954: (2) • 1966: (1)

Steudel, Ernst Gottlieb von (1783-1856) Bioscript

Steven, Christian von (1781-1863) Finnish-born botanist, physician in Russia Bioscript

Stevens, Bioscript

Stevens, Edward Bioscript

Stevens, Henry English bookseller Correspondence • 1983: (1)

Stevens, Jane Tulane U., Howard-Tilton Library Correspondence $\bullet$ 1969: (1)

Stevens, Joan The J's Nest, Barton, Cambridge Correspondence $\bullet 1970$ : (1)

Stevens, Neil Everett (1887-1949) American plant pathologist; U. of Illinois, Urbana, Dept. of Botany Correspondence $\bullet$ 1943: (1)

Stevens, Orin Alva (1885-1979) American plant ecologist, phytogeographer; North Dakota Agricultural College Botanical Album Correspondence • 1943: (4) • 1944: • 1947: (2)

Stevens, $\mathbf{R}$. H. Bioscript

Stevens, Russell Bradford (1915-) American plant pathologist; National Academy of Sciences, Secretary Correspondence $\bullet$ 1956: (1) 1977 : (2)

Stevens, Thomas Jones Bioscript

Stevens, Warren Douglas (1944) American taxonomist; Missouri Botanical Garden, St. Louis, MO, Director of Research Bioscript Daniels Keck $\bullet 1989$ : (1) 


\section{Guide to the Ewan Papers}

Stevens, William Chase (1861-) Botanical Album

Stevenson, C. G. Bioscript

Stevenson, James Bioscript

Stevenson, John Albert (1890-) American plant pathologist, mycologist; U. S. Dept. of Agriculture, Bureau of Plant Industry, Beltsville, MD, Principal Mycologist Correspondence $\bullet$ 1950: (2) -1951 : (2) Species -1952 : (2) $\bullet$ 1954: (1) • 1964: (1)

Stevenson, John James (1841-1924) Bioscript

Stevenson, Matilde Coxe (Evans) (1850-1915) Bioscript

Stevenson, Paul H. Correspondence • 1962 : (2)

Stevenson, Robert Louis Botanical Album

Stewart, Alban N. (1875-1940) Bioscript

Stewart, Ewell Sale (1924-1987) Philadelphia writer and traveller Biofile

Stewart, Florence R. Northwestern U. Correspondence • 1950: (1)

Stewart, James Torrence (1824-1901) American amateur botanist, physician in Illinois Botanical Album

Stewart, Laurie U. of North Carolina, Herbarium Correspondence • 1940: (1)

Stewart, Margaret Grover (1911-) Bioscript

Stewart, Maria Santa Barbara, CA; husband is William Stewart Correspondence • 1974: (7) • 1978: (4)

Stewart, Maryalice H. Archives of the Canadian Rockies, Director Correspondence • 1969: (2)

Stewart, Ralph Randles (1890-) American taxonomist Botanical Album

Stewart, Robert G. Smithsonian Institution, National Portrait Gallery

Correspondence • 1966: (6) • 1967: (2)

Stewart, William Sheldon (1914) American plant physiologist, horticulturist; Pacific Tropical Botanic Garden, Loloa, Kauai, HI; nickname "Bill"

Bioscript $\bullet$ Botanical Album Correspondence • 1937: (1) • 1938: (1) • 1943: (1) • 1945: (1) • 1956: (2) • 1961: (4) Epling, Carl Clawson, 1894-1968 • 1962:(2) • 1965: (1) • 1966: (2) • 1967: (1) - 1970: (8) Book collectors - 1971: (4) Barton, Benjamin Smith, 1766-1815 - 1972: (5) - 1973: (10) Letter to Mrs. Harry T. Howard, Jr. • 1979: (2) • 1980: (1) • 1981: (1) - 1985: (1) • 1986: (1)

Stewart, Wilson Nichols (1917-) American plant morphologist; U. of Alberta, Edmonton, Canada; Chairman, Dept. of Botany

Correspondence • 1969: (0) Filed under John G. Packer
Steyermark, Julian Alfred (1909-1988) American plant taxonomist

Bioscript $\bullet$ Botanical Album

Correspondence • 1929-1933: (5) • 1934: (1) • 1942: (1)

- 1946: (2) • 1949: (2) • 1950: (1) • 1951: (5) Species; Vismia $\bullet 1952:(3) \bullet 1958:(2) \bullet 1959:(2)$

Stibbs, John Henry Tulane U., New Orleans, LA, Director of Student Activities, Dept. of English Correspondence $\bullet$ 1954: (1)

Stibbs, Phyllis Husband is John Henry Stibbs Correspondence • 1977: (1)

Stieber, Michael Thomas (1943-) Archivist; Hunt Library Correspondence • 1983: (1) • 1986: (1) • 1993: (2)

Stigall, B. M. Paseo High School, Kansas City, MO Correspondence $\bullet$ 1939: (2) • 1940: (1) • 1941: (5) Species • 1945: (2)

Stigliano, Charles Bioscript

Stiles, Charles Wardell (1867-1933) American zoologist Biofile

Stiles, Karl Amos Botanical Album

Stillingfleet, Benjamin (1702-1771) English naturalist, physician Bioscript

Stillman, Jacob Davis Babcock (1819-1888) American physician, botanical collector Bioscript

Stine, Perna M. Zephyrhills, FL Correspondence • 1955: (1) • 1956: (2) • 1971: (3) Species

Stinehour Press Lunenberg, VT, Roderick Stinehour, proprietor

Correspondence $\bullet$ 1974: (3) • 1975: (2) • 1977: (1)

- 1979: (0) Filed under Mills Lane IV $\bullet$ 1981: (1)

Stinehour, Roderick

Botanical Album

Correspondence - 1979: (2) Postcard from Freeman Keith filed under Stinehour $\bullet$ 1984: (1)

Stivers, Charles Austin Bioscript

Stix, Judith S. St. Louis, MO Correspondence $\bullet$ 1977: (2)

Stockard, A. H. U. of Michigan, Ann Arbor, Biological Station Correspondence • 1949: (2) • 1950: (2) • 1951: (1)

Stocker, Erik Correspondence $\bullet$ 1988: (1)

Stockholm, Naturhistoriska Rijksmuseum Correspondence • 1950: (1) • 1967: (1)

Stocks, J. E. Bioscript

Stockwell, Palmer (1898-) Botanical Album

Stoddard, 0. U. Bioscript

Stokes, Susan Gabriella (1868-) Bioscript • Botanical Album Correspondence • 1949: (2) 
Stokey, Alma Gracey (1877-1968) American plant collector, plant anatomist, morphologist Biofile

Stolz, Robert Botanical artist Bioscript • Botanical Album

Stolze, Robert G. (1927- ) American pteridologist; Field Museum of Natural History, Dept. of Botany Correspondence $\bullet$ 1973: (1)

Stomps, Theodoor Johann (1885- ) Dutch plant geneticist, cytologist

Bioscript $\bullet$ Botanical Album

Stone, Benjamin (1933-1994) American taxonomist, phytogeographer; Malaysia

Correspondence $\bullet$ 1983: (1)

Stone, Beverly Organization for Tropical Studies Correspondence • 1982: (1) • 1989: (1) • 1990: (1) • 1991: (3) $\bullet$ 1993: (1)

Stone, Donald Eugene (1930- ) American plant geneticist; Duke U., Dept. of Botany

Botanical Album

Correspondence $\bullet$ 1959: (2) • 1960: (3) $\bullet$ 1961: (5) • 1962: (2) • 1963: (2) • 1964: (7) Filed with T. Richard Fisher correspondence $\bullet$ 1965: (3) • 1966: (2) • 1968: (4) Copy of correspondence from Wayne E. Manning $\bullet$ 1969:

(3) $\bullet$ 1970: (3) $\bullet$ 1971: (2) $\bullet$ 1972: (1) $\bullet$ 1973: (2) Species - 1974: (1) • 1977: (7) • 1978: (3) Correspondence to Delzie Demaree and Donald Stone $\bullet$ 1980: (1) $\bullet$ 1983: (2) - 1990: (1)

Stone, Doris Zemurray (1909-)

Correspondence • 1980: (1) • 1983: (1)

Stone, Edna L. American Fern Society

Correspondence $\bullet$ 1951: (1)

Stone, George H. (1841-1917) Bioscript

Stone, Lois Chambers Ornithologist; U. of California, Berkeley, Museum of Vertebrate Zoology

Bioscript $\bullet$ Botanical Album

Correspondence • 1938: (1) • 1942: (1) • 1946: (1) 1957

(1) 1 1958: (3) • 1963: (6) • 1964: (1) • 1965: (2) • 1967:

(3) 1968: (2) Copy of correspondence to Clif Smith 1970: (3) • 1971: (4) • 1974: (2) • 1975: (15) • 1976: (5) - 1977: (14) Rocky Mountain Naturalists; Book collectors; letters from Leon E. Brooks in correspondence $\bullet$ 1978: (12) - 1979: (10) Book collectors • 1980: (15) $\bullet$ 1981: (16) 1982: (10) - 1983: (18) • 1984: (8) • 1985: (10) - 1986: (8) - 1987: (3) Book collectors • 1988: (3) • 1989: (7) • 1990: (4) • 1991: (3) • 1992: (2) • 1993: (1)

Stone, Marjorie Williams Bibliographer; Harvard U., Gray Herbarium Botanical Album

Correspondence • 1935: (1) • 1941: (1) • 1943: (1) • 1946: (1) - 1951: (3) • 1952: (5)

Stone, Peter A. Flood Control District, Central and Southern Florida Correspondence $\bullet$ 1976: (2)

Stone, Robert Tulane U., New Orleans, LA, Sociology Correspondence 1965: (1)

Stone, W. G. M.

Bioscript

Stone, Witmer (1866-1939) Bioscript
Stones, Elsie Margaret (1921-) Australian-born botanical artist

Biofile $\bullet$ Bioscript $\bullet$ Botanical Album

Correspondence • 1978: (1) • 1979: (7) • 1980: (6) • 1982: (2) • 1983: (7) • 1985: (2) • 1986: (2) • 1988: (2) Letter re. M. Stones $\bullet$ 1990: (3) $\bullet$ 1991: (7) $\bullet$ 1992: (3) 1993: (2)

Stool, Sylvan E. Children's Hospital of Pittsburg, PA Correspondence • 1982: (1) • 1983: (1)

Stopes, Marie Charlotte Carmichael (1880-1958) Scottishborn paleobotanist; England Bioscript

Storer, David Humphreys (1804-1891) American obstetrician, lichenologist Biofile • Bioscript

Storer, Robert W. U. of Michigan, Ann Arbor, Museum of Vertebrate Zoology

Correspondence $\bullet$ 1950: (2) • 1951: (1) • 1989: (1)

Stork, William

Bioscript

Stotler, Raymond Eugene (1940-) Southern Illinois U., Carbondale, Dept. of Botany

Correspondence • 1989: (1)

Stout, Arlow Burdette (1876-1957) American botanist, plant geneticist, plant breeder Botanical Album

Stout, Arthur B. Bioscript

Stout, George H. U. of Washington, Seattle, Dept. of Chemistry

Correspondence • 1965: (2) • 1966: (1)

Stout, William Bioscript

Stoutamire, Warren Petrie (1928-) American taxonomist; Indiana U., Bloomington

Correspondence • 1954: (1) • 1958: (2)

Stowe Gardens Botanical Album

Stowe, Stephanie H. Denver Museum of Natural History, CO, Librarian

Correspondence • 1976: (0) Filed under Denver Museum of Natural History

Strachan, D. J. Correspondence $\bullet$ 1992: (1)

Strachey, Richard, Sir (1817-1908) Bioscript

Strahlenberg, Bioscript

Strand, Embrick Botanical Album

Strangways, William Thomas Horner Fox (1795-1865) English botanist Bioscript

Strasburger, Eduard Adolf (1844-1912) Russian-born botanist; Germany

Bioscript

Stratton, Elenore New York, NY Correspondence $\bullet$ 1959: (11)

Stratton, Robert (1893-) Oklahoma Agriculural and Mechanical College Correspondence $\bullet 1942:$ (4) 1948: (1) 


\section{Guide to the Ewan Papers}

Stratton, W. S.

Bioscript

Straus, Robert Ware The Accokeek Foundation Inc., Washington, D.C.

Correspondence • 1960: (4) • $1962:(1) \bullet 1966:(3) \bullet$ 1967: (1)

Strauss, Harold Alfred A. Knopf, Inc. Correspondence $\bullet$ 1968: (8) $\bullet$ 1970: (2) $\bullet$ 1971: (1)

Streator, Clark P.

Bioscript

Correspondence • 1950: (4) Rocky Mountain Naturalists

Street, Joseph A. Bioscript

Street, Lillian F. Bioscript

Stressmann, E. Correspondence $\bullet$ 1955: (3)

Stretch, Richard Harper (1837-1923?) Bioscript

Stringham, Emerson (fl. 1948) Bioscript $\bullet$ Biofile

Strobbe, Connie Bioscript

Strobel, Pellegrino Bioscript

Stromberg, Oliver A. Plattsburgh, NY Correspondence $\bullet$ 1991: (1)

Strommen, Sue Gibson Missouri Botanical Garden, St. Louis, MO, Public Relations Manager Correspondence • 1987: (0) Letter to Nick Howes, filed under Missouri Botanical Garden

Strother, John Lance (1941- ) American taxonomist; U. of California, Berkeley

Botanical Album

Correspondence • 1968: (2) • 1980: (3) • 1982: (3)

Stroud, Patricia Tyson Academy of Natural Sciences, Friends of the Library, Philadelphia, PA; nickname "Patsy;" husband is Noel J. Tyson

Correspondence 1985: (2) • 1987: (3) Bartram, William 1739-1823

Struik, Dirk J. Massachusetts Institute of Technology, Dept. of Mathematics Correspondence $\bullet$ 1967: (2)

Strzelecki, Bioscript

Stuaffer, John H. C. V. Mosby Company, St. Louis, MO Correspondence • 1959: (1)

Stuart, George

Botanical Album

Correspondence $\bullet$ 1984: (1)

Stuart, John

Bioscript

Stuart, John, Earl of Bute (1713-1792) Scottish-born statesman, botanist in England Bioscript

Stuart, John McDouall (1815-1866) English surveyor and plant collector in Australia Bioscript
Stuckey, Ronald Lewis (1938- ) American taxonomist, botanical historian; Ohio State U., Columbus

Biofile • Bioscript $\bullet$ Botanical Album

Correspondence $\bullet$ 1964: (6) $\bullet$ 1966: (12) $\bullet$ 1967: (2) $\bullet$ 1969: (2) • 1970: (1) - 1971: (7) • 1972: (14) $\bullet$ 1975: (1) - 1976: (1) • 1977: (5) • 1978: (9) • 1979: (1) • 1980: (1) - 1982: (7) - 1983: (10) 1984: (0) Filed under Charles Boewe • 1984: (6) • 1985: (1) $\bullet$ 1986: (2) $\bullet$ 1987: (5) $\bullet$ 1988: (5) $\bullet$ 1989: (3) $\bullet$ 1990: (2) $\bullet$ 1991: (9) Letters from C. Zacher, T. Stuessy and D. Crawford $\bullet$ 1992: (8) See also letter to Dale E. Johnson filed with Council on Botanical and Horticultural Libraries • 1993: (3)

Studd, Gretchen Eugene, OR; student in Ewan's classes, Plant Systematics and History of Biology

Correspondence • 1978: (2)

Studer, William J. Ohio State U., Columbus, Director of Libraries Correspondence $\bullet$ 1986: (1)

Stuessy, Tod Falor (1943- ) American taxonomist; Ohio State U., Columbus

Biofile $\bullet$ Bioscript $\bullet$ Botanical Album

Correspondence $\bullet$ 1979: (6) $\bullet$ 1980: (2) Letter from Stuessy to Heiser in Bretting correspondence $\bullet$ 1983: (2) 1984: (4) • 1985: (6) • 1986: (5) • 1988: (3) • 1991: (0) Filed under R. Stuckey

Stull, Florence Federated Garden Clubs of Michigan, Inc.; husband is Charles R. Stull Correspondence $\bullet$ 1975: (1)

Sturgis, R. A. Bioscript

Sturgis, William Codman (1862-1942) American botanist Bioscript $\bullet$ Botanical Album

Sturm, Johann Wilhelm (1808-1865) German taxonomist, editor Bioscript

Sturtevant, Alfred Henry (1891-1970) American biologist Botanical Album

Sturtevant, Edward Lewis (1842-1898) American . agriculturist and botanist Biofile $\bullet$ Bioscript $\bullet$ Botanical Album

Sturtevant, William C. National Museum of Natural History, Division of Ethnology Correspondence $\bullet$ 1976: (2) - 1979: (1) Barton, Benjamin Smith, 1766-1815

Stuve, W. E. Bioscript

Suckley, George (1830-1869) Naturalist, surgeon; U. S. Army Biofile • Bioscript

Sudds, R. H. U. of Connecticut, Dept. of Plant Science Correspondence • 1957: (2)

Sudler, Olive W. Milford Mill High School, Baltimore, MD Correspondence • 1959: (2)

Sudworth, George Bishop (1864-1927) Bioscript $\bullet$ Botanical Album

Sugden, Jane Time-Life Books Correspondence • 1972: (2)

Suksdorf, Wilhelm Nikolaus (1850-1932) German-born American botanist and plant collector Biofile $\bullet$ Bioscript $\bullet$ Botanical Album Correspondence • 1929-1933: (2) 
Sulgrove, Leslie

Bioscript

Sulgrove, Sabina Mueller (1940-) American plant geneticist, taxonomist; The American Ivy Society Correspondence $\bullet$ 1980: (2)

Sullivan, , Dr. Correspondence $\bullet$ 1973: (1)

Sullivant, Eliza Griscom (Wheeler) (1817-1850) Bioscript

Sullivant, William Starling (1803-1873) American businessman and bryologist Biofile $\bullet$ Bioscript $\bullet$ Botanical Album

Sumner, Francis Bertody (1874-1945) American zoologist Biofile • Bioscript

Sumner, George (1793-1855) Physician, botanist Bioscript

Sundararaj, B. I.

Botanical Album

Correspondence $\bullet$ 1967: (2)

Sundell, Eric (1942- ) U. of Arkansas, Monticello Correspondence • 1974: (1) • 1975: (1) • 1976: (2) • 1983: (3) • 1984: (3) • 1985: (1) • 1987: (1)

Suno, Gordon Bioscript

Superintendent of Documents, U. S. Government Printing Correspondence $\bullet$ 1944: (1)

Suringar, William Frederick Reiner (1832-1898) Dutch botanist

Bioscript $\bullet$ Botanical Album

Sussman, Alfred Sheppard (1919-) American botanist, microbiologist, mycologist

Botanical Album

Sutherland, James (c. 1639-1719) British botanist Bioscript

Sutliffe, Dorothy (1866-1952) American bryologist; husband is E. C. Sutliffe

Biofile • Bioscript

Sutro, Adolph (1863-1933) Private and instructional collector Biofile

Suttkus, Royal Dallas Tulane U., New Orleans, LA, Museum of Natural History, Director

Botanical Album

Correspondence • 1952: (2) • 1954: (2) - 1955: (1)

- 1962 : (1) • 1966: (1) • 1969: (1) • 1981: (1) Book collectors $\bullet$ 1983: (1) $\bullet$ 1987: (2)

Sutton, George Miksch (1898-) American bird painter, ornithologist

Biofile $\bullet$ Bioscript $\bullet$ Botanical Album

Correspondence $\bullet$ 1965: (10) $\bullet$ 1977: (2)

Sutton, Joseph G. New York Botanical Garden Correspondence $\bullet$ 1972: (1)

Sutton, Raymond M. Jr. Book dealer, Williamsburg, KY

Correspondence $\bullet$ 1988: (2)

Sutton, Stephanne B. Harvard U., Arnold Arboretum Correspondence • 1966: (9) • 1967: (4) • 1968: (7) • 1969: (1)

Suzuki, Howard K. U. of Florida, Gainesville Botanical Album

Correspondence $\bullet 1973$ :
Svenson, Henry Knute (1897-1986) Swedish-born taxonomist; U. S. A.

Botanical Album

Correspondence • 1935: (1) American Fern Society • 1939: (1) American Fern Society $\bullet 1940$ : (4) American Fern Society • 1944: (1) • 1951: (3) • 1953: (1) • 1954: (2) 1 1957: (3) $\bullet 1960:(2)$

Swain, C. G. U. S. Dept. of Agriculture Correspondence • 1939: (4) Species

Swain, Ralph Brownlee Entomologist Botanical Album

Correspondence $\bullet 1948:(2)$

Swaine, Charles

Bioscript

Swainson, Geoffrey M.

Correspondence $\bullet$ 1993: (1)

Swainson, William (1789-1855) British naturalist and explorer

Biofile $\bullet$ Bioscript

Swallen, Jason Richard (1903-1991) American agrostologist, taxonomist; Smithsonian Institution Bioscript $\bullet$ Botanical Album Correspondence $\bullet$ 1937: (7) • 1939: (1) • 1941: (2) • 1942: (2) • 1943: (2) • 1956: (2) • 1957: (1) • 1958: (2) Vismia • 1959: (7) Vismia $\bullet$ 1960: (4) • 1961: (3) • 1962 : (2) $\bullet 1980:(3)$

Swallow, Alan U. of Denver Press Correspondence $\bullet$ 1948: (2) • 1949: (7) • 1950: (12) Rocky Mountain Naturalists; Verdoorn, Frans Antonie, 1906-1984 - 1951: (11) Rocky Mountain Naturalists; Verdoorn, Frans Antonie, 1906-1984 • 1952: (4) • 1953: (5) • 1954: (2) • 1956: (1) Rocky Mountain Naturalists • 1964: (0) Filed under George Booth Van Schaack

Swallow, George Clinton (1817-1899) State geologist; Missouri Bioscript

Swan, Charles Walter (1838-1921) Bioscript

Swann, Anthony H. Wheldon and Wesley Ltd. booksellers; nickname "Tony"

Correspondence $\bullet$ 1992: (6) Book collectors $\bullet$ 1993: (2)

Swann, Charles Kirke Wheldon \& Wesley Limited Correspondence $\bullet$ 1957: (2) Book collectors $\bullet$ 1958: (2) $\bullet$ 1961: (3) • 1972: (1) - 1977: (1) Book collectors

Swann, Christopher K. Wheldon and Wesley Ltd. booksellers Correspondence • 1975: (10) - 1993: (1)

Swann, Howard Kirke (1919-1993) English natural history bookseller; Wheldon and Wesley Ltd.

Biofile

Correspondence $\bullet$ 1960: (7) $1962:(1) \bullet 1964:$ (1) $\bullet$ 1971: (2) Book collectors - 1974: (1) Book collectors • 1975: (0) Filed under Mills Lane IV $\bullet 1977$ : (5) $\bullet 1978$ : (2) - 1979: (5) Book collectors • 1980: (9) - 1981: (1) Book collectors • 1982: (4) • 1983: (11) • 1984: (5) • 1985: (9) Book collectors • 1986: (3) - 1987: (1) Book collectors • 1988: (14) • 1989: (2) • 1990: (1) • 1991: (2)

Swanson, Audrey New Orleans, LA Correspondence - 1952: (1) 


\section{Guide to the Ewan Papers}

Swanson, Carl P. Quarterly Review of Biology; Johns Hopkins U., Baltimore, MD, Dept. of Biology Correspondence $\bullet$ 1951: (2) $\bullet$ 1957: (1)

Swanson, Linnea Former student Correspondence $\bullet 1941:$ (2)

Swarth, Harry Schelwaldt (1878-1935) American ornithologist Biofile • Bioscript

Swarthmore College, Friends Historical Library Albert W. Fowler, Assistant Director

Correspondence • 1978: (3)

Swartz, Olaf Peter (1760-1818) Bioscript

Sweet Briar College Dept. of Botany, Margaret Simpson, Chairman

Correspondence • 1980: (1)

Sweet, Helen Elizabeth Bioscript

Sweet, Robert (1783-1835) Nurseryman, botanical author Bioscript

Sweetland, Correspondence $\bullet$ 1980: (1)

Swem, E. G. College of William and Mary, Librarian Emeritus

Botanical Album

Correspondence $\bullet$ 1955: (2) $\bullet$ 1956: (4) $\bullet$ 1958: (4) 1959: (4) Banister, John, 1650-1692 • 1962 : (3) • 1963 : (1)

Swenson, Lowell E. U. of Colorado Museum Correspondence $\bullet$ 1950: (2)

Swetman, Glenn L. People's Bank of Biloxi Correspondence • 1976: (4)

Swift, Charles Henry (1881-1966) American naturalist and physician Biofile

Swift, J. Otis Botanical Album

Swingle, Deanne Bret (1879-1944) American taxonomist, plant pathologist; Montana State College

Correspondence • 1942: (4)

Swingle, John Alta California Bookstore Botanical Album

Correspondence • 1965: (1) Letter from George Lawrence - 1972: (1) • 1973: (1) • 1974: (1)

Swingle, Mary Belle Alta California Bookstore; husband is John Swingle

Correspondence $\bullet$ 1972: (1) $\bullet$ 1981: (2) Book collectors 1988: (3)

Swingle, Walter Tennyson (1871-1952) American botanist Biofile $\bullet$ Botanical Album

Swink, Floyd Allen (1921-) American taxonomist; Morton Arboretum

Bioscript

Correspondence • 1969: (1)

Switzer, Stephen (1682?-1745) Bioscript

Swoger, Arthur Correspondence • 1975: (1) Bartram, William, 1739-1823

Sydney, Cedric Botanical Album
Symmes, Mabel

Correspondence • 1957: (0) Filed under John Thomas Howell

Synge, Patrick Millington (1910-) American horticulturist, phytogeographer, editor; Royal Horticultural Society, London, Editor

Botanical Album

Correspondence • 1960: (3) 1962 : (2) $\bullet$ 1968: (10)

Taylor, George, Sir, 1904- 1969: (1) Bartram, William, 1739-1823

Syracuse University Library

Correspondence - 1954: (1) Book collectors; Pursh, Frederick, 1774-1820

Syracuse University Press Walda Metcalf, Managing Editor

Correspondence $\bullet$ 1982: (3) $\bullet$ 1983: (7) $\bullet$ 1986: (1) • 1990: (1)

Szeles, Robert E. Kean College of New Jersey, Union Correspondence $\bullet$ 1975: (2)

Szewczyk, David M. Bookseller; Philadelphia Rare Books and Manuscripts

Correspondence $\bullet$ 1984: (4) $\bullet$ 1985: (6) $\bullet$ 1986: (1) 1989: (2) • 1990: (3) • 1991: (1)• 1992: (2)

\section{T}

Tabley, Lord de See also John Byrne Leicester Warren Bioscript

Tabor, Paul Correspondence • 1967: (2) • 1972: (3)

Täckholm, Vivi Laurent (1898-1978) Swedish-born taxonomist; Egypt Botanical Album

Tacoma Audubon Society Operation "Nature Guide," Tacoma, WA Correspondence 1977 : (4) Biology-Instruction and study

Tacoma School Board Tacoma, WA Correspondence 1958: (1)

Tagawa, Motozi (1908-1977) Japanese pteridologist; Kyoto Imperial U., Japan Correspondence • 1937: (2) • 1938: (1) • 1939: (1)

Taihoku Imperial University, Formosa Botanical Album

Takhtajan, Armen Leonovich (1910- ) Russian plant morphologist, phylogeneticist Bioscript • Botanical Album

Talbot, Murrel Williams Botanical Album

Talmadge, Robert L. Tulane U., Howard-Tilton Memorial Library, New Orleans

Bioscript

Correspondence • 1961: (4) • 1962: (4) • 1963: (1) $\bullet$ 1964: (2) • 1965: (5) • 1966: (2)

Tanaka, Tyôzaburô (1885-) Japanese taxonomist, economic botanist Botanical Album

Tancin, Charlotte Hunt Botanical Library, Librarian Correspondence • 1992: (1) 
Tanis, Janis Bryn Mawr College Library, Bryn Mawr, PA, Director

Correspondence • 1977: (1) Book collectors

Tanner, Lois

Correspondence $\bullet$ 1950: (1)

Tanner, Vasco M. Brigham Young U.

Correspondence • 1949: (2)

Tanneu, Robert

Correspondence • 1983: (1)

Tansley, Arthur George (1871-1955) English plant taxonomist

Bioscript $\bullet$ Botanical Album

Tapley, Harriet S. Librarian; Essex Instiute, Salem, MA Correspondence • 1952: (1)

Targioni Tozzetti, Antonio (1785-1856) Italian botanist Bioscript

Targioni Tozzetti, Ottaviano (1755-1829) Italian physician, botanist

Bioscript

Tate, George Henry Hamilton (1894-1953) British-born American zoologist and collector Biofile • Bioscript

Tatnall, Edward (1818-1898) American plant explorer Bioscript

Tatnall, Robert Richardson (1870-1856) Plant collector Bioscript - Botanical Album

Taubenhaus, Esther Texas A \& M, College Station, S. M. Tracy Herbarium; husband is Jacob Joseph Taubenhaus Correspondence • 1941: (2)

Taverner, P. A. Bioscript

Taxon Journal, Int'l Assoc. for Plant Taxonomy; Botanical Garden \& Museum, Berlin, Germany

Correspondence • 1983: (1) • 1986: (2) • 1991: (1) • 1992: (3) • 1993: (1)

Taylor, Adelaide Correspondence $\bullet$ 1929-1933

Taylor, Aline Tulane U., Newcomb College, New Orleans, LA

Correspondence • 1961: (1)

Taylor, Bayard Bioscript

Taylor, Douglas H. Miami University, $\mathrm{OH}$ Correspondence $\bullet$ 1978: (1) Book collectors

Taylor, E. M. Correspondence $\bullet$ 1936: (1)

Taylor, Floyd B. National Park Service, Superintendent Correspondence $\bullet$ 1970: (7)

Taylor, Garland Forbes Tulane U., Howard-Tilton Library, New Orleans, LA, Librarian Correspondence - 1948: (4) Book collectors $\bullet$ 1949: (4) 1950: (10) Book collectors $\bullet$ 1951: (4) $\bullet$ 1952: (2) $\bullet$ 1953: (1) • 1954: (7) • 1955: (3) • 1956: (6) Book collectors - 1957: (5) Book collectors $\bullet$ 1958: (3) • 1959: (2)

Taylor, George, Sir (1904-1993) Scottish plant collector, taxonomist; British Museum Natural History, Kew, England; Stanley Smith Horticultural Trust Biofile - Bioscript $\bullet$ Botanical Album Correspondence 1937: Nutrall, Thomas, 1786-1859 Species • 1939: (6) Species • 1947: (1) • 1954: (2) •
Taylor, George, Sir (1904-1993) (continued) 1955: (4) • 1956: (7) • 1957: (5) Royal Botanic Gardens, Kew $\bullet$ 1958: (4) $\bullet$ 1960: (2) $\bullet$ 1961: (19) Book collectors; some letters from Wilson Callendar 1961 (0) Filed under Wilson Callendar • 1962: (10) Royal Botanic Gardens, Kew $\bullet$ 1963: (6) • 1964: (6) • 1965: (4) • 1966: (9) - 1967: (8) Letters to Mrs. V. Fleming • 1968: (9) • 1968: (0) Some correspondence filed under Patrick Millington Synge • 1969: (6) Royal Botanic Gardens, Kew - 1969: (0) Letter filed under Harry D. Lubrecht • 1970: $(4) \bullet 1971:(2) \bullet 1972:(1) \bullet 1974:(2) \bullet 1975:$ (1) $\bullet$ 1977: (6) • 1978: (6) • 1979: (3) • 1980: (3) • 1981: (1) • 1983: (1) • 1984: (3) • 1985: (1) • 1987: (1) Bartram, William, 1739-1823 - 1988: (11) Barton, Benjamin Smith, 1766-1815 • 1989: (3) • 1990: (4) • 1991: (2) Barton, Benjamin Smith, 1766-1815

Taylor, H. A. Liverpool Record Office, Archivist Correspondence $\bullet$ 1954: (1)

Taylor, Henry Reed Bioscript

Taylor, James Herbert (1916-) American plant geneticist Botanical Album

Taylor, Kenneth L. U. of Oklahoma, Norman Correspondence • 1969: (5)

Taylor, Knowland Branch, LA Correspondence $\bullet$ 1959: (1)

Taylor, Lois Chambers See Stone, Lois Chambers

Taylor, Mary Agnes (1885-1976) Bioscript

Taylor, Mary Susan (1951-) American botanical bibliographer; Missouri Botanical Garden Botanical Album Correspondence • 1979: (1) • 1980: (1) • 1989: (1) - 1990: (1)

Taylor, R. M. Bioscript

Taylor, Raymond Leech (1901-) Correspondence • 1946: (2)

Taylor, Richard Cowling (1789-1861) Plant collector Bioscript

Taylor, Roy Lewis (1932-) Canadian taxonomist, plant collector; U. of British Columbia, Vancouver Bioscript • Botanical Album Correspondence $\bullet 1977$ : (3)

Taylor, Sue See Taylor, Mary Susan (1951-)

Taylor, Thomas Mayne Cumningham (1904-1983) U. of British Columbia, Vancouver, Professor Correspondence 1967 : (2)

Taylor, Thomas N. (1937-) American paleobotanist; Ohio State U., Columbus, Professor

Correspondence $\bullet 1977$ : (0) Filed with Ronald Lewis Stuckey $\bullet 1978$ : (0) Filed with Ronald Lewis Stuckey

Taylor, Tom Artist Botanical Album

Taylor, V. U. of Arizona, Tucson Correspondence $\bullet$ 1954: (1)

Taylor, Walter K. U. of Central Florida, Orlando, Dept. of Biology, Professor Correspondence $\bullet$ 1993: (5) 


\section{Guide to the Ewan Papers}

Taylor, William Randolph (1895-1990) American phycologist; U. of Michigan, Ann Arbor Botanical Album

Correspondence • 1956: (2) • 1965: (4) $\bullet$ 1967: (5) $\bullet$ 1968: (12) • 1969: (8) • 1970: (2) • 1971: (1)

Teed, Terry Correspondence • 1973: (2)

Teijsmann, J. E. Botanical Album

Tempest, H. G. Bioscript

Temple University Cornelia S. King, Rare Book Bibliographer Correspondence $\bullet$ 1983: (2)

Templeton, Bonnie Carolyn (1910-) Plant collector; Los Angeles Museum of Art, History and Science Bioscript Correspondence • 1934: (1) • 1935: (2) Species • 1959: (5)

Templeton, Kenneth S. Lilly Endowment, Inc. Correspondence • 1967: (1) Banister, John, 1650-1692

Tempsky, F. Bioscript

Tennent, John, Sir ( -1748) Virginia physician, botanist Biofile • Bioscript

Tenney, Sanborn (1827-1877) Bioscript

Tenore, Michele (1780-1861) Italian botanist Bioscript

Tenore, Vincenzo (1825-1886) Italian plant collector; nephew of Michele Tenore Bioscript

Teodorescu, Emmanuel Constantin (1866-1949) Romanian plant collector Bioscript

Tepfer, Gary Botanical Album Correspondence • 1982: (2)

Tepfer, Sanford Samuel (1918-) American plant morphologist; U. of Oregon, Eugene; Valgandemer, France; nickname "Sandy"

Correspondence • 1977: (3) • 1979: (2) • 1987: (1)

Tepler, Bert Correspondence • 1990: (1)

Terborgh, John W. Duke University, Center for Tropical Conservation Correspondence $\bullet 1990:(1)$

Terra, Hellmut de See DeTerra, Hellmut (1900-)

Terracciano, Nicola (1837-1921) Italian biologist, taxonomist Bioscript

Terrell, Clyde B. Pasadena, TX Correspondence • 1953: (3) • 1953: (0) Letter filed under John Lynch

Terrell, Edward E. Botanist; U. S. Dept. of Agriculture, Beltsville, MD Correspondence • 1957: (5) • 1960: (1) • 1971: (1)

Tessier, Bioscript
Teurckaninow, $\mathbf{N}$.

Bioscript

Teuscher, Henry (1891-1984) German-born horticulturist, orchidologist, plant collector; U. S. A., Canada Correspondence $\bullet$ 1968: (5)

Texas A \& M Correspondence $\bullet$ 1986: (1)

Texas State Historical Association Correspondence • 1982: (2) • 1991: (2) • 1992: (2)

Teysmann, Johannes Elias (1809-1882) Dutch-born plant explorer; Java

Bioscript

Thacher, G. W., Mrs. Bioscript

Thackray, John C. Society for the Bibliography of Natural History, Honorary Secretary; Geological Museum, British Museum (Natural History), London, England Botanical Album Correspondence - 1976: (0) Filed under Society for the Bibliography of Natural History $\bullet$ 1980: (3) $\bullet$ 1987: (2)

Thaly, Daniel Correspondence • 1951: (1) Pursh, Frederick, 1774-1820

Tharp, Benjamin Carroll (1885-1964) American taxonomist, plant ecologist; U. of Texas, Austin Bioscript $\bullet$ Botanical Album

Correspondence • 1939: (2) • 1940: (1) 1941: (3) 1944: (3) • 1949: (2) • 1950: (1)

Thatcher, Edward P. U. of Oregon, Museum of Natural History, Eugene, Map Librarian

Correspondence $\bullet$ 1970: (3) Book collectors $\bullet$ 1974: (3) 1975: (3) • 1976: (5) • 1977: (7) Van Schaack, George Booth, 1903-1983 • 1978: (6) • 1979: (1) • 1983: (1)

Thaxter, Roland (1858-1932) American mycologist Biofile $\bullet$ Bioscript

Thayer, Emma Homan (1842-1908) Bioscript

Thedenius, C. G. H. Botanical Album

Theerman, Paul Smithsonian Institution, Joseph Henry Papers, Asst. Editor Correspondence • 1991: (4)

Theobald, William Louis (1936- ) American taxonomist; Pacific Tropical Botanical Garden, HI Correspondence • 1974: (1) • 1975: (1) • 1976: (2) • 1977: (7) • 1979: (4) • 1980: (1) • 1981: (1) • 1982: (1)

Thien, Leonard (1938-) Tulane U., Dept. of Botany Bioscript $\bullet$ Botanical Album

Correspondence • 1973: (1) • 1975: (1) • 1976: (15) • 1977: (2) • 1980: (1) • 1982: (2) • 1983: (7) • 1984: (1) - 1985: (3) • 1987: (2) • 1988: (4) • 1989: (2) • 1990: (2) - 1991: (1) - 1992: (1) •

Thien, Loraine Husband is Leonard Thien Correspondence • 1976: (0) Letters filed under Leonard Thien 1977: (2) Tulane U., Dept. of Botany

Thieret, John Williams (1926-) American taxonomist, economic botanist; book editor for Society for Economic Botany; U. of Southwestern Louisiana, Lafayette; North Kentucky State College, Highland Heights Bioscript Correspondence $\bullet$ 1963: (11) $\bullet$ 1964: (2) $\bullet$ 1965: (0) Letter filed under J. J. Wurdack • 1965: (13) • 1966: (8) • 1967: 
Thieret, John Willams (1926-) (continued)

(15) • 1968: (11) • 1970: (2) • 1971: (8) • 1972: (1) •

1973: (6) - 1974: (3) • 1976: (2) Correspondence with R.

(Dick) E. Schultes • 1977: (3) • 1978: (4) • 1980: (1)

Thiers, Barbara M. New York Botanical Garden.

Bronx, NY, Associate Editor

Correspondence • 1991: (1)

Thimann, Keaneth Vivian (1904) English-born American botanist, plant physiologist; U. of California, Santa Cruz, Professor of Biology

Botanical Albwm

Correspondence • 1968: (3)

Thin, Hilary James Thin / Booksellers, Edinburgh, Scodand Correspondence $\bullet 1991$ : (3)

Thistel, Jerrie H. Qwarterly Review of Biology, Bditorial Asst.

Correspondence $\bullet 1964:$ (1)

Thoma, Roger F. Ohio State U., Entomology Dept. Correspondence • 1983: (1)

Thomas, Alan G. Alan G. Thomas, Bookseller, Bournemouth. England

Correspondence $\bullet$ 1961: (2)

Thomas, Cyrus (1825-1910) Plart collectior, entomologist; U. S. A.

Bioscript

Thomas, George Henry (1816-1870)

Bioscript

Thomas, Isalah Bloscript

Thomas, John Hunter (1928-) German-bom American taxonomist; Stanford U., Dudiey Herbarium. Assistant Curator

Botanical Album

Correspondence • 1959: (6) • 1962: (2) • 1963: (2) •

1964: (2) • 1966: (5) • 1969: (2) • 1977: (1) • 1979: (1) • 1991: (3) • 1992: (2)

Thomas, Maurice E. Univeridad del Valle, Cali, Division de Ciencias

Correspondence • 1968: (1) • 1969: (1)

Thomas, Roy Dale (1936-) American taxonomist, plant ecologist: Northeast Louisiana U., Dept. of Biologe Biofle • Bioscript

Correspondence • 1966: (2) • 1968: (2) • 1972: (4) •

1974: (1) Bartram, William, 1739-1823 • 1975: (2)

Thomas, W. Stephan American Ptillosophical Society Correspondence • 1942: (1)

Thompson, A., Mrs. Bioscript

Thompson, Bruce Allen Tulane U., gradoate stadert Correspondence • 1969: (2)

Thorapson, D'Arcy Wentworth, Sir (IS66-1968) Botanical Album

Tompson, Eiten Nellie) Powell (A. 1872) Far collector; Unined States

Bioscript

Thompson, Harol Turner Heflin High School, Heflin, LA; basband is Delmar $R$. Thompeon Correspondence $\bullet 1973 ;(2)$

Thompson, Jacqueline Alejers, New Orleans, LA Correspondence $\bullet$ 1961: (1)
Thompson, James P., Jr. Correspondence $\bullet 1956$ : (1)

Thompson, James W. Correspondence $\bullet 1966$ : (1)

Thompson, Jim Correspondence • 1965: (1)

Thompson, John Herndon Tulane U., New Orleans, LA

Correspondence $\bullet$ 1954: (2)

Thompson, John McLean (1877-1977) English plant collector

Botanical Album

Correspondence • 1954: (2)

Thompson, John Vaughan (1779-1841) British surgeon, zoologist

Biofile

Thompson, John William (1890-) American plant collector, botanist; Seattle, WA

Botanical Album

Correspondence $\bullet$ 1929-1933: Book collectors $\bullet$ 1934: (1) - 1937: (4) • 1938: (3) Delphinium • 1939: (1) • 1940: (6) - 1941: (2)

Thompson, Michael R. Zeitlin \& VerBrugge, Booksellers, Los Angeles, CA

Correspondence • 1969: (0) filed under Zeitlin \& VerBrugge $\bullet 1971:$ (2) $\bullet$ 1972: (2)

Thompson, Nancy Missouri Botanical Garden, St. Louis, MO, Garden Guides

Correspondence $\bullet 1988:$ (1)

Thompson, Paul U. of Colorado Correspondence $\bullet$ 1947: (2)

Thompsen, Sandra S. State of Louisiana, Trails Advisory Council

Correspondence • 1976: (0) Filed under Louisiana Trails Advisory Council • 1976: (4) • 1977: (2) • 1978: (0) Campaign newsletter

Thompson, Stephen Everly, Mrs. American Rhododendron Society member Correspondence $\bullet 1979$ : (2)

Thompson, Sue Carnegie Museum of Natural History, Section of Botany, Pitsburgh, PA Correspondence • 1988: (2)

Thompson, W. Bioscript

Thempson, W. M., Mrs. South Shore Garden Club, Chicago, II Correspondence $\bullet 1950:(2)$

Thompson, Zadock Bioscript

Thomson, Andrew D. (1930-) New Zealand plant virologist; Dept. of Scientific and Industrial Research Botany Division, Christchurch, New Zealand Correppondence • 1980: (2) • 1981: (2) • 1984: (1)

Domson, Charles (1729-1824) Bioscript

Thomson, John W. Museum of Natural History, NY, School Nanure League Correppondence $\bullet 1941:(6)$

Tnemsen, S. Harrisen U. of Colorado, History Dept. Cornespondence * 1942: (2) 


\section{Guide to the Ewan Papers}

Thomson, T.

Bioscript

Thone, Frank Ernest Aloyisius (1891-) Plant collector Correspondence • 1946: (1)

Thorburn, Grant (1773-1863) Scottish-born American seedsman, gardener

Bioscript

Thoreau, Henry David (1817-1862) Bioscript

Thornber, John James (1872-1962) American taxonomist, plant ecologist, plant collector; U. of Arizona

Botanical Album

Correspondence • 1929-1933: (7) • 1937: (2) • 1939: (1) • 1940: (1)

Thorne, Robert Folger (1920-) American taxonomist; State U. of Iowa, Iowa City; Rancho Santa Ana Botanic Garden, Claremont, CA

Biofile - Botanical Album

Correspondence • 1954: (3) • 1956: (2) • 1962: (1) • 1967: (2) • 1970: (4) • 1971: (1) • 1972: (4)

Thornton, J. B. U. of New South Wales, Kensington, School of History

Correspondence 1974-75: (2)

Thornton, Robert John (1768-1837) English botanist, physician

Bioscript

Thornton, William Bioscript

Thorup, Oscar A., Jr. Correspondence $\bullet$ 1956: (1)

Thouars, Abel Aubert du Petit (1793-1864) Bioscript

Thouin, André (1747-1824) French horticulturist, agronomist Bioscript

Thrall, Will H. Trails Magazine, Editor Correspondence • 1938: (5) • 1939: (2)

Threadgil, Paul U. of Kentucky, Lexington, T. H. Morgan School of Biological Sciences, Graduate student Correspondence $\bullet$ 1977: (2)

Threlkeld, Caleb (1676-1728)

Bioscript

Threlkeld, G. B. Botanical Album

Threlkeld, Niki Flora Publishing, Santa Fe, NM Correspondence • 1978: (2)

Thrower, Norman J. W. U. of California, Los Angeles Botanical Album

Correspondence • 1979: (2) • 1983: (3) • 1984: (2) • 1991: (2)

Thunberg, Carl Peter (1743-1828) Swedish botanist, physician

Biofile • Bioscript

Thurber, E. Correspondence $\bullet$ 1954: (1)

Thurber, Eugene Carleton Bioscript

Thurber, George (1821-1890) American botanist, horticulturist Bioscript • Botanical Album
Thurber, Robert K. U. S. Commercial Company Correspondence • 1947: (1)

Thuret, G. Bioscript

Thurmann, J. Bioscript

Thurow, Friedrich Wilhelm (1852-) German-born plant collector, USA

Bioscript

Thwaites, George Henry Kendrick (1812-1882) Englishborn entomologist, botanist, plant collector; Ceylon Bioscript • Botanical Album

Thyne, Ena W. Correspondence $\bullet$ 1954: (1)

Tice, John $\mathbf{H}$. Bioscript

Tice, $\mathbf{L}$. Bioscript

Tidestrom, Ivar Frederick (1864-1956) Swedish-born American botanist, agriculturist, plant explorer Biofile • Bioscript Correspondence • 1949: (1)

Tiehm, Arnold Correspondence $\bullet$ 1990: (3)

Tight, William G. (1865-1910) Denison University, Professor of Botany and Geology Botanical Album

Tilden, Josephine Elizabeth (1869?-1957) Bioscript $\bullet$ Botanical Album Correspondence • 1938: (1) • 1949: (2)

Tilden, Richard (f. 18141825) Bioscript

Tiling, Heinrich Sylvester Theodore (1818-1871) Bioscript

Tillett, Stephen Szlatenyi (1930-) American-born taxonomist, botanist, plant collector; Herbarium Ovalles, Caracas, Venezuela Correspondence $\bullet$ 1985: (3)

Tillman, John Bioscript

Tim, Stephen K. M. (1937-) Brooklyn Botanic Garden Correspondence $\bullet$ 1973: (1)

Timber Press Portland, OR Correspondence $\bullet$ 1988: (1)

Time, Inc. Barbara Heywood Motilones Correspondence $\bullet$ 1952: (3)

Time Magazine Correspondence • 1948: (1)

Times, The Correspondence $\bullet$ 1961: (1)

Times-Picayune Publishing Corporation New Orleans, George Healy, Editor Correspondence • 1971: (3) Species

Tindall, Charles W. Correspondence $\bullet$ 1929-1933: (4)

Tineo, Vincenzo (1791-1856) Italian botanist Bioscript

Tinker, Edward Larocque Bioscript

Tinkle, Donald U. Michigan, Ann Arbor, Zoology Correspondence • 1979: (1) 
Tinkle, Lon (f. 1967) Southern Methodist U., Dallas, TX Botanical Album

Tinsdale, Mary

Bioscript

Tinsley, John D.

Bioscript

Tiranti, D. C. London book dealer

Correspondence • 1941: (1)

Tiranti, John

Correspondence - 1940: (1) Book collectors

Tisdale, William Burleigh (1890-) Plant collector; U. of Florida, Gainesville

Correspondence • 1947: (2)

Tising, Ralph Dixon and Co. Correspondence $\bullet$ 1940: (1)

Titford, Anthony R.

Correspondence • 1968: (1) • 1969: (8) Banks, Joseph, Sir, 1743-1800 • 1971: (3) - 1972: (3)

Titford, William Jowett (1784-1823/7) Bioscript

Titsingh, Isaac Bioscript

Titus, Edward Gaige (1873-) Bioscript

Tobe, John David Clemson U., Clemson, SC, Biology Dept.

Correspondence • 1993: (2)

Todaro, Agostino (1818-1892) Italian botanist, taxonomist Bioscript

Todd, Anne Ophelia (1907-) Botanical artist; American Museum of Natural History

Correspondence • 1962: (1) Correspondence with Donald A. Johansen

Todd, Clement Charies (-1828) Naturalist, plant collector; Canada

Biofile

Todd, J. E. Bioscript

Todd, St. E. Clyde Carnegie Museum, Curator Emerims of Birds

Correspondence - 1962: (0) Letter filed under Averil

Margaret Lysaght

Todzia, Carol A. U. of Texas at Austin, Assoc. Curator Correspondence • 1989: (3) • 1992: (2)

Tokyo National Science Museum, Library Botanical Album

Toledano, Roulhac Correspondence • 1968: (2) • 1969: (1) Bartram, Walliam, 1739-1823 - 1970: (3)

Tolmie, William Fraser (1812-1886) Scottish-born botanical collector: Canada

Bioscript

Tolstead, William Lawrence Botanist, plant collector, U. of Nebraska

Correspondence • 1942: (5) • 1943: (6) Delphiniam * 1948: (1)

Tomaselli, Eugenie

Correspondence • 1982: (2)
Tomaselli, Ruggero (1920-1982) Italian plant ecologist, phytogeographer; Istituto de Orto Botanico, U. di Pavia, Pavia, Italy

Bioscript - Botanical Album

Correspondence • 1953: (9) • 1954: (6) • 1955: (5) •

1956: (1) • 1969: (1) • 1970: (1) - 1974: (1) • 1975: (4)

Tomassy, Raymond (1810-1863) French hydraulic engineer, geologist in Louisiana Biofile

Tomb, Andrew Spencer (1943-) U. of Illinois, Chicago Circle, Chicago, II, Dept. Biological Sciences

Correspondence $\bullet$ 1970: (1)

Tomlinson, O. A.

Correspondence • 1937: (1)

Tommasini, Giuseppe Murio Spirito (1794-1879) Italian botanist, taxonomist

Bioscript

Tonduz, Ad

Botanical Album

Toner, Joseph M. (1825-1896) American physician, medical historian

Biofile - Bioscript

Tongue, James Bioscript

Topping, David Leroy (1861-1939) American plant collector, pteridologist, taxonomist Botanical Album

Torok, Denes de Harvard U., Cambridge, MA, The Biological Laboratories Correspondence • 1961: (1)

Toronto, Joseph B. Bioscript

Torrens, Hugh S. U. of Keele, Staffordshire, Engiand, Dept. of Geology; British Society for the History of Science Correspondence • 1984: (1) • 1987: (1) • 1988: (2) • 1990: (2)

Torres, Andrew Marion (1931-) American cytogeneticist, taxonomist; U. of Kansas, Lawrence, Dept. of Botany Correspondence • 1968: (2)

Torrey Botanical Chub Staten Island Instinte of Arts and Sciences Botanical Album Correspondence • 1929-1933: (1) • 1974: (2) • 1981: (3) • 1990: (1)

Torrey, Bradford Bioscript

Torrey, Harry Beal (1873-) Bioscript

Torrey, John (1796-1873) American botanist, chemist, physician See Torrey Botanical Club Biofile • Bioscript • Botanical Album

Torrey, John Gordon (1921-) American plant physiologist Botenical Album

Torrey, Raymond Hezekiah (1880-1938) American botanist, plant morphologist Boturical Album

Touchstone, Blake Tulane U., New Orleans Correspondence • 1968: (1) • 1971: (1) • 1984: (1) 


\section{Guide to the Ewan Papers}

Toumey, James William (1865-1932) American plant collector, forester, dendrologist Bioscript $\bullet$ Botanical Album

Tournefort, Joseph Pitton de (1656-1708) French botanist Biofile - Bioscript

Touro, Judah (1775-1854) Physician Biofile

Tower Grove Park St. Louis, MO Botanical Album

Tower Grove Park, Friends of Correspondence $\bullet$ 1993: (2)

Towers, G. H. N. U. of British Columbia, Dept. of Botany Correspondence $\bullet$ 1975: (2)

Towers, Neil Correspondence $\bullet$ 1975: (1)

Towle, Margaret Harvard U., Botanical Museum Correspondence - 1968: (3)

Towles, Thomas Bioscript

Towne, Rosa M. (1827-1909) Plant collector, South Carolina Bioscript

Towner, H. C. Bioscript

Townley, Wayne C. McLean County Court House, Hall of Records, Bloomington, IL Correspondence • 1949: (3)

Townsend, Alexander Cockburn (1905-1964) British Museum (Natural History), London, Librarian Correspondence • 1951: (1) • 1957: (7) • 1958: (3) • 1959: (5) • 1960: (2) • 1961: (1) $\bullet$ 1962: (5) $\bullet$ 1963: (5) $\bullet$ 1964: (1)

Townsend, Charles Haskins (1859-1944) Bioscript

Townsend, Charles Wendell (1859-1934) American physician, ornithologist Biofile

Townsend, David Bioscript

Townsend, David (1787-1858) American bocanist Biofile

Townsend, Hal E., Jr. Northwestern State College of Louisiana, Dept. of Agriculture Correspondence $\bullet$ 1970: (1)

Townsend, J. L. Bioscript

Townsend, John Kirk (1809-1851) American naturalist Bioscript

Townsend, Norton Strange (1815-1895) Bioscript

Townsend, Washington (1813-1894) Bioscript

Tracy, Hiram Harwood (1883-) Plant collector; American Fern Society

Bioscript Correspondence • 1936: (2) - 1937: (1)

Tracy, Joseph Prince (1879-1953) Botanical Album

Tracy, Samuel Mills (1847-1920) American botanist, horticulturist Bioscript
Tradescant, John

Bioscript

Tradescant, John (the Younger) (1608-1662) British naturalist, traveller, collector, gardener; Virginia Biofile $\bullet$ Botanical Album

Tradescant Trust, London Correspondence $\bullet$ 1980: (1) • 1981: (2) • 1985: (5)

Traill, Catherine Parr (1802-1899) (nee Strickland) British naturalist, plant collector, writer; husband is Thomas Traill Biofile $\bullet$ Botanical Album

Traill, Thomas Stewart Bioscript

Train, Agnes Scott (1905-) American plant collector, ethnobotanist; husband is John Train Botanical Album

Train, Percy (1876-1942) American paleobotanist, plant collector, ethnobotanist Bioscript

Train, Russell E. World Wildlife Fund Correspondence $\bullet$ 1984: (1)

Transeau, Edgar Nelson (1875-1960) American plant ecologist, phycologist, plant physiologist Botanical Album

Transylvania University Correspondence $\bullet$ 1967: (3) • 1983: (1)

Traphagen, Frank Weiss (1861-1941) Plant collector Bioscript • Botanical Album

Trask, Blanche (1865-1916) Plant collector Bioscript

Trask, John Boardman (1824-1879) Bioscript

Trattinnick, Leopold (1764-1849) Austrian naturalist Bioscript

Traub, Hamilton Paul (1890-1983) American botanist, plant breeder; California

Biofile

Correspondence • 1970: (3) • 1975: (2)

Traylor, Preston New Orleans, LA Correspondence • 1961: (1)

Treat, Mary (1830-1923) American botanist, naturalist Biofile - Bioscript

Trécul, Auguste Adolphe Lucien (1818-1896) Plant collector Bioscript

Trelease, Frank J. Correspondence • 1989: (2)

Trelease, Richard W. Arizona State U., Dept. of Botany Correspondence $\bullet$ 1972: (2)

Trelease, Sam Farlow (1892- ) Bioscript

Trelease, Sidney B.

Correspondence • 1991: (0) Letter filed under Emanuel David Rudolph

Trelease, William (1857-1945) American botanist, plant morphologist, taxonomist; Missouri Botanical Garden; U. of Illinois, Urbana

Biofile $\bullet$ Bioscript $\bullet$ Botanical Album

Trenary, Carlos Correspondence • 1985: (1)

Trent, J. A. State Teachers College, Pittsburg, KS Correspondence $\bullet$ 1938: (2) 
Treubig, Ronald J. Louisiana Forestry Commission, Woodworth, LA

Correspondence • 1976: (0) Letter filed under Louisiana Forestry Commission • 1976: (1)

Treviranus, Ludolf Christian (1779-1864) German botanist

Biofile • Bioscript

Trew, Christopher (1695-1769) German botanist, physician Bioscript • Botanical Album

Triana, José Jerónimo (1834-1890) Colombian plant collector, taxonomist

Biofile - Bioscript

Tribe, Christoph Jacob

Correspondence • 1992: (1)

Trice, Mildred $\mathbf{M}$. Correspondence • 1981: (1)

Trimble, H. E. Correspondence • 1934: (1)

Trimen, $\mathbf{H}$. Bioscript

Trinity College Hartford, CT Correspondence • 1969: (1) • 1982: (1)

Trinity College Library Correspondence • 1966: (1)

Trinius, Carl Bernhard von (1778-1844) German physician. botanist

Bioscript

Tripp, Henry Boolsseller, scientific books and periodicals Correspondence • 1964: (2) • 1965: (2)

Trippe, $\mathbf{T}$.

Bioscript

Troll, Carl (1899-1975) German phytogeographer, botanist; brother of W. J. G. H. Troll; Biofile

Troll, Ralph Augustana College, Rock Island, II, Depe. of Biology, Professor Correspondence • 1978: (1)

Trollope, Anthony Botanical Album

Trollope, Fanny Bioscript

Troost, Gerard (1776-1850) Bloscript

Trowbridge, William Pett (1828-1892) Bioscript

Troy, Sylvia Correspondence • 1976: (0) Filed under Srve the Dunes Council

Trucksess, Mrs

Correspondence $\bullet$ 1943: (1)

Trudeau, James De Berty (1817-1887) American physician, ornithologist, artist

Biofile • Bioscript

Trudeau, Tancred $\mathbf{R}$. Correspondence • 1982: (2) • 1983: (3)

Trudell, Harry William (18s4) Plant collector; Abington. PA

Correspondence - 1952: (1)

True, A. C.

Bioscript
True, Rodney Howard (1866-1940) American plant physiologist; Morris Arboretum

Correspondence • 1939: (1)

Trueblood, Emily Walcott Emmart (1898-) American cytologist, botanical historian; Harvard U., Botanical Museum; husband is Charles Kingsiey Correspondence • 1970: (4) • 1973: (3) • 1979: (1)

Truitt, E. D.

Correspondence $\bullet$ 1947: (1)

Trumbell, Ellen J. U. S. National Museum Correspondence $\bullet$ 1958: (2)

Trumbull, J. H.

Bioscript

Tryon, Alice Faber (1920-) American taxonomist, cytologist, pteridologist; U. of California, Berkeley; Harvard U., Gray Herbarium; husband is Rolla Milton Tryon

Correspondence • 1957: (1) - 1958: (1) • 1962: (4) • 1964: (2) • 1966: (3) • 1967: (3) • 1968: (0) Filed under Rolla Milton Tryon • 1969: (0) Filed under Rolla Milton Tryon • 1970: (2) • 1972: (4) • 1973: (1) • 1975: (3) • 1976: (0)

Tryon, G. S., Jr. Bioscript

Tryon, Rolla Milton (1916-) American taxonomist, pteridologist; U. of Minnesota, Minneapolis; Missouri Botanical Garden, St. Louis, MO; Harvard University, Gray Herbarium

Bioscript • Botanical Album

Correspondence • 1940: (2) • 1942: (4) • 1945: (1) • 1946: (1) • 1947: (2) American Fern Society • 1948: (2) • 1949: (6) • 1950: (1) • 1951: (10) American Fern Society - 1952: (2) • 1956: (2) • 1959: (1) • 1963: (3) • 1966: (5) - 1967: (5) • 1968: (8) • 1969: (2) • 1974: (1) • 1976: (2) - 1977: (1) • 1979: (3) • 1983: (2) • 1985: (3) • 1990: (1)

Tsai, Loh Seng Botanical Album

Tschernikh, George Bioscript

Tubeuf, Simon Karl Freiherr von (1862-1941) German plant physiologist Biascript

Tucher, Andrea J. Natural history bibliographer; Library Company of Philadelphia Correspondence $\bullet$ 1978: (2)

Tuchman, Barbara W. Botenical Album

Tucker, Arthur O. (1945-) Plant collector; Delaware State College

Correspondence • 1981: (2) • 1983: (2) • 1984: (1)

Tucker, Gary Arikansas Tech U., Assoc. Prof. of Biology Correspondence $\bullet 1977$ : (1)

Tucker, H. M. Zoologist, plant collector; College of Idaho, Caldwell

Bioscript

Tucker, John Maurice (1916-) American taxonomist; U. of California, Davis, Div. of Botany, Assistant Botanist Comespondence - 1951: (2) Biology-Instruction and swady; Delphiniwn • 1964: (1) 1967 : (10) $\bullet 1968$ : (2) 


\section{Guide to the Ewan Papers}

Tucker, Shirley Cotter (1927-) American plant anatomist, plant ecologist; U. of Louisiana, Baton Rouge

Correspondence • 1968: (3) • 1972: (2) • 1973: (1) • 1975: (2) • 1977: (1) • 1985: (5) • 1987: (1) • 1992: (2) 1993: (1)

Tuckerman, Edward (1817-1886) American lichenologist, plant collector

Biofile • Bioscript

Tulane Alumni Association Correspondence • 1973: (1) • 1978: (1)

Tulane University New Orleans, LA Botanical Album

Correspondence • 1980: (8) • 1981: (4) • 1982: (1) • 1983: (3) • 1984: (6) • 1986: (6) • 1987: (1) • 1988: (2)

Tulane University Dept. of Pharmacology, William J. George, Professor

Correspondence • 1979: (1) Filed under Pharmacology

Tulane University Herbarium Correspondence • 1948: (1)

Tulane University, Howard-Tilton Memorial Library Correspondence • 1970: (1) • 1976: (1) • 1987: (1) • 1990: (2) • 1991: (2)

Tulasne, Louis René (Edmond) (1815-1885) French plant collector, mycologist

Bioscript

Tull, Jethro (1674-1740) English agriculturist Biofile

Tully, W. Bioscript

Tuomey, Michael (1808-1857) Irish-born geologist, agriculturist Bioscript

Tupper, F. G. New Orleans, LA Correspondence $\bullet$ 1951: (2) $\bullet$ 1952: (1)

Turk, Rudy H. Arizona State U., Tempe, University Art Museum, Director Correspondence $\bullet$ 1990: (2)

Turnbull Library Wellington, NZ Correspondence • 1983: (1)

Turner, Anne L. Librarian; North Carolina State College, Raleigh Correspondence $\bullet$ 1955: (2)

Turner, Billie Lee (1925- ) American taxonomist; U. of Texas, Austin, Herbarium, Dept. of Botany

Botanical Album

Correspondence • 1964: (3) • 1967: (3) • 1970: (3) • 1980: (1)

Turner, Dawson (1775-1858) English banker, botanist Biofile - Bioscript

Turner, E(dward?)

Bioscript

Turner, Frederick Jackson (1861-1932) American historical geographer Biofile

Turner, George Harrison (1877-1970) Canadian physician, botanist Biofile

Turner, James Pediatrician, St. Louis, MO Correspondence • 1992: (1) • 1993: (2)

Turner, Joanna Correspondence • 1989: (1) • 1990: (1)
Turner, Ruth D. Harvard U., Museum of Comparative Zoology Correspondence $\bullet$ 1972: (7)

Turner, S. William

Bioscript

Turner, W. V. Bioscript

Turner, William Bioscript

Turpin, Pierre Jean Francois (1775-1840) French botanist, botanical artist

Bioscript

Turrill, William Bertram (1890-1961) English taxonomist, phytogeographer; Royal Botanic Gardens, Kew Correspondence $\bullet$ 1940: (2) $\bullet$ 1955: (2) $\bullet$ 1959: (2)

Turtox News Chicago, IL Correspondence • 1959: (2)

Tweedie, John (1775-1862) Plant collector of Argentina Bioscript

Tweedy, Frank (1854-1937) Plant collector of Rocky Mountains Bioscript

Tweney, George $\mathbf{H}$. Correspondence • 1969: (5) • 1971: (1)

Twisselmann, Ernest Christian (1917-1972) Plant collector of California Correspondence $\bullet$ 1972: (2)

Twitty, Victor Chandler American zoologist Biofile

Twohig, Dorothy Papers of George Washington, Co-Editor Correspondence • 1989: (1)

Tyler Ecology Award U. of California, Berkeley Correspondence $\bullet 1974$ : (3)

Tyler, Harriet Griswold (Burr) (1873- ) Bioscript

Tyler, Morris New Haven, CT Correspondence • 1971: (5)

Tyrrell, Robert A. Botanical Album

\section{$\boldsymbol{U}$}

Ubach, Levi M. Botanical Album

Udvardy, Miklos D. F. (1919- ) U. of British Columbia, Vancouver, Dept. of Zoology Correspondence • 1964: (5) - 1965: (1)

Ugent, Donald (1933-) American taxonomist Botanical Album

Ugent, $\mathbf{V}$. Botanical Album

Ugolini, Ugolino (1856-1942) Italian naturalist, plant morphologist Biofile

Uhde, Adolph Bioscript

Uhlan, Edward Exposition Press, Inc. Correspondence • 1969: (1)

Uhvits, Rachel Correspondence • 1959: (1) • 1973: (1) 
Ule, Ernst Heinrich George (1854-1915) German botanist, plant collector, taxonomist in Brazil Bioscript

Ulke, Titus (1866-1961) American botanist Bioscript

Correspondence • 1949: (4)

Ulrich, Edward Oscar (1857-1944) American paleontologist Biofile

Umbach, Levi Menger (1853-1918) Plant collector Bioscript

Underwood, Lucien Marcus (1853-1907) American botanist

Biofile - Bioscript - Botanical Album

Unger, Donald Oregon State U., Corvallis Correspondence • 1970: (2)• 1974: (2) Rocky Mountain Naturalists

Unger, Franz Joseph Andreas Nicholaus (1800-1870)

German phytogeographer, physician; Austria

Biofile - Bioscript

Union Printing and Publishing Co.

Correspondence $\bullet$ 1945: (1)

United Fruit Company J. A. Marquette

Correspondence • 1952: (2)

United States Army Corps of Engineers Colonel Richard Hunt

Correspondence • 1972: (1) • 1974: (1)

United States Department of Agriculture Correspondence • 1941: (2) • 1950: (1) • 1975 (1) • 1980; (2)

United States Department of the Interior Rolland B. Handley

Correspondence • 1979: (1)

United States National Arboretum Botanical Album

Correspondence - 1964: (2) Re: dedication ceremony

United States Post Office Dept. of Design Correspondence • 1967:

United States Senate

Correspondence $\bullet$ 1979: (3)

Universidad Nacional Antbooma de Mexico Correspondence • 1983: (1)

University of Alabama (Filed under Alabuma) Botanical Album

University of Alabama Press Lester D. Stephens Correspondence • 1987: (0) Letter filed under Emest Charles Nelson

University of Arizona (Filed under Arizora) Botanical Album

University of Arkansas Library Andrea Cantrell, Fayetteville (Filed under Arkansas)

Correspondence • 1989: (1)

University of Bath Library (Fi) Comespondence * 1975: (1)

aiversity of California, Berkeley (Filed under California) Botanical Album

Correspondence • 1963 : (1) correspondent is Roger Hahn - 1976: (1)

University of California Botanical Gardea Berkeley Botanical Album
University of California, Los Angeles (Filed under California)

Botanical Album

Correspondence $\bullet$ 1977: (2)

University of California Press

Correspondence $\bullet 1956:$ (1)

University of Chicago (Filed under Chicago)

Botanical Album

University of Colorado Boulder, Library (Filed under Colorado)

Correspondence • 1976: (1) Rocky Mountain Naturalists

University of Delaware Newark, Center for Science and Culture, D. Heyward Brock \& Ann M. Harward, Editors (Filed under Delaware)

Correspondence • 1981: (2)

University of Delaware Library Newark, DE (Filed under Delaware

Correspondence $\bullet 1990$ : (2)

University of Denver Press

Correspondence • 1951: (0) Filed under Frans Antonie Verdoorn

University of Georgia Press Athens, GA (Filed under Georgia)

Correspondence $\bullet 1977$ : (1) • 1985: (2)

University of Glasgow Keeper of the Herbarium (Filed under Glasgow)

Correspondence $\bullet 1955$ : (1)

University of Hawaii Honolulu (Filed under Hawaii) Botanical Album

Correspondence $\bullet 1974$ : (1)

University of Ilinois Urbana (Filed under Illinois) Correspondence 1971 (1)

University of Illinois Press Urbana (Filed under Illinois) Botanical Album

Correspondence • 1965: (2) • 1970: (1) Banister, John, 1650-1692 • 1971 (1) • 1973: (1) • 1974: (2) • 1975: (2)

- 1976: (2) • 1979: (1) • 1980: (2) • 1981: (1) • 1983: (1) Banister, John, 1650-1692 • 1984: (1) Banister, John, 1650-1692 • 1985: (1) • 1986: (1) Banister, John, $1650-1692$

University of Kansas Libraries Lawrence (Filed under Kansas)

Correspondence • 1964: (1) • 1967: (1)

University of Kentucky Press Lexington; Kenneth W. Elliott (Filed under Kentucky)

Correspondence • 1961: (1)

University of Leiden Botanic Garden Botanical Album

University of Malawi Librarian (Filed under Malawi) Correspondence $\bullet 1968$ : (1)

University of Malaya (Filed under Malaya) Correspondence $\bullet$ 1980: (4) Letters to/from Dr.

Kuthubutheen Ahmed Jalaludin, Deputy Dean, Facuity of Science

University of Michigan Ann Arbor, Herbarium; Robert L. Shaffer, Director (Filed under Michigan)

Botanical Album

Correspondence $\bullet 1962:$ (1) $\bullet 1977$ : (1) $\bullet 1978:(1)$

University of Missouri (Filed under Missouri)

Correspondence $\bullet 1985$ (3) 


\section{Guide to the Ewan Papers}

University of Missouri, St. Louis

Botanical Album

University of Nebraska Lincoln, Dept. of Entomology (Filed under Nebraska)

Correspondence • 1959: (1)

University of Nebraska Press Stephen F. Cox, Executive Editor (Filed under Nebraska) Correspondence • 1979: (1) Rocky Mountain Naturalists • 1980: (1) Rocky Mountain Naturalists

University of Nevada Reno, College of Arts and Science; correspondent is Catherine S. Fowler, Professor (Filed under Nevada)

Correspondence $\bullet$ 1990: (1)

University of Newcastle (Filed under Newcastle) Correspondence $\bullet$ 1974: (1)

University of New Mexico Librarian (Filed under New Mexico)

Correspondence • 1949: (1)

University of New Orleans (Filed under New Orleans) Correspondence 1984 : (1).

University of North Carolina Press Chapel Hill (Filed under North Carolina)

Correspondence • 1961: (8) Banister, John, 1650-1692 • 1975: (1) - 1976: (2)

University of Oklahoma James R. Estes (Filed under Oklahoma)

Correspondence $\bullet$ 1980: (1)

University of Oklahoma Library Norman; Sul H. Lee, Director (Filed under Oklahoma)

Correspondence • 1978: (1)

University of Oklahoma Press John N. Drayton, Editor-inChief (Filed under Oklahoma)

Botanical Album

Correspondence • 1977: (3) Rocky Mountain Naturalists

University of Oregon Eugene (Filed under Oregon) Correspondence • 1969: (1)

University of Pennsylvania Botanical Album

University of South Carolina (Filed under South Carolina) Correspondence $\bullet$ 1950: (1)

University of South Florida Richard P. Wunderlin (Filed under South Florida)

Correspondence $\bullet$ 1976: (1)

University of (the) South Pacific Suva (Filed under South Pacific

Correspondence • 1974: (4)

University of Southern Mississippi Hattiesburg; Dept. of Biology (Filed under Mississippi)

Correspondence • 1977: (5)

University of Southwestern Louisiana Lafayette, Library (Filed under Southwestern Louisiana)

Correspondence • 1975: (1)

University of Strathclyde Glasgow, Scotland (Filed under Strathclyde)

Correspondence • 1977: (1) Book collectors

University of Texas Austin (Filed under Texas) Botanical Album

Correspondence • 1968: (1) • 1987: (0) Filed under Ernest Charles Nelson

University of Toronto Library (Filed under Toronto) Correspondence • 1989: (1)
University of Virginia Charlottesville, Rare Books, Alderman Library (Filed under Virginia)

Correspondence $\bullet$ 1985: (1) $\bullet$ 1987: (3)

University of Wisconsin Press (Filed under Wisconsin) Botanical Album

Correspondence • 1962: (6) Banister, John, 1650-1692 • 1964: (2) • 1965: (5) Banister, John, 1650-1692

University of Wyoming (Filed under Wyoming) Correspondence • 1965: (2) • 1986: (2) • 1992: (1)

Unzicker, , Mr.

Correspondence • 1945: (1)

Uphof, Johannes Cornelius Theodorus (1886-) Dutch-born plant breeder, economic botanist; U. of Tampa, FL Correspondence • 1929-1933: (1) • 1952: (8)

Upingham, John Bioscript

Uppsala, Botanical Album

Urban, Ignatz (1848-1931) German botanist, horticulturist Bioscript $\bullet$ Botanical Album

Urbatsch, Lowell Edward (1942- ) American taxonomist; Louisiana State U., Baton Rouge, Dept. of Botany Correspondence $\bullet$ 1977: (2) • 1979: (3) • 1980: (2) • 1982: (1)

Urness, Carol U. of Minnesota, Minneapolis, James Ford Bell Library, Assistant Curator

Correspondence • 1971: (3) - 1979: (2) Barton, Benjamin Smith, 1766-1815 • 1980: (2) • 1981: (1) • 1985: (1) • 1989: (2) • 1993: (1)

Urville, Dumont d' (1790-1842) Biofile

Usher, Ethel W. Tulane U., New Orleans, Howard-Tilton Memorial Library Correspondence $\bullet$ 1950: (3) • 1951: (2) • 1954: (3)

Ussery, $\mathbf{H}$. Correspondence • 1975: (1)

USSR Academy of Sciences Correspondence $\bullet$ 1976: (1)

Usteri, Paul J. (1768-1831) Swiss botanical historian, statesman, physician Bioscript

Utrecht U. Botanical Garden Botanical Album

Uttal, Leonard J. Virginia Polytechnic Institute, Blacksburg Correspondence • 1982: (4) Letter to Steven Darwin

\section{$\boldsymbol{V}$}

Vahl, Martin Hendrickson (1869-1946) Danish botanist Bioscript

Vail, Anna Murray (1863-1955) Botanist, plant collector Bioscript

Vaillant, Bioscript

Vaillant, Sébastien (1669-1772) French plant collector, Rocky Mountains Bioscript

Vale, V. T. Colesworthy's Book Store Correspondence $\bullet$ 1954: (1)

Valenciennes, Achille Bioscript 
Valentin, Louis

Bioscript

Vallance, Hilary

Correspondence $\bullet$ 1993: (2)

Vallance, Thomas George U. of Sydney, Australia, Dept. of Geology and Geophysics

Correspondence $\bullet$ 1974: (6) • 1976: (2) • 1977: (3) • 1978: (1) • 1979: (4) • 1980: (2) • 1982: (3) • 1983: (3) • 1984: (3) • 1985: (1) • 1986: (7) • 1988: (3) • 1989: (10) - 1990: (2) • 1991: (3)

Van Bruggen, Theodore ( 1926- ) American plant taxonomist, phytogeographer

Botanical Album

Van Brunt, Charles Gershom (1870- ) Bioscript

Van Denburgh, John (1872-1924) Herpetologist, ornithologist; California Academy of Sciences, Dept. of Herpetology Bioscript

Van der Bosch, R. B. Botanical Album

Van der Pas, P. W. Tulane U., New Orleans Correspondence • 1953: (2) • 1962: (1) • 1963: (1)

Van der Schott, Joseph Bioscript

Van Devanter, Willis Correspondence $\bullet$ 1963: (2)

Van Dugee, Edward Payson (1861-1940) Bioscript

Van Dyke, Edwin Cooper (1869-) Entomologist Biofile - Botanical Album

Van Ek, Jacob U. of Colorado, Boulder, Dean Correspondence $\bullet$ 1940: (1) $\bullet$ 1942: (1)

Van Horn, John C. Maryland Historical Society, Latrobe Papers

Correspondence • 1977: (0) Filed under Edward C. Carter II

Van Ravenswaay, Charles (1917-1990) Missouri Historical Society, St. Louis, MO

Bioscript $\bullet$ Botanical Album

Correspondence • 1946: (1) • 1950: (2) • 1955: (2) • 1977: (1) • 1982: (2) • 1984: (4) • 1986: (3) • 1987 (3) • 1988: (6) • 1989: (2)

Van Rensselaer, Maunsell (1897- ) Santa Barbara Botanic Garden

Botanical Album

Correspondence - 1941: (2) Species Van Riper, Walker 1942: (1)

Van Schaack, George Booth (1903-1983) American mathematician, librarian, botanist; Missouri Botanical Garden, St. Louis; Morton Arboretum, Lisle, IL Biofile • Bioscript $\bullet$ Botanical Album

Correspondence • 1946: • 1947: (16) "Rocky Mountain Vegetation" • 1948: (8) • 1949: (28) • 1950: (3) • 1951: (12) 1952: (16) Academy of Namural Sciences of Philadelphia; Pennell, Francis Whittier, 1886-1952 - 1953: (9) "Rocky Mountain Vegetation" - 1954: (9) • 1955: (12) Bartram, William, 1739-1823 • 1956: (4) • 1957: (12) • 1958: (14) - 1959: (29) Book collectors 1 1960: (20) Letter from Hubert W. Deam to Ewan is filed here $\bullet 1961$ : (29) $\bullet$ 1962: (31) Banister, John, 1650-1692; Banks, Joseph, Sir,
Van Schaack, George Booth (1903-1983) (continued) 1743-1800; Langman, Ida Kaplan; letter to Ronald Press and John S. Gilmour - 1963: (15) Letters to W. C. Steere and to Harry Lubrecht • 1964: (42) $\bullet$ 1965: (45) Lawrence, George Hill Mathewson, 1910; 5 letters to/from Cambridge University Press; letter to Edwin T. Coman • 1966: (15) • 1967: (32) Letters to Gordon Carruth - 1968: (28) Banister, John, 1650-1692; Bartram, William, 1739-1823; Letter of Ohio State University Press; Letter with A. C. Drysdale • 1969: (34) Banister, John, 1650-1692 • 1970: (25) • 1971: (16) - 1972: (14) - 1973: (20) Leters to George H. M. Lawrence and Hayward Cirker $\bullet 1974:$ (21) $\bullet 1975:(9)$ Book collectors • 1976: (10) • 1977: (5) • 1978: (8) Book collectors • 1979: (6) • 1980: (6) • 1981: (2) • 1982: (2)

Van Trump, James D. The Rachel McMasters Miller Hunt Botanical Library Correspondence • 1962: (1)

Van Vleck, Jacob (1751-1831) Plant collector, USA Bioscript

Van Waters, S. P. U. S. Dept. of Agriculture, Bureau of Plant Industry Correspondence • 1939: (1) • 1942: (1)

Van Winkle, Jane U. of North Carolina Press Correspondence • 1949: (1)

Vance, Gladys B. Academy of Natural Sciences, Philadelphia, PA

Correspondence $\bullet$ 1952: (2)

Vancouver, George (1758-1798) Bioscript

Vander Meer, Robert K. U. of the South Pacific, Suva Correspondence $\bullet 1974:$ (1)

Vanzolini, P. E. Museu de Zoologia de Universidade de São Paulo, Brazil

Bioscript

Correspondence $\bullet$ 1985: (1)

Varea Quevedo a Traves, Marco T. Bioscript

Vargas, José Marie (1786-1854) Venezuelan-born botanist Bioscript

Varian, Lester, Mrs. Denver, CO Correspondence • 1978: (1) Rocky Mountain Naturalists

Vasey, George (1822-1893) English-born American botanist, agrostologist Biofile $\bullet$ Bioscript $\bullet$ Botanical Album

Vasey, George Richard (fl. 1881) Son of George Vasey Bioscript

Vaucher, Jean Pierre E. (1763-1841) Bioscript

Vaughan, Alice Esther Plant collector; U. of Colorado, Boulder, Dept. of Biology

Bioscript Correspondence • 1939: (1) • 1940: (2)

Vaughan, Benjamin Brother of John Vaughan (1855-1922) Bioscript

Vaughan, Elizabeth W. Correspondence $\bullet$ 1954: (2)

Vaughan, J. E. Central State U., Edmond, OK, Dept. of Biology Correspondence • 1979: (1) 


\section{Guide to the Ewan Papers}

Vaughan, John (1855-1922) Plant collector Biofile

Vaughan, Josephine C. San Bernadino, CA Correspondence • 1969: (2) • 1970: (2) Jones, Marcus Eugene, 1852-1934

Vaughan, Samuel Bioscript

Vaughan, Samuel (n. 1770) Biofile

Vaurie, Charles American Museum of Natural History, Bird Dept., New York, NY; Société Ornithologique de France et de L'union Française Correspondence • 1961: (4) • 1962: (2)

Vavilov, Nikolai Ivanovich (1887-1943) Russian botanist, agronomist, geneticist Biofile $\bullet$ Bioscript $\bullet$ Botanical Album

Veale, Francis (n. 1730) Ethnologist of North Carolina Biofile

Veatch, John Allen (1808-1870) American naturalist Biofile • Bioscript

Veazie, Walter B. U. of Colorado, Dept. of Philosophy Correspondence • 1942: (1)

Veitch, H. L. Bioscript

Velez, Pilar de (1911-) Ecuadorian taxonomist Correspondence $\bullet$ 1964: (1)

Velie, Jacob W. (1829-1908) Bioscript

Velkinburgh, C. J. Van Village de Lest Garden Club Correspondence $\bullet$ 1968: (3)

Velloso, Frei José Marianno da Conceiç Botanist; Brazil (c. 1800) Biofile

Venegas, Miguel (1680-1764) Bioscript

Venning, Frank Denmire (1920-) American plant anatomist and morphologist; U. of Miami, Swingle Research Laboratory Correspondence $\bullet$ 1953: (1)

Ventenat, Étienne Pierre (1751-1808) French clergyman, librarian, botanist Biofile - Bioscript

Venton, K. W. Canal Zone Junior College, Balboa Heights Correspondence - 1952: (1)

Ventura, Arnoldo K. Institute of Jamaica, Natural History Division

Correspondence $\bullet$ 1980: (0) Letter filed under Peter Konrad Bretting

Verano, Luis F. U. of Oregon, Eugene, Dept. of Romance Languages

Correspondence $\bullet$ 1978: (2)

Verdoorn, Frans Antonie (1906-1984) Dutch botanist, botanical editor, biohistorian; Institute of the U. of Utrecht, Netherlands, Chronica Botanica

Biofile - Bioscript - Botanical Album

Correspondence • 1941: (5) - 1942: (4) • 1943: (10) • 1944: (2) • 1946: (11) "Rocky Mountain Vegetation"; Jepson, Willis Linn, 1867-1946 • 1947: (3) • 1948: (11) "Rocky Mountain Vegetation" • 1948: (0) Letter filed under Frantisek Horavka $\bullet$ 1949: (30) $\bullet$ 1950: (13) Pennell, Francis Whittier, 1886-1952; Pursh, Frederick, 1774-1820
Verdoorn, Frans Antonie (1906-1984) (continued) - 1951: (18) Chronica Botanica; Rocky Mountain Naturalists • 1952: (22) "Rocky Mountain Vegetation"; Pennell, Francis Whittier, 1886-1952; Academy of Natural Sciences of Philadelphia 1 1953: (15) "Rocky Mountain Vegetation" • 1954: (16) Biology-Instruction and study; "Rocky Mountain Vegetation"; letter from Frank Morton Jones - 1955: (9) "Rocky Mountain Vegetation" - 1956: (12) Merrill, Elmer Drew, 1876-1956 • 1957: (12) "Rocky Mountain Vegetation" • 1959: (1) • 1960: (1) "Rocky Mountain Vegetation" • 1962: (1) • 1964: (2) • 1966: (10) - 1967: (7) • 1969: (3) Banister, John, 1650-1692 • 1971: (2) • 1974: (1) • 1976: (2) • 1977: (1) • 1978: (1) $\bullet 1981:$ (3) 1982: (1) - 1983: (5) Letters to/from R. P. W. Visser re: Verdoorn

Verdoorn-Hunik, Johanna F. (1909-) Husband is Frans Antonie Verdoorn ; nickname "Joop"

Biofile

Correspondence $\bullet$ 1965: (2) $\bullet$ 1984: (2) • 1985: (1) • 1987: (2) • 1988: (2)

Verey, Rosemary Husband is David Verey Correspondence • 1986: (2)

Verhoek, Susan Bailey Hortorium, Visiting Fellow Correspondence 1982 : (2)

Verity, David S. (1930-) U. of California, Los Angeles, Mathias Botanical Garden, Herbarium Correspondence • 1977: (5) • 1979: (4) • 1980: (3) • 1984: (3) - 1986: (2) • 1987: (2)

Vernon, Ethel S. New Orleans, LA Botanical Album

Correspondence • 1966: (1) • 1969: (1) • 1975: (2) • 1977: (1) • 1979: (1)

Vernon, Tom Botanical Album

Vernon, William Bioscript

Verret, Joey Northwestern State U. of Louisiana, Natchitoches Correspondence • 1976: (2)

Verschaffelt, Amboise Alexander (1825-1886) Bioscript

Vestal, Arthur Gibson (1888-1964) Ecologist Bioscript Correspondence • 1949: (1)

Vianna, Fernando Segadas Museu Nacional Divisao de Botânica, Rio de Janeiro, Brazil Correspondence $\bullet$ 1955: (2)

Viccars, Marion U. of West Florida, Pensacola Correspondence • 1976: (4) • 1977: (4) • 1978: (2) • 1979: (5) • 1980: (1)

Vick, James (1818-1882) American seedsman, publisher; Rochester, NY; Vick's Illustrated Monthly Magazine Bioscript - Botanical Album

Vickery, Robert Kingston (1922-) American plant geneticist; U. of Utah, Salt Lake City, Professor of Biology Correspondence $\bullet$ 1970: (4) • 1971: (1)

Vickey Book Service Correspondence • 1977: (1)

Victoria and Albert Museum London, England Correspondence • 1971: (1) Species 
Vieillot, Louis Pierre (1748-1831) French naturalist, ornithologist

Biofile - Bioscript

Vienna Natural History Museum Christa Riedl-Dorn Correspondence • 1992: (3)

Vignoles

Bioscript

Vigors, Nicholas Aylward (1785-1840) Bioscript

Viguerie, Joseph Correspondence • 1969: (1)

Villar, Domenique Bioscript

Vilman, M. (fl. 1930) McGregor Museum, Kimberley Botanical Album

Vilmorin, Eliza de Bioscript

Vilmorin, Henri L. de Bioscript

Vilmorin, Louis L. de Bioscript

Vilmorin, Maurice L. de Bioscript

Vincent, Edith M. (1885-1963) Librarian; Field Museum of Natural History, Chicago, IL, Dept. of Botany Correspondence • 1941: (3) • 1943: (3) • 1944: (4) Delphinium

Vincent, John Huntington Hartford Foundation Correspondence $\bullet$ 1964: (1)

Vines, Robert A. Museum of Natural History of Houston, TX

Botanical Album

Correspondence • 1938: (1) • 1949: (2) • 1950: (2) • 1951: (2) • 1952: (1)

Vines, Sidney $\mathbf{H}$. Bioscript

Vinzent, Charles (1816-1882) German botanist in Texas Biofile

Viosca, Paul Percy (1892-1961) Naturalist, author; New Orleans, LA

Bioscript - Botanical Album

Correspondence • 1953: (1)

Vira, Raghu Advancing Frontiers of Science Series, New Delhi, India Correspondence • 1961: (1)

Virginia Museum of Natural History Martinsville, Richard L. Hoffiman, Curator, Recent Invertebrates Correspondence • 1991: (4) Banister, John, 1650-1692

Virginia State Library Brent Tarter, Dictionary of Virginia Biography, Editor

Correspondence - 1990: (2)

Virville, Adrien Davy de Bryologist, phytogeographer; France; Secretaire de la Section Histoire de la Botanique VIII Congress International de Botanique Correspondence • 1953: (3)

Visser, Dorothy D. Correspondence $\bullet$ 1972: (2)

Viviani, Domenico Bioscript

Voallaire, Marc

Bioscript
Voeller, Bruce Raymond (1934) American plant physiologist; Rockefeller U.

Correspondence $\bullet 1968$ : (2)

Vogel, Benedict Christian (1745-1825) German botanist, physician

Bioscript

Vogel, Jacob

Bioscript

Vogel, Theodore

Bioscript

Vogelenzang, L. Librarian; Rijksherbarium, Leiden, Holland

Correspondence • 1958: (1) • 1979: (0) Letter filed under J. Cramer

Vogelmann, Hubert Walker (192\& ) American taxonomist, plant ecologist; U. of Vermont, Burlington, Botany Dept. Correspondence $\bullet 1958$ : (2)

Vogt, George L. Correspondence $\bullet$ 1980: (2)

Vogts, Marie Murray South African horticulturist, author Botanical Album

Voise, Waldemar XI International Congress of the History of Science

Correspondence • 1965: (3) Bartram, William, 1739-1823 - 1966: (1) Bartram, William, 1739-1823

Vokes, Emily Hoskins Tulane University, New Orleans, Geology

Bioscript

Correspondence $\bullet$ 1972: (1) $\bullet$ 1978: (0) Letter filed under Harold Vokes

Vokes, Harold

Correspondence $\bullet 1978$ : (2)

Vollmer, Albert Michael Bioscript

Volney, Constantin-Francois (1757-1820) Bioscript

Volodarsky, Alexander XIII Congres International D'Histoire Des Sciences, Moscow, Russia, Secretary Correspondence $\bullet 1971$ :

Volozay, D. A. Bioscript

Volpe, Carolyn Correspondence $\bullet$ 1977: (3)

Volpe, Erminio Peter (1927-) Zoologist; Tulane U., New Orleans, Dept. of Biology

Bioscript $\bullet$ Botanical Album

Correspondence $\bullet$ 1965: (5) $\bullet$ 1965: (0) Letter filed under Conway Zirkle • 1972: (1) • 1976: (1) • 1977: (1) • 1978: (2) - 1980: (1)

Volpe, John A. Dept. of Transportation, Washington, D.C. Correspondence $\bullet 1971$ : (1)

Voltaire Bioscript

Volz, Friedrich Bioscript

Vosper, Robert Librarian; U. of California Correspondence $\bullet 1965$ : (1) $\bullet$ 1967: (3) Letter to Henry Roemol 
Voss, Edward Grosebeck (1929-) American botanist; U. of Michigan, Ann Arbor, Herbarium

Biofile • Bioscript

Correspondence • 1957: (2) • 1961: (1) • 1966: (2) • 1968: (3) Letter to Reed C. Rollins • 1969: (1) Bartram, William, 1739-1823 • 1969: (0) Letter filed under Michael Woldenberg • 1972: (2) • 1976: (3) • 1977: (2) Barton, Benjamin Smith, 1766-1815 • 1978: (1) • 1979: (1) • 1980: (3) • 1981: (1) Pursh, Frederick, 1774-1820 • 1990: (1)

Voss, John W. (1912-)

Bioscript

Voznsenskii, I'ia Gavrilovich (1816-1871) Russian naturalist in California and Mexico

Biofile - Bioscript • Botanical Album

Vreeland, Benjamin (-1866) Plant collector, Greenland Biofile - Bioscript

Vreeland, Frederick King (1874 ) Bioscript

Vries, Hugo de (1848-1935) Dutch plant anatomist, plant physiologist, plant breeder Biofile $\bullet$ Botanical Album

Vrolik, Wilhelm

Bioscript

Vrugtman, Freek Royal Botanic Gardens, Hamilton, Ontario, Curator of Collections Correspondence • 1979: (3)

Vrugtman, Ina Correspondence • 1989: (2)

\section{W}

W. \& G. Foyle Ltd. The World's Greatest Bookshop, Charing Cross Road, London Correspondence • 1951: (1)

Wachtel, Harvey J. Harvard University Correspondence - 1956: (2)

Wade, David H. U. of Oregon, Eugene, Herbarium, Dept. of Biology Correspondence • 1979: (2)

Wade, Joseph M. Bioscript

Wade, L. Keith Botanic Garden, Vancouver, B.C. Correspondence • 1978: (1) • 1979: (8) Wade, Walter (1740/60?-1825) Irish taxonomist, surgeon
Biofile - Bioscript

Wagener, Willis Westlake (1892-) Plant pathologist; U.S.D.A. Correspondence • 1935: (1)

Wagenknecht, Burdette L. Taxonomist; U. of Kansas, Lawrence; Arnold Arboretum Correspondence • 1957: (1) - 1958: (3)

Waggeman, Jane F. Tulane University, New Orleans, Graduate School Correspondence $\bullet$ 1948: (0) Letter filed under Armando
Dugand Wagner, Adam S. Colorado Federation of Garden Clubs,
President

Correspondence • 1941: (3)
Wagner, David H. U. of Oregon, Eugene, Herbarium, Botanical Album

Correspondence • 1978: (1) • 1980: (2) • 1982: (1) • 1983: (3) Van Schaacks, George Booth, 1903-1983 • 1984 (1)

Wagner, Florence S. (1919- ) American pteridologist Correspondence • 1973: (4) Letter from Connie G. Griffith

Wagner, Warren Herbert (1920- ) U. of Michigan, Dept. of Botany; nickname "Herb"

Bioscript • Botanical Album

Correspondence • 1951: (1) • 1952: (1) • 1953: (2)

American Fern Society $\bullet$ 1956: (2) • 1958: (12) • 1961:

(4) $\bullet$ 1963: (6) • 1966: (2) Species $\bullet$ 1970: (4) $\bullet$ 1971: (2)

Species - 1974: (3) - 1976: (2) Copy of letter between

Warren Wagner and F. H. Sargent • 1977: (2) - 1978: (3)

- 1985: (1) • 1988: (1)

Wagoner, Bill

Correspondence 1954 (0) Filed under June Wagoner

Wagoner, June

Correspondence • 1954: (1)

Wagstaffe, R. City of Liverpool Public Museums, Curator of Birds

Correspondence $\bullet$ 1955: (4) $\bullet$ 1957: (2)

Waibel, Leo

Biofile

Wailes, Benjamin Leonard Covington (1797-1862) Bioscript

Wait, Lucita Fairchild Tropical Garden Correspondence • 1969: (1)

Waits, Leslie $\mathbf{M}$.

Correspondence • 1951: (2) - 1952: (1)

Wakefield, G. R. Stonehurst Estates

Correspondence • 1965: (2) • 1966: (1)

Wakefield, Priscilla (1751-1832) British author, philanthropist

Biofile • Bioscript

Wakeley, Philip Carman (1902-) U. S. Dept. of Agriculture, Forest Service, New Orleans, LA Correspondence • 1950: (2) • 1963: (1) • 1964: (3) • 1965: (1)

Wakeley, William Henry (1857-1925) Taxidermist; CA Biofile

Walckenear, Charles Athanase (1771-1852) Bioscript

Walcott, Charles Doolittle (1850-1927) American paleontologist

Bioscript

Wald, George Harvard U., Professor of Botany Bioscript

Correspondence $\bullet$ 1969: (1) Copy of letter sent to Hugh Downs, NBC

Waldrop, A. Gayle U. of Colorado, Boulder Correspondence • 1940: (1) • 1942: (4) • 1956: (2)

Walford, George W. London bookseller Correspondence • 1959: (1)

Walgreen, Myrtle (1879-) Phytogeographer Biofile

Walker, Charles A., Jr.

Correspondence • 1992: (3) 
Walker, Charles Ralph (1897- ) American biologist; Western State College, Gunnison, CO Bioscript Correspondence • 1949: (1)

Walker, D. Australian National U., Dept. of Biogeography \& Geomorphology Correspondence • 1979: (2)

Walker, Egbert Hamilton (1899-1991) American botanist, botanical bibliographer; Smithsonian Institution Biofile • Bioscript • Botanical Album Correspondence $\bullet$ 1947: (0) Letter filed under John E. Flynn • 1947: (2) • 1948: (2) $\bullet$ 1950: (4) $\bullet$ 1951: (6) $\bullet$ 1952: (2) • 1957: (4) • 1958: (1) - 1962: (1) • 1963: (0) Letter filed under Frans Antonie Stafleu $\bullet$ 1963: (6) - 1966: (4) Bartram, William, 1739-1823 • 1969: (1) • 1970: (1) • 1971: (1) • 1974: (1) • 1975: (1) • 1976: (3) Merrill, Elmer Drew, 1876-1956 - 1986: (1) Letter from daughter, Jeanne Walker Houghton $\bullet$ 1990: (1)

Walker, Ernest Pillsbury (1891-) American mammalogist, conservationist, botanical collector; National Zoological Park, Washington, D.C.

Bioscript $\bullet$ Botanical Album

Correspondence • 1949: (3)

Walker, Harriet Bioscript $\bullet$ Botanical Album

Walker, Henry U. of Alabama, Dept. of Biology Correspondence $\bullet$ 1962: (3)

Walker, James Willard, Jr. (1943- ) American taxonomist, plant collector; Harvard U., Gray Herbarium Botanical Album Correspondence $\bullet$ 1966: (1)

Walker, John (1731-1803) Scottish botanist, clergyman Biofile

Walker, Joseph Henry U. of Alabama, Tuscaloosa, Dept. of Biology Correspondence • 1959: (2)

Walker, Louise U. of Oregon, Science Library Correspondence $\bullet$ 1981: (1)

Walker, Mary Husband is Eugene Walker Correspondence 1982: (2)

Walker, Sarah Bennett Amateur botanist, Colorado Bioscript Correspondence $\bullet$ 1942: (1)

Walker, Thelma Retcliffe Bioscript

Walker, William C. Bioscript

Wall, Bennett H. Tulane U., Dept. of History Correspondence $\bullet$ 1974: (2)

Wall, Faye A. Colorado Federation of Garden Clubs; husband is G. A. Wall Correspondence $\bullet$ 1942: (1)

Wall, J. R. Texas Tech, Lubbock Correspondence $\bullet$ 1967: (2)

Wallace, Alexander Doniphon (1905-) Tulane U., New Orleans, LA, Mathematics Dept. Correspondence $\bullet 1961:$ (1) $\bullet 1963:$ (1)

Wallace, Alfred Russel (1823-1913) British explorer, zoologist, botanist Biofile $\bullet$ Bioscript $\bullet$ Botanical Album
Wallace, Don Tulane U., New Orleans, LA Correspondence • 1962: (1)

Wallace, Gary Dean (1946-) American plant taxonomist, phytogeographer; Arboreta and Botanic Gardens, County of Los Angeles, CA; Natural History Museum, Los Angeles. Botany

Bioscript

Correspondence • 1976: (1) • 1989: (1) • 1990: (3) • 1991: (1)

Wallace, James Westwood ( -1838) Bioscript

Wallace, Robert ( - 1838) British gardener, plant collector Bioscript

Wallace, William Allen (1815-1893) American farmer, historian, freelance writer, amateur botanist Biofile - Bioscript

Waller, Adolph Edward (1892-) American plant ecologist, plant pathologist; Ohio State U., Columbus Bioscript

Correspondence • 1946: (1) • 1957: (3) • 1967: (0) Letters filed under Herbert George Baker

Wallich, Nathaniel (1786-1854) (nee Nathan Wulff [Wolff]) Danish physician, botanist

Bioscript • Botanical Album

Wallihan, A. G., Mrs. Bioscript

Wallroth, [Carl] Friedrich William (1792-1857) German botanist Bioscript

Walmsley, Thomas Bioscript

Walmsley, William Mason Bioscript

Walpole, Frederick Andrews (1861-1904) American botanical artist Biofile • Bioscript

Walpole, Michael Correspondence • 1975: (1) • 1977: (3) Book collectors

Walsh, Hu Botanical Album

Walsh, Thomas J. National Botanic Garden, Dublin Correspondence • 1969: (1) Royal Botanic Gardens, Kew

Walsh, Wendy Botanical Album

Walsingham, Lord [Thomas De Grey] (1843-1919) English entomologist Biofile

Walter, Ray Limestone County Historian Correspondence $\bullet 1966$ : (1)

Walter, Thomas (1740-1789) British-born botanist in North America Biofile • Bioscript

Walters, Stuart Max (1920-) English taxonomist, plant collector; U. of Cambridge, Botany School Botanical Album Correspondence $\bullet$ 1963: (1)

Walther, Eric (1892-1959) German-born horticulturist; Golden Gate Park, San Francisco, CA Bioscript $\bullet$ Botanical Album 
Walther, Henry, Jr. Chamber of Commerce, New Orleans, LA

Correspondence $\bullet$ 1959: (1) • 1960: (0) Letter filed under De Lesseps S. Morrison

Walton, Robert (1875-1893) Bioscript

Walton, Thomas E. Botanical Album

Wamsley, James S. The Commonwealth: The Magazine of Virginia Correspondence $\bullet 1961$ : (1)

Wang, Arthur W. Hill and Wang, publisher Correspondence • 1986: (3)

Wangenheim, Friedrich Adam Julius von (1749-1800) German forester Bioscript

Warburg, Edmund Frederic (1908-1966) English botanist; U. of Oxford, Dept. of Botany, Oxford, England Botanical Album Correspondence • 1956: (1) • 1957: (1) • 1961: (1)

Ward, B. O. Mississippi Southern College, Hattiesburg, Dept. of Biology Correspondence $\bullet$ 1959: (3)

Ward, Brenda Missouri Botanical Garden, St. Louis, MO Botanical Album (Filed under Brian Ward)

Ward, Brian Missouri Botanical Garden, St. Louis, MO Botanical Album

Ward, Daniel Bertram (1928-) American taxonomist, plant collector; U. of Florida, Gainesville, Herbarium Correspondence $\bullet$ 1970: (3) • 1976: (1)

Ward, Francis Kingdon See Kingdon-Ward, Francis (1885-1958)

Ward, George B. Texas State Historical Association Correspondence • 1992: (0) Letter filed under Bernard L. (Barney) Lipscomb

Ward, Grace Mandeville, LA Correspondence • 1968: (1) • 1969: (1) • 1977: (1) • 1979: (1)

Ward, H. G. British attache in Mexico Bioscript

Ward, Henry Augustus (1834-1906) American natural history merchant Biofile - Bioscript - Botanical Album

Ward, Henry Baldwin (1865-1945) American parasitologist Bioscript

Ward, Ivor Jesmond (1908-1947) English-born Canadian entomologist Bioscript

Ward, James Bioscript $\bullet$ Botanical Album

Ward, Lester Frank (1841-1913) American botanist, philosopher, sociologist Biofile - Bioscript - Botanical Album

Ward, Malthus A. Ward (17941863) American botanist, physician Biofile

Ward, Nathaniel Bagshaw (1791-1868) British physician, botanist, horticulturist Biofile $\bullet$ Bioscript $\bullet$ Botanical Album
Ward, O. J. (Jim) Edgar B. Stern Estate, Metairie,

LA

Correspondence $\bullet$ 1960: (1) • 1961: (2) • 1963: (2) $\bullet$

1965: (3) - 1968: (1) 1969: (1) Biology-Instruction and study • 1970: (2) • 1973: (1) • 1975: (1) • 1976: (3) • 1977: (2) • 1987: (1)

Warden, David Bailie (1772-1845) Irish-born American Consul in the diplomatic service Biofile

Wards Natural Science Establishment Botanical Album

Ware, Bioscript

Ware, George Northwestern State College, Natchitoches, LA Correspondence $\bullet$ 1962: (4)

Ware, John White (1795-1864) American physician, professor of medicine Biofile • Bioscript

Ware, Nathaniel A. (1780-1854) Bioscript

Ware, Robert A. Correspondence • 1929-1933: (5)

Warfel, Harry R. Scholars Facsimiles \& Reprints, Gainesville, FL, editor Correspondence $\bullet$ 1959: (1)

Waring, J. J., Mrs. Denver, CO Correspondence $\bullet$ 1940: (1)

Waring, James U. of Colorado, Denver, School of Medicine Correspondence $\bullet$ 1946: (1)

Waring, Nell-Pape Correspondence $\bullet$ 1969: (2)

Warming, Johannes Eugenius Bülow (1841-1924) Danish botanist Biofile - Bioscript $\bullet$ Botanical Album

Warne, Henry A. Bioscript

Warner, Dwain W. Bioscript

Warner, Mary B. Correspondence $\bullet$ 1941: (1)

Warner, Ralph U. of Colorado, Dept. of Spanish Correspondence • 1942: (2)

Warnock, Barton Holland (1911-) American plant taxonomist; Sul Ross State College, Alpine, TX Botanical Album Correspondence $\bullet$ 1956: (1)

Warnock, Michael J. (1956-) Sam Houston State U., Huntsville, TX Correspondence $\bullet$ 1986: (1) $\bullet$ 1989: (0) Letter filed under Nancy Ruth Morin

Warp, Oscar Warp Publishing Correspondence $\bullet$ 1942: (2)

Warre, Henry James (1819-1898) Topographical artist, British Army

Bioscript

Warren, Edward R., Mrs. Correspondence • 1963: (1) 
Warren, Edward Royal (1860-1942) American ornithologist, mammologist Bioscript

Warren, Fred Adelbert (1902- ) American botanist; Belfair, WA

Correspondence • 1941: (3) Delphinium • 1948: (2) Species

Warren, Gouverneur Kemble (1830-1882) American engineer, explorer, topographer Biofile

Warren, I. W. P. Summer student Correspondence • 1943: (1)

Warren, I. W. P., Mrs. Correspondence $\bullet$ 1942: (1)

Warren, J. A. Correspondence $\bullet$ 1940: (2)

Warren, John Bioscript

Warren, John Byrne Leicester (Lord de Tabley) (1835-1895)

Bioscript - Botanical Album

Warren, Joy F. Tougaloo, MS; husband is Harold C. Warren

Correspondence $\bullet$ 1952: (1)

Warren, Patricia J. Jefferson County Historical Society, Port Townsend, WA

Correspondence $\bullet$ 1992: (1) $\bullet$ 1992: (0) Filed with Eric William Groves

Warren Wilson College Correspondence • 1970: (1)

Warsaw

Botanical Album

Warszewicz, Joseph von Rawicz (1812-1866)

Lithuanian-born gardener and plant collector Biofile

Warwickshire County Records Office Deputy County Archivist, Monica Ory

Correspondence • 1983: (5)

Waserman, Manfred Correspondence $\bullet$ 1984: (1)

Washington, George (1732-1799) First president of the U.S., plant collector Biofile - Bioscript

Washington State University, Pullman Botanical Album

Wasshausen, Dieter Carl (1938- ) German-born botanist, taxonomist; Smithsonian, Dept. Botany Correspondence • 1978: (1) • 1983: (1)

Waterfall, Umaldy Theodore (1910-1971) American taxonomist; U. of Oklahoma, Norman, Dept. of Plant Sciences; Texas A \& M; Oklahoma State U., Stillwater Correspondence • 1948: (1) Delphinium • 1957: (7) • 1967: (3)

Waterfield, Robin E. David Low, Booksellers, Ltd. Correspondence 1943 : (1)

Waterhouse, Benjamin (1754-1846) American physician and naturalist Biofile - Bioscript

Waterhouse, John Fothergill ( -1817)
Waterman, George Arthur (1872-1960) American psychotherapist, shell collector

Biofile

Waterman, Robert

Correspondence • 1938: (13)

Waterman, Warren Gookin (1872-1952) American plant ecologist; Northwestern U., Professor of Botany Correspondence $\bullet$ 1950: (3)

Waterton, Charles (1782-1865) English traveler, naturalist Biofile • Bioscript $\bullet$ Botanical Album

Watkins, Charles Alan Appalachian Cultural Center Correspondence $\bullet$ 1985: (3)

Watkins, G. M. Texas A \& M U., Dept. of Plant Sciences Correspondence $\bullet$ 1969: (1)

Watkins, John Bioscript

Watson, George E. Smithsonian Institution, Div, of Birds Correspondence $\bullet$ 1966: (1) $\bullet$ 1985: (1) $\bullet$ 1990: (1)

Watson, H. Clay New Orleans Jazz Museum and Archives Correspondence $\bullet$ 1963: (2)

Watson, Henri W. Correspondence $\bullet$ 1954: (1) $\bullet$ 1957: (4)

Watson, Hewett Cottrell (1804-1881) British botanist and phrenologist Biofile - Bioscript

Watson, Joseph Ralph (18741946) Bioscript

Watson, Julie Correspondence $\bullet$ 1962: (2)

Watson, _ Mrs. Correspondence $\bullet$ 1957: (3)

Watson, Samuel Harding (1837-1928) English-born American botanist Biofile

Watson, Sereno (1826-1892) American botanist, taxonomist, bibliographer Bioscript

Watson, William Kew Books Correspondence $\bullet 1975:$ (4)

Watt, James Bioscript

Watts, Elizabeth Tulane U., New Orleans, LA Correspondence $\bullet 1979$ : (1)

Wauchope, Robert (1910?-1979) American anthropologist; Tulane U., New Orleans, Middle American Research Institute

Bioscript

Correspondence • 1953: (1) • 1957: (2) • 1958: (2) • 1964: (1)

Wawra, Heinrich Ritter von Fornsee (1831-1887) Austrian physician, botanist, plant collector Bioscript

Wayer, Natalie Tulane U., New Orleans, Howard-Tilton Library Correspondence $\bullet$ 1970: (1)

Weatherby, Charles Alfired, Mrs. Cambridge, MA Correspondence $\bullet$ 1952: (1)

Weatherby, Charles Alfred (1875-1949) American pteridologist; Harvard, Gray Herbarium; American Fern Society, President Bioscript $\bullet$ Botanical Album 


\section{Guide to the Ewan Papers}

Weatherby, Charles Alfred (1875-1949) (continued) Correspondence • 1929-1933: (13) Delphinium • 1935: (2) Species • 1936: (1) • 1938: (3) • 1939: (6) Species • 1940: (6) letters from Jacques Cattrall, American Fern Society • 1941: (3) • 1942: (3) • 1943: (16) • 1944: (6) American Fern Society • 1945: (3) - 1946: (4) Letter from Harrold G. Rugg • 1947: (2)

Weathers, Philip Syon House, Brentford, Middlesex Correspondence • 1969: (0) Filed under Ray Desmond

Weatherwax, Paul (1888-1976) American botanist Biofile - Botanical Album

Weaver, J. Calvin Correspondence • 1954: (1)

Weaver, John Ernst Botanical Album

Weaver, John Bioscript

Weaver, Mabel Dudley Herbarium Correspondence • 1942: (1) • 1944: (1)

Weaver, Marvin B. U. S. Commercial Company Correspondence • 1948: (1)

Weaver, Richard Harvard University, Arnold Arboretum Jamaica Plain, MA

Correspondence $\bullet$ 1971: (1) $\bullet$ 1982: (4)

Webb, David Allardice (1912-) Irish plant ecologist; Trinity College, Dublin

Botanical Album

Correspondence • 1955: (4) • 1956: (2) • 1964: (2) • 1969: (1) • 1991: (1) Stafleu, Frans Antonie, 1921-

Webb, Homer L.

Correspondence • 1961: (0) Filed under Waldemar H. Fries

Webb, Mildred L. U. of Pennsylvania, Philadelphia Correspondence • 1945: (1)

Webb, Philip Barker (1793-1854) British naturalist, traveler, geologist

Biofile - Bioscript

Webb, R. G. U. S. Dept. of Agriculture, Beltsville, MD

Correspondence • 1984: (1)

Webb, Robert G. U. of Texas, El Paso Correspondence • 1986: (2)

Webb, Thompson, Jr. U. of Wisconsin Press, Madison Correspondence • 1961: (2) • 1962: (6) Banister, John, 1650-1692; British Museum (Natural History) • 1964: (4) Banister, John, 1650-1692

Webber, David Gould (1809-) Bioscript

Webber, Herbert John (1865-1940) American botanist Bioscript

Webber, John

Bioscript

Weber, Jeff Rare bookseller Correspondence • 1990: (2)

Weber, Neal A. Entomologist; Tallahassee, FL Correspondence • 1991: (1) • 1992: (1)

Weber, William Alfred (1918-) American taxonomist; U. of Colorado, Boulder, Museum Bioscript • Botanical Album Correspondence • 1940: (5) • 1941: (4) • 1943: (5) • 1946: (6) • 1947: (9) • 1948: (9) • 1949: (7) • 1950: (5) Delphinium $\bullet$ 1951: (7) Cockerell, Theodore Dru Alison,
Weber, William Alfred (1918- ) (continued) 1866-1948; Delphinium • 1952: (2) Cockerell, Theodore Dru Alison, 1866-1948 • 1956: (3) • 1961: (1) • 1962: (1) - 1966: (1) Cockerell, Theodore Dru Alison, 1866-1948 • 1967: (2) • 1972: (4) • 1973: (3) • 1974: (1) • 1976: (5) Rocky Mountain Naturalists • 1977: (3) Rocky Mountain Naturalists • 1980: (1) • 1981: (5) • 1983: (3) • 1990: (5) - 1991: (2) Reference to Steve Darwin $\bullet$ 1992: (2) U. of Colorado, Boulder, Dept. of Biology

Weber-van Bosse, Anna Antoinette (1852-1942) Botanical Album

Weberbauer, August Bioscript

Webert, Henry Francis T. Nicholls State College, Thibodaux, Dept. of Biological Sciences Correspondence $\bullet$ 1968: (1)

Webster, Barbara D. U. of California, Davis, Dept. of Agronomy and Range Sciences

Correspondence $\bullet$ 1976: (3) $\bullet$ 1978: (0) Letters filed under Ronald Lewis Stuckey $\bullet$ 1978: (2)

Webster, Charles D. The Horticultural Society of New York Correspondence $\bullet$ 1969: (5) $\bullet$ 1975: (3)

Webster, Grady Linder (1927-) American botanist, taxonomist, plant collector; Purdue U., Lafayette, IN, Dept. of Biological Sciences; U. of California, Davis, Dept. of Botany; National Science Foundation

Bioscript • Botanical Album

Correspondence $\bullet$ 1962: (3) • 1976: (1) • 1977: (3) • 1981: (1) • 1982: (1) • 1983: (1) • 1988: (1) • 1991: (2)

Weddell, Hugh Algernon (1819-1877) British-born French botanist, physician

Bioscript

Wedgewood, Julia Bioscript

Weed, Bioscript

Weed, Amos (1828-1918) American plant collector, carpenter and farmer Biofile

Weed, Charles L. Bioscript

Weeks, Josephine Correspondence $\bullet$ 1970: (1)

Weeks, Mangum Alexandria, VA Botanical Album Correspondence $\bullet$ 1961: (3) • 1963: (10) • 1965: (8) • 1966: (6) Bartram, William, 1739-1823 - 1968: (5) 1969: (1) Royal Botanic Gardens, Kew • 1971: (4) • 1972: (1) $\bullet$ 1975: (2)

Weese, Asa Orrin (1885-1955) American zoologist; National Research Council Correspondence $\bullet$ 1939: (1)

Weidner, Earl Louisiana State U., Baton Rouge, Dept. of Zoology Correspondence $\bullet$ 1991: (1)

Weimerskirch, Philip J. U. of Rochester Medical Center, Edward G. Miner Library Correspondence • 1979: (1) 
Weinglass, D. H. U. of Missouri, Kansas City, Dept. of English Correspondence $\bullet$ 1983: (2) $\bullet$ 1984: (1)

Weishaught, Clara Gertrude (1898-1991) American taxonomist; Ohio State U., botany teacher Biofile - Bioscript

Weismann, August (1834-1914) German zoologist and geneticist

Biofile

Weisner, Conrad Bioscript

Weiss, Caroline Correspondence $\bullet$ 1977: (2)

Weiss, Grace M. Ziegler Husband is Harry B. Weiss Correspondence • 1964: (1)

Weiss, Harry B. Entomologist; The Past Times Press, Trenton, NJ

Bioscript

Correspondence - 1959: (4) - 1960: (1) Banister, John, 1650-1692 • 1963: (2) Banister, John, 1650-1692

Weiss, Leon C. Correspondence $\bullet$ 1981: (1)

Welch, Winona Hazel (1896-1991) American taxonomist, plant ecologist, bryologist; DePauw U., Greencastle, IN, Professor of Botany

Correspondence • 1958: (4)

Weld, Isaac

Bioscript

Welden, Arthur Luna (1927- ) American mycologist; Tulane U., Newcomb College, New Orleans, LA Bioscript • Botanical Album Correspondence • 1956: (1) • 1957: (5) • 1958: (2) • 1959: (4) • 1963: (1) • 1964: (3) • 1967: (3) • 1969: (2) • 1971: (1) • 1972: (12) • 1973: (3) • 1974: (1) • 1975: (1) 1977: (0) Letter filed under John Wesley Grear and Howard Samuel Irwin, Jr. $\bullet$ 1980: (1) • 1987: (4) • 1988: (5) Copy of letter from Reba Alpert $\bullet$ 1990: (3) 1991: (3) - 1992: (5) - 1993: (1)

Welden, Charles W. Ft. Collins, CO, graduate student; son of Arthur Luna Welden

Correspondence $\bullet$ 1979: (2)

Welden, Frances Husband is Arthur L. Welden Correspondence • 1963: (1) • 1972: (1) • 1979: (1) • 1986: (1)

Welden, Franz Ludwig von (1782-1853) Bioscript

Weldon, George Percival (1880-) American economic botanist

Bioscript

Wellcome Historical Medical Library London, England Correspondence - 1960: (2) • 1991: (1)

Welle, Ben J. H. ter Symposium Tropical Botany, Secretary Correspondence 1986: (8)

Wells, Ellen B. Smithsonian Institution Libraries Bioscript

Correspondence - 1985: (5)

Wells, Francis

Correspondence • 1973: (1)

Wells, Jane Alumni Relations, U. of Colorado

Correspondence $\bullet$ 1941: (1)
Wells, John

Bioscript

Correspondence 1971: (1)

Wells, Lester G. Syracuse U. Library, Syracuse, NY Curator

Correspondence • 1951: (2) • 1952: (0) Letters filed under Frans Antonie Verdoorn

Wells, William Charles Bioscript

Welwitsch, Friedrich Martin Josef (1806-1872) Austrian botanist

Biofile - Bioscript

Wemple, Don Kimberly (1929-) American taxonomist: Iowa State U., Ames

Correspondence $\bullet$ 1964: (2) $\bullet$ 1965: (1)

Wendell, William Tulane U., Dept. of Biochemistry Correspondence • 1968: (1)

Wendland, Hermann (1825-1903) German botanist, gardener

Bioscript

Went, Frits Warmolt (1903-1990) Dutch-born American plant physiologist; California Institute of Technology; Missouri Botanical Garden, St. Louis, MO Biofile $\bullet$ Bioscript $\bullet$ Botanical Album Correspondence • 1949: (1) • 1961: (1)

Wentworth, Edward N. Armour and Company, Chicago. IL

Correspondence $\bullet$ 1951: (2)

Wernecke, J. B. E. Botanical Album

Werthner, William Bioscript

Wesmael, Alfred (1832-1905) Belgian botanist, horticulturist

Bioscript

West Chester State College West Chester, PA Correspondence $\bullet$ 1951: (1) $\bullet$ 1981: (2)

West, Erdman (1894-1965) American botanist, mycologist; U. of Florida, Gainesville

Botanical Album

Correspondence • 1946: (2) Species • 1947: (1)

West, Hugh Stanford U. Correspondence • 1974: (2)

West, Robert C. Louisiana State U., Baton Rouge, Anthropology

Correspondence $\bullet 1984:(1)$

Westbrook, Bruce Correspondence $\bullet$ 1990: (1)

Westfeldt, Thomas D. New Orleans, LA Botanical Album Correspondence $\bullet$ 1963: (1) $\bullet$ 1964: (1)

Weston, Richard (1733-1806) British agricultural writer. thread-hosier Bioscript

Wetherill, John W. (1866-1944) American archeologist Bioscript

Wetmore, (Frank) Alexander (1886-1978) American ornithologist and biologist, Smithsonian Institution, Administrator, Washington, D.C.; National Academy of Science; nicknames "Alex"

Biofile $\bullet$ Bioscript $\bullet$ Botanical Album 


\section{Guide to the Ewan Papers}

Wetmore, (Frank) Alexander (1886-1978) (continued) Correspondence - 1929-1933: Maxon, William Ralph, 1877-1948 • 1947: (3) Vismia • 1949: (1) • 1958: (4) • 1960: (2) Banks, Joseph, Sir, 1743-1800 • 1961: (3) • 1969: (3) Banister, John, 1650-1692; Bartram, William, 1739-1823; Royal Botanic Gardens, Kew • 1974: (4)

Wetmore, Rae Wetmore Declamation Bureau Correspondence • 1949: (2)

Wettstein, Richard Bioscript $\bullet$ Botanical Album

Wheeler, Alwyne C. British Museum (Natural History), Dept. of Zoology; nickname "Wyn"

Botanical Album

Correspondence • 1974: (6) $\bullet$ 1980: (1) $\bullet$ 1981: (2) • 1982: (5) • 1983: (3) • 1984: (3) • 1985: (1) • 1986: (1) • 1987: (2) • 1988: (3) • 1991: (8)

Wheeler, Benjamin Ide Botanical Album

Wheeler, Betty Barnes Foundation Arboretum Correspondence $\bullet$ 1974: (1)

Wheeler, George Montague (1842-1905) Bioscript

Wheeler, Helen-Mar U. of California, Berkeley, Dept. of Botany

Correspondence $\bullet$ 1950: (3) Jepson, Willis Linn, 1867-1946

Wheeler, Herbert Newell

Bioscript $\bullet$ Botanical Album

Wheeler, Leota Husband is Louis Cutter Wheeler Correspondence • 1982: (2) • 1983: (2) • 1984: (1)

Wheeler, Leston Ansel Botanical Album

Wheeler, Louis Cutter (1910-1980) American botanist; U. of Southern California, Los Angeles Biofile - Bioscript - Botanical Album Correspondence $\bullet$ 1929-1933: (16) Munz, Philip Alexander, 1892-1974 • 1934: (0) Letter filed under Robert J. Kessler - 1935: (14) • 1936: (14) • 1937: (13) • 1938: (12) • 1939: (8) Species • 1940: (6) • 1941: (2) • 1942: (13) Jepson, Willis Linn, 1867-1946 • 1945: (3) • 1948: (3) • 1951: (1) • 1952: (1) • 1957: (1) • 1959: (2) Includes letter to Robert J. Kessler from Ewan - 1960: (2)• 1963: (0) Letter filed under John William Thieret and reply from J. Ewan • 1968: (1) Jepson, Willis Linn, 1867-1946 • 1971: (5) - 1973: (1) - 1974: (3) Jepson, Willis Linn, 1867-1946 - 1975: (2) • 1976: (2) • 1977: (6) • 1978: (2) • 1979: • 1980: (2)

Wheeler, Otis B. Louisiana State U., Baton Rouge, Acting Chancellor Correspondence $\bullet$ 1981: (1)

Wheeler, P. R., Mrs. New Orleans, LA Correspondence $\bullet$ 1961: (1)

Wheeler, W. F., Mrs.

Bioscript

Wheeler, Walter Bioscript

Wheeler, William Morton (1865-1937) American zoologist Biofile - Bioscript • Botanical Album
Wheeler, Willis Hayes U. S. Dept. of Agriculture, Plant Quarantine Branch; brother Louis Cutter Wheeler Botanical Album

Correspondence $\bullet$ 1956: (3) $\bullet$ 1965: (1) $\bullet$ 1983: (1)

Wheellock, W. D. Royal Palm State Park, FL Correspondence • 1929-1933: (1)

Wheldon \& Wesley, Limited Correspondence $\bullet$ 1946: (1) Book collectors $\bullet$ 1947: (2) • 1956: (2) • 1973: (3)

Wherry, Edgar Theodore (1885-1982) American mineralogist, chemist and botanist; U. of Pennsylvania, Philadelphia

Biofile $\bullet$ Bioscript $\bullet$ Botanical Album

Correspondence $\bullet$ 1937: (4) American Fern Society; Species • 1938: (5) • 1939: (2) • 1940: (1) • 1941: (5) • 1942: (2) • 1943: (4) • 1946: (1) American Fern Society $\bullet$ 1948: (3) Species $\bullet$ 1950: (2) $\bullet$ 1952: (4) $\bullet$ 1969: (2) • 1973: (1) • 1976: (2) • 1979: (1)

Whetzel, Herbert Hice (1877-1944) American mycologist and phytopathologist Biofile

Whipple, Amiel Weeks (1816-1863) U.S. Army officer, topographer

Bioscript

Whitaker, Robert H. U. of California, Irvine, Dept. of Population and Environmental Biology

Correspondence $\bullet$ 1968: (2)

Whitaker, Thomas Wallace (1905-1993) American plant geneticist; U. S. Dept. of Agriculture, Horticultural Field Station, La Jolla, CA

Biofile - Bioscript

Correspondence 1966: (3) Bartram, William, 1739-1823 - 1967: (3) - 1968: (2) • 1969: (5) Bartram, William, 1739-1823 1975 : (1) Copy of correspondence with Fredi Chiappeli 1 1978: (3) 1987 : (2)

Whitam, Kem Plant pathologist; Louisiana Cooperative Extension, Baton Rouge Correspondence $\bullet$ 1978: (1)

Whitcraft, Thos. E. Dept. of Interior Correspondence $\bullet$ 1936: (1)

White, Charles Abiathar (1826-1910) Bioscript

White, David (1862-1935) American paleontologist Biofile

White, David A. Loyola U., New Orleans, LA, Biological Sciences

Bioscript $\bullet$ Botanical Album

Correspondence $\bullet$ 1975: (4) • 1987: (1) • 1988: (5) • 1990: (1) • 1991: (7) • 1992: (1) • 1993: (2)

White, George Albert (1930-) American agronomist; U. S. Dept. of Agriculture, Germplasm Resources Laboratory Correspondence $\bullet$ 1973: (4)

White, George Willard (1903- ) American geologist; U. of Illinois, Urbana, Dept. of Geology

Biofile

Correspondence - 1963: (3) Letter to Jeannette Graustein • 1964: (1) • 1965: (5) 1 1966: (7) Banister, John, 1650-1692 • 1967: (6) • 1969: (8) Rocky Mountain Naturalists • 1970: (2) • 1974: (3) • 1976: (2) • 1977: (3) - 1981: (1) • 1982: (1) • 1984: (1) 
White, Gilbert (1720-1793) British curate and naturalist Biofile $\bullet$ Bioscript

White, James (fl. 1867) Bioscript

White, James J. National Museum of Natural History, Smithsonian Institution, Washington, D.C.; Hunt Institute, Curator of Art; Hunt Botanical Library

Correspondence $\bullet$ 1971: (4) $\bullet$ 1974: (2) • 1975: (2) • 1976: (1) • 1978: (2) • 1979: (0) Filed under Harry McCall, Jr. • 1981: (3) • 1985: (4) • 1986: (1)

White, John (1756-1832) Irish naval surgeon and botanist Biofile

White, John (of Virginia) Bioscript

White, Joseph J. Bioscript

White, Joshua Elder Bioscript

White, Lynn U. of California, Los Angeles, Dept. of History

Botanical Album

Correspondence $\bullet$ 1969: (2)

White, Mary

Bioscript

White, , Mrs.

Correspondence $\bullet$ 1921-1928: (1)

White, Orland Emile

Bioscript $\bullet$ Botanical Album

White, Philip R.

Botanical Album

White, Stephen S. Agricultural Experiment Station; Servicio Cooperativo Inter-Americano de Agricultura, Quito, Ecuador

Botanical Album

Correspondence • 1949: (1) • 1952: (1) • 1953: (3)

White, Taylor Bioscript

White, Terence Hanbury Indian-born British author Biofile

White, W. H. Correspondence $\bullet$ 1947: (1)

Whiteaves, Joseph Frederick (1835-1909) British-born Canadian paleontologist, conchologist Bioscript

Whitehead, Jack Botanical Album

Whitehead, Peter P. J. Biologist, ichthyologist; British Museum (Natural History); Centro de Investigaciones, Biológicas, Mexico

Biofile - Bioscript $\bullet$ Botanical Album Correspondence $\bullet$ 1973: (1) • 1974: (8) $\bullet$ 1975: (1) 1977: (6) • 1978: (4) • 1979: (6) • 1980: (3) $\bullet$ 1982: (2) 1983: (2) • 1984: (2) $\bullet$ 1987: (2) • 1990: (1) $\bullet 1991:(1) \bullet$ 1992: (1)

Whitehill, Walter Muir (1905-) Librarian; Boston Athenaeum Bioscript

Whitehouse, Eula (1892-1974) Southern Methodist U., Dallas, TX, Dept. of Botany Correspondence $\bullet$ 1958: (3)
Whitelock, Otto V. St. Encyclopedia Americana Correspondence • 1961: (0) Filed under Encyclopedia Americana

Whitfield, Mary Croom Tallahassee, FL Correspondence $\bullet$ 1952: (1)

Whitford, Harry Nichols (1872-1941) Bioscript

Whitley, Gilbert Percy (1903-1975) British-born Australian zoologist; Australian Museum, Sydney

Biofile Correspondence • 1974: (2) • 1975: (1) • 1976: (1) • 1976: (0) Letter filed under John R. Paxton

Whitlow, Charles (1776-1829) Bioscript

Whitman, C. Bioscript

Whitman, Charles Otis (1842-1910) American zoologist Biofile

Whitmire, Mildred E. Correspondence $\bullet$ 1971: (1)

Whitmore, Frank C. Correspondence $\bullet$ 1977: (1)

Whitney, Elsie Gibson American Fern Society, Secretary Bioscript Correspondence $\bullet$ 1939: (1) $\bullet$ 1940: (7) • 1941: (5) 1942: (3) • 1943: (1) • 1944: (2) $\bullet$ 1945: (4) • 1946: (1) - 1947: (5) - 1949: (1) $\bullet$ 1950: (1) - 1970: (1)

Whitney, Gordon G. Ecologist; Harvard U., Harvard Forest, Petersham, MA Correspondence $\bullet$ 1988: (1)

Whitney, Jane Husband is Morgan Whitney Botanical Album Correspondence • 1948: (1) • 1976: (3) • 1983: (2) • 1984: (2)

Whitney, Josiah Dwight (1819-1896) American geologist, paleontologist Biofile - Bioscript

Whitney, Leo D. U. of Hawaii Bioscript Correspondence $\bullet$ 1936: (3) • 1937: (2)

Whittaker, Robert H. (1920-1980) American plant ecologist Biofile

Whittemore, Alan T. Bioscript Correspondence $\bullet$ 1993: (2)

Whittemore, Dorothy J. Tulane U., Howard-Tilton Memorial Library, Acting Library Director Correspondence • 1968: (1) • 1976: (3) • 1978: (2)

Whittemore, Thomas Correspondence $\bullet$ 1976: (1)

Whittlesey, Charles (1808-1886) American soldier. engineer, geologist Biofile

Who Knows and What Correspondence $\bullet$ 1949: (2) Who's Who in America Chicago, IL Correspondence $\bullet$ 1948: (1)

Who's Who in Science and Engineering Wilmette,

$$
\text { II } \text { Correspondence } \bullet \text { 1991: (1) }
$$




\section{Guide to the Ewan Papers}

Whyte, Margaret H. U. of Chicago; Botanical Gazette Correspondence • 1935: (1)

Wickerby, Harry Forbes Botanical Album

Wickham, Henry Frederick (1866-1933) Bioscript

Wickson, G. C. California Historical Society Quarterly, Editor Correspondence $\bullet$ 1956: (1)

Wickstrom, Johannes E. Bioscript

Widmann, Berthold Author; Missouri Correspondence • 1921-1928: (2)

Widmann, Otto Botanical Album

Wiebelt, Ralph G. Metairie, LA Correspondence • 1958: (1)

Wied-Neuwied See Maximilian, Alexander Philip, Prince of Neuwied (1782-1867)

Wiedorn, Mr. Correspondence • 1957: (1)

Wiegand, Karl McKay (1873-1942) American botanist; Cornell U. Botanical Album Correspondence • 1937: (1)

Wiehe, Paul Octave ( -1975) Floreal, Mauritius, Indian Ocean and Paris, France; Agricultural Research Station, Likuni, Lilongew, Nyasaland, Africa

Botanical Album

Correspondence • 1945: (4) - 1946: (2) • 1947: (1) • 1948: (5) • 1949: (1) • 1952: (1)

Wieland, George Reber (1865-1953) American paleobotanist

Biofile $\bullet$ Botanical Album

Wiencke, Gus Minerals Museum, Blue Ridge Parkway Correspondence $\bullet$ 1978: (2)

Wientraut, Frances Botanical Album

Wiesener, Otto A. Charlottenlund, Denmark Correspondence $\bullet$ 1958: (1) $\bullet$ 1959: (2)

Wiggins, Dorothy Menlo Park, CA Correspondence $\bullet$ 1988: (4)

Wiggins, Ira Loren (1899-1987) American botanist: Stanford U., Stanford, CA, Dudley Museum; American Fern Society

Biofile • Bioscript $\bullet$ Botanical Album Correspondence • 1942: (2) • 1943: (1) • 1948: (1) • 1950: (1) - 1957: (3) • 1958: (3) • 1959: (0) Letter filed under Lyman David Benson • 1959: (1) • 1962: (3) Beaglehole, John Cawte 1963 : (4) Copy with note to Lois Stone • 1964: (2) • 1966: (1) • 1974: (1)

Wight, Robert

Bioscript

Wilbur, Robert Lynch (1925-) Duke U., Durham, Herbarium Curator; U. of Michigan, Ann Arbor, Curator, Herbarium

\section{Botanical Album}

Correspondence $\bullet$ 1951: (1) $\bullet$ 1952: (7) $\bullet$ 1960: (3) $\bullet$ 1961: (4) • 1967: (1) • 1968: (3) • 1978: (2) • 1988: (4)
Wilcox, Earley Vernon (1869-) American botanist entomologist Bioscript

Wilcox, Ernest N. Bioscript

Wilcoxson, E. M. Field Museum of Natural History Correspondence $\bullet$ 1935: (1) • 1939: (1)

Wilczek, Ernst Botanical Album

Wild, Gwendolin State College of Washington, Pullman Correspondence $\bullet$ 1954: (1)

Wilde, Walter Botanical Album

Wildenow, C. Bioscript

Wilder, Charlotte May Bioscript Correspondence • 1936: (2) • 1938: (2)

Wilder, Marshall P. C Bioscript

Wiley, Farida A. American Museum of Natural History Botanical Album Correspondence 1949: (1)

Wiley, $\mathbf{H}$. Correspondence $\bullet$ 1993: (1)

Wilken, Dieter H. (1944-) Occidental College, Dept. of Biology, Assistant Professor Correspondence $\bullet$ 1972: (4)

Wilkens, Hans (1898-) German-born amateur botanist; Reading, PA

Botanical Album

Correspondence • 1941: (3) • 1942: (3) • 1948: (1) 1950: (3) - 1953: (3) Rocky Mountain Naturalists; Pennell, Francis Whittier, 1886-1952 • 1969: (4)

Wilkes, Charles (1798-1877) Bioscript

Wilkes, Hilbert Garrison (1937- ) U. of Massachusetts, Boston

Bioscript $\bullet$ Botanical Album

Correspondence - 1966: (1) - 1968: (3) Book collectors; copy to Eric Lundberg; $\bullet$ 1970: (11) $\bullet 1971$ : (6) $\bullet 1972$ : (6) $\bullet$ 1973: (5) $\bullet$ 1974: (3) $\bullet$ 1975: (16) Correspondence w/ Arthur Herschman and w/ Carol L. Rogers $\bullet 1976:$ (14) 1977: (7) • 1978: (6) • 1979: (2) • 1980: (2) • 1981: (1) • 1982: (7) • 1983: (1) • 1985: (1) • 1992: (1)

Wilkes, Susan Husband is Hilbert Garrison Wilkes Correspondence • 1968: (1) • 1973: (1) • 1974:

Wilkins, Alice London, England; husband is Guy Wilkins Correspondence $\bullet$ 1957: (15) $\bullet$ 1958: (6)

Wilkins, Guy L. Conchologist; British Museum (Natural History)

Correspondence • 1956: (4) • 1957: (1)

Wilkinson, Edward (1846-) American sheet metal worker, naturalist, plant collector Biofile

Wilkinson, John Botanical Album

Wilkinson, Norman B. Correspondence $\bullet$ 1972: (1) 
Wilkinson, Ronald Sterne British Museum (Natural History). London, England; Library of Congress. Washington, D.C.

Bioscript

Correspondence • 1975: (4) • 1976: (18) • 1977: (6) Harper, Francis, 1886-1972 • 1983: (5) • 1984: (5) • 1985: (2)

Willard, Alex Bioscript

Willdenow, Karl Ludwig (1765-1812) German bounist Biofile - Bloscript

Willet, George Los Angeles Museum Natural Histony Correspondence • 1944: (1)

Willey, Basil Bloscript

William, Coxe (1762-1831) Bioscript

William H. Allen Co. Bookseller; Philadelphih, PA Correspondence • 1952: (2)

William \& Wilkins $\mathrm{C}_{0}$. Correspondence • 1949: (1)

Williams, Austin B. Systematic zologist; U. S. National Museum

Correspondence • 1973: (4)

Williams, Benjamin J. Correspondence • 1975: (1)

Williams, Carolyn E. National Museum of Natural History, Dept. of Botany. Washington, D.C

Correspondence • 1990: (2) • 1991: (2)

Williams, Damaris D. Bed and Brcalfast proprietor aear Kew Gardens

Correspondence • 1956: (4) • 1958: (1) • 1959: (2) • 1960: (2) • 1962: (1) • 1963: (1)

Willams, Emile Francis (1858-1929) American civi engineer, collector Biofile

Willams, Francis Xavier (1882-) Bioscripi

Walliams, Frank Correspondence • 1929-1933: (1)

Willams, Frank B., Mrs. Garden Stady Cab of New Orleans, Metaire, LA

Correspondence • 1970: (3)

Willams, Iolo Aneurin (1890-1962) Englink journalist, bibliographer, amateur botanist, ornithologist Biofile

Williams, John Lee

Bioscripi

Williams, L. H. John Botanist; British Museen (Navural History)

Correspondence • 1958: (2)

Williams, Lee Terry Many, LA Correspondence • 1952: (4) - 1953: (0) American Ferm Society: Filed with Ralph Curtis Benodict

Wulliams, Loring R. Reno, NV; Northern Nevada Native Plant Society (NNNPS)

Correcpondence • 1979: (1) • 1980: (1) • 1992: (3)

Willians, Louks G. Furman U.

Correspondence - 1950: (I5)
Williams, Louis Otho (1908-1991) American botanist; Harvard U. Museum; Escuela Agricola Panamericana, Tegucigalpa, Honduras; Chicago Natural History Museum, II

\section{Botanical Album}

Correspondence • 1938: (1) • 1951: (2) • 1953: (2) Pursh, Frederick, 1774-1820 • 1956: (4) • 1963: (2) • 1964: (1) • 1966: (1) • 1976: (1) • 1978: (2) Merrill, Elmer Drew, 1876-1956 • 1982: (1)

Williams, Margaret J. Reno, NV; Northern Nevada Native Plant Society (NNPS); husband is Loring Williams Botanical Album

Correspondence • 1978: (3) • 1981: (3) • 1986: (2)

Williams, R. N., 2nd Historical Society of Pennsylvania Correspondence $\bullet 1959$ : (1)

Williams, Richmond D. Eleutherian Mills Historical Library, Greenville, DE Correspondence $\bullet 1965$ : (2)

Williams, Robert Statham (1859-1945) American bryologist, businessman, miner, explorer, ornithologist Biofile • Bioscript $\bullet$ Botanical Album

Williams, Roger L. U. of Wyoming, Laramie, Prof. of History Correspondence $\bullet$ 1982: (2)

Williams, S. Wells Bioscript

Williams, Thomas Albert (1865-1900) American botanist Bioscript

Williams, W. D. Botanical Album

Williams, William P. California School of Natural Science Correspondence $\bullet$ 1937: (6)

Wiliamsburg Botanical Album

Williamsburg, Colonial Foundation Thomas B. Schlesinger, Director Correspondence $\bullet 1977$ : (1)

Williamson, Bruce G. Louisiana State Library, Baton Rouge Correspondence $\bullet$ 1983: (1)

Whiliamson, C. S. Bioscript

Willianson, E. Tulane U., New Orleans Correspondence $\bullet 1957$ : (1)

Willianson, G. B. Correspondence • 1982: (1)

Williamson, Flugh Biascript

Willianson, John Biascript

Williamson, Karen D. Artist; Fox Sudio, Denver, CO Correspondence • 1979: (0) Letter filed under Sidney Dillon Ripiey $\bullet$ 1982: (1)

Williamsoa, Robert Stockton (1824-1882) Topographer;

U. S. Army, Pacific R. R. Survey Bioscript

Whatharson, Velma Middleturg. FL Correspondence $\bullet$ 1958: (2)

Willamsen, W. C. Bioseript 


\section{Guide to the Ewan Papers}

Willis, John Christopher (1868-1958) British botanist Bioscript • Botanical Album

Willis, Oliver Rivington (1815-1902) American school teacher, botanist Bioscript

Willmott, Ellen Ann Bioscript

Wills, Morris U. of California, Berkeley, Dept. of Geography Correspondence • 1979: (3)

Willson, E. J. Correspondence • 1963: (3) • 1964: (4) • 1969: (2)

Willughby, Francis Bioscript

Wils, Bourse Bioscript

Wilson, Albert Botanist; Garden Consultant, Menlo Park, CA Correspondence • 1951: (1) • 1952: (1)

Wilson, Alexander (1766-1813) Scottish-born American ornithologist Biofile - Bioscript

Wilson, Belle Correspondence • 1951: (2)

Wilson, Burt Francis T. Nocholls State College, Thibodaux, LA

Correspondence • 1970: (1)

Wilson, Charles R. Encyclopedia of Southern Culture, Baton Rouge, LA Correspondence • 1982: (4)

Wilson, Christopher U. of Wisconsin, Madison, Dept. of Botany

Correspondence • 1978: (2)

Wilson, Clifford The Beaver, Editor; Hudson Bay House, Winnipeg, Manitoba, Canada Correspondence • 1951: (1)

Wilson, Conrad Correspondence • 1980: (5) Bartram, William, 1739-1823

Wilson, D. V. Royal Botanic Gardens Correspondence - 1974: (1) Royal Botanic Gardens, Kew

Wilson, Edward Osborn Botanical Album

Wilson, Ernest Henry (1876-1930) British-born American botanist, plant collector, traveller Biofile - Botanical Album

Wilson, George ([?]1814-1894) Bioscript

Wilson, George Warren Attorney for Ynex Mexia Correspondence • 1941: (3)

Wilson, Hollis R. Southeastern Louisiana College, Hammond, LA; nickname "Roomie" Correspondence $\bullet$ 1952: (2)

Wilson, Hugh D. Indiana U., Bloomington, Graduate Student; U. of Wyoming, Laramie, Rocky Mountain Herbarium

Correspondence • 1972: (1) • 1977: (2) BiologyInstruction and study

Wilson, J. Canterbury Museum Correspondence • 1974: (1)
Wilson, Kate Penguin Books, Ltd.

Correspondence $\bullet$ 1976: (0) Correspondence filed with David Elliston Allen

Wilson, Leonard G. Journal of the History of Medicine and Allied Sciences, Book Review Editor; U. of Minnesota, Minneapolis, Division of Medical History

Correspondence • 1962: (4) Beaglehole, John Cawte • 1964: (4) • 1967: (2) • 1978: (1) • 1979: (1)

Wilson, Louise Palmer (1903-) Wellesley College, Wellesley, MA, Dept. of Biology Correspondence • 1964: (2) • 1965: (7)

Wilson, M. F. U. of Illinois, Urbana-Champaign Correspondence • 1974: (1)

Wilson, N. Manager, Hotel Latham, NY Correspondence $\bullet$ 1956: (1)

Wilson, Norman C. Bioscript - Botanical Album

Wilson (of Washington, D.C.) Bioscript

Wilson, Percy (1879-1944) American botanist; New York Botanical Garden Botanical Album

Wilson, Rene New Orleans, LA Correspondence • 1951: (1) • 1977: (1) • 1987: (1) - 1988: (2)

Wilson, Robert Gardner (1911-1989) American horticulturist Biofile

Wilson, Sam Prescott, AK Correspondence • 1987: (1)

Wilson, Thomas B. Bioscript

Wilson, W. Bioscript

Wilson, William Bioscript

Wilson, William P. Bioscript

Winberry, Carolyn J. U. of South Carolina, Columbia, Science Librarian Correspondence $\bullet$ 1986: (1) • 1987: (1)

Winge, Ovind (1886-1964) Danish botanist Bioscript

Wingfield, Edmund D. New Orieans, LA Correspondence $\bullet$ 1970: (0) Filed under Longue Vue Gardens

Winslow, Charles Frederick (1811-1877) Geologist, physicist

Bioscript

Winsor, Mary P. U. of Toronto, Toronto, Canada Correspondence $\bullet$ 1991: (2)

Winter, Howard A. U. of Southern California, Los Angeles; Allan Hancock Foundation Correspondence • 1955: (1) • 1956: (1)

Winterbotham, William Bioscript

Wirt, Elizabeth American author Biofile

Wisdom, William B. New Orleans, LA Correspondence • 1967: (2) 
Wiser, Vivian Historian; United States Dept. of Agriculture, Washinton, D.C.

Correspondence • 1970: (3)

Wislizenus, Friedrich Adolph (1810-1889) German-born American physician, naturalist Bioscript

Wisner, Herb Correspondence • 1981: (1)

Wisniewski, Tad Archives of Plant Cartography, Warsaw, Poland Correspondence • 1938: (1)

Wistar, Charles Bioscript

Wistar, Isaac Jones (1827-1905) Bioscript

Withee, L. V. Kansas State, Manhattan, Professor of Agronomy Correspondence • 1992: (2)

Withering, William Bioscript

Withers, Robert Bioscript

Wittrock, Gustave Ludwig New York Botanical Garden Botanical Album

Correspondence • 1939: (3) Delphinium; Species • 1940: (1) • 1941: (6) • 1942: (2) • 1943: (4) Delphinium

Wittrock, Viet Brecher (1839-1914) Swedish taxonomist, plant collector, physiologist Bioscript

Woburn Abbey Librarian; J. Barton Correspondence • 1965: (2)

Wodehouse, Roger Philip (1889-1978) Canadian botanist, palynologist, allergist Biofile

Woldenberg, Michael Harvard Center for Environmental Design Studies

Correspondence - 1969: (4) Copy of correspondence with Edward G. Voss

Wolf, Carl Brandt (1905-1974) American botanist; Santa Ana Botanic Gardens

Biofile • Bioscript • Botanical Album

Correspondence • 1937: (1) • 1939: (1) • 1940: (3) Lyon, John, 1764-1814; Cockerell, Theodore Dru Alison, 1866-1948 • 1941: (2) • 1942: (3) • 1943: (4) Delphinium - 1945: (3)

Wolf, Clarence Correspondence $\bullet 1977$ : (1)

Wolf, Constance Paulette (1943-) American librarian; Missouri Botanical Garden, St. Louis, MO; nickname "Connie" Bioscript Correspondence • 1984: (1) • 1986: (5) • 1987: (4) • 1988: (2) • 1989: (0) Letter filed under Bi-Jun Yao • 1990: (0) Correspondence filed under Dale Edwin Johnson • 1990: (1) • 1991: (1) • 1993: (1)

Wolf, Edwin, II (1911-1991) American librarian; Library Company of Philadelphia Biofile - Bioscript

Correspondence • 1963: (1) • 1973: (1) • 1978: (1) • 1981: (3) • 1983: (1) • 1984: (2) $\bullet$ 1985: (2)
Wolf, Frederick T. Vanderbilt U., Nashville, TN, Dept. of Botany Correspondence $\bullet$ 1958: (2)

Wolf, John (1820-1897) American botanist, naturalist; member of Wheeler Geological Survey, 1873 Bioscript $\bullet$ Botanical Album

Wolf, John E., Jr. Baylor College of Medicine Correspondence $\bullet$ 1982: (1)

Wolf, Martha Leigh Historic Bartram's Garden, Philadelphia, PA

Correspondence $\bullet$ 1991: (1) $\bullet$ 1993: (2)

Wolfe, Elmer Ellsworth Botanical Album

Wolfe, Frederick J., Jr., Mrs. Sophie Gumbel School, Association for Retarded Children, New Orleans, LA Correspondence • 1971: (1) • 1972: (1)

Wolfe, James Jacob (1875-1920) American biologist Biofile

Wolfe, John N. Botanical Album

Wolfe, Leon Audubon Park Commission, New Orleans Correspondence • 1965: (1) Taylor, George, Sir, 1904

Wolfe, Mary Lou Librarian; Pennsylvania Horticultural Society Correspondence • 1976: (3) • 1977: (1) • 1980: (2)

Wolfe, Richard J. Francis A. Countway Library of Medicine Bioscript Correspondence $\bullet$ 1966: (2)

Wolf, Kaspar Friedrich (1733-1794) Bioscript

Wolfle, Dael Scientific Monthly, Editor Correspondence • 1955: (1)

Wollaston, A. F. R. Bioscript

Wolle, Francis U. of Colorado, Boulder, English Literature Correspondence $\bullet$ 1973: (1)

Wolle, Christian Jacob (1788-1863) Jamaican-born American botanist Bioscript • Botanical Album

Wolle, Muriel Sibell Artist, historian; Boulder, CO Botanical Album

Wong, Stephen Willis Correspondence • 1967: (1)

Wood, Alice Correspondence $\bullet$ 1942: (1)

Wood, Alphonso (1810-1881) American botanist and teacher Biofile - Bioscript

Wood, Carieton B. Correspondence • 1991: (4)

Wood, Carroll E., Jr. (1921-) American taxonomist; Harvard University, Arnold Arboretum Bioscript Correspondence • 1959: (2) • 1960: (3) • 1966: (8) • 1967: (4)

Wood, Casey A. Botanical Album 


\section{Guide to the Ewan Papers}

Wood, Charles B., III Antiquarian book collector and seller; Green South Woodstock, CT Correspondence • 1971: (6) • 1972: (5) • 1974: (2) • 1975: (2) • 1977: (2) • 1979: (1) Barton, Benjamin Smith, 1766-1815 • 1980: (2) • 1992: (2) • 1993: (1)

Wood, Fergus J. Environmental Science Services Administration, Coast and Geodetic Survey Correspondence • 1968: (1)

Wood, Horatio Bioscript

Wood, John Correspondence • 1985: (3) • 1986: (1)

Wood, Leonard E. U. S. Dept. of Defense, Advanced Research Projects Agency, Research and Development Field Unit, Dept. of Defense Correspondence • 1966: (1)

Wood, Raymond Donovan (1902-1964) American attorney, photographer of alpine wildflowers Biofile

Wood, Richard Dawson (1918- ) U. of Rhode Island, Kingston Correspondence $\bullet$ 1964: (2) $\bullet$ 1965: (3)

Wood, Virginia S. Boston U., Boston, Mugar Memorial Library Correspondence • 1977: (2) Banister, John, 1650-1692

Wood, Wallace Flockert (1909-) Mammologist Bioscript - Botanical Album Correspondence • 1939: (2)

Wood, William S. Zoological collector, taxidermist Bioscript

Woodbridge, Grace Correspondence • 1982: (1) • 1983: (1)

Woodburn, Elisabeth H. (1912-1990) American bookseller, collector; Booknoll Farm Bioscript • Botanical Album Correspondence • 1967: (2) • 1968: (5) Bartram, William, 1739-1823 • 1969: (5) Bartram, William, 1739-1823 • 1971: (3) • 1972: (3) - 1973: (2) • 1974: (6) • 1975: (1) • 1976: (1) • 1977: (6) • 1978: (1) • 1979: (1) • 1980: (2) • 1981: (3) - 1982: (3) • 1983: (1) 1984: (4) Lawrence, George Hill Mathewson, 1910-1978 • 1985: (3) • 1986: (8) - 1987: (6) - 1988: (2) - 1989: (0) Letter filed under John J. Baxevanis • 1989: (1) • 1990: (1) • 1992: (1) Letter from Ms. Brad Lyon

Woodbury, Angus M. (1886-) Bioscript

Woodcock, Fidella G. Botanical Album

Woodford, Charles Morris Bioscript

Woodhouse, James (1770-1890) Bioscript

Woodhouse, Samuel Washington (1821-1903) American physician, naturalist, assistant surgeon; U.S. Army Bioscript

Woodruff, Bess Botanical Album

Woodruff, Herbert A. Henry E. Huntington Library and Art Gallery

Correspondence • 1962: (1) Lyon, John, 1764-1814 ; Banister, John, 1650-1692; Banks, Joseph, Sir, 1743-1800
Woodruff, Lindsay Botanical Album

Woods, Dorothy E. Laguana, CA Correspondence $\bullet$ 1970: (5)

Woods, Frank U. of Tennessee, Knoxville, Dept. of Botany Correspondence $\bullet$ 1952: (1)

Woods Hole Marine Biological Laboratory Correspondence $\bullet$ 1974: (2)

Woodson, Robert Everard, Jr. (1904-1963) American plant taxonomist and plant geographer; Missouri Botanical Garden, St. Louis, MO Biofile • Bioscript $\bullet$ Botanical Album Correspondence • 1946: (2) • 1949: (5) • 1950: (6) • 1951: (2) • 1952: (2) • 1960: (1) • 1963: (1) Banister, John, 1650-1692

Woodward, Augustus Brevoort Bioscript

Woodward, Carol H. New York Botanical Garden, Editor; Brookdyn Botanic Garden, Editor

Botanical Album

Correspondence • 1942: (5) • 1944: (3) $\bullet$ 1945: (3) • 1946: (3) • 1947: (10) • 1948: (10) • 1949: (8) $\bullet 1950$ (13) - 1951: (9) • 1952: (3) • 1953: (4) Eastwood, Alice, 1859-1953 • 1954: (1) • 1955: (1) • 1956: (1) • 1968: (1) - 1974: (1)

Woodward, John (fl. 1695) Naturalist Biofile

Woodward, Ralph Correspondence $\bullet$ 1981: (1)

Woodward, Richard William (1846-1931) American chemist, amateur botanist Bioscript

Woodward, Shingo Bioscript Correspondence • 1986: (1)

Woodwell, George Masters (1928- ) American plant ecologist

Botanical Album

Woodworth, Charles William (1865-1940) Bioscript

Woody, Norman C. Tulane U., Professor of Pediatrics Correspondence • 1973: (0) Filed under James Polk Morris

Woolf, Harry U. of Washington, Seattle; Editor of Sources of Science

Correspondence • 1960: (1) • 1966: (2)

Woolward, Florence Botanical Album

Wooton, Elmer Ottis (1865-1945) American botanist Biofile - Bioscript $\bullet$ Botanical Album

Worcester County Horticulture Fayre Nason, Secretary Correspondence • 1962: (2)

Worcester, P. G. U. of Colorado, Boulder, Acting Dean Correspondence • 1943: (1)

Word, Ola Mae Long Vue Gardens, Metairie, LA, Public Relations Correspondence $\bullet$ 1970: (0) Correspondence filed under Longue Vue Gardens

Work, Marian McLellan Daughter of Dr. McLellan Correspondence • 1992: (1)

World and I Linda Joyce Forristal, Science Editor Correspondence • 1986: (1) 
World Magazine

Correspondence • 1973: (1)

World Wildife Fund Beth Ann Baillie, World Wildlife Fund, Peru, IN

Correspondence $\bullet$ 1987: (1) $\bullet$ 1990: (1)

Worley, Clair L. Botanical Album

Wormser, Richard S. Bookseller Correspondence $\bullet$ 1972: (1) Book collectors $\bullet$ 1973: (1)

Wormskjold, Morten (1783-1845) Bioscript

Worthington, Frank $\mathbf{R}$. Correspondence $\bullet$ 1954: (2)

Worthley, Elmer George (1921-) American agrostologist, bryologist

Bioscript

Correspondence • 1976: (1)

Woytkowski, Feliks (1892-1966) Polish naturalist in Peru Biofile

Wrangel, C. M. Bioscript

Wrangell, Ferdinand Petrovich von baron (1796-1870) Bioscript

Wray, Thomas Jarram (1781-1852) Bioscript

Wreden, William P. Bookseller Correspondence • 1990: (1) • 1992: (2) • 1993: (1)

Wright, Albert Allen (1846-1905) American botanist, geologist, zoologist; Oberlin College Bioscript

Wright, Albert Hazen American zoologist; Cornell U.; wife is Anna Allen Wright Botanical Album Correspondence $\bullet$ 1940: (1)

Wright, Anna Allen Husband is Albert Hazen Wright See Wright, Alben Hazen

Wright, Charles (1811-1889) American botanical collector Biofile - Bioscript - Botanical Album

Wright, E. Perceval Bioscript

Wright, Fred Correspondence • 1963: (1)

Wright, G. Fred Bioscript

Wright, Herbert E., Jr. U. of Minnesota, Minneapolis, School of Earth Sciences Correspondence • 1963: (2) • 1964: (5)

Wright, J. K. Bioscript

Wright, Louis B. Folger Shakespeare Library, Washington, D.C.

Correspondence $\bullet$ 1960: (1)

Wright, Samuel (1793-1841) Bioscript

Wright, Samuel Hart (1825-1905) American botanist, plant collector Bioscript • Botanical Album

Wright, James, Sir Bioscript
Wright, William Greenwood (ca. 1830-1912) American plant collector Bioscript • Botanical Album

Wright, William $\mathbf{H}$. Bioscript

Wright, Winthrop R. Swarthmore College, Swarthmore, PA, Dept. of Physics Correspondence $\bullet$ 1950: (4) $\bullet 1951:(9) \bullet 1952:(5) \bullet$ 1953: (1)

Wuerttemberg, Duke Friedrich Paul Wilhelm von Herzog (1797-1860) Bioscript

Wunderlin, Richard Paul (1939-) American taxonomist; U. of South Florida, Tampa, Curator of the Herbarium Correspondence $\bullet 1976$ : (0) Filed under University of South Florida • 1980: (2) • 1987: (7)

Wunner, R. (Bob) Arcata, CA Correspondence $\bullet$ 1988: (1)

Wurdack, John Julius (1921-) American taxonomist; New York Botanical Garden, New York, NY; Smithsonian Institution

Botanical Album

Correspondence $\bullet$ 1951: (1) $\bullet$ 1958: (2) $\bullet$ 1964: (1) $\bullet$ 1965: (9) Letter filed under John William Thieret $\bullet 1966$ :

(0) Letter filed under Marie Wurdack $\bullet$ 1967: (5) - 1968:

(1) $\bullet$ 1969: (4) $\bullet$ 1971: (14) $\bullet$ 1972: (5) Book collectors; Pennell, Francis Whittier, 1886-1952 - 1973: (1) • 1975:

(1) Book collectors $\bullet$ 1976: (1) $\bullet$ 1978: (1) - 1979: (3) $\bullet$ 1985: (1) • 1986: (3) • 1987: (1) • 1991: (1) • 1992: (1)

Wurdack, Kenneth John (1946-) Beltsville, MD Bioscript • Botanical Album Correspondence • 1984: (1) • 1987: (3) • 1988: (10) • 1989: (1) - 1990: (3)

Wurdack, Marie Correspondence $\bullet$ 1966: (1) $\bullet$ 1967: (2) • 1968: (1) $\bullet$ 1969: (1) • 1970: (7) • 1972: (1) • 1974: (3) Book collectors • 1975: (10) Book collectors • 1976: (1) • 1978: (1)

Wurdemann, John George F. (1810-1849) American physician, merchant Biofile • Bioscript

Wydler, Heinrich (1800-1883) Swiss botanist Bioscript

WYES New Orleans Public Broadcast Station Director of Broadcasting. Julius Cain; New Orleans, LA Correspondence • 1970: (1) • 1972: (1) • 1973: (5) • 1983: (2)

Wyeth, John B. Bioscript

Wyeth, Nathaniel Jarvis (1802-1856) Americain explorer Biofile • Bioscript

Wylie, Margery Correspondence $\bullet$ 1975: (1)

Wylly, Molly Audubon House and Gardens Correspondence $\bullet 1986$ : (1)

Wyman, Donald Bioscript

Wyman, Jeffiries (18141874) American physician, paleontologist Biofile - Bioscript 


\section{Guide to the Ewan Papers}

Wyne, Addison Correspondence • 1984: (1)

Wynne, Ross Brombaugh Botanical Album

Wyss-Dunant, Biofile

Wystrach, V. P. Wilton, CT Correspondence $\bullet 1978:$ (3)

Wythe, Margaret W. The Condor, Cooper Ornitholgical Club

Correspondence • 1935: (1) Delphinium

\section{$X-Y$}

Xantus, Louis John (1825-1894) Bioscript • Botanical Album

Yadon, Vernal L. Museum of Natural History, Pacific Grove, CA Correspondence • 1976: (4) Delphinium • 1982: (1)

Yale Forestry School Botanical Album

Yale University Press Jane Olsen, Editor Correspondence • 1965: (3) Banister, John, 1650-1692

Yao, BiJun Academia Sinica, Institutum Botanicum, Peking, China

Bioscript • Botanical Album

Correspondence - 1989 (3) Letter from Constance Wolf

Yama, George H. Pulaski, VA Correspondence • 1987: (1)

Yarrell, William Bioscript

Yarrow, Harry Crecy (1840-) Bioscript

Yarwood, C. E. Bioscript

Yatabe, $\mathbf{R}$. Bioscript

Yates, Harris Oliver (1934) American plant morphologist, plant cytologist; Vanderbilt University, Dept. of Biology Correspondence • 1963: (3)

Yates, Lorenzo Gordin (1837-1909) British-born American naturalist, pteridologist Biofile • Bioscript

Yates, Stanley Iowa State U., Ames, Library Correspondence $\bullet$ 1977: (2)

Yatskievych, George American botanist; Missouri Dept. of Conservation, St. Louis, MO

Botanical Album

Correspondence - 1988: (1) copy of letter from Alfred E. Schuyler • 1990: (0) Correspondence filed under Harriet Jansma • 1990: (3) • 1992: (1)

Yatskierych, Kay

Botanical Album

Correspondence • 1991: (1)

Yatsu, Lawrence Bioscript

Yen, Teng-Chien

Botanical Album

Yerbury, John William Bioscript
Yerkes, Roberta U. of Wisconsin Press Correspondence • 1963: (5) Banister, John, 1650-1692 • 1964: (1)

Yochelson, Ellis L. (fl. 1985) Paleontologist; United States Geological Survey

Biofile

Correspondence $\bullet$ 1972: (2) $\bullet$ 1973: (1) $\bullet$ 1977: (2) Copy of correspondence between Ellis L. Yochelson and Hemphill $\bullet$ 1985: (1) Letter to A.C. Rocha-Campos

Yocum, L. Edwin George Washington U., Washington, D.C. Correspondence $\bullet$ 1947: (0) Letter filed under Robert L. Stearns • 1947: (1)

Yongboonkird, Umpai (1928- ) Thai taxonomist; U. of Florida, Gainesville Correspondence $\bullet$ 1957: (2)

York, Harlan Harvey (1875-1961) American botanist Biofile

Youmans, E. L. Bioscript

Young, David (1942-) Correspondence • 1990: (1)

Young, Delor W. Correspondence $\bullet$ 1960: (1)

Young, John R. Bioscript

Young, Mary Sophie Bioscript

Young, Robert Thompson Bioscript

Young, Roy A. Oregon State U., Corvallis Correspondence • 1965: (1)

Young, Roy Alton(?) Botanical Album

Young, Thomas Botanical Album

Young, William, Jr. (1742-1785) German-born American botanist Biofile $\bullet$ Bioscript

Youngberg, Florence Pomona College, Herbarium Correspondence $\bullet$ 1938: (4) • 1939: (1) Species

Yu, Hsiew Correspondence • 1984: (2)

Yuncker, Truman George (1891-1964) American botanist; DePauw U., Greencastle, IN Biofile $\bullet$ Botanical Album Correspondence • 1939: (3) • 1940: (1) • 1941: (5) • 1942: (2) • 1943: (2) Delphinium • 1945: (1) • 1947: (4) - 1948: (1) • 1951: (5) • 1952: (2) • 1955: (1) • 1961: (6) - 1962: (1) - 1973: (1)

Yust, Walter Encyclopaedia Britannica Correspondence $\bullet$ 1958: (3)

\section{Z}

Zacher, Christian K. Ohio State U., Columbus, Columbian, Quincentenary Committee, Chairman Correspondence • 1991: (0) Filed under Ronald Lewis Stuckey

Zafer, Andrea Sierra Club Centennial Campaign Correspondence • 1993: (1) 
Zahm, John Bioscript

Zahrobsky, Adrienne Glen Ellyn, IL Correspondence • 1988: (1)

Zaninovich, Mark U. of California, Davis, Dept. of Viticulture and Oenology Correspondence $\bullet 1962$ :

Zantedeschi, Francesco (1797-1873) Bioscript

Zardini, Elsa Missouri Botanical Garden, St. Louis, MO, Assoc. Curator Botanical Album

Zaremba, Bohdan Bookseller; Cambridge, MA Correspondence • 1987: (1)

Zarn, D. Correspondence • 1976: (0) Filed under Simon Fraser University; Executive Secretary, Cook Conference

Zarucchi, James Lee Correspondence • 1990: (1)

Zeile, Elsie M. California Botanical Society Botanical Album Correspondence • 1929-1933: (2)

Zeitlin, Jacob Israel (1902-1986) Antiquarian book dealer; Zeitlin and VerBrugge Booksellers, Los Angeles, CA; Nickname "Jake" Biofile • Bioscript • Botanical Album Correspondence • 1965: (4) - 1966: (11) Book collectors • 1967: (11) Book collectors - 1968: (5) Lawrence, George Hill Mathewson, 1910-1978; Science-Instruction and study - 1973: (3) • 1974: (8) Book collectors • 1975: (14) Book collectors - 1976: (2) Book collectors $\bullet$ 1977: (2) $\bullet$ 1978: (6) $\bullet$ 1979: (3) letter to Jack and Josephine [VerBrugge]; Book collectors $\bullet$ 1980: (1) $\bullet$ 1981: (1) Book collectors $\bullet$ 1982: (1) • 1983: (5) • 1984: (4) • 1986: (3) • 1969: (8) • 1970: (1)

Zeitlin \& VerBrugge Booksellers; Los Angeles, CA Correspondence • 1969: (8) Book collectors • 1970: (1)

Zeky, Mohammed Amin Royal Egyptian Embassy Correspondence • 1947: (1)

Zemurray, Samuel (1877-1961) United Fruit Company, President Biofile Correspondence • 1948: (3)

Zetterstedt, Johann E. Bioscript

Zeyher, Johann Michael (1770-1843) Bioscript

Ziegler, Charles W., III Correspondence • 1965: (3) • 1968: (1)

Ziegler, Charles W., Mrs. Correspondence • 1973: (2)

Ziegler, Deborah C. New Orleans, LA Correspondence • 1966: (2) • 1969: (6) • 1970: (2) • 1971: (3) Species $\bullet 1972:(7) \bullet 1974:$ (1) $\bullet 1975$ : (3) $\bullet$ 1976: (2) • 1977: (3) Rocky Motuntain Naturalists; correspondence between Deborah Ziegler and Mrs. Ira Nelson

Zielinskd, Quentin (1919-) American plant geneticist; U. of Virginia, Boyce, Blandy Experimental Farm Correspondence • 1945: (1)
Zierer, Clifford M. U. of California, Dept. of Geography Correspondence • 1938: (1)

Zimman, Michael The Haydn Foundation Correspondence $\bullet$ 1976: (1)

Zimmer, John T. American Museum of Natural History Correspondence • 1943: (2)

Zimmerman, E. A. W. von Bioscript

Zimmerman, Elwood C. Entomologist, natural history, bookseller; Books for Naturalists, Peterborough, NH; Hunter House, Peterborough, NH; Bishop Museum; Newton Centre, MA; Nickname "Zimmie"

Botanical Album

Correspondence $\bullet$ 1943: (2) $\bullet$ 1957: (9) $\bullet$ 1957: (0)

Letter filed under Alice Wilkins - 1960: (9) Book collectors • 1961: (9) • 1963: (3) • 1964: (5) • 1965: (9) Book collectors - 1966: (3) Book collectors

Zimmerman, James H. Naturalist; U. of Wisconsin, Madison, Arboretum

Botanical Album

Correspondence • 1956: (4) Species • 1957: (1) • 1962:

(2) 1 1967: (1)

Zimmerman, Lois (Lampe) (1896-1971) Bioscript

Zimmerman, P. W. Boyce Thompson Institute for Plant Research, Inc., Yonkers, NY

Correspondence • 1948: (1) • 1952: (3) • 1953: (2)

Zimmerman, William

Botanical Album

Zimmermann, Martin H. Harvard U., Harvard Forest Correspondence • 1975: (1) • 1976: (1)

Zimmet, A. Office of the War Bond Office, Panama Canal Dept., Headquarters Correspondence • 1945: (1)

Zinsser, Hans (1878-1940) American physician, bacteriologist Biofile

Zirkle, Conway (1895-) American plant cytologist, science historian; U. of Pennsylvania, Philadelphia, Biology Dept. Bioscript Correspondence • 1948: (1) • 1965: (1) Letter to Peter Volpe • 1966: (6) Bartram, William, 1739-1823 • 1967: (6) 1968: (5) 1969: (6) Bartram, William, 1739-1823

Zobel, Henrietta L. U. of Colorado, Denver, Dept. of Botany Bioscript Correspondence • 1944: (1) • 1981: (1) Rocky Mountain Naturalist

Zomlefer, Wendy B. Florida Museum of Natural History, Gainesville Bioscript - Botanical Album Correspondence $\bullet$ 1991: (2)

Zoological Society New Orleans, LA Correspondence - 1971: (1)

Zorn, Jacob Bioscript

Zouche, Edward la Baron (ca. 1556-1625) English botanist Bioscript

Zucearini, Joseph Gerhard Bioscript 
Guide to the Ewan Papers

Zundel, George Lorenzo (1885-1950) American botanist Biofile

Zula, Floyd M. Tulane U., Howard-Tilton Library Botanical Album

Zwackh, W. von

Bioscript

Zwanziger, Gustav Adolf

Bioscript

Zwinger, Ann Haymond American traveler, writer, illustrator; Colorado Springs, CO

Bioscript - Botanical Album

Correspondence $\bullet$ 1985: (2)

Zwisohn, Jane Bookseller

Botanical Album

Correspondence • 1982: (1) • 1984: (3) • 1985: (9) •

1986: (1) 